Stephan Seiter

\title{
Der Beitrag Nicholas Kaldors zur Neuen Wachstumstheorie
}




\section{Der Beitrag Nicholas Kaldors zur Neuen Wachstumstheorie}

In den zentralen Modellen der Neuen Wachstumstheorie (NWT) wird durch die Einführung von Externalitäten bei der Akkumulation von Sach- und Humankapital endogenes Wachstum des Pro-Kopf-Einkommens möglich. Diese Vorgehensweise basiert auf Kenneth Arrows Konzept des learning by doing. Während dabei nachfrageseitige Aspekte des Wachstumsprozesses ausgeblendet werden, finden sich diese in den wachstumstheoretischen Arbeiten von Nicholas Kaldor. Die von ihm betonte Rolle steigender Skalenerträge und des damit verbundenen Verdoorn-Zusammenhangs bilden eine wichtige Parallele zur NWT. Anhand einer kritischen Darstellung und Gegenüberstellung beider Sichtweisen verdeutlicht der Autor die Relevanz der Beiträge Kaldors für die NWT und die Notwendigkeit einer integrierten Erklärung von Wachstumsprozessen.

Stephan Seiter, geboren 1963 in Stuttgart, studierte Wirtschaftswissenschaften an der Universität Hohenheim, wo er seit 1990 als wissenschaftlicher Mitarbeiter von Prof. Dr. Harald Hagemann am Institut für Volkswirtschaftslehre tätig ist. Forschungsinteressen: Wachstumstheorie, Technischer Fortschritt, Produktivitätsentwicklung und Beschäftigungstheorie. 
Der Beitrag Nicholas Kaldors zur Neuen Wachstumstheorie 


\title{
Hohenheimer Volkswirtschaftliche Schriften
}

\author{
Herausgegeben von \\ Prof. Dr. Rolf Caesar, Prof. Dr. Harald Hagemann, \\ Prof. Dr. Klaus Herdzina, Prof. Dr. Jörn Kruse, \\ Prof. Dr. Renate Ohr, Prof. Dr. Walter Piesch, Prof. Dr. Ingo Schmidt, \\ Prof. Dr. Peter Spahn, Prof. Dr. Gerhard Wagenhals, \\ Prof. Dr. Helmut Walter
}

\section{Band 27}

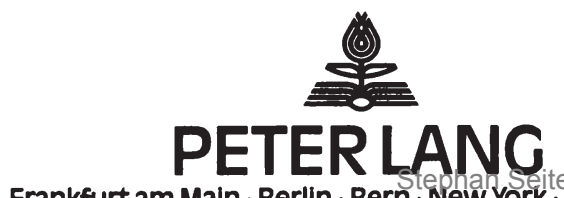

Frankfurt am Main . Berlin. Bern. New York. Parris. Wien 5 22:48AM 


\section{Stephan Seiter}

\section{Der Beitrag Nicholas Kaldors zur Neuen Wachstumstheorie}

Eine vergleichende Studie vor dem Hintergrund der Debatte über den Verdoorn-Zusammenhang

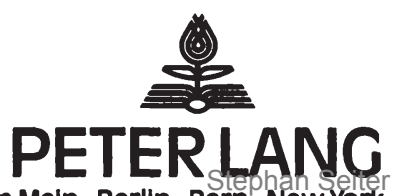

Frankfurt am Main. Berlin. Bern. New York, Parls i Mjen 22:48AM 
Die Deutsche Bibliothek - CIP-Einheitsaufnahme Seiter, Stephan:

Der Beitrag Nicholas Kaldors zur Neuen Wachstumstheorie : eine vergleichende Studie vor dem Hintergrund der Debatte über den Verdoorn-Zusammenhang / Stephan Seiter. - Frankfurt am Main ; Berlin ; Bern ; New York ; Paris ; Wien : Lang, 1997 (Hohenheimer volkswirtschaftliche Schriften ; Bd. 27) Zugl.: Hohenheim, Univ., Diss., 1996 ISBN 3-631-31672-0

NE: GT

Open Access: The online version of this publication is published on www.peterlang.com and www.econstor.eu under the international Creative Commons License CC-BY 4.0. Learn more on how you can use and share this work: http://creativecommons. org/licenses/by/4.0.

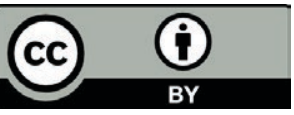

This book is available Open Access thanks to the kind support of ZBW - Leibniz-Informationszentrum Wirtschaft.

\author{
D 100 \\ ISSN 0721-3085 \\ ISBN 3-631-31672-0 \\ ISBN 978-3-631-75406-1 (eBook) \\ (C) Peter Lang GmbH \\ Europäischer Verlag der Wissenschaften \\ Frankfurt am Main 1997 \\ Alle Rechte vorbehalten.
}

Das Werk einschließlich aller seiner Teile ist urheberrechtlich geschützt. Jede Verwertung außerhalb der engen Grenzen des Urheberrechtsgesetzes ist ohne Zustimmung des Verlages unzulässig und strafbar. Das gilt insbesondere für

Vervielfältigungen, Übersetzungen, Mikroverfilmungen und die Einspeicherung und Verarbeitung in elektronischen Systemen. 
Meinem Sohn Tim, 21/2, den dieses Buch wohl im Augenblick nicht interessieren wird, da es weder Bilder von Baggern noch von Eisenbahnen enthält. 
Stephan Seiter - 978-3-631-75406-1

Downloaded from PubFactory at 01/11/2019 05:22:48AM

via free access 


\section{Vorwort}

Die Erstellung einer Dissertationsschrift ist normalerweise durch eine Reihe konjunktureller Schwankungen der Arbeitsmotivation gekennzeichnet, die durch den Einfluß vieler Menschen geglättet werden. Mein erster Dank gilt an dieser Stelle meinem Doktorvater Prof. Dr. Harald Hagemann, der mit seiner Politik der "offenen Tür" und seinem persönlichem Engagement nicht nur die Entstehung dieser Arbeit ermöglicht hat, sondern auch meinen Blick über den Tellerrand des eigenen Themas hinaus gelenkt hat. Herm Prof. Dr. Helmut Walter und Herm Prof. Dr. Klaus Herdzina danke ich für anregende Diskussionen und ihre Mitwirkung an meinem Promotionsverfahren.

Einen wichtigen Beitrag haben auch meine Kollegen am Institut für Volkswirtschaftslehre der Universität Hohenheim durch ihre Diskussionsbereitschaft im offiziellen Doktorandenseminar und in den Kaffeepausen geleistet. Ganz besonders danke ich Christian Braun, Dr. Rolf Daxhammer, Hans-Ulrich Eßlinger, Bernhard Hübner, Karin Knottenbauer, Margit Kraus, Thomas Keil, Barbara Link, Dr. Gerhard Mauch, Bertram Melzig-Thiel, Jürgen M. Schechler, Dr. Burkhard Scherer, Dr. Olaf Schneider, Dr. Sabine Stützle-Leinmüller, PD Dr. HansMichael Trautwein, Christof Rühl und Klaus Weißenberg sowie Muriel Dal-Pont am LATAPSES in Nizza und Dr. Georg Erber vom Deutschen Institut für Wirtschaftsforschung.

U I I I T T T T T T DU G U I (1) S T I F I U I 6
Danken möchte ich auch der Stiftung Ausbildung, Fort- und Weiterbildung der Landesgirokasse Stuttgart, die mit einem großzügigen Druckkostenzuschuß, die Veröffentlichung dieser Arbeit unterstützt hat.

Mein letzter und besonders herzlicher Dank gilt meiner Familie, die die sozialen Kosten meiner Doktorarbeit zu tragen hatte. So mußte zum einen meine Frau Ute mich längere Zeit mit einem Notebook teilen und zum anderen mein Sohn Tim so manche Runde mit seiner Holzeisenbahn alleine drehen. 
Stephan Seiter - 978-3-631-75406-1

Downloaded from PubFactory at 01/11/2019 05:22:48AM

via free access 


\section{Inhaitsverzeichnis}

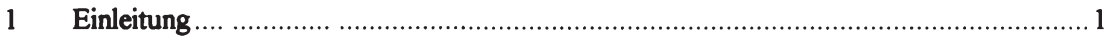

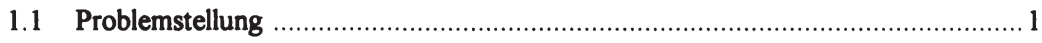

1.2 Aufbau der Arbeit...................................................................................... 5

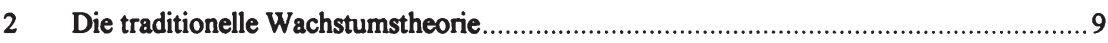

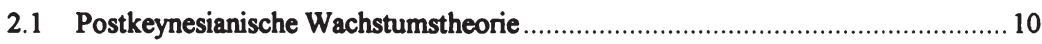

2.1.1 Die Rolle des Kapazitătseffekts ....................................................... 10

2.1.2 Investitionsentscheidungen und Wachstumsgleichgewicht..................... 12

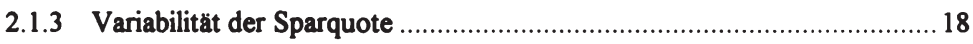

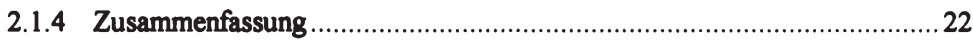

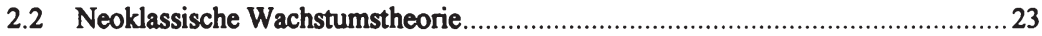

2.2.1 Erste Ansätze: Die Überlegungen von Jan Tinbergen ............................2 24

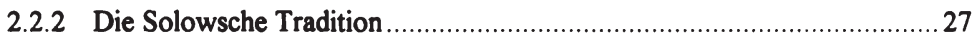

2.2.3 Das langfristige Gleichgewicht: Steady State ..................................... 30

2.2.4 Der Begriff der Konvergenz im neoklassischen Grundmodell .................. 37

2.2.5 Überwindung des Nullwachstums: Exogener technischer Fortschritt ........ 38

2.2.6 Goldene Regeln gleichgewichtigen Wachstums.....................................46

2.2.7 Der Standardfall der Cobb-Douglas-Produktionsfunktion ...................... 53

2.2.8 Eine Erweiterung: Gebundener technischer Fortschritt ......................... 55

2.2.9 Zusammenfassung der Ergebnisse des neoklassischen Grundmodells ....... 58

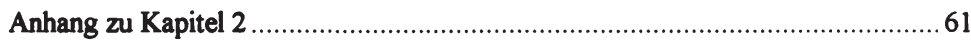

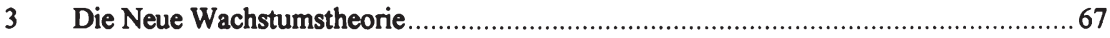

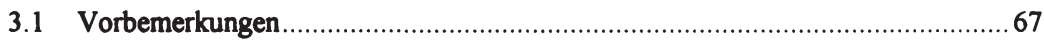

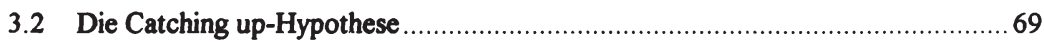

3.2.1 Inhalt der Catching up-Hypothese ..................................................69

3.2.2 Empirische Ergebnisse zur Konvergenz- und Catching up-Hypothese ...... 73

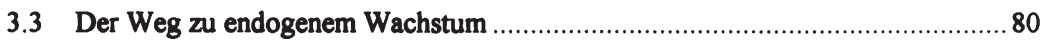

3.3.1 Investitionen - Lemen - Externalitäten............................................... 80

3.3.2 Der erste Schritt: Steigende Skalenerträge..........................................89

3.3.2.1 Wissensexternalitäten und steigende Skalenerträge ..................89

3.3.2.2 Endogenes Wachstum - nicht ohne steigende Skalenerträge? .... 94

3.3.2.3 Steigende Skalenertrăge und Spezialisierung ...........................99

3.3.2.4 Öffentlicher Kapitalstock und endogenes Wachstum ............... 103

3.3.2.5 Fazit ............................................................................ 104 
3.3.3 Humankapital als Wachstumsmotor............................................... 104

3.3.3.1 Vorbemerkungen............................................................. 104

3.3.3.2 Der Impuls: Das Humankapitalmodell von Lucas (1988)......... 106

3.3.3.3. Weitere Anwendungen des Humankapitalkonzepts ................ 114

3.3.3.4 Humankapital und learning by doing ................................ 117

3.3.3.5 Fazit ........................................................................... 120

3.3.4 Produktinnovationen und endogenes Wachstum ............................... 121

3.3.4.1 Vorbemerkungen......................................................... 121

3.3.4.2 Die Basisideen .............................................................. 121

3.3.4.3 Innovationen und Humankapital: Ursachen und Folgen des technischen Fortschritts ................................................... 123

3.3.4.4 Wachstum als Folge neuer und besserer Güter ..................... 128

3.3.4.5 Fazit........................................................................ 137

3.4 Kritische Anmerkungen zur Neuen Wachstumstheorie ................................... 137

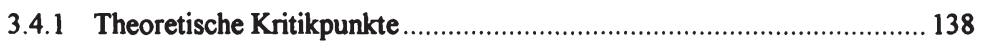

3.4.1.1 Kritik an den zugrundegelegten Funktionen ........................ 138

3.4.1.2 Weitere Mängel ............................................................ 142

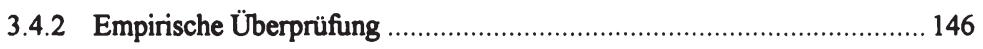

3.4.2.1 Konvergenz- und Divergenz-Tests..................................... 147

3.4.2.2 Determinanten des Pro-Kopf-Wachstums ............................ 150

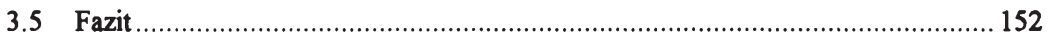

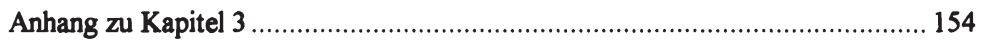

$4 \quad$ Nicholas Kaldors Beitrag zur Erklänung wirtschaftlichen Wachstums ........................ 157

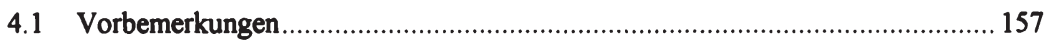

4.2 Das Konzept der technical progress function.................................................. 159

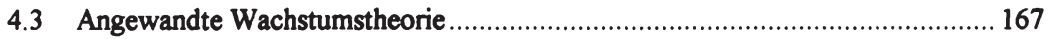

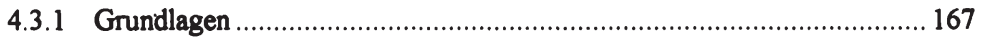

4.3.1.1 Das Prinzip der kumulativen Verursachung ............................ 167

4.3.1.2 Steigende Skalenerträge in der Tradition von Allyn Young ...... 169

4.3.1.3 Der Verdoorn-Zusammenhang........................................... 176

4.3.2 Die Renaissance des Verdoorn-Zusammenhangs:

Kaldors Inaugural Lecture.............................................................. 182

4.3.2.1 Der Verdoom-Zusammenhang im Verarbeitenden Gewerbe..... 182

4.3.2.2 Die Rolle des Arbeitsangebots ............................................ 186

4.3.2.3 Die Wahl der richtigen exogenen Variable:

Beschäftigung oder Output? ................................................. 189

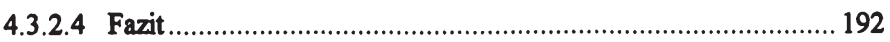


4.4 Zur Erklärung des Verdoom-Zusammenhangs

4.4.1 Theoretische Begründungen des Verdoorn-Zusammenhangs auf Basis der Cobb-Douglas-Produktionsfunktion

4.4.1.1 Begriffliche Klärung:

Verdoorn-Elastizität versus Verdoom-Koeffizient

4.4.1.2 Der Einfluß der Kapitalbildung auf den Verdoom-Zusammenhang

4.4.1.3 Statischer versus dynamischer Verdoorn-Zusammenhang......... 204

4.4.2 Eine kaldorianische Begründung des Verdoorn-Zusammenhangs

4.4.2.1 Der Verdoom-Zusammenhang und die technical progress function

4.4.2.2 Die technical progress function versus die Cobb-Douglas-Produktionsfunktion

4.4.3 Der Verdoorn-Zusammenhang und Zeitreihenuntersuchungen ............... 215

4.4.4 Weitere Ergebnisse für den Verdoorn-Zusammenhang ........................ 220

4.4.5 Verdoorn-Zusammenhang - was bleibt? ........................................... 222

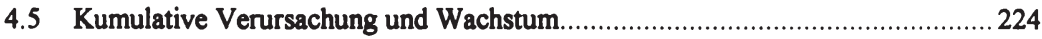

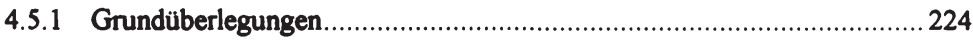

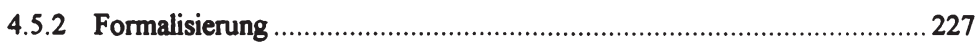

4.5.3 Weitere Überlegungen zu den Bedingungen ..................................... 231 der kumulativen Verursachung

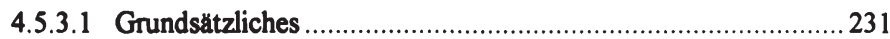

4.5.3.2 Nachfrageinduziertes Produktivitätswachstum ...................... 233

4.5.3.3 Produktivitătsinduzierte Nachfrageveränderungen.................. 235

4.5.3.4 Empirische Ergebnisse furr das erweiterte Modell der kumulativen Verursachung ....................................... 237

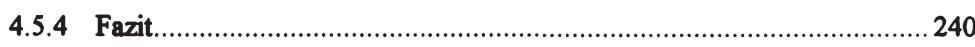

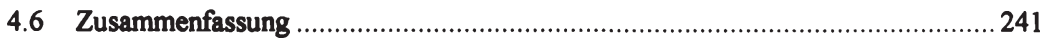

5 Neue Wachstumstheorie und Kaldors Beitrag zur Erklärung wirtschaftichen Wachstums im Vergleich ......................................................... 245

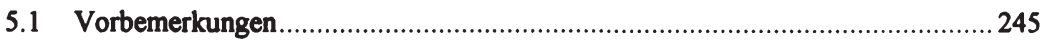

5.2 Unterschiede und Gemeinsamkeiten........................................................ 245

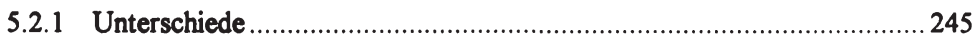

5.2.1.1 Die Vorstellungen über die Produktionsstruktur................... 245

5.2.1.2 Individuum versus Gesellschaft ........................................ 248

5.2.1.3 Konstante versus variable Wachstumsraten ........................... 249

5.2.1.4 Determinanten des Wachstumsprozesses.............................. 251 


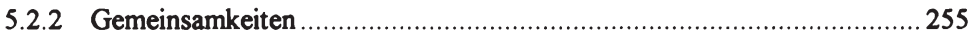

5.2.2.1 Die Rolle der Externalitäten............................................ 255

5.2.2.2 Die Rolle der Investitionen ............................................. 257

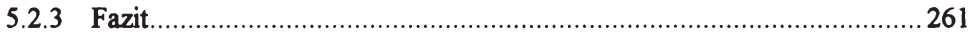

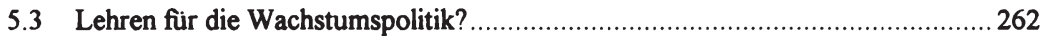

5.3.1 Die Neue Wachstumstheorie .................................................... 262

5.3.2 Die kaldorianische Sichtweise .......................................................... 267

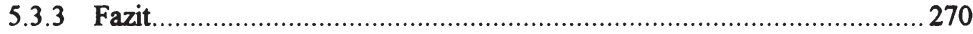

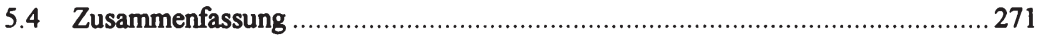

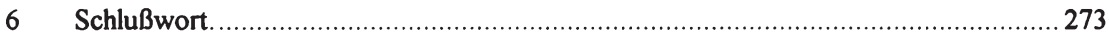

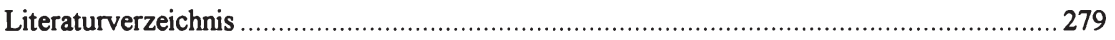

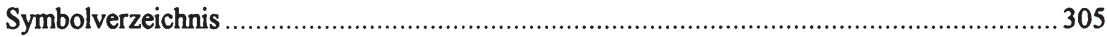




\section{Abbildungsverzeichnis}

Abb. 2.1 Stabilität im neoklassischen Grundmodell.................................................. 33

Abb. 2.2 Veränderung der Kapitalintensität bei Erhöhung der Sparquote...........................34

Abb. 4.1 Die technical progress function ................................................................. 160

Abb. 4.2 Die Bestimmung der Beschäftigungsschwelle ............................................ 217

Abb. 4.3 Produktivitäts- und Nachfragegleichung im Modell der kumulativen Verursachung

Abb. 5.1 Graphische Darstellung der Wachstumsrate der Kapitalintensität im AK-Modell

\section{Tabellenverzeichnis}

Tab. 2.1 Tinbergens und Solows Modell im Vergleich ................................................. 26

Tab. 3.1-A Wachstumsraten des Bruttoinlandsprodukts pro Kopf 1950-1973 ...................... 74

Tab. 3.1-B Wachstumsraten des Bruttoinlandsprodukts pro Kopf 1973-1989 ..................... 75

Tab. 3.2-A Relatives Pro-Kopf-Einkommen 1950 und 1989 (USA=100) ........................... 76

Tab. 3.2-B Mittelwert, Standardabweichung und Variationskoeffizient des relativen Pro-Kopf-Einkommens

Tab. 3.3 Rangkorrelation zwischen anfänglichen Produktivitätsniveaus und den Produktivitätswachstumsraten 1950-1986.

Tab. 4.1 Jährlicher Anstieg der Produktion und der Arbeitsproduktivität in der Industrie (Ländervergleich).

Tab. 4.2 Jährlicher Anstieg der Produktion und der Arbeitsproduktivität in der Industrie (Regressionsrechnung) .......................................................... 178

Tab. 4.3 Kaldors Wachstumsgesetze..................................................................... 183

Tab. 4.4 Diverse Spezifikationen des Verdoorn-Zusammenhangs................................. 190

Tab. 4.5 Der einfache und der erweiterte Verdoorn-Zusammenhang im Industriesektor für 12 OECD Länder (1953/54-1963/64) ........................... 200

Tab. 4.6 Schätzungen für den statischen Verdoorn-Zusammenhang ............................... 206

Tab. 4.7 Schätzungen des dynamischen und statischen Verdoom-Zusammenhangs (US-amerikanische Bundesstaaten 1963-1973 bzw. 1963 und 1973).

Tab. 4.8 Die Entwicklung der Beschäftigungsschwelle in der Bundesrepublik Deutschland

Tab. 4.9 Der Verdoorn-Zusammenhang im Verarbeitenden Gewerbe in zwölf OECD-Ländern 1950-1988

Tab. 4.10 Der Verdoom-Zusammenhang im Verarbeitenden Gewerbe in zwölf OECD-Ländern 1950-1980 
Stephan Seiter - 978-3-631-75406-1

Downloaded from PubFactory at 01/11/2019 05:22:48AM

via free access 


\section{$1 \quad$ Einleitung}

\subsection{Problemstellung}

Lange Zeit schien es, als sei das Interesse der ökonomischen Wissenschaft an wachstumstheoretischen Fragestellungen fast vollständig verloren gegangen. Während man die fünfziger und sechziger Jahre guten Gewissens als die Hochzeit dieses Teils der Wirtschaftswissenschaften bezeichnen kann, standen in den siebziger und achtziger Jahren vor allem Themen der Konjunkturtheorie sowie, insbesondere in den USA, die Erklärung des sog. productivity slowdowns im Mittelpunkt vieler theoretischer und empirischer Arbeiten. Das Phänomen deutlich zurückgehender Wachstumsraten der Arbeitsproduktivität wurde mit Hilfe weitgehend bekannter Theorien zu erklären versucht. Eine umfassende Weiterentwicklung der neoklassischen und postkeynesianischen Wachstumstheorie erfolgte in dieser Zeit jedoch nicht. Erst in den achtziger Jahren setzte eine Welle von Publikationen ein, die schnell unter dem Sammelbegriff Neue bzw. Endogene Wachstumstheorie zusammengefaßt wurde. Das Hauptziel der Vertreter dieses jungen Zweigs der ökonomischen Theorie ist die Überwindung der vermeintlichen Schwächen der traditionellen neoklassischen Wachstumstheorie, die sich in weiten Teilen auf das Modell von Solow aus dem Jahre 1956 stützt. Sowohl die theoretischen Ergebnisse als auch deren empirische Überprüfung werden in der Neuen Wachstumstheorie als unbefriedigend angesehen.

Die neoklassische Wachstumstheorie Solowscher Prägung wurde als Antwort auf die postkeynesianischen Arbeiten von Harrod $(1939,1948)$ und Domar $(1946,1947)$ seit Mitte der fünfziger Jahre entwickelt, wobei grundlegende Beiträge von Solow/Samuelson (1953), Tobin (1955), Solow (1956) und Meade (1961) geleistet wurden. Während die Überlegungen der postkeynesianischen Autoren zu langfristigen Wachstumsprozessen durch die Eindrücke der schweren Wirtschaftskrisen der Vorkriegszeit geprägt waren und deshalb eine gleichgewichtige Entwicklung einer marktwirtschaftlich organisierten Volkswirtschaft als eher unwahrscheinlich ansahen, wurde innerhalb der Modelle der Neoklassik bei Erfüllung einer Reihe von Annahmen über die Produktionstechnik sowie über das Spar- und Investitionsverhalten die Existenz und Stabilităt eines langfristigen, von Joan Robinson (1956) als "goldenes" Zeitalter bezeichneten Wachstumsgleichgewichts nachgewiesen. Die Entstehung der neoklassischen Wachstumstheorie diente jedoch nicht nur der Kritik an den postkeynesianischen Ansätzen, sondern auch der Erklärung der sehr hohen Wachstumsraten des Sozialprodukts vieler Volkswirtschaften in der Nachkriegszeit. Relativ schnell wurden in dieser Zeit Weiterentwicklungen des Basismodells durchgefuhrt, die z.B. im Rahmen des sog. VintageAnsatzes die Annahme eines homogenen Kapitalstocks durch die Berücksichtigung der Altersstruktur der Kapitalgüter ersetzten. Hierbei sei z.B. auf die Arbeiten von Solow (1958), Johansen (1959), Phelps (1962) und Solow/Tobin/Weizsăcker/Yaari (1966) verwiesen. Jedoch 
nicht nur die Produktionsfaktoren für sich alleine genommen standen im Zentrum der Diskussion, sondern auch die Beziehungen zwischen Arbeit und Kapital. So zeigten Arrow/Chenery/Minhas/Solow (1961) die Rolle der Substitutionselastizität im Rahmen der neoklassischen Theorie, indem sie das im Vergleich zur Cobb-Douglas-Produktionsfunktion allgemeinere Konzept der Constant-Elasticity-of-Substitution-Produktionsfunktion (kurz: CES-Produktionsfunktion) einführten.

Die verschiedenen Modifikationen konnten jedoch ein zentrales Ergebnis der neoklassischen Wachstumstheorie nicht verändern, so lange die Eigenschaften der neoklassischen Produktionsfunktion nicht wesentlich verändert wurden. Die langfristige Wachstumsrate des Pro-Kopf-Einkommens wird durch die Höhe des exogenen technischen Fortschritts bestimmt und kann durch Veränderungen des Spar- und Investitionsverhaltens nicht beeinflußt werden. Die Dynamik der langfristigen Entwicklung unterliegt somit nicht ökonomischen Entscheidungen, sondern ist das Resultat im Modell nicht-erklärter Zusammenhänge und Parameter. Dieses unbefriedigende Ergebnis regte verschiedene Arbeiten (z.B. Arrow (1962), Sheshinski (1967) oder Shell (1967a, b)) an, welche die Endogenisierung des technischen Fortschritts zum Inhalt hatten. Darüber hinaus bewirkten eine empirische Untersuchung von Solow (1957), die einen überwältigenden Anteil des unerklärten technischen Fortschritts am Wachstum des ProKopf-Einkommens in den USA für die Zeit zwischen 1909 und 1949 ergab, und eine Studie von Abramovitz (1956) eine zunehmende Anwendung neoklassischer Modelle auf empirische Fragestellungen im Rahmen des sog. Growth-Accounting-Ansatzes. Vor allem die Studien von Denison (1962, 1967, 1972, 1974, 1979, 1984, 1985), Jorgenson/Griliches (1967) oder Jorgenson (1995) erhielten große Beachtung.

Trotz dieser Bemühungen gelang es nicht, die exogene Bestimmung des langfristigen Wachstumsprozesses zu durchbrechen. Es läßt sich auch spekulieren, ob die in den sechziger Jahren ausgefochtene Cambridge-Kontroverse über die Definition und die Messung des Produktionsfaktors Kapital die Kräfte der neoklassischen Theorie von der Entwicklung endogener Wachstumsmodelle abgehalten hat. Tatsächlich erlebte die Diskussion über die Endogenität der Wachstumsrate erst seit Mitte der achtziger Jahre einen gewissen Aufschwung, dessen Ausgangspunkt die Veröffentlichung eines Beitrags von P. Romer im Jahre 1986 zum Zusammenhang zwischen Skalenerträgen und wirtschaftlichem Wachstum ist. Romers erste Publikation und die Arbeiten vieler seiner Nachfolger haben nicht nur die theoretische Kritik am traditionellen neoklassischen Wachstumsmodell zum Inhalt, sondern befassen sich auch mit dessen Implikationen für die Wachstumsraten von Volkswirtschaften. Bei Gültigkeit der neoklassischen Prämissen müßten kapitalärmere Volkswirtschaften schneller als industrialisierte wachsen. Tatsächlich ist dem aber nicht so.

Im Rahmen der neuen Modelle wird nun versucht, diese Nachteile zu überwinden, indem die Wachstumsrate und/oder der technische Fortschritt endogenisiert, d.h. von ökonomischen Entscheidungen abhängig gemacht werden. $\mathrm{Zu}$ diesem $\mathrm{Zweck}$ führen die Autoren diverse 
Wachstumsfaktoren, wie z.B. steigende Skalenerträge, Humankapitalakkumulation oder den Prozeß der schöpferischen Zerstörung in der Tradition Schumpeters, ein. Ein Kernelement sind dabei mögliche externe Effekte der Investitionsentscheidung einzelner Unternehmen auf die Situation anderer Firmen. Sehr deutlich wird dies am Beispiel von Forschungs- und Entwicklungsausgaben. Durch den Versuch ein neues Produkt oder ein neues Herstellungsverfahren zu entwerfen, wird das unternehmensinterne Know-how mit Sicherheit steigen. Darüber hinaus können auch Außenstehende von den gewonnenen Forschungsergebnissen profitieren. Kommt z.B. ein neues Produkt auf den Markt, können andere Firmen dieses analysieren und selbst ein ähnliches oder verbessertes Gut anbieten. Die Nachahmer in Schumpeters Vorstellungen zur Rolle des innovativen Unternehmers sind ein Beispiel für solche externen Effekte. Die der japanischen Industrie von westlichen Unternehmen vorgeworfene Strategie der kostengünstigen Kopie erfolgreicher Produkte, die unter hohem Aufwand von Firmen der USA oder Europa entwickelt worden sind, stellt ein empirisches Beispiel für diesen Zusammenhang dar.

Die Diskussion von Externalitäten dieser Art wird nicht erst seit dem Aufkommen der Neuen Wachstumstheorie gefürt, sondern findet sich z.B. schon bei Marshall (1920), Young (1928) oder Arrow (1962). Vor allem die Arbeit von Arrow bildet ein Kernstück der aktuellen Modelle. Interessanterweise erscheinen in vielen Beiträgen zur Neuen bzw. Endogenen Wachstumstheorie Hinweise auf die Arbeiten dieser Autoren. Sehr viel seltener wird die Verbindung zu einem anderen Strang der ökonomischen Theorie dargestellt, die zum einen ebenfalls auf den Überlegungen Marshalls und Youngs basiert, zum anderen sehr eng mit Arrows Konzept des learning by doing verwandt ist. Etwas früher als Arrow stellte Nicholas Kaldor (1957) aufbauend auf seiner Kritik am neoklassischen Paradigma, die insbesondere die Verwendung gesamtwirtschaftlicher Produktionsfunktionen betraf, die Idee der technical progress function vor. Nach Kaldors Auffassung sind die Kapitalakkumulation und die Realisierung des technischen Fortschritts nicht trennbar. Vielmehr wird durch die Kapitalintensivierung der Produktion das vorhandene technologische Wissen ausgenutzt, und die verbesserte Kapitalausstattung eines Arbeitnehmers erhöht dessen Produktivität. Aus diesem Grunde ist nicht eine sich aufgrund von exogenem technischen Fortschritt in ihrer Lage verändernde Produktionsfunktion, sondern vielmehr ein funktionaler Zusammenhang zwischen der.Wachstumsrate des Kapitals pro Arbeitsplatz und der Wachstumsrate der Produktivität in ein Wachstumsmodell zu integrieren. Darüber hinaus schaffen Investitionen die Voraussetzung für Lerneffekte und die Entstehung neuen Wissens. Dieses von Kaldor später modifizierte Konzept ist ein erster Schritt zur Endogenisierung des technischen Fortschritts.

Neben seinen wachstumstheoretischen Arbeiten regte Kaldor Mitte der sechziger Jahre eine Diskussion über den sog. Verdoorn-Zusammenhang und dessen Relevanz für die im internationalen Vergleich geringen Wachstumsraten der britischen Volkswirtschaft in der Zeit nach dem Zweiten Weltkrieg an. Das zentrale Thema Kaldors in seiner Antrittsvorlesung in Cambridge im Jahre 1966 ăhnelt demjenigen der Neuen Wachstumstheorie der ausgehenden 
achtziger und frühen neunziger Jahren. Warum realisieren einige Volkswirtschaften hohe Wachstumsraten des Pro-Kopf-Sozialprodukts, während anderen Volkswirtschaften hingegen nur geringes oder gar kein Wachstum der Güterversorgung pro Einwohner erreichen? Kaldor sah eine zentrale Ursache für die Divergenz der Wachstumsprozesse und mögliche kumulative Entwicklungsprozesse in den sektoralen Strukturen der jeweiligen Volkswirtschaften. Die einzelnen Sektoren (primärer, sekundärer und tertiärer) unterscheiden sich durch ihre produktionstechnischen Gegebenheiten. Innerhalb des primären Sektors unterliegt der Einsatz der Produktionsfaktoren abnehmenden Skalenerträgen, wodurch das Wachstum der Produktivität begrenzt ist. Der sekundäre, d.h. industrielle Bereich dagegen weist steigende Skalenerträge bei der Produktion auf. Die Erhöhung des Faktoreinsatzes führt somit zu überproportionalen Outputsteigerungen. Dies impliziert eine zunehmende Produktivităt der Produktionsfaktoren. Diese Eigenschaft des sekundären Sektors kommt nach Kaldor (1966) im Verdoorn-Zusammenhang zum Ausdruck, der eine lineare Beziehung zwischen den Wachstumsraten der Produktion und der Arbeitsproduktivität zum Inhalt hat.

Diese von Kaldor immer wieder betonte und für den Wachstumsprozeß einer Volkswirtschaft als entscheidend angesehene Beziehung wurde vielfach untersucht und diskutiert. Im Zentrum steht vorrangig die Frage nach den zugrundeliegenden Produktionsverhältnissen, d.h. es soll geklärt werden, welche Produktionsfunktion den empirisch feststellbaren Zusammenhang bedingen kann. Nicht zwangslăufig muß eine Cobb-Douglas-Produktionsfunktion die richtige Antwort auf diese Frage sein. Auch Lernkurven oder Kaldors technical progress function bieten sich an. Steigende Skalenerträge können unterschiedliche Gründe haben, die einerseits z.B. in der Massenproduktion und andererseits in externen Effekten unternehmerischer Entscheidungen zu finden sind. Einfache Rückschlüsse auf die tatsächlich vorliegende Produktionsstruktur sind aus dem ermittelten Verdoorn-Zusammenhang nicht eindeutig möglich, zu groß ist die Zahl der Interpretationsmöglichkeiten. Aus diesem Grund scheint es sinnvoller, den Verdoorn-Zusammenhang als Ausdruck der Bedingungen innerhalb einer Volkswirtschaft zu verstehen, die einen positiven Einfluß der Produktionsentwicklung auf das Produktivitätswachstums bewirken. Tatsächlich ist das Vorliegen induzierter Produktivitätssteigerungen nicht ausreichend für die Existenz sich selbsttragenden Wachstums bzw. kumulativer Entwicklungen, die von Kaldor als so wichtig angesehen worden sind. Die Reaktionen der Nachfrage auf Produktivitätssteigerungen sind dem Verdoorn-Zusammenhang gegenüberzustellen.

Die Vorstellungen innerhalb der Neuen Wachstumstheorie und die Überlegungen Kaldors zu den treibenden Kräften wirtschaftlichen Wachstums im Sinne steigender Produktivităt und Pro-Kopf-Versorgung weisen vergleichbare Strukturen auf. Dennoch liegen zwischen beiden Sichtweisen Unterschiede vor. Insbesondere die Rolle angebots- und nachfrageseitiger Entwicklungen wird unterschiedlich diskutiert und beurteilt, obwohl realiter für den Wachstumsprozeß einer Volkswirtschaft beide Seiten des Marktes von Bedeutung sind. In der vorlie- 
genden Arbeit werden deshalb anhand ausgesuchter Modelle der Neuen Wachstumstheorie sowie anhand der Beiträge von Kaldor zu wachstumsrelevanten Fragestellungen und der Diskussion über die Bestimmungsgründe des Verdoorn-Zusammenhangs die Kerninhalte beider Sichtweisen herausgearbeitet und gegenübergestellt. Insbesondere der Weg zu endogenem Wachstum steht hierbei im Vordergrund. Ziel ist es, den vernachlässigten Beitrag von Nicholas Kaldor zur Neuen Wachstumstheorie darzustellen und durch eine Kombination der Erkenntnisse der untersuchten Modellstrukturen wachstumspolitische Schlußfolgerungen zu ziehen. Auch dieser Versuch einer Integration kann selbstverständlich nur einen Ausschnitt aus der großen Zahl der Faktoren erfassen, die für die Entstehung von Wachstumsprozessen und technischem Fortschritt von Bedeutung sind. Weder die Modelle der Neuen Wachstumstheorie noch die kaldorianische Sichtweise geben ein exaktes Abbild aller in der Realität relevanten Strukturen. Der Vorteil eines Vergleichs bzw. einer Synopse dieser Modelle besteht primär in der Berücksichtigung angebots- und nachfrageseitiger Determinanten des Wachstumsprozesses.

\subsection{Aufbau der Arbeit}

Zur Lösung der gestellten Fragen, d.h. zur Ermittlung des Beitrags von Nicholas Kaldor zur Neuen Wachstumstheorie, wurde folgende Vorgehensweise gewählt.

Im anschließenden zweiten Kapitel werden die Grundmodelle der postkeynesianischen und der neoklassischen Wachstumstheorie erläutert und die für den weiteren Verlauf der Arbeit wichtigen Ergebnisse dieser Ansătze zusammengefaßt. Insbesondere die Resultate des traditionellen neoklassischen Wachstumsmodells waren und sind für die Entwicklung der Neuen Wachstumstheorie von besonderer Bedeutung. Diese Darstellung hat des weiteren den Zweck, die unterschiedlichen Auffassungen über die Stabilität marktwirtschaftlicher Systeme in den jeweiligen Modellen in Erinnerung zu rufen. Der pessimistischen Sichtweise des Postkeynesianismus in dieser Frage steht die Vorstellung der Neoklassik gegenüber, daß ein flexibles Preissystem auf allen Märkten ein Gleichgewicht zwischen Angebot und Nachfrage bewirken kann, solange keine exogene Schocks aufteten. Darüber hinaus dient die Behandlung der traditionellen Wachstumstheorie auch der Vollständigkeit, da nach Auffassung des Verfassers eine Arbeit zur Wachstumstheorie nicht ohne eine Besprechung der Modelle auskommen kann, die das Denken von Generationen von Ökonomen geprägt haben.

Das dritte Kapitel ist der Neuen Wachstumstheorie gewidmet, die die Überwindung der Schwächen der traditionellen neoklassischen Wachstumstheorie zum Ziel hat. Vor allem die in den Ansătzen der füfziger und sechziger Jahre vorherrschende Bestimmung der langfristigen Wachstumsrate des Pro-Kopf-Einkommens durch exogenen technischen Fortschritt soll durch endogene Mechanismen ersetzt werden. Aufbauend auf den Überlegungen zur sog. Catching 
up-Hypothese, die sich mit den Bedingungen eines erfolgreichen Aufholprozesses von Volkswirtschaften gegenüber dem technologisch führenden Land befaßt, werden anhand der zentralen Modelle die Grundüberlegungen und die Wege zu endogenem Wachstum innerhalb der Neuen Wachstumstheorie diskutiert. Im Mittelpunkt stehen dabei insbesondere Skalenerträge der akkumulierbaren Produktionsfaktoren. Während im neoklassischen Grundmodell für den Produktionsfaktor Kapital abnehmende Grenzerträge angenommen wurden, werden in den aktuellen Ansätzen mit Hilfe von externen Effekten, die durch Investitionen entstehen, konstante Skalenerträge des Kapitals bei der Herstellung des Endprodukts unterstellt. Die Neue Wachstumstheorie beschränkt sich nicht nur auf das Sachkapital, sondern befaßt sich auch mit den Folgen von Investitionen in das Humankapital einer Volkswirtschaft und den Konsequenzen von Produktinnovationen für den Wachstumsprozeß: Die Diskussion der jeweiligen Ansätze wird die herausragende Rolle der Kapitalintensivierung der Produktion für die Existenz endogenen Wachstums der Pro-Kopf-Größen zeigen, wobei der Begriff des Kapitals in diesem Zusammenhang sehr weit gefaßt ist. Den Abschluß des Kapitels bildet die Besprechung wichtiger Kritikpunkte an der Neuen Wachstumstheorie. Es werden dabei zum einen theoretische Einwände und zum anderen die mangelnde empirische Überprüfung angesprochen.

Der Beitrag von Nicholas Kaldor zur Erklärung von ökonomischen Wachstumsprozessen ist Inhalt des vierten Kapitels. Entsprechend der chronologischen Reihenfolge seiner Veröffentlichungen wird zu Anfang dieses Teils der Arbeit das Konzept der technical progress function diskutiert sowie die relative Nähe der Ergebnisse der frühen kaldorianischen Wachstumsmodelle zur Neoklassik aufgezeigt. Daran anschließend wird auf die angewandte Wachstumstheorie im Werk von Kaldor eingegangen. Ausgangspunkt sind hierbei die Inhalte seiner Antrittsvorlesung an der University of Cambridge aus dem Jahre 1966, in der er vor allem die Rolle des Verdoorn-Zusammenhangs für endogene Wachstumsprozesse diskutierte. Im Gegensatz zu den Ansätzen der Neoklassik sah Kaldor aufgrund der Existenz von steigenden Skalenerträgen in der industriellen Produktion ungleichgewichtige, divergierende Wachstumsprozesse als sehr wahrscheinlich an. Aufgrund der herausragenden Bedeutung des Verdoorn-Zusammenhangs, der als Beleg für steigende Skalenerträge verstanden wird, wird dieser im Hinblick auf mögliche theoretische Erklärungen hin untersucht. Wie schon oben angesprochen, sind mehrere Interpretationsmöglichkeiten für diese Beziehung zwischen Produktions- und Produktivitätswachstum denkbar. Neben einer Cobb-Douglas-Produktionsfunktion sind auch eine Lernkurve oder eine technical progress function kaldorianischer Prägung als Grundlage vorstellbar. In einem weiteren Schritt wird die Relevanz des Verdoorn-Zusammenhangs im kaldorianischen Verständnis des Wachstumsprozesses noch einmal aufgegriffen, das sich sehr deutlich in einem Beitrag von ihm zu regionalen Wachstumsunterschieden zeigt. Die Diskussion der verbalen Ausführungen Kaldors werden durch eine von Dixon/Thirlwall (1975) durchgefuhrte Formalisierung sowie eine Gegenüberstellung mit einem endogenen Wachs- 
tumsmodell von Lucas (1988) ergänzt. Daran anschließend kommt ein allgemeineres Modell der kumulativen Verursachung zur Sprache, das die für endogenes Wachstum erforderlichen Feedback- und Spillover-Effekte zwischen der Nachfrage- bzw. Produktions- und der Produktivitätsentwicklung zum Inhalt hat. Den Abschluß des Kapitels bildet eine Zusammenfassung der wichtigsten Ergebnisse.

Im fünten Kapitel werden die Aussagen und Ergebnisse der Neuen Wachstumstheorie den Ansichten Kaldors gegenübergestellt. Beide Sichtweisen unterscheiden sich vor allem bzgl. der Behandlung produktionstechnischer und nutzentheoretischer Zusammenhänge und der Frage nach den wirklichen Determinanten bzw. Beschränkungen von Wachstumsprozessen sowie der Stabilität langfristiger Wachstumsraten. Gemeinsamkeiten finden sich zum einen in der Rolle, die externen Effekten bzw. investitionsinduzierten Produktivitätsfortschritten zugewiesen wird, zum anderen in der damit eng verbundenen Relevanz von Investitionen für den Wachstumsprozeß. Der zweite Schwerpunkt dieses Kapitels umfaßt die Diskussion möglicher wachstumspolitischer Schlußfolgerungen, die sich aus beiden Ansätzen ziehen lassen. Die Berücksichtigung der Angebots- und der Nachfrageseite von Wachstumsprozessen bietet eine Möglichkeit zur Überwindung einseitiger wirtschaftspolitischer Handlungsweisen.

Den Abschluß der Arbeit bildet ein Schlußwort zum Vergleich zwischen der Neuen Wachstumstheorie und der kaldorianischen Sichtweise von Wachstumsprozessen. 
Stephan Seiter - 978-3-631-75406-1

Downloaded from PubFactory at 01/11/2019 05:22:48AM

via free access 
Die Anfänge der in der Zeit ihrer Entstehung als modern bezeichneten Wachstumstheorie werden normalerweise auf die Zeit von den späten dreißiger bis Ende der vierziger Jahre datiert. In dieser Zeit erschienen die vielzitierten Arbeiten von Domar (1946, 1947) und Harrod $(1939,1948)$, die als Ursprünge der postkeynesianischen Wachstumstheorie angesehen werden. Sowohl Harrods als auch Domars Beiträge waren sehr pessimistisch im Hinblick auf die Stabilität des wirtschaftlichen Systems und stehen somit in der Tradition von Keynes. Geprägt durch die schweren wirtschaftichen Krisen in der Zeit nach dem Ersten Weltkrieg erschien ihnen ein gleichgewichtiger Wachstumsprozeß mit ausgelasteten Kapazitäten und vor allem Vollbeschäftigung eher ein Ergebnis des Zufalls denn des ökonomischen Prozesses. Diese Sichtweise des wirtschaftlichen Systems spiegelt sich in den Wachstumsmodellen dieser Zeit wider und wird wohl am besten mit Begriff des Wachstums auf des Messers Schneide umschrieben, der insbesondere mit dem Wachstumsmodell von Harrod in Verbindung gebracht wird. ${ }^{1}$

Im Gegensatz hierzu sind die Modelle der neoklassischen Wachstumstheorie, die hauptsächlich mit den Namen Solow (1956), Swan (1956) und Meade (1961) verbunden sind, durch den Glauben an die inhärente Stabilität eines marktwirtschaftlich organisierten Wirtschaftssystems gekennzeichnet. Die Flexibilität der Preise auf allen Märkten erlaubt zu jeder Zeit die Anpassung der Ökonomie an veränderte Parameterwerte, so daß prinzipiell immer ein Gleichgewicht auf den Märkten erreicht wird und ein gleichgewichtiger Wachstumspfad möglich ist, zu dem die Volkswirtschaft konvergiert. Die Konzentration auf Wachstumsmodelle, die stabile Gleichgewichtspfade abbilden, ist nicht nur durch die Kritik an der postkeynesianischen Theorie, sondern auch durch die realen Wachstumsprozesse in den funfziger und sechziger Jahren zu erklären. Die hohen, teilweise sogar zweistelligen Wachstumsraten des Sozialprodukts vieler Volkswirtschaften und das steigende Beschäftigungsniveau in dieser Zeit verlangten nach einer theoretischen Begründung.

Im weiteren Verlauf werden beide Stränge der damals modernen Wachstumstheorie skizziert. Dies geschieht zum einen einer gewissen Vollstăndigkeit willens, zum anderen der Vorbereitung der Diskussion neuerer Entwicklungen auf dem Gebiet der neoklassischen Wachstumstheorie sowie des Einflusses Kaldors auf diese aktuellen Ansätze. Beide Theoriegebäude sind in ihren prinzipiellen Einstellungen gegenüber langfristigen Wachstumsprozessen in den jeweiligen ursprünglichen Ansätzen verwurzelt, so daß eine kurze Rückbesinnung auf die wichtigsten Zusammenhänge in diesen Modellen von Nutzen scheint.

1 Vgl. auch Harrod (1960). Harrod selbst lehnte diesen Begriff vehement ab. Die Losung fur die gleichgewichtige Situation in seinem Modell ist zwar instabil, aber nicht in dem Maße, daß es sich um ein Wachstum auf des Messers Schneide handelt. Dieser Begriff impliziert seiner Meinung nach, daB nur geringe Krafte ausreichen, das System aus seinem Gleichgewicht zu bringen. Tatsachlich mußten aber großBere Abweichungen von den Idealwerten vorliegen, damit dies geschieht. Vgl. Harrod (1973, Kap. 3). 


\subsection{Postkeynesianische Wachstumstheorie}

\subsubsection{Die Rolle des Kapazitätseffekts}

Die kurzfristige Analyse von John Maynard Keynes (1936) zu den Ursachen von Wirtschaftskrisen schreibt den Investitionen eine maßgebliche Rolle zu. Veränderungen der Investitionstätigkeit führen via Multiplikatorprozeß zu gleichgerichteten, wesentlich größeren Veränderungen des Gleichgewichtseinkommens der Volkswirtschaft. Investitionen werden jedoch ausschließlich im Hinblick auf den mit ihnen verbundenen Einkommenseffekt analysiert. Aufgrund der Orientierung der Untersuchung an der kurzen Frist wird der zweite Effekt von Nettoinvestitionen vernachlässigt. Positive Nettoinvestitionen erhöhen die Produktionskapazitäten der Volkswirtschaft. Ob diese in der Zukunft ausgelastet werden oder nicht, bleibt in den traditionellen keynesianischen Modellen außen vor. Im kurzfristigen Gleichgewicht gewährleistet die Erfüllung der IS-Bedingung zwar die Übereinstimmung von Güterangebot und Güternachfrage, die Auslastung der durch die Nettoinvestitionen entstandenen Kapazitäten ist jedoch nur bei einer ständig steigenden Nachfrage möglich, d.h. die Nettoinvestitionen müssen genauso wie das Sozialprodukt ständig zunehmen. Diese Zusammenhänge wurden von Domar (1946) sehr deutlich dargestellt. ${ }^{2}$

Die notwendigen Bedingungen für einen gleichgewichtigen Anstieg aller Größen lassen sich am einfachsten an einer dreipoligen keynesianischen Modellwirtschaft zeigen. ${ }^{3}$ Vernachlässigt man den autonomen Konsum, sind marginale und durchschnittliche Sparneigung identisch. ${ }^{4}$ Das gesamtwirtschaftliche Sparen (S) stellt einen konstanten Teil (s) des Outputs dar, d.h. S=sY. Es soll immer die IS-Bedingung erfüllt sein. Bei autonomen Investitionen (I) gilt in diesem Fall für den Gleichgewichtsoutput (Y): 5

$$
\mathrm{Y}=\frac{1}{\mathrm{~S}} \mathrm{I}
$$

bzw. für die Veränderungen beider Größen

$$
\dot{\mathrm{Y}}=\frac{1}{\mathrm{~s}} \dot{\mathrm{I}}
$$

2 Domars Aufsatz ist zwar mit dem Titel "Capital Expansion, Rate of Growth, and Employment" überschrieben, eine explizite Modellierung des Arbeitsmarktes erfolgt aber nicht. Vielmehr wird angenommen, daß die Beschăftigung eine Funktion des Verhaltnisses zwischen Sozialprodukt und Produktionskapazităt, d.h. des Auslastungsgrads ist (vgl. Domar 1946, S. 139).

3 Vgl. zu dieser Art der Darstellung z.B. Bombach (1965), Walter (1983) oder Rose (1987).

4 Dies gilt selbstverständlich auch für die marginale und durchschnittliche Konsumneigung. Punkte auf den Variablen kennzeichnen Veränderungen dieser.

5 Von Abschreibungen auf den Kapitalstock wird an dieser Stelle abgesehen. Wenn von Investitionen gesprochen wird, sind Nettoinvestitionen gemeint. 
Darüber hinaus schaffen die Investitionen neue Produktionskapazitäten $(\dot{\Omega})$. Die entsprechende Veränderung ergibt sich aus:

$$
\dot{\Omega}=\mathfrak{\imath} \dot{\mathbf{K}}=\mathfrak{\imath I}
$$

Hierbei bezeichnet ( 1 ) die Durchschnittsproduktivität der Investitionen, die als konstant angenommen wird. ${ }^{6}$ Im langfristigen Gleichgewicht müssen sich die Veränderungen des Einkommens und der Produktionskapazität entsprechen, d.h. $\dot{\mathbf{Y}}=\dot{\Omega}$. Es folgt

$$
\frac{1}{\mathrm{~S}} \dot{\mathrm{I}}=\mathbf{I I} \quad \text { bzw. } \quad \frac{\dot{\mathrm{I}}}{\mathrm{I}}=\mathbf{s i} .
$$

Die Wachstumsrate der Investitionen ist somit aufgrund der Konstanz der Sparquote und der Durchschnittsproduktivität ebenfalls konstant. Wenn (l) sich nicht verändert, muß auch der Kapitalkoeffizient (v) konstant sein, wobei $v=\mathrm{l}^{-1}$ ist. Aufgrund von (2.1) und (2.1') wächst im Gleichgewicht auch der Output mit der Wachstumsrate der Investitionen. Die Konstanz des Kapitalkoeffizienten bedingt gleichzeitig, daß auch der Kapitalstock mit dieser Rate wächst. Aufgrund der Annahmen über die Spar- und Konsumfunktion wachsen auch Konsum und Sparen mit derselben Wachstumsrate. Eine Situation, in der alle Variablen mit konstanten Raten wachsen, wird als Steady State bezeichnet.

Die Identität der Wachstumsraten aller Variablen sowie deren Konstanz sind eine Folge der getroffenen Annahmen. Inwieweit das Domarsche System ein Abbildung realer Zusammenhänge darstellt, ist fraglich. Insbesondere das Fehlen des Produktionsfaktors Arbeit sowie die Nichtbeachtung des technischen Fortschritts lassen das Modell unvollständig erscheinen. Im Rahmen einer langfristigen Analyse müssen diese beiden Komponenten Eingang in die Modellstruktur finden. Steigende Bevölkerungszahlen stellen einerseits das Potential für mehr Produktion dar, andererseits erfordert eine gleichgewichtige Entwicklung der Volkswirtschaft die Auslastung des zunehmenden Arbeitsangebots. Der technische Fortschritt ist durch dieselben Eigenschaften gekennzeichnet. Aufgrund seiner effizienzsteigernden Wirkungen kann mit gegebenem Faktoreinsatz mehr produziert werden. Gleichzeitig jedoch setzt er Pro-

6 Domar bezeichnet ( $\iota$ ) als "potential social average investment productivity" (Hervorhebung im Original, Domar 1946, S. 140). Davon ist das Verhăltnis des rein technisch moglichen Kapitalkoeffizienten der getătigten Investitionen zu unterscheiden. Der Koeffizient (l) wird in der Realităt hăufig unter den technischen Möglichkeiten liegen, da jede Investition in Konkurrenz zu bestehenden Kapazităten tritt. Werden Produktionsfaktoren und-mittel dort abgezogen, sinkt entsprechend das Produktionspotential (vgl. Domar 1946, S. 139f.). Die Realisierung aller technisch möglichen Kapazitătssteigerungen ist nur denkbar, wenn die Nettoinvestitionen mit zusătzlichen anderen Produktionsfaktoren kombiniert werden und keine Einschrănkungen in anderen Bereichen auftreten. Eine Möglichkeit wäre die Auflosung von versteckter Arbeitslosigkeit im primaren Sektor der Volkswirtschaft, um den Arbeitskräftebedarf für die Ausweitung der industriellen Produktion zu decken. Für Domar trägt der Agrarbereich zwar dazu bei, die Existenz überschulssiger Arbeitskrafte zu verschleiern, die Chance hoherer Produktionsmoglichkeiten werden von ihm jedoch nicht weiter diskutiert (vgl. z.B. Domar 1946, Fn 15, S. 145). 
duktionsfaktoren frei, so daß der Output entsprechend gesteigert werden muß, um die Vollbeschäftigung der Produktionsfaktoren zu gewährleisten. Die wohl angemessenste Auslegung der Modellergebnisse ist, sie als Bedingungen für einen gleichgewichtigen Wachstumsprozeß bei Gültigkeit der getroffenen Annahmen zu sehen. Offen ist, ob die Volkswirtschaft diese gleichgewichtige Situation erreichen kann. ${ }^{7}$ Probleme können insbesondere durch das Verhalten der Investoren auftreten. Auf ihre Reaktionen bei Unterauslastung der Kapazitäten kommt es an, ob der gleichgewichtige Wachstumspfad erreicht wird. Diese Überlegung wird im Modell von Harrod, das im năchsten Abschnitt im Mittelpunkt steht, wesentlich besser abgebildet als im Ansatz von Domar. ${ }^{8}$

\subsubsection{Investitionsentscheidungen und Wachstumsgleichgewicht 9}

Wie am Ende des letzten Abschnitts angesprochen, bleiben in Domars Modell die Entscheidungen der Unternehmen außen vor. Dieses Manko wird durch eine andere Interpretation des Verhältnisses zwischen Produktionskapazitäten und Output in Harrods Überlegungen geschlossen. Die Investitionsfunktion soll dem Akzeleratorprinzip unterliegen, d.h. die Nettoinvestitionen stellen die Reaktion der Unternehmen auf erwartete Nachfrageänderungen dar:

$$
I=\beta_{\mathbf{r}} \dot{Y}
$$

Der Akzelerator $\left(\beta_{\mathrm{r}}\right)$ wird hierbei als ein von den Unternehmen angestrebtes Verhältnis zwischen Investition und erwartetem Outputwachstum verstanden. Die Sparquote wird auch hier als konstant angenommen, so daß gilt

$$
\mathbf{S}=\mathbf{s} \mathbf{Y} \text {. }
$$

Durch Gleichsetzen von (2.4) mit (2.5) ergibt sich die Lösung für die sog. gewünschte bzw. befriedigende Wachstumsrate $\left(\mathrm{w}_{\mathrm{d}}\right)$ des Outputs:

7 Vgl. Walter $(1983$, S. 18).

8 Etwaige Folgen unterausgelasteter Kapazităten werden bei Domar berucksichtigt, jedoch erfolgt deren Ableitung nicht so klar wie bei Harrod. Zwei Zitate sollen als Beleg dieser Aussage dienen:

"Thus the failure of the economy to grow at the required rate creates unused capacity and unemployment." (Hervorhebung im Original), (Domar 1946, S. 143.)

"Consequently, the rate of growth may well be depressed below the required rate st, and unused capacity will be develop. Our present model does not allow us to separate unused capacity into idle capital and idle men, though most likely both will be present. Because of humanitarian considerations, we are more concerned with unemployed men. But unemployed capital is extremely important, because its presence inhibits new investment. (Hervorhebung im Original) It presents a grave danger to a full-employment equilibrium in a capitalist society." (Domar 1946, S. 145) Hier folgen Domars Überlegungen vollstandig denen von Harrod.

9 Vgl. zu der Ableitung des Gleichgewichts bei Harrod z.B. Bombach (1965), Walter (1983) oder Rose (1987). 


$$
w_{d}=\frac{\dot{Y}}{Y}=\frac{s}{\beta_{r}}
$$

Die tatsächliche Wachstumsrate $\left(w_{t}\right)$ ergibt sich bei Konstanz des Kapitalkoeffizienten aus der ex post-Identität von Sparen und Investieren, d.h. mit

$\mathrm{Y}=\frac{1}{\mathrm{~S}} \mathrm{~S} \quad$ und $\quad \dot{\mathrm{Y}}=\frac{1}{\mathrm{~V}} \mathrm{I}$

folgt:

$$
\mathrm{w}_{\mathrm{t}}=\frac{\dot{\mathrm{Y}}}{\mathrm{Y}}=\frac{\mathrm{s}}{\mathrm{v}}
$$

Die gleichgewichtige Situation ist erreicht, wenn sich ex post- und ex ante-Größen entsprechen. Harrod beschreibt diese Situation des dynamischen Gleichgewichts folgendermaßen:

"The line of output traced by the warranted rate of growth is a moving equilibrium, in the sense that it represents the one level of output at which producers will feel in the upshot that they have done the right thing, and which will induce them to continue in the same line of advance." 10

Die tatsächliche und die gewünschte Wachstumsrate müssen in diesem Falle identisch sein. Eine solches Akkumulationsgleichgewicht ist wieder entsprechend den Überlegungen zum Modell von Domar durch die Gleichheit der Wachstumsraten aller Variablen gekennzeichnet.

$\mathrm{Zu}$ fragen bleibt, welche Prozesse einsetzen, wenn Abweichungen zwischen gewünschter und befriedigender Wachstumsrate auftreten. Hauptakteure sind die Unternehmen, die mittels ihrer Investitionsentscheidungen die Entwicklung der Volkswirtschaft bestimmen. Geht man von einer konstanten Sparquote aus, können sich Unterschiede in den Wachstumsraten nur durch Differenzen beim tatsächlichen Kapitalkoeffizienten und befriedigenden Akzelerator ergeben. Wenn $v<\beta_{r}$ ist, stellen die Unternehmen fest, daß ihre Kapazitäten nicht ausreichen, die Güternachfrage zu bedienen. Konsequenterweise werden sie versuchen, entsprechende Kapazitäten aufzubauen und deshalb ihre Investitionen auszudehnen. Diesem Akzeleratoreffekt schließt sich die Wirkung des Investitionsmultiplikators an. Die erhöhten Nettoinvestitionen bedingen steigenden Output und somit mehr Einkommen. Dies bedeutet jedoch eine weitere Übernachfrage. Die Investoren werden erneut feststellen, daß sie im Vergleich zur tatsächlichen Nachfrage zu wenig produzieren können. Die weitere Ausdehnung der Kapazität wird

$10 \operatorname{Harrod}(1939$, S. 22). 
erforderlich. Der Unterschied zwischen tatsächlicher und befriedigender Wachstumsrate wird ständig zunehmen. Ist die ursprüngliche Situation durch eine im Verhältnis zum Gleichgewicht zu kleine tatsächliche Wachstumsrate gekennzeichnet, wird der Prozeß in die andere Richtung ablaufen. In beiden Fällen werden Ungleichgewichte durch die Reaktion der Unternehmen verstärkt, d.h. die gleichgewichtige Situation wird nicht mehr selbständig erreicht. Entsprechendes gilt, wenn man einen Anstieg oder eine Senkung der Sparquote unterstellt. Verändert sich diese, falls sich die Volkswirtschaft auf einem Gleichgewichtspfad befindet, werden im Moment der Änderung tatsächliche und befriedigende Wachstumsrate auseinanderfallen und den zuvor angesprochenen Prozeß anstoßen. 11

Die Folgen einer Abweichung der tatsächlichen von der befriedigenden Wachstumsrate werden als konjunkturelles Stabilitätsproblem oder Harrod-Paradoxon bezeichnet. Es entsteht durch die inadäquate Reaktionen der Investoren. Sie schätzen permanent die Folgen ihres Handelns falsch ein, da ihnen vollkommene Voraussicht fehlt. Gelänge es ihnen, das richtige Investitionsniveau zu ermitteln, würde das Gleichgewicht wieder erreicht. ${ }^{12}$ Der Ablauf in Harrods Modell ist ein Fall einer zirkulären, kumulativen Verursachung. Eine kleine Abweichung $\left(w_{d} \neq w_{t}\right)$ vom Idealzustand ruft Reaktionen (Multiplikatoreffekte) hervor, die wiederum Rückwirkungen auf die ursprünglich abweichende Größe haben und das System somit weiter vom Gleichgewicht entfernen anstelle es wieder zu diesem zurückzubringen. Eine Rückkehr zum Gleichgewicht kann nur durch einen exogenen Impuls erfolgen. Im Falle der konjunkturellen Instabilität bei Harrod müßten z.B. die Erwartungen der Investoren geändert oder per Fiskalpolitik eingegriffen werden.

Inwieweit eine kleine Abweichung der tatsächlichen von der befriedigenden Wachstumsrate $z u$ dem geschilderten Prozeß führt, ist fraglich. Harrod selbst ging davon aus, daß solange sich die Unterschiede zwischen beiden Raten innerhalb eines Korridors bewegen, das Instabilitätsproblem nicht auftaucht. Es müssen seiner Meinung nach schon sehr große Fehleinschätzungen bzw. deutliche Differenzen zu dem als optimal angesehenen Auslastungsgrad vorliegen, damit die Unternehmen entsprechend der oben getroffenen Annahmen mit einer Veränderung der Investitionen reagieren. ${ }^{13}$ Eng mit dieser Überlegung verbunden ist die Frage nach der Ober- und Untergrenze der Entwicklung. Gibt es einen Punkt, ab dem das System in Richtung Gleichgewicht umkehrt? Das rein formale Modell von Harrod kann eine solche Entwicklung nicht abbilden. Tatsächlich kann man sich vorstellen, daß ein expansiver Prozeß z.B. durch Engpässe bei den Arbeitskräften oder in relevanten Industriezweigen gestoppt werden kann. In einer Depression kann dagegen das Ausbleiben von Ersatzinvestitionen unterausgelastete Kapazitäten abbauen, da der Kapitalstock aufgrund von technischem Verschleiß

11 Vgl. z.B. Harrod (1939, S. 22f.).

12 Vgl. z.B. Walter (1983, S. 23) bzw. Rose (1987, S. 45ff.).

13 Vgl. Harrod (1973, Kap. 3). 
sinkt. Der ab einem gewissen Zeitpunkt notwendige Ersatzbedarf kann wiederum den Anstoß zu einem neuen Aufschwung sein. 14

Die möglichen Schwierigkeiten, die durch eine Abweichung der tatsächlichen von der befriedigenden Wachstumsrate entstehen können, sind primär konjunktureller Art. Die langfristige Stabilität des Wachstumsprozesses erfordert aber die Berücksichtigung des Arbeitseinsatzes (L). Im Harrodschen Modell wird von einem mit der exogenen Rate (n) wachsendem Arbeitsangebot ausgegangen. Hierbei wird (n) auch als natürliche Wachstumsrate bezeichnet. Vollbeschäftigung ist dann gegeben, wenn die angebotene Arbeitsmenge von den Unternehmen vollständig nachgefragt wird. Unterstellt man ein fixes Einsatzverhältnis zwischen Arbeit und Kapital, was nicht zwangsläufig eine linear-limitationale Produktionsfunktion bedingt, sondern auch die Folge eines nicht ausreichend flexiblen Preissystems sein kann, ergibt sich als langfristige Gleichgewichtsbedingung für den Arbeitsmarkt: ${ }^{15}$

$$
u Y_{t}=L_{0} e^{n t}
$$

Für die Wachstumsraten folgt dann durch Logarithmieren und Differenzieren nach der Zeit: ${ }^{16}$

$$
\frac{\dot{\mathrm{Y}}}{\mathrm{Y}}=\mathbf{n}
$$

Die Wachstumsrate des Outputs muß der Wachstumsrate der Bevölkerung entsprechen. Zur Erhaltung des Akkumulationsgleichgewichts, d.h. zur Befriedigung der Erwartungen der Investoren zeigte sich oben, daß der Output mit der Rate $(s / v)$ wachsen muß, d.h. $v=\beta_{r}$ ist. Ein langfristiges Gleichgewicht liegt somit vor, wenn gilt: 17

$$
\frac{\mathbf{s}}{\mathrm{v}}=\mathrm{n}
$$

Alle Variablen wachsen in dieser Situation mit derselben Rate, d.h. es liegt eine Steady StateSituation vor. Die Höhe der natürlichen Rate stellt dabei eine Obergrenze der langfristigen Wachstumsmöglichkeiten dar. Da alle Variablen mit der natürlichen Rate wachsen, liegt im

14 Vgl. Bombach (1965, S. 780ff.) und Hicks (1949).

15 Hierbei stellt (u) den Arbeitskoeffizienten dar. Vgl. zur Diskussion der zugrundeliegenden Produktionsfunktion z.B. Bombach (1965).

16 Die entsprechenden $Z$ wischenschritte sind:

1.) $\ln u+\ln Y_{t}=\ln L_{0}+\ln e^{n t}$ und 2.) $\frac{d \ln u}{d t}+\frac{d \ln Y}{d t}=\frac{d \ln L_{0}}{d t}+\frac{d(n t)}{d t}$

17 Erweitert man das Modell um den technischen Fortschritt, umfaBt die natüliche Rate neben dem Wachstum der Bevollkerung auch die produktivitătssteigernden Effekte technischer Neuerungen. Zu einer ausfuhrlicheren Besprechung der Arten des technischen Fortschritts sei an dieser Stelle auf die Ausfihrungen zur neoklassischen Theorie verwiesen, da die Einführung dieser Komponente an den qualitativen Aussagen des Harrodschen Wachstumsmodells nichts ăndert. 
Steady State ein Nullwachstum der Pro-Kopf-Größen vor. Die Ökonomie befindet sich damit in einer Art stationärem Zustand höherer Ebene. Nur technischer Fortschritt kann zu einer steigenden Güterversorgung führen. Wie sich weiter unten zeigen wird, unterscheidet sich damit das postkeynesianische Modell nicht von den Ergebnissen der neoklassischen Wachstumstheorie. Problematisch sind in diesem Modell mögliche Ungleichgewichtssituationen, die entstehen können, wenn die befriedigende Wachstumsrate von der natürlichen Rate abweicht. Langfristige Instabilität erfordert deshalb nicht zwingend das Vorliegen eines konjunkturellen Ungleichgewichts. Die tatsächliche und die befriedigende Rate können durchaus identisch sein, und dennoch kommt es nicht zur Übereinstimmung aller Wachstumsraten. Man ist geneigt, entsprechend des Sprachgebrauchs in der kurzfristigen keynesianischen Analyse, von einem Ungleichgewichtsgleichgewicht zu sprechen.

Aufgrund der notwendigen Übereinstimmung aller drei Wachstumsraten im langfristigen Gleichgewicht sind unterschiedliche Konstellationen denkbar, die zu einem säkularen Instabilitätsproblem führen können. Liegt z.B. die befriedigende Wachstumsrate der Investoren über der natürlichen Rate, die der tatsächlichen entsprechen soll, entstehen Überkapazitäten beim Kapitalstock, während das Arbeitsangebot ausgelastet ist. Diese Überkapazitäten werden bei den Unternehmen Reaktionen erzeugen, die zu einem Rückgang der Kapazitätsauslastung führen. Die Volkswirtschaft tendiert zu einer Stagnation bei Überkapazităt. Dauerhaft kann dies aber Rückwirkungen auf die Arbeitsnachfrage haben, so daß auch eine Unterbeschäftigungssituation auftreten kann. Ist die Volkswirtschaft nun durch die Identität der tatsächlichen und der befriedigenden Wachstumsrate gekennzeichnet, besteht bei einer Abweichung dieser von der natürlichen Rate nach unten das Problem der Unterbeschăftigung bei Akkumulationsgleichgewicht. Eine mögliche Ursache hierfür kann in einer zu geringen Investitionsneigung der Unternehmen liegen. 18

Die Realisierung eines langfristigen Gleichgewichts kann nun aufgrund der Exogenităt der relevanten Größen nur ein Ergebnis des Zufalls sein. In Harrods Modell gibt es keine endogenen Prozesse, die langfristige Stabilität garantieren. M.a.W. es ist ein Steady State-Wachstumspfad unter den getroffenen Annahmen zwar möglich, er ist jedoch unwahrscheinlich. Harrods Modell zeigt wie die Ausführungen von Domar notwendige Bedingungen auf, die erfüllt sein müssen, damit die Volkswirtschaft sich auf einem Steady State-Pfad befindet. Der Vorteil der Harrodschen Überlegungen liegt in der expliziten Berücksichtigung des Investorenverhaltens. Sie sind es, die letztendlich mit ihren Entscheidungen und Reaktionen über die Stabilität des Gleichgewichts bestimmen. Ihre "Unfähigkeit", die richtigen Schlüsse aus der Nichterfullung ihrer Vorstellungen zu ziehen, verursacht zum einen das konjunkturelle Stabilitätsproblem und zum anderen bewirken ihre Investitionen mögliche Abweichungen der befriedigenden von der natürlichen Wachstumsrate. Würden die Unternehmen in der Lage sein, aus

$18 \mathrm{Zu}$ weiteren Kombinationsmoglichkeiten der relevanten Wachstumsraten vgl. z.B. Eltis (1987), Walter (1983) oder Rose (1987). 
Fehlern zu lernen oder hätten sie rationale Erwartungen, müßte es nicht zwangsläufig zu konjunkturellen bzw. säkularen Fehlentwicklungen kommen. 19

Aus den Ergebnissen des skizzierten Modells lassen sich weitere Schlußfolgerungen ziehen. Im Harrodschen Modell bestimmt die Sparquote zusammen mit dem befriedigenden Kapitalkoeffizienten die befriedigende Wachstumsrate, die langfristig mögliche Wachstumsrate ist jedoch die natürliche. $\mathrm{Da}$ beide nicht zwangsläufig übereinstimmen müssen, hat die Sparquote langfristige Konsequenzen für den Wachstumsprozeß und nicht nur für das Niveau des Einkommens. Wirtschaftspolitische Maßnahmen zur Erhöhung des Sparens können deshalb dauerhafte Wirkungen zeigen. Dies ist ein Unterschied zu dem weiter unten zu besprechenden traditionellen neoklassischen Wachstumsmodell.

Die Abhängigkeit der befriedigenden Wachstumsrate von der gesamtwirtschaftlichen Sparquote sowie von dem gewünschten Kapitalkoeffizienten wirft die Frage auf, ob das Harrodsche Modell ein "endogenes" Wachstumsmodell darstellt. Deren Beantwortung hängt davon ab, wie man den Begriff endogen in Verbindung mit Wachstumsmodellen definiert. Normalerweise wird unter endogen die Bestimmung einer Variablen durch das Modell selbst verstanden. Die Sparquote und der Kapitalkoeffizient werden jedoch beide nicht erklärt, sondern als gegeben angenommen. Die Wachstumsrate wiederum ergibt sich durch die Erfüllung der vorgegebenen Gleichgewichtsbedingungen, so daß hier scheinbar nicht von Endogenität gesprochen werden kann. Eine Erhöhung der Sparquote hat dauerhafte Effekte, da sich keine Anpassung ans Gleichgewicht ergibt. Versteht man unter endogenem Wachstum langfristiges Pro-Kopf-Wachstum, das keine exogene Determinante wie z.B. den technischen Fortschritt benötigt, muß die Aussage relativiert werden. Der Steady State-Pfad ist bei Harrod durch die Identität der Wachstumsraten aller Variablen gekennzeichnet. Damit ist Pro-KopfWachstum ohne technischen Fortschritt unmöglich. Nur wenn das System sich nicht im Steady State befindet, d.h. die befriedigende und die tatsächliche Wachstumsrate über der natürlichen liegen, kommt es zu positivem Pro-Kopf-Wachstum, da die Wachstumsrate des Kapitalstocks über derjenigen der Bevölkerung liegt. Dauerhaft wird eine solche Situation nicht Bestand haben, wenn in Harrods Modell der Kapitaleinsatz abnehmenden Grenzerträgen unterliegt, so daß sich die Wachstumsraten aller Variablen anpassen. Bleibt der Kapitalkoeffizient konstant, kommt es zu dauerhaftem Pro-Kopf-Wachstum. Berücksichtigt man diese Überlegungen, läßt sich festhalten, daß der Ansatz von Harrod zur Gruppe endogener Wachstumsmodelle gerechnet werden kann.

Zwar scheint das konjunkturelle Instabilitătsphänomen kein Problem der wachstumstheoretischen Analyse zu sein, da es nur kurzfristige Zusammenhänge beleuchtet. Dennoch sorgt das Fehlen eines endogenen Mechanismus, der bewirkt, daß die Unternehmen sich richtig 
verhalten, für anhaltende Abweichungen vom Gleichgewichtspfad. ${ }^{20} \mathrm{Zu}$ fragen ist deshalb, wo endet die kurze und wo beginnt die lange Frist? Zieht man zusätzlich Hysterese-Effekte und Pfadabhängigkeiten in die Überlegungen mit ein, gewinnt das kurzfristige Instabilitätsproblem eine wichtige Bedeutung für die langfristige Entwicklung der Volkswirtschaft. Die Entscheidung über das aktuelle Investitionsniveau legt fest, welchen Kapitalstock die Volkswirtschaft in den nachfolgenden Perioden zur Verfügung hat. Nimmt man mit Arrow (1962) und Kaldor (1957) an, daß Investitionen nicht nur den Stand des technischen Wissens, das im Moment der Investition verfugbar ist, beinhalten, sondern auch die Gelegenheit zu neuem Lernen und somit weiteren Produktivitätsfortschritten bieten, determinieren die Investitionsentscheidungen der Unternehmen die weiteren Entwicklungschancen der Volkswirtschaft. ${ }^{21}$ Die unterlassenen Investitionen im Falle einer Abweichung der tatsächlichen von der befriedigenden Wachstumsrate nach unten können das $\mathrm{Maß}$ des erreichbaren technischen Fortschritts reduzieren. Diese Konsequenzen konjunktureller Instabilität erfordern selbstverständlich eine Erweiterung des ursprünglichen Modells von Harrod (und auch von Domar). Sie zeigen aber, daß der kategorische Verweis der Folgen einer Divergenz zwischen befriedigender und tatsächlicher Wachstumsrate in das Aufgabengebiet der Konjunkturtheorie nicht ohne weiteres befolgt werden sollte.

\subsubsection{Variabilität der Sparquote}

Das säkulare Instabilitätsproblem im Modell von Harrod kann ohne die Modifikation der Annahmen nicht gelöst werden. Einer der Parameter (s, v und n) müßte endogenisiert werden. Prinzipiell kann dies bei jeder dieser Größen erfolgen. Die Diskussion der Folgen eines variablen und endogenen Kapitalkoeffizienten sind Inhalt der neoklassischen Wachstumstheorie, die in Abschnitt (2.2) erläutert wird. Die von Harrod unterstellte Konstanz dieser Größe steht z.B. im Mittelpunkt der Kritik von Solow (1956) am Harrodschen Wachstumsmodell. Zur Endogenisierung der natürlichen Wachstumsrate läßt sich sagen, daß hierbei zwischen dem Bestandteil des Bevölkerungswachstums und des technischen Fortschritts unterschieden werden muß. Die Abhängigkeit beider Faktoren von ökonomischen Zusammenhängen wurde auf unterschiedliche Art und Weise herzustellen versucht. $\mathrm{Da}$ in den nachfolgenden Kapiteln der Arbeit immer wieder auf die Entstehung und die Konsequenzen des technischen Fortschritts eingegangen wird, bleibt diese Komponente der natürlichen Wachstumsrate im Moment außen vor.

20 Dieses Problem wurde in Arbeiten, die auf Harrods Überlegungen aufbauten, zu losen versucht. Zu nennen ist vor allem Hicks (1949).

21 Die Konzepte von Arrow und Kaldor zum induzierten technischen Fortschritt werden weiter unten ausfuhrlicher besprochen. 
Die Endogenisierung der Wachstumsrate der Bevölkerung soll nur kurz erwähnt werden, da sie nicht Gegenstand der weiteren Untersuchungen sein wird. Die Reproduktionsrate kann z.B. an die Entwicklung des Subsistenzlohnes oder an eine Abweichung des tatsächlichen Reallohnes von diesem gekoppelt werden. ${ }^{22}$ Unterstellt man einen positiven Zusammenhang zwischen Reallohnhöhe und Bevölkerungsentwicklung, stehen diese Überlegungen in der Tradition von Malthus' Aussagen über die "Endogenität" der Bevölkerungsentwicklung. Verändern sich die Knappheitsrelationen zwischen Arbeit und Kapital, wird es zu induzierten Lohnreaktionen kommen, die einerseits positive, $d . h$. erhöhende Wirkungen oder andererseits negative, d.h. senkende Effekte auf die Reproduktionsrate haben, die sich in der Höhe der natürlichen Wachstumsrate niederschlagen.

Diese Art der Endogenisierung des Bevölkerungswachstums stellt eine recht einfache Vorgehensweise dar. Fraglich ist z.B., ob der klassische positive Zusammenhang zwischen Bevölkerungszunahme und Reallohnentwicklung in modernen Industrienationen noch Gültigkeit hat. Alle entwickelten Volkswirtschaften weisen trotz gestiegenem Wohlstand rückläufige Geburtenraten mit stagnierenden bzw. sinkenden Bevölkerungszahlen auf. Dies spricht mehr für eine inverse Beziehung zwischen Reallohn und Reproduktionsrate. Verläßt man das Bild einer geschlossenen Volkswirtschaft, muß diese Aussage wieder relativiert werden. Die Bevölkerung eines Landes wächst nicht nur durch den Geburtenüberschuß, sondern auch aufgrund eines positiven Zuwanderungssaldos mit dem Ausland. Hier kann man argumentieren, daß Lohndifferenzen zwischen einzelnen Volkswirtschaften einen Anreiz für die Arbeitskräfte darstellen, im Hochlohnland ihre Arbeit anzubieten. ${ }^{23}$ Ein historisches Beispiel hierfür ist ohne Zweifel der Zustrom von Gastarbeitern nach Westdeutschland seit Anfang der sechziger Jahre. Diese Zuwanderung war nicht allein das Ergebnis von Lohndifferenzen, sondern wurde aufgrund des Arbeitskräftemangels in Westdeutschland durch Anwerbemaßnahmen unterstützt.

Eine Anmerkung zur Endogenisierung des Bevölkerungswachstums sei an dieser Stelle noch erlaubt. In Zeiten zunehmender Umweltbelastungen, die u.a. durch die stark zunehmende Weltbevölkerung verursacht werden, gewinnt dieser Teil der natürlichen Rate innerhalb eines Wachstumsmodells eine neue Qualität. In den Modellen von Harrod und später in denen der neoklassischen Wachstumstheorie stellt (n) bei Nichtexistenz von technischem Fortschritt eine

22 Vgl. z.B. zur Integration des Bevolkerungswachstums auf diese Art Niehans (1963), der weiter unterscheidet in ein Ein- und Zwei-Klassen-Modell bei der Reproduktion. Die (neoklassische) Untersuchung von Steinmann (1974) zur Endogenisierung des Bevolkerungswachstums zeigt den engen Zusammenhang zwischen der Kapitalakkumulation und dem Bevollkerungswachstum, wenn von exogenem technischen Fortschritt abgesehen wird. Durch die Endogenisienung des Bevolkerungswachstum wird in diesem Falle die Abhangigkeit der langfristigen Wachstumsrate des Pro-Kopf-Einkommens von der Sparquote ermoglicht. Das Bevolkerungswachstum kann somit zum Wachstumsmotor werden. Wird zusatzlich autonomer technischer Fortschritt unterstellt, weist das Sparen nur noch einen Einflub auf das Niveau des langfristigen Wachstumspfads auf. Sowohl bei Kaldor (1957), der das Wachstum der Bevolkerung nach oben begrenzt (S. 614f.), als auch bei Solow (1956) findet sich ein kurzer Abschnitt uber die Folgen eines endogenen Arbeitsmengenwachstums.

23 Vgl. Rose (1987, S. 58ff.). Zu Fragen des Zusammenhangs zwischen Bevolkerungswachstum und wirtschaftlichem Wachstum siehe z.B. Coale (1976) und die enthaltenen Beitrage sowie Pitchford (1974). 
Obergrenze für die Wachstumsmöglichkeiten der Volkswirtschaft dar. Vor dem Hintergrund des Problems der Überbevölkerung wird die Forderung nach der Begrenzung der Reproduktionsrate immer wieder aufgestellt, d.h. (n) müßte aufgrund dessen als eine exogen festgelegte Variable eingebaut werden, die nicht von anderen Beziehungen im Modell verändert werden kann und darf. Eine Endogenisierung des Bevölkerungswachstums müßte deshalb ausgeschlossen werden, um untersuchen zu können, wie sich die anderen Variablen entwickeln müssen, um das Ziel einer begrenzten Wachstumsrate der Bevölkerung erreichen zu können.. Die Vorgehensweise der traditionellen Wachstumstheorie erhält bei Berücksichtigung ökologischer Aspekte somit wieder eine gewisse Berechtigung.

Die Auflösung des säkularen Instabilitätsproblems mit Hilfe einer veränderlichen natürlichen Wachstumsrate unterliegt, wie sich aus den vorangegangenen Abschnitten ablesen läßt, einigen Schwierigkeiten. Als letzte Möglichkeit, zum Gleichgewicht zu kommen, bietet sich die Sparquote an. Wäre sie endogen bestimmt und würde sie richtig auf ungleichgewichtige Situationen reagieren, könnte sich auch unter Aufrechterhaltung eines konstanten Kapitalkoeffizienten die befriedigende Wachstumsrate an den Wert der natürlichen Rate anpassen. Liegt die befriedigende Wachstumsrate über der natürlichen, müßte die gesamtwirtschaftliche Sparquote fallen, in der umgekehrten Konstellation steigen. Das postkeynesianische Modell von Nicholas Kaldor (1955/56, 1957) beinhaltet einen Weg zur Generierung eines solchen Ergebnisses.

Ausgehend von einem Vollbeschäftigungsgleichgewicht spaltet Kaldor das gesamtwirtschaftiche Sparen in zwei Komponenten auf: Sparen aus Profiten und Sparen aus Löhnen. Die Sparquote aus Löhnen soll geringer sein als die aus Profiten. Es gilt:24

$$
\begin{aligned}
& Y \equiv W+P \\
& I \equiv S \\
& S \equiv S_{W}+S_{p}=s_{w} W+s_{p} P
\end{aligned}
$$

Aus der IS-Identität folgt für die Investitionsquote und somit für die gesamtwirtschaftliche Sparneigung:

$$
\frac{I}{Y}=\left(s_{p}-s_{w}\right) \frac{P}{Y}+s_{w}
$$

24 Vgl. Kaldor (1955/56, S. 221). Hierbei steht (W) für die Lohnsumme, (P) für die Profitsumme, $S_{i}$ bzw. $s_{i}$ furr das Sparen aus bzw. die Sparneigung bezogen auf die Einkommensart i. 
Die Investitionsquote hängt von der Profitquote und den Sparquoten der jeweiligen Einkommensarten ab. Setzt man (2.10) in die Gleichgewichtsbedingung im Harrodschen Modell ein, ergibt sich:

$$
n v=\left(s_{p}-s_{w}\right) \frac{P}{Y}+s_{w}
$$

Dieses Resultat impliziert, daß "...the 'warranted' and the 'natural' rates of growth are not independent of one another; if profit margins are flexible, the former will adjust itself to the latter through a consequential change in P/Y."25 Kommt es zu Unterschieden zwischen den beiden Raten, wird eine Veränderung der Einkommensverteilung, d.h. sinkende oder steigende Profitquote (Lohnquote), eine Veränderung des gesamtwirtschaftlichen Sparens induzieren. Das System tendiert von dieser Seite aus zum Gleichgewicht, aber "this does not mean that there will be an inherent tendency to a smooth rate of growth in a capitalist economy, only that the causes of cyclical movements lie elsewhere - not in the lack of an adjustment mechanism between s and Gv." 26

An dieser Stelle ist anzumerken, daß Kaldor mit $\mathrm{G}$ nicht die natürliche Wachstumsrate meint, sondern die befriedigende, d.h. er ist primär der Auffassung, daß die Variabilität der Sparquote das kurzfristige Instabilitätsproblem löst und nicht direkt das langfristige, denn

"I/Y will therefore tend to equal Gv, not G'v."27

Die Anpassung der Sparquote durch eine sich ändernde Einkommensverteilung kann jedoch auf die säkulare Fragestellung übertragen werden, da auch die befriedigende Wachstumsrate mit der Sparquote variiert. Es ist somit zumindest theoretisch möglich, daß es immer eine Einkommensverteilung gibt, die Identität zwischen den Wachstumsraten erzeugen kann. ${ }^{28}$

Wie kann man sich nun einen Anpassungsprozeß vorstellen, der Abweichungen zwischen den Raten, insbesondere der befriedigenden und der natürlichen, beseitigt? Ist die Situation durch $(\mathrm{s} / \mathrm{v})>$ n gekennzeichnet, wächst der Kapitalstock schneller als die verfügbare Arbeitsmenge. Sind die Preise kurzfristig variabler als die Nominallöhne, d.h. die Reallöhne sind flexibel, müßte der Mangel an Arbeitskräften zu einer Steigerung der Lohnquote und folglich zu einem Rückgang der gesamtwirtschaftlichen Sparquote führen. Weicht die befriedigende Rate nach unten von der natürlichen $a b$, bedingen steigende Preise (und damit verbunden

25 Kaldor (1955/56, S. 224).

26 Kaldor (1955/56, S. 224).

27 Kaldor (1955/56, S. 224, Fn 2). Hier steht G furr "...the entrepreneurs' desired growth rate..." und G' für "...the natural growth rate..." (Kaldor, 1955/56, S. 224)

28 In Kaldors Wachstumsmodellen zeigt sich im Steady State, daß die Investitionsquote das Ergebnis der naturlichen Wachstumsrate und des Kapitalkoeffizienten ist. Die Einkommensverteilung und die Sparquote ergeben sich als Restgroße aus den exogen vorgegebenen Parameter. Vgl. Kaldor (1957, S. 611), Kurz (1984, S. 167ff.) sowie Kapitel 4 dieser Arbeit. 
sinkende Reallöhne) im kaldorianischen Vollbeschäftigungsmodell eine Zunahme der Profitquote, die Kapitalbildung nimmt zu. Die befriedigende Wachstumsrate paßt sich an die natürliche an. 29

\subsubsection{Zusammenfassung}

Die postkeynesianische Wachstumstheorie baut im wesentlichen auf den Ansätzen von Harrod und Domar auf, in denen zum einen die notwendigen Gleichgewichtsbedingungen für einen gleichgewichtigen, vollbeschäftigungskonformen Wachstumspfad abgeleitet wurden, zum anderen Hindernisse diskutiert wurden, welche die Erreichung eines Steady States unmöglich machen können. Insbesondere die Erwartungen der Unternehmen können ungleichgewichtige Prozesse bedingen.

Ein gleichgewichtiger Wachstumspfad erfordert unter den getroffenen Annahmen die Identität der Wachstumsraten von Kapital, Output und (effizienter) Arbeitsmenge, wobei die sog. natürliche Wachstumsrate eine Obergrenze für die langfristig erreichbare Veränderungsrate bildet. Diese Situation kann sich nur per Zufall einstellen. Jegliche Abweichungen vom Gleichgewicht führen immer weiter von ihm weg. Vor allem die von Harrod getroffene Unterscheidung in eine tatsächliche, eine für die Investoren befriedigende und die natürliche Wachstumsrate hat gezeigt, wo mögliche Schwierigkeiten auf dem Weg zum Gleichgewicht liegen können. Es können sowohl konjunkturelle als auch säkulare Instabilitäten auftreten, die durch die Inflexibilität des Systems entstehen. So stellt die Unfähigkeit zum Lemen der Unternehmen die zentrale Ursache für die persistente Ungleichheit zwischen tatsächlicher und befriedigender Wachstumsrate dar. Im Rahmen des langfristigen Stabilitätsproblems sorgte die exogen vorgegebene Konstanz aller drei involvierten Parameter (Sparquote, Kapitalkoeffizient und natürliche Wachstumsrate) für Differenzen zwischen den Wachstumsraten.

Vor allem die Arbeiten von Kaldor und Pasinetti haben einen Weg gewiesen, wie durch die Verbindung verteilungstheoretischer Aspekte mit dem Harrodschen Wachstumsmodell eine Anpassung der befriedigenden an die natürliche Wachstumsrate ermöglicht werden kann. ${ }^{30}$

29 Die Vollbeschăftigungsannahme ist notwendig, damit bei einem Anstieg der Nachfrage Preis- und keine Mengenreaktionen auftreten, wie es bei unterausgelasteten Kapazităten der Fall wăre. Vgl. Kurz (1984, S. 167). Im Hinblick auf die notwendigen Existenzbedingungen sei auf Kaldors eigene Ausfithrungen sowie z.B. auf Kalmbach (1972), Walter (1983), Müller/Strobbele (1985) oder Rose (1987) verwiesen. Auch auf die Weiterentwicklung durch Pasinetti (1962), der insbesondere die Konsequenzen einer positiven Sparneigung aus Lohneinkommen für die Querverteilung problematisiert hat, soll hier nicht eingegangen werden. Vgl. hierzu auch Kalmbach (1972). Für die Anwendung einer kaldorianischen Sparfunktion in einem zweisektoralen Modell vgl. Hagemann (1991).

30 Von Meade (1962a) wurde eine kaldorianische Sparfunktion in ein neoklassisches Wachstumsmodell integriert, d.h. es werden unterschiedliche Sparquote für die verschiedenen Einkommensarten unterstellt. Die Verteilung des Einkommens auf Lohne und Profite bestimmt sich nach den Knappheitsverhaltnissen bei den Produktionsfaktoren. Vgl. Meade (1962a, S. 31ff. und Anhang 1). Im Falle einer Cobb-DouglasProduktionsfunktion mit einer Substitutionselastizităt zwischen Arbeit und Kapital in Hơhe von Eins wird 
Ungleichgewichtige Situationen, wie sie von Harrod diskutiert wurden, bedingen Veränderungen der Einkommensverteilung, die wiederum die gesamtwirtschaftliche Sparquote beeinflussen. Dieser Anpassungsprozeß stellt jedoch keine Garantie für eine gleichgewichtige Entwicklung der Volkswirtschaft per se dar. Die postkeynesianischen Modelle sind vielmehr durch den typisch keynesianischen Zweifel an der Fähigkeit marktwirtschaftlich organisierter Systeme zur Erreichung einer Vollbeschäftigungssituation ohne staatliche Einflußnahme geprägt. Die angesprochenen Arbeiten von Nicholas Kaldor scheinen eine Ausnahme zu bilden, da er in seinen Modellen zur Verteilungs- und Wachstumstheorie von einer Vollbeschäftigungssituation ausgeht. Das zentrale keynesianische Element in Kaldors Beiträgen ist das Vorliegen einer eigenständigen Investitionsfunktion, die unabhängig von den Sparentscheidungen ist. 31

Diese Grundeinstellung innerhalb der postkeynesianischen Wachstumstheorie unterscheidet die in diesem Abschnitt skizzierten Modelle von denen der Neoklassik, die im nächsten Teilkapitel im Mittelpunkt des Interesses stehen sollen. Wie schon oben angesprochen, sind letztere durch die Variabilität des Kapitalkoeffizienten gekennzeichnet, die maßgeblich für die Realisierung eines langfristigen Gleichgewichts verantwortlich ist.

\subsection{Neoklassische Wachstumstheorie}

In Antwort auf die wachstumstheoretischen Arbeiten von Domar $(1946,1947)$ und Harrod (1939, 1948), die im vorigen Abschnitt dargestellt wurden, entstanden vor allem in den 50er Jahren die grundlegenden Modelle der neoklassischen Wachstumstheorie von Solow (1956), Tobin (1955), Swan (1956) und später Meade (1962a). ${ }^{32}$ Hierbei stand einerseits die Annahme fixer Einsatzverhältnisse in der Produktion im Zentrum der Kritik. Würde Substituierbarkeit der Produktionsfaktoren Arbeit und Kapital zugelassen, würde das Problem des Wachstums auf des Messers Schneide hinfällig. Andererseits würde die (post)keynesianische Theorie langfristige Phänomene mit Hilfe eines für die kurze Frist entworfenen Instrumentariums analysieren. Multiplikator, Akzelerator und die Konstanz des Kapitalkoeffizienten sind zentrale Elemente der (post)keynesianischen Untersuchung konjunktureller Erscheinungen. Die Diskussion wachstumstheoretischer Fragestellungen mache aber Methoden notwendig, die einer langfristigen Entwicklung angepaßt sind ${ }^{33}$

die Einkommensverteilung durch veränderte Knappheitsverhăltnisse nicht berührt. Folglich bleibt auch die gesamtwirtschaftliche Sparquote konstant. Die Annahme einer kaldorianischen Sparfunktion fuhrt deshalb nicht zu einer variablen gesamtwirtschaftichen Sparquote.

31 Vgl. Kaldor (1955/56, S. 220). In Kapitel vier wird auf die Rolle der Investitionsfunktion in Kaldors Wachstumsmodellen eingegangen.

32 Die erste Auflage von Meades Buch erschien im Jahre 1961. Im folgenden wird jedoch die zweite Auflage verwendet, die 1962 veroffentlicht wurde.

33 Vgl. hierzu z.B. Solow (1956, S. 161f.). 


\subsubsection{Erste Ansätze: Die Überlegungen von Jan Tinbergen}

Im allgemeinen werden die Ursprünge der neoklassischen Wachstumstheorie in den oben angeführten Arbeiten von Tobin, Solow, Swan und Meade gesehen. Tatsächlich lassen sich aber schon in Marshalls Principles Ansätze eines solchen Modells entdecken. Während des Zweiten Weltkriegs entstand des weiteren ein Wachstumsmodell, das sehr große Ähnlichkeit mit den Strukturen hat, die heute als neoklassisches Grundmodell firmieren. In einem in deutscher Sprache erschienenen Aufsatz aus dem Jahre 1942 diskutiert Tinbergen einen Beitrag zur Theorie der Trendbewegung. Wie nach ihm Solow (1956) blendet Tinbergen die kurzfristigen, konjunkturellen Schwankungen aus, um sich ausschließlich auf die langfristige Analyse konzentrieren zu können.

"Das Hauptproblem der Trendbewegung, vor allem der »Realreihen« der Wirtschaft, ist jedoch ein anderes, und zwar die Erklärung des fortwährenden Anstiegs dieser Reihen. Auf die Bestimmungsgründe dieses Anstiegs und auf ihre mögliche Beeinflussung wird sich unsere Analyse insbesondere richten." 34

Die generelle Zielsetzung seiner Untersuchung gilt dabei dem genaueren Verständnis des langfristigen Entwicklungsprozesses, um diesen möglicherweise beeinflussen, d.h. die Wachstumspotentiale einer Volkswirtschaft besser nutzen zu können. ${ }^{35}$

Das zentrale Element der Analyse ist eine linear-homogene Cobb-Douglas-Produktionsfunktion (u):

$$
u=\varepsilon^{t} a^{\lambda} K^{1-\lambda}
$$

Hierbei steht (a) für die eingesetzte Arbeitsmenge, (K) für die Kapitalmenge, die auch Boden umfaßt, und $\left(\varepsilon^{t}\right)$ für den technischen Stand in der Volkswirtschaft zum Zeitpunkt $(t)$. Im heutigen Sprachgebrauch würde man von Hicks-neutralem technischen Fortschritt sprechen, der sich gleichmäßig auf beide Produktionsfaktoren auswirkt. ${ }^{36}$

Die Nachfrage nach Arbeit und Kapital resultiert aus dem Gewinnmaximierungskalkül der Unternehmer, d.h. es werden die Mengen an Produktionsfaktoren eingesetzt, bei denen die Grenzproduktivität des jeweiligen Faktors genau dem Reallohn ( ${ }^{\mathrm{r}}$ ) bzw. dem Realzins ( $\mathrm{i}^{\mathrm{r}}$ ) entspricht. Für die Nachfragegleichungen folgt somit: 37

34 Tinbergen (1942, S. 512)

35 Vgl. Tinbergen (1942, S. 517).

36 Dieses Konzept des technischen Fortschritts wurde von Hicks 1932 in seinem Buch The Theory of Wages entwickelt.

37 Vgl. Tinbergen (1942, S. 523f.). 


$$
\frac{\partial \mathrm{u}}{\partial \mathrm{a}}=\lambda\left(\frac{\mathrm{K}}{\mathrm{a}}\right)^{1-\lambda} \varepsilon^{\mathrm{t}}=\mathrm{l}^{\mathrm{r}} \text { und } \frac{\partial \mathrm{u}}{\partial \mathrm{K}}=(1-\lambda)\left(\frac{\mathrm{a}}{\mathrm{K}}\right)^{\lambda} \varepsilon^{\mathrm{t}}=\mathrm{i}^{\mathrm{r}}
$$

Für das Kapitalangebot wird von einem vollkommen unelastischen Angebot ausgegangen, so daß der Zinssatz keinen Einfluß auf die Angebotsseite hat. Beim Arbeitsangebot wird dagegen eine Abhängigkeit vom Lohnsatz unterstellt. Dabei soll folgender Zusammenhang gelten:

$$
\frac{1^{r}}{1^{\circ}}=\left(\frac{a}{b}\right)^{\gamma}
$$

In dieser Gleichung stehen $\left(1^{\circ}\right)$ für einen sog. Normallohn, (b) für die Bevölkerungsmenge und $(\gamma)$ für die Lohnflexibilität. Die Einführung eines Normallohns dient in diesem Zusammenhang dazu, auch Veränderungen der Lohnansprüche über die Zeit hinweg zuzulassen. ${ }^{38}$ Der Normallohn kann sich aufgrund des technischen Fortschritts oder veränderter sozialer Bewertungen der Arbeit verändern. Dies wird Folgen für die Bereitschaft zu arbeiten haben. Die Erwerbsquote würde bei jedem Reallohn c.p. zurückgehen. Tinbergen bezieht sich bei seinen Ausfuhrungen in großem Maße auf die Untersuchung von Douglas (The Theory of Wages) aus dem Jahre 1934. Aufgrund der dort angefuhrten Ergebnisse, die keinen eindeutigen Schluß auf den tatsächlichen Wert der Lohnflexibilităt des Arbeitsangebots erlauben, läßt er diesen offen.

Die Bevölkerung wächst entsprechend der Gleichung:

$$
b=b_{0} \beta^{t}
$$

d.h. die Bevölkerung wächst jedes Jahr mit einer konstanten Rate ( $\beta-1)$.

Die Investitionen pro Zeitraum sind durch

$$
\dot{\mathrm{K}}=\frac{\mathrm{dK}}{\mathrm{dt}}=\mathrm{I}=\chi \mathrm{u}
$$

bestimmt. Es gilt immer I=S, wobei das Sparen einen konstanten Teil des Sozialprodukts $(\chi)$ darstellt. ${ }^{39}$

38 Der Normallohn entspricht somit einem von der Gesellschaft als angemessen angesehenes Lohnniveau. Tinbergen fuhrt ein konstantes Wachstum des Normallohns ein:

$1^{\circ}=1^{\circ} \theta^{t}$ mit $\left(1^{\circ \circ}\right)$ als Konstante und $(\theta)$ als Steigerungsrate des Normallohns (vgl. Tinbergen 1942, S. 525).

39 Neben diesem Modell diskutiert Tinbergen noch andere, modifizierte Modelltypen, die sich bei der zugrundegelegten Produktionsfunktion (Komplementarităt anstelle von Substitutionalităt), der Wachstumsfunktion der Bevolkerung (logistisch anstelle von exponentiell), der Verwendung des Kapitalstocks (Voll- versus Unterauslastung) und der Frage, ob die Nachfrage- oder die Angebotsseite beschrankend wirkt, unterscheiden. 
Die Struktur des Modells von Tinbergen entspricht somit vollständig derjenigen, welche später zum Zentrum der neoklassischen Wachstumstheorie Solowscher Prägung wurde. ${ }^{40} \mathrm{Im}$ Unterschied zu den späteren Arbeiten konzentriert sich Tinbergen nicht auf die Analyse eines Steady State-Wachstumspfades, sondern ermittelt nur für die mittlere Periode $(t=0)$ die jeweiligen Wachstumsraten der Produktion, der Kapitalmenge und der Beschäftigung bei spezifischen Annahmen über die Lohnelastizität des Arbeitsangebots. Durch diese Betrachtungsweise werden die Einflüsse der unterschiedlichen Variablen auf den Wachstumsprozeß während einer Ungleichgewichtssituation deutlich. Tinbergen hatte also weniger die Referenzgröße Steady-State als vielmehr den Weg dorthin im Sinn.

Tab. 2.1: Tinbergens und Solows Modell im Vergleich

\begin{tabular}{|c|c|c|}
\hline Modellelement & Tinbergen (1942) & Solow (1956) \\
\hline Produktionsfunktion & $u=\varepsilon^{t} a^{\lambda} K^{1-\lambda}$ & $\begin{array}{l}\quad \mathrm{Y}=\mathrm{F}(\mathrm{K}, \mathrm{L}) \\
\text { nicht genau spezifiziert, muß je- } \\
\text { doch Inada-Bedingungen er-fullen. } \\
\text { Cobb-Douglas-PF somit moglich }\end{array}$ \\
\hline Sparen & $\mathrm{S}=\chi \mathrm{u}$ & $\mathrm{S}=\mathrm{sF}(\mathrm{K}, \mathrm{L})$ \\
\hline Investition & $\frac{\partial \mathrm{u}}{\partial \mathrm{K}}=(1-\lambda)\left(\frac{\mathrm{a}}{\mathrm{K}}\right)^{\lambda} \varepsilon^{\mathrm{t}}=\mathrm{i}^{\mathrm{r}}$ & $\begin{array}{l}\text { formal nicht spezifiziert, jedoch } \\
\text { auch Vorstellung der Grenzpro- } \\
\text { duktivitätsentlohnung }\end{array}$ \\
\hline Arbeitsangebot & $\frac{1^{r}}{1^{\circ}}=\left(\frac{a}{b}\right)^{\gamma}$ & $\mathrm{L}_{\mathrm{t}}=\mathrm{L}_{0} \mathrm{e}^{\mathrm{nt}}$ \\
\hline Arbeitsnachfrage & $\frac{\partial \mathrm{u}}{\partial \mathrm{a}}=\lambda\left(\frac{\mathrm{K}}{\mathrm{a}}\right)^{1-\lambda} \varepsilon^{\mathrm{t}}=1^{\mathrm{r}}$ & $\begin{array}{l}\text { formal nicht spezifiziert, jedoch } \\
\text { auch Vorstellung der Grenzpro- } \\
\text { duktivitätsentlohnung }\end{array}$ \\
\hline Bevölkerungsentwicklung & $b=b_{0} \beta^{t}$ & $\mathrm{~L}_{\mathrm{t}}=\mathrm{L}_{0} \mathrm{e}^{\mathrm{nt}}$ \\
\hline $\mathrm{I}=\mathrm{S}$ & erfüllt & erfüllt \\
\hline
\end{tabular}

Der Beitrag Tinbergens zur Wachstumstheorie geriet lange Zeit in Vergessenheit. Dies hat mit Sicherheit mit dem Erscheinungsort (Deutschland im Zweiten Weltkrieg) und der Sprache (deutsch anstelle von englisch) zu tun. Kurz nach dem Krieg wurde Tinbergens Modell von P.J. Verdoorn (1949) wieder aufgegriffen, um die Zusammenhänge zwischen dem langfristigen Output- und Produktivitätswachstum zu bestimmen. ${ }^{41}$ Darüber hinaus hat Verdoom (1959) gezeigt, daß die Lösung des Tinbergen-Modell für die Steady State-Wachstumsrate des

$40 \mathrm{Vgl}$. auch Tabelle 2.1.

41 Vgl. das Kapitel über den Verdoorn-Zusammenhang weiter unten. 
Outputs und des Kapitalstocks derjenigen des neoklassischen Modells mit einer Cobb-DouglasProduktionsfunktion entspricht. Diese kurzen Überlegungen zeigen, daß Tinbergen in der Ahnenreihe der neoklassischen Wachstumstheorie zumindest chronologisch vor den oben genannten Protagonisten stehen sollte. 42

\subsubsection{Die Solowsche Tradition}

Als Ausgangspunkt für eine ökonomische Analyse, welche den Anforderungen der langfristigen Betrachtungsweise besser angepaßt ist, dient in den neoklassischen Arbeiten der 50er und 60er Jahre die Einführung der neoklassischen Produktionsfunktion. In einer Ein-Gut-Welt wird unter Verwendung der zueinander annahmegemäß substitutionalen Produktionsfaktoren Arbeit (L) und Kapital (K) der gesamtwirtschaftliche reale Output (Y) erzeugt. ${ }^{43}$ Es soll gelten:

$$
\mathrm{Y}=\mathrm{F}(\mathrm{K}, \mathrm{L})
$$

Hierbei soll die Funktion $F(\cdot)$ linear homogen sein, d.h. die Produktion weist konstante Skalenerträge auf, wobei die Grenzproduktivitäten positiv sind, aber mit steigendem Faktoreinsatz abnehmen. ${ }^{44}$ In Verbindung mit der Annahme der Grenzproduktivitätsentlohnung der Produktionsfaktoren ist eine konsistente Ableitung der funktionalen Einkommensverteilung im neoklassischen System möglich. ${ }^{45}$ Für Solow stellen konstante Skalenerträge "...the natural assumption to make a theory of growth"46 dar. Würde ein konstanter, nicht vermehrbarer Produktionsfaktor (z.B. Land) vorliegen, kann es zu abnehmenden Skalenerträgen kommen. Interessanterweise diskutiert Solow an dieser Stelle nur diese Möglichkeit, nicht jedoch die

42 Vor allem in der deutschsprachigen Literatur findet man Hinweise auf Tinbergens Papier (z.B. in Bombach 1965, S. 778; Walter 1969, S. 116; 1983, S. 11).

43 Im weiteren werden reale Situationen betrachtet. Fragen des Preisniveaus bzw. dessen Änderungen sind nicht Inhalt der Untersuchung. Der Zeitindex wird im allgemeinen vernachlasssigt und nur an Stellen verwendet, wenn es erforderlich ist. Solow selbst diskutiert nicht nur den Fall einer substitutionalen Produktionsfunktion, sondern geht auch auf die Implikationen fixer Produktionskoeffizienten ein (vgl. Solow (1956, S. 170ff.).

44 Die Produktionsfunktion hat formal gesprochen folgende Eigenschaften:

1.) $\frac{\partial \mathrm{Y}}{\partial \mathrm{K}}>0$ und $\frac{\partial \mathrm{Y}}{\partial \mathrm{L}}>0$, d.h. positive Grenzproduktivitaten.

2.) $\frac{\partial^{2} \mathrm{Y}}{\partial \mathrm{K}^{2}}<0$ und $\frac{\partial^{2} \mathrm{Y}}{\partial \mathrm{L}^{2}}<0$, d.h. sinkende Grenzproduktivităten.

45 Vgl. z.B. Rose (1987), Külp (1994) oder Preiser (1970a, b)

46 Solow $(1956$, S. 163). 
Folgen steigender Skalenerträge. Insgesamt soll die Produktionsfunktion die bekannten InadaBedingungen erfüllen, d.h. well-behaved sein. ${ }^{47}$

Die Bruttoinvestitionen ( $\mathrm{I}^{\mathrm{br}}$ ) entsprechen zu jedem Zeitpunkt den Ersparnissen und stellen im einfachen Grundmodell einen konstanten Teil (s) des Einkommens dar:

$$
S=s Y=s F(K, L)
$$

Berücksichtigt man noch zusätzlich Abschreibungen auf den Kapitalstock ( $\delta \mathrm{K})$, gilt für die Veränderung des Kapitalstocks:

$$
\dot{\mathrm{K}}=\mathrm{I}^{\mathrm{br}}-\delta \mathrm{K}=\mathrm{sF}(\mathrm{K}, \mathrm{L})-\delta \mathrm{K}
$$

Für die Produktionsfaktoren wird unterstellt, daß sie zu jedem Zeitpunkt vollbeschäftigt sind. Die Bevölkerung soll exponentiell mit der Rate (n) wachsen, so daß die verfügbare Arbeitsmenge zu einem bestimmten Zeitpunkt $\left(\mathrm{L}_{\mathrm{t}}\right)$ durch

$$
\mathrm{L}_{\mathrm{t}}=\mathrm{L}_{0} \mathrm{e}^{\mathrm{nt}} \quad \text { mit } \mathrm{n} \geq 0
$$

gegeben ist. Bei einer konstanten Erwerbsquote wächst auch der Arbeitsinput mit der gleichen Rate (n) wie die Bevölkerung. Gleichung (2.20) kann auch direkt als Arbeitsangebotsfunktion interpretiert werden, die ein unelastisches Arbeitsangebot bzgl. des Reallohns implizieren würde. Ein nach oben und nach unten flexibler Reallohn ermöglicht in beiden Fällen den Ausgleich zwischen Arbeitsangebot und Arbeitsnachfrage, so daß die Volkswirtschaft sich immer im Vollbeschäftigungsgleichgewicht befindet. ${ }^{48}$

Aus den Annahmen für die Entwicklung der Beschäftigung und des Kapitalstocks, folgt für die Bewegungsgleichung des letzteren die Abhängigkeit von der vorhandenen Arbeitsmenge. Die Gleichung (2.19) gibt den Wert der vollbeschäftigungskonformen Kapitalbildung an, die aussagt, wie hoch die Nettoinvestitionen pro Periode sein müssen, damit die angebotene Arbeit beschäftigt werden kann. In jeder Periode bestimmt die vorhandene Arbeitsmenge und der von der Vorperiode übernommene Kapitalstock die Produktionsmöglichkeiten. Der Faktorpreismechanismus garantiert die Vollbeschäftigung beider Faktoren, so daß mit Hilfe der Produktionsfunktion $\mathrm{F}(\mathrm{K}, \mathrm{L})$ die hergestellte Gütermenge und über die Sparfunktion die geplanten Investitionen ermittelt werden können.

47 Für die jeweiligen Grenzwerte muB deshalb gelten:

$\lim _{i \rightarrow 0} \frac{\partial Y}{\partial i}=\infty$ und $\lim _{i \rightarrow \infty} \frac{\partial Y}{\partial i}=0$ mit $i=K, L$

Vgl. Inada (1963).

$48 \mathrm{Vgl}$. Solow $(1956$, S. 163.) 
Die Produktionsfaktoren beeinflussen neben der Höhe des Outputs auch dessen Wachstumsrate. Differenziert man die Produktionsfunktion nach der Zeit, zeigt sich, daß sich das Wachstum des Outputs zusammensetzt aus:

$$
\frac{\mathrm{dY}}{\mathrm{dt}}=\frac{\partial \mathrm{Y}}{\partial \mathrm{K}} \frac{\mathrm{dK}}{\mathrm{dt}}+\frac{\partial \mathrm{Y}}{\partial \mathrm{L}} \frac{\mathrm{dL}}{\mathrm{dt}}
$$

Durch Division der Gleichung mit ( $\mathrm{Y}$ ) folgt:

$$
\frac{\mathrm{dY}}{\mathrm{dt}} \frac{1}{\mathrm{Y}}=\frac{\partial \mathrm{Y}}{\partial \mathrm{K}} \frac{\mathrm{dK}}{\mathrm{dt}} \frac{1}{\mathrm{Y}}+\frac{\partial \mathrm{Y}}{\partial \mathrm{L}} \frac{\mathrm{dL}}{\mathrm{dt}} \frac{1}{\mathrm{Y}}
$$

Mit $F_{K}=\frac{\partial Y}{\partial K}$ bzw. $F_{L}=\frac{\partial Y}{\partial L}$ und partiellem Erweitern mit $K / K$ sowie mit $L / L$ ergibt sich für die Wachstumsrate des Outputs:

$$
\hat{\mathrm{Y}}=\mathrm{F}_{\mathrm{K}} \frac{\mathrm{dK}}{\mathrm{dt}} \frac{\mathrm{K}}{\mathrm{K}} \frac{1}{\mathrm{Y}}+\mathrm{F}_{\mathrm{L}} \frac{\mathrm{dL}}{\mathrm{dt}} \frac{\mathrm{L}}{\mathrm{L}} \frac{1}{\mathrm{Y}}
$$

Unter Verwendung des Kapitalkoeffizienten $v=\frac{K}{Y}$ und des Pro-Kopf-Outputs $y=\frac{Y}{L}$ kann (2.22) weiter umgeschrieben werden in:

$$
\hat{\mathrm{Y}}=\frac{1}{\mathrm{v}^{-1}} \mathrm{~F}_{\mathrm{K}} \frac{\mathrm{dK}}{\mathrm{dt}} \frac{1}{\mathrm{~K}}+\frac{1}{\mathrm{y}} \mathrm{F}_{\mathrm{L}} \frac{\mathrm{dL}}{\mathrm{dt}} \frac{1}{\mathrm{~L}}
$$

bzw. in

$$
\hat{\mathbf{Y}}=\mathrm{E}_{\mathrm{YK}} \hat{\mathbf{K}}+\mathrm{E}_{\mathrm{YL}} \hat{\mathbf{L}}
$$

wobei $E_{Y K}=\frac{F_{K}}{v^{-1}}$ und $E_{Y L}=\frac{F_{L}}{y}$ den partiellen Produktionselastizitäten des Kapitals bzw. der Arbeit entsprechen. Die Wachstumsrate des Outputs ist somit eine gewichtete Summe der Wachstumsraten der Inputs. Wird eine Komponente für den technischen Fortschritt hinzugefügt, erhöht sich die Wachstumsrate entsprechend.

Eine Produktionsfunktion mit den oben dargestellten Eigenschaften kann durch Division mit $(\mathrm{L})$ in eine Pro-Kopf-Produktionsfunktion überfuhrt werden. Der Pro-Kopf-Output (y) wird in diesem Falle von der Kapitalintensität (k) bestimmt:49

49 Die Moglichkeit der Uberfuhrung von $\mathrm{F}(\mathrm{K}, \mathrm{L})$ in $\mathrm{f}(\mathrm{K})$ ist eine Konsequenz der konstanten Skalenerträge der Produktionsfunktion. Deutlich wird dies anhand einer Cobb-Douglas-Produktionsfunktion. Wenn 


$$
y=f(k)
$$

Die Bewegungsgleichung des Kapitalstocks (2.19) kann nun ebenfalls in Pro-Kopf-Größen dargestellt werden. Gleichung (2.19) wird $\mathrm{zu}^{50}$

$$
\dot{k}=\operatorname{sf}(k)-(n+\delta) k
$$

Hierbei gibt $\mathbf{s f}(\mathbf{k})$ das Sparen pro Kopf und $(\mathrm{n}+\delta) \mathbf{k}$ die notwendige Investitionshöhe pro Kopf an, um eine konstante Kapitalintensität aufrechtzuerhalten. Die Kapitalintensität würde ansonsten aufgrund der Zunahme der Bevölkerung und/oder der technischen Abnutzung der Anlagen sinken, d.h. die Produktionskapazitäten der Volkswirtschaft würden sich reduzieren. Man könnte deshalb bei $(n+\delta)$ von einer effektiven Abschreibungsrate des Kapitals sprechen. ${ }^{51}$ Erhöht sich die Kapitalintensität im Zeitablauf wird auch von capital-deepening gesprochen. Analog hierzu wird $(\mathrm{n}+\delta) \mathrm{k}$ als capital widening bezeichnet.

\subsubsection{Das langfristige Gleichgewicht: Steady State}

Die neoklassische Wachstumstheorie konzentriert sich auf die Analyse von langfristigen Gleichgewichtssituationen. Hierbei muß zwischen einem dynamischen Gleichgewicht (DG) und einem dynamischen Steady State-Gleichgewicht (DSSG) unterschieden werden. Unter einem dynamischen Gleichgewicht wird eine Situation verstanden, in der die Produktionskapazitäten ausgelastet sind, die Wachstumsraten der Variablen aber nicht konstant sind. In einer dynamischen Steady State-Situation herrscht Vollauslastung, und die Wachstumsraten der Variablen sind konstant. Innerhalb des neoklassischen Wachstumsmodells kann aufgrund der Annahmen immer von einer DG-Situation ausgegangen werden, auch wenn die Volkswirtschaft sich nicht im dynamischen Steady State-Gleichgewicht befindet. Im postkeynesianischen Modell dagegen können beide Gleichgewichte nur zusammen auftreten, da die Vollauslastung der Kapazitäten nur im Falle der DSSG-Situation möglich ist. Im folgenden wird für die DSSG-Situation der Begriff des Steady States verwendet. Die Situation des Steady State ist damit durch die Konstanz der Wachstumsraten der Modellvariablen gekennzeichnet. Dies

$Y=L^{\alpha} K^{1-\alpha}$ gegeben ist, dann gilt: $y=\frac{Y}{L}=\frac{K^{1-\alpha}}{L^{1-\alpha}}=k^{1-\alpha}$. $Y$ ist somit durch $L y=L k^{1-\alpha}$ bestimmt. Auch für die Pro-Kopf-Produktionsfunktion sind die Inada-Bedingungen erfullt.

50 In einem ersten Schritt würde sich $\frac{\dot{K}}{L}=s f(k)-\delta k$ ergeben. Mit $\dot{k}=\frac{d\left(\frac{K}{L}\right)}{d t}=\frac{\dot{K}}{L}-n k$ ergibt sich daraus (2.14). Vgl. hierzu z.B. Barro/Sala-i-Martin (1995, S. 17).

51 Vgl. z.B. Walter (1983, S. 39) und Barro/Sala-i-Martin (1995, S. 18f.) 
impliziert jedoch nicht automatisch die Identität aller Wachstumsraten. Vielmehr kann gezeigt werden, daß sich bei der Forderung der Konstanz der Wachstumsraten aufgrund der Annahmen über die Produktionstechnik und die Sparfunktion die Identität der Veränderungsraten als Konsequenz ergibt. Da auch die Kapitalintensität mit einer konstanten Rate wachsen muß, lassen sich aus (2.14) weitere Bedingungen für den Steady State ableiten.

Teilt man Gleichung (2.14) durch (k), steht für die Wachstumsrate der Kapitalintensität:

$$
\frac{\dot{k}}{k}=\frac{s f(k)}{k}-(n+\delta)
$$

Die Kapitalintensität verändert sich mit einer konstanten Rate, wenn $\left(\frac{\mathrm{sf}(\mathrm{k})}{k}-(\mathrm{n}+\delta)\right)$ konstant ist. Annahmegemäß sind die Sparquote und die Wachstumsrate der Bevölkerung konstant. Deshalb muß auch $\mathrm{f}(\mathrm{k}) / \mathrm{k}$ konstant sein. Differenziert man diesen Term nach der Zeit, folgt:

$$
\left(-\frac{\left[\mathrm{f}(\mathrm{k})-\mathrm{kf}^{\prime}(\mathrm{k})\right]}{\mathrm{k}}\right) \frac{\dot{\mathrm{k}}}{\mathrm{k}}
$$

Der Term in der eckigen Klammer $\left[f(k)-k f^{\prime}(k)\right]$ entspricht der Grenzproduktivität der Arbeit, die definitionsgemäß immer größer Null ist. Der Wert in der runden Klammer wird mit steigender Kapitalintensität sinken und bei einer unendlichen Kapitalintensität Null sein. Für einen endlichen Wert von (k) kann der gesamte Ausdruck nur gleich Null sein, wenn die Wachstumsrate der Kapitalintensität Null ist. Die Forderung nach Konstanz der Wachstumsraten im Steady State bedingt die Konstanz der Kapitalintensität. 52

Für den gleichgewichtigen Wachstumspfad folgt somit aufgrund dieses Ergebnisses aus (2.25):

$$
\dot{k}=\operatorname{sf}(k)-(n+\delta) k=0
$$

bzw.

52 Schreibt man $Y=L f(k)$, so ist aufgrund der Produktregel $\frac{\partial Y}{\partial L}=f(k)+\frac{\partial f(k)}{\partial L} L$. Hierbei ergibt sich für den zweiten Teil des Terms auf der rechten Seite: $\frac{\partial f(k)}{\partial L} L=\frac{\partial f(k)}{\partial k} \frac{d k}{d L}=f^{\prime}(k) \frac{-K}{L^{2}} L=-k f^{\prime}(k)$. Insgesamt ergibt sich für die Grenzproduktivităt der Arbeit: $\mathbf{f}(\mathbf{k})-\mathbf{k f} \mathbf{f}^{\prime}(\mathbf{k})$.

Die runde Klammer kann auch als $\frac{f(k)}{k}-f^{\prime}(k)$ geschrieben werden. Dies entspricht der Differenz aus der Durchschnitts- und der Grenzproduktivităt des Kapitals. Für $\mathbf{k} \rightarrow \infty$ streben beide gegen null. Für die Grenzproduktivităt ergibt sich dies aus den Inada-Bedingungen. Die Durchschnittsproduktivitat des Kapitals sinkt bei steigender Kapitalintensităt aufgrund der sinkenden Grenzproduktivităt des Kapitals. 


$$
\operatorname{sf}(k)=(n+\delta) k \text { bzw. } \quad \frac{\operatorname{sf}(k)}{k}=\frac{s}{v}=n+\delta
$$

Im Steady State entspricht somit der Quotient aus Sparquote (s) und Kapitalkoeffizient (v) der Summe aus der Wachstumsrate der Bevölkerung (n) und der Abschreibungsrate ( $\delta$ ). Dasselbe Ergebnis hätte auf anderem Wege über die notwendige Gleichgewichtsbedingung I=S erreicht werden können, wobei $\mathrm{sf}(\mathrm{k})=(\mathrm{n}+\delta) \mathrm{k}$ sein muß.53

Da die Wachstumsrate der Kapitalintensität auf einem gleichgewichtigen Wachstumspfad Null sein muß, müssen sich die Wachstumsraten des Kapitalstocks und der Bevölkerung entsprechen. Dies wird auch deutlich, wenn man Gleichung (2.19) durch den Kapitalstock dividiert:

$$
\frac{\dot{\mathrm{K}}}{\mathrm{K}}=\frac{\mathrm{I}}{\mathrm{K}}-\delta=\mathrm{s} \frac{\mathrm{F}(\mathrm{K}, \mathrm{L})}{\mathrm{K}}-\delta=\frac{\mathrm{s}}{\mathrm{v}}-\delta \quad \text { bzw. } \quad\left(2.28^{\prime}\right) \frac{\mathrm{s}}{\mathrm{v}}=\frac{\dot{\mathrm{K}}}{\mathrm{K}}+\delta
$$

Vergleicht man (2.28) mit (2.27), folgt

$$
\frac{\dot{\mathbf{K}}}{\mathbf{K}}=\mathrm{n}
$$

Wenn aber der Kapitalstock und die Bevölkerung bzw. die eingesetzte Arbeitsmenge mit derselben Rate wachsen, wird auch der Output nach Gleichung (2.23) im Falle einer linearhomogenen Produktionsfunktion mit dieser Rate zunehmen. Eine Bestätigung dieser Aussage erfolgt, wenn die Konstanz der Wachstumsrate des Outputs als Startpunkt gewählt wird. Soll im Steady State $(\hat{Y})$ fix sein, wird der Kapitalstock aufgrund der Annahme einer konstanten Sparfunktion ebenfalls mit dieser Rate wachsen. Nach (2.28) gilt:

$$
\frac{\dot{\mathrm{K}}}{\mathrm{K}}=\frac{\mathrm{s}}{\mathrm{v}}-\delta=\frac{\mathrm{sY}}{\mathrm{K}}-\delta
$$

Damit $(\hat{\mathrm{K}})$ konstant ist, muß auch der Kapitalkoeffizient konstant bleiben, d.h. es ist notwendig, daß $\hat{\mathbf{Y}}=\hat{\mathbf{K}}$ ist. Aufgrund der unterstellten Produktionstechnik muß auch hier letztendlich gelten:

$$
\hat{\mathbf{Y}}=\hat{\mathbf{K}}=\mathbf{n}=\frac{\mathrm{s}}{\mathrm{V}}-\delta
$$

53 Vgl. Barro/Sala-i-Martin (1995, S. 17f.). Walter (1983, S. 40f.) und Rose (1987, S. 92f.). 
Das oben abgeleitete Steady State Gleichgewicht ist als Folge der Eigenschaften der neoklassischen Produktionsfunktion stabil. Liegt z.B. die Kapitalintensität unter ihrem langfristigen Gleichgewichtswert, wird ihre Wachstumsrate positiv sein, d.h. die Kapitalausstattung pro Arbeitseinheit wird zunehmen. Dies wird deutlich, wenn die Gleichung der Wachstumsrate in eine Grafik umgesetzt wird (vgl. Abb. 2.1).

\section{Abb. 2.1 Stabilität im neoklassischen Grundmodell}

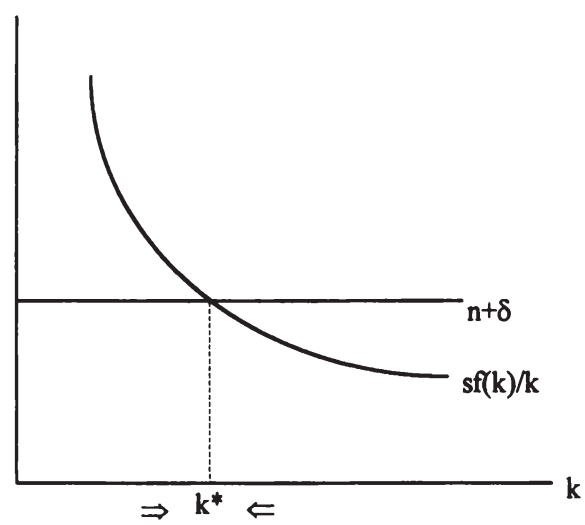

Quelle: Barro/Sala-i-Martin 1995, S. 23.

Der erste Term der Wachstumsratengleichung der Kapitalintensität ist eine Funktion mit negativer Steigung. Für $k<k^{*}$ ist $s f(k) / k$ größer als $n+\delta$, d.h. daß die Bruttoinvestition über dem effektiven "Kapitalverzehr" durch Bevölkerungswachstum und technischer Abnutzung liegt. Der Kapitaleinsatz pro Kopf steigt. Bei einer höheren Kapitalintensität wird die Grenzproduktivität des Kapitals gesunken sein. Der Kapitalkoeffizient wird deswegen steigen, was wiederum $s f(k) / k$ reduziert. Im Steady State schließlich verändert sich die Kapitalintensität nicht mehr, da die Bruttoinvestitionen genau ausreichen, den "Kapitalverzehr" zu decken. Die Grenzproduktivität des Kapitals bleibt in dieser Situation konstant und somit auch der Kapitalkoeffizient. 54

Konsequenterweise resultiert aus der Identität der Wachstumsraten aller Variablen im Steady State die Konstanz der Pro-Kopf-Größen. Sowohl der Pro-Kopf-Output als auch der Pro-Kopf-Konsum wachsen mit der Rate Null. Das neoklassische Grundmodell liefert aus diesem Grunde keine Erklärung für die wachsende Güterversorgung pro Kopf in der Realität.

54 Damit dieser Anpassungsprozeß møglich ist, müssen die Faktorpreise ausreichend flexibel sein. Verăndern sich die relativen Faktorpreise aufgrund des im Vergleich zur Beschätigung schneller gewachsenen Kapitalangebots zuungunsten des Realzinses, kommt es zur Absorption des Kapitalmehrangebots im ProduktionsprozeB. Vgl. zur Stabilităt des Gleichgewichts Barro/Sala-i-Martin (1995, S. 22ff.), Walter (1983, S. 37ff.) und Rose (1987, S. 85ff.) 
Darüber hinaus folgt aus der Gleichheit aller Wachstumsraten ein Prozeß des strukturkonstanten Wachstums und somit ein weiteres Ergebnis, das nicht den realen Entwicklungen entspricht.

Ein weiterer Mangel ist die Unabhängigkeit der langfristigen Wachstumsrate von den Spar- und Investitionsentscheidungen der Wirtschaftssubjekte. Erhöht sich z.B. die Sparquote in der Volkswirtschaft, so liegt die Wachstumsrate des Kapitalstocks über der natürlichen Wachstumsrate (n). Es tritt derselbe Anpassungsprozeß ein, wie er oben bei der Abweichung der tatsächlichen Kapitalintensität von der gleichgewichtigen erläutert wurde. Das größere Kapitalangebot führt zu einem Rückgang des Zinssatzes. Die Investitionen steigen. Aufgrund der Gültigkeit der Gesetzes des abnehmenden Grenzertrags bei der partiellen Variation eines Produktionsfaktors wird der Kapitalkoeffizient zunehmen und gleicht somit die wachstumsbeschleunigenden Effekte der höheren Sparquote wieder aus. Die tatsächliche Wachstumsrate (s/v) sinkt stetig auf das Niveau der natürlichen Rate (n) ab. Die neue Gleichgewichtssituation ist durch eine gestiegene Kapitalintensität gekennzeichnet (vgl. Abb. 2.2).

\section{Abb. 2.2 Veränderung der Kapitalintensität bei Erhöhung der Sparquote}

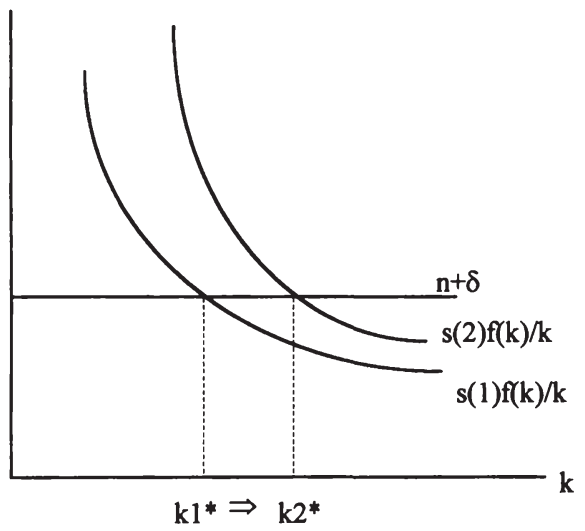

Quelle: Barro/Sala-i-Martin 1995, S. 25.

Während des Anpassungsprozesses weichen die Wachstumsraten der Variablen voneinander ab, wobei gilt:

$$
\hat{\mathbf{K}}>\hat{\mathrm{Y}}>\mathbf{n}
$$


Aufgrund dieser Differenzen steigt durch eine Erhöhung der Spar- bzw. der Investitionsquote neben der Kapitalintensität auch der Pro-Kopf-Output. Der neue Gleichgewichtspfad weist ein höheres Niveau der Kapitalintensität und des Outputs auf. Die gleichgewichtige Wachstumsrate $(n)$ verändert sich jedoch nicht, d.h. das langfristige Wachstum kann nicht beschleunigt werden. Die Bereitschaft, dauerhaft mehr zu sparen, bietet die Möglichkeit, während des Anpassungsprozesses die tatsächliche Wachstumsrate zu steigern und das Aktivitätsniveau der Volkswirtschaft zu erhöhen, aber nur eine ständige Erhöhung der Sparquote kann diesen Vorgang aufrechterhalten. Verharrt die Sparquote auf einem bestimmten Niveau, sorgt die Erhöhung des Kapitalkoeffizienten für die Anpassung der tatsächlichen an die gleichgewichtige Wachstumsrate. 55

Wie gezeigt, ist die Variabilität des Kapitalkoeffizienten (v) verantwortlich für die Erreichung des Steady State-Pfades. Würde (v), d.h. würde die Kapitalproduktivität bei einer Erhöhung der Sparquote konstant bleiben, käme es zu keiner Annäherung der Kapital- und der Outputwachstumsrate an die natürliche Wachstumsrate. ${ }^{56}$ Der Rückgang der durchschnittlichen Kapitalproduktivität ist nun das Ergebnis der abnehmenden Grenzproduktivität des Kapitals. Würde der Kapitaleinsatz z.B. konstante Grenzerträge aufweisen, würde der Output immer, d.h. auch außerhalb des Steady States, mit derselben Rate wie der Kapitalstock wachsen. Eine Veränderung der Sparquote hätte in diesem Falle dauerhafte Konsequenzen für die tatsächliche Wachstumsrate des Systems.

Der Kapitalkoeffizient ist gegeben durch:

$$
v=\frac{k}{f(k)}
$$

Der Kapitalkoeffizient ist über die Zeit konstant, wenn dessen Veränderung ( $\dot{v})$ Null ist. Differenziert man (20) nach der Zeit, folgt:

$$
\dot{v}=\frac{d v}{d t}=\frac{\dot{k f}(k)-k f^{\prime}(k) \dot{k}}{[f(k)]^{2}}=\frac{\dot{k}\left[f(k)-k f^{\prime}(k)\right]}{[f(k)]^{2}}
$$

55 Vgl. hierzu z.B. Solow (1956, S. 166f.), Barro/Sala-i-Martin (1995, S. 24f.) und Walter (1983, S. 46ff.). In seinem hăufig zitierten Aufsatz zu den Möglichkeiten einer Fiskalpolitik im neoklassischen Wachstumsmodell zeigt Sato unter Verwendung einer Cobb-Douglas-Produktionsfunktion und Hicksneutralem technischen Fortschritt, wie groß der Zeitraum ist, bis eine Volkswirtschaft $90 \%$ des Anpassungsprozesses hinter sich gebracht hat. Dieser Zeitraum wird um so größer, je hơher die Sparquote in der Ausgangssituation war und ist um so kürzer, je größer die neue Sparquote, die Rate des technischen Fortschritts und die Rate des Bevollkerungswachstums sind. Die Anpassungszeit wird auch durch eine hohe Produktionselastizităt der Arbeit verkürzt (vgl. Sato 1968, S. 180).

56 Die Situation entspricht dem Harrod-Modell. 
Damit $\dot{v}=0$ ist, muß $\dot{k}\left[\mathbf{f}(\mathbf{k})-k f^{\prime}(\mathbf{k})\right]=0$ sein. Soll dies immer, $d . h$. auch außerhalb des Steady States, der Fall sein, muß $f(k)-k f^{\prime}(k)=0$ gelten. Hieraus ergibt sich als Bedingung für die Konstanz von (v):

$$
\frac{f(k)}{k}=f^{\prime}(k)
$$

Die Durchschnittsproduktivität des Kapitals muß, wie oben gezeigt, der Grenzproduktivität entsprechen. Dieses Ergebnis hat jedoch weitere Implikationen für die Produktionstechnik. Die Differenz $f(k)-k f^{\prime}(k)$ entspricht der Grenzproduktivität der Arbeit. Die Bedingung für die Konstanz des Kapitalkoeffizienten erfordert somit, daß die Grenzproduktivität der Arbeit gleich Null ist. Dies ist aber aufgrund der Eigenschaften der zugrundegelegten Produktionsfunktion nur möglich, wenn der Arbeitseinsatz gegen unendlich geht. Für alle anderen Situationen $(\mathrm{L}<\infty)$, ist die Grenzproduktivität der Arbeit größer Null.

Eine weitere Möglichkeit zur Erfüllung von (2.33), ist die Wahl einer Produktionsfunktion, die keinen Arbeitseinsatz aufweist, d.h. als einzigen Produktionsfaktor Kapital benötigt. Ist die mögliche Produktionsmenge unabhängig vom Arbeitseinsatz, ist

$$
\frac{\partial \mathrm{Y}}{\partial \mathrm{L}}=\frac{\partial \mathrm{f}(\mathrm{k})}{\partial \mathrm{L}}=0
$$

Dieser Kunstgriff ermöglicht somit eine dauerhafte Differenz zwischen der Wachstumsrate des Kapitalstocks bzw. des Outputs und der natürlichen Wachstumsrate. Die ausschließliche Abhängigkeit des Produktionsergebnisses vom Kapitaleinsatz ist jedoch nur eine notwendige und noch keine hinreichende Bedingung hierfür. Die Identität von Durchschnitts- und Grenzproduktivität erfordert darüber hinaus, daß die Produktionsfunktion konstante Skalenerträge aufweist. Diese Eigenschaften werden z.B. von einer limitationalen Produktionsfunktion mit einem Kapitalkoeffizienten $v=1$ erfüllt.:

$$
\mathbf{Y}=\mathbf{K}
$$

Im Rahmen der Neuen Wachstumstheorie wurden entsprechende Produktionsfunktionen als eine Möglichkeit zur Generierung endogenen, dauerhaften Pro-Kopf-Wachstums diskutiert. Aus diesem Grund wird auf diese weiter unten noch einmal eingegangen (vgl. Abschnitt 3.3). 


\subsubsection{Der Begriff der Konvergenz im neoklassischen Grundmodell}

Die Annahmen über die neoklassische Produktionsfunktion, insbesondere die Variabilität des Kapitalkoeffizienten, sorgten für die Anpassung des Wachstumspfades nach einer Parameteränderung an den Steady State-Pfad. Darüber hinaus zeigte sich ein inverser Zusammenhang zwischen der Abweichung der tatsächlichen von der gleichgewichtigen Kapitalintensität $\left(\Delta \mathrm{k}=\mathrm{k}_{\mathrm{tat}}-\mathrm{k}^{*}\right)$ und der Wachstumsrate des Kapitalstocks $(\hat{\mathrm{K}})$ bzw. derjenigen der Kapitalintensität $(\hat{\mathbf{k}})$. Es gilt:

$$
\hat{\mathrm{k}}=\Phi\left(\mathrm{k}_{\text {tat }}-\mathrm{k}^{*}\right)=\Phi(\Delta \mathrm{k}) \quad \text { mit } \quad \Phi(0)=0 \text { und } \frac{\partial \Phi}{\partial \Delta \mathrm{k}}<0
$$

Je weiter eine Volkswirtschaft vom Steady State entfernt ist, d.h. je mehr $\left(\mathrm{k}_{\text {tat }}\right)$ nach unten von $\left(\mathrm{k}^{*}\right)$ abweicht, desto höher ist ihre tatsächliche Wachstumsrate des Kapitalstocks und folglich auch diejenige des Outputs. Dies legt auf den ersten Blick die Schlußfolgerung nahe, daß kapitalarme Volkswirtschaften schneller wachsen müssen als kapitalreiche. Dieser Zusammenhang wird auch als absolute Konvergenz bezeichnet. ${ }^{57}$ Diese Aussage ist aber nur bedingt richtig. Eine im internationalen Vergleich geringe Kapitalintensität hat nicht zwangsläufig eine hohe Wachstumsrate zur Folge. Nur wenn alle Volkswirtschaften dieselben Parameterwerte aufweisen, d.h. Sparquoten, Produktionsfunktionen, Bevölkerungswachstum und Abschreibungsrate müßten identisch sein, gilt diese These uneingeschränkt. Im Falle unterschiedlicher Parameterwerte kann nicht ohne weiteres von der Höhe der Kapitalintensität auf die Wachstumsrate des Outputs und des Kapitalstocks geschlossen werden. Vielmehr entscheidet der Abstand vom eigenen Steady State-Wert über die Höhe des tatsächlichen Wachstums. Je größer der Abstand von der spezifischen Steady State-Kapitalintensität ist, desto höher wird die Wachstumsrate sein. Man spricht dann auch von bedingter Konvergenz. Eine hohe Kapitalausstattung pro Arbeitseinheit kann deshalb mit hohen Wachstumsraten einhergehen. 58

In diesem Zusammenhang kommt der Sparquote eine große Bedeutung zu. Weist eine Volkswirtschaft eine hohe Kapitalintensität und eine hohe Sparquote auf, wird sie u.U. höhere Wachstumsraten realisieren können als eine vergleichsweise kapitalarme Volkswirtschaft, die eine geringe Sparneigung besitzt. Die obigen Aussagen zur Konvergenz an den Steady State gelten ohne Einschränkung für geschlossene Volkswirtschaften, wo die Sparquote eines Landes durch die Präferenzen der inländischen Bevölkerung bestimmt wird. Sie müssen jedoch relativiert werden, wenn internationale Kapitalströme zugelassen werden. In diesem Fall attrahieren kapitalarme Volkswirtschaften aufgrund ihrer im Vergleich zu anderen Ökonomien

57 Ein Begriff, bei dem Sala-i-Martin fur sich in Anspruch nimmt, ihn in die wirtschaftstheoretische Literatur eingefuhrt zu haben. Vgl. Sala-i-Martin (1996, S. 1020).

58 Vgl. zur Frage des Konvergenzbegriffs Barro/Sala-i-Martin (1995, S. 26ff.) und Sala-i-Martin (1996, S. 1020ff.). 
hohen Grenzproduktivität des Kapitals ausländische Ersparnisse. Aufgrund dieses Zustroms an Kapital erhöht sich die Spar- und damit die Investitionsquote des Landes bezogen auf das eigene Sozialprodukt. Die Wachstumsrate des Kapitalstocks nimmt zu. In Abbildung (2.1) würde dies einer Verschiebung der $\mathrm{sf}(\mathrm{k}) / \mathrm{k}$-Kurve entsprechen.

Die Annahmen des neoklassischen Grundmodells bedingen somit nicht automatisch höhere Wachstumsraten für kapitalarme Volkswirtschaften, sondern implizieren nur eine Konvergenz zum jeweiligen, länderspezifischen Steady State-Pfad, dessen Steigung sich nicht, dessen Niveau sich durch Variationen der Sparquote und/oder internationale Kapitalverflechtungen verändern kann. Darüber hinaus bleibt festzuhalten, daß die Konvergenz an den Steady State nicht zwangsläufig die Verringerung der Streuung der Pro-Kopf-Outputs aller Länder zur Folge hat. Die Zunahme aller Pro-Kopf-Einkommen bedeutet nicht zwangsläufig eine Abnahme der Streuung der Einkommensniveaus zwischen den Ländern. Je nach Ausgangssituation kann diese zu- oder abnehmen. 59

\subsection{5 Überwindung des Nullwachstums: Exogener technischer Fortschritt}

Der Steady State-Wachstumspfad im Grundmodell der neoklassischen Wachstumstheorie ist, wie oben gezeigt, durch die Identität der Wachstumsraten aller Variablen gekennzeichnet. Dies impliziert die Konstanz aller Pro-Kopf-Größen, d.h. die betrachtete Volkswirtschaft wächst in Höhe der natürlichen Wachstumsrate (n), die Güterversorgung der Wirtschaftssubjekte verbessert sich jedoch nicht. Diese Aussage gilt allerdings nur fur die gleichgewichtige Situation. Hat die Volkswirtschaft diesen Pfad z.B. aufgrund einer Erhöhung der Sparquote verlassen, kommt es zur Veränderung der Pro-Kopf-Größen. Dauerhaft stellt das exogen vorgegebene Wachstum der Bevölkerung eine Schranke für die Entwicklung dar, so daß ohne einen weiteren Faktor die empirisch feststellbare Entwicklung der realen Pro-Kopf-Einkommen durch das neoklassische Modell nicht erklärt werden kann. Eine Möglichkeit hierzu ist die Einführung des Stands der Technik als zusätzlicher, kostenloser Produktionsfaktor. Die während einer Periode mögliche Produktionsmenge hängt dann neben den eingesetzten Mengen von Arbeit und Kapital auch vom erreichten Wissen der Volkswirtschaft ab. Technischer Fortschritt, d.h. Veränderungen des Stands der Technik, kann in der Realität in Form von Prozeß- und Produktinnovationen aufteten bzw. in vielen Fällen eine Mischung aus beiden sein. Die Wachstumstheorie, wie sie in diesem Kapitel vorgestellt wird, konzentriert sich maßgeblich auf Prozeßinnovationen.

In den traditionellen neoklassischen Wachstumsmodellen wird gemeinhin die Unabhängigkeit des technischen Wissens von den anderen Variablen des Systems angenommen. Die

59 Vgl. für den formalen Nachweis Barro/Sala-i-Martin (1995, S. 32f.). In Kapitel drei wird im Rahmen der Besprechung der Catching up-Hypothese auf diese Überlegungen noch einmal eingegangen werden. 
einzige Größe, die Einfluß auf die absolute Höhe und die Veränderung des Wissens nimmt, ist die Zeit. Die Effizienz der Produktionstechnik ist somit zeitabhängig. Die Erhöhung des technischen Know-hows ermöglicht eine Steigerung der Outputmenge, ohne die Quantität der anderen Produktionsfaktoren (Arbeit und Kapital) auszudehnen. Die Produktion kann nun durch die quantitative und qualitative Veränderung des Faktoreinsatzes gesteigert werden.

Um die Zeitabhängigkeit des technischen Fortschritts abbilden zu können, wird im allgemeinen von einem exponentiellen Wachstum des Wissens mit einer exogen vorgegebenen Rate $(\gamma)$ ausgegangen. Mögliche Bestimmungsgründe des technischen Fortschritts bleiben in den einfachen Wachstumsmodellen außen vor, d.h. auch die Höhe von $(\gamma)$ wird nicht erklärt. Eine solche von ökonomischen Zusammenhängen losgelöste Generierung von neuem Know-how könnte in einem eigenen Forschungs- und Entwicklungssektor der Volkswirtschaft geschehen, in dem in jeder Periode ( $t$ ) Forschungsergebnisse entstehen, die zu einer Steigerung der Produktion um $\gamma \%$ pro Periode führen. Diese Art der Behandlung des technischen Fortschritts als reine exogene Größe führte zum Bild des Manna vom Himmel. Ähnlich der Speisung der Kinder Israels in der Wüste Sinai fällt im einfachen neoklassischen Modell die Steigerung der Effizienz der Produktionsfaktoren vom Himmel. ${ }^{60}$ Aber nicht nur die Determinanten des technischen Wandels werden nicht integriert, auch der Weg des technischen Wissens in den Produktionsprozeß wird im einfachsten Fall vernachlässigt. Trotz der Tatsache, daß die Resultate von Forschungs- und Entwicklungsanstrengungen ein Vehikel benötigen, um zur Anwendung zukommen, wurde anfänglich sog. ungebundener technischer Fortschritt unterstellt. Aktuelle Forschungsergebnisse werden dabei nicht nur in den neuesten Generationen der Produktionsfaktoren zu finden sein, sondern die Produktivität aller im Produktionsprozeß eingesetzten Faktoren unabhängig von deren Alter verbessert sich. Diese Art von technischem Fortschritt kann durch folgende Funktion dargestellt werden:

$$
A_{t}=A_{0} e^{\gamma t}
$$

Die durch technischen Fortschritt möglich gewordenen Prozeßverbesserungen können auf drei verschiedene Arten wirken: 61

60 Das biblische Original erinnert in manchem an den technischen Fortschritt des neoklassischen Wachstumsmodells. Nachdem das Volk Israels auf seiner Flucht aus der agyptischen Gefangenschaft das Schilfmeer durchschritten hatte, mußte es in der Wüste von Sinai hungern: "Und es murrte die ganze Gemeinde der Kinder Israel wider Mose und Aaron in der Wüste. Sie sprachen: Wollte Gott, wir wären in Ägypten gestorben durch des HERRN Hand, als wir bei den Fleischtopfen saßen und hatten Brot die Fülle zu essen. Denn ihr habt uns dazu herausgefuhrt in diese Wüste, dab ihr diese ganze Gemeinde an Hunger sterben labt. Da sprach der HERR zu Mose: Siehe, ich will euch Brot vom Himmel regnen lassen, und das Volk soll hinausgehen und tăglich sammeln, was es für den Tag bedarf, daß ich's prüfe, ob es in meinem Gesetz wandle oder nicht." (2. Buch Mose, 16, 1-4).

61 Die Konzepte des technischen Fortschritts sind eng mit den Namen der Autoren verbunden, die die jeweiligen Definitionen entwickelten. Vgl. Hicks (1932), Harrod (1948) und Solow (1959). Zur Klassifikation der Fortschrittswirkungen siehe des weiteren Walter (1969, S. 58ff. und 1977). 
1.) Hicks-neutral: Der technische Fortschritt wirkt symmetrisch auf beide Produktionsfaktoren, d.h. sowohl die Kapital- als auch die Arbeitsproduktivität nehmen in gleichem Maße zu. Das Faktoreinsatzverhältnis bleibt konstant. In die oben diskutierte Produktionsfunktion fließt der technische Fortschritt als eigene Komponente ein:

$$
Y=\Gamma(K, L, t)=A_{t} F(K, L)
$$

2.) Harrod-neutral: Der technische Fortschritt erhöht nur die Produktivität der Arbeit, während der Kapitalkoeffizient konstant bleibt. Das Faktoreinsatzverhältnis verändert sich zugunsten des Kapitals. Es wird auch vom labour-augmenting technical progress gesprochen. Die Steigerung der Arbeitsproduktivität wirkt wie eine Erhöhung der Arbeitskräftezahl. Da der technische Fortschritt nur die Effizienz der Arbeit verbessert, wird er in der Produktionsfunktion ausschließlich diesem Faktor zugeordnet:

$$
Y=F\left(K, A_{t} L\right)
$$

3.) Solow-neutral: Der technische Fortschritt erhöht nur die Produktivität des Kapitals. Das Faktoreinsatzverhältnis verändert sich zugunsten der Arbeit. Gleichzeitig bleibt die Arbeitsproduktivität konstant. Analog zum arbeitsvermehrenden technischen Fortschritt wird hier vom capital-augmenting technical progress gesprochen. Die Zuordnung der Fortschrittskomponente erfolgt in der Produktionsfunktion beim Kapital:

$$
\mathrm{Y}=\mathrm{F}\left(\mathbf{A}_{\mathbf{t}} \mathrm{K}, \mathrm{L}\right)
$$

Nachdem die unterschiedlichen Arten des technischen Fortschritts angesprochen worden sind, stellt sich die Frage nach dessen Wirkungen auf das Wachstum des Outputs, insbesondere dessen Folgen für den Steady State-Pfad. Aufgrund der Exogenität des technischen Wandels im Modell der neoklassischen Wachstumstheorie liegt die Schlußfolgerung nahe, daß die Rate des technischen Fortschritts zur Wachstumsrate der Bevölkerung analoge Wirkungen aufweist. ${ }^{62}$ Es ist zu erwarten, daß die Fortschrittskomponente in irgendeiner Form als additive Komponente $\mathrm{zu}(\mathrm{n})$ in der Gleichgewichtslösung hinzukommen wird.

Geht man z.B. von Hicks-neutralem technischen Fortschritt aus, kann die Produktionsfunktion geschrieben werden als:

$$
Y^{r}=\Gamma(K, L, t)=A_{t} F(K, L)
$$

62 Die Summe aus der Bevollkerungswachstumsrate und der Rate des technischen Fortschritts bildet die natürliche Wachstumsrate. 
Hieraus folgt für das Wachstum des Outputs:

$$
\frac{d Y}{d t}=\frac{d A}{d t} F(K, L)+A\left[\frac{\partial F}{\partial K} \frac{d K}{d t}+\frac{\partial F}{\partial L} \frac{d L}{d t}\right]
$$

Geht man entsprechend dem Fall ohne technischen Fortschritt vor, ergibt sich durch Division mit $\mathrm{Y}=\mathrm{AF}(\mathrm{K}, \mathrm{L})$ :

$$
\frac{\mathrm{d} Y}{\mathrm{dt}} \frac{1}{\mathrm{Y}}=\frac{\mathrm{dA}}{\mathrm{dt}} \frac{1}{\mathrm{~A}}+\mathrm{A}\left[\frac{\partial \mathrm{F}}{\partial \mathrm{K}} \frac{\mathrm{dK}}{\mathrm{dt}} \frac{1}{\mathrm{Y}}+\frac{\partial \mathrm{F}}{\partial \mathrm{L}} \frac{\mathrm{dL}}{\mathrm{dt}} \frac{1}{\mathrm{Y}}\right]
$$

Durch dieselben Umformungen wie im Falle ohne technischen Fortschritt und mit $\mathrm{A} \frac{\partial \mathrm{F}}{\partial \mathrm{i}}=\frac{\partial \Gamma}{\partial \mathrm{i}}$ und unter der Annahme exponentiellen Wachstums des technischen Wissen gilt schließlich für die Wachstumsrate des Outputs: 63

$$
\hat{\mathrm{Y}}=\gamma+\mathrm{E}_{\mathrm{YK}} \hat{\mathrm{K}}+\mathrm{E}_{\mathbf{Y L}} \hat{\mathrm{L}}
$$

Wird dagegen Harrod-neutraler oder Solow-neutraler technischer Fortschritt unterstellt, wirkt der technische Fortschritt faktorvermehrend. Arbeitsvermehrender technischer Fortschritt mit einer Rate von $2 \%$ z.B. hat dieselbe Wirkung auf das Wachstum des Outputs wie eine Erhöhung des Arbeitseinsatzes um 2\%. Die Rate des technischen Fortschritts kann deshalb abhängig davon, welcher Produktionsfaktor betroffen ist, zu dessen Wachstumsrate hinzugerechnet werden. Die Wirkung des technischen Fortschritts auf den Output ergibt sich dann durch die Gewichtung der Fortschrittsrate mit der faktorspezifischen Produktionselastizität. Es resultieren deshalb folgende tatsächlichen Wachstumsraten des Outputs:

1.) Arbeitsvermehrender technischer Fortschritt:

$$
\hat{\mathrm{Y}}=\mathrm{E}_{\mathrm{YK}} \hat{\mathrm{K}}+\mathrm{E}_{\mathrm{YL}}\left(\hat{\mathrm{L}}+\gamma_{\text {Harrod }}\right)
$$

2.) Kapitalvermehrender technischer Fortschritt:

$$
\hat{\mathrm{Y}}=\mathrm{E}_{\mathrm{YK}}\left(\hat{\mathrm{K}}+\gamma_{\text {Solow }}\right)+\mathrm{E}_{\mathrm{YL}} \hat{\mathrm{L}}
$$

Die Wirkungen des technischen Fortschritts im Steady State werden durch Gleichungen (2.37)(2.39) nicht erfaßt. Als Steady State wurde die Situation bezeichnet, in der alle Variablen mit

63 Hierbei gilt: $\frac{\mathrm{dA}}{\mathrm{dt}} \frac{1}{\mathrm{~A}}=\frac{\mathrm{d}\left(\ln \mathrm{e}^{\gamma t}\right)}{\mathrm{dt}}=\frac{\mathrm{d} \gamma t}{\mathrm{dt}}=\gamma \cdot\left(2.23^{\prime}\right)$ stellt somit nur eine Erweiterung von (2.23) dar. 
einer konstanten, nicht zwangsläufig identischen Rate wachsen. Von den drei skizzierten Fortschrittskonzepten läßt sich jedoch nur der Harrod-neutrale technische Fortschritt mit dieser Definition des gleichgewichtigen Wachstums vereinbaren. Ein Kennzeichen dieser Art des neutralen technischen Fortschritts ist die Konstanz des Kapitalkoeffizienten, d.h. der Output und der Kapitaleinsatz wachsen mit der gleichen Rate. Ausgehend von einer gleichgewichtigen Situation würde technischer Fortschritt Hicks-neutraler Art bei konstantem Faktoreinsatzverhältnis, sprich Kapitalintensität, zum Anstieg der Produktivität und damit der Rentabilität beider Produktionsfaktoren führen. Können die Unternehmen aufgrund des technischen Fortschritts aus einer Investition einen höheren Ertrag erzielen, werden die Investitionen zunehmen. Mehr Kapitalbildung bedeutet aber aufgrund der Annahmen über die Produktionstechnik, daß die Grenzproduktivität des Kapitals mit steigendem Kapitaleinsatz abnehmen wird, die Wachstumsrate des Outputs somit kleiner als die des Kapitals ist. Dieser Prozeß führt zum Rückgang der Kapitalproduktivität und des Investitionsanreizes bis die Grenzproduktivität denselben Wert wie in der Anfangssituation erreicht hat. Die Ökonomie ist dann auch zum alten Kapitalkoeffizienten bei gleichzeitig gestiegener Kapitalintensität zurückgekehrt. Diese Situation entspricht jedoch genau den Bedingungen des Harrod-neutralen technischen Fortschritts. ${ }^{64}$

Die ausschließliche Vereinbarkeit des Steady State-Wachstums mit Harrod-neutralem technischen Fortschritt läßt sich auch auf anderem Wege nachweisen. ${ }^{65}$ Unterstellt man eine Produktionsfunktion, in die sowohl kapital- als auch arbeitsvermehrender technischer Fortschritt einfließt, so kann diese folgendermaßen dargestellt werden:

$$
\mathrm{Y}=\mathrm{F}\left[\mathrm{KS}_{\mathrm{t}}, \mathrm{LH}_{\mathrm{t}}\right]
$$

Die Funktion $S_{t}$ erfaßt die Wirkungen des technischen Fortschritts Solow-neutraler Art, $\mathrm{H}_{t}$ diejenigen des Harrod-neutralen technischen Fortschritts. Es soll gelten: 66

$$
\begin{aligned}
& S_{t}=e^{\gamma_{s} t} \\
& H_{t}=e^{\gamma_{H} t}
\end{aligned}
$$

64 Vgl. Walter (1969, S. 123ff. und 1977, S. 576).

65 Vgl. im folgenden Barro/Sala-i-Martin (1995, S. 54f.).

66 Hier bezeichnet $\left(\gamma_{S}\right)$ die Rate des Solow-neutralen und $\left(\gamma_{H}\right)$ die Rate des Harrod-neutralen technischen Fortschritts. 
Die Kapitalproduktivität $\mathrm{Y} / \mathrm{K}$ errechnet sich durch Division von (2.29) mit K:

$$
\frac{Y}{K}=e^{\gamma_{s} t} F\left[1, \frac{L e^{\gamma_{H} t}}{K^{\gamma_{s} t}}\right]=e^{\gamma_{s} t} \phi\left[\frac{L}{K} e^{\left(\gamma_{H}-\gamma_{s}\right) t}\right]
$$

Der Kapitalstock und die Beschäftigungsmenge zum Zeitpunkt $(t)$ sind durch $K_{t}=K_{0} e^{\hat{K} t}$ und $\mathrm{L}_{\mathrm{t}}=\mathrm{L}_{0} \mathrm{e}^{\mathrm{nt}}$ gegeben. Normiert man $\left(\mathrm{L}_{0}\right)$ und $\left(\mathrm{K}_{0}\right)$ gleich Eins und geht man von einer Steady State-Situation aus, in der $(\hat{\mathbf{K}})$ der gleichgewichtigen, konstanten Wachstumsrate des Kapitalstocks entspricht, folgt für die Kapitalproduktivität:

$$
\frac{Y}{K}=e^{\gamma_{s} t} \phi\left[e^{\left(n+\gamma_{H}-\gamma_{s}-\hat{K}\right) t}\right]
$$

Im Steady State müssen sich nach Gleichung (2.19) die Wachstumsrate des Outputs und des Kapitalstocks entsprechen, d.h. der Kapitalkoeffizient muß konstant sein. Entsprechend Gleichung (2.30) kann dies nur der Fall sein, wenn $\left(e^{\gamma_{s}}\right)$ und $\left(e^{\left(n+\gamma_{H}-\gamma_{s}-\hat{K}\right) t}\right)$ konstant sind. Hierzu muß $\left(\gamma_{S}\right)$ gleich Null sein, d.h. es darf nur Harrod-neutraler technischer Fortschritt vorliegen. Im Steady State muß dann gelten:

$$
\hat{\mathbf{K}}=\hat{\mathbf{Y}}=\mathrm{n}+\gamma_{\mathbf{H}}
$$

Die Kapitalproduktivität kann auch konstant bleiben, wenn sich die Wirkungen von $\left(\mathrm{e}^{\gamma} \mathrm{s}\right)$ und $\phi(\bullet)$ gegenseitig aufheben. Die notwendige Bedingung erhält man aus der Differentiation von Gleichung (2.41) nach der Zeit, die Null sein muß. Es gilt:

$$
\frac{\partial \frac{Y}{K}}{\partial t}=\gamma_{S} e^{\gamma_{s} t_{\phi}}(\omega)+e^{\gamma_{s} t}\left(n+\gamma_{H}-\gamma_{S}-\hat{K}\right) \omega \frac{\partial \phi(\omega)}{\partial \omega}
$$

Es steht dabei $(\omega)$ für $\left(e^{\left(n+\gamma_{H}-\gamma_{S}-\hat{K}\right) t}\right)$. Setzt man die erste Ableitung gleich Null und formt um, kommt man zu:67

$$
\frac{\partial \phi(\omega)}{\partial \omega} \omega \frac{1}{\phi(\omega)}=-\frac{\gamma_{S}}{n+\gamma_{S}-\gamma_{S}-\hat{K}}
$$

67 Man teilt durch $\left(\mathrm{e}^{\gamma_{\mathrm{s}}}\right)$, das immer grober Null ist. 
Die Funktion $\phi(\bullet)$ kann nach Integration geschrieben werden als:

$$
\phi(\omega)=C \omega^{1-\alpha}
$$

$\mathrm{Da} \phi(\bullet)$ die Produktion pro effizienter Kapitaleinheit angibt, kann unter Rückbesinnung auf die Annahme konstanter Skalenerträge auf die Produktionsfunktion aus Gleichung (2.29) geschlossen werden:

$$
Y=C\left(K^{\gamma_{s} t}\right)^{\alpha}\left(L^{\gamma_{H} t}\right)^{1-\alpha}
$$

Die den Überlegungen zugrundegelegte Produktionsfunktion muß somit eine Cobb-DouglasFunktion sein, falls es kapitalvermehrenden technischen Fortschritt gibt. Für Funktionen dieser Art ist es aber möglich, die drei Fortschrittskonzepte (Harrod-, Hicks-, und Solow-neutral) zu vereinbaren. ${ }^{68} \mathrm{Im}$ vorliegenden Fall wäre obige Cobb-Douglas-Funktion äquivalent $\mathrm{zu}$ :

$$
\mathrm{Y}=\mathrm{CK}^{\alpha}\left(\mathrm{Le}^{\gamma_{\mathrm{H}}^{*} \mathrm{t}}\right)^{1-\alpha} \quad \text { mit } \quad \gamma_{\mathrm{H}}^{*}=\frac{\gamma_{\mathrm{S}} \alpha+\gamma_{\mathrm{H}}(1-\alpha)}{1-\alpha}
$$

Steady State-Wachstum bedingt deshalb, daß der technische Fortschritt als ausschließlich arbeitsvermehrend betrachtet werden kann.

Steady State-Wachstum war gekennzeichnet durch die Konstanz der Wachstumsraten aller Variablen. Durch die Einführung von Harrod-neutralem technischen Fortschritt in die Produktionsfunktion verändern sich die Investitionsgleichungen (2.19) und (2.25) zu:

$$
\dot{\mathrm{K}}=\mathbf{s F}\left(\mathrm{K}, \mathrm{LH}_{\mathrm{t}}\right)-\delta \mathrm{K}
$$

für Absolutgrößen und für Pro-Kopf-Größen zu:

$$
\dot{\mathrm{k}}=\mathrm{sF}\left(\mathrm{k}, \mathrm{H}_{\mathrm{t}}\right)-(\mathrm{n}+\delta) \mathrm{k}
$$

Die Wachstumsrate der Kapitalintensität ergibt sich nun aus:

$$
\frac{\dot{k}}{k}=\frac{s F\left(k, H_{t}\right)}{k}-(n+\delta)
$$

Wie im Fall ohne technischen Fortschritt darf sich auch hier die Kapitalproduktivität im Steady State nicht ändern, damit die Wachstumsrate der Kapitalintensität konstant ist. Nach Gleichung (2.41) und (2.42) muß auf dem gleichgewichtigen Wachstumspfad der Kapitalstock und der

68 Vgl. Walter (1969, S. 96ff.) 
Output mit derselben Rate wachsen, die sich aus der Summe des Bevölkerungswachstums und der Rate des technischen Fortschritts ergibt. Dies impliziert jedoch folgende Ungleichheiten:
1.) $\hat{\mathrm{Y}}>\mathrm{n}$
2.) $\hat{\mathbf{K}}>\mathrm{n}$

Kapitalstock und Output wachsen deshalb stärker als die Bevölkerung, so daß die Pro-KopfGrößen eine von Null verschiedene Wachstumsrate, nämlich $\left(\gamma_{\mathrm{H}}\right)$ aufweisen. Dies impliziert eine steigende Kapitalintensität. Konstant bleibt dagegen die in Effizienzeinheiten gemessene Kapitalintensität, d.h. das Verhältnis zwischen dem Kapitalstock und der fiktiven Arbeitsmenge $L^{\text {eff }}=L e^{\left(n+\gamma_{H}\right) t}$. Harrod-neutraler technischer Fortschritt mit einer Rate von $\gamma_{H} \%$ kann ja mit einer Vermehrung der Arbeitsmenge um dieselbe Prozentzahl gleichgesetzt werden. Wächst der Kapitalstock und ( $L^{\text {eff }}$ ) mit derselben Rate bleibt der Quotient $k^{\text {eff }}=K / L^{\text {eff }}$ konstant. Sämtliche Aussagen, die über das Grundmodell im Hinblick auf die Wirkungen von Veränderungen der Sparquote gemacht wurden, haben auch bei Erweiterung um Harrodneutralen technischen Fortschritt weiter Gültigkeit. Anstelle der auf die Arbeitsmenge bezogenen Kapitalintensität muß nur die ( $k^{\text {eff }}$ ) eingesetzt werden. 69

Die Relevanz des technischen Fortschritts für den tatsächlichen Wachstumsprozeß wurde von Solow (1957) für die Volkswirtschaft der USA gezeigt. Er konnte nachweisen, daß 7/8 des Pro-Kopf-Wachstums der US-amerikanischen Ökonomie im Zeitraum 1909-1949 nicht durch das Wachstum der Menge an Produktionsfaktoren bestimmt war, sondern durch eine Komponente des Hicks-neutralen technischen Fortschritts erfaßt wurde. Dieses Ergebnis führte zu der Entstehung eines eigenen Forschungsgebiets: dem Growth-Accounting. Insbesondere in Arbeiten von Denison und Jorgenson/Griliches wurde versucht, diese Residualgröße, die von Abramovitz auch als "measure of ignorance" bezeichnet wurde, besser erklären zu können. 70 Das um den technischen Fortschritt erweiterte Grundmodell ist somit in der Lage, dauerhaftes Pro-Kopf-Wachstum abzubilden. Die langfristige gleichgewichtige Wachstumsrate bleibt aber weiterhin von exogenen Größen bestimmt. Es wird weder eine ökonomische Erklärung der Entstehung des technischen Fortschritts noch der Bevölkerungswachstumsrate gegeben. Diese Unabhängigkeit der entscheidenden Wachstumsdeterminanten von den ökonomischen Entscheidungsprozessen kann nicht befriedigen. Mögliche Ansätze zur Endogenisierung der langfristigen Wachstumsrate werden deshalb im Mittelpunkt der weiteren Untersuchungen im Rahmen dieser Arbeit stehen. Bevor auf die neuesten Entwicklungen innerhalb der neoklassischen Wachstumstheorie eingegangen wird, werden im nächsten Abschnitt die Bedin-

69 Vgl. hierzu z.B. Barro/Sala-i-Martin (1995, 32ff.), Walter (1983, S.109ff.) und Solow (1971, S. 45ff.).

70 Vgl. Abramovitz (1956), Solow (1957) sowie Denison (1962, 1967, 1972, 1974, 1979, 1984, 1985), Jorgenson (1995) und Jorgenson/Griliches (1967). Anzumerken ist, daß Denison die Existenz steigender Skalenertragge explizit zulaBt und somit von den Standardannahmen der neoklassischen Theorie abweicht. 
gungen für optimales Wachstum im Grundmodell diskutiert sowie die Folgen der Aufgabe einer exogen gegebenen Sparquote erläutert.

\subsubsection{Goldene Regeln gleichgewichtigen Wachstums ${ }^{71}$}

In den bisherigen Ausführungen wurden die zentralen Eigenschaften des neoklassischen Grundmodells erläutert. Die sehr allgemeinen Ergebnisse lassen keine Beantwortung der Frage nach der Optimalität des erreichten Wachstumsgleichgewichts zu. Die Ausführungen über die Folgen einer Veränderung der Sparquote zeigten die Existenz einer Vielzahl möglicher gleichgewichtiger Wachstumspfade, die zwar alle dieselbe Steigung aber unterschiedliche Niveaus der Absolutgrößen aufweisen. Welcher Pfad für eine Volkswirtschaft der beste ist, blieb bisher offen. Da eine Nutzenfunktion ausgeschlossen wurde, kann nur der Konsum als Maß für die Qualität eines Pfades gewählt werden. Die Bereitschaft zu sparen stellt den Verzicht auf Konsum in der laufenden Periode dar. Eine Erhöhung der Sparquote reduziert somit die aktuellen Konsummöglichkeiten zugunsten zukünftiger. Die für die Kapitalakkumulation einsetzbaren Ressourcen erhöhen die Produktionskapazitäten nachfolgender Perioden und damit des Konsums.

Aufgrund der Annahme einer dauerhaft konstanten Sparquote kann der Konsum pro Kopf (c) als

$$
c=(1-s) f(k)=f(k)-s f(k)
$$

geschrieben werden. Im Steady State entspricht das Sparen pro Kopf der effektiven Abschreibung des Kapitals durch Bevölkerungswachstum und technischen Verzehr, so daß in diesen Situationen für den Pro-Kopf-Konsum gilt:72

$$
c=f(k)-(n+\delta) k
$$

Die Konsummöglichkeiten sind in ihrer Höhe von der gleichgewichtigen Kapitalintensität abhängig, die wiederum selbst eine Funktion der Sparquote ist, d.h.

$$
c(s)=f[k(s)]-(n+\delta) k(s)
$$

71 Der Diskussion über die goldene Regel der Kapitalakkumulation verdankt die okonomische Theorie mit Sicherheit einen ihrer lesenswerten Beitrăge. Phelps' Mărchen über die Einwohner im Kơnigreich Solovia, insbesondere den Held der Geschichte Oiko Nomos, gibt auf amüsante Art Einblick in die Welt optimalen Wachstums (vgl. Phelps 1961 und 1966).

72 Vgl. z.B. Barro/Sala-i-Martin (1995, S. 19). 
Maximiert man (2.49), erhält man die Bedingung für den konsummaximalen Wachstumspfad: ${ }^{73}$

$$
f^{\prime}\left(k^{g}\right)=n+\delta
$$

(Goldene Regel der Kapitalakkumulation)

Die Annahme der Grenzproduktivitätsentlohnung impliziert in Verbindung mit (2.49), daß die Bruttorealrendite auf den Kapitaleinsatz der Summe aus Bevölkerungswachstumsrate und Abschreibungsrate entsprechen muß. Es ist die Sparquote zu wählen, bei der die Grenzproduktivität des Kapitals der Summe aus Wachstumsrate der Bevölkerung und Abschreibungsrate entspricht. Nach Gleichung (2.16) ist im Steady State $(n+\delta) v=s$. Erweitert man (2.50) mit dem Kapitalkoeffizienten, ergibt sich die optimale Sparquote: ${ }^{74}$

$$
s^{g}=(n+\delta) v=f^{\prime}\left(k^{g}\right) v=E_{Y K}
$$

Die Goldene Regel der Kapitalakkumulation läßt sich auch auf eine andere Art und Weise ermitteln. Meade (1962a) berechnet für eine Cobb-Douglas-Produktionsfunktion die Elastizität des Konsumniveaus bzgl. der Sparquote im Steady State, die durch Gleichung (2.52) gegeben ist. ${ }^{75}$

$$
\frac{\partial C}{\partial s} \frac{s}{C}=\frac{\alpha-s}{(1-\alpha)(1-s)}
$$

Für den Fall, daß die Produktionselastizität des Kapitals ( $\alpha$ ) größer als (s) ist, kann durch eine Sparquotenerhöhung das Konsumniveau gesteigert werden. Im umgekehrten Fall ( $\alpha<$ s), senkt eine Erhöhung der Sparquote das Konsumniveau. ${ }^{76}$ Das maximale Konsumniveau wird erreicht, wenn die Sparquote so groß wie die Produktionselastizität des Kapitals ist.

$\mathrm{Da}$ die Produktionselastizität des Kapitals der Profitquote entspricht, muß die Volkswirtschaft zur Erreichung eines konsumoptimalen Gleichgewichts soviel sparen, wie die Kapitaleigner verdienen. Die Goldene Regel der Kapitalakkumulation gibt Auskunf, welche Kapitalintensität für die betrachtete Volkswirtschaft effizient ist. Alle Situationen, in denen $\left(\mathrm{kg}^{\mathrm{g}}\right)$ nicht erreicht worden ist, scheinen somit ineffizient zu sein. Je nachdem, ob die tatsächliche Kapitalintensität größer oder kleiner als die "goldene" Kapitalintensität ist, müßte die Volkswirtschaft den Kapitalstock abbauen oder vergrößern. Beide Maßnahmen führen auf den ersten

73 Vgl. z.B. Walter (1983, S. 53f.) und Barro/Sala-i-Martin (1995, S. 20).

74 Anzumerken bleibt, daB die Erweiterung des Modells um exogenen Harrod-neutralen technischen Fortschritt nichts an den prinzipiellen Aussagen des Modells zum konsummaximalen Wachstumspfad andert. Anstelle der auf den eigentlichen Arbeitseinsatz bezogenen Kapitalintensităt tritt die effektive Kapitalintensitat ( $\mathbf{k}^{\mathrm{eff}}$ ).

$75 \mathrm{Vgl}$. Meade (1962a). Diese Gleichung gilt für jede allgemeine neoklassische Produktionsfunktion. Zur Herleitung dieser Gleichung für eine allgemeine neoklassische Produktionsfunktion siehe den Anhang zu diesem Kapitel.

76 Der Nenner der Gleichung ist immer positiv. 
Blick zu einer Steigerung der Effizienz. Bei genauerem Hinsehen wird deutlich, daß nur im Falle der notwendigen Desinvestition eindeutige Rückschlüsse im Hinblick auf das erreichbare Konsumniveau gezogen werden können. Legt die Goldene Regel einen Kapitalabbau nahe, erreicht die Volkswirtschaft zum einen ein dauerhaft höheres Konsumniveau, zum anderen steigen die Konsummöglichkeiten im Zeitraum, in dem der Kapitalstock reduziert wird. Muß dagegen die Volkswirtschaft ihren Kapitaleinsatz erhöhen, steht der dauerhaften Konsumsteigerung in der Zukunft der Rückgang der Konsummöglichkeiten während der Investitionserhöhung gegenüber. Beide Effekte müssen miteinander verglichen werden, um die Konsequenzen auf die Effizienz im Sinne des Konsummaximums beurteilen zu können. ${ }^{77}$

Der zeitliche Unterschied in den Wirkungen einer Investitionserhöhung erfordert einen Maßstab, mit dem diese intertemporal vergleichbar gemacht werden können. Zur Beantwortung der Frage nach der Optimalität eines Wachstumspfades kann auf eine intertemporale Nutzenfunktion zurückgegriffen werden, wobei die über den Betrachtungszeitraum anfallenden Nutzen abdiskontiert werden müssen. Die Spar- und Investitionsentscheidung der Wirtschaftssubjekte muß entsprechend den Erfordernissen der Maximierung des Gegenwartswerts der Nutzenströme erfolgen. ${ }^{78}$ Die Verwendung einer gesamtwirtschaftlichen Nutzenfunktion ist jedoch nicht unproblematisch. Falls die Wirtschaftssubjekte sich in ihren Nutzenfunktionen unterscheiden, ist die Ermittlung einer gesamtwirtschaftlichen Nutzenfunktion nach dem Arrowschen Unmöglichkeitstheorem unter Einhaltung gewisser Kriterien beim gesellschaftlichen Entscheidungsprozeß nicht immer durchführbar. Als Möglichkeit bleibt entweder die Einführung eines (hoffentlich) wohlwollenden Diktators oder die Rückbesinnung auf das repräsentative Individuum. Im letzteren Fall deckt sich die gesamtwirtschaftliche mit der individuellen Nutzenfunktion. 79

Geht man davon aus, daß sich der Nutzen eines repräsentativen Individuums durch den Konsum pro Kopf bestimmt, d.h. $u=u\left(c_{t}\right)$, dann ist der Gegenwartswert der Nutzenströme zwischen $\mathrm{t}=0$ und $\mathrm{t}=\infty$ gegeben durch:

77 Vgl. Frey (1970, S. 13ff.). Aufgrund dieser Asymmetrie wird auch von der falschen Seite (zuviel Investition in der Vergangenheit) und der richtigen Seite der Goldenen Regel gesprochen. Vgl. zu den Goldenen Regeln bzw. dem Begriff des optimalen Wachstums auch Schumann (1969), der einen Überblick über verschiedene Modelle (Ramsey, von Neumann und Phelps) gibt.

78 Die Abdiskontierung zukünftiger Nutzenströme ist nicht unumstritten. Im Papier von Ramsey aus dem Jahre 1928, das den Ausgangspunkt der modernen Optimierungsmodelle darstellt, wird 2.B. diese Vorgehensweise kritisch betrachtet:

"One point should perhaps be emphasised more particularly; it is assumed that we do not discount later enjoyments in comparison with earlier ones, a practice which is ethically indefensible and arises merely from the weakness of the imagination." (Ramsey 1928, S. 543.)

Im weiteren Verlauf seiner Untersuchung behandelt Ramsey dennoch die Konsequenzen der Abdiskontierung (vgl. Ramsey 1928, S. 553ff.). Grundlegende Beiträge zur Ableitung optimaler Wachstumspfade unter Einbeziehung von Nutzenfunktionen wurden von Cass (1965) und Koopmans (1970) geleistet.

79 Vgl. Arrow (1963). Die geforderten Eigenschaften des Entscheidungsmechanismus sind z.B. bei Varian (1991, S. 506ff.) anschaulich erläutert. Die Aufgabe einer der Anforderungen an den Entscheidungsprozeß macht die Aufstellung einer sozialen Wohlfahrtsfunktion moglich. 
(2.53.1) $U=\int_{t=0}^{\infty} u\left(c_{t}\right) e^{-\rho t} d t \quad$ mit $\quad \frac{\partial u}{\partial c}>0$ und $\frac{\partial^{2} u}{\partial c^{2}}<0$

Hierbei steht $u\left(c_{t}\right)$ für den Nutzen, der durch den Pro-Kopf-Konsum im Zeitraum ( $t$ ) entsteht und $(\rho)$ gibt die Zeitpräferenzrate des repräsentativen Individuums an. 80 Das Ziel ist die Maximierung von (U) unter der Nebenbedingung der Investitionsfunktion, die sich durch

$$
\dot{k}=f(k)-(n+\delta) k-c
$$

ergibt. Das sich stellende Maximierungsproblem zur Ermittlung des optimalen Wachstumspfades ist eine der zentralen Anwendungen des Pontryagin-Maximums-Prinzips. ${ }^{81}$ Als weitere notwendige Nebenbedingung darf hierbei der mit dem durchschnittlichen Zinssatz abdiskontierte Kapitalstock am Ende des Planungshorizonts nicht negativ sein. Formal heißt dies:

$$
\lim _{t \rightarrow \infty}\left(k_{t} e^{-\bar{r}_{t} t}\right) \geq 0
$$

Aus dem Optimierungsproblem ergibt sich die Hamilton-Funktion $(\mathrm{H})$ :

$$
H=u(c) e^{-\rho t}+\tau[f(k)-(n+\delta) k-c]
$$

Hierbei ist $(\tau)$ der Schattenpreis für eine Einheit Kapital. Die notwendigen Bedingungen zur Maximierung dieser Funktion sind:

$$
\begin{aligned}
& \frac{\partial H}{\partial c}=\frac{\partial U}{\partial c} e^{-\rho t}-\tau=0 \\
& \frac{d \tau}{d t}=-\frac{\partial H}{\partial k}=-\tau\left[f^{\prime}(k)-(n+\delta)\right] \\
& \lim _{t \rightarrow \infty}\left(k_{t} \tau_{t}\right)=0
\end{aligned}
$$

80 Im weiteren wird der Index $t$ bis auf die Falle, in denen die zeitliche Markierung unerlaßlich ist, vernachlăssigt.

81 Das Pontryagin-Maximum-Prinzip stellt eine dynamische Erweiterung des Lagrange-Ansatzes dar. Die Entwicklung und Beweise dieses Prinzips finden sich in Pontryagin (1962) und Pontryagin/Boltyanskii/Gamkrelidze/Mischchenko (1962). Weitere Erlăuterungen, wie z.B. zum Begriff der Hamiltonfunktion, und Anwendungsbeispiele für okonomische Fragestellungen, insbesondere der Wachstumstheorie, finden sich in Frey (1969), Müller/Ströbele (1985) Blanchard/Fischer (1989), Dixit (1990), Tu (1994), Barro/Sala-i-Martin (1995) und Romer, D. (1996). 
Bedingung (2.55.3) stellt die sog. Transversalitätsbedingung dar, die sich aus der Bedingung eines nicht negativen Kapitalstocks am Ende des betrachteten Zeitraums der Optimierung ergibt (vgl. Gleichung 2.53.3). Ist der Kapitalstock zu diesem Zeitpunkt positiv, muß dessen Schattenpreis Null sein. Wird dem Kapital aber ein positiver Wert $(\tau)$ pro Einheit zugeordnet, darf kein Kapital mehr verbleiben. Für den Fall eines endlichen Planungshorizonts ist diese Bedingung einfach zu interpretieren. Würde am Ende der letzten Planungsperiode ein Kapitalstock mit positivem Wert, d.h. Nutzen verbleiben, der nicht dem Verbrauch zugeführt würde, wäre dies Verschwendung. Die Optimierung wäre nicht gelungen, da der Konsum dieses Kapitalstocks den Nutzen steigern würde. Ist dagegen der Planungshorizont unendlich, muß die Transversalitätsbedingung nicht notwendigerweise immer erfullt sein, damit die sich aus den anderen Bedingungen ergebende Lösung optimal ist. ${ }^{82}$

Differenziert man nun (2.55.1.) nach der Zeit ergibt sich eine weitere Bedingung für die zeitliche Veränderung des Schattenpreises des Kapitals $(\tau)$ :

$$
\frac{\partial^{2} \mathrm{U}}{\partial \mathrm{c}^{2}} \frac{\mathrm{dc}}{\mathrm{dt}} \mathrm{e}^{-\rho t}-\rho \mathrm{e}^{-\rho t} \frac{\partial \mathrm{U}}{\partial \mathrm{c}}-\frac{\mathrm{d} \tau}{\mathrm{dt}}=0 \quad \text { bzw. } \quad \frac{\mathrm{d} \tau}{\mathrm{dt}}=\left[\frac{\partial^{2} \mathrm{U}}{\partial \mathrm{c}^{2}} \frac{\mathrm{dc}}{\mathrm{dt}}-\rho \frac{\partial \mathrm{U}}{\partial \mathrm{c}}\right] \mathrm{e}^{-\rho t}
$$

Aus (2.55.1) folgt des weiteren eine Bedingung für $(\tau)$ :

$$
\frac{\partial U}{\partial c} e^{-\rho t}=\tau
$$

Durch Gleichsetzen von (2.56 mit (2.55.2) unter Verwendung von (2.57) folgt für die zeitliche Veränderung des Pro-Kopf-Konsums: 83

$$
\frac{d c}{d t}=-\left[f^{\prime}(k)-\delta-n-\rho\right] \frac{\partial u}{\partial c}\left(\frac{\partial^{2} u}{\partial c^{2}}\right)^{-1}
$$

Teilt man schließlich durch (c), folgt für die Wachstumsrate des Pro-Kopf-Konsums ( $\hat{c})$ im optimalen Fall:

82 Konvergiert die Zielfunktion, in unserem Falle die Nutzenfunktion, und erfolgt eine Abdiskontierung der zeitlich unterschiedlich anfallenden Nutzen, wird im allgemeinen davon ausgegangen, dab die Transversalitătsbedingung erfüllt sein muß. Vgl. Blanchard/Fischer (1989, S. 43); Barro/Sala-i-Martin (1995, S. 507f.) und vertiefend Michel (1982).

83 In einem ersten Schritt ergibt sich:

$$
\left[\frac{\partial^{2} U}{\partial c^{2}} \frac{d c}{d t}-\rho \frac{\partial U}{\partial c}\right] e^{-\rho t}=-\frac{\partial U}{\partial c} e^{-\rho t}\left[f^{\prime}(k)-(n+\delta)\right]
$$

Teilt man durch $-\frac{\partial U}{\partial c} \mathrm{e}^{-\rho t}$, kommt man $z u(2.48)$. 


$$
\hat{\mathbf{c}}=\frac{\mathrm{f}^{\prime}(\mathrm{k})-\delta-\mathrm{n}-\rho}{\lambda} \quad \text { mit } \quad \lambda=-\mathrm{c} \frac{\partial^{2} \mathrm{u}}{\partial \mathrm{c}^{2}} / \frac{\partial \mathrm{u}}{\partial \mathrm{c}}
$$

Die optimale Wachstumsrate des Pro-Kopf-Konsums wird bestimmt durch die Grenzproduktivität des Kapitals, die Abschreibungsrate, die Bevölkerungswachstumsrate und die Diskontrate sowie durch die Grenznutzenelastizität $(\lambda)$. Letztere gibt an, wie sich der Nutzen bei steigendem Pro-Kopf-Konsum verändert. Sie steht in enger Beziehung zur intertemporalen Substitutionselastizität des Konsums, die allgemein durch das Verhältnis

$$
\sigma=-\frac{d\left(c_{1} / c_{2}\right)}{c_{1} / c_{2}}: \frac{d\left(u_{1}^{\prime} / u_{2}^{\prime}\right)}{u_{1}^{\prime} / u^{\prime}{ }_{2}}
$$

definiert ist. ${ }^{84} \mathrm{Da} \mathrm{u^{ \prime }} / \mathrm{u}_{2}{ }_{2}$ der Steigung der Indifferenzkurve entspricht, kann sie auch als Kehrwert der prozentualen Veränderung des Grenznutzenverhältnisses bei einer 1\%igen Änderung des Verhältnisses zwischen heutigem und morgigem Konsum interpretiert werden. Ist der Abstand zwischen $t=1$ und $t=2$ gering, geht $(\sigma)$ über in

$$
\sigma=-u^{\prime} / \mathrm{cu}^{\prime \prime}
$$

Sie stellt somit den Kehrwert der Grenznutzenelastizität dar. Je niedriger $(\sigma)$ ist, desto geringer ist die Bereitschaft der Wirtschaftssubjekte heutigen Konsum durch morgigen zu ersetzen.

Um einen optimalen Steady State-Pfad mit Hilfe von Gleichung (2.59) bestimmen zu können, muß die Annahme der Konstanz der intertemporalen Substitutionselastizität getroffen werden, da ein gleichgewichtiger Wachstumspfad durch die Konstanz der Wachstumsraten der Variablen definiert ist. Nach Gleichung (2.59) ist die Wachstumsrate des Pro-Kopf-Konsums konstant, wenn neben den anderen annahmegemäß konstanten Größen auch die Grenzproduktivität des Kapitals konstant ist. Diese ändert sich nur dann nicht, wenn die Kapitalintensität nicht steigt oder nicht fältt. ${ }^{85}$ Folglich müssen die eingesetzte Kapital- und Arbeitsmenge mit derselben Rate wachsen. Bei einer Produktionsfunktion mit konstanten Skalenerträgen ohne technischen Fortschritt bedeutet dies, daß auch der Output mit dieser Rate wachsen muß. Nach Gleichung (2.53) ist der Pro-Kopf-Konsum pro Periode gleich

84 Hierbei steht $\left(c_{\mathfrak{i}}\right)$ für den Konsum im Zeitraum (i) und $\left(u_{\mathfrak{i}}^{\prime}\right)$ für den Grenznutzen beim Konsums $\left(c_{\mathfrak{i}}\right)$. Vgl. Barro/Sala-i-Martin (1995, S.64).

85 Die Bedingung der Konstanz der Kapitalintensitat folgt ebenfalls, wenn man die Wachstumsrate des ProKopf-Konsums nach der Zeit differenziert und gleich Null setzt. Als notwendige Bedingung ergibt sich:

$$
\frac{\mathrm{dc}}{\mathrm{dt}}=\frac{\partial \mathbf{f}^{\prime}(\mathbf{k})}{\partial \mathbf{k}} \frac{\mathrm{dk}}{\mathrm{dt}} \lambda^{-1}=\mathbf{f}^{\prime \prime}(\mathbf{k}) \frac{\mathrm{dk}}{\mathrm{dt}} \lambda^{-1}=0
$$

Da $\mathrm{f}^{\prime}(\mathrm{k})<0$ und $\lambda$ konstant und ungleich Null ist, darf sich im Steady State die Kapitalintensităt nicht verăndern. 


$$
c=f(k)-(n+\delta) k-\dot{k}
$$

Ist die Kapitalintensität konstant und wachsen Output, Beschäftigung und Kapitalstock mit identischen Raten, verändern sich ( $(\mathrm{f}(\mathrm{k})$ ) und $(\mathrm{k})$ nicht. Konsequenterweise ist die Differenz in Gleichung (2.60), d.h. der Pro-Kopf-Konsum immer gleich. Dessen Wachstumsrate ist somit im Steady State gleich Null. Hieraus folgt zwangsläufig aus Gleichung (2.59) für den optimalen gleichgewichtigen Wachstumspfad:

$$
f^{\prime}\left(k^{g}\right)=\delta+n+\rho
$$

(Goldene Nutzenregel)

Wird das Modell um Harrod-neutralen technischen Fortschritt erweitert, ergibt sich als Bedingung für die Optimalität des Steady States 86

$$
f^{\prime}\left(k^{g}\right)=\delta+n+\rho+\lambda^{-1} \gamma_{H}
$$

Die optimale Situation ist durch die Konstanz aller in Effizienzeinheiten gemessenen Pro-KopfGrößen gekennzeichnet. Die in natürlichen Einheiten gemessenen Variablen wachsen mit der Rate des exogenen technischen Fortschritts. Liegt eine positive Zeitpräferenz der Wirtschaftssubjekte vor, muß die Grenzproduktivität des Kapitals nach der Goldenen Nutzenregel im Vergleich zur Goldenen Regel der Kapitalakkumulation auf dem optimalen Pfad höher sein. Dies impliziert eine niedrigere Kapitalintensität und somit bei gegebener Ausgangssituation eine geringere Investitionsquote. Auch der exogene technische Fortschritt fürt bei Gleichung $\left(2.61^{\prime}\right)$ zu einer geringeren Investitionsquote. Der heutige Konsumverzicht kann geringer ausfallen, wenn einerseits zukünftiger Konsum geringer geschätzt wird und andererseits Folgegenerationen von den Segnungen des technischen Fortschritts profitieren können. 87

Bei endogenem technischen Fortschritt können sich Veränderungen dieser Aussagen ergeben, da die Rate des technischen Fortschritts von der Investitionstätigkeit abhängt. Dies legt auf den ersten Blick nahe, daß die Volkswirtschaft im Vergleich zu einer Situation ohne technischen Fortschritt ebenfalls weniger investieren muß, um ihren optimalen Pfad zu erreichen. Da der technische Fortschritt positive Zuwächse bei zukünftigem Konsum zur Folge hat, trifft diese Vermutung auch zu. Bei identischen Zeitpräferenzen muß deshalb weniger

86 Mit $\mathrm{kg}^{\mathrm{g}}$ ist bei Vorliegen von technischem Fortschritt die auf Effizienzeinheiten bezogene Kapitalintensităt gemeint. Vgl. hierzu auch Frey (1970, S. 16).

87 Vgl. Frey (1970, S. 18f.). Auch bei Ramsey findet sich ein Hinweis auf die Auswirkungen des technischen Fortschritts auf die Frage der optimalen Sparquote:

"On the other hand, the probability that future inventions and improvements in organisation are likely to make income obtainable with less sacrifice than at present is a reason for saving less. The influence of inventions thus work in two opposite ways: they give us new needs which we can better satisfy if we have saved up beforehand, but they also increase our productive capacity and make preliminary saving less urgent." (Ramsey 1928, S. 549.) 
gespart werden. Vergleicht man Situationen, von denen die eine exogenen und die andere endogenen technischen Fortschritt aufweist, fallt diese Aussage nicht mehr so eindeutig aus. Entscheidend ist der Beitrag, den der Konsumverzicht zur Rate des technischen Fortschritts leistet. Im Falle mit exogenem technischen Fortschritt muß der zusätzliche Produktionsbeitrag, den der höhere Kapitaleinsatz und der technische Fortschritt erbringen, die negativen Effekte des Konsumverzichts, gemessen durch die Zeitpräferenz, ausgleichen. Im Falle endogenen technischen Fortschritts gilt dies auch. Je nachdem, wie groß die investitionsinduzierten Steigerungen des technischen Fortschritts sind, muß mehr oder weniger investiert werden als im Falle des exogenen technischen Fortschritts. Erbringt die Investition nur sehr geringe Zuwächse beim technischen Fortschritt, muß dies über eine höhere Grenzproduktivität ausgeglichen werden, die Volkswirtschaft wird weniger investieren als in der Situation mit exogenem technischen Fortschritt. Erbringt dagegen die Investition hohe Zuwächse beim technischen Fortschritt, wird sie tendenziell mehr investieren, damit die Wirkungen der Zeitpräferenz ausgeglichen werden. Endogener technischer Fortschritt kann somit eine höhere oder eine geringere Investitionsquote zur Folge haben als im Falle mit exogenem technischen Fortschritt.

Die abgeleiteten Regeln für die Optimalität des Wachstumspfads lassen sich nicht nur unter der impliziten Annahme eines allwissenden sozialen Planers, sondern auch bei rational handelnden Wirtschaftssubjekten gewinnen. Je nach unterstellter Nutzenfunktion, die zu maximieren ist, verändert sich die Zahl der einfließenden Variablen in Gleichung (2.61) und $\left(2.61^{\prime}\right){ }^{88}$ Das prinzipielle Ergebnis bleibt dennoch erhalten. Die Grenzproduktivität des Kapitals muß an exogen vorgegebene Variablen angepaßt werden, um optimales Investieren zu erreichen. Obwohl eine Zielfunktion für die Wirtschaftssubjekte eingeführt wurde, wodurch die Annahme einer exogenen Sparquote aufgegeben werden konnte, wird die langfristige Wachstumsrate nicht modellendogen bestimmt. Die Volkswirtschaft kann aufgrund der getroffenen Annahmen über die Produktionstechnik nicht über ihren "Schatten" springen. Daran ändert auch die Verfeinerung des Auswahlverfahrens für einen optimalen Wachstumspfad nichts.

\subsubsection{Der Standardfall der Cobb-Douglas-Produktionsfunktion}

Die wohl am häufigsten verwendete Spezifikation einer neoklassischen Produktionsfunktion ist die Cobb-Douglas-Produktionsfunktion. ${ }^{89}$ Geht man von dem Fall ohne technischen Fortschritt aus, ergibt sich

$$
Y=K^{\alpha} L^{1-\alpha}
$$

88 Vgl. z.B. Barro/Sala-i-Martin (1995, Kap. 2) und Romer, D. (1996, Kap. 2).

89 Vgl. den Anhang zu diesem Kapitel für den allgemeineren Fall der Constant-Elasticity-of-SubstitutionProduktionsfunktion. 
und als Pro-Kopf-Funktion mit $\mathrm{k}=\mathrm{K} / \mathrm{L}$

$$
f(k)=y=k^{\alpha}
$$

Für die Grenzproduktivität des Kapitals gilt:

$$
\frac{\partial Y}{\partial K}=\alpha L^{1-\alpha} K^{\alpha-1}=\alpha \frac{Y}{K} \quad \text { bzw. } \quad f^{\prime}(k)=\alpha k^{\alpha-1}
$$

Die Veränderungsgleichungen des Kapitals bzw. der Kapitalintensität sind dann entsprechend

$$
\dot{\mathrm{K}}=\mathrm{sK}^{\alpha} \mathrm{L}^{1-\alpha}-\delta \mathrm{K} \quad \text { bzw. } \quad \dot{\mathrm{k}}=\mathrm{sk}^{\alpha}-(\mathrm{n}+\delta) \mathrm{k}
$$

Der Steady State Wert für die Kapitalintensität ist gegeben durch

$$
k=\left(\frac{s}{n+\delta}\right)^{1 /(1-\alpha)}
$$

Die tatsächliche Wachstumsrate ist durch

$$
\frac{s}{v}=s\left(\frac{L}{K}\right)^{1-\alpha}=s k^{\alpha-1}
$$

bestimmt. Im Steady State gilt somit

$$
\mathrm{sk}^{\alpha-1}=\mathrm{n}+\delta . \quad \text { bzw. } \quad \mathrm{sk}^{\alpha-1}-\delta=\mathrm{n}
$$

Die Goldene Regel der Kapitalakkumulation verlangte, daß die Grenzproduktivität des Kapitals der Summe aus der Wachstumsrate der Bevölkerung und der Abschreibungsrate entspricht. Im Falle der Cobb-Douglas-Produktionsfunktion bedeutet dies

$$
\alpha \mathrm{k}^{\alpha-1}=\mathrm{n}+\delta \quad \text { bzw. } \quad \alpha \mathrm{k}^{\alpha-1}-\delta=\mathrm{n}
$$

Die Einführung von Harrod-neutralem technischen Fortschritt ändert an den Ergebnissen nichts. ${ }^{90}$ Es steht jedoch anstelle von $(k$ )die Kapitalintensität bezogen auf Effizienzeinheiten der Arbeit (k $\left.\mathbf{k}^{\mathrm{eff}}\right)$.

90 In (2.65) steht in diesem Falle: $k=\left(\frac{s}{n+\delta+\gamma_{H}}\right)^{1 /(1-\alpha)}$ 


\subsubsection{Eine Erweiterung: Gebundener technischer Fortschritt}

Im oben erläuterten neoklassischen Wachstumsmodell wurde davon ausgegangen, daß von technischen Neuerungen alle im Produktionsprozeß eingesetzten Kapitalgüter und/oder Arbeitseinheiten betroffen sind. Tatsächlich bedarf die Einführung technologischer Neuerungen in die Produktion eines Mediums. Eine Möglichkeit ist die Bindung des technischen Fortschritts an die Kapitalgüter, wobei die im Zeitraum ( $t$ ) investierten Kapitalgüter den aktuellen Stand des technischen Wissens beinhalten. Aus dem ursprünglich homogenen Kapitalstock wird eine Summe von heterogenen Anlagen, die sich entsprechend des Zeitpunkts ihrer Fertigung in ihrer Effizienz unterscheiden. Aus diesem Grund werden diese Ansätze als VintageModelle bezeichnet. Die ansonsten unterstellte beliebige Verformbarkeit des Kapitals geht somit verloren. Konsequenterweise ist hiervon auch die Produktionsfunktion betroffen, die nun jahrgangsbezogen aufgestellt werden müßte.

Bei der Analyse der Konsequenzen der Verbindung der Wirkungen des technischen Fortschritts mit der Investitionsentscheidung werden im allgemeinen drei Fälle bei der Variabilität des Faktorproportionen unterschieden:

1.) Putty-Putty: Ex ante- und ex post-Substitutionalität zwischen Arbeit und Kapital

2.) Putty-Clay: Ex ante-Substitutionalität und ex post-Limitationalität zwischen Arbeit und Kapital

3.) Clay-Clay: Ex ante- und ex post-Limitationalität zwischen Arbeit und Kapital

Mit ex ante und ex post ist jeweils die Situation vor und nach der Einführung des Kapitalgutes in den Produktionsprozeß gemeint. Ex ante- und ex post-Substitutionalität bedeutet z.B., daß die Ausstattung einer Anlage mit Arbeit dauernd variiert werden kann. Die unterschiedlichen Annahmen über die Substituierbarkeit der Produktionsfaktoren haben vor allem Folgen für die ökonomische Lebensdauer der Maschinen. Im Putty-Putty-Fall ermöglichen die technischen Gegebenheiten eine friktionslose Reaktion der Unternehmen auf Faktorpreisveränderungen, während in den beiden anderen Fällen steigende Faktorpreise den Zeitraum der effizienten Verwendung eines Kapitalgutes beeinflussen. ${ }^{91}$

An dieser Stelle ist eine andere Konsequenz der Vintage-Modelle von größerem Interesse. Wenn die Einführung technischer Neuerungen in den Produktionsprozeß an die Investitionen gebunden ist, liegt auf den ersten Blick die Abhängigkeit der Wachstumsrate des Systems von der Investitionsentscheidung nahe. Eine höhere Sparquote würde eine höhere Investitionsquote und somit eine größere Zahl neuer Maschinen im Produktionsprozeß implizieren. Der Anteil moderner Anlagen würde steigen, das Wachstum des Pro-Kopf-Einkommens zunehmen.

91 Vgl. fur einen Überblick zu diesen Fragestellungen z.B. Walter (1969, S. 132ff.), Hahn/Matthews (1964, S. 836ff.) und Rose (1987, S. 184ff.). 
Besitzt die Volkswirtschaft eine Putty-Putty-Technologie, gelten diese Überlegungen jedoch nur für einen Übergangszeitraum. Langfristig zeigt sich die Unabhängigkeit der Steady StateWachstumsrate von der Spar- und Investitionsquote bei exogenem technischen Fortschritt.

Ausgehend von einer Cobb-Douglas-Produktionsfunktion, die einerseits Hicks-neutralen technischen Fortschritt mit der Rate $\left(\gamma_{\mathrm{HI}}\right)$ und andererseits kapitalgebundenen technischen Fortschritt mit der Rate ( $\xi$ ) beinhaltet, zeigt z.B. Phelps (1962), daß die langfristige gleichgewichtige Wachstumsrate unabhängig von der Sparquote ist und folgendermaßen bestimmt wird: 92

$$
\hat{\mathbf{Y}}=\frac{\zeta+\gamma_{\mathrm{HI}}}{1-\alpha}+\mathbf{n}
$$

Sparquotenerhöhungen führen wie im Grundmodell zu einem höheren Level des Wachstumspfades. Dessen Steigung, d.h. die langfristige Wachstumsrate, wird nicht beeinflußt. Die Ursache für dieses Ergebnis liegt in der Altersstruktur des Kapitalstocks, die nur von der Abschreibungsrate und der langfristigen Wachstumsrate abhängt. Da beide Raten konstant und unabhängig von der Spar-Investitionsquote sind, haben Änderungen der letzteren keine Konsequenzen. Zwar kommen direkt nach der Erhöhung der Investitionsquote überproportional viel junge Kapitalgüter in den Produktionsprozeß, die unveränderliche technische Lebensdauer sorgt dafür, daß sich durch das Absterben der alten weniger effizienten Jahrgänge das Durchschnittsalter wieder auf seinen Gleichgewichtswert einpendelt. ${ }^{93}$ Nur ständige Erhöhungen der Investitionsquote bewirken eine anhaltende Modernisierung, wobei $s=1$ die natürliche Grenze für diese Wachstumspolitik in einer geschlossenen Volkswirtschaft bildet. Konsequenterweise ergibt sich für diesen Grenzfall eine Konsumquote von Null, was für die Konsumenten sicher keine optimale Situation ist.

Das Ergebnis von Phelps weist jedoch nur eingeschränkte Relevanz auf. Innerhalb des Putty-Putty-Falls verändert sich der Arbeitsbesatz älterer Maschinenjahrgänge aufgrund der eintretenden Reallohnsteigerungen ständig. Es wird deshalb eine Gewichtung der Jahrgänge mit der Arbeitsmenge pro Anlage vorgeschlagen. Die durchschnittliche Lebensdauer wird bei einer Substitutionselastizität zwischen Arbeit und Kapital ungleich eins von der Spar-Investitionsquote abhängig. Die Annahme einer Cobb-Douglas-Produktionsfunktion determiniert somit maßgeblich das Resultat einer von der Sparquote unabhängigen langfristigen Wachstumsrate. ${ }^{94}$ Wird an dieser Art der Produktionsfunktion festgehalten, bleibt das Standardergebnis der neoklassischen Wachstumstheorie erhalten: die langfristige Wachstumsrate kann durch eine Veränderung der Sparquote nicht erhöht werden.

92 Vgl. Phelps (1962, S. 556).

93 Vgl. Phelps (1962, S. 558f.)

94 Vgl. Matthews $(1964$, S. 165ff.) 
Auch unter den Annahmen anderer Substitutionsverhältnisse ändern sich die prinzipiellen Aussagen über die Unabhängigkeit des langfristigen Wachstumspfads nicht. Nur während der Übergangsphase von einem Gleichgewichtspfad zu einem anderen nimmt die Sparquote Einfluß auf die Wachstumsrate. Ist ein neues Gleichgewicht erreicht, bestimmen weiterhin die exogen vorgegebenen Parameter (technischer Fortschritt und Bevölkerungswachstum) die gleichgewichtige Wachstumsrate. ${ }^{95}$ Dieses Resultat ist nicht weiter verwunderlich, denn auch im Modell mit inkorporiertem technischen Fortschritt bleiben die prinzipiellen Annahmen der abnehmenden Grenzproduktivitäten der Produktionsfaktoren sowie der Exogenität des technischen Fortschritts erhalten. Die Verwendung eines Trägermediums für den technischen Fortschritt ändert nichts an der Tatsache, daß dessen Entstehung nicht erklärt wird. Die treibende Kraft des Pro-Kopf-Wachstums bleibt eine exogene Größe, die nicht von modellendogenen Variablen beeinflußt wird. Aus diesem Grund können die Vintage-Ansätze die Konsequenzen einer Investitionsbeschleunigung während des Übergangs zu einem neuen Gleichgewicht besser erklären als das Standardmodell. Eine Endogenisierung der langfristigen Wachstumsrate ist jedoch nicht gelungen.

Neben den Kapitalgütern kann auch der zweite Produktionsfaktor das Vehikel des technischen Fortschritts bilden. Der aktuelle Stand des Wissens kann an die Arbeit gebunden werden. Die Vorstellung wäre z.B., daß jüngere Arbeitnehmer, die in den Arbeitsprozeß eintreten, aufgrund des technischen Fortschritts und der Ausbildung über mehr Wissen und Fähigkeiten, auch Humankapital genannt, verfügen als die bisher Erwerbstätigen. So wie die Investitionen in den zuvor skizzierten Ansätzen den technischen Fortschritt transportieren und den Kapitalstock produktiver machen, erhöhen hier die Ausbildungsinvestitionen die Produktivität der Arbeit. Eine solche Vorgehensweise stellt aber letztendlich keinen Fortschritt gegenüber den Kapital-Vintage-Modellen dar. Denn weiterhin wäre die Entwicklung des technischen Fortschritts außerhalb des ökonomischen Systems angesiedelt.

Eine Gleichbehandlung des Human- und des physischen Kapitals ist auch aufgrund eines weiteren Sachverhalts schwierig, der sich durch den Satz: "Kapital denkt nicht" ausdrücken läßt. Der Unterschied zwischen Investitionen in die menschlichen Făhigkeiten und Sachkapitalinvestitionen liegt bei ihrer Beziehung zur Entstehung des technischen Fortschriţts. Zur Gewinnung neuer Erkenntnisse ist ein gewisser Bestand an Know-how vonnöten, so daß Humankapital sowohl Voraussetzung als auch Resultat des technischen Fortschritts ist. Investitionen in Sachkapital hingegen bedingen für sich genommen keine technische Neuerung. An dieser Stelle lassen sich selbstverständlich die Konzepte des investitionsinduzierten technischen Fortschritts anfuihren, wie sie von Kaldor und Arrow vorgestellt wurden. Doch auch bei diesen Ansätzen kann neues Wissen und somit zukünttige Produktivitätsfortschritte nicht ohne den komplementären Faktor Mensch entstehen. Eine Trennung zwischen technischem

95 Vgl. zu einem Überblick Walter (1969, S. 142ff.) sowie speziell zum Clay-Clay- bzw. Putty-Clay-Fall Weizsăcker (1966). 
Fortschritt und Humankapitalinvestitionen ist somit problematisch. Wenn davon ausgegangen wird, daß neues technisches Wissen zu seiner Realisierung im Produktionsprozeß die Verbesserung des Ausbildungsstandes der arbeitenden Menschen benötigt, müssen auch die Feedback-Effekte des erhöhten Wissenstandes auf das insgesamt verfügbare Know-how berücksichtigt werden. 96

\subsubsection{Zusammenfassung der Ergebnisse des neoklassischen Grundmodells}

Bevor im nächsten Kapitel auf weitere Entwicklungen innerhalb der neoklassischen Wachstumstheorie eingegangen wird, werden an dieser Stelle die wesentlichen Aussagen der traditionellen neoklassischen Wachstumstheorie sowie die für die weitere Untersuchung relevanten Sachverhalte noch einmal zusammengefaßt.

Eines der wichtigsten Resultate ist die Unabhängigkeit der langfristigen Steady StateWachstumsrate sowohl der Absolut- als auch der Pro-Kopf-Größen von den Spar-Investitionsentscheidungen der Wirtschaftssubjekte. Veränderungen des Sparverhaltens, welche via Realzinsmechanismus automatisch zu äquivalenten Veränderungen der Investitioen führen, haben langfristig keinen Einfluß auf die gleichgewichtige Wachstumsrate, sondern bedingen nur eine Verschiebung des Wachstumspfades. Weder die Einführung einer von den Wirtschaftssubjekten zu maximierenden Nutzenfunktion noch die Berücksichtigung der Heterogenität des Kapitalstocks bzw. des Arbeitseinsatzes im Vintage-Ansatz konnten dieses Ergebnis entscheidend modifizieren. Diese Unabhängigkeit bedeutet jedoch nicht, daß die Investitionsquote ohne Einfluß auf den Wachstumsprozeß bleibt. Berücksichtigt man, daß der durch Veränderungen des Investitionsverhaltens angeregte Anpassungsproze $B$ an ein neues Wachstumsgleichgewicht einen unendlich langen Zeitraum benötigt, gewinnt die Kapitalbildung sehr wohl an Bedeutung. ${ }^{97}$ Dies gilt insbesondere, wenn man die Konsequenzen eines höheren Konsumverzichts auf das Niveau des Wachstumspfades berücksichtigt. Investitionen steigern sehr wohl das Pro-Kopf-Einkommen einer Volkswirtschaft, indem sie den Gleichgewichtspfad nach oben verschieben. Sie können nur nicht auf Dauer die Dynamik des Wachstumsprozesses erhöhen. Darüber hinaus stellt das Konzept des Steady State ein Referenzmodell dar, das zeigt, wohin sich eine Volkswirtschaft bei vollkommener Flexibilität der Faktorpreise und einer Produktionstechnik mit konstanten Skalenerträgen sowie exogen gegebenem technischen Fort-

96 Vgl. zu diesen Überlegungen auch Walter (1969, S. 156ff.), der auch auf die Probleme der Messung des Humankapitals hinweist sowie die Tatsache betont, daß die Effizienz der Ausbildung auch von den individuellen angeborenen Anlagen abhängt. Es unterscheiden sich deshalb nicht nur die einzelnen Humankapital-Jahrgănge in der Produktivităt voneinander, sondern auch die Jahrgange in sich selbst. Die Rolle des Humankapitals im Wachstumsprozeß wird im Abschnitt über die Neue Wachstumstheorie noch einmal aufgegriffen werden.

97 Genauer gesagt, dauert eine 100\%ige Anpassung unendlich lange. Vgl. hierzu den schon angesprochenen Beitrag von Sato (1968). 
schritt und Bevölkerungswachstum bewegt, wenn sie sich selbst überlassen bleibt. Solows Ausgangspunkt war der Nachweis der theoretisch möglichen Stabilität einer kapitalistisch organisierten Volkswirtschaft und nicht die linientreue Abbildung tatsächlicher Wachstumsprozesse.

Die Irrelevanz der Investitionsentscheidungen für die Dynamik des Wachstumsprozesses hat ihren Grund in der unterstellten Produktionstechnik. Für beide Produktionsfaktoren wurde eine sinkende Grenzproduktivität bei steigendem Faktoreinsatz angenommen. Diese Eigenschaft impliziert eine sinkende Kapitalrendite und somit einen sinkenden Investitionsanreiz. Im Falle konstanter Sparquoten führt jede zusätzliche Kapitaleinheit zu einem unterproportionalen Zuwachs des Outputs. Die durch den Produktionszuwachs induzierten Investitionen fallen nun geringer aus als die vorige Investition. Im Steady State-Gleichgewicht gibt es für die Unternehmen keinen Anreiz, den Kapitaleinsatz pro Arbeitskraft weiter auszudehnen. Die tatsächlichen Investitionen pro Kopf entsprechen den Investitionen, die notwendig sind, die aktuelle Kapitalausstattung pro Kopf konstant zu halten. Die gewünschte Wachstumsrate entspricht somit der natürlichen Wachstumsrate.

Die Konsequenz der abnehmenden Grenzproduktivität des Kapitals wird besonders deutlich, wenn man die Modelle des optimalen Wachstums betrachtet, die eine Nutzenfunktion beinhalten. Es zeigt sich, daß im Fall ohne technischen Fortschritt im Steady State die Grenzproduktivität des Kapitals der Summe aus Bevölkerungswachstum und Diskontrate entspricht. Solange $f^{\prime}(k)>n+\rho+\delta$, lohnt es sich für die Volkswirtschaft auf Konsum zu verzichten. Mit zunehmender Investition sinkt $\mathbf{f}(\mathbf{k})$ ab. Auf dem Steady State-Pfad entspricht der Ertrag des Kapitaleinsatzes genau der Summe aus dem Aufwand, der für die Aufrechterhaltung der Kapitalintensität notwendig ist, und der von den Wirtschaftssubjekten geforderten Entschädigung für ihren Konsumverzicht. Eine Ausdehnung des Kapitaleinsatzes über das gleichgewichtige Niveau hinaus lohnt sich nicht. Die Kosten dieses Vorgehens würden die Erträge überwiegen. Die Volkswirtschaft verbleibt auf ihrem Steady State-Pfad. Die Kapitalakkumulation erfolgt in der Höhe, welche die Konstanz der Produktionsverhältnisse sichert.

Die Einfürung des technischen Fortschritts wirkt den unterstellten abnehmenden Grenzerträgen des Kapitals entgegen. Der Anreiz, mehr zu akkumulieren als zur Aufrechterhaltung einer konstanten Kapitalintensität erforderlich ist, bleibt dauerhaft bestehen. Kapitalstock und Output wachsen demnach schneller als der Arbeitseinsatz, das Pro-Kopf-Einkommen muß konsequenterweise zunehmen. Der technische Fortschritt erfüllt aufgrund dieses Zusammenhangs die Funktion eines Wachstumsmotors. Würde die Rate des technischen Fortschritts auf Null sinken, würde die Annahme der abnehmenden Grenzproduktivität des Kapitals wieder zu einem Nullwachstum der Pro-Kopf-Größen im Steady State führen. Das neoklassische Grundmodell benötigt somit exogene Faktoren, die eine steigende Güterversorgung pro Kopf erklären können. 
Eine weitere wichtige Implikation der traditionellen neoklassischen Wachstumstheorie ist mit dem Begriff der Konvergenz verknüpft. Einerseits konvergiert eine Volkswirtschaft, die den getroffenen Annahmen entspricht und keine exogene Störungen zu verzeichnen hat, gegen ihren Steady State-Wachstumspfad, d.h. die Kapitalintensität, gemessen in realen bzw. effizienten Arbeitseinheiten, und die tatsächliche Wachstumsrate erreichen ihren Gleichgewichtswert. Weisen alle Volkswirtschaften identische Parameterwerte auf, müssen alle demselben Gleichgewicht zustreben. Diejenigen Volkswirtschaften, die geringe Kapitalintensitäten aufweisen, müßten dann höhere tatsächliche Wachstumsraten realisieren als diejenigen mit hoher Kapitalausstattung pro Kopf. Unterscheiden sich die Volkswirtschaften bei den Modellparametern, ist die Höhe der Wachstumsrate abhängig von der relativen Position zum Steady State. Je größer die Abweichung zum Gleichgewicht ist, desto höher müßte die Wachstumsrate sein. Internationale Kapitalmobilität und weltweite Verfügbarkeit des technischen Fortschritts hätten des weiteren aufgrund der Anpassung der Produktionsverhältnisse die Konvergenz der ProKopf-Wachstumsraten sowie die Anpassung der Pro-Kopf-Einkommen zur Folge, da letztendlich nur die Rate des technischen Fortschritts die langfristige Wachstumsmöglichkeiten bestimmt. Keines dieser Phänomene konnte jedoch empirisch festgestellt werden. Nur für Länder, die in enger Beziehung stehen und sich sehr ähnlich sind, konnten Konvergenzprozesse nachgewiesen werden. 98

Die traditionelle neoklassische Wachstumstheorie weist somit drei, für die weitere Untersuchung entscheidende Mängel auf:

1.) Die gleichgewichtige Wachstumsrate wird nur von exogenen Faktoren bestimmt.

2.) Die Entscheidungen der Wirtschaftssubjekte über Sparen und Investieren können nur das Niveau des Wachstumspfades verändern, nicht jedoch dessen Steigung.

3.) Die Implikationen des Modells für den Entwicklungsprozeß real existierender Volkswirtschaften werden in der Realität nicht erfullt.

In den nächsten Kapiteln werden Ansätze vorgestellt, die versuchen diese Mängel zu überwinden, um einerseits eine Endogenisierung der Wachstumsrate und andererseits eine bessere Übereinstimmung der Modellaussagen mit empirisch feststellbaren Entwicklungen zu gewährleisten.

98 Vgl. z.B. Barro (1991), Barro/Sala-i-Martin (1991, 1992a, 1995), Baumol (1986), Baumol/Wolff (1988), Chatterji (1992), DeLong (1988), Dowrick/Gemmell (1991), Dowrick/Nguyen (1989). 


\section{Anhang zu Kapitel 2}

a) Die Elastizităt des Konsums in Bezug auf die Sparquote im Steady State nach Meade (1962a)

Die Goldene Regel der Kapitalakkumulation kann auch auf eine andere Art und Weise als im Haupttext abgeleitet werden. Im Steady State gilt für die gleichgewichtige Wachstumsrate:

$$
\text { (A.2.1) } \frac{\mathrm{sY}}{\mathrm{K}}=\mathrm{n}
$$

Löst man diese Gleichung nach (K) auf, folgt:

$$
\text { (A.2.2) } \quad K=\frac{s Y}{n}
$$

Setzt man (A.2.2) in eine neoklassische Produktionsfunktion ein, steht 99

$$
\text { (A.2.3) } \quad \mathrm{Y}=\mathrm{F}\left(\frac{\mathrm{sY}}{\mathrm{n}}, \mathrm{L}\right)
$$

Aus (A.2.4) kann die Reaktion des Steady State-Einkommens auf Veränderungen der Sparquote ermittelt werden:

$$
\text { (A.2.4) } \frac{\partial \mathrm{Y}}{\partial \mathrm{s}}=\frac{\partial \mathrm{F}}{\partial \mathrm{K}} \frac{\mathrm{dK}}{\mathrm{ds}}+\frac{\partial \mathrm{F}}{\partial \mathrm{L}} \frac{\mathrm{dL}}{\mathrm{ds}}
$$

Der zweite Term der rechten Seite ist unter Annahme, daß die Arbeitsmenge nicht von Veränderungen der Sparquote berührt wird, gleich Null, d.h. unter Verwendung von (A.2.2) folgt:

(A.2.5') $\frac{\partial \mathrm{Y}}{\partial \mathrm{s}}=\frac{\partial \mathrm{F}}{\partial \mathrm{K}} \frac{\mathrm{dK}}{\mathrm{ds}}=\frac{\partial \mathrm{F}}{\partial(\mathrm{sY} / \mathrm{n})} \frac{\mathrm{d}(\mathrm{sY} / \mathrm{n})}{\mathrm{ds}}$

damit gilt:

(A.2.6) $\frac{\partial \mathrm{Y}}{\partial \mathrm{s}}=\alpha \frac{\mathrm{Y}}{\mathrm{K}} \frac{\mathrm{dK}}{\mathrm{ds}}$

99 Meade (1962a) verwendet eine spezifizierte Cobb-Douglas-Produktionsfunktion. 
Für die Lösung von Gleichung (A.2.6) muß (dK/ds) ermittelt werden. Hierbei gilt:

(A.2.7) $\frac{d K}{d s}=\frac{d(s Y / n)}{d s}=\frac{1}{n}\left(Y+s \frac{d Y}{d s}\right)$

In Gleichung (A.2.7) muß aber (dY/ds) der Lösung von (A.2.6) entsprechen, so daß bei Einsetzen von (A.2.6) in (A.2.7) folgt:

(A.2.8) $\quad \frac{\mathrm{dK}}{\mathrm{ds}}=\frac{\mathrm{d}(\mathrm{sY} / \mathrm{n})}{\mathrm{ds}}=\frac{1}{\mathrm{n}}\left(\mathrm{Y}+\mathrm{s} \alpha \frac{\mathrm{Y}}{\mathrm{K}} \frac{\mathrm{dK}}{\mathrm{ds}}\right)$

Nun kann man (A.2.8) nach (dK/ds) auflösen:

(A.2.9) $\quad \frac{\mathrm{dK}}{\mathrm{ds}}\left(1-\frac{\mathrm{s}}{\mathrm{n}} \alpha \frac{\mathrm{Y}}{\mathrm{K}}\right)=\frac{1}{\mathrm{n}} \mathrm{Y}$

bzw. mit (A.2.2) folgt

(A.2.10) $\frac{\mathrm{dK}}{\mathrm{ds}}=\frac{\mathrm{Y}}{\mathrm{n}} \frac{1}{1-\frac{\mathrm{s}}{\mathrm{n}} \alpha \frac{\mathrm{Y}}{\mathrm{K}}}=\frac{\mathrm{Y}}{\mathrm{n}} \frac{1}{1-\alpha}$.

Eingesetzt in (A.2.6) folgt unter Verwendung von (A.2.2)

(A.2.11) $\frac{\partial \mathrm{Y}}{\partial \mathrm{s}}=\alpha \frac{\mathrm{Y}}{\mathrm{K}} \frac{\mathrm{Y}}{\mathrm{n}} \frac{1}{1-\alpha}=\frac{\alpha}{1-\alpha} \frac{\mathrm{Y}}{\mathrm{s}}$

Die Elastizität des Einkommens bzgl. der Sparquote ist dann nach (A.2.12) bestimmt.

(A.2.12) $\frac{\partial \mathrm{Y}}{\partial \mathrm{s}} \frac{\mathrm{s}}{\mathrm{Y}}=\frac{\alpha}{1-\alpha}$

Für den Konsum gilt immer

(A.2.13) $\quad \mathrm{C}=(1-\mathrm{s}) \mathrm{Y}$

Will man nun die Reaktion des Konsums auf die Veränderung der Sparquote darstellen, folgt:

(A.2.14) $\frac{\partial \mathrm{C}}{\partial \mathrm{s}}=(1-\mathrm{s}) \frac{\partial \mathrm{Y}}{\partial \mathrm{s}}-\mathrm{Y}$ 
Man kann aus (A.2.14) unter Verwendung des Ergebnisses von (A.2.13) nun (A.2.15) ermitteln.

$$
\frac{\partial C}{\partial s}=(1-s) \frac{\alpha}{1-\alpha} \frac{Y}{s}-Y=\frac{(1-s) \alpha Y}{(1-\alpha) s}-\frac{(1-\alpha) s Y}{(1-\alpha) s}=\frac{\alpha-s}{(1-\alpha) s} \frac{Y}{s}
$$

Für die Elastizität des Konsum bzgl. der Sparquote folgt daraus:

(A.2.16) $\quad \frac{\partial C}{\partial s} \frac{s}{C}=\frac{\alpha-s}{(1-\alpha) s} \frac{Y}{s} \frac{s}{(1-s) Y}=\frac{\alpha-s}{(1-\alpha)(1-s)}$

Die Reaktion des Konsumniveaus im Steady State wird von der Produktionselastizität des Kapitals und der Sparquote beeinflußt. Dasselbe Ergebnis hätte auch über die Wachstumsratengleichung für die Sparquote und des Konsums im Steady State ermittelt werden können. Die hier vorgestellte Vorgehensweise folgt jedoch derjenigen von Meade (1962).

b) Die Constant-Elasticity-of-Substitution-Produktionsfunktion

Die neoklassische Produktionsfunktion kann als Spezialfall der Gruppe der ConstantElasticity-of-Substitution-Produktionsfunktionen (CES-Produktionsfunktion) gesehen werden, die von Arrow/Chenery/Minhas/Solow (1961) eingeführt wurde. Eine mögliche allgemeine Form der CES-Produktionsfunktion ist: 100

(A.2.17) $\quad \mathrm{Y}=\mathrm{A}\left\{\mathrm{a}(\mathrm{bK})^{\varphi}+(1-\mathrm{a})[(1-\mathrm{b}) \mathrm{L}]^{\varphi}\right\}^{1 / \varphi}$ mit $\varphi<1$

und in Pro-Kopf-Schreibweise

$$
f(k)=A\left\{a(b k)^{\varphi}+(1-a)(1-b)^{\varphi}\right\}^{1 / \varphi}
$$

Die CES-Produktionsfunktion ist linear-homogen und weist somit konstante Skalenerträge auf. Für die Grenzproduktivität des Kapitals gilt:

$$
\text { (A.2.19) } \quad f^{\prime}(k)=\operatorname{Aab}^{\varphi}\left\{a b^{\varphi}+(1-a)(1-b)^{\varphi} k^{-\varphi}\right\}^{1 / \varphi}
$$

100 Vgl. Barro/Sala-i-Martin (1995, S. 43). 
und für die Durchschnittsproduktivität des Kapitals folgt:

$$
\frac{f(k)}{k}=A\left\{a b^{\varphi}+(1-a)(1-b)^{\varphi} k^{-\varphi}\right\}^{1 / \varphi}
$$

Welche Form die Produktionsfunktion und welchen Verlauf die Funktionen der Durchschnittsund der Grenzproduktivităt annehmen, hängt vom Substitutionsparameter ab $(\varphi)$. Die CobbDouglas-Produktionsfunktion ergibt sich, wenn $(\varphi)$ gegen Null strebt. Die Substitutionselastizität ergibt sich durch $[1 /(1-\varphi)]$, d.h. für die Cobb-Douglas-Produktionsfunktion folgt ein Wert von Eins. Falls $0<\varphi<1$, resultieren für die Durchschnitts- und die Grenzproduktivität folgende Grenzwerte:

(A.2.21) $\quad \lim _{k \rightarrow \infty} f^{\prime}(k)=\lim _{k \rightarrow \infty} \frac{f(k)}{k}=\mathrm{Aba}^{1 / \varphi}>0$

(A.2.22) $\quad \lim _{k \rightarrow 0} f^{\prime}(k)=\lim _{k \rightarrow 0} \frac{f(k)}{k}=\infty$

Die CES-Produktionsfunktion erfullt damit nicht die Inada-Bedingungen, die für eine typische neoklassische Produktionsfunktion unterstellt worden sind. Die Wachstumsrate der Kapitalintensität konvergiert gegen einen konstanten Wert für (k) gegen unendlich:

$$
\lim _{\mathbf{k} \rightarrow \infty} \hat{\mathbf{k}}=\mathrm{sAba}^{1 / \varphi}-(\mathrm{n}+\delta)
$$

Falls $\mathrm{sAba}{ }^{1 / \varphi}>(\mathrm{n}+\delta)$ gilt, wächst die Kapitalintensität auch ohne technischen Fortschritt mit einer positiven Rate und somit auch das Pro-Kopf-Einkommen. Wenn sAba ${ }^{1 / \varphi}<(n+\delta)$, wird wieder eine konstante Kapitalintensităt und folglich auch eine Steady State-Situation erreicht. Das Ergebnis ändert sich, wenn $(\varphi)<0$ ist. Die Grenzwerte für die Durchschnitts- und Grenzproduktivität ergeben sich dann nach:

(A.2.24) $\quad \lim _{k \rightarrow \infty} f^{\prime}(k)=\lim _{k \rightarrow \infty} \frac{f(k)}{k}=0$

(A.2.25) $\quad \lim _{k \rightarrow 0} f^{\prime}(k)=\lim _{k \rightarrow 0} \frac{f(k)}{k}=A b a^{1 / \varphi}<\infty$

Auch in diesem Fall sind die Inada-Bedingungen verletzt. Jedoch konvergiert die Grenz- und Durchschnittsproduktivität gegen Null, konsequenterweise ergeben sich die aus dem neoklassischen Modell bekannten Ergebnisse. Endogenes Wachstum ist ausgeschlossen. Falls 
$s A b a^{1 / \varphi}<(n+\delta)$, sind die Wachstumsraten der Kapitalintensität und des Pro-Kopf-Wachstums negativ. Der Fall einer schrumpfenden Volkswirtschaft liegt vor, die keine gleichgewichtige Situation erreicht.

Unter Verwendung einer CES-Produktionsfunktion kann, wenn Arbeit und Kapital gute Substitute sind, endogenes Wachstum erreicht werden. Dies verlangt aber die Verletzung der Inada-Bedingung, daß der Grenzwert des Kapitals bei steigender Kapitalintensität gegen Null konvergiert. Neben den Konsequenzen fur den Wachstumsprozeß hat die CES-Produktionsfunktion auch Folgen für die Verteilungssituation. Das Verhältnis zwischen dem Profit- und Lohneinkommen ist nicht konstant, sondern ist abhängig von dem Substitutionsparameter der CES-Produktionsfunktion, der Parameter (a) und (b) sowie der Kapitalintensität. Im Gegensatz zur Cobb-Douglas-Produktionsfunktion variiert die Einkommensverteilung mit der Kapitalintensität. 101

101 Vgl. Rose (1987, S. 104ff.). 
Stephan Seiter - 978-3-631-75406-1

Downloaded from PubFactory at 01/11/2019 05:22:48AM

via free access 
Die Neue Wachstumstheorie

\subsection{Vorbemerkungen}

Im vorigen Kapitel wurden die Standardmodelle der modernen Wachstumstheorie skizziert und besprochen. Trotz der großen Euphorie, mit der wachstumstheoretische Fragestellungen noch in den sechziger Jahren diskutiert wurden, wendete sich die ökonomische Wissenschaft seit Anfang der siebziger Jahre verstärkt konjunkturellen Fragestellungen zu. Nicht mehr die Erklärung der langfristigen Entwicklung des Sozialprodukts war en vogue, sondern die kurzfristigen Schwankungen waren von Interesse. Auch die signifikanten Rückgänge bei der Wachstumsrate der Arbeitsproduktivität in vielen Volkswirtschaften ab 1973, dem sog. productivity slowdown, wurden zumindest aus wachstumstheoretischer Sicht mehr mit vorhandenen Instrumenten zu erklären versucht, als daß neue Konzepte entworfen wurden.

Seit Mitte der achtziger Jahre hat jedoch das Interesse an der Wachstumstheorie wieder zugenommen. Innerhalb der neoklassischen Wachstumstheorie wurden neue Aspekte in das traditionelle Modell integriert, die dazu dienen sollen, die am Ende des letzten Kapitels zusammengefaßten Mängel zu beheben und eine bessere Übereinstimmung der modelltheoretischen Aussagen mit der Realität zu erreichen. Diese Ansätze werden unter dem Begriff der Neuen bzw. Endogenen Wachstumstheorie zusammengefaßt. ${ }^{1}$ Insbesondere die Abhängigkeit der Steady State-Wachstumsrate von exogenen Parametern bzw. ihre Unabhängigkeit von der Spar- und Investitionsentscheidung sollen überwunden werden. Dies bedeutet, daß einerseits Pro-Kopf-Wachstum langfristig möglich sein soll, ohne daß auf exogenen technischen Fortschritt zurückgegriffen werden muß und andererseits, daß das langfristige Wachstum durch ökonomische Entscheidungen dauerhaft beeinflußt werden kann.

Die Zielsetzung, die sich die Vertreter der Neuen Wachstumstheorie gesetzt haben, läßt sich am besten mit einer Reihe von Sachverhalten darstellen, die innerhalb eines Wachstumsmodells ihren Platz haben sollen. Ähnlich den berühmten Stylized Facts von Kaldor (1957) stellt Romer (1994) solche Zusammenhänge dar. Die erste Tatsache ist die Existenz vieler Firmen innerhalb einer Marktwirtschaft, d.h. das Ergebnis eines Modells darf nicht die Konzentration der gesamten Güterproduktion auf einen einzigen Monopolisten sein. Es muß, mit anderen Worten, noch ein gewisses Maß an Wettbewerb bestehen. Zweitens sind technische Neuerungen im Gegensatz zu anderen Produktionsfaktoren oft durch die Nichtrivalität bei der Nutzung gekennzeichnet. Grundlagenwissen stellt z.B. ein quasi-öffentliches Gut dar, das von vielen Anwendern gleichzeitig genutzt werden kann, ohne daß gegenseitige Einbußen bestehen. Drittens können alle physischen Aktivitäten reproduziert werden. Für die Produktionstechnik

1 Zur Neuen Wachstumstheorie wurden aus unterschiedlicher Sichtweise heraus Überblicksartikel verfaßt. Vgl. z.B. Boltho/Holtham (1992), Stolpe (1992), Verspagen (1992), Flemmig/Gotz (1993), Ramser (1993), Schneider/Ziesemer (1995). 
bedeutet dies konstante Skalenerträge, d.h. wenn die Inputs Arbeit und Kapital verdoppelt werden, wird sich auch der Output verdoppeln. Hierfür müssen die nicht-rivalisierenden Inputs nicht verdoppelt werden. Skalenerträge aufgrund von Größenvorteilen sind hiermit ausgeschlossen. Viertens ist technischer Fortschritt ein Ergebnis menschlichen Handelns. Dies ist eine klare Absage gegen das Instrument des exogenen technischen Fortschritts. Nur wenn Anstrengungen in Form von Ressourceneinsatz unternommen werden, können Weiterentwicklungen beim technologischen Know-how entstehen. Fünftens gibt es trotz der ersten und zweiten Tatsache das Phänomen der Marktmacht und Monopolrenten aufgrund von Erfindungen. Die Existenz dieser Abweichungen vom Modell der vollkommenen Konkurrenz ist das Resultat der teilweisen Ausschließbarkeit anderer Unternehmen von der Nutzung technischer Neuerungen. ${ }^{2}$

Die beiden letzten Aspekte werden vom traditionellen neoklassischen Wachstumsmodell nicht erklärt. Einerseits ist der technische Fortschritt exogener Art, andererseits sind monopolistische Strukturen annahmegemäß ausgeschlossen. Aufgrund dieser Einschränkung auf nur drei von fünf realiter relevanten Eigenschaften des Wachstumsprozesses von Volkswirtschaften scheint ein Scheitern der neoklassischen Wachstumstheorie der alten Prägung vorgezeichnet. Eine Integration der beiden letzten Sachverhalte könnte hier Abhilfe schaffen.

Die Modelle der Neuen Wachstumstheorie beinhalten diverse Mechanismen, um die von Romer aufgelisteten stilisierten Fakten aufzunehmen und die Konsequenzen daraus für den langfristigen Wachstumsprozeß abzuleiten. Zentrale Punkte sind dabei steigende Skalenerträge bei der Produktion des Endprodukts aufgrund von Wissensexternalitäten, die Bildung und Verwendung von Humankapital, Forschungs- und Entwicklungsinvestitionen sowie Infrastrukturinvestitionen im weitesten Sinne. Die Ansätze der Neuen Wachstumstheorie bringen somit keine grundlegend neuen Aspekte in die Wirtschaftstheorie ein. Schon in der Entwicklungstheorie wurden viele dieser Sachverhalte diskutiert. Auch die sog. Neue Handelstheorie bzw. die Strategische Handelspolitik zeigte z.B. die Konsequenzen steigender Skalenerträge für den Außenhandel auf. Die neuen Modelle der Wachstumstheorie integrieren vielmehr allgemein Bekanntes in den vorhandenen Rahmen der neoklassischen Wachstumstheorie und gelangen teilweise zu Ergebnissen und Schlußfolgerungen, die man bei neoklassisch orientierten Autoren nicht unbedingt erwarten würde.

Da innerhalb der Neuen Wachstumstheorie auch die Frage nach der Konvergenz bzw. Divergenz zwischen Volkswirtschaften von Interesse ist, wird im folgenden zuerst die Catching up-Hypothese diskutiert. Diese kann in gewissem Sinne als Vorläufer der Neuen Wachstumstheorie verstanden werden, obwohl sie weniger auf mathematischem Wege, sondern vielmehr verbal die Gründe für erfolgreiche Entwicklungsprozesse von Volkswirtschaften analysiert. Die sich anschließenden Abschnitte werden sich mit den unterschiedlichen Ansätzen zur Endogenisierung der Wachstumsrate beschäftigen sowie die Kritik an der Neuen Wachstumstheorie

2 Vgl. Romer (1994, S. 12f.). 
beinhalten. Mögliche wirtschaftspolitische Schlußfolgerungen werden im Anschluß an die Gegenüberstellung mit den kaldorianischen Überlegungen diskutiert. Den Abschluß dieses Kapitels bildet eine Zusammenfassung der wichtigsten Ergebnisse.

\subsection{Die Catching up-Hypothese}

\subsubsection{Inhalt der Catching up-Hypothese}

Innerhalb des neoklassischen Wachstumsmodells ergab sich das Ergebnis, daß die Wachstumsraten des Pro-Kopf-Outputs in den Volkswirtschaften höher sein müssen, die eine Kapitalintensität weit unterhalb ihres Steady State-Wertes aufweisen. Diese Aussage impliziert jedoch nicht, daß sich alle Länder in ihren Einkommensniveaus anpassen. Erst internationale Kapitalmobilität und die internationale Verfügbarkeit des technologischen Wissens können dafür sorgen, daß alle Länder dieselben Steady State-Werte erreichen können. Bisher unterentwickelte Länder hätten dann die Chance, gegenüber industrialisierten Volkswirtschaften beim Wohlstandsniveau aufzuholen.

Eine vergleichbare Schlußfolgerung läßt sich aus der sog. Catching up-Hypothese gewinnen, die maßgeblich von Abramovitz $(1979,1986,1990,1994)$ vertreten wird und zur Erklärung divergierender und konvergierender Wachstumsprozesse von Volkswirtschaften dienen soll. Von Interesse ist z.B., warum es nach dem Zweiten Weltkrieg einigen Ländern gelungen ist, sich beim Niveau der Arbeitsproduktivität an die USA anzunähern, die im internationalen Vergleich bei diesem Indikator den höchsten Wert zu verzeichnen hat, andere Länder dagegen im wahrsten Sinne des Wortes abgehängt worden sind. ${ }^{3}$ Entsprechend der Catching up-Hypothese weisen Volkswirtschaften, die im Vergleich zu den führenden Industrienationen ein niedriges Produktivitätsniveau besitzen, ein großes Potential für einen schnellen Aufholprozeß auf. Je geringer die Produktivität in einer Volkswirtschaft ist, desto höhere Wachstumsraten müßten sie deshalb verzeichnen. Die zentrale Begründung für diese Aussage liegt im Zusammenhang zwischen Forschungs- und Entwicklungsaktivitäten einerseits und dem Kapitalstock als Trägermedium des Wissensstandes einer Ökonomie andererseits. Es wird wie im Vintage-Ansatz davon ausgegangen, daß die neuesten Kapitalgüter immer den aktuellen Stand des technischen Know-hows beinhalten, den eine Volkswirtschaft zum Zeitpunkt der Investition erreicht hat. Länder, die führend bei der Forschung und Entwicklung sind, wie z.B. die USA, werden deshalb auch das höchste Produktivitätsniveau erreichen.

3 Vgl. z.B. Abramovitz (1990). Auch im Rahmen der hier nicht im Mittelpunkt stehenden Erklărung des sog. productivity slowdowns, worunter die seit 1973 plotzlich deutlich gesunkenen Wachstumsraten der Arbeitsproduktivităt verstanden wird, kommt die Catching up-Hypothese zur Anwendung. Vgl. zu den verschiedenen Erklărungsansătzen zu diesem Phănomen z.B. Giersch/Wolter (1983), Lindbeck (1983), Bombach (1985), Gehrke/Rühl (1988). 
Wollen diese Volkswirtschaften weitere Produktivitätsfortschritte realisieren, müssen sie den Stand der Forschung erhöhen, d.h. neben der Investition in das reale Kapital müssen auch Forschungsausgaben getätigt werden. Volkswirtschaften, die dagegen beim Know-how Rückstände aufweisen, werden zum einen eine geringere Produktivität als die führende Volkswirtschaft aufweisen, zum anderen aber können sie größere Produktivitätssprünge machen, indem sie moderne Kapitalgüter importieren. Gelingt es gegenüber dem führenden Land aufzuholen, verringert sich gleichzeitig das Potential für Produktivitätsfortschritte dieser Art. Die Folgen dieses Vintage-Effektes werden u.U. durch weitere induzierte Prozesse verstärkt. Eine direkte Konsequenz einer solchen Strategie ist die Erhöhung der Kapitalintensität bedingt durch relativ sinkende Kapitalkosten aufgrund des technischen Fortschritts, die auch bei Vorliegen abnehmender Grenzerträge des Kapitals zu einer Erhöhung des Outputs pro Kopf führt, wie es das neoklassische Grundmodell gezeigt hat. Steigendes Produktivitätsniveau bedingt Steigerungen der Realeinkommen, wodurch aufgrund einer zunehmenden Nachfrage eine Erhöhung der Absatzmengen und folglich Skaleneffekte realisiert werden können. Produktivitätssteigerungen können darüber hinaus auch durch einen Abbau versteckter Arbeitslosigkeit im primären Sektor, insbesondere der Landwirtschaft ermöglicht werden. Die entsprechenden Arbeitskräfte können im wachsenden industriellen Sektor eingesetzt werden, was einen Anstieg der gesamtwirtschaftichen Produktivität zur Folge hat. Letztlich schaffen Investitionen die Möglichkeit zu weiteren technologischen Fortschritten aufgrund von Lerneffekten, wie sie von Arrow (1961) und Kaldor (1957) diskutiert wurden. ${ }^{4}$

Die Catching up-Hypothese scheint auf den ersten Blick auf einer monokausalen Beziehung zwischen dem Rückstand beim technologischen Know-how und dem Wachstumspotential von Volkswirtschaften aufzubauen. Jedes Land, das einen hohen Produktivitätsrückstand gegenüber dem "Leader" besitzt, müßte dann auch hohe Wachstumsraten bei der Produktivität zeigen. Das dem nicht so ist, bedarf keiner weiteren Erklärung. Tatsächlich ist es nur wenigen Volkswirtschaften gelungen, ihr Aufholpotential zu nutzen. Andere Faktoren spielen somit eine Rolle, die den Entwicklungsprozeß hemmen können. Der Erfolg einer Modernisierungsstrategie, um mit Hilfe von Investitionen Produktivitätslücken auszugleichen, setzt eine ausreichende Befähigung der Volkswirtschaft zur Übernahme des technologisch neuesten Stands voraus. Innerhalb der Diskussion über die Catching up-Hypothese werden die notwendigen Bedingungen hierfurr mit dem Begriff der social capability umschrieben. Hierunter werden neben dem Bildungsstand der Bevölkerung und der Leistungsfähigkeit des Bildungssystems auch politische und finanzwirtschaftliche Institutionen, der sektorale Aufbau der Volkswirtschaft, die Struktur des Arbeitsangebots sowie kulturelle und andere gesellschaftiche Gegebenheiten verstanden. ${ }^{5}$ Diese Aufzählung macht deutlich, daß es sich bei diesem Begriff

4 Vgl. Abramovitz (1979, S. 199; 1986, S. 221ff.)

5 Vgl. Abramovitz (1979, S. 188; 1986, S. 222f.). 
um eine Catch-all-Variable in der Catching up-Hypothese handelt. Eine solch umfassende Definition ist nicht operational.

Ein erfolgreicher Catching up-Prozeß hängt nicht nur von der Fähigkeit ab, eine Technologie anzunehmen. Technologischer Wandel hat im allgemeinen auch sektorale und gesellschaftliche Umwälzungen zur Folge, d.h. die social capability muß auch die Bereitschaft beinhalten, bestehende Strukturen aufzubrechen und an ihre Stelle neue zu setzen. Der Begriff "Eurosklerose" sei hier nur als Beispiel genannt. Als Folge des technischen Fortschritts muß sich somit auch die social capability selbst verändern. Es ist somit durchaus möglich, daß der durch den technischen Fortschritt induzierte Wandel bzw. die angeregte Weiterentwicklung eines Landes ein neues bei der Technologieentwicklung führendes Land entstehen lassen, das die bisher führende Nation ablöst. ${ }^{6}$ Die Geschichte belegt diese Überlegung z.B. durch die Übernahme der Führungsposition Großbritanniens durch die USA. Aufgrund des rasanten Aufholprozesses Japans und der Bundesrepublik Deutschlands seit dem Zweiten Weltkrieg stellt sich die Frage, wie lange die Vereinigten Staaten ihre Stellung als wirtschaftlich fuhrendes Land behaupten können.

Die social capability eines Landes muß ein System von Institutionen und Fähigkeiten bilden, das die Übernahme des technologischen Know-hows der führenden Volkswirtschaft erlaubt. Notwendig ist ein Humankapitalbestand, der die Arbeitskräfte in die Lage versetzt, mit den neuesten Kapitalgütern umzugehen bzw. schnell ihre Bedienung zu erlernen. Ergänzend muß ein gesellschafticher Konsens bestehen, den notwendigen Wandel zu vollziehen. Darüber hinaus bedarf es eines zu den privaten Investitionen komplementären öffentlichen Kapitalstocks sowie Bedingungen, die eine rasche Diffusion von Wissen und Forschungsergebnissen ermöglichen und eine hohe Investitionstätigkeit anregen. ${ }^{7}$. Eine Volkswirtschaft wird erfolgreich sein, falls für sie folgende Aussage gilt:

"Countries that are technologically backward have a potentiality for generating growth more rapid than that of more advanced countries, provided their social capabilities are sufficiently developed to permit successful exploitation of technologies already employed by the technological leaders. The pace at which potential for catchup is actually realized in a particular period depends on factors limiting the diffusion of knowledge, the rate of structural change, the accumulation of capital, and the expansion of demand. The process of catching up tends to be self-limiting, but the strength of the tendency may be weakened or overcome, at least for limited periods, by advantages connected with the convergence of production patterns as followers advance towards leaders or by an endogenous enlargement of social capabilities."8

Neben den länderspezifischen Rahmenbedingungen sind für die Ausnutzung des Produktivitätspotentials auch die Beziehungen der Volkswirtschaften untereinander wichtig. Ohne Handel

6 Vgl. Abramovitz (1986, S. 224).

7 Vgl. Abramovitz (1989a, S. 2ff.).

8 Abramovitz (1986, S. 225). 
zwischen dem führenden und den nachfolgenden Ländern können die letzteren nicht von den Wissensvorsprüngen profitieren. Darüber hinaus müssen sich den unterentwickelten Volkswirtschaften Absatzchancen für die Produkte bieten, die sie mit der neuen Technologie herstellen können. Außenwirtschaftliche Beziehungen mit den Verfolgern bieten der führenden Volkswirtschaft auch Vorteile. Entwickeln sich international neue Produktionsstandorte, können z.B. entsprechend der Produktlebenszyklus-Hypothese von Vernon (1966) standardisierte Produkte nun von Ländern hergestellt werden, die geringere Kosten aufweisen. Den Käufern der Produkte kommt diese Entwicklung zugute und setzt andererseits in der führenden Volkswirtschaft Ressourcen frei, die in anderen produktiveren Verwendungen beschäftigt werden können. Dies setzt jedoch im führenden Land die Fähigkeit zum Wandel voraus. Können sich die Unternehmen nicht an die gewandelten Erfordernisse anpassen, kann Arbeitslosigkeit in der am weitesten fortgeschrittenen Volkswirtschaft die Folge des erfolgreichen Aufholprozesses sein. ${ }^{9}$ Die Beziehung zwischen dem Führenden und seinen Verfolgern beim Produktivitätsrennen ist einer der Inhalte der durch das Aufkommen südostasiatischer Produzenten angeregten Debatte über die Wettbewerbsfähigkeit von Volkswirtschaften. Einerseits ist es japanischen und später südkoreanischen Unternehmen gelungen, Güter, deren Herstellungsverfahren durch eine hohe Kapitalintensität gekennzeichnet sind, aufgrund ihrer Lohnkostenvorteile konkurrenzfähig auf den Weltmärkten anzubieten, während andererseits vor allem in den Volkswirtschaften Westeuropas sich das Problem einer zu geringen Zahl an neuentwickelten, marktfähigen Produkten manifestiert hat. Parallel hat sich auch die social capability in den aufholenden Ländern verbessert, so daß sich z.B. Japan in manchen Bereichen aufmacht, selbst zur führenden Volkswirtschaft aufzusteigen. 10

Ein erfolgreicher Aufholprozeß eines Landes mit geringem Produktivitätsniveau erfordert im Sinne der Catching up-Hypothese das Zusammenspiel zahlreicher Faktoren, die sich nicht ohne weiteres quantifizieren lassen und sich deswegen einer direkten ökonometrischen Überprüfung entziehen. Wie soll der Beitrag einer religiösen Weltanschauung, wie z.B. des Kalvinismus, auf die Entwicklung einer Volkswirtschaft formal erfaßt und berechnet werden?11 Der Begriff der social capability stellt deshalb gleichzeitig Vor- und Nachteil der Catching upHypothese dar. Einerseits ermöglicht er die Berücksichtigung von Faktoren, die für einen erfolgreichen Entwicklungsprozeß notwendig sind, andererseits aber verhindert er die Quantifizierung des Beitrags dieser Faktoren zum Wirtschaftswachstum. Darüber hinaus werden die exakten Anforderungen an den Aufbau einer potentiell erfolgreichen Volkswirtschaft nicht genauer spezifiziert. Wie sehen die Bedingungen aus, die eine schnelle Diffusion des Wissens

9 Vgl. z.B. Abramovitz (1986, S. 233).

$10 \mathrm{Vgl}$. zum japanischen EntwicklungsprozeB z.B. Ito (1992), Kosai (1988), Morishima (1982), Tsurata (1988), Tsuru (1993) und Uekusa (1988), und zum Entwicklungsprozeß Südkoreas z.B. Amsden (1989), Kim (1989), Chowdhury/Islam (1993), und Weltbank (1987).

11 In diesem Zusammenhang sind selbstverständlich die Arbeiten von Max Weber $(1904,1905) \mathrm{zu}$ diesem Thema zu nennen. Für eine zusătzliche Berücksichtigung asiatischer ethischer Vorstellungen vgl. z.B. McClelland (1961). 
zulassen? Welches sind die richtigen Anforderungsprofile beim Humankapitalstock? Für die Beantwortung dieser Fragen bleibt die Catching up-Hypothese in zu allgemeinen Konzepten verhaftet. Sie kann deshalb auf notwendige Rahmenbedingungen hinweisen, ihre exakte Ausgestaltung bedarf einer weitergehenden Untersuchung.

Die Catching up-Hypothese ist eng mit der in Kapitel 2 diskutierten Konvergenz zwischen Volkswirtschaften verwandt. Entsprechend dem neoklassischen Grundmodell konvergieren Volkswirtschaften zu ihrem Steady State. Weisen sie identische Parameterwerte auf, werden sich auch die Steady State-Werte entsprechen. Stellt der technische Fortschritt ein weltweit öffentliches Gut dar, wird es zu identischen Wachstumsraten der Pro-Kopf-Größen in allen Ökonomien kommen. Bei international mobilem Kapital, ist darüber hinaus denkbar, daß alle Volkswirtschaften zum gleichen Pro-Kopf-Output konvergieren. Die Catching up-Hypothese wiederum hat die Konvergenz der Produktivitätsniveaus zum Inhalt. Wenn ein Land die notwendige social capability besitzt, wird es ihm gelingen, zur führenden Volkswirtschaft aufzuschließen. Sowohl die neoklassischen Modellüberlegungen als auch die mehr wirtschaftshistorisch orientierte Herangehensweise im Rahmen der Catching up-Hypothese kommen bei internationaler Kapitalmobilität zum selben Ergebnis. Während jedoch die mathematischformale Methode der neoklassischen Wachstumstheorie auf starke Vereinfachungen angewiesen ist, erlaubt die Catching up-Hypothese die Einbeziehung vieler Faktoren, die der formalen Methode verschlossen bleiben. Die Existenz dieser Faktoren ist mit für das potentielle Scheitern der neoklassischen Aussagen an der Realität verantwortlich. Die Überlegungen der Catching up-Hypothese können in diesem Zusammenhang als eine Art Brainstorming und Briefing verstanden werden, die mehr Klarheit darüber verschaffen, welche Aspekte in die formalen Modelle zu integrieren sind. Die späteren Abschnitte über die Neue Wachstumstheorie werden zeigen, auf welchen Wegen dies der Fall sein kann. 12

\subsubsection{Empirische Ergebnisse zur Konvergenz- und Catching up-Hypothese}

Die sehr weite Fassung des Begriffs der social capability macht eine umfassende Überprüfung der Catching up-Hypothese sehr schwierig. Die formal orientierten Ansätze der Neoklassik geben mehr Hinweise auf überprüfbare Zusammenhänge. Erste Belege auf Catching upProzesse kann ein Blick auf die Wachstumsraten des Pro-Kopf-Einkommens oder der Arbeitsproduktivităt geben. Die Entwicklung beider Variablen muß nicht unbedingt gleich verlaufen. Die Arbeitsproduktivität kann z.B. sehr stark ansteigen, während das Pro-Kopf-Einkommen beinahe stagniert. Der Grund liegt in unterschiedlichen Erwerbsquoten. Nur wenn diese über

12 Die Diskussion um Catching up-Phămonene hat jedoch nicht nur innerhalb der neoklassischen Theorie zur Entwicklung neuerer Ansătze gefüht, sondern es wurden auch Modelle vorgestellt, die evolutionäre Vorstellungen in die Wachstumstheorie integrieren. Vgl. z.B. Soete/Verspagen (1994), Dosi/Freeman/Fabiani/Aversi (1994), Verspagen (1994), Dosi/Fabiani (1994), Nelson (1995). 
die Zeit hinweg konstant sind, laufen beide Indikatoren gleich. Für den Wohlstand einer Bevölkerung ist das Pro-Kopf-Einkommen relevant, da es Auskunf über die für den einzelnen zur Verfügung stehende Gütermenge gibt. ${ }^{13}$ Betrachtet man die Daten über einen längeren Zeitraum hinweg, zeigen sich internationale Unterschiede bei den Wachstumsraten des Pro-KopfEinkommens gemessen als Bruttoinlandsprodukt pro Einwohner. Die Verteilung der Wachstumsraten zeigt jedoch eher die industrialisierten als die armen Länder an der Spitze. Erstellt man eine Rangliste für die Zeit zwischen 1950-1973 und 1973-1989 ergibt sich folgende Reihenfolge.

Tab. 3.1-A Wachstumsraten des Bruttoinlandsprodukts pro Kopf 1950-1973

\begin{tabular}{|c|l|c|c|l|c|}
\hline Rang & \multicolumn{1}{|c|}{ Land } & $\begin{array}{c}\text { Wachstums- } \\
\text { rate } \\
\mathbf{1 9 5 0 - 1 9 7 3}\end{array}$ & Rang & \multicolumn{1}{|c|}{ Land } & $\begin{array}{c}\text { Wachstums- } \\
\text { rate } \\
\mathbf{1 9 5 0 - 1 9 7 3}\end{array}$ \\
\hline 1 & Japan & $\mathbf{8 , 0}$ & 18 & Dänemark & 3,1 \\
\hline 2 & Griechenland & 6,2 & 19 & Irland & 3,1 \\
\hline 3 & Taiwan & 6,2 & 20 & Mexiko & 3,1 \\
\hline 4 & Portugal & 5,6 & 21 & Schweden & 3,1 \\
\hline 5 & Korea & 5,2 & 22 & Kanada & 2,9 \\
\hline 6 & Spanien & 5,1 & 23 & Peru & 2,5 \\
\hline 7 & Deutschland & 5,0 & 24 & Großbritannien & 2,5 \\
\hline 8 & Italien & 5,0 & 25 & Australien & 2,4 \\
\hline 9 & Österreich & 4,9 & 26 & USA & 2,2 \\
\hline 10 & Finnland & 4,3 & 27 & Argentinien & 2,1 \\
\hline 11 & Frankreich & 4,0 & 28 & Kolumbien & 2,1 \\
\hline 12 & Brasilien & 3,8 & 29 & Indonesien & 2,1 \\
\hline 13 & China & 3,7 & 30 & Afrika & 1,9 \\
\hline 14 & Belgien & 3,5 & 31 & Pakistan & 1,8 \\
\hline 15 & Niederlande & 3,4 & 32 & Indien & 1,6 \\
\hline 16 & Norwegen & 3,2 & 33 & Chile & 1,2 \\
\hline 17 & Thailand & 3,2 & 34 & Bangladesch & $-0,7$ \\
\hline
\end{tabular}

Quelle: Maddison (1994, S. 26).

Der Zeitraum 1950-1973 war für alle Volkswirtschaften der Stichprobe durch hohe Wachstumsraten des Pro-Kopf-Einkommens gekennzeichnet. Gegenüber den USA konnten sämtliche anderen OECD-Länder sowie einige lateinamerikanische und asiatische Länder höhere Wachstumsraten aufweisen. Volkswirtschaften mit großen Produktivitätsrückständen fielen sogar in dieser Zeit des Aufschwungs zurück. Die vorderen Positionen innerhalb dieser Reihen-

13 Natürlich gibt dieses Maß keinerlei Auskunft über die Verteilung des Wohlstands. 
folge für Länder wie Japan, Korea, Griechenland oder Portugal scheinen eine gewisse Bestätigung furr Catching up-Hypothese zu liefern. In der Zeit nach 1973 bis zum Beginn der politischen Umbrüche in Osteuropa und der damit einhergehenden Veränderung der politischen und wirtschaftlichen Weltordnung, haben sich zwar die Wachstumsraten des Pro-KopfEinkommens weltweit verringert, die Zahl der Länder, die relativ zu den USA höhere Wachstumsraten zu verzeichnen hatten, ist gering gestiegen. Dieses Phänomen muß jedoch nicht zwingend einen wesentlich gestiegenen Lebensstandard für die Aufholländer bedeuten. Tatsächlich hat sich auch das Produktivitätsniveau in den USA nicht sehr stark erhöht. Der auf den ersten Blick vorliegende Catching up-Prozeß kann seine Gründe auch in der Schwäche der führenden Volkswirtschaft haben.

Tab. 3.1-B Wachstumsraten des Bruttoinlandsprodukts pro Kopf 1973-1989

\begin{tabular}{|c|l|c|c|l|c|}
\hline Rang & Land & $\begin{array}{c}\text { Wachstums- } \\
\text { rate } \\
1973-1989\end{array}$ & Rang & Land & $\begin{array}{c}\text { Wachstums- } \\
\text { rate } \\
1973-1989\end{array}$ \\
\hline 1 & Korea & 6,4 & 18 & Deutschland & 1,9 \\
\hline 2 & Taiwan & 6,1 & 19 & Großbritannien & 1,9 \\
\hline 3 & China & 5,7 & 20 & Spanien & 1,8 \\
\hline 4 & Thailand & 5,2 & 21 & Kolumbien & 1,8 \\
\hline 5 & Indonesien & 3,4 & 22 & Dänemark & 1,7 \\
\hline 6 & Norwegen & 3,1 & 23 & Schweden & 1,7 \\
\hline 7 & Japan & 3,0 & 24 & Australien & 1,7 \\
\hline 8 & Irland & 2,9 & 25 & Griechenland & 1,7 \\
\hline 9 & Finnland & 2,8 & 26 & Portugal & 1,7 \\
\hline 10 & Pakistan & 2,8 & 27 & Brasilien & 1,7 \\
\hline 11 & Indien & 2,7 & 28 & USA & 1,6 \\
\hline 12 & Italien & 2,6 & 29 & Chile & 1,5 \\
\hline 13 & Kanada & 2,4 & 30 & Niederlande & 1,3 \\
\hline 14 & Österreich & 2,3 & 31 & Mexiko & 1,0 \\
\hline 15 & Bangladesch & 2,2 & 32 & Afrika & $-0,3$ \\
\hline 16 & Belgien & 2,0 & 33 & Argentinien & $-1,2$ \\
\hline 17 & Frankreich & 1,9 & 34 & Peru & $-1,2$ \\
\hline
\end{tabular}

Quelle: Maddison (1994, S. 26).

Ein erster einfacher Test, ob ein Aufholprozeß bei der Arbeitsproduktivität zwischen den verschiedenen Ländern feststellbar ist, besteht in der Ermittlung des Mittelwertes der Produktivitätsniveaus bezogen auf die führende Volkswirtschaft innerhalb einer Stichprobe von Ländern zu zwei ausgesuchten Zeitpunkten. Steigt der Mittelwert an, ist dies ein erster Hinweis darauf, 
daß die Verfolgerstaaten ihre Position verbessern konnten. Zusätzlich kann der Variationskoeffizient als weiterer Indikator verwendet werden. Ein steigender Mittelwert bei gleichzeitiger Abnahme des Variationskoeffizienten deutet auf einen erfolgreichen Catching upProzeß bei gleichzeitiger Annäherung der Länder beim Produktivitätsniveau untereinander hin. Führt man eine solche Berechnung für die Länder aus Tabelle (3.1) im Zeitraum 1950-1989 durch, ergibt sich ein Ansteigen des Mittelwertes, aber auch eine Zunahme der Standardabweichung. Der Variationskoeffizient blieb in etwa konstant. Betrachtet man nur die Volkswirtschaften, die 1950 ein überdurchschnittliches relatives Pro-Kopf-Einkommen (in Tab. 3.2A mit Stern) hatten, zeigt sich ein Ansteigen des Mittelwerts und der Standardabweichung, während der Variationskoeffizient sank. Nimmt man aus dieser Gruppe den "Ausreißer" Argentinien heraus, ergibt sich genau das erwartete Resultat: Zunahme des Mittelwertes bei gleichzeitigem Rückgang der Streuungsmaße. Diese Ländergruppe realisierte einen Catching up-Prozeß gegenüber den USA. ${ }^{14}$

Tab. 3.2-A Relatives Pro-Kopf-Einkommen 1950 und 1989 (USA=100)

\begin{tabular}{|l|c|c|l|c|c|}
\hline \multicolumn{1}{|c|}{ Land } & $\begin{array}{c}\text { Relatives } \\
\text { Pro-Kopf- } \\
\text { Einkommen } \\
\mathbf{1 9 5 0}\end{array}$ & $\begin{array}{c}\text { Relatives } \\
\text { Pro-Kopf- } \\
\text { Einkommen } \\
\mathbf{1 9 8 9}\end{array}$ & \multicolumn{1}{|c|}{ Land } & $\begin{array}{c}\text { Relatives } \\
\text { Pro-Kopf- } \\
\text { Einkommen } \\
\mathbf{1 9 5 0}\end{array}$ & $\begin{array}{c}\text { Relatives } \\
\text { Pro-Kopf- } \\
\text { Einkommen } \\
1989\end{array}$ \\
\hline USA & 100,0 & 100,0 & Kolumbien & 21,8 & 21,8 \\
\hline Kanada* & 71,0 & 94,3 & Peru & 21,0 & 14,2 \\
\hline Australien* & 69,8 & 74,1 & Japan & 18,8 & 83,9 \\
\hline Schweden* & 65,9 & 81,2 & Portugal & 18,7 & 40,4 \\
\hline Großbritannien* & 65,7 & 73,9 & Mexiko & 18,5 & 17,9 \\
\hline Dänemark* & 60,7 & 75,6 & Griechenland & 16,9 & 41,4 \\
\hline Niederlande* & 54,7 & 69,3 & Brasilien & 16,7 & 24,1 \\
\hline Norwegen* & 52,6 & 83,1 & Afrika & 12,1 & 9,1 \\
\hline Belgien* & 49,1 & 70,4 & Thailand & 10,2 & 21,9 \\
\hline Frankreich* & 48,5 & 76,3 & Korea & 8,8 & 35,6 \\
\hline Finnland* & 40,5 & 76,7 & Taiwan & $\mathbf{8 , 2}$ & 39,7 \\
\hline Deutschland* & 38,3 & 75,2 & Indonesien & 7,6 & 9,8 \\
\hline Argentinien* & 36,2 & 22,3 & Pakistan & 6,3 & 7,0 \\
\hline Österreich* & 33,3 & 68,5 & Indien & 5,8 & 6,0 \\
\hline Italien* & 33,0 & 71,0 & Bangladesch & 5,4 & 3,0 \\
\hline Irland & 30,2 & 45,3 & Chile & 5,3 & 13,9 \\
\hline Spanien & 27,9 & 55,1 & China & 5,3 & 13,9 \\
\hline
\end{tabular}

Quelle: Maddison (1994, S. 26), eigene Berechnungen.

14 Die Resultate zeigen, wie sehr die Aussagen der Berechnung von der Wahl der Stichprobe abhăngen. Vgl. Tabelle 3.2-B. 
Tab. 3.2-B Mittelwert, Standardabweichung und Variationskoefíizient des relativen Pro-Kopf-Einkommens

\begin{tabular}{|c|c|c|c|}
\hline Jahr & Mittelwert & $\begin{array}{c}\text { Standard- } \\
\text { abweichung }\end{array}$ & $\begin{array}{c}\text { Variations- } \\
\text { koefirient }\end{array}$ \\
\hline 1950 & 30,8 & 20,8 & 0,66 \\
\hline 1989 & 46,4 & 28,6 & 0,62 \\
\hline 1950 & 51,4 & 13,2 & 0,26 \\
\hline 1989 & 72,3 & 15,3 & 0,21 \\
\hline $1950^{*}$ & 52,5 & 13,0 & 0,25 \\
\hline $1989^{*}$ & 76,1 & 6,7 & 0,09 \\
\hline
\end{tabular}

Quelle: eigene Berechnungen.

Da die Catching up-Hypothese einen Zusammenhang zwischen dem anfänglichen Produktivitätsniveau $\left(\mathrm{P}_{0}\right)$ und der potentiellen Wachstumsrate der Produktivität $\left(\mathrm{p}_{\mathrm{pot}}\right)$ behauptet, ist der Rangkorrelationskoeffizient zwischen $\left(\mathrm{P}_{0}\right)$ und der tatsächlichen Wachstumsrate der Produktivität $\left(\mathrm{p}_{\mathrm{tat}}\right)$ ein anderes $\mathrm{Maß}$ zur Überprüfung dieser These. Ein Wert dieses Koeffizienten nahe minus Eins legt den Schluß nahe, daß die Aussage der Catching up-Hypothese bestätigt werden kann. Tabelle (3.3) zeigt die Ergebnisse einer derartigen Berechnung von Abramovitz.

Tab. 3.3: Rangkorrelation zwischen anfänglichen Produktivitätsniveaus und den Produktivitätswachstumsraten 1950-1986

\begin{tabular}{|l|c|}
\hline Zeitraum & Rangkorrelationskoeffizient \\
\hline $1950-1979^{*}$ & $-0,92$ \\
\hline $1950-1960^{*}$ & $-0,81$ \\
\hline $1960-1973^{*}$ & $-0,90$ \\
\hline $1973-1979^{*}$ & $-0,13$ \\
\hline $1950-1973^{*}$ & $-0,96$ \\
\hline $1973-1986^{\#}$ & $-0,53$ \\
\hline
\end{tabular}

15 Die Produktivitat wurde auf Stundenbasis ermittelt. Die Ursprungsdaten stammen von Maddison (1982 und 1989), wobei die Stichprobe die Lănder Australien, Belgien, Bundesrepublik Deutschland, Dănemark, Frankreich, Finnland, Großbritannien, Italien, Japan, Niederlande, Norwegen, Schweden, Schweiz, Osterreich und die USA umfaBte. In den Berechnungen von Abramovitz (1994) ist die Schweiz nicht enthalten. 
Dieses Ergebnis weist insbesondere im Zeitraum von 1960-1973 auf die Ausnutzung der vorhandenen Wachstumspotentiale in den ausgesuchten Volkswirtschaften hin und scheint auf den ersten Blick die Gültigkeit der Catching up-Hypothese zu beweisen.

Im Kapitel zur traditionellen neoklassischen Theorie wurde zwischen der sog. absoluten und der bedingten Konvergenz unterschieden, wobei unter absoluter Konvergenz das schnellere Wachstum des Pro-Kopf-Einkommens ärmerer gegenüber reicheren Volkswirtschaften verstanden wird, während der Begriff der bedingten Konvergenz für die Überlegung steht, daß die Wachstumsrate einer Volkswirtschaft mit dem Abstand von ihrem Steady State korreliert ist. Das Vorliegen dieser Arten von Konvergenz bedeutet nicht die Annäherung der Pro-Kopf-Einkommen auf absolutem Niveau, sondern sie sind dafür nur notwendige, keine hinreichende Bedingungen. ${ }^{16}$

Eine Möglichkeit Konvergenz zu überprüfen, besteht in der Durchführung einer Regressionsrechnung zwischen dem anfänglichen logarithmierten Pro-Kopf-Einkommen der betrachteten Volkswirtschaften und ihren durchschnittlichen Wachstumsraten des Pro-KopfEinkommens im Betrachtungszeitraum:

$$
\hat{y}_{i, t, t+T}=\alpha-\beta \log y_{i, t}+u_{i, t, t+T}
$$

Kann nachgewiesen werden, daß $(\beta)$ statistisch signifikant von Null verschieden ist, kann auf absolute Konvergenz geschlossen werden.

Unter Verwendung dieser Methode zeigen Untersuchungen, daß nur innerhalb einer Gruppe von Volkswirtschaften, die annähernd identische Strukturen aufweisen, dieses Phänomen feststellbar ist. Erste Studien von Baumol (1986) legten zwar den Schluß nahe, daß Volkswirtschaften mit geringem Pro-Kopf-Output schneller wachsen. Dieses Resultat ist jedoch in hohem Maße von der getroffenen Stichprobe abhängig. Baumol hatte sich nur auf erfolgreiche Volkswirtschaften konzentriert. Daran anschließende Untersuchungen wiesen auf das Vorliegen sog. Konvergenz-Clubs hin. Hierunter wird eine Gruppe von Volkswirtschaften verstanden, innerhalb der es zu Konvergenzprozessen kommt. ${ }^{17}$

Bedingte Konvergenz und Konvergenz-Clubs stehen in enger Beziehung zueinander. Volkswirtschaften, die sich in vielen Variablen sehr ähnlich sind, wie z.B. dem Bildungsniveau, dem Kapitalstock, der sektoralen Struktur oder dem technologischen Wissen, werden u.U. auch ähnliche Steady States aufweisen. Bezieht man nur solche Länder in eine Stichprobe zur Abschätzung von Konvergenzprozessen ein, müßten sich statistisch signifikante Ergebnisse für $(\beta)$ ergeben. Untersuchungen zur Konvergenz sollten sich deshalb auf homogene Länder

16 Vgl. Sala-i-Martin (1996, S. 1020ff.).

17 Vgl. z.B. Baumol/Wolff (1988), Baumol/Blackman/Wolff (1989, Kap. 5), Baumol/Nelson/Wolff (1994) und DeLong (1988). 
konzentrieren und nicht versuchen, eine möglichst große Zahl an Volkswirtschaften zu berücksichtigen.

Beispiele für diese Vorgehensweise finden sich bei Barro/Sala-i-Martin (1992, 1995), die für 48 US-Bundesstaaten, japanische Präfekturen und europäische Regionen Regressionsrechnungen durchführten. ${ }^{18}$ Tatsächlich ergaben sich für den gesamten Betrachtungszeitraum bei allen Stichproben statistisch signifikante, von Null verschiedene Werte für $(\beta)$. Trotz der Unterschiede zwischen den betrachteten Stichproben stellten die Autoren zwei auffälige Ergebnisse fest. Erstens war die Geschwindigkeit der Anpassung nicht konstant. Teilt man die Zeiträume in Zehn-Jahres-Schritten auf, schwanken die Werte für den Regressionskoeffizienten. In der amerikanischen und der japanischen Stichprobe tauchen sogar Zeiträume auf, in denen eine negative Entwicklung bei der Konvergenz auftritt. Der Annäherungsprozeß der Regionen unterliegt damit Schwankungen. Zweitens ermittelten Barro/Sala-i-Martin für die drei Stichproben ( $\beta$ )-Werte, die für den kompletten Zeitraum und für die meisten Teilperioden um einen Wert von ca. $2 \%$ schwanken. Dies legt den Schluß nahe, daß dem Konvergenzprozeß in allen Regionen ähnliche Gesetzmäßigkeiten zugrunde liegen.

Sala-i-Martin (1996) referiert Resultate für 110 Länder. ${ }^{19}$ Hier konnte für den Zeitraum 1960-1990 keinerlei absolute Konvergenz festgestellt werden. Vielmehr ergab sich ein negativer Wert für den Regressionskoeffizienten. Wird jedoch das Konzept der bedingten Konvergenz angewandt, ändert sich das Ergebnis. $\mathrm{Da}$ in einer Stichprobe von 110 Ländern nicht von ähnlichen Volkswirtschaften ausgegangen werden kann, muß eine "Korrektur" der Daten durchgeführt werden, die die Steady States angleicht. Aus diesem Grunde muß eine Reihe von zusätzlichen Variablen eingeführt werden, die charakteristisch für eine Steady State Situation sind. Werden diese konstant gehalten, muß sich für $(\beta)$ ein positiver Wert ergeben. Bei Modifizierung der Daten auf diese Art und Weise wurde wieder das erhoffe Ergebnis erreicht. Es lag bedingte Konvergenz vor, da ( $\beta$ ) signifikant größer Null war. ${ }^{20}$

Die diversen empirischen Ergebnisse zur Konvergenz lassen den Schluß zu, daß zumindest innerhalb einer Gruppe von Ländern eine negative Beziehung zwischen dem anfänglichen ProKopf-Einkommen und dessen Wachstumsrate besteht. Dies bedeutet jedoch nicht zwangsläufig die Angleichung der Pro-Kopf-Einkommen, sondern stellt nur eine notwendige Bedingung für diese dar. Tatsächliches Catching up liegt jedoch nur vor, falls sich auch die Pro-KopfEinkommen angleichen. Hierauf deuten die Ergebnisse von Abramovitz und Maddison hin. Die

18 Der Betrachtungszeitraum betrug für die US-Bundesstaaten 1880-1990, für die japanischen Prăfekturen 1930-1990 und für die europăischen Regionen 1950-1990. Die geschătzte Regressionsgleichung hatte folgendes Aussehen:

$\frac{1}{T} \ln \frac{y_{i, t}+T}{y_{i, t}}=\alpha-\frac{1-e^{-\beta t}}{T} \ln y_{i, t}+u_{i, t, t+T}$

Diese Gleichung kann aus der traditionellen neoklassischen Produktionsfunktion abgeleitet werden. Vgl. Barro/Sala-i-Martin (1995, S. 80ff. und S. 383ff.).

19 Er arbeitet mit derselben Schătzgleichung wie zuvor Barro/Sala-i-Martin (1992a, 1995).

20 Vgl. Sala-i-Martin (1996, S. 1028). 
Catching up- und die Konvergenzhypothese müssen somit im Hinblick auf ihre Inhalte unterschieden werden. Empirisch besitzen sie aber beide nur für Gruppen von Ländern und nicht für alle Volkswirtschaften gleichzeitig Gültigkeit. Aus diesem Grunde bleibt weiterhin die Notwendigkeit einer Theorie bestehen, die sowohl Aufholprozesse als auch DivergenzKonvergenz-Phänomene erklären kann.

\subsection{Der Weg zu endogenem Wachstum}

\subsubsection{Investitionen - Lernen - Externalitäten}

Das zweite Faktum, das nach Auffassung von P. Romer in einem wachstumstheoretischen Modell enthalten sein soll, ist die Nichtrivalität des technischen Know-hows. ${ }^{21}$ Der exogene technische Fortschritt innerhalb des neoklassischen Grundmodells erfüllte diese Eigenschaft vollständig. Das technologische Wissen der Volkswirtschaft wächst zeitabhängig mit einer konstanten Rate und erhöht die Effizienz der Produktion ohne zusätzliche Kosten. Der technische Fortschritt ist in diesem Falle ein rein öffentliches Gut. Die damit verbundenen kostenlosen Produktivitätssteigerungen wirken den abnehmenden Grenzproduktivităten bei zunehmender Kapitalintensivierung entgegen und halten damit den Anreiz zur Investition aufrecht. Dauerhaftes Wachstum des Pro-Kopf-Outputs wird ermöglicht.

Neben öffentlichen Gütern können weitere kostenlose Faktoren die Effizienz der Produktion steigern. Wenn innerhalb einer Volkswirtschaft Externalitäten auftreten, die von der Handlung eines Wirtschaftssubjekts auf die Nutzen- oder Produktionsfunktion eines anderen wirken, wird das Verhältnis zwischen den Inputs und dem Output verändert. Der bekannteste Fall sind mit Sicherheit negative Externalitäten der Produktion, die bei einem Unternehmen A entstehen (z.B. Wasserverschmutzung) und in Folge die Kosten eines anderen Unternehmens B erhöhen. Unternehmen B muß für dasselbe Produktionsresultat mehr Inputs aufwenden als ohne die Externalität. Externe Effekte sind auf vielerlei Arten denkbar. Es wird darüber hinaus in pekuniäre und technologische externe Effekte unterschieden. Pekuniäre Externalitäten sind Preiseffekte, die sich zwischen Unternehmen ergeben, wenn ein Unternehmen z.B. mehr Inputs verwendet und damit die Kosten für andere Unternehmen verändert. Technologische Externalitäten werden dagegen nicht über das Preissystem erfaßt, d.h. sie liegen vor, wenn sich nicht alle sozialen Grenzkosten bzw. Grenzerträge im Marktpreis wiederfinden. Beide Arten von externen Effekten können Konsequenzen für den Wachstums-

21 Vgl. oben S. 65. 
prozeß von Volkswirtschaften haben, da sie die Kosten- und Ertragssituation von Firmen und damit die Grenzproduktivität des investierten Kapitals beeinflussen. ${ }^{22}$

Innerhalb der Neuen Wachstumstheorie hat im Hinblick auf die Wirkungen externer Effekte insbesondere eine Überlegung an Einfluß gewonnen, die mit dem Konzept des learning by doing von Kenneth Arrow (1962) verbunden ist. Arrow wollte eine endogene Theorie des technischen Fortschritts aufstellen, da die alleinige Abhängigkeit des Pro-Kopf-Wachstums von diesem Faktor in der neoklassischen Wachstumstheorie der fünfziger und sechziger Jahre sowohl aus intellektuellen als auch aus empirischen Gründen unbefriedigend sei:

"Nevertheless a view of economic growth that depends so heavily on an exogenous variable, let alone one so difficult to measure as the quantity of knowledge, is hardly satisfactory. From a quantitative, empirical view, we are let with time as an explanatory variable. Now trend projections, however necessary they may be in practice, are basically a confession of ignorance, and, what is worse from a practical viewpoint, are not policy variables. 23

Den Ausgangspunkt stellten in Arrows Überlegungen die Veränderungen des Wissens dar. Wirtschaftssubjekte eignen sich Wissen durch Lernen an, wobei letzteres zu einem gewissen Teil eine Folge von gesammelten Erfahrungen und erst möglich ist, wenn Probleme gelöst werden müssen. Für Arrow kann Lemen nur während der Ausübung von Aktivitäten geschehen. Aus diesem Grunde auch der Begriff learning by doing. ${ }^{24}$

Eine Möglichkeit zu Lernen tritt bei der wiederholten Durchführung einer Tätigkeit auf. Je häufiger eine bestimmte Handlung erfolgt, desto leichter fallt sie. Säuglinge und Kleinkinder lernen auf diese Art und Weise und den Erwachsenen macht z.B. die Zeit in der Fahrschule diesen Aspekt wieder deutlich. Bei dieser Form der Wissensaneignung zeigt sich aber auch, daß die erzielten Fortschritte immer mehr abnehmen. Lernen unterliegt abnehmenden Grenzerträgen. Will man dauerhaft Fortschritte erreichen, müssen ständig neue Situationen geschaffen werden, die Lernprozesse ermöglichen. ${ }^{25}$ Ähnlich wie der technische Fortschritt im neoklassischen Grundmodell die abnehmende Grenzproduktivität des Kapitals bei anhaltenden Investitionen verhindert, muß bei der Akkumulation von Wissen ständig ein Faktor vorhanden sein, der den Anreiz zur "Investition" aufrechterhält. Zur Verdeutlichung der Relevanz seiner Aussagen über die Zusammenhänge zwischen Lernen und Erfahrung führt Arrow das Konzept der Lernkurve an, die z.B. in der Produktion von Flugzeugen beobachtet werden konnte. Die Zahl der zur Herstellung eines Flugzeugrahmens notwendigen Arbeitsstunden war negativ von der Zahl der bisher produzierten Einheiten abhängig. Die Beziehung zwischen einzusetzender

$22 \mathrm{Vgl}$. zur Unterscheidung in pekuniäre und technologische externe Effekte Viner (1932), Meade (1952) und Scitovsky (1954). Die Differenzierung zwischen diesen beiden Arten externer Effekte spielt insbesondere fur die Frage wirtschaftspolitischer Implikationen eine Rolle. Vgl. Kapitel 5.

23 Arrow (1962, S. 155).

24 Vgl. Arrow (1962, S. 155).

25 ebenda (S. 155f.), 
Arbeit (L) und gefertigten Stückzahlen $(N)$ zeigte dabei einen hyperbolischen Verlauf, d.h. es liegen abnehmende Grenzproduktivitäten bei steigenden Stückzahlen vor:

$$
\mathrm{L}=\mathrm{N}^{-1 / 3}
$$

Einen weiteren Beleg für diese Überlegungen lieferten Beobachtungen in den schwedischen Horndahlwerken, wo während eines Zeitraums von 15 Jahren die Arbeitsproduktivität pro Jahr um $2 \%$ zunahm, ohne daß investiert wurde. Allein aufgrund der gewonnenen Erfahrungen der Arbeitskräfte stieg die Produktivität an. 26

Produktivitätssteigerungen bedingt durch die Repetition einer Handlung sind eng mit den potentiellen Zunahmen der Arbeitsproduktivität durch eine sich intensivierende Arbeitsteilung verwandt. Das bekannte Beispiel der Nadelherstellung bei A. Smith macht dies deutlich. ${ }^{27}$ Die Unterteilung eines Produktionsprozesses in einzelne Schritte hat positive Effekte auf die Effizienz, da ein und dieselbe Tätigkeit wiederholt wird und somit die Realisierung von Lerneffekten erlaubt. Tatsächlich aber sind diese Wirkungen der Arbeitsteilung begrenzt, wie die Diskussion über den Taylorismus gezeigt hat. Darüber hinaus gewinnen Transaktionskosten eine steigenden Bedeutung bei zunehmender Arbeitsteilung. ${ }^{28}$

Aufbauend auf diesen Überlegungen entwickelt Arrow ein Modell, das die produktivitätssteigernden Effekte des learning by doing abbilden und die Folgen für die Wachstumsrate des Systems verdeutlichen soll. Eine zentrale Rolle spielt hierbei die Funktion, welche die Lerneffekte abbilden soll. Entgegen der naheliegenden Wahl des Outputs, besser gesagt des kumulierten Outputs, wählt Arrow die Summe der bisher getätigten Bruttoinvestitionen als Maß für die gewonnenen Erfahrungen. Unterstellt man Identität von Investitionen und gesamtwirtschaftichem Sparen, kann das Konzept des kumulierten Outputs in das der kumulierten Bruttoinvestitionen überführt werden. Es muß dazu nur wie im neoklassischen Grundmodell die Annahme einer dauerhaft konstanten Sparquote getroffen werden. Die Summe der kumulierten Bruttoinvestitionen entspricht in diesem Fall einem konstanten Anteil des kumulierten Outputs. ${ }^{29}$

Die Verwendung der kumulierten Bruttoinvestitionen hat jedoch zwei Vorteile gegenüber der Outputgröße. Zum einen enthalten die Investitionen, die zu einem bestimmten Zeitpunkt getätigt werden, den neuesten Stand des Wissens, d.h. Arrows Modell steht in der Tradition

26 ebenda ( $S .156)$.

27 Vgl. Smith (I.i., [1993, S. 11ff.]).

28 Das Phănomen der Arbeitsteilung hat nicht nur Folgen für die Produktionsprozesse, sondern berührt auch andere soziale Beziehungen. Nimmt aufgrund steigender Spezialisierung das Wissen des einzelnen Individuums zu, ergeben sich Konsequenzen für die Kommunikation zwischen Individuen mit unterschiedlichen Wissensstănden. Vertrauen in die Richtigkeit der Aussagen des anderen muß entstehen, da es für einen Menschen alleine nicht mehr moglich ist, alles zu wissen. Vgl. hierzu z.B. Arrow (1979, S. 77ff.). Weitere interessante Aspekte bieten auch Marschak/Radner (1972).

29 Vgl. zu dieser Überlegung z.B. Hacche (1979, S. 12 und S. 143). 
der Vintage-Ansätze. Zum anderen schaffen Investitionen neue Rahmenbedingungen, d.h. der oben angesprochene notwendige Impuls, der Lernen aufrechterhält, ist damit gesichert. ${ }^{30}$ Investitionen weisen somit insgesamt drei Wirkungen auf. Erstens den aus der keynesianischen Analyse bekannten Einkommenseffekt, zweitens den bei der traditionellen Wachstumstheorie besprochenen Kapazitätseffekt und drittens den Produktivitätseffekt im Sinne des Arrowschen Modells. Letzterer entsteht durch das inkorporierte Wissen und durch die Schaffung neuer Lernimpulse.

Um sich ausschließlich auf die Effekte des Lernens zu konzentrieren, wählt Arrow eine jahrgangsspezifische Produktionstechnik mit fixen Koeffizienten. Das Einsatzverhältnis zwischen Arbeit und Kapital ist festgelegt und kann nicht verändert werden, d.h. es liegt ein Clay-Clay-Fall vor. Neue Maschinen benötigen jedoch für dieselbe Outputmenge immer weniger, höchstens gleich viel an Arbeit, als es ältere tun. Im weiteren wird unter (G) die Summe der kumulierten Bruttoinvestitionen der Volkswirtschaft verstanden, die bis dato getätigt wurden. (G) steht auch für die Seriennummer der letzten Investitionen. Unterstellt man technischen Verschleiß, werden innerhalb der Ökonomie zu einem bestimmten Zeitpunkt nicht mehr alle bisher investierten Kapitalgüter im Produktionsprozeß enthalten sein.

Der gesamtwirtschaftliche Output ergibt sich aus der Summe der mit diesen Maschinen produzierten Mengen:

$$
Y=\int_{G^{\prime}}^{G} f(G) d G \quad \text { wobei ein Spezialfall ist: } \quad f(G)=a
$$

Hierbei ist $\mathrm{G}-\mathrm{G}^{\prime}=\mathrm{T}$ die technische Lebensdauer der Maschinen, die konstant sein soll. ${ }^{31}$

Der gesamte Arbeitsbedarf läßt sich aus (3.3) ermitteln:

$$
L=\int_{G^{\prime}}^{G} h(G) d G \quad \text { wobei ein Spezialfall ist: } h(G)=b G^{-n} \text { mit } 0<n<1
$$

Die Parameter (b) und (n) sind konstant und folgen den empirischen Ergebnissen wie in (3.1). Verwendet man im weiteren Verlauf die jeweils bei (3.2) und (3.3) angeführten Spezialfälle kommt man zu:

$$
Y=\int_{G^{\prime}}^{G} \operatorname{adG}=a G-a G^{\prime}
$$


und für die Arbeitsmenge

$$
L=\int_{G^{\prime}}^{G} b G^{-n} d G=\frac{b}{1-n}\left(G^{1-n}-G^{(1-n)}\right) .
$$

Man kann nun $\left(G^{\prime}\right)$ in $(Y)$ durch die Lösung für die Arbeitsmenge bestimmen.

$$
G^{\prime}=\left(G^{1-n}-\frac{L(1-n)}{b}\right)^{1 /(1-n)}
$$

Eingesetzt in (3.4) folgt: 32

$$
Y=a G-a\left[G^{1-n}-\frac{L(1-n)}{b}\right]^{1 /(1-n)}=a G-a G\left[1-\frac{L(1-n)}{b G^{1-n}}\right]^{1 /(1-n)}
$$

Diese Produktionsfunktion weist steigende Skalenerträge auf. Multipliziert man die Einsatzmengen der Produktionsfaktoren mit $(\lambda)$, kommt man zu:

$$
Y_{\lambda}=a \lambda G\left(1-\left[1-\frac{\lambda}{\lambda^{1-n}} \frac{L(1-n)}{b G^{1-n}}\right]^{1 /(1-n)}\right)
$$

bzw.

$$
Y_{\lambda}=\lambda\left(\mathrm{aG}-\mathrm{aG}\left[1-\lambda^{\mathrm{n}} \frac{\mathrm{L}(1-\mathrm{n})}{\mathrm{bG}}\right]^{1 /(1-n)}\right)
$$

Um nun beurteilen zu können, ob konstante oder steigende Skalenerträge vorliegen, muß überlegt werden, wie sich der Wert des Terms in der eckigen Klammer bei einer proportionalen Faktorvariation verändert. Ohne den Faktor $\left(\lambda^{n}\right)$ würde der Klammerwert dem ohne Faktorvariation entsprechen. Die Produktionsfunktion hätte konstante Skalenerträge. Da $\lambda^{n>1}$ ist, wird der Term in der eckigen Klammer durch die proportionale Erhöhung des Faktoreinsatzes kleiner, d.h. von (aG) wird weniger abgezogen. Insgesamt ist somit der Wert in der runden Klammer größer als vor der Faktorerhöhung. Die Produktionsfunktion unterliegt steigenden Skalenerträgen. Diese Art der Skalenerträge sind dynamischer Art, d.h. sie entstehen durch Veränderungen des Wissens, z.B. Lemprozesse und nicht durch die Ausnutzung von Größenvorteilen, wie sie z.B. bei hohen Fixkostenanteilen in der Produktion entstehen können.

32 Diese Gleichung entspricht der Produktionsfunktion im Beitrag von Arrow (1962, S. 159). Die Ableitung dieser Gleichung wurde hier etwas anders durchgeführt. Vgl. auch Levhari (1966a, S. 31). 
Die produktivitätssteigernden Effekte des learning by doings bedingen steigende Skalenerträge. Im weiteren Verlauf seiner Überlegungen zeigt Arrow, daß bei rationalen Erwartungen und der Annahme von Vollbeschäftigung im Wettbewerbsgleichgewicht der Output und die kumulierten Bruttoinvestitionen bzw. die Seriennummer der Investitionen mit der Rate 33

$$
\hat{\mathrm{Y}}=\hat{\mathrm{G}}=\frac{\sigma}{1-\mathrm{n}}
$$

wachsen, wobei $(\sigma)$ der Rate des Bevölkerungswachstums entspricht. Die Pro-Kopf Größen wachsen dann mit der Rate

$$
\hat{\mathrm{y}}=\frac{\sigma}{1-\mathrm{n}}-\sigma=\frac{\mathrm{n}}{1-\mathrm{n}}
$$

Es liegt somit ohne exogenen technischen Fortschritt positives Pro-Kopf-Wachstum vor. Dieses Ergebnis resultiert aus den steigenden Skalenerträgen.

Scheinbar ist mit Hilfe von Lerneffekten das Problem der Exogenität der gleichgewichtigen Wachstumsrate des neoklassischen Grundmodells überwunden. Tatsächlich aber weist das Ergebnis für die Wachstumsrate einen interessanten Aspekt auf. Wachstum, absolutes und Pro-Kopf-Wachstum, ist nur möglich, wenn die Bevölkerung wächst, d.h. wenn $\sigma>0$ ist. Sobald die Arbeitsmenge nicht wächst, kann auch die Volkswirtschaft nicht mehr wachsen. Der Grund hierfür liegt in der Entwicklung der Arbeitsproduktivität, die der Kehrwert von (3.3) ist. Für den hier betrachteten Spezialfall gilt für die Arbeitsproduktivität

$$
P=b^{-1} G^{n}
$$

Im Steady State wachsen alle Größen mit der durch (3.8) gegebenen Rate. Die Arbeitsproduktivität entwickelt sich somit aufgrund $n<1$ mit einer geringeren Rate. Die Wachstumsrate des Outputs ist die Summe der Wachstumsraten der Arbeitsmenge und der Arbeitsproduktivität. Falls die Beschäftigungsmenge nicht zunimmt, wächst auch der Output nicht. Konsequenterweise kann auch die Produktivität nicht steigen. In Verbindung mit Gleichung (3.10), nach der die Wachstumsrate der Arbeitsproduktivität geringer als die des Outputwachstums sein soll, ist dies das einzige logische Ergebnis. ${ }^{34}$ Das Bevölkerungswachstum gibt somit einen entscheidenden Impuls für den Wachstumsprozeß.

Das Ergebnis von Arrow entspricht in gewissem Sinne den Aussagen des neoklassischen Wachstumsmodells. Zwar ermöglichen die durch Lernen bedingten steigenden Skalenerträge eine Wachstumsrate des Pro-Kopf-Outputs im Steady State, die größer Null ist, dennoch ist

34 Vgl. Scott (1991, S. 113) und Hahn/Matthews (1964, S. 846). 
weiterhin dafür Bevölkerungswachstum notwendig. Der Grund liegt in der Abhängigkeit der Lerneffekte vom Investitionsprozeß. Nur wenn investiert wird, können auch die diskutierten Produktivitätseffekte auftreten. Steigt die Bevölkerungszahl an, müssen die zusätzlichen Arbeitskräfte aufgrund der Vollbeschäftigungsannahme auch Arbeit finden. Die getroffene Annahme fixer Produktionskoeffizienten macht deshalb eine Ausdehnung der Investitionen notwendig. Learning by doing wird möglich. Die Ausstattung der zusätzlichen Arbeitskräfte führt somit im Gegensatz zum neoklassischen Grundmodell aufgrund der steigenden Skalenerträge zu steigendem Pro-Kopf-Einkommen.

Obwohl den Investitionen also eine Beziehung zum Produktivitätswachstum zugestanden wird, ist die Sparquote weiterhin ohne Einfluß auf die Wachstumsrate des Systems. Der Grund liegt in den abnehmenden Grenzerträgen des Kapitals. Es gilt: ${ }^{35}$

$$
\frac{\partial Y}{\partial G}=a\left[1-\left(G^{1-n}-\frac{L(1-n)}{b}\right)^{n /(1-n)} G^{-n}\right]
$$

bzw.

$$
\frac{\partial Y}{\partial G}=a\left[1-\frac{\left(G^{1-n}-\frac{L(1-n)}{b}\right)^{n /(1-n)}}{G^{n}}\right]=a\left[1-\left(\frac{G^{\prime}}{G}\right)^{n}\right]
$$

Berücksichtigt man $G^{\prime}=G-T$, folgt:

$$
\frac{\partial Y}{\partial G}=a-a\left[1-\frac{T}{G}\right]^{n}
$$

Die Grenzproduktivität sinkt bei steigendem $\mathrm{G}$, denn

$$
\frac{\partial^{2} Y}{\partial G^{2}}=- \text { an }\left[\frac{G-T}{G}\right]^{n-1} \frac{T}{G^{2}}<0
$$

Die Profitabilität des Kapitaleinsatzes nimmt ständig ab. Es entsteht dieselbe Konsequenz für die gleichgewichtige Wachstumsrate wie im neoklassischen Grundmodell. ${ }^{36}$

Des weiteren tritt bei Arrows Modell das Phänomen auf, daß die heutige Investitionsentwicklung positive Externalitäten für zukünftige Investitionen hat. Jede Investition erhöht

35 Vgl. Levhari (1966a, S. 32).

36 Vgl. zu dieser Aussage auch Romer (1986a, S. 1006), der seine Behauptung jedoch nicht belegt. 
bzw. erhält den Sachkapitalbestand einerseits und führt gleichzeitig über die induzierten Lerneffekte zu einer Erhöhung der Produktivität der nachfolgenden Maschinen. In der Unternehmensplanung werden jedoch nur die individuellen Erträge aus der Investition berücksichtigt. Externe Effekte fließen nicht in die Kalkulation ein. Die private Ertragsrate einer Investition ist deshalb geringer als die soziale. ${ }^{37}$

Fallen beide Ertragsraten auseinander, bedeutet dies, daß die Volkswirtschaft nicht in der optimalen Höhe spart bzw. zu geringe Investitionen tätigt. Ein allwissender Planer würde alle Effekte der Investitionstätigkeit, d.h. auch die intertemporalen Produktivitätseffekte berücksichtigen und ein höheres Investitionsniveau realisieren. An dieser Stelle kann die Frage aufgeworfen werden, ob die dezentralisierte Entscheidung der privaten Unternehmer wirklich suboptimal ist. Hierbei kann zwischen der mikroökonomischen und der gesamtwirtschaftlichen Ebene unterschieden werden. Der einzelne Investor würde seine optimale Situation erreichen, wenn er auch die in der Zukunft entstehenden Erträge seiner Aktivität erhalten würde, d.h. entsprechend der wahren Grenzproduktivität entlohnt werden würde. Da seine Investition bis ans Ende aller Tage $(t=\infty)$ in den nachfolgenden Kapitalgütern wirkt, müßten auch die Zahlungen an ihn bis in diese Zeit reichen. Diese nicht realisierbare Schlußfolgerung ist das Ergebnis der Übertragung mikroökonomischer Resultate auf makroökonomische Zusammenhänge. Auf gesamtwirtschaftlicher Ebene wird im Fall steigender Skalenerträge immer zu wenig investiert. Durch Konsumverzicht kann die Volkswirtschaft immer mehr zukünftigen Konsum erreichen. Sinnvoll wäre die komplette Ersetzung des Konsums durch Investitionen, was jedoch immer noch nicht optimal wäre. 38

Dieser Ansicht ist zuzustimmen, wenn man die Übertragung der Modellergebnisse auf reale Situationen als Maßstab wählt. Ohne Zweifel ist es nicht möglich, Unternehmen dauerhaft alle Erträge ihrer Investitionen zukommen zu lassen. Modelltheoretisch ist diese Anforderung jedoch kein Problem. Durch Einfürung eines unendlich langen Planungshorizonts für den allwissenden Planer kann dies berücksichtigt werden. Darüber hinaus erlaubt die Einführung einer gesamtwirtschaftlichen Nutzenfunktion die Bestimmung eines optimalen Wachstumspfads, auf dem Zeitpräferenz und soziale Ertragsrate in Einklang gebracht sind. Das Optimierungsproblem ist zumindest theoretisch lösbar. Die Ermittlung der Bedingungen für ein unter den jeweils getroffenen Annahmen optimales Gleichgewicht sagt jedoch nichts darüber aus, ob

37 Der Unterschied zwischen der privaten und der sozialen Ertragsrate wurde schon immer von den Kommentatoren des Arrow-Modells betont. Vgl. Arrow (1962, S. 159 und S. 168ff.), Hahn/Matthews (1964, S. 846), v. Weizsacker (1966, S. 68) und Walter (1969, S. 225). Bei Levhari (1966a) findet sich der ausfuhrliche formale Nachweis, daB die von den Unternehmen berucksichtigte Grenzproduktivităt kleiner als die soziale ist.

38 Vgl. zu dieser Sichtweise z.B. Walter (1969, S. 229f.) 
den Investoren tatsächlich alle Erträge zufließen oder nicht andere Gruppen von den Externalitäten profitieren. 39

Neben den positiven Effekten der Investitionstätigkeit auf nachfolgende Generationen haben Investitionen zu jeder Zeit aber auch negative Wirkungen. Sie zerstören die Rentabilität vorhandener Kapitalgüter und lassen diese obsolet werden. Trotzdem ist die Investition in neue Kapitalgüter sinnvoll, da sie die modernste Produktionstechnologie verbessert. Der positive Effekt auf die Produktivitätsverhältnisse bedingt ja erst, daß die alten Kapitalgüter nicht mehr rentabel sind. Die späteren Ausführungen zur Neuen Wachstumstheorie werden zeigen, daß die Mischung zwischen den schöpferischen und den zerstörerischen Elementen von Investitionen in neues Wissen für die Existenz dauerhaften Wachstums entscheidend sein kann. ${ }^{40}$

Zusammenfassend läßt sich festhalten, daß das Konzept des learning by doing in der Form, wie es von Arrow diskutiert wurde, es ermöglichte, auch ohne technischen Fortschritt Pro-Kopf-Wachstum im Steady State zu generieren. Aufgrund der abnehmenden Grenzerträge des Kapitals ist aber auch in diesem Modell die langfristige Wachstumsrate unabhängig von der Spar- und Investitionsneigung. Für Steady State-Wachstum ist weiterhin positives Bevölkerungswachstum notwendig. Die langfristige Gleichgewichtssituation ist wie zuvor durch die Erfullung der Erwartungen der Investoren gekennzeichnet. Die Unternehmen werden in einer Situation ohne allwissenden Planer soviel investieren, wie furr eine konstante Ertragsrate notwendig ist. Berücksichtigt man den sozialen Planer, wird die soziale Ertragsrate an die Zeitpräferenz angeglichen. 41

Für die weiteren Überlegungen ist vor allem jedoch der externe Effekt der Investitionstätigkeit relevant. Investitionen verändern nicht nur die Situation der Investoren, sondern erhöhen auch die Rentabilität nachfolgender Investitionen, da sie die Voraussetzung für die Schaffung neuen Wissens bilden. Die Erhöhung des Know-hows ist dabei das Nebenprodukt der Investitionstätigkeit, d.h. der technische Fortschritt ist kein Ergebnis einer gezielten Entscheidung. Im nächsten Abschnitt wird dargestellt werden, daß die Vorstellung Arrows über die Wirkungen von Investitionen und deren externe Effekte ein zentraler Bestandteil in den Modellen der Neuen Wachstumstheorie ist.

39 Vgl. z.B. Levhari (1966b, S. 127). Das angedeutete Verteilungsproblem wird durch die Annahme eines repräsentativen Individuums ausgeschlossen. Auf die Probleme zwischen dieser Annahme und der Frage nach der Existenz von Externalităten wird im Abschnitt zum Romer-Modell eingegangen werden.

40 Vgl. Walter (1969, S. 231).

41 Vgl. Arrow (1962, S. 168ff.) sowie Levhari (1966a, b), der in beiden Beitrăgen die formalen Eigenschaften des Modells vertiefend darstellt. 


\subsubsection{Der erste Schritt: Steigende Skalenerträge}

\subsubsection{Wissensexternalitäten und steigende Skalenerträge}

Im vorigen Abschnitt hatte sich gezeigt, daß die Einführung steigender Skalenerträge in die Produktionsfunktion zwar positives Pro-Kopf-Wachstum im Steady State erreichen kann, dem Bevölkerungswachstum jedoch weiterhin eine entscheidende Bedeutung zukommt. Ohne Bevölkerungswachstum konnte kein Wachstum stattfinden. Eine Weiterentwicklung dieser Überlegungen von Arrow erfolgte in den ersten Modellen der Neuen bzw. Endogenen Wachstumstheorie. Insbesondere die Arbeit von P. Romer aus dem Jahre (1986) ist hierbei zu nennen. Von Arrow wird vor allem die Annahme übernommen, daß unternehmerische Entscheidungen über die Kapitalakkumulation nicht nur Konsequenzen für das jeweilige Unternehmen selbst, sondern auch positive externe Effekte für andere Unternehmen haben.

Ausgangspunkt der Überlegungen Romers ist das Problem der abnehmenden Grenzerträge des Kapitals, die im neoklassischen Grundmodell dafür verantwortlich sind, daß der Wachstumsprozeß der Pro-Kopf-Größen ohne exogenen technischen Fortschritt zum Erliegen kommt bzw. bei Arrow für die Abhängigkeit der langfristigen Wachstumsrate vom Bevölkerungswachstum entscheidend sind. Endogenes Wachstum wird durch die Konvexität der Produktionstechnologie verhindert. Am besten wird dies deutlich, wenn man sich innerhalb der Produktionsfunktion einen nicht vermehrbaren Produktionsfaktor vorstellt, der für die Produktion notwendig ist. Ein solcher Faktor kann Land und/oder Arbeit sein. Die Kapitalbildung einer Volkswirtschaft $(\dot{\mathbf{K}})$ kann dann durch Gleichung (3.11) dargestellt werden. ${ }^{42}$

$$
\dot{\mathrm{K}}=\mathrm{F}(\mathrm{K}, \mathrm{E})-\mathrm{C}
$$

Unter (E) wird hierbei die Menge der begrenzten Faktoren verstanden. Die maximal mögliche Investition wird durch den maximalen Output beschränkt, so daß

$$
\dot{\mathrm{K}}_{\mathrm{t}}^{\max }=\mathrm{F}\left(\mathrm{K}_{\mathrm{t}}^{\max }, \mathrm{E}\right)
$$

Dies würde bedeuten, daß das komplette Sozialprodukt investiert würde. Dies ist zwar für die Wirtschaftssubjekte sicher nicht optimal, da sie nur auf zukünftigen Konsum hoffen können, erlaubt aber die maximal mögliche Investitionsquote. Geht man von einer linear-homogenen Produktionsfunktion aus, gilt für die maximale Wachstumsrate des Kapitalstocks:

$$
\frac{\dot{\mathrm{K}}_{\mathrm{t}}^{\max }}{\mathrm{K}^{\max }}=\mathrm{F}\left(1, \frac{\mathrm{E}}{\mathrm{K}^{\max }}\right)
$$

42 Vgl. Romer (1991a, S. 92) 
Da ohne (E) keine Produktion möglich ist, wird die Wachstumsrate des Kapitalstocks bei steigendem Kapitaleinsatz abnehmen. Dies ist die Folge der Inada-Bedingungen. Im Extremfall $\mathrm{K}^{\max } \rightarrow \infty$ stellt sich eine Wachstumsrate von Null ein. Der Kapitalstock und mit ihm der Output bleiben konstant. ${ }^{43}$

Die Konsequenzen endlicher, aber für die Produktion notwendiger Produktionsfaktoren kann z.B. im Modell von Smith die Zunahme der Arbeitsteilung zum Erliegen bringen sowie in Ricardos Überlegungen zur Einkommensverteilung den Wachstumsprozeß begrenzen. Während Smith eine positive Beziehung zwischen der Wachstumsrate der Bevölkerung und der Rate der Kapitalakkumulation annahm und damit die Wachstumsgrenze nach außen verschob, sah Ricardo einen Ausweg im exogenen Fortschritt. ${ }^{44}$ Innerhalb des neoklassischen Grundmodells, könnte nun eingewandt werden, ist die Menge der Arbeitskräfte nicht begrenzt, da sie mit der Rate (n) wächst. Diese Wachstumsrate der Bevölkerung ist aber exogen gegeben und somit begrenzt. Sie paßt sich nicht an die Kapitalakkumulation an, wie es bei Smith der Fall war. Vielmehr führte die steigende Kapitalintensität zu einer Anpassung an (n). Die statische Begrenzung (konstante Bevölkerung) wird nur durch eine "quasi-dynamische" Begrenzung (konstante Wachstumsrate der Bevölkerung) ersetzt. Eine prinzipielle Veränderung ergibt sich daraus nicht.

Eine Lösung dieses Problems ist die Überwindung der Konvexität der Produktionstechnologie mit Hilfe nicht-rivalisierender Inputfaktoren, wie z.B. Wissen. Wie oben angesprochen, können solche Produktionsfaktoren gleichzeitig in mehreren Produktionsprozessen eingesetzt werden, ohne den jeweiligen Ertrag einzuschränken. Die Konsequenzen solcher nicht-rivalisierender Inputs sind bei einer Produktionsfunktion, die bzgl. der "normalen" Produktionsfaktoren konstante Skalenerträge aufweist, steigende Skalenerträge. Würde man die Einsatzmengen aller traditionellen, rivalisierenden Produktionsfaktoren (R) z.B. verdoppeln, würde sich der Output verdoppeln. Erhöht sich gleichzeitig der Einsatz der nichtrivalisierenden Produktionsfaktoren (N), kommt es zu steigenden Skalenerträgen. Formal stellt sich dies folgendermaßen dar:45

$$
F(\lambda R, \lambda N)>F(\lambda R, N)=\lambda F(R, N)
$$

Steigende Skalenerträge stellen für die neoklassische Theorie aus verteilungstheoretischer Sicht heraus ein Problem dar. Geht man von Grenzproduktivitätsentlohnung aus, folgt bei einem Skalenniveau der Produktionsfaktoren von größer als Eins eine Überausschöpfung des Sozialprodukts. Die Ansprüche an das Sozialprodukt fallen zu groß aus. Um dennoch eine konsistente Bestimmung der Einkommensverteilung zu erreichen, wird zur Generierung

43 Vgl. Romer (1991a, S. 92).

$44 \mathrm{Vgl}$. Kurz/Salvadori (1995b).

45 Vgl. Romer (1986a, S. 1015 und 1990a, S. 98). 
steigender Skalenerträge auf einen "Trick" zurückgegriffen. Die Ursache steigender Skalenerträge sind positive Externalitäten bei der Investition. Am deutlichsten wird dies, wenn als einziger akkumulierbarer Produktionsfaktor Wissen in die Produktion eingeführt wird. Im neoklassischen Grundmodell wird diese Aufgabe vom exogenen technischen Fortschritt übernommen.

In seinem ersten Modell zur Neuen Wachstumstheorie unterstellt Romer eine Produktionsfunktion auf Unternehmensebene, die von drei Produktionsfaktoren bestimmt wird: dem unternehmensspezifischen Wissen $\left(\mathbf{k}_{\mathbf{i}}\right)$, dem Vektor aller als konstant angenommenen, traditionellen Produktionsfaktoren (physisches Kapital und Arbeit) $\left(x_{i}\right)$ und dem gesamtwirtschaftichen Wissen (K). Wenn die Volkswirtschaft aus (n) Unternehmen besteht, soll $\mathrm{K}=\sum_{\mathrm{i}}^{\mathrm{n}} \mathbf{k}_{\mathrm{i}}$ sein. Der gesamtwirtschaftliche Wissensstock ergibt sich somit als Summe der unternehmensspezifischen Wissensbestände. Für die Produktionsfunktion des Unternehmens folgt hieraus: 46

$$
\mathrm{F}\left(\lambda \mathrm{k}_{\mathrm{i}}, \lambda \mathrm{K}, \lambda \mathrm{x}_{\mathrm{i}}\right)>\mathrm{F}\left(\lambda \mathrm{k}_{\mathrm{i}}, \mathrm{K}, \lambda \mathrm{x}_{\mathrm{i}}\right)=\lambda \mathrm{F}\left(\mathrm{k}_{\mathrm{i}}, \mathrm{K}, \mathrm{x}_{\mathrm{i}}\right)
$$

Ein Vergleich mit (3.14) macht die Rolle des gesamtwirtschaftlichen Wissens (K) als nichtrivalisierender Produktionsfaktor deutlich. $\mathbf{F}(\bullet)$ ist bzgl. des unternehmensspezifischen Wissens und der traditionellen Produktionsfaktoren homogen vom Grade eins. Der Einfluß von (K) auf die Effizienz des einzelnen Unternehmens entsteht durch die Tatsache, daß Unternehmen, die neues Wissen generieren, dieses sich nicht vollständig aneignen können. Nicht alles Know-how kann z.B. geheimgehalten werden. Andere Unternehmen können von den Anstrengungen des innovativen Unternehmens profitieren, ohne Ressourcen einsetzen zu müssen. Jede Vermehrung eines unternehmensspezifischen Wissensbestands führt zu einer Erhöhung des gesamtwirtschaftlichen Wissens.

Diese Vorgehensweise beinhaltet jedoch Inkonsistenzen. Wenn die Erhöhung des individuellen Wissensbestandes automatisch das gesamtwirtschaftliche Wissen steigert, stellt sich die Frage, wie man sich die Vervielfachung der Produktionsfaktoren $\left(x_{i}\right)$ und $\left(k_{i}\right)$ auf Unternehmensebene vorzustellen hat. Zum einem nämlich soll Wissen ein Faktor sein, der auf Unternehmensebene bei einer Faktorvariation vervielfacht werden kann, ohne (K) zu steigern. Investiert ein Unternehmen in die Wissensgenerierung, erhöhen sich zum anderen sein eigenes und das gesamtwirtschaftliche Know-how. Die Faktorvariation kann deshalb entweder nur ein Gedankenexperiment sein oder Wissen hat in den beiden Fällen unterschiedlichen Charakter. Interessant sind auch die Konsequenzen, die sich aus der Annahme identischer Unternehmen für das Modell ergeben. Wenn aufgrund der Modellannahmen (Homogenität der Produktionsfunktion bzgl. der zu entlohnenden Faktoren) davon ausgegangen wird, daß alle Unternehmen

46 Vgl. Romer (1986a, S. 1015). 
auf demselben Aktivitätsniveau tätig werden, stellt sich die Frage, ob nicht alle Unternehmen dasselbe Wissen aufweisen. Falls dem so ist, fält das gesamtwirtschaftliche Wissen mit dem unternehmensspezifischen Wissen zusammen. Vermehrt nun ein Unternehmen sein Know-how, erhöht es automatisch $(K)$. In $F(\bullet)$ erhöht sich bei jedem Unternehmen $\left(x_{i}\right)$ und $\left(k_{i}\right)$ entsprechend. Wie kann es bei identischen Unternehmen aber externe Effekte geben? Es bleibt unklar, wo die Ursache dieser Externalitäten liegt, wenn $k_{i}=K$ ist. Nur falls $k_{i} \neq K$, ist es vorstellbar, daß Unternehmen zusätzlich von $(\mathrm{K})$ profitieren können. ${ }^{47}$ Die Verwendung externer Effekte als Ursache steigender Skalenerträge auf Ebene der gesamtwirtschaftlichen Produktion steht somit auf tönernen Füßen. Ein Ausweg aus diesem Dilemma ist ein weiteres Mal die Rückbesinnung auf Arrows Modell, in dem heutige Investitionen positive Wirkungen auf die Effizienz zukünftiger Investitionen haben und somit ein intertemporaler externer Effekt vorliegt. Morgige Investitionen sind aufgrund der Handlungen der Vorgănger effizienter geworden.

Läßt man diese Überlegungen für einen Moment außen vor, kann festgehalten werden, daß das zentrale Element in Romers Modell von 1986 die externen Effekte der Wissensgenerierung sind, die bei der gesamtwirtschaftlichen Produktion zu steigenden Skalenerträgen führen. Darüber hinaus nimmt Romer ein steigendes soziales Grenzprodukt für das Wissen an. Je höher die vorhandene Menge an technologischem Wissen ist, desto höher wird der Outputzuwachs durch eine weitere Einheit Wissen. Die Produktionselastizität dieses Inputfaktors ist somit größer als eins. ${ }^{48}$ Eine solche Annahme umgeht das Problem der Unabhängigkeit der Wachstumsrate von der Investitionsquote, wie es sich in der traditionellen neoklassischen Theorie bzw. in Arrows Ansatz aufgrund der abnehmenden Grenzproduktivität des akkumulierbaren Faktors Kapital ergeben hat. 49

Die Existenz steigender Skalenerträge bedingt aber gleichzeitig eine große Schwierigkeit für die Eigenschaften des Systems. Im traditionellen Modell führte die Annahme sinkender Grenzproduktivität des Kapitals, d.h. des akkumulierbaren Produktionsfaktors zu einer Steady State-Situation, weil während des Anpassungsprozesses das Sozialprodukt unterproportional zum Kapitalstock wuchs. Steigende Skalenerträge jedoch lassen die Wachstumsrate immer höher werden, je mehr von dem akkumulierbaren Produktionsfaktor eingesetzt wird. Zum einen kann die Wachstumsrate des Kapitalstocks und damit auch des Outputs einen unendlich hohen Wert zu einem endlichen Zeitpunkt erreichen, zum anderen kann gezeigt werden, daß der Bestand des akkumulierbaren Produktionsfaktors und mit ihm der Output zu einem end-

47 Dieses Problem taucht auch bei Lucas (1988) wieder auf, wobei es jedoch dort keine Rolle für die Existenz endogenen Wachstums spielt.

$48 \mathrm{Vgl}$. Romer (1986a, S. 1020).

49 Vgl. Romer (1986a, S. 1006). 
lichen Zeitpunkt einen unendlich hohen Bestand aufweisen. 50 Vor allem letzteres hat eine interessante Implikation, die am besten mit einem Zitat von Solow verdeutlicht werden kann:

"It is one thing to say that a quantity will eventually exceed any bound. It is quite another to say that it will exceed any stated bound before Christmas." 51

Die Annahme steigender Skalenerträge ohne weitere Nebenbedingungen führt somit zum "...end of scarcity as we know it." 52 Wird jedoch das Problem der Knappheit überwunden, wäre auch die Wissenschaft, die sich mit diesem Themenkomplex beschäftigt, d.h. die Ökonomie überflüssig. Damit die Situation unendlicher großer Kapitalbestände jedoch erreicht werden kann, müssen die steigenden Skalenerträge auch ausreichend lange vorliegen. Die dauerhafte Existenz solcher Produktionsbedingungen wird mit Sicherheit nicht gegeben sein.

Um dieses Problem zu vermeiden, baut Romer eine "Bremse" ein, indem er abnehmende Grenzprodukte bei der Wissensgenerierung unterstellt. ${ }^{53}$ Die Schaffung neuen Wissens stellt einen eigenständigen Prozeß dar, der von den Unternehmen angeregt wird. Darüber hinaus wird deutlich, daß Wissen nur durch Konsumverzicht entstehen kann, d.h. Ressourcen müssen einer investiven Verwendung zugeführt werden. Im Unterschied zu den Ausführungen von Arrow ist Wissen im vorliegenden Fall ein Produktionsfaktor, dessen Herstellung immer mehr Input pro Einheit notwendig macht. Romer selbst hat die Betonung der intendierten Wissensbildung später wieder aufgegeben und sich an Arrows Vorgehensweise orientiert, in der Wissen ein Kuppelprodukt der Investitionstätigkeit ist. ${ }^{54}$ Dieser Wandel erklärt mit Sicherheit die mitunter anzutreffende nicht ganz korrekte Wiedergabe des Modells von 1986 in der Literatur. ${ }^{55}$

Im weiteren Verlauf seines Papiers zeigt Romer daß eine sozial optimale Lösung einer intertemporalen Nutzenmaximierung bei Existenz von steigenden Skalenerträgen, die durch die geschilderten Wissensexternalitäten entstehen, möglich ist. Dies stellt eine Modifikation bzw. Erweiterung der Vorgehensweise dar, wie sie bei der Ableitung der Goldenen Nutzenregel angesprochen wurde bzw. der Beiträge Levharis (1966a, b) zu den Implikationen des learning by doing. ${ }^{56}$ Aufgrund der Komplexität der zugrundeliegenden Mathematik verzichtet Romer auf die Ableitung gleichgewichtiger Wachstumsraten. Für den Fall einer linearen intertemporalen Nutzenfunktion zeigt sich die asymptotische Annäherung der Wachstumsrate des

$50 \mathrm{Vgl}$. zu den Aussagen bzgl. der Wachstumsrate des akkumulierbaren Produktionsfaktors insbesondere Meade (1962b), zur Unendlichkeit des Kapitalstocks bzw. Outputs Solow (1994a) bzw. den Anhang zu diesem Kapitel.

51 Solow (1994a, S. 50).

52 Solow (1994a, S. 50, Fn 1 und 2).

53 Vgl. Romer (1986a, S. 1019).

54 Vgl. Romer (1991, S. 109).

55 Vgl. für die Interpretation Romers als Learning by doing-Modell z.B. Boltho/Holtham (1992) oder Aghion (1994).

56 Weitere Grundlagen zur Losung eines solchen Optimierungsproblems finden sich auch bei Cass/Shell (1976a, b). 
unternehmensspezifischen Wissens an den Wert, der als Obergrenze für die Wachstumsrate des Wissens aufgrund der Annahme abnehmender Grenzproduktivitäten gesetzt wurde. Die Wachstumsraten des Konsums und des Outputs nähern sich dem Produkt aus dieser maximalen Wachstumsrate und der Summe der Produktionselastizitäten des privaten und gesamtwirtschaftichen Wissens an. ${ }^{57}$ Die Wachstumsrate des Wissens und die Effizienz des Wissens determinieren somit den Wachstumsprozeß.

Des weiteren ergeben sich durch die Existenz von Externalitäten der Wissensbildung wie im Modell Arrows (1962) Konsequenzen für das Verhältnis zwischen sozialer und privater Ertragsrate der Investitionen. Die Unternehmen gehen von einer zu geringen Grenzproduktivität ihres Wissens aus und neigen deshalb dazu, zu wenig in die Wissensbildung zu investieren. Eine dem freien Spiel der Kräfte überlassene Lösung tendiert deshalb zu einer suboptimalen Lösung im Hinblick auf die Investitionshöhe. Die Wachstumsrate der Volkswirtschaft wird in diesem Falle im Vergleich zur Situation, in der alle Effekte berücksichtigt werden, zu gering ausfallen. 58 Die Frage, inwieweit dies Spielraum für wirtschaftspolitische Maßnahmen, insbesondere die Einführung von Subventionen, läßt, wird später diskutiert werden. Im Moment steht primär die Frage nach der Art und Weise, wie endogenes Wachstum generiert wird, im Vordergrund des Interesses.

\subsubsection{Endogenes Wachstum - nicht ohne steigende Skalenerträge?}

Sehr häufig wird der Kernpunkt der Neuen Wachstumstheorie in der Existenz steigender Skalenerträge gesehen. Sind diese tatsächlich die Voraussetzung für endogenes Wachstum? Im Abschnitt zur neoklassischen Wachstumstheorie alter Prägung wurde gezeigt, daß die Flexibilität des Kapitalkoeffizienten (v) für die Anpassung der tatsächlichen Wachstumsrate an die natürliche Wachstumsrate sorgt. Bleibt (v) konstant, wird die tatsächliche Wachstumsrate dauerhaft über der natürlichen liegen. Kapitalstock und Output werden schneller wachsen als die Bevölkerung bzw. die Arbeitsmenge. Das Pro-Kopf-Einkommen steigt stetig an. Eine Produktionsfunktion, die diese Bedingung erfüllt, ist $Y=K$ mit $v=1$. Es liegt somit endogenes Wachstum vor, ohne daß steigende Skalenerträge angenommen werden müssen. Die Konstanz der Grenzproduktivität des akkumulierbaren Produktionsfaktors ist eine hinreichende Bedingung für endogenes Wachstum. So verstanden ist auch das Harrod-Modell ein endogenes Wachstumsmodell.

Eine Verbindung zwischen dieser Überlegung und den Ausführungen zu Romers Modell von 1986 läßt sich durch folgende Vorgehensweise verdeutlichen. Unterstellt man eine Produktionsfunktion, die neben den Produktionsfaktoren Arbeit $(L)$ und physischem Kapital 
(K) auf Unternehmensebene auch das unternehmensspezifische Wissen ( $\mathrm{k}$ ) enthält und nimmt man des weiteren einen positiven Einfluß des gesamtwirtschaftichen Wissens auf die unternehmerische Produktionsfunktion an, kann die unternehmensspezifische Produktionsfunktion durch (3.16) dargestellt werden. 59

$$
\mathrm{Y}_{\mathrm{i}}=\mathrm{F}_{\mathrm{i}}\left(\mathrm{K}_{\mathrm{i}}, \mathrm{L}_{\mathrm{i}}, \mathrm{K}_{\mathrm{i}}\right) \Phi(\mathrm{K})
$$

Bildet man eine Analogie zu Arrows Modell, in dem die Investitionen Träger und Ursache neuen Wissens und die Summe der kumulierten Bruttoinvestitionen der Maßstab für das Wissen in einer Volkswirtschaft waren, kann man den Kapitalstock in einem Unternehmen bzw. in der Gesamtwirtschaft als Ausdruck des Know-hows des Unternehmens bzw. der Volkswirtschaft interpretieren. Die Produktionsfunktion der Gesamtwirtschaft, d.h. in der Summe für alle Unternehmen ist dann:

$$
\mathrm{Y}=\mathrm{F}(\mathrm{K}, \mathrm{L}) \Phi(\mathrm{K})
$$

Für eine Cobb-Douglas-Produktionsfunktion $F(\bullet)=K^{\alpha} L^{1-\alpha}$ und $\Phi(K)=K^{\theta}$ folgt dann

$$
\mathbf{Y}=\mathbf{K}^{\alpha} \mathbf{L}^{1-\alpha} \mathbf{K}^{\theta}
$$

Die Annahme einer solchen Produktionstechnik spiegelt die Vorstellung einer Zweiteilung des Wissens wider. Einerseits entwickeln Unternehmen neue Produkte und Verfahren, die nur sie selbst verwenden und sich vollständig zu eigen machen können, andererseits fließt dieses Wissen in das allgemein verfügbare Know-how der Gesellschaft. Auf gesamtwirtschaftlicher Ebene spielt deswegen nur das gesamtwirtschaftliche Wissen eine Rolle, in den Unternehmen aber auch das individuelle. Problematisch bleibt weiterhin die Annahme identischer Unternehmen. Selbst wenn eine Einheit mehr privates Wissen eine davon verschiedene Einheit öffentliches Wissen erzeugt, können externe Effekte nur bei unterschiedlichen Unternehmen auftreten. Die für die Aggregation notwendige Identitätsannahme steht somit im Gegensatz zur Existenz von Externalitäten.

Der Kapitalstock gewinnt hier auf zwei Wegen Einfluß auf die gesamtwirtschaftliche Produktion. Zum einen als traditioneller Produktionsfaktor, zum anderen als Indikator für das angesammelte produktionstechnische Wissen. Sobald der Kapitalstock insgesamt eine Produktionselastizität größer als Eins besitzt, ist aufgrund der nicht abnehmenden Grenzproduktivitäten endogenes Wachstum möglich.

Am besten wird dies an einem Spezialfall, dem sog. AK-Modell deutlich, bei dem es nur einen Produktionsfaktor, nämlich Kapital (K) mit einer Produktionselastizităt von Eins gibt und

59 Vgl. Romer (1987b, S. 170). 
(A) für das Niveau des technischen Wissens steht. ${ }^{60}$ Arbeit fließt nicht in die Produktionsfunktion ein

$$
\mathrm{Y}=\mathbf{A K}
$$

Der Kapitalstock kann hier als Summe des privaten und des öffentlichen Kapitals verstanden werden. Bei Konstanz von (A) folgt für die Durchschnitts- und die Grenzproduktivität des Kapitals:

$$
\frac{\mathrm{Y}}{\mathrm{K}}=\frac{\partial \mathrm{Y}}{\partial \mathrm{K}}=\mathbf{A}
$$

Beide Größen sind identisch und konstant. In Pro-Kopf-Größen ausgedrückt bedeutet dies:

$$
\begin{aligned}
& \text { (3.21.1) } f(k)=A k \\
& (3.21 .2) \quad f^{\prime}(k)=A \\
& (3.21 .3) \quad \frac{f(k)}{k}=A
\end{aligned}
$$

Die Wachstumsrate der Kapitalintensität ist somit

$$
\frac{\dot{\mathrm{k}}}{\mathrm{k}}=\mathrm{sA}-(\mathrm{n}+\delta)
$$

wenn weiterhin Bevölkerungswachstum und technisch bedingte Abschreibungen auftreten. Eine Steady State-Situation ist definiert durch die Konstanz der Wachstumsraten aller Größen. Da die Sparquote (s), der Stand des technischen Wissens (A), die Wachstumsrate der Bevölkerung ( $n$ ) und die Abschreibungsrate $(\delta)$ als konstant angenommen wurden, ist auch die Wachstumsrate der Kapitalintensität $(\hat{\mathbf{k}})$ konstant. Aufgrund dessen wächst auch der ProKopf-Output mit dieser Rate. ${ }^{61}$

Durch die Konstanz von (3.22) ist jedoch nicht automatisch positives Pro-KopfWachstum garantiert. Die Bedingung für dauerhaft von Null verschiedenes Pro-KopfWachstum ist:

$$
\mathrm{sA}>\mathrm{n}+\delta
$$

60 Vgl. Rebelo (1991).

61 Vgl. Barro/Sala-i-Martin (1995, S. 39ff.) 
Das Sparen pro Kopf muß über dem effektiven Kapitalverzehr liegen. Entsprechendes galt aber auch schon für Situationen außerhalb des Steady States im neoklassischen Grundmodell. Dort kam das Pro-Kopf-Wachstum zum Erliegen, als die Investitionen genau die Menge erreichten, die den Kapitalverzehr ausglichen. Im vorliegenden Fall liegt die Ursache des endogenen Wachstums in der Kapitalintensivierung der Produktion. Da die Grenzproduktivităt des Kapitals nicht sinken kann, bleibt der Anreiz zur Investition erhalten, d.h. die Profitrate nimmt nicht ab. Der Gund für dieses Phänomen sind die Externalitäten der Wissensbildung bzw. der Investition.

Die Unterschiede zwischen dem traditionellen neoklassischen und dem AK-Wachstumsmodell sind sehr vage. Im letzteren bleibt die Investition pro Kopf konstant. Dies war im neoklassischen Wachstumsmodell auch möglich, falls man exogenen technischen Fortschritt unterstellt. Er erfüllt dort dieselbe Funktion wie die Wissensexternalitäten im AK-Modell. Beide Erscheinungen wirken den abnehmenden Grenzerträgen des Kapitals entgegen. Es darf nicht übersehen werden, daß auch im neoklassischen Grundmodell im Steady State die Kapitalintensität gemessen in realen Arbeitseinheiten ständig zunahm. Der Unterschied zwischen den Modellen ist somit graduell. Die Annahme der Existenz von Wissensexternalitäten ist genauso willkürlich wie die des exogenen technischen Fortschritts. Das AK-Modell kehrt im Gegensatz zum traditionellen Modell die Relevanz der Investitionen für einen dauerhaften Wachstumsprozeß hervor, weist aber gleichzeitig dem Produktionsfaktor Arbeit eine vernachlässigbare Rolle zu.

Die exogen gegebene Konstanz des Kapitalkoeffizienten hat eine weitere Implikation, die einen deutlichen Unterschied zum traditionellen Modell ausmacht. Die Sparquote als Ausdruck der Präferenzen der Wirtschaftssubjekte beeinflußt die langfristige Wachstumsrate, d.h. die Bereitschaft mehr zu sparen erhöht nicht nur das Niveau des Wachstumspfades, sondern auch dessen Steigung, M.a.W.: Maßnahmen zur Veränderung des Sparverhaltens haben Konsequenzen für den Wachstumsprozeß. Da alle Variablen mit der von den vorgegebenen Parametern determinierten Rate wachsen, besitzt das Modell keine inhärente Tendenz zu Konvergenzprozessen. Die Wachstumsraten. zweier oder mehrerer Volkswirtschaften sind entweder identisch, falls sie dieselben Parameterwerte aufweisen, oder sie unterscheiden sich dauerhaft. ${ }^{62}$ Eine Volkswirtschaft furr sich genommen weist ebenfalls keine Konvergenz zu einem spezifischen Steady State auf. Die tatsächliche und die langfristige Wachstumsrate sind zu jedem Zeitpunkt identisch, da die Grenzproduktivität nicht variiert. Wären alle Volkswirtschaften durch identische Produktionstechnologien gekennzeichnet, bliebe den armen Ökonomien der Weg zu schnellem Wachstum mit Hilfe von internationalem Kapital verschlossen. Die Rentabilität des Kapitaleinsatzes wäre in allen Ländern gleich. Wird für Produktionselastizität des Kapitals ein Wert größer als eins zugelassen, kann es sogar zur Divergenz kommen, da sich die Wachstumsraten immer weiter voneinander entfernen.

62 Vgl. Barro/Sala-i-Martin (1995, S. 40). 
Trotz seiner Einfachheit gibt das AK-Modell einen Hinweis auf die Probleme in unterentwickelten Ländern. Die notwendige Bedingung für von Null verschiedenes Pro-KopfWachstums war $\mathbf{s A}>\mathbf{n}+\delta$. Gelingt es einer Gesellschaft nicht, diese Voraussetzung zu erfullen, ist z.B. das Sparen im Vergleich zum Kapitalverzehr zu gering, wird die Volkswirtschaft nicht wachsen können. Hier wird noch einmal die Rolle der Kapitalakkumulation für den Wachstumsprozeß deutlich.

Die Aussagen gelten auch bei Verwendung einer intertemporalen Nutzenfunktion anstelle einer konstanten Sparquote. Die tatsächliche, langfristige Wachstumsrate ist nun abhängig von der Zeitpräferenz der Wirtschaftssubjekte. Da $\mathrm{f}^{\prime}(\mathrm{k})=\mathrm{A}$ ist, wird (2.48) aus Kapitel 2 zu

$$
\hat{\mathbf{c}}=\frac{\mathrm{A}-\mathrm{n}-\delta-\rho}{\lambda}
$$

und ist somit immer konstant. Für $\mathbf{A}>\mathbf{n}-\delta-\rho$ liegt bei $\lambda>0$ positives Pro-Kopf-Wachstum vor. Dies verlangt, daß die Nettoerträge aus einer zusätzlichen Kapitaleinheit über der Zeitpräferenzrate liegen, d.h. die Investition mehr Ertrag bringen muß, als durch den Konsumverzicht an Nutzen verloren geht. Auch in dieser Situation wird deutlich, daß der Investitionsanreiz für endogenes Wachstum dauerhaft erhalten bleiben muß.

Die Verwendung der AK-Funktion stellt eine Verletzung der Inada-Bedingungen dar, die abnehmende Grenzerträge der Produktionsfaktoren verlangten. Muß nun zur Erzielung von dauerhaftem endogenen Wachstum mindestens eine der Inada-Bedingungen verletzt werden? Die Antwort auf diese Frage kann auf den ersten Blick nicht mit einem klaren ja oder nein erfolgen. Wie gezeigt, macht endogenes Wachstum die dauerhafte Existenz eines Investitionsanreizes notwendig. Die potentiellen Erträge aus der Investition müssen über den Kosten liegen. Die Überlegungen zum AK-Modell haben ergeben, daß dies durch die Konstanz der Grenzproduktivität des Kapitals gewährleistet ist. Dauerhaftes Pro-Kopf-Wachstum kann aber auch möglich sein, wenn die Grenzproduktivität des Kapitals zwar fällt, die Durchschnittsproduktivität im Falle konstanter Sparquoten über $[(n+\delta) / s]$, bzw. im Fall der intertemporalen Nutzenmaximierung die Grenzproduktivität des Kapitals über $(n+\delta+\rho)$, liegt. Eine solche Produktionsfunktion wäre z.B. durch (3.25) gegeben. 63

$$
\begin{aligned}
& \mathrm{Y}=\mathrm{AK}+\mathrm{BK}^{\alpha} \mathrm{L}^{1-\alpha} \quad \text { mit } \alpha<1 \\
& \mathrm{f}(\mathrm{k})=\mathrm{Ak}+\mathrm{Bk}^{\alpha}
\end{aligned}
$$

63 Vgl. Jones/Manuelli (1990) und Barro/Sala-i-Martin (1995, S. 41f.). 
Die Durchschnittsproduktivität ist in diesem Fall:

$$
\frac{f(k)}{k}=A+B k^{\alpha-1}
$$

Da $\alpha<1$ ist, sinkt $\mathrm{Bk}^{\alpha-1}$ mit steigender Kapitalintensität (k), wobei für den Grenzwert der Durchschnittsproduktivität gilt:

$$
\lim _{\mathbf{k} \rightarrow \infty} \mathbf{f}(\mathbf{k}) / \mathbf{k}=\mathbf{A}
$$

Betrachtet man die Grenzproduktivitätsfunktion dieser Produktionsfunktion, steht

$$
f^{\prime}(k)=A+\alpha B k^{\alpha-1}
$$

Auch die Grenzproduktivität konvergiert gegen den konstanten Wert (A), wenn die Kapitalintensität wächst. Die Inada-Bedingungen sind somit ein weiteres $\mathrm{Mal}$ verletzt. Zwar sinken Durchschnitts- und Grenzproduktivität des Kapitals ab, der Fall eines Grenzwertes von Null für die Grenzproduktivität des Kapitals wird nicht erfüllt. Damit dauerhaftes Wachstum der Pro-Kopf-Größen möglich ist, müssen wieder die entsprechenden Bedingungen für (A) wie im einfachen AK-Modell erfült sein.

Aufgrund der abnehmenden, nach unten begrenzten Grenzproduktivität weist (3.24) andere Konvergenzeigenschaften auf als das einfache AK-Modell. Eine Volkswirtschaft mit einer Produktionstechnik, wie sie durch (3.24) gegeben ist, strebt einem endlichen Wert der Kapitalintensität zu und wird um so höhere Wachstumsraten besitzen, je stärker die tatsächliche Kapitalintensität nach unten von diesem Wert abweicht. ${ }^{64}$

\subsubsection{Steigende Skalenerträge und Spezialisierung}

Im vorigen Kapitel wurde als ein Weg, um steigende Skalenerträge in der Produktion zu ermöglichen, auf Investitionsexternalitäten zurückgegriffen. Eine andere Ursache von steigenden Skalenerträgen können Lerneffekte sein, die durch eine zunehmende Arbeitsteilung erzeugt werden. Bei Adam Smith findet sich das berühmte Beispiel der Nadelherstellung, an dem er deutlich macht, welche Folgen die wachsende Zergliederung von Produktionsprozessen in viele einzelne Arbeitsschritte hat. $\mathrm{Da}$ diese Überlegungen insbesondere in den Beiträgen von Kaldor eine wichtige Rolle spielen, wird in Kapitel 4 vertieft auf diese Fragestellung einge-

64 Zur Konvergenz in diesem Modell vgl. Barro/Sala-i-Martin (1995, S. 42f.) 
gangen. An dieser Stelle steht die modelltheoretische Behandlung dieses Aspekts in der Neuen Wachstumstheorie durch Romer (1986b, 1987a, b) im Mittelpunkt.

Steigende Spezialisierung führt durch Ausnutzung von Größenvorteilen und Lerneffekten aufgrund von learning by doing zu statischen und dynamischen Skalenerträgen. Eine wichtige Eigenschaft eines Spezialisierungsprozesses ist die zunehmende Zahl an Zwischenprodukten, die in der Produktion Verwendung finden. Dieses Phänomen kann in einer Produktionsfunktion vom Typ Cobb-Douglas erfaßt werden, indem man an Stelle des traditionellen Faktors Kapital die Summe der Zwischenprodukte setzt. Die Produktionstechnik hätte dann folgendes Aussehen: 65

$$
Y=L^{1-\alpha} \int_{R_{+}} Z(i)^{\alpha} \text { di } \quad \text { mit } \quad 0<\alpha<1
$$

Hierbei gibt Z(i) die Menge des Zwischenproduktes (i) an. Je höher die Zahl der Zwischenprodukte ist, desto höher wird der Wert des Integrals. Folglich wird auch die Produktivität bei jedem gegebenen Arbeitseinsatz höher sein. Die Produktionsfunktion weist trotz dieser Modifikation konstante Skalenerträge auf. ${ }^{66}$ Der endogene Wachstumseffekt, falls es denn einen gibt, entsteht durch die zunehmende Spezialisierung in der Produktion. Anstelle des in den vorigen Modellen notwendigen dauerhaften Investitionsanreizes tritt nun die Notwendigkeit der fortwährenden Erhöhung des Spezialisierungsgrades.

Für die Herstellung der Zwischenprodukte soll eine bestimmte Ressource (R) benötigt werden. Diese kann entweder in ihrer Menge als begrenzt angesehen werden oder aber als stetig wachsend. Im ersten Falle wird durch die vorgegebene Menge der Ressource der Wachstumsprozeß begrenzt. Sobald alle verfügbaren Reserven für die Herstellung von Zwischengütern verbraucht sind, kann kein weiteres Zwischenprodukt hergestellt werden. Die Volkswirtschaft verharrt in einem stationären Gleichgewicht. Der Fall wachsender Ressourcenreservoirs ist für den Wachstumsprozeß der interessantere. Für die Produktion der Zwischengüter werden fallende Durchschnittskosten angenommen, d.h. es liegt ein gewisser Fixkostenanteil vor. 67

$$
G(Z(i))=g_{0}+g_{1} Z(i)
$$

Nimmt man darüber hinaus an, daß die Kosten in realen Größen, d.h. in verbrauchten Einheiten der Ressource (R) gemessen werden können, unterliegen die gesamten Kosten der Beschränkung:

65 Vgl. Romer (1986b, S. 7 und 1987b, S. 172). Eine ahnliche Vorgehensweise findet sich bei Romer (1990b, c) und Grossman/Helpman (1991).

66 Vgl. Romer (1987b, S. 172).

67 Vgl. Romer (1986b, S. 8, 1987a, S. 57 und 1987b, S. 173). 


$$
\int_{\mathbf{R}_{+}} G(Z(i)) \leq R_{t}
$$

Die in (3.31) enthaltene Bedingung macht noch einmal das zuvor über die Wirkung der Ressourcenbeschränkung in puncto Wachstumsmöglichkeiten gesagte deutlich. Sobald alle Bestände verbraucht worden sind, besteht kein Spielraum mehr für eine weitere Expansion. Wir sind somit zurück bei dem schon von Smith und Ricardo erkannten Problem einer stationären Volkswirtschaft aufgrund von Mengenbeschränkungen bei den Produktionsfaktoren, sei es die Arbeitsmenge bei Smith oder sei es die Lage und Qualität der Böden bei Ricardo.68 Romer nimmt in seinem Modell an, daß die Ressource (R) durch Konsumverzicht vermehrt werden kann:

$$
\dot{\mathrm{R}}_{\mathrm{t}}=\mathrm{Y}_{\mathrm{t}}-\mathrm{C}_{\mathrm{t}}
$$

Die Bewegungsgleichung für die Ressource (R) entspricht somit vollständig derjenigen des Produktionsfaktors Kapital im traditionellen Modell. Der Unterschied liegt in der Effizienz des Faktoreinsatzes. Aufgrund der Spezialisierung werden neue Kapitaleinheiten immer produktiveren Verwendungen zugeführt. Nimmt man des weiteren an, daß $Z(i)=N / M$ für alle $i \in[0$, $\mathrm{M}$ ] ist, wobei $\mathrm{N}$ für die Menge der Inputs und $\mathrm{M}$ die Zahl der Zwischenprodukte, d.h. den Grad der Spezialisierung angibt, kann die Produktionsfunktion als

$$
Y=M^{1-\alpha} N^{\alpha} L^{1-\alpha}
$$

geschrieben werden. 69

Die Modellwirtschaft hat nun i+1 Märkte, da ein Endprodukt (Y) und (i) Zwischengüter hergestellt werden. Auf dem Markt für das Endprodukt liegt vollständige Konkurrenz vor, während es auf den Zwischenproduktmärkten als Folge der sinkenden Durchschnittskosten nur einen Anbieter geben wird, der den Preis für das Gut setzen kann. Für jedes Produkt ergibt sich eine fallende Nachfragefunktion, die aus den Gewinmaximierungsbestrebungen der Produzenten des Endprodukts folgt: $p_{i}=Z_{i}^{\alpha-1}$. Die Nachfrager nach den Investitionsgütern müssen den Preis für die Zwischenprodukte als gegeben hinnehmen. Ohne auf den Beweis für die gleichgewichtige Lösung eingehen zu wollen, werden im weiteren die Folgen einer solchen Marktstruktur für den Wachstumsprozeß kurz skizziert. ${ }^{70}$ Für den Fall einer fixierten Menge

$68 \mathrm{Vgl}$. zu diesen Aspekten in den Überlegungen von Smith und Ricardo Kurz/Salvadori (1995b).

69 Vgl. Romer (1986b, S. 7).

70 Der ausfuhrliche Beweise findet sich bei Romer (1986b, S. 6ff.) und in verkürzter Form bei Romer (1987a, S. 57ff.). 
der Ressource (R), d.h. $\dot{R}=0$, stellt sich als Lösung für die produzierte Menge des Zwischengutes (i)

$$
\mathrm{Z}_{\mathrm{i}}=\overline{\mathrm{Z}}=\frac{\mathrm{g}_{0}}{\mathrm{~g}_{1}} \frac{\alpha}{1-\alpha}
$$

ein und für den Spezialisierungsrad (M)

$$
M=R \frac{1-\alpha}{g_{0}} .
$$

Daraus folgt $N=\bar{Z} M=\frac{\alpha R}{g_{1}}$. Die Produktionsfunktion (3.32) kann umgeschrieben werden in (3.34):

$$
Y=\left(\frac{1-\alpha}{g_{0}}\right)^{1-\alpha}\left(\frac{\alpha}{g_{1}}\right)^{\alpha} R L^{1-\alpha}=B R L^{1-\alpha}
$$

Die Produktionsfunktion für das Endprodukt unterliegt damit steigenden Skalenerträgen in (R) und $(\mathrm{L})$ und erinnert deshalb sehr stark an den Fall mit Wissensexternalităten. Die Ursache für steigende Skalenerträge sind zum einen die sinkenden Durchschnittskosten bei der Herstellung der Zwischengüter $\left(Z_{\mathfrak{i}}\right)$ und zum anderen die positive Abhängigkeit des Outputs bei der Produktion des Gutes (Y) von der Zahl der Zwischengüter, d.h. dem Spezialisierungsgrad. Die Ausdehnung der Zahl der Zwischengüter durch ein Unternehmen hat deshalb mehrere Implikationen. Ein potentieller Anbieter wird zwar den Monopolpreis für sein Gut verlangen können, er wird jedoch nicht für alle positiven Wirkungen des neuen Zwischengutes entlohnt. Die sozialen Erträge eines neuen Zwischengutes liegen über den privaten, die durch die Monopolpreisbildung berücksichtigt werden. Durch die Erfindung eines neuen Zwischenprodukts erhöht er die Produktivität der Arbeit mit. Aufgrund der gesamtwirtschaftlich konstanten Grenzproduktivität des akkumulierbaren Faktors (R) kommt es in diesem Modell ebenfalls zu positivem endogenen Wachstum. ${ }^{71}$ Eine Weiterführung dieser Überlegungen stellt das Modell von Romer (1990b, c) zu den Wirkungen neuer Kapitalgüter dar.

71 Zur Bestimmung der gleichgewichtigen Wachstumsrate unter Verwendung einer spezifischen Nutzenfunktion in diesem Modell siehe Romer (1986b). 


\subsubsection{4 Öffentlicher Kapitalstock und endogenes Wachstum}

In den obigen Abschnitten trug das Wissen innerhalb einer Volkswirtschaft, das als öffentliches Gut angesehen werden kann, maßgeblich zum endogenen Wachstum bei. Dies wirf die Frage auf, ob nicht auch andere öffentliche Güter diese Funktion erfüllen können. Staatliche Leistungen können tatsächlich die Effizienz privater Produktionsaktivitäten erhöhen, wenn man z.B. an Verkehrs- oder Kommunikationsnetze denkt. ${ }^{72}$ Ein Weg diese Effekte abzubilden, stellt die Integration des öffentlichen Kapitalstocks in die private Produktionsfunktion dar. Ähnlich wie das gesamtwirtschaftliche Wissen bei Romer (1986) verbessert dann die Bereitstellung eines öffentlichen Gutes die Effizienz der Produktionsfaktoren. Auf den ersten Blick scheint sich hierdurch kein entscheidender Unterschied zu den oben getätigten Überlegungen zu ergeben. Tatsächlich aber sind Staatsausgaben für eine Volkswirtschaft nicht kostenlos. Zur Finanzierung müssen Steuereinnahmen getätigt werden, welche die Ertragsrate der privaten Investitionen reduzieren. Dem produktivitätssteigernden Effekt des öffentlichen Gutes steht der investitionshemmende Effekte der Steuerfinanzierung gegenüber. Dieser Trade-off wirft das Problem der optimalen Menge an öffentlichen Gütern auf.

Dieses Problem wird noch deutlicher, wenn die realistischere Annahme des Vorliegens von Überfüllungsproblemen getroffen wird. Der Nutzen, den das bereitgestellte öffentliche Gut für die privaten Unternehmen erbringt, wird dabei normalerweise durch die Zahl der Nutzer negativ beeinflußt. Eine andere Möglichkeit zur Erfassung dieser Effekte mittels produktionstechnischer Zusammenhänge besteht darin, den Nutzen des öffentlichen Gutes von der Outputmenge abhängig zu machen. Die Existenz solcher Phänomene erzeugt genau das Gegenteil wie im Modell mit Wissensexternalitäten. Die individuelle Produktions- und Investitionstätigkeit erzeugt negative externe Effekte für andere Unternehmen und senkt somit die gesamtwirtschaftliche Rendite privater Investitionen.

Barro (1990) und Barro/Sala-i-Martin (1992, 1995) haben für beide Fälle gezeigt, daß bei einer proportionalen Besteuerung des Einkommens zwischen der Wachstumsrate im Wettbewerbsgleichgewicht und dem Steuersatz keine monotone Beziehung besteht. Bei niedrigen Steuersätzen überwiegen die positiven Effekte des öffentlichen Gutes, während bei hohen Steuersätzen die Ertragsrate privater Investitionen durch die Steuerzahlung mehr belastet wird als das öffentliche Gut Nutzen erbringt. Führt man einen allwissenden Planer ein, kann dieser die Steuerpolitik optimal wählen. Im Überfullungsfall können somit auch die negativen externen Effekte der steigenden Nutzung des öffentlichen Gutes berücksichtigt werden. ${ }^{73}$

72 Schon Adam Smith betonte die Rolle einer funktionierenden offentlichen Infrastruktur für die Effizienz privatwirtschaftlicher Produktionsaktivitaten. (V.i., 1993, S. 393).

73 Vgl. Barro (1990), Barro/Sala-i-Martin (1992b und 1995 Kap. 4). 


\subsubsection{Fazit}

Trotz einer anderen Art der Generierung der steigenden Skalenerträge kann der zuletzt genannte Ansatz derselben Gruppe wie das AK-Modell bzw. das zuvor vorgestellte Modell mit Wissensexternalitäten zugerechnet werden. In all diesen Ansätzen weisen die akkumulierbaren Produktionsfaktoren zusammen eine Produktionselastizität $\geq 1$ auf, d.h. die Ertragsrate dieser Faktoren darf nicht sinken bzw. unter einen bestimmten Wert fallen, damit endogenes Wachstum der Pro-Kopf-Größen stattfinden kann. Die vorgestellten Überlegungen sind ein erster Schritt die fünf neuen stilisierten Fakten, wie sie oben erläutert wurden, zu erfassen. Insbesondere die Ursache technischen Wandels im menschlichen Handeln (Fakt 4) sowie das Problem von Marktunvollkommenheiten (Fakt 5) wurden in den Modellen von Romer erfaßt. Dennoch weisen diese Ansätze neben den schon angesprochenen Schwierigkeiten, die sich durch die Annahme des repräsentativen Individuums und das Vorliegen steigender Skalenerträge ergeben können, weitere kritische Eigenschaften auf. Diese werden in einem weiteren isolierten Abschnitt besprochen werden, da auch die im folgenden darzustellenden Modellansätze sich dieser Kritik ausgesetzt sehen.

\subsubsection{Humankapital als Wachstumsmotor}

\subsubsection{Vorbemerkungen}

In den im vorigen Abschnitt dargestellten endogenen Wachstumsmodellen spielte der Produktionsfaktor Wissen eine herausragende Rolle. Das Know-how einer Volkswirtschaft wurde als beliebig vermehrbar angesehen und entstand entweder als Nebenprodukt der Investition in Sachkapital oder durch gezielten Ressourceneinsatz. Der abstrakte Begriff Wissen ist eng mit einem anderen Begriff verwandt, dem des Humankapitals, der schon kurz im Rahmen der Besprechung des Vintage-Ansatzes und der Catching up-Hypothese auftauchte. Im allgemeinen werden unter Humankapital die Fähigkeiten und das produktiv verwendbare Wissen eines Menschen bzw. der Bevölkerung einer Volkswirtschaft verstanden. Humankapital steht somit nicht nur für das Wissen, das an einer Bildungseinrichtung erworben wird, sondern umfaßt auch die praktischen Kenntnisse eines Menschen. Es ist primär ein personengebundenes, ein Embodiment-Konzept, während das in einer Volkswirtschaft generell verfügbare Wissen unabhängig von den Wirtschaftssubjekten gesehen werden kann. Die Diskussion über den Themenkomplex Humankapital kann an dieser Stelle nicht angemessen wiedergegeben werden, sondern es wird ausschließlich auf die Fragestellungen eingegangen, die für die Beurteilung der Verwendung dieses Konzepts in der Neuen Wachstumstheorie vorrangig sind. 
Als primäre Quelle des Humankapitals werden Aus- und Weiterbildung gesehen, d.h. Wirtschaftssubjekte investieren Zeit und Geld, um ihre Făhigkeiten zu verbessern. Darüber hinaus tragen auch die schon in den Ausführungen zu Arrows Modell besprochenen learning by doing-Effekte zur Vergrößerung des Humankapitals bei. Die Fähigkeiten des einzelnen bzw. aller produktiv tätigen Wirtschaftssubjekte werden des weiteren von Größen wie Gesundheit und Ernährung beeinflußt. Für den gesamtwirtschaftlichen Humankapitalstock spielt auch die Lebenserwartung eine Rolle. Je älter die Menschen werden, desto länger können sie einen aktiven Beitrag zur Produktion leisten. ${ }^{74}$ Sowohl für das Individuum als auch für die Volkswirtschaft insgesamt ist ein hoher Bestand an Humankapital von Vorteil. ${ }^{75}$ Prinzipiell wird von einem positiven Zusammenhang zwischen dem persönlichen Arbeitseinkommen und dem Humankapital, das ein Mensch besitzt, ausgegangen. ${ }^{76}$ Auf gesamtwirtschaftlicher Ebene wird ein analoge Beziehung unterstellt: Je mehr Humankapital eine Volkswirtschaft aufweisen kann, desto produktiver ist sie, d.h. um so höher wird ihr Wohlstandsniveau sein.

Die endlose Vergrößerung des individuellen bzw. des gesamtwirtschaftlichen Humankapitals ist jedoch nicht zwingend sinnvoll. Die Kosten und Erträge der Investition müssen miteinander verglichen werden. Insbesondere Becker (1964) hat auf mikroökonomischer Ebene die Bedingungen für ein rationales Investitionsverhalten in diesem Bereich dargestellt. ${ }^{77}$ Auch auf makroökonomischer Ebene müssen die Kosten der Humankapitalbildung, insbesondere von Aus- und Weiterbildung, den Gewinnen gegenübergestellt werden. Ressourcen, die in den Bildungssektor fließen, stehen nicht der Produktion von Konsum- und physischen Kapitalgütern zur Verfügung. Gleichzeitig vergrößern bessere Fähigkeiten der Arbeitnehmer das Produktionspotential in der Zukunt. Diese Überlegungen dürfen nicht vergessen lassen, daß Humankapital und physisches Kapital trotz dieser Ähnlichkeit bei der Wirkung auf das Produktionspotential sich weiterhin unterscheiden. Tatsächlich können Eigentumsrechte am Humankapital im Gegensatz zum physischen Kapital aufgrund der Abhängigkeit vom Individuum nicht veräußert werden. ${ }^{78}$ Konsequenterweise ist auch die Beleihung des Humankapitals nicht möglich, d.h. die Finanzierung von Humankapitalinvestitionen kann nicht ohne weiteres auf Kredit erfolgen. Im Unterschied zu einer Maschine geht das Humankapital mit seinem

74 Vgl. z.B. Schultz (1961) und Rosen (1987, S. 682f).

75 Die positiven Effekte eines hohen individuellen und gesamtwirtschaftlichen Humankapitalbestands wurden bereits von A. Smith betont. Er forderte eine allgemeine Schulpflicht, da die zunehmende Arbeitsteilung die Arbeitnehmer dequalifiziert und gleichzeitig die Wehrfahigkeit der jungen Mănner gesenkt wird. Vgl. Smith (V.i.2, 1993, S. 420ff.).

76 Eine der ersten Arbeiten zu diesem Themenkomplex wurde von Mincer (1958) durchgefuhrt. Vgl. für die Zusammenhänge zwischen Humankapital und der Verteilung des Arbeitseinkommens Wegner (1981).

77 Vgl. z.B. Becker (1964, Kap. II und III).

78 Dies gilt natürlich nur in einer freien Gesellschaft. Die historischen Beispiele der Sklaverei, sei es im alten Rom oder in den USA, zeigen die Moglichkeit des Utbergangs der Eigentumsrechte am individuellen Humankapital auf ein anderes Wirtschaftssubjekt auf. 
Eigentümer bei dessen Tod verloren. Einem Kreditgeber bleiben deshalb keine Sicherheiten, wie es im Fall von Sachkapital der Fall ist. ${ }^{79}$

Sehr interessant ist die von Becker (1964) getroffene Unterscheidung in allgemeine und spezifische Fähigkeiten bzw. Humankapital. Allgemeines Humankapital kann von einem Wirtschaftssubjekt in verschiedenen Tätigkeiten eingesetzt werden und ist nicht von dem Unternehmen oder der Stelle abhängig, wo das Wissen erworben wurde. Becker führt z.B. die Ausbildung eines Maschinisten in der Armee an, der nach seiner Dienstzeit sein Know-how auch in der privaten Wirtschaft anwenden kann. ${ }^{80}$ Spezifisches Humankapital kann nur dort produktiv eingesetzt werden, wo es gebildet wurde. Auch hier dient das Militär als Beispiel. Die Ausbildung zum Kanonier ist fast vollständig ohne Wert in zivilen Verwendungen. ${ }^{81}$ Die Trennung zwischen beiden Arten von Humankapital kann nicht vollständig erfolgen. Jede Ausbildung z.B. trägt spezifische und allgemeine Elemente in sich. Doch es wird der eine oder der andere Anteil je nach Inhalt der Ausbildung überwiegen. Aus mikroökonomischer Sicht beeinflußt die Zusammensetzung des individuellen Humankapitals aus spezifischen und allgemeinen Fähigkeiten die Produktivität und die Beschäftigungschancen eines Wirtschaftssubjekts und somit auch dessen zu erwartende Einkommensströme. Gesamtwirtschaftich wird die Struktur des Humankapitals ebenfalls die Produktionsmöglichkeiten und die Flexibilität der Arbeitskräfte bestimmen. Ein hoher Anteil von Arbeitnehmern mit überwiegend spezifischen Humankapitalelementen wird bei schnellem Strukturwandel u.U. zu Friktionen führen. Ein wachstumstheoretisches Modell müßte diese Strukturkomponente berücksichtigen.

Im weiteren wird auf verschiedene Formen der Behandlung des Humankapitals in der Neuen Wachstumstheorie eingegangen werden. Die oben angesprochenen Aspekte werden dabei weitgehend von den Autoren bei der Modellierung nicht berücksichtigt. Zumeist wird von dem Humankapital als ganzem gesprochen, das ein Analogon zum Sachkapital darstellt. Unterschiede ergeben sich z.B. bei der Frage, ob Externalitäten der Investition vorliegen oder bei den Annahmen über den Verlauf der Grenzproduktivitätskurve des jeweiligen Kapitals.

\subsubsection{Der Impuls: Das Humankapitalmodell von Lucas (1988)}

Einer der zentralen Aufsätze der Neuen Wachstumstheorie, und sicher neben dem von Romer aus dem Jahr (1986) der am häufigsten zitierte, ist der Beitrag von Lucas aus dem Jahr 1988. Konzentrierten sich die im vorigen Abschnitt besprochenen Modelle auf die Externalitäten als Ursache endogenen Wachstums, führt Lucas neben diesen einen expliziten Wachstumsmotor

79 Vgl. zu den Unterschieden zwischen Humankapital und Sachkapital z.B. Rosen (1987, S. 682ff.) und Walter (1969, S. 156ff.) sowie Kapitel zwei dieser Arbeit.

$80 \mathrm{Vgl}$. Becker (1964, S. 11f.).

81 Vgl. Becker (1964, S. 18ff.). 
ein, um unterschiedliche Wachstumsraten in verschiedenen Ländern zu erklären. ${ }^{82} \mathrm{Zu}$ den traditionellen Produktionsfaktoren Kapital und Arbeit kommt das Humankapital hinzu, das alle Fähigkeiten eines Individuums umfassen soll. Die Wachstumsrate des Modells wird sowohl von der Höhe der externen Effekte als auch von der Entscheidung der Wirtschaftssubjekte Wissen zu akkumulieren beeinflußt.

Durch die Einführung des Humankapitals als zusätzlichen Produktionsfaktor verändert sich auch die Rolle der Arbeit im Produktionsprozeß. Jeder Arbeitnehmer ist mit einer bestimmten Menge an Humankapital (h) ausgestattet, die proportional zu seiner Produktivität ist. Ein Individuum kann durch den Einsatz von Zeit für Aus- und Weiterbildung sein Humankapital erhöhen. Durch die differierende Ausstattung mit Humankapital wird der Produktionsfaktor Arbeit zu einer heterogenen Größe. ${ }^{83} \mathrm{Um}$ die effektive Arbeitsmenge erfassen zu können, muß deshalb eine Gewichtung des einzelnen Arbeitnehmers mit seinem Humankapital erfolgen. Der Lucassche Ansatz besitzt damit Elemente eines Vintage-Modells, in dem die unterschiedlichen Jahrgänge des Sachkapitals ebenfalls entsprechend ihrer Produktivität bewertet wurden. Ein weiterer zu berücksichtigender Faktor ist die Arbeitszeit, die Wirtschaftssubjekte für die Produktion aufwenden. Geht man von einem begrenzten Zeitbudget eines Individuums aus, das es für Produktions- und Bildungszwecke verwenden kann, wird jede Stunde, die in die Produktion von Gütern fließt, nicht der Bildung zugute kommen und umgekehrt. ${ }^{84}$ Der einzelne steht somit vor dem Problem der optimalen Aufteilung seiner knappen Ressource Zeit. In der Güterproduktion ist nun wichtig, wie lange jeweils die unterschiedlichen Qualitätsstufen der Arbeit eingesetzt werden, d.h. die Arbeitnehmer müssen nicht nur mit ihrem Humankapitalbestand, sondern zusätzlich auch mit ihrer Arbeitszeit gewichtet werden. Aus diesen Überlegungen heraus ergibt sich der effektive Arbeitseinsatz $\left(\mathrm{L}^{\mathrm{e}}\right)$ als

$$
\mathrm{L}^{\mathrm{e}}=\int_{0}^{\infty} \mathrm{u}_{\mathrm{h}} \mathrm{L}_{\mathrm{h}} \mathrm{hdh},
$$

wobei $\left(u_{h}\right)$ für den Anteil der Nichtfreizeit steht, die von Arbeitskräften mit dem Humankapitalbestand (h) für die Produktion verwendet wird, $\left(L_{h}\right)$ der Anzahl der Arbeitskräfte mit einer Humankapitalausstattung (h) entspricht. Leider wird diese Überlegung zur Heterogenität der Arbeitnehmer von Lucas sehr schnell wieder fallengelassen, da er im weiteren Verlauf seines Beitrags identische Individuen unterstellt. ${ }^{85}$

Die Erhöhung des individuellen Humankapitals hat seiner Auffassung nach zwei Effekte. Zum einen erhöht eine Bildungsmaßnahme die Produktivităt des Wirtschaftssubjekts. Dies wird

82 Das Modell von Lucas ist dennoch das einer geschlossenen Volkswirtschaft.

83 Vgl. Lucas (1988, S. 17).

84 Lucas nennt dieses Zeitbudget Nichtfreizeit. Vgl. Lucas (1988, S. 17).

$85 \mathrm{Vgl}$. Lucas (1988, S. 17f.). 
als interner Effekt der Humankapitalbildung bezeichnet. Zum anderen erhöht jede individuelle Investition in das persönliche Humankapital auch das durchschnittliche Niveau des Humankapitals. Bildet sich ein Wirtschaftssubjekt weiter, während alle anderen auf dem individuell gleichen Stand verharren, steigt das gesamtwirtschaftliche Ausbildungsniveau. Der durchschnittliche Humankapitalbestand (ha) beeinflußt die Produktivität der anderen Produktionsfaktoren, die bei der Herstellung des Endprodukts Verwendung finden, was als externer Effekt des Humankapitals bezeichnet werden kann. Der durchschnittliche Humankapitalbestand stellt somit ein öffentliches Gut dar. ${ }^{86}$ Externalitäten dieser Art können durch die Kommunikation zwischen den Mitgliedern einzelner Gruppen entstehen. Erhöht z.B. in einer Arbeitsgruppe ein Individuum sein Wissen, kann es dadurch die Produktivität der anderen Mitglieder erhöhen, ohne daß diese sich selbst weiterbilden müssen bzw. die gleichen Anstrengungen unternehmen müssen, wie es das erste Individuen mußte:

"Most of what we know we learn from other people. We pay tuition to a few of these teachers, either directly or indirectly by accepting lower pay so we can hand around them, but most of it we get for free, and often in ways that are mutual without a distinction between student and teacher. Certainly in our own profession, the benefits of colleagues from whom we hope to learn are tangible enough, to lead us to spend a considerable fraction of our time fighting over who they shall be, and another fraction travelling to talk with those we wish we could have as colleagues but cannot. We know this kind of external effect is common to all the arts and sciences - the 'creative professions'. All of the intellectual history is the history of such effects." 87

In gewissen Sinne entsteht der externe Effekt des Humankapitals durch eine Art Fixkostendegression, da sich die einmalig anfallenden Kosten für die Erhöhung des individuellen Humankapitals auf mehrere Köpfe verteilen.

Ein Weg, solche Externalitäten zu nutzen, besteht z.B. durch die Bildung von Technologieparks oder die Durchfuhrung von Konferenzen. Lucas selbst verweist auf die Entstehungsgeschichte von Städten bzw. Handelszentren. Die Ansiedlung in Ballungszentren bietet trotz hoher Kosten für Grund und Boden, die Möglichkeit, mit anderen Menschen zu kommunizieren und von deren Humankapital zu profitieren. Diese Argumentationsweise findet sich schon in Marshalls Principles (1920), so daß die Überlegungen von Lucas zu den externen Effekten des Humankapitals nicht so neu sind, wie es auf den ersten Blick scheint. Interessant ist, daß in Lucas' Papier kein Hinweis auf Marshall zu finden ist, obwohl es furr die Marshall Lectures an der University of Cambridge geschrieben wurde. ${ }^{88}$

Trotz der eingängigen Diskussion der Relevanz externer Effekte des Humankapitals stellt sich die Frage, wie man die Existenz solcher Externalitäten aufrechterhalten kann, wenn gleichzeitig identische Individuen unterstellt werden. Eine Erhöhung des individuellen

86 Vgl. Lucas (1988, S. 18).

87 Lucas (1988, S. 38.)

88 Siehe zu den Überlegungen Marshalls Kapitel 4 dieser Arbeit. 
Humankapitals kann nicht mehr losgelöst von den anderen Wirtschaftssubjekten gesehen werden. Bildet sich ein Wirtschaftssubjekt weiter, müssen entweder alle anderen den Ausbildungsstand dieses Einzelnen erlangen, jeder interne Effekt des Humankapitals wäre gleichzeitig vollständig extern, oder das sich weiterbildende Individuum erlangt ein höheres Humankapitalniveau und unterscheidet sich von den anderen. Dann muß aber die Annahme identischer Wirtschaftssubjekte aufgehoben werden. Im ersten Fall bleibt offen, weshalb man noch eine explizit externe Komponente einführen soll. Eine gegebene Investition in Humankapital wäre einfach sehr produktiv in dem Sinne, daß sie bei gegebenem Ressourceneinsatz eine Einheit individuelles Humankapital schaff plus die Erhöhung des Humankapitalbestands der anderen Wirtschaftssubjekte. Um diese Effekte zu erfassen, muß die Produktionsfunktion jedoch nicht zwei Variablen für das Humankapital beinhalten, sondern es würde eine einzige gemeinsame ausreichen. Zur Verteidigung der Unterscheidung zwischen externen und internen Folgen der Humankapitalbildung kann vorgebracht werden, daß im gewissen Sinne auch bei identischen Wirtschaftssubjekten externe Effekte vorliegen können, wenn der Einzelne die Folgen seiner Investition für Dritte nicht berücksichtigt. Das einzelne Wirtschaftssubjekt erhöht nicht indirekt die Produktivität der anderen, sondern direkt indem es die Wirtschaftssubjekte ändert. ${ }^{89}$ Wenn dem aber so ist, schließt sich sofort die Frage nach den Informationen, über die die Wirtschaftssubjekte verfügen, an.

Für die Produktionstechnologie ergibt sich aus den Überlegungen zur Rolle des Humankapitals und dessen externen Effekten folgendes Aussehen:90

$$
Y=A K_{t}^{\alpha}\left(u_{t} h_{t} L_{t}\right)^{1-\alpha} h a_{t}^{\gamma}
$$

Hierbei erfaßt (A) den als konstant angenommenen Stand des technischen Wissens und (K) steht für die Menge des physischen Kapitals. Der externe Effekt des Humankapitals wird durch $(\gamma)$ erfaßt. Die Produktionsfunktion besitzt bzgl. des effektiven Arbeitseinsatzes $\left(u_{t} h_{t} L_{t}\right)$ und dem physischen Kapital $\left(\mathrm{K}_{t}\right)$ konstante Skalenerträge. Durch die Hinzunahme des externen Humankapitaleffekts kommt es insgesamt zu steigenden Skalenerträgen. Betrachtet man (3.36) genauer und multipliziert man den Term für den effektiven Arbeitseinsatz aus, ergibt sich

$$
Y=A K_{t}^{\alpha} h_{t}^{1-\alpha}\left(u_{t} L_{t}\right)^{1-\alpha} h a_{t}^{\gamma}
$$

89 Ein solches Argument kann auch das analoge Problem auf Unternehmensebene 10 sen.

90 Vgl. Lucas (1988, S. 18). 
Aufgrund der Annahme identischer Wirtschaftssubjekte entsprechen sich der durchschnittliche und der individuelle Humankapitalbestand. Damit unterliegt die Produktionsfunktion bzgl. der akkumulierbaren Produktionsfaktoren im vorliegenden Fall steigenden Skalenerträgen. Setzt man $(\gamma)$ gleich Null, liegen immer noch konstante Skalenerträge der vermehrbaren Inputs vor. Das Modell von Lucas besitzt eine große Nähe zum AK-Modell, auf die weiter unten noch einmal eingegangen wird.

Die Bildung des physischen und des Humankapitals soll wie folgt definiert sein:91

$$
\dot{K}_{t}=A K_{t}^{\alpha}\left(u_{t} h_{t} L_{t}\right)^{1-\alpha} h a_{t}^{\gamma}-L_{t} c_{t}
$$

und

$$
\dot{h}_{t}=h_{t} \varepsilon\left(1-u_{t}\right)
$$

Die Bewegungsgleichung des physischen Kapitals (3.38) entspricht der gewohnten Vorgehensweise. Die Investition entspricht der Differenz aus Güterproduktion und Konsum, wobei (c) für den Pro-Kopf-Konsum steht. Die Veränderung des Humankapitals wird durch die vorhandene Menge an diesem Produktionsfaktor, den für Bildung verwendeten Anteil der Nichtfreizeit sowie einen Effizienzparameter $(\varepsilon)$ bestimmt. ${ }^{92}$ Die unterstellte Funktion weist konstante Skalenerträge und konstante Grenzproduktivität bzgl. des Humankapitalstocks auf, d.h. unabhängig von der Höhe des schon erreichten Niveaus erfordert jede zusätzliche Einheit Humankapital dieselbe Anstrengung. Würde die Grenzproduktivität abnehmen, würde die Humankapitalbildung irgendwann zum Erliegen kommen. ${ }^{93}$ Lucas weist somit explizit auf diese entscheidende Bedingung hin. Endogenes Wachstum ist eine Folge der Annahmen. Die Endogenität des Wachstumsprozesses entsteht durch die Existenz eines weiteren Produktionsfaktors, nämlich der Zeit, die für Bildungszwecke verwendet wird. Auch sie hat eine konstante Grenzproduktivität. Mehr Zeit für Studienzwecke würde c.p. das Wachstum des Humankapitals erhöhen und auf das gesamtwirtschaftliche durchschlagen. Insgesamt weist (3.39) steigende Skalenerträge auf. Die Zeitaufteilung der Wirtschaftssubjekte entsprechend ihrer Präferenzen ist das endogene Element an dieser Stelle. ${ }^{94}$

91 Vgl. Lucas (1988, S. 18ff.). Die Akkumulationsgleichung des Humankapitals geht auf Uzawa (1965) zurück. In einem Modell von Stokey (1991), in dem die Autorin eine Verbindung zwischen der Humankapitalausstattung der Wirtschaftssubjekte, die eine begrenzte Lebenszeit haben, und der Qualităt der produzierten Güter analysiert, kommt dieselbe Funktion zur Anwendung. Darüber hinaus liegt auch im Ansatz von Stokey ein externer Effekt der Humankapitalbildung vor, der für positives Steady StateWachstum sorgen kann.

92 Dieser Effizienzparameter gibt gleichzeitig die maximale Wachstumsrate des Humankapitals vor, die erreicht wird, wenn alle Nichtfreizeit in die Bildung gesteckt wird.

93 Vgl. Lucas (1988, S. 19).

94 Vgl. auch Solow (1992a, S.21). 
Die Gleichungen (3.38) und (3.39) lassen erkennen, wo die Erweiterung zum traditionellen Modell Solowscher Prägung liegen. Die Einführung eines zusätzlichen Produktionsfaktors stellt die Wirtschaftssubjekte vor eine weitere Entscheidung. Sie müssen nicht nur die produzierte Gütermenge auf Investition und Konsum verteilen, sondern sie müssen auch bestimmen, welchen Anteil ihrer Nichtfreizeit sie für die Herstellung von Gütern und welchen Anteil sie für die Humankapitalbildung verwenden wollen. Sie haben deshalb eine zweistufige Investitionsentscheidung $\mathrm{zu}$ treffen: zuerst über die Verteilung der produktiv verwendbaren Zeit, dann über die Verwendung des nach dieser Entscheidung möglichen Outputs.

Zur Bestimmung einer Steady State Wachstumsrate maximiert Lucas vergleichbar mit der Goldenen Nutzenregel bzw. dem Vorgehen von Romer eine intertemporale Nutzenfunktion unter Einhaltung der Nebenbedingungen für die Kapitalbildung (3.38) und (3.39). Aufgrund des externen Effekts des Humankapitals muß zwischen der sozial optimalen und der Laisserfaire-Wachstumsrate unterschieden werden. Die gleichgewichtige Wachstumsrate des Konsums bzw. des Kapitalstocks pro Kopf bestimmt sich dann nach (3.40)

$$
\hat{\mathrm{c}}=\hat{\mathrm{k}}=\left(\frac{1-\alpha+\gamma}{1-\alpha}\right) \mathrm{v} \quad \text { mit } \mathrm{v}=\delta(1-\mathrm{u})
$$

Sieht man von dem externen Effekt der Humankapitalbildung ab, d.h. $\gamma=0$, entsprechen sich die Wachstumsrate des Konsums pro Kopf und die des Humankapitals (v). Die Unterschiede zwischen der sozial optimalen Situation und dem Laisser-faire-Gleichgewicht spiegelt sich in (v) wider. Berücksichtigt man die Bedingungen ersten Grades für die Lösung des zugrundeliegenden Optimierungsproblems ergeben sich folgende Gleichungen für die Wachstumsrate des Humankapitals: 95

$$
\begin{array}{ll}
v^{\mathrm{opt}}=\sigma\left[\varepsilon-\frac{1-\alpha}{1-\alpha+\gamma}(\rho-\mathrm{n})\right] & \text { (Sozial optimales Wachstum) } \\
v^{\text {lf }}=\frac{(1-\alpha)(\varepsilon-[\rho-n])}{\sigma^{-1}(1-\alpha+\gamma)-\gamma} & \text { (Laisser-faire Wachstum) }
\end{array}
$$

Sowohl (3.41.1) als auch (3.41.2) werden von der intertemporalen Substitutionselastizität ( $\sigma$ ), der sozialen Diskontrate $(\rho)$, dem Bevölkerungswachstum (n), der Produktionselastizität des physischen Kapitals $(\alpha)$, dem externen Effekt des Humankapitals $(\gamma)$ sowie dem Effizienzparameter des Bildungssektors $(\varepsilon)$ bestimmt. Wie zu erwarten, beeinflussen $(\sigma)$ und $(\gamma)$ die Wachstumsrate des Humankapitals positiv, während $(\rho)$ negativ mit der Wachstumsrate korreliert ist. Dies impliziert, daß "geduldige" Volkswirtschaften höhere Wachstumsraten zu

95 Vgl. Lucas (1988, S. 22f.). 
verzeichnen haben als Volkswirtschaften, deren Wirtschaftssubjekte eine sehr hohe Präferenz für heutigen Konsum haben. Darüber hinaus läßt sich festhalten, daß die Wachstumsrate des Humankapitals in beiden Fällen mit der steigender Effizienz des Bildungssystems ( $\varepsilon$ ) zunimmt. Bemerkenswert dagegen ist der positive Effekt des Bevölkerungswachstums auf die Wachstumsrate. Dies läßt sich einerseits auf die Ausnutzung des externen Effekts des Humankapitals zurückführen, andererseits führt eine Erhöhung der Arbeitnehmer in der Produktionsfunktion (3.37) ebenfalls zu steigenden Skalenerträgen. Dies wird deutlich, wenn man den Term für den effektiven Arbeitseinsatz ausmultipliziert.

Sieht man vom externen Effekt ab, fallen alle drei Wachstumsraten zusammen, d.h. daß

$$
\hat{\mathrm{c}}=\hat{\mathrm{k}}=\mathrm{v}^{\text {opt }}=\mathrm{v}^{\text {lf }}=\sigma[\varepsilon-(\rho-\mathrm{n})]
$$

Damit positives Wachstum vorliegt, muß $\varepsilon+n>\rho$ sein. Die Effizienz des Bildungssystems und die Wachstumsrate der Bevölkerung müssen insgesamt größer sein als die Zeitpräferenzrate der Wirtschaftssubjekte. Die Erträge des Konsumverzichts müssen ausreichend groß sein, um die Individuen zur Investition in Humankapital zu bewegen.

Anhand Gleichung (3.40) wird eine Voraussetzung für endogenes Wachstum deutlich. Die Bewegungsgleichung des Humankapitals, die ja eine Produktionsfunktion für zusätzliche Einheiten dieses Guts darstellt, muß eine konstante Grenzproduktivität des Humankapitals aufweisen. In allgemeiner Form kann (3.39) als

$$
\dot{h}_{t}=h_{t}^{\beta} \varepsilon\left(1-u_{t}\right)
$$

geschrieben werden. ${ }^{96}$ Die Wachstumsrate des Humankapitals ist somit allgemein

$$
\hat{h}_{t}=h_{t}^{\beta-1} \varepsilon\left(1-u_{t}\right)
$$

Nimmt man als Grenzfall die maximal mögliche Wachstumsrate des Humankapitals an, muß $\left(u_{t}\right)=0$ sein, d.h. alle Nichtfreizeit fließt in die Produktion von Humankapital. Gleichung (3.44) würde zu

$$
\hat{h}_{t}=h_{t}^{\beta-1} \varepsilon
$$

Für den Fall $\beta<1$ wird $\hat{h}_{t}$ mit steigendem Humankapital gegen Null konvergieren. Das Wachstum des Humankapitals kommt zum Erliegen und folglich wird auch die Wachstumsrate

96 Vgl. Lucas (1988, S. 18). 
des Pro-Kopf-Konsums gegen Null gehen. Die Volkswirtschaft kann kein endogenes Wachstum erreichen.

Bei der Besprechung des AK-Modells wurden als eine Bedingung für positives gleichgewichtiges Wachstum konstante Skalenerträge bzgl. aller akkumulierbaren Produktionsfaktoren genannt. Diese Eigenschaft erfullt das Modell von Lucas, wie weiter oben gezeigt wurde. Tatsächlich ist diese Bedingung nicht hinreichend, denn auch die Produktionsfunktionen der akkumulierbaren Inputs muß insgesamt konstante Skalenerträge aufweisen.

Sind diese Bedingungen erfullt, ist im Modell von Lucas auch ohne exogenen technischen Fortschritt dauerhaftes Pro-Kopf-Wachstum möglich. Die Entscheidungen der Wirtschaftssubjekte über Sparen und Investieren spiegeln sich in beiden gleichgewichtigen Wachstumsraten, im Einfluß der intertemporalen Substitutionselastizität und der Diskontrate wider. Treten bei diesen Größen Veränderungen ein, wird dies anhaltende Konsequenzen für die Wachstumsrate des Modells haben. Nimmt man z.B. den Standardfall mit $(\sigma=1)$ an und steigt die Bereitschaft zum Konsumverzicht, d.h. sinkt die Zeitpräferenzrate ab, wird c.p. (3.42) zunehmen. Die Verbindung zwischen Sparen-Investieren und Steady State-Wachstumsrate ist hergestellt.

Welche Konsequenzen ergeben sich nun für die Konvergenzüberlegungen? Unterscheiden sich Volkswirtschaften in ihren Parameterwerten, z.B. unterschiedliche Effizienz des Bildungswesens, werden sie unterschiedliche, langfristige Wachstumsraten realisieren. Sind aber alle Parameter identisch, kann es keinen Unterschied hierbei geben. In solch einer Situation spielt der Startpunkt, d.h. das anfägliche Einkommen und die Faktorausstattung eine Rolle. Aufgrund der Annahme konstanter Skalenerträge bzgl. des Humankapitals bei der Produktion von selbigem, gibt es keinen Grund für schnelleres Wachstum von humankapitalarmen Volkswirtschaften. Das Modell kann dauerhafte Lücken zwischen den Einkommensniveaus erklären, nicht jedoch Unterschiede bei den Wachstumsraten. ${ }^{97}$

Bei dem neoklassischen Grundmodell hat sich gezeigt, daß internationale Kapitalflüsse Konvergenz zwischen einzelnen Volkswirtschaften erzeugen können. In Gleichung (3.39) führt eine Modifikation der Humankapitalbildung zu einer Verbindung zwischen verschiedenen Volkswirtschaften. Als weiteres Argument kann ein externer Effekt des durchschnittlichen Humankapitalbestandes aller Volkswirtschaften der Erde oder der führenden Volkswirtschaften eingeführt werden. Unterstellt man in allen Ländern die gleiche Aufteilung der Nichtfreizeit, kann gezeigt werden, daß sich die Humankapitalbestände der Volkswirtschaften annähern und somit auch die Einkommensniveaus. Die Veränderung des Humankapitals eines Landes könnte in der Form

$$
\dot{h}_{t}=\varepsilon\left(1-u_{t}\right) h_{t}^{1-\theta} H_{t}^{\theta}
$$

97 Vgl. Lucas (1988, S. 25ff.). In einem kurzen Papier aus dem Jahre (1990) verwendet Lucas die Grundstruktur des hier vorgestellten Modells, um zu skizzieren, warum unterschiedliche Bestände an Humankapital die Durchfuhrung von Direktinvestitionen verhindern kann. Vgl. Lucas (1990). 
geschrieben werden, wobei $\left(\mathbf{H}_{t}\right)$ den Wert des durchschnittlichen Humankapitals aller Länder darstellt. Für die Wachstumsrate des länderspezifischen Humankapitals würde folgen:

$$
\frac{\dot{h}_{t}}{h_{t}}=\varepsilon\left(1-u_{t}\right)\left(\frac{H_{t}}{h_{t}}\right)^{\theta}
$$

Je höher der relative individuelle Bestand des Humankapitals ist, desto geringer wird die Wachstumsrate des Humankapitalbestandes sein. Die relative Position der Volkswirtschaften mit unterdurchschnittlicher Ausstattung mit Humankapital verbessert sich.98

Der von Lucas gewählte Ansatz stellt somit einen Schritt zur Endogenisierung der Steady State-Wachstumsrate dar. Die explizite Berücksichtigung des Humankapitals als eigenständigen Produktionsfaktor scheint auf den ersten Blick einen Unterschied zu den Ansätzen auszumachen, die im vorigen Abschnitt behandelt wurden. Tatsächlich aber stellt die Vorgehensweise von Lucas nur eine Aufgliederung des weiten Begriffs Kapital dar. Die Trennung zwischen Sach- und Humankapital als die beiden akkumulierbaren Inputfaktoren im Modell verlangt weiterhin die Existenz konstanter Skalenerträge bzgl. des Aggregats beider Variablen, d.h. die Summe der Produktionselastizitäten muß Eins sein. Darüber hinaus muß auch die Herstellung dieser Inputs bzgl. der vermehrbaren Produktionsfaktoren konstanten Skalenerträgen unterliegen. Der Vorteil dieser Disaggregierung, wenn man sie denn so nennen möchte, liegt bei der Möglichkeit, den Einfluß weiterer Größen auf die langfristige Wachstumsrate zu zeigen. Der grundsätzliche Weg zu endogenem Wachstum wird hiervon nicht berührt.

\subsubsection{Weitere Anwendungen des Humankapitalkonzepts}

Das Papier von Lucas (1988) hat eine wahre Flut von Veröffentlichungen ausgelöst, in denen die Rolle des Humankapitals für endogene Wachstumsprozesse und die Generierung des technischen Fortschritts diskutiert und untersucht werden. An dieser Stelle sollen einige wesentliche Aussagen verschiedener Modelle dargestellt werden, ohne im einzelnen auf die exakten formalen Strukturen einzugehen. Im Vordergrund stehen vielmehr die jeweiligen Vorstellungen über die Wirkungsweise der Humankapitalakkumulation.

98 Vgl. hierzu Lucas (1993, S. 254f.). 


\section{Humankapital und technischer Fortschritt}

Die Produktionsfunktion (3.36) enthielt als Lageparameter den Stand des technischen Wissens (A), der als konstant angenommen wurde. Eine realistischere Annahme wäre jedoch, nicht nur die Güterproduktion, sondern auch das technologische Wissen vom Humankapitaleinsatz abhängig zu machen. Eine Möglichkeit wäre hierzu, vergleichbar mit der Akkumulationsgleichung des Humankapitals bei Lucas (1988), eine Produktionsfunktion für den Stand der Technik bzw. dessen Veränderungen einzuführen. Ein Beispiel hierfür sind die Ansätze von Ziesemer $(1987,1991)$, in denen das Verhältnis zwischen dem vorhandenen Humankapital in einer Volkswirtschaft und dem Bestand an unqualifizierten Arbeitnehmern sowie das vorhandene Wissen Inputfaktoren in der linear-homogenen Funktion zur Generierung neuen technischen Wissens sind. ${ }^{99}$ Diese Modellierung berücksichtigt die Tatsache, daß neues Wissen nur durch den Einsatz von Know-how und qualifiziertem Personal entstehen kann. Das Humankapital wiederum entsteht durch den Einsatz von unqualifizierter Arbeit, dem Stand des technischen Wissens und dem davon zu unterscheidenden Bestand an wissenschaftichem Know-how. Auch diese Produktionsfunktion ist linear-homogen.

Die Annahmen über die jeweiligen Akkumulationsgleichungen der diversen Kapitalarten führen zur Abhängigkeit der langfristigen Wachstumsrate des System von den Investitionen in die Grundlagenforschung und das Humankapital. Des weiteren ist die Rate des technischen Fortschritts abhängig von der Verteilung der traditionellen Produktionsfaktoren (Arbeit und Kapital) auf Produktions- und Bildungszwecke.

\section{Humankapital und Armutsfallen}

Interessante Schlußfolgerungen ergeben sich aus der Kombination der Überlegungen von Lucas (1988) und von Romer (1986a), d.h. der Aggregation von Humankapital und die Existenz von Externalitäten. Unterstellt man z.B., daß die Ertragsrate von Investitionen in Humankapital mit dem schon vorhandenen Bestand an Humankapital positiv korreliert sind, d.h. daß steigende Grenzerträge vorliegen, oder wird angenommen, daß externe Effekte der Humankapitalbildung erst ab einer bestimmten Höhe des Humankapitalstocks eintreten, sind unterschiedliche Steady State-Lösungen möglich. 100 M.a.W., es sind multiple Gleichgewichte denkbar. Die Annahme steigender Ertragsraten der Investitionen in Humankapital läßt sich mit der Überlegung rechtfertigen, daß gute Ausbildung den Einsatz von qualifizierten Lehrkräften erfordert bzw. für das Erlernen komplizierter Zusammenhänge ein gewisses Basiswissen vorliegen muß. Die Abhängigkeit der Externalitäten vom Überschreiten eines gewissen Grenzwertes kann z.B. durch die Notwendigkeit, einen bestimmten absoluten Wissensstand besitzen

99 Die erste Arbeit Ziesemers wurde also vor der von Lucas veroffentlicht.

$100 \mathrm{Vgl}$. zur ersten Annahme Becker/Murphy/Tamura (1990), zur zweiten Azariadis/Drazen (1990). 
zu müssen, um erst von dem Humankapital anderer profitieren zu können, modelliert werden. Ursache hierfür ist die Wahlmöglichkeit der Volkswirtschaft zwischen verschiedenen Formen der Investition. Entweder lassen die Wirtschaftssubjekte Ressourcen in die Bildung von Sachkapital oder von Humankapital fließen. ${ }^{101}$ Befindet sich nun eine Volkswirtschaft in einer Situation, in der die Ertragsrate des Sachkapitals über derjenigen des Humankapitals liegt, werden die investiven Mittel in den erstgenannten Bereich fließen. Geht man des weiteren von der traditionellen Annahme der abnehmenden Grenzproduktivität des Kapitals aus, d.h. werden die Inada-Bedingungen für diesen Produktionsfaktor erfüllt, schwenkt die Volkswirtschaft auf einen Wachstumspfad ein, wie er aus dem neoklassischen Grundmodell bekannt ist. Hat die Volkswirtschaft dagegen den entscheidenden Schwellenwert für das Humankapital erreicht, d.h. ist der kritische Wert überschritten, bei dem Externalitäten des Humankapitals auftreten, und liegt dessen Ertragsrate über derjenigen des physischen Kapitals, konzentriert sich die Ökonomie auf die Investition in Humankapital und kann aufgrund der steigenden Grenzerträge des Humankapitals endogenes Wachstum realisieren. Die Implikationen dieser Ansätze stehen somit in der Tradition von Rostows "Take-off"-Hypothese. 102

Modellüberlegungen dieser Art legen nahe, daß der Bestand an Humankapital eine notwendige Voraussetzung für erfolgreiches Wachstum ist. Azariadis/Drazen (1990) überprüften ihr Modell mit Schwellenexternalitäten an 32 Ländern. Als Humankapitalvariable wurde die Alphabetisierungsrate gewählt. Es zeigte sich, daß nur Länder mit hohen Werten bei diesem Indikator hohe Wachstumsraten erzielen konnten. Die Aussagen dieser Ansätze harmonieren auch mit dem Konzept der social capability, das die Erfüllung gewisser Voraussetzungen forderte, um vorliegende Produktivitätspotentiale ausnutzen zu können. Ein weiteres Anwendungsbeispiel ist die Situation der Bundesrepublik Deutschland nach dem Zweiten Weltkrieg. Die bundesdeutsche Volkswirtschaft hatte einerseits einen hohen Humankapitalstock und andererseits einen Sachkapitalstock zur Verfügung, der durch geringe (Reparatur)Investitionen zur Überwindung von Flaschenhälsen hoch produktiv war, wodurch weitere Investitionen in beide Kapitalarten hohe Ertragsraten brachten, so daß ein selbsttragender Wachstumsprozeß entstehen konnte. Selbstverständlich spielten noch eine Reihe anderer Faktoren eine wichtige Rolle im Wirtschaftswunder, ganz von der Hand zu weisen sind die Überlegungen jedoch nicht. 103

Die angeführten Beispiele machen deutlich, wie vielfältig die Anwendungsmöglichkeiten der Überlegungen zum Humankapital im Rahmen eines endogenen Wachstumsmodells sind.

101 Becker/Murphy/Tamura (1990) beziehen eine dritte Moglichkeit mit ein, die insbesondere in unterentwickelten Volkswirtschaften eine Rolle spielt: die Erhöhung der Kinderzahl als Mittel zur Alterssicherung.

102 Vgl. Rostow (1990b).

103 Vgl. zur Situation des Kapitalstocks Westdeutschlands in der Nachkriegszeit vor allem Krengel (1958, 1963). Die marginale Kapitalproduktivităt betrug $1949 / 50$ z.B. 1, wăhrend ansonsten ein Wert von 0,25 normal ist. 
Angemerkt werden muß, daß die Funktion dieses Inputfaktors auch von einer anderen Größe erfült werden kann, welche die entsprechenden Eigenschaften besitzt.

\subsubsection{Humankapital und learning by doing}

In den bisherigen Modellen zum Humankapital wurde dessen beabsichtigte Akkumulation angenommen, d.h. die Wirtschaftssubjekte standen vor der Entscheidung, ihr Zeitbudget auf die verschiedenen Aktivitäten aufzuteilen. Im Ansatz von Arrow spielt dagegen das learning by doing-Konzept die entscheidende Rolle für den Wachstumsprozeß. Auch "beiläufiges" Lernen erhöht die Fähigkeiten der Arbeitnehmer. Ein weiterer Schritt wäre nun beide Arten der Humankapitalgenerierung zusammen zu betrachten. Ein erster Ansatz in diese Richtung wurde von Lucas (1988) gemacht. 104

Ausgangspunkt ist die Überlegung, daß durch die Produktionsmenge (x) eines Gutes (i) das sektorspezifische Humankapital bestimmt wird. Das durch Lerneffekte entstandene Knowhow kann nun nur in dem Bereich eingesetzt werden, in dem es entstanden ist. Die produzierte Menge des Gutes wiederum ist abhängig vom vorhandenen Bestand an Humankapital (h), der Zeit, die für die Produktion verwendet wird, und der Menge der eingesetzten Arbeitnehmer mit einem bestimmten Niveau an Humankapital $(\mathrm{N})$. Zur Vereinfachung nimmt Lucas an, daß nur die durchschnittlichen Bestände des Humankapitals eine Rolle spielen. Eine Unterscheidung zwischen internen und externen Effekten entfällt. ${ }^{105}$ Formal können diese Überlegungen durch folgende Gleichungen ausgedrückt werden:106

$$
\begin{aligned}
& x_{i}=h_{i} u_{i} N \\
& \dot{h}_{i}=h_{i} \varepsilon_{i} u_{i}
\end{aligned}
$$

(Produktionsmenge des Gutes i)

(Veränderung des Humankapitals in Sektor i)

Die Akkumulation des Humankapitals beinhaltet neben der Arbeitszeit $\left(u_{i}\right)$ und dem schon vorhandenen Bestand an Humankapital $\left(h_{i}\right)$ auch einen sektorspezifischen Effizienzparameter $\left(\varepsilon_{\mathrm{i}}\right)$, der den Unterschied zwischen den Sektoren ausmacht. Er stellt das Maß für die Lern

104 Das zweite von Lucas (1988) behandelte Modell wird im Gegensatz zu dem zuvor diskutierten in der Literatur vielfach ubergangen.

$105 \mathrm{Vgl}$. Lucas (1988, S. 27f.). Interessant ist, daß Lucas an dieser Stelle auf die Tatsache hinweist, daß die Beachtung des durchschnittlichen Humankapitalniveaus den Unterschied zwischen internen und externen Effekten hinfallig macht, wahrend er ihn zuvor einfach ubergeht.

106 Die Zeitindizes wurden zwecks übersichtlicher Darstellung weggelassen. 
effekte dar. Je höher $\left(\varepsilon_{\mathrm{i}}\right)$ ist, desto schneller wächst das Humankapital in einem Sektor, um so höhere Produktivitätswirkungen entstehen wiederum in der Herstellung des entsprechenden Gutes. 107

Gleichung (3.48) besitzt keine abnehmenden Grenzerträge des Lernens, sondern die Wachstumsrate des Humankapitals bleibt dauerhaft konstant. Wie zuvor gesehen, ist diese Annahme wichtig für die Existenz dauerhaften Wachstums. Tatsächlich aber nehmen die Lerneffekte entsprechend dem Lernkurvenkonzept mit der Zeit ab. Ein Trick dennoch die getroffene Annahme aufrechterhalten zu können, ist die Vorstellung ständig neu hinzu kommender Produkte, die immer wieder neue Impulse geben. 108

Unter der Annahme der Profitmaximierung der Unternehmen und einer Nutzenfunktion mit konstanter Substitutionselastizität ${ }^{109}$ ergeben sich für das Spezialisierungsmuster der Volkswirtschaft folgende Aussagen:

$$
\begin{aligned}
& \frac{1-u_{1}}{u_{1}}=\left(\frac{\alpha_{2}}{\alpha_{1}}\right)^{\varphi}\left(\frac{h_{2}}{h_{1}}\right)^{\varphi-1} \\
& \frac{x_{2}}{x_{1}}=\left(\frac{\alpha_{2}}{\alpha_{1}}\right)^{\varphi}\left(\frac{h_{2}}{h_{1}}\right)^{\varphi}=\left(\frac{\alpha_{2}}{\alpha_{1}}\right)^{\varphi} \mathrm{q}^{-\varphi}
\end{aligned}
$$

Gleichung (3.49) gibt die Aufteilung der Arbeitszeit auf die Produktion beider Güter an, Gleichung (3.50) zeigt das Verhältnis zwischen den produzierten Mengen. Der Faktor (q) steht für das Preisverhältnis zwischen den Gütern im Gleichgewicht, das gleichzeitig durch das umgekehrte Verhältnis der anfänglichen Humankapitalausstattungen bestimmt ist. Mit Hilfe des Entwicklungspfades für das Preisverhältnis lassen sich nun Schlußfolgerungen für den weiteren Verlauf der Entwicklung treffen. Es gilt:

$$
\hat{\mathrm{q}}=\left(\varepsilon_{1}+\varepsilon_{2}\right)\left[1+\left(\frac{\alpha_{2}}{\alpha_{1}}\right)^{\varphi} \mathrm{q}^{1-\varphi}\right]^{-1}-\varepsilon_{2}
$$

107 Das von Lucas angewandte Konzept geht auf Krugman (1987) zuruck, der mit Hilfe eines Modells der komparativen Vorteile, zum einen die Rolle der Lernkurve für die Wettbewerbsfahigkeit von Industrien zeigen wollte, zum anderen die Probleme der niederlăndischen verarbeitenden Industrie untersuchen wollte (S. 114ff.), die durch die Erdolfunde auftraten. Auch die Geldpolitik der Thatcher-Ära kommt bei ihm zur Sprache (S. 116ff.).

108 Vgl. Lucas (1988, S. 28).

109 Die Nutzenfunktion hat die Form: $U\left(x_{1}, x_{2}\right)=\left[\alpha_{1} x_{1}^{-\rho}+\alpha_{2} x_{2}^{1-\rho}\right]^{-1 / p}$, wobei bekanntermaßen die

Substitutionselastizităt $(\varphi)$ durch $1 /(1+\rho)$ bestimmt ist. Es wurde extra eine andere Variable für die Substitutionselastizităt gewăhlt, um die inhaltlichen Unterschiede zur intertemporalen Substitutionselastizităt in Erinnerung zu rufen. Natürlich entspricht $(\rho)$ auch nicht der Diskontrate der Wirtschaftssubjekte. Vgl. auch Lucas (1988, S. 28). 
Je nach Substitutionselastizität ergeben sich unterschiedliche Ergebnisse. Für den Fall $\varphi=1$, bestimmen $\left(\alpha_{1}\right)$ und $\left(\alpha_{2}\right)$ die Verteilung der Arbeitskräfte auf die einzelnen Sektoren. Das Verhältnis der Preise wächst mit konstanter Rate und somit auch das Verhältnis der produzierten Güter. Für den Fall $\varphi<1$, wird sich eine stabile Allokation einstellen, in der das Arbeitsvolumen entsprechend der Bedingung $u_{1} \varepsilon_{1}=\left(1-u_{1}\right) \varepsilon_{2}$ aufgeteilt wird. Gilt $\varphi>1$, wird sich die Volkswirtschaft auf das eine oder andere Gut spezialisieren. Aufgrund von Gleichung (3.51) läßt sich ein "kritischer" Wert ( $q^{\text {krit }}$ ) für das anfängliche Preisverhältnis $\left(q_{0}\right)$ ermitteln, bei dem die Ökonomie in einer stabilen Spezialisienungssituation verharrt, da $\hat{q}=0$ ist. Liegt $\left(q_{0}\right)$ über

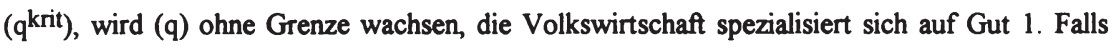
$\left(\mathrm{q}_{0}\right)<\left(\mathrm{q}^{\mathrm{krit}}\right)$, wird Gut 2 sich durchsetzen. Entscheidend sind somit die Erstausstattungen an sektorspezifischen Humankapital. Eine Volkswirtschaft wird sich auf das Gut konzentrieren, bei dem es Vorteile hat. ${ }^{110}$

Gibt man die Annahme einer geschlossenen Volkswirtschaft auf und fürt Freihandel sowie eine Reihe kleiner Volkswirtschaften ein, übernimmt das Verhältnis der Weltmarktpreise beider Güter die Rolle von (q) im geschlossenen Modell. Wieder werden sich die Länder mit einem anfänglichen Humankapitalverhältnis (h1/h2) $>\mathrm{p}$ zugunsten des Produkts eins entscheiden, die anderen für Produkt zwei. Schließt man die Möglichkeit zum Wechsel der Spezialisierung aus, d.h. sind Entscheidungen für ein Gut nicht mehr umkehrbar, wird unter der Annahme $\varphi>1$ zu dauerhaft höheren Wachstumsraten der Volkswirtschaften führen, die sich auf das Gut mit hohen Lerneffekten konzentriert haben. Die durch den komparativen Vorteil entstandene Spezialisierung bedingt eine Verbesserung dieses Vorteils. Die Wachstumsraten einzelner Volkswirtschaften können sich somit unterscheiden, während sie für jedes einzelne Land aufgrund der vorgegebenen Produktionstechnologien für das Endprodukt und die Lerneffekte konstant sind. ${ }^{111}$

Die Ergebnisse dieses Modells haben Konsequenzen für die Bedeutung des Freihandels. Länder mit komparativen Vorteilen bei Gütern, die nur geringe Produktivitätsfortschritte erlauben, werden dauerhaft nicht gegenüber technologisch führenden Volkswirtschaften aufholen können. Das einmal festgelegte Handelsmuster determiniert die langfristige relative Stellung einer Ökonomie. Tatsächlich lassen sich aber Veränderungen im Länderranking feststellen. Die südostasiatischen Volkswirtschaften sind Beispiele für erfolgreiche Aufholprozesse. Um diese Entwicklungen besser erfassen zu können, sind Modifikationen des einfachen Modells erforderlich. Insbesondere die Modellierung der Lerneffekte bietet sich hier an. Die Vorteile, die aus einer ständigen Wiederholung von Produktionsprozessen möglich sind, sind begrenzt, d.h. die Rate des Lernens nimmt ab. Wenn dem aber so ist, kann auch das Wachstum der Volkswirtschaft gegen Null gehen. Eine Möglichkeit zur Überwindung dieses Problems besteht in der Einführung ständig neuer Güter, die vergleichbar mit den Investitionen im 
Ansatz von Arrow (1962) die Gelegenheit zum Lernen bieten. ${ }^{112}$ In dieselbe Richtung gehen Überlegungen, die von einem Spektrum von Gütern ausgehen, von dem entsprechend der Präferenzen der Wirtschaftssubjekte immer nur ein Teil produziert wird. Pro Gut sind zwar die Lerneffekte begrenzt, jedoch entsteht aufgrund externer Effekte des Lernens innerhalb einer Industrie auf alle anderen Industrien sowie der Verschiebung der Güterstruktur dauerhaftes Wachstum. ${ }^{113}$

All diesen Ansätzen ist gemeinsam, daß sie zum selben Ergebnis kommen wie das Ursprungsmodell von Lucas (1988). Die Faktorausstattungen der Länder bestimmen dauerhaft die Position der einzelnen Volkswirtschaften, während Aufholprozesse nicht möglich sind und Freihandel die Länder mit schlechten Startvoraussetzungen bei den möglichen Wachstumsraten benachteiligt. ${ }^{114}$ Die Modelle zeigen, daß Freihandel zwar ganz im Sinne der traditionellen neoklassischen Handelstheorie die Wohlfahrt aller beteiligten Länder erhöht, die relativen Positionen beim Einkommen sich aber verschlechtern können. ${ }^{115}$

\subsubsection{Fazit}

Der Überblick über die Behandlung des Produktionsfaktors Humankapital im Rahmen der Neuen Wachstumstheorie hat gezeigt, daß sowohl die geplante Investition in Humankapital als auch Lerneffekte einen Beitrag zu endogenem Wachstum liefern können. Eine strikte Trennung zwischen beiden Sachverhalten kann zwar im Modell aufrechterhalten werden, realiter finden beide Formen der Humankapitalbildung parallel statt. Auch ist eine genaue Abgrenzung von den Ansätzen, die sich mit Wissensexternalitäten befaßt haben, nur schwer möglich, da Analogien zwischen Sach- und Humankapital gemacht werden. Die Ansätze mit expliziter Berücksichtigung des Humankapitals gehen jedoch einen Schritt weiter als die hoch aggregierten Modelle, da ein zweiter Sektor eingeführt wird, dessen Produktionsbedingungen mit entscheidend für die Existenz von endogenem Wachstum sind. Sie bieten somit einen besseren Einblick in und tiefergehende Erklärungsmöglichkeiten von Wachstumsprozessen. Dies könnten jedoch auch Modelle mit verschiedenen Wissensarten (gebunden-ungebunden, rivalisierend-nicht-rivalisierend) leisten. Die Einführung des zusätzlichen Inputfaktors Humankapital verändert deshalb in einem ersten Schritt nichts an den Möglichkeiten des Modells, sondern stellt primär eine begriffliche Veränderung dar. Der große Vorteil gegenüber den Modellen, die in der Tradition von Romer (1986a) stehen, ist die Behandlung des Wissens als unabhängigen

112 Vgl. z.B. Stokey $(1988,1991)$

113 Vgl. hierzu Young (1991, insbesondere S. 376f.).

114 Im Modell von Young sind bei geringen Abständen zwischen entwickelten und nicht entwickelten Lăndern mit Hilfe der Skalenerträge, die sich aus großen Bevölkerungszahlen ergeben, theoretisch Aufholprozesse vorstellbar. (Vgl. Young 1991, S. 396ff.)

$115 \mathrm{Zu}$ einer Kombination dieser Überlegungen siehe Lucas (1993). 
Produktionsfaktor. Die Generierung und die Wirkungen des Humankapitals bzw. Wissens werden unabhängig von Sachkapitalinvestitionen, was einen Schritt zu mehr Realitätsnähe bedeutet.

\subsubsection{Produktinnovationen und endogenes Wachstum}

\subsubsection{Vorbemerkungen}

In den bisher dargestellten Modellen wurde endogenes Wachstum maßgeblich durch die Existenz zumindest konstanter Skalenerträge der gesamtwirtschaftlichen Produktionsfunktion bzgl. der vermehrbaren Produktionsfaktoren erreicht. Insbesondere das Vorliegen von Externalitäten bei der Akkumulation dieser Inputfaktoren bildete die Ursache für die Aufrechterhaltung dieser Annahme. Darüber hinaus wurde bei der Diskussion der Modelle, die neben dem Sachkapital einen weiteren akkumulierbaren Produktionsfaktor, also Humankapital, einführten, die Notwendigkeit konstanter Skalenerträge bei der Herstellung dieses zusätzlichen Inputs deutlich. Sämtlichen Ansätzen dieser Art ist die Steigerung der Produktivität des Faktoreinsatzes bei anhaltendem Akkumulationsprozeß gemein. Das entstehende Wachstum stellt aber nicht zwangsläufig technischen Fortschritt dar, sondern ist das Ergebnis einer zunehmenden Kapitalintensivierung. Nur wenn Rückwirkungen auf den Bestand des technologischen Wissens unterstellt werden, kann von technischem Fortschritt im traditionellen Sinne gesprochen werden. Diese Problematik wird bei der Diskussion der allgemeinen Kritik an den Ansätzen der Neuen Wachstumstheorie noch einmal angesprochen. Im Moment bleibt festzuhalten, daß sich die Endogenisierung des technischen Fortschritts in den bis an diese Stelle besprochenen Modellen auf Prozeßinnovationen bezog. Tatsächlich ist die technologische Entwicklung nicht ausschließlich durch die Steigerung der Produktivität der Produktionsfaktoren gekennzeichnet, sondern kommt auch durch das Entstehen neuer Produkte zum Ausdruck. Oft gehen beide Prozesse Hand in Hand. Vor allem neuentwickelte Kapitalgüter stellen Produktinnovationen dar, die in den Sektoren, in denen sie zum Einsatz kommen, Prozeßinnovationen sind. Die Erfindung des Rads ist zum einen eine Produktinnovation, zum anderen aber auch eine Verbesserung der Produktion des Gutes Transportleistung. Die Konsequenzen von Produktinnovationen bilden den Inhalt einer weiteren Gruppe von Modellen, die innerhalb der Neuen Wachstumstheorie entstanden ist und im folgenden dargestellt werden soll.

\subsubsection{Die Basisideen}

Die im folgenden zu untersuchenden Ansätze versuchen den Zusammenhängen zwischen der Entwicklung neuer Produkte, d.h. Produktinnovationen, und der langfristigen Wachstumsrate 
einer Volkswirtschaft besser gerecht zu werden als die Modelle mit gesamtwirtschaftlichen Produktionsfunktionen. Die zentrale Idee dabei ist, daß Forschungs- und Entwicklungsaktivitäten zum Entstehen neuer Güter führen, die als Ursache eines dauerhaften Wachstumsprozesses zu sehen sind. Die Vertreter dieses Zweigs der Neuen Wachstumstheorie stehen somit in der Tradition von Joseph A. Schumpeter (1934) und seinen Überlegungen zum Proze $\beta$ der schöpferischen Zerstörung.

Produktinnovationen können in verschiedener Form aufteten. Zum einen kann zwischen Kapital- und Konsumgütern unterschieden werden, d.h. die Modellierung der Effekte von neuentwickelten Produkten kann durch Einführung eines Sektors für Investitionsgüter oder durch die Aufspaltung des Outputs in viele einzelne Konsumgüter erfolgen. Neu hinzukommende Kapitalgüter werden die Produktivitätsverhältnisse der Unternehmen verbessern, die das Endprodukt herstellen, während neue Konsumgüter die Nutzenfunktion der Haushalte beeinflussen. Produktinnovationen und Prozeßinnovationen lassen sich somit nicht genau trennen. In beiden Fällen ergeben sich daraus unter der Annahme gewinn- bzw. nutzenmaximierender Wirtschaftssubjekte Impulse für den Wachstumsprozeß. Zum anderen schlagen sich Forschungs- und Entwicklungstätigkeiten in der Verbesserung der Qualität schon bestehender Güter nieder, d.h. das Ergebnis von FuE-Anstrengungen sind nicht neue, sondern bessere Produkte. Auch unter dieser Annahme lassen sich Wirkungen auf die Gewinn- bzw. Nutzenfunktion der Wirtschaftssubjekte darstellen. Die Grenze zwischen Erhöhung der Anzahl der produzierten Güter und der Verbesserung der Qualität der Güter ist dabei fließend. Insbesondere die Arbeiten von Grossman/Helpman (z.B. 1991) haben die große Übereinstimmung zwischen den Modellergebnissen bei beiden Annahmen gezeigt.

Ein gemeinsames Element aller Modelle ist die schon oben angesprochene Rückbesinnung auf den Prozeß der schöpferischen Zerstörung, der von Schumpeter als die treibende Kraft der wirtschaftlichen Entwicklung innerhalb einer kapitalistisch organisierten Volkswirtschaft angesehen wurde. Die Abbildung einer solchen Entwicklung bedingt gewisse Veränderungen der Annahmen gegenüber den bisher angesprochenen Modellen. Gelingt es einem innovativen Unternehmer, ein neues Produkt zu entwickeln und es erfolgreich auf den Markt zu bringen, wird er zumindest temporär ein Monopol auf dieses Gut erhalten, d.h. er wird Extraprofite realisieren können. Sind keine Marktzutrittsschranken vorhanden, werden Imitatoren mit dem anfänglichen Monopolisten in Konkurrenz treten. Dieser wird mehr und mehr seine Position verlieren. Dies entspricht dem fünten Faktum auf der in den Vorbemerkungen zu diesem Kapitel vorgestellten Liste stilisierter Fakten. Aufgrund dieser Überlegung müssen die Modelle zumindest temporäre Monopolsituationen abbilden können, d.h. die Annahme der vollständigen Konkurrenz kann nicht aufrechterhalten werden, zumindest nicht für alle Märkte.

Ein weiterer wichtiger Punkt ist das zerstörerische Element des Innovationsprozesses. Neue Produkte, die sich durchsetzen, schränken je nachdem, ob es sich um additive oder substitutive Produkte handelt, die Nachfrage nach vorhandenen Produkten ein, wenn sie sie 
sogar nicht ganz verdrängen. Die Gewinnsituationen bisheriger Produzenten werden dadurch verschlechtert. Um die Folgen von Produktinnovationen beurteilen zu können, darf deshalb nicht nur der Vorteil für die Konsumenten und Hersteller der neuen Güter gesehen werden, sondern es müssen auch die Verluste der betroffenen Unternehmen aufgerechnet werden.

Als weiteres wichtiges Element ist die Tatsache $\mathrm{zu}$ sehen, daß Innovationen Volkswirtschaften aus einer Ruhelage, d.h. einer gleichgewichtigen Situation bringen können und. Anpassungsprozesse entstehen lassen. ${ }^{116}$ Trotz dieser Überlegung konzentrieren sich die Ansätze auf die Analyse von Steady State-Pfaden. Schwankungen im Wachstumsprozeß werden außen vor gelassen, so daß eine zentrale Konsequenz des FuE-Prozesses nicht berücksichtigt wird, sondern vielmehr außerhalb des Interesses der Autoren liegt.

Neben diesen direkt mit dem Untersuchungsgegenstand "Produktinnovationen" verbundenen Charakteristika, die in verschiedener Form und Intensität in den Strukturen der Modelle berücksichtigt werden, weisen diese auch Elemente auf, die in Verbindung mit den Überlegungen zum learning by doing und der Humankapitalbildung stehen. Auch in den in diesem Abschnitt zur Sprache kommenden Ansätzen der Neuen Wachstumstheorie werden explizit Externalitäten des Investitionsprozesses, hier der FuE-Investitionen, bzw. der öffentliche Gutscharakter des technologischen Wissens berücksichtigt. Die Existenz dieser kostenlosen Produktivitätseffekte bildet eine der zentralen Ursachen für dauerhaftes Wachstum. Führte das Vorliegen externer Effekte in den Modellen, für die stellvertretend die Namen Lucas und Romer stehen, zum Ergebnis, daß in einem Laisser-faire-Gleichgewicht die Wachstumsrate des Systems unterhalb der sozial optimalen Rate liegt, kann hier aufgrund der Effekte der Produktinnovationen auf die Ertragssituation bereits bestehender Unternehmen die umgekehrte Situation eintreten.

Im folgenden werden drei Möglichkeiten besprochen, wie Produktinnovationen in ein Modell endogenen Wachstums integriert werden können. Der Schwerpunkt wird hierbei vor allem auf der Frage nach den Gründen für endogenes Wachstum liegen, d.h. die Analyse wird sich auf die zentralen Gleichungen der Modelle konzentrieren.

\subsubsection{Innovationen und Humankapital: Ursachen und Folgen technischen Fortschritts}

Eines der ersten Modelle, das die oben angeführten Überlegungen abbilden soll, wurde von Romer $(1990 \mathrm{~b}, \mathrm{c})$ vorgestellt. Unter Verwendung eines dreisektoralen Modells greift Romer den schon häufiger diskutierten Aspekt rivalisierender und nicht-rivalisierender Inputs auf und kombiniert dieses Element mit der Überlegung, daß als Ergebnis von FuE-Investitionen eine

116 Die Reaktionen des okonomischen Systems auf exogene Stơrungen stehen im Mittelpunkt der Traversenanalyse. Hier sind insbesondere die Arbeiten von Lowe (1976) und von Hicks (1973) zu nennen. Vgl. hierzu auch Hagemann $(1990 \mathrm{c}, 1992,1996)$. 
steigende Zahl von Kapitalgütern entsteht. Romers Modell stellt deswegen eine Brücke zwischen den Arbeiten aus den Abschnitten (3.2.2) und (3.2.3) und denjenigen des folgenden Abschnitts dar und kommt seinen eigenen Ansprüchen an ein endogenes Wachstumsmodell am nächsten. 117

Der zentrale Produktionsfaktor in diesem Ansatz ist das Wissen, das sich in zwei Kategorien aufteilen läßt: Humankapital $(\mathrm{H})$ und allgemeines technologisches Wissen (A). Während das erste in seiner Nutzung rivalisiert, d.h. sich wie die anderen traditionellen Produktionsfaktoren verhält, ist das technologische Wissen ein öffentliches Gut, das beliebig hăufig eingesetzt werden kann. Das Humankapital kann dagegen als personengebunden interpretiert werden, das nur dort zur Anwendung kommen kann, wo sein Träger tătig wird. Die gesamte Menge des Humankapitals entspricht immer der Summe aus den in den verschiedenen Sektoren eingesetzten Mengen, d.h. $H=\Sigma_{i} H_{i}$. Ein Indikator für (A) stellt die $Z$ ahl der verschiedenen Kapitalgüter dar, die eine Volkswirtschaft aufgrund ihres Wissens produzieren kann. ${ }^{118}$

Die primäre Produktionsstufe ist ein Forschungssektor, in dem unter Einsatz des vorhandenen allgemeinen technischen Wissens und dem hier eingesetzten Humankapital neues Knowhow entsteht. Das Ergebnis eines erfolgreich abgeschlossenen FuE-Projekts ist ein sog. Design, das das Wissen über ein Herstellungsverfahren für ein neues Kapitalgut umfaßt. Die Zahl der möglichen neuen Herstellungsverfahren ist abhängig von der Menge an Humankapital, die in diesen Bereich fließt und der Höhe des schon vorhandenen Wissens. Je mehr Know-how eine Generation der nächsten hinterläßt, um so mehr Designs kann diese mit einer gegebenen Menge an Produktionsfaktoren entwerfen. Heutige Forschung hat positive externe Effekte auf morgige Forschungsprojekte. Die Arrowsche Vorstellung über die Externalităten bei Investitionsvorhaben fließt auch hier ein. Die Produktionsfunktion für neues Wissen wird folgendermaßen dargestellt, wobei $(\varepsilon)$ ein Effizienzparameter ist: 119

$$
\dot{\mathrm{A}}=\varepsilon \mathrm{H}_{\mathrm{A}} \mathrm{A}
$$

Diese Funktion weist konstante Skalenerträge bzgl. des Humankapitals allein und steigende bzgl. beider Inputs auf und erfüllt damit die weiter oben angesprochene Bedingung für endogenes Wachstum, die an die Akkumulationsfunktion der vermehrbaren Produktionsfaktoren gestellt wird. Insbesondere die Linearität in bezug auf das Wissen ist für endogenes Wachstum relevant. Abnehmende Grenzerträge von (A) würden zu einer ständig sinkenden Wachstums-

117 Vgl. Romer (1994).

$118 \mathrm{Vgl}$. Romer (1990b, S. S79). Diese Unterscheidung erinnert an die von Gary Becker getroffene Differenzierung zwischen spezifischem und allgemeinem Humankapital.

119 Vgl. Romer (1990b, S. S83; 1990c, S. 345). Eine vergleichbare Darstellung findet sich bei dem schon unter dem Aspekt der Humankapitalwirkungen angesprochenen Modell von Ziesemer $(1987,1991)$. 
rate des Wissens führen, um im Steady State schließlich Null zu sein. Dies entspricht exakt den Anforderungen an die Humankapitalbildung im Lucas-Modell (1988). ${ }^{120}$

Die zweite Produktionsstufe bildet die Herstellung der Kapitalgüter, für die einerseits Konsumverzicht geübt werden, andererseits das Know-how über das jeweilige Produktionsverfahren vorhanden sein muß. Jedes einzelne Kapitalgut soll nur von einem einzigen Unternehmen produziert werden können, d.h. es gibt nur einen Eigentümer eines speziellen Designs. Das Unternehmen erwirbt dieses aus dem ersten Sektor. Aufgrund dieser Annahme ist der Markt für jedes Kapitalgut durch ein Monopol gekennzeichnet. Die Nachfragekurve für jedes Gut wird sich aus der Gewinnmaximierung im Endproduktsektor ergeben. Die tatsächlich produzierte Menge des Kapitalgutes (i) ergibt sich aus der Gewinnmaximierung des jeweiligen Monopolisten. Für die Akkumulationsgleichung des Kapitalstocks, d.h. das Aggregat aller unterschiedlichen Kapitalgüter ergibt sich somit ganz allgemein:

$$
\dot{\mathrm{K}}_{\mathrm{t}}=\mathrm{Y}_{\mathrm{t}}-\mathrm{C}_{\mathrm{t}}
$$

Der Kapitalstock (K) ergibt sich durch:

$$
K=\eta \sum_{i=1}^{A} x_{i} \quad \text { für den stetigen Fall: } \quad K=\eta \int_{i=1}^{A} x_{i} d i
$$

wobei $\left(\mathbf{x}_{\mathbf{i}}\right)$ für die Menge des Kapitalgutes (i) steht und $(\eta)$ den notwendigen Konsumverzicht angibt, der für die Herstellung eines Kapitalguts notwendig ist. Im weiteren wird die Unabhängigkeit von ( $\eta$ ) von der Art des Kapitalgutes angenommen, d.h. es muß für jede Kapitaleinheit im selben Umfang Konsumverzicht geübt werden. Da für alle Kapitalgüter dieselben Überlegungen bzgl. der Produktionstechnik und dem Verhalten der Produzenten gelten, werden von jedem Kapitalgut identische Mengen $(\bar{x})$ hergestellt. Da die Höhe des technologischen Wissens (A) durch die Zahl der Kapitalgüter definiert ist, d.h. A=n, kann (3.54) auch in folgender Form geschrieben werden: 121

$$
K=\overline{\mathbf{x}} \eta \mathbf{A}
$$

Die Produktion des Endprodukts ( $\mathrm{Y}$ ) wiederum macht den Einsatz von Arbeit (L), Humankapital $\left(\mathrm{H}_{\mathrm{Y}}\right)$ und aller bekannten Kapitalgüter notwendig: 


$$
Y=H_{Y}^{\alpha} L^{\beta} \int_{i=1}^{\infty} x_{i}^{1-\alpha-\beta} d i
$$

Die Produktionsfunktion unterliegt somit konstanten Skalenerträgen bzgl. aller Produktionsfaktoren. Die Unternehmen dieses Sektors stellen die Nachfrager der Kapitalgüter dar, die im zweiten Sektor produziert werden. Ihre Nachfragekurven für diese Güter werden sich entsprechend ihrer Zielsetzung der Gewinnmaximierung ergeben. 122

Im Gegensatz zum traditionellen neoklassischen Modell fließt das technologische Wissen (A) nicht direkt in die Produktion der letzten Stufe ein, sondern nur indirekt über seinen Beitrag zur Entwicklung neuer Designs und zur Herstellung eines spezifischen Kapitalgutes. An dieser Stelle kommt zur Nichtrivalität der Anwendung von (A) noch das Phänomen der teilweisen Nichtausschließbarkeit hinzu. Jedes Unternehmen der zweiten Produktionsstufe kontrolliert den Einsatz des jeweiligen Designs, d.h. niemand anderes darf mit diesem Kapitalgüter herstellen. Die Anwendung des Wissens im Forschungssektor entzieht sich aber dem Zugriff des Designeigentümers. Die Wirkungen des technologischen Wissens auf die Herstellung des Endprodukts werden deutlich, wenn (3.54') in (3.55) eingesetzt wird: 123

$$
Y=H_{Y}^{\alpha} L^{\beta} A \bar{x}^{1-\alpha-\beta}=H_{Y}^{\alpha} L^{\beta} A\left(\frac{K}{\eta A}\right)^{1-\alpha-\beta}
$$

bzw.

$$
\mathrm{Y}=\left(\mathrm{H}_{\mathrm{Y}} \mathrm{A}\right)^{\alpha}(\mathrm{LA})^{\beta} \mathrm{K}^{1-\alpha-\beta} \eta^{\alpha+\beta-1}
$$

Die Modellstruktur weist sowohl Merkmale des traditionellen neoklassischen als auch des Modells von Romer zu den Effekten der Spezialisierung auf. Es liegt Humankapital- und arbeitsvermehrender technischer Fortschritt vor. Wäre die Wachstumsrate von (A) exogen und konstant, würde sich keine Veränderung zu den Modellen aus Kapitel 2 ergeben. ${ }^{124}$ Die Erweiterungen bestehen in der zusätzlichen Berücksichtigung des Humankapitals und der genaueren Spezifizierung der Entstehung neuen Wissens bzw. neuer Kapitalgüter. Konsequenterweise werden sich zusätzliche Einflußgrößen für die gleichgewichtige Wachstumsrate des Outputs ergeben.

Unter Verwendung einer intertemporalen Nutzenfunktion sowie der Annahmen eines konstanten Bestands an Humankapital und Nullwachstum der Bevölkerung ermittelt Romer eine Steady State-Lösung für eine Laisser-faire- und eine sozial optimale Situation. Letztere

122 Vgl. Romer (1990b, S. S83 und S86). In seinem zweiten Papier zu dieser Modellstruktur wathlt Romer keine Cobb-Douglas-Produktionsfunktion, sondern eine allgemeine CES-Funktion. Vgl. (Romer, 1990c, S. 343f.). 
berücksichtigt die externen Effekte im Forschungssektor sowie die Konsequenzen der monopolistischen Strukturen im Kapitalgütersektor. Aufgrund der Preisstruktur in diesem Bereich erhält der Erfinder des Designs nicht den vollen sozialen Ertrag seiner Erfindung. Die Entlohnung des für Forschungszwecke eingesetzten Humankapitals fällt deshalb ohne einen allwissenden Planer zu gering aus. Konsequenterweise wird zu wenig Humankapital für FuEZwecke eingesetzt, die Wachstumsrate des technologischen Wissen ist niedriger als im sozialen Optimum. Die gleichgewichtigen Wachstumsraten des Outputs, des Kapitalstocks und des technologischen Wissens ergeben sich für die beiden Situationen wie folgt:

$$
\begin{array}{ll}
\hat{y}=\varepsilon H_{A}=\frac{\varepsilon H-\Lambda \rho}{\sigma \Lambda+1} & \text { mit } \Lambda=\frac{\alpha}{(1-\alpha-\beta)(\alpha+\beta)} \\
\hat{y}=\varepsilon H_{A}=\frac{\varepsilon H-\Theta \rho}{\sigma \Theta+(1-\Theta)} & \text { mit } \Theta=\frac{\alpha}{\alpha+\beta}
\end{array}
$$

(Laisser-faire)

(Soziales Optimum)

Die Höhe des Humankapitals $(\mathrm{H})$, die Effizienz der Forschung $(\varepsilon)$ bzw. das für Forschungszwecke eingesetzte Humankapital $\left(\mathrm{H}_{\mathrm{A}}\right)$ beeinflussen die Wachstumsrate positiv. Volkswirtschaften, die im Vergleich zu anderen einen hohen Humankapitalbestand haben, können bei gleicher Zeitpräferenz und intertemporaler Substitutionselastizität ein höheres Wachstum erreichen. Die Allokation der gegebenen Menge an Humankapital determiniert die Wachstumsmöglichkeiten. Eine wichtige Rolle spielt dabei der Zinssatz, d.h. die Ertragsrate des Kapitals. Die Wirtschaftssubjekte werden die Erträge des Humankapitals in der Forschung, die durch die zukünttigen Nettoeinkünfte aus einem Design gegeben sind, mit den Erträgen der Verwendung des Humankapitals in der Güterproduktion vergleichen. Aufgrund der notwendigen Abzinsung der Erträge aus der Forschung, mindert ein hoher Zinssatz deren Gegenwartswert und es wird lohnender mehr Humankapital in der Produktion einzusetzen. ${ }^{125}$ Diese Überlegung tauchte in gleicher Form schon im Modell von Becker/Murphy/Tamura (1990) auf. Auch ein Vergleich zum Modell von Lucas (1988) bietet sich an. Hier wie dort determinieren die Anfangsausstattungen der Ökonomie mit Produktionsfaktoren die mögliche Wachstumsrate.

Betrachtet man die Lösungen für die gleichgewichtigen Wachstumsraten (3.57) und (3.58), fält des weiteren die große Ähnlichkeit, insbesondere beim Zähler, mit der Lösung des AK-Modells auf. Die Position von ( $\varepsilon$ ) nimmt dort die Sparquote (s) ein, diejenige von $(\mathrm{H})$ hat dort der Stand des technologischen Wissens (A) inne. Trotz der anfänglichen Disaggregierung des gesamtwirtschaftlichen Kapitalstocks in (n) verschiedene Kapitalgüter ergibt sich inhaltlich kein großer Unterschied zum einfachen AK-Modell. Der Grund liegt im identischen Verhalten

125 In einem anderen Papier (1990c) ergeben sich aufgrund einer anderen gesamtwirtschaftlichen Produktionsfunktion Einflusse der Arbeitskraftezahl und der Hơhe des zur Herstellung eines Kapitalgutes notwendigen Konsumverzichts $(\eta)$. 
aller Kapitalgüterproduzenten, da durch diese Symmetrie jeder dieselbe Menge an $(\bar{x})$ produziert. In Verbindung mit Gleichung (3.54') wird nămlich wieder eine aggregierte Kapitalgröße in die gesamtwirtschaftliche Produktionsfunktion aufgenommen. In beiden Modellen ist das gleichgewichtige Wachstum um so höher, je höher der Bestand an Humankapital (Romer 1990b) bzw. das technologische Wissen (AK-Modell) ist. Fallen beide Größen zu gering aus, d.h. wird der Zähler der Wachstumsraten Null, wird auch kein positives Wachstum aufteten.

Die qualitativen Resultate scheinen somit kaum die Komplexität des Modells zu rechtfertigen. Tatsächlich berücksichtigt Romers Ansatz die Entwicklung des technologischen Wissens und geht somit einen Schritt weiter zu mehr Erklärungsgehalt. Im nächsten Abschnitt wird auf Modelle eingegangen werden, die weitere Eigenschaften von FuE-Prozessen zu berücksichtigen versuchen und hierbei auf Romers Modell (1990b, c) aufbauen.

\subsubsection{Wachstum als Folge neuer und besserer Güter}

Der Themenkomplex Produktinnovationen als Ursache endogenen Wachstum kann nicht nur an der Zahl der Kapitalgüter anknüpfen, sondern auch an deren Qualität bzw. an der Vielzahl und/oder Qualităt der Konsumgüter. Wichtige Beiträge wurden auf diesem Gebiet insbesondere von Grossman/Helpman (z.B. 1991) und Aghion/Howitt (z.B. 1992a,b) geleistet. 126 Während sich der Ansatz von Grossman/Helpman auf die Entwicklung von neuen bzw. besseren Konsumgütern konzentriert, befassen sich Aghion/Howitt mit den Folgen der Qualitätsverbesserungen von Kapitalgütern. Im folgenden wird zuerst die Basisstruktur des Modells zur Steigerung der Produktzahl bei Grossman/Helpman skizziert, um daran anschließend die sich ähnelnden Modelle zur Qualitätsverbesserung zu beleuchten.

\section{Die Enweiterung der Produktvielfalt}

Ähnlich zum oben besprochenen Modell von Romer wird im Modell von Grossman/Helpman zur Erweiterung der Produktvielfalt das Ergebnis des Innovationsprozesses in neuen Gütern, jedoch neuen Konsumgütern, gesehen. Die Zahl der in einer Volkswirtschaft vorhandenen Konsumgüter ist dabei ein Argument in der Nutzenfunktion der Konsumenten. Mehr Güter

126 Beide Autorenpaare haben aufbauend auf Basismodellen verschiedene Modifikationen durchgefuhrt, die an dieser Stelle nicht alle behandelt werden können. Hier interessieren vor allem die Grundstrukturen der Modelle, die für endogenes Wachstum verantwortlich sind. Ein kurzer Uberblick uber die diversen Ansatze von Grossman/Helpman findet sich bei Helpman (1992). Der Ansatz von Aghion/Howitt (1992a, b) hătte auch zusammen mit dem Modell von Romer $(1990 \mathrm{~b}, \mathrm{c})$ behandelt werden können. Die Ähnlichkeit der Modellergebnisse legt aber eine Besprechung zusammen mit den Überlegungen von Grossman/Helpman nahe. 
führen dabei zu einem höheren Nutzenniveau. Die Nutzenfunktion für einen repräsentativen Konsumenten ist wie folgt bestimmt:

$$
U_{t}=\int_{t}^{\infty} e^{-\rho(\tau-t)} \log D(\tau) d \tau
$$

In Gleichung (3.59) steht $D(\tau)$ für einen Konsumindex zum Zeitpunkt ( $\tau$ ), die Zeitpräferenz ist durch $(\rho)$ gegeben. Für die ansonsten in einer intertemporalen Nutzenfunktion enthaltene intertemporale Substitutionselastizität wurde der Wert eins angenommen. ${ }^{127}$ Der Konsumindex (D) ist dabei abhängig von der Zahl der vorhandenen Güter $(n)$ und jeweiligen Menge $\left(x_{i}\right)$, die von einem Gut produziert wird: 128

$$
D=\left[\int_{0}^{n} x_{i}^{\alpha} d i\right]^{1 / \alpha} \text { mit } 0<\alpha<1
$$

Die Modellwirtschaft weist einen Forschungs- und einen Produktionssektor auf. Der einzige Input in der Forschung ist Arbeit, die konstante Skalenerträge besitzt. Ein Unternehmen wird in die Forschung investieren, wenn der Gegenwartswert der zukünftigen Erträge aus einer erfolgreichen Entwicklung zumindest die Arbeitskosten deckt. Alle Güter, die bis zu einem bestimmten Zeitpunkt entworfen worden sind, werden im Produktionssektor der Volkswirtschaft ebenfalls unter Verwendung von Arbeit bei Vorliegen konstanter Skalenerträge produziert. Das Engagement in diesem Bereich hängt vom Verhältnis des erzielbaren Gewinns zu den möglichen Erträgen einer sicheren Kapitalanlage ab. Für den Arbeitsmarkt wird ein ständiges Gleichgewicht angenommen, d.h. die Summe der Arbeitsmenge im Forschungs- und Produktionssektor entspricht genau der angebotenen Menge an Arbeitskräften. Auch hier liegt eine weitere Parallele zu Romers Ansatz vor. An die Stelle der Arbeitskräfte tritt bei ihm die Menge des verfügbaren Humankapitals.

Gelingt einem Unternehmen die Entwicklung eines neuen Produkts, wird es auf dieses ein Monopol erhalten, das es bis zum Auftreten zusätzlicher Anbieter auch behält. Die Sicherung des Monopols kann durch einen umfassenden Patentschutz erfolgen oder durch das Vorliegen von hohen Kosten, die bei der Nachahmung eines Gutes entstehen, gewährleistet sein. Durch den erfolgreichen Abschluß eines FuE-Projekts werden jedoch nicht nur die innovierenden

127 Vgl. Grossman/Helpman (1991, S. 27 Fn 7).

128 Diese Form eines Konsumindexes geht auf Dixit/Stiglitz (1977) zurück. Sowohl in Romers Modell zur Spezialisierung als auch in seinem Modell zur Produktinnovation wird für den aggregierten Kapitalstock dieser Index verwendet. (D) kann auch als Produktionsfunktion bzw. Teil einer solchen verstanden werden. Vgl. auch Ethier (1982), Judd (1985) und Grossman/Helpman (1991, S. 47) 
Unternehmen berührt, sondern auch die Konsumenten und die Produzenten anderer Güter sind betroffen. Bei den Konsumenten stellt sich der sog. Konsumentenrenteneffekt ein, der durch das höhere Nutzenniveau aufgrund der gestiegenen Produktvielfalt entsteht. Die Wohlfahrt wird dadurch gesteigert. Auf der anderen Seite werden sich die Hersteller der bis zum Zeitpunkt des Entstehens eines neuen Produkts vorhandenen Güter einem zusätzlichen Konkurrenten um die Nachfrage der Konsumenten gegenübersehen. Bei einer als konstant angenommenen nominalen Nachfrage werden sie Rückgänge in der Produktion zu verzeichnen haben. Ihre Produzentenrente wird geschmälert. Diese Wirkung wird als Profitzerstörungseffekt bezeichnet. Je nach unterstellter Nutzenfunktion neutralisieren sich diese Wirkungen. ${ }^{129}$

Das Modell von Grossman/Helpman wäre kein Modell der Neuen Wachstumstheorie, wenn es nicht auch einen externen Effekt des Investierens aufweisen würde. Die Verwendung von Ressourcen für FuE-Zwecke stellt eine Investition, d.h. Konsumverzicht, dar. Jede Forschungsaktivität eines Unternehmens erhöht das gesamtwirtschaftliche Know-how, das sich aus produktspezifischem und allgemeinem Wissen zusammensetzt. Ist die Entwicklung neuer Güter wiederum vom bestehenden allgemeinen Know-how (A) abhängig, bedeutet mehr Wissen eine höhere Produktivität der in der Forschung tätigen Arbeitskräfte. Dies kann durch Gleichung (3.61) verdeutlicht werden: 130

$$
\dot{\mathrm{n}}=\frac{\mathrm{L}_{\mathrm{F}} \mathrm{A}}{\mathrm{a}} \text { mit } \quad \mathrm{A}=\mathrm{n}
$$

Die Veränderung der Anzahl der verschiedenen Güter $(\dot{\mathrm{n}})$ ist wiederum eine lineare Funktion von (A) bzw. (n) und weist somit konstante Skalenerträge bzgl. dieses Produktionsfaktors auf. Mittlerweile kennen wir diese Funktion und ihre Bedeutung für den endogenen Wachstumsprozeß aus den Überlegungen von Lucas (1988) und Romer (1990b,c). Morgige Forschergenerationen profitieren somit von den Anstrengungen ihrer Vorgänger. Es liegt ein learning by doing-Effekt in der Forschung vor. Die Parallelen zur Vorgehensweise von Romer sind gegeben. Wie dieser wählen auch Grossman/Helpman die Zahl der schon entwickelten Güter als Maß für das vorhandene Wissen der Volkswirtschaft. ${ }^{131}$

Diese Überlegung hat auch Konsequenzen für den Vergleich von Volkswirtschaften. Aus einer Gruppe von Ländern würde dasjenige mit der höchsten Zahl an entwickelten Produkten die höchste Produktivität in der Forschung aufweisen. Analog zu den Konsequenzen bei Arrow kann eine Volkswirtschaft nur durch eigene Forschungsaktivitäten ein bestimmtes Effizienzniveau erreichen. Die einfache Einführung von Gütern aus anderen Volkswirtschaften würde

129 Vgl. Grossman/Helpman (1991, S. 70 und den Anhang zu Kapitel 3). Weist die Nutzenfunktion eine konstante Substitutionselastizitat auf, kommt die angesprochene Neutralisierung zustande.

130 Vgl. Grossman/Helpman (1991, S. 58).

131 Vgl. Grossman/Helpman (1991, S. 57f.). 
nur das Nutzenniveau der Konsumenten vermehren, die erfahrungsabhängigen externen Effekte in der Forschung würden nicht realisiert.

Das Vorliegen dieser Wissensexternalitäten ist entscheidend für dauerhaftes Wachstum innerhalb des Modells. Kann sich ein Unternehmen alle Ergebnisse seiner FuE-Aktivitäten aneignen, d.h. stellt Wissen ein privates Gut dar, wird die langfristige Wachstumsrate gegen Null konvergieren. Die Begründung für diese Aussage erfolgt analog zum neoklassischen Wachstumsmodell. Die Rentabilität der Forschung geht mit steigender Zahl an Produkten zurück, da die nominale Nachfrage nach jedem einzelnen Gut sinkt. Die Ertragsrate von FuEInvestitionen nähert sich mit steigender Produktvielfalt der sozialen Diskontrate an, so daß der für die Forschung zu leistende Konsumverzicht der Gesellschaft nicht mehr angemessen erscheint. ${ }^{132}$ Die unterstellten Externalitäten dienen wie schon so häufig der Aufrechterhaltung des Investitionsanreizes.

Die Externalität im Forschungssektor hat noch weitere Konsequenzen für die langfristige Wachstumsrate im Laisser-faire- und im sozial optimalen Gleichgewicht. Da die Unternehmen die positiven Konsequenzen ihrer Forschungsanstrengungen auf nachfolgende Forschergenerationen nicht berücksichtigen, werden sie zu wenig Ressourcen in die Entwicklung neuer Güter stecken. Die Innovationsrate als Maß für die Veränderung der Produktvielfalt innerhalb einer Periode wird im Wettbewerbsgleichgewicht im Vergleich zum sozialen Optimum zu gering ausfallen. Gleichen sich der Konsumentenrenten- und der Profitzerstörungseffekt nicht aus, würden sie ebenfalls eine Differenz zwischen den beiden Situationen zur Folge haben. Die Nichtbeachtung des Nutzenzuwachses für die Konsumenten aufgrund eines neuen Produkts hat zu geringe, die Vernachlässigung der Zerstörung der Produzentenrente zu hohe Forschungsinvestitionen in einer sich selbst überlassenen Volkswirtschaft zur Folge. ${ }^{133}$

Für die jeweiligen Situationen ergeben sich im Steady State folgende Innovationsraten, d.h. Wachstumsraten für die Veränderung der Güterzahl:

$$
\begin{aligned}
& \hat{\mathrm{n}}_{\mathrm{Lf}}=(1-\alpha) \frac{\mathrm{L}}{\mathrm{a}}-\alpha \rho \\
& \hat{\mathrm{n}}_{\mathrm{opt}}=\frac{1}{1-\alpha}\left[(1-\alpha) \frac{\mathrm{L}}{\mathrm{a}}-\alpha \rho\right]
\end{aligned}
$$

(Laisser-faire-Situation)

(Soziales Optimum)

Da $\alpha<1$ ist, ist die Wachstumsrate im Optimum größer als im Wettbewerbsgleichgewicht. Die Veränderungsrate der Güterzahl einer Volkswirtschaft ist somit positiv abhängig von der verfügbaren Menge an Arbeitskräften $(\mathrm{L})$ und der Produktivität der Arbeit im Forschungssektor (1/a). Das negative Vorzeichen der Diskontrate $(\rho)$ spiegelt den Einfluß der Ungeduld auf die Innovationsrate der Volkswirtschaft wider. Eine höhere Bereitschaft zum Konsum- 
verzicht wird mehr neue Güter ermöglichen. Interessant ist insbesondere der Einfluß von $(\alpha)$, der ein Maß für die Wertschätzung der Haushalte für die Zahl der verschiedenen Güter darstellt. Legen die Haushalte großen Wert auf möglichst viele verschiedene Produkte bzw. sind die Güter schlechte Substitute, d.h. ist $(\alpha)$ klein, werden die Unternehmen in ihrem Bereich mehr Monopolmacht haben. Die Profite aus der Entwicklung und Produktion eines Gutes werden hoch sein, und der Anreiz zur Investition in FuE-Projekte damit auch. ${ }^{134}$

Der Einfluß der angeführten Variablen scheint bis auf die Zahl der Arbeitskräfte plausibel. Die positive Wirkung von (L) auf die Wachstumsmöglichkeiten der Volkswirtschaft würde bedeuten, daß bevölkerungsreiche Länder innovativer sein müßten als bevölkerungsarme. Der Grund für dieses Ergebnis liegt in der Annahme der Vollbeschäftigung. Wenn der Arbeitsmarkt immer geräumt ist, d.h. die Gleichung als Bedingung ins Modell eingeführt wird, müssen die flexiblen Teile des Modells sich daran anpassen. Besitzt nun eine Volkswirtschaft eine große Menge an Arbeitskräften, die auch beschäftigt werden, bedeutet dies auch mehr Ressourcen für die Entwicklung neuer Güter. In Kombination mit der konstanten Grenzproduktivität in der Produktionsfunktion für neue Produkte kann kein anderes Ergebnis als die positive Abhängigkeit der Innovationsrate von der Größe der Volkswirtschaft resultieren.

\section{Die Steigerung der Produktqualität}

Neben der Entwicklung neuer Güter kann das Ergebnis von FuE-Anstrengungen auch die Verbesserung der Qualität schon vorhandener Produkte sein. Das tägliche Leben zeigt hierfür viele Beispiele. Recht anschaulich wird dieser Prozeß mit dem Bild der Qualitätsleitern. Für jedes Gut in einer Volkswirtschaft gibt es eine Leiter, deren Sprossen für die Qualitätsstufen des Gutes stehen. Jede Innovation bei einem Gut führt zum Erklimmen einer weiteren Sprosse. Hiervon sind nicht nur Konsumgüter, sondern auch Kapitalgüter betroffen. Die Konsequenz einer Qualitätssteigerung ist die Obsoleszenz des bisherigen Standards. Im Gegensatz zum Modell mit steigender Produktvielfalt liegen somit nur temporäre Monopole vor. Der Prozeß der schöpferischen Zerstörung wird somit besser abgebildet.

Die Modelle von Aghion/Howitt (1992) und Grossman/Helpman (1991) weisen über weite Strecken Gemeinsamkeiten auf. Unternehmen streben in beiden Fällen die Realisierung von Monopolprofiten an, indem sie FuE-Ausgaben tätigen, um neue Qualitătsstandards zu entwickeln. Bei dieser Entscheidung müssen die Unternehmen berücksichtigen, daß ihr Monopol nicht von Dauer ist. Die Möglichkeit sein Monopol zu verlieren, kann Rückwirkungen auf die Forschungsanstrengungen haben. Erwarten die Unternehmen nicht ausreichend hohe Profite aus ihrer Innovation, werden sie keine FuE-Investitionen tätigen. Die Höhe der

$134 \mathrm{Vgl}$. Grossman/Helpman (1991, S. 63). Die Autoren zeigen in ihren Ausfuhrungen, daB die Wachstumsrate des realen Outputs und der Konsumindex proportional zur Innovationsrate sind, so dab die Betrachtung dieser für die Darstellung der wichtigsten Zusammenhănge ausreichend ist. 
möglichen Gewinne hängt maßgeblich vom Zeitpunkt ab, wann ein neuer Qualitătsstandard auftritt. Ist die Eintrittswahrscheinlichkeit für dieses Ereignis von der Höhe der Ausgaben im Forschungssektor abhängig, wird sich der Zeitraum für das eigene Monopol um so mehr verkürzen, je mehr andere in die Forschung investieren. Die Gefahr seine Monopolstellung früh zu verlieren steigt, die erwarteten Gewinne sinken. Dies kann u.U. zur Aufgabe aller Forschungsanstrengungen führen, was Nullwachstum zur Folge haben könnte. ${ }^{135}$

Es könnte gefragt werden, warum der Monopolist nicht den Standard selbst zu erhöhen versucht. Geht man davon aus, daß die Erlöse für jedes innovative Unternehmen aus einer Erfindung für sich genommen gleich groß sind, wären die Erträge aus einer neuen Qualitätsstufe für den aktuellen Monopolisten geringer als für einen neuen Produzenten, da er seine eigenen Gewinne vernichten würde.. Der Anreiz ist für ihn im Vergleich zu anderen Unternehmen zu gering. Das Vorantreiben des State-of-the-Arts könnte auch eine strategische Komponente aufweisen. Zeigt der Monopolist seine Stärke, könnte er potentielle Konkurrenten auf Distanz halten. Dies erfordert aber ausreichend Informationen der Konkurrenten über die scheinbare Aussichtslosigkeit eigener Forschungsaktivitäten. ${ }^{136}$

Der Ansatz von Grossman/Helpman (1991) zur Verbesserung der Produktqualität ist ähnlich wie das oben besprochene Modell zur Produktvielfalt aufgebaut. Während jedoch im letzteren ein neues Produkt das gesamtwirtschaftliche Know-how vergrößert hat, haben Qualitätsverbesserungen nur Einfluß auf das produktspezifische Wissen. Als Resultat weist das Modell dieselben Bestimmungsgrößen der Innovationsrate im Laisser-faire-Gleichgewicht wie zuvor auf. Anstelle des Parameters für die Wertschätzung der Produktvielfalt $(\alpha)$ tritt hier ein Maß für die Größe der Qualitätsschritte. Auch hier treten externe Effekte im Forschungssektor auf, die für zuküntige Generationen die Forschung effizienter machen. Unterschiede ergeben sich bei der Frage nach der Optimalität des Ergebnisses, die weiter unten behandelt werden. ${ }^{137}$

Interessante Aspekte bietet dagegen die Vorgehensweise von Aghion/Howitt (1992), die innerhalb eines Drei-Sektoren-Modells die Wirkungen von Qualitätsverbesserungen bei Kapitalgütern untersuchen. Im Unterschied zum Grossman/Helpman-Modell werden drei Arten von Arbeit betrachtet. a) unqualifizierte Arbeit ( $\mathrm{L}^{\mathrm{uq}}$ ), die ausschließlich im Konsumgütersektor zum Einsatz kommt, b) qualifizierte Arbeit ( $\left(\mathrm{L}^{q}\right)$, die bei der Herstellung der Kapitalgüter und im Forschungssektor Eingang findet und c) spezialisierte Arbeit $\left(\mathrm{L}^{\mathrm{S}}\right)$, die nur für Forschungszwecke verwendet wird. Zur Herstellung des Konsumgutes müssen zusätzlich zur Arbeit auch Kapitalgüter unter der Annahme konstanter Skalenerträge eingesetzt werden. Je besser die Qualität dieser ist, desto produktiver wird auch die unqualifizierte Arbeit sein. Prinzipiell wird Vollbeschäftigung aller Arten von Arbeit unterstellt. Der Kapitalgutsektor produziert ausschließlich mit qualifizierter Arbeit, wobei auch hier konstante Skalenerträge vorliegen.

$135 \mathrm{Vgl}$. zu diesen Uberlegungen z.B. Aghion/Howitt (1992a, S. 323f.).

$136 \mathrm{Vgl}$. zu solchem strategischen Verhalten Grossman/Helpman (1991, S. 93 und Fn 4 auf dieser Seite). 137 Vgl. Grossman/Helpman (1991, S. 86ff.). 
Während die Produktionsergebnisse im Konsumgüter- und Kapitalgutsektor in Abhängigkeit von den eingesetzten Mengen der Inputs fest determiniert sind, unterliegt der Output des Forschungssektors einem Zufallsprozeß. Die Zahl der qualifizierten und der spezialisierten Arbeitskräfte beeinflußt die Eintrittswahrscheinlichkeit einer erfolgreichen Innovation, wobei die qualifizierte Arbeit die entscheidende Größe ist. Die Eintrittswahrscheinlichkeit geht gegen Null, wenn (Lq) gleich Null ist. Aghion/Howitt gehen von einer Poissonverteilung aus, wobei Innovationen voneinander unabhängige Ereignisse sind. Die Wahrscheinlichkeit einer Innovation wird nicht von vorigen Innovationen bestimmt. Dennoch gibt es eine Beziehung zwischen heutigen und morgigen Erfindungen. Da jede Innovation die Produktivität der Kapitalgüter im Konsumgüterbereich dauerhaft um einen bestimmten Prozentsatz erhöht, wird auch die Rentabilität der nachfolgenden Innovationen erhöht. Somit hat auch der Ansatz von Aghion/Howitt seine Verbindung mit Arrows Überlegungen. 138

Die Volkswirtschaft steht in diesem Ansatz vor dem Problem, die verfügbare Menge der qualifizierten Arbeit zwischen dem Kapitalgut- und dem Forschungssektor aufzuteilen. Die gleiche Entscheidung mußte im Modell von Romer (1990b,c) für das Humankapital getroffen werden. Mehr (Lq) in der Forschung, eine höhere Wahrscheinlichkeit für eine Innovation und weniger Kapitalgüter und damit weniger Konsumgüterproduktion. Auf der Kostenseite der Beschäftigung von (Lq) stehen der Produktionsverlust in den beiden Gütersektoren sowie der Rentenverlust des Monopolisten bei einer früheren Innovation. Die Erträge sind durch die höheren Profite der nächsten Qualitätsverbesserung gegeben. Die Menge der Facharbeiter in der Forschung wird durch vier Größen bestimmt. Erstens hat die insgesamt verfügbare Menge an $(\mathrm{Lq})$ einen positiven Einfluß. Dies entspricht genau den Überlegungen, die zum Modell von Grossman/Helpman gemacht wurden. Zweitens bewirkt ein größeres Ausmaß der Innovation größeren Produktivitätsfortschritt im Kapitalgütersektor und somit eine höhere Rentabilität von (Lq) in der Forschung. Der Einsatz dieser Art von Arbeit wird attraktiver. Drittens senkt ein hohes Zinsniveau den Gegenwartswert erwarteter Monopolrenten, weshalb der Anteil von (Lq) in der Forschung sinkt. Viertens sorgt nach Meinung der Autoren ein höherer Parameter der Poissonverteilung für einen höheren Einsatz an qualifizierter Arbeit im Forschungsbereich, wobei der Einfluß dieses Parameters ambivalent ist. Einerseits impliziert eine höhere Eintrittswahrscheinlichkeit eine effizientere Forschung, andererseits steigt jedoch die Rate der schöpferischen Zerstörung mit all ihren Folgen für die Monopolrenten. Für die Wachstumsrate der Outputs ergeben sich dieselben Determinanten. Aufgrund der Zufalligkeit der Innovationen können die Aussagen nur die durchschnittlichen Größen betreffen, d.h. die Wachstumsraten können über oder unter dem ermittelten Wert liegen. ${ }^{139}$

$138 \mathrm{Vgl}$. Aghion/Howitt (1992a, S. 330).

$139 \mathrm{Vgl}$. Aghion/Howitt (1992a, S. 334f.). Leider wird das Überwiegen des positiven Effekts von den Autoren nur behauptet und nicht bewiesen. 
Während in allen bisher besprochenen Wachstumsmodellen das Vorliegen von positiven Externalităten zu einer Abweichung der gleichgewichtigen Laisser-faire-Wachstumsrate von derjenigen im sozialen Optimum nach unten führte, kann in den Modellen von Aghion/Howitt und Grossman/Helpman keine eindeutige Aussage getroffen werden. Die Ursache hierfür liegt in den verschiedenen Formen des Marktversagens. Der Ansatz von Grossman/Helpman beinhaltet den schon bekannten Profitzerstörungs-, den Konsumentrenten- und den Spill-overEffekt. Der Saldo zwischen den positiven und negativen Wirkungen hängt ab von der Höhe der Qualitätssprünge, die durch die Innovationen entstehen. Sehr große und sehr kleine Qualitätssprünge bedingen zu große Innovationsanreize im Wettbewerbsgleichgewicht. ${ }^{140}$ Im Modell von Aghion/Howitt sind vier Formen des Marktversagens denkbar. Die Unternehmen berücksichtigen bei ihrer Entscheidung über die Höhe der Investitionen im Forschungsbereich nicht die Konsequenzen eines erfolgreichen Forschungsprojekts für die Profite des bisherigen Monopolisten. Dieser Effekt wird durch die Fehleinschätzung der tatsächlichen Opportunitätskosten - nur die unternehmensinternen und nicht die gesamtwirtschaftlichen werden ins Kalkül gezogen - des Einsatzes von qualifizierter Arbeit verstärkt. Die Vernachlässigung dieser Aspekte impliziert eine zu hohe Wachstumsrate im Vergleich zum sozialen Optimum. Die Unternehmen machen aber auch den Fehler, daß sie nicht die innovationsbedingten dauerhaften Produktivitätssteigerungen beachten, und deshalb eine zu hohe Diskontrate bei der Abzinsung der erwarteten Profite ansetzen. Darüber hinaus orientieren sie sich nur an den eigenen Gewinnen und nicht am gesamtwirtschaftlichen Produktionsniveau. Beide Effekte führen zu einer tendenziell zu niedrigen Wachstumsrate im Laisser-faire-Gleichgewicht. In der Summe sind eindeutige Schlußfolgerungen für das Verhältnis zwischen den Wachstumsraten in den Situationen mit und ohne allwissenden Planer nicht möglich. ${ }^{141}$

\section{Erweiterungen}

Die zuvor geschilderten Modelle bilden die Basis für mehrere Erweiterungen, insbesondere der Ansatz von Grossman/Helpman wurde von den Autoren auf vielfache Weise ausgebaut. ${ }^{142}$ In einem weiteren Schritt wird die Akkumulation von physischem Kapital, in einem anderen verschiedene Qualitäten des Humankapitals berücksichtigt. Durch die Integration einer Sachkapitalkomponente ändert sich an der prinzipiellen Bestimmung der gleichgewichtigen Innovationsrate nichts. Weiterhin determinieren die Parameter, die Innovationen begünstigen, den langfristigen Wachstumsprozeß. Die Wachstumsrate des Outputs und des Kapitalstocks sind

$140 \mathrm{Vgl}$. Grossman/Helpman (1991, S. 101ff.)

141 Vgl. Aghion/Howitt (1992a, S. 338f.)

142 Die Erweiterungen von Aghion/Howitt befassen sich z.B. mit Patentwettbewerb unterschiedlicher Qualitătsniveaus sowie einem Mehrgüterfall und den Konsequenzen von strategischem Verhalten des Monopolisten. Daruber hinaus werden auch variable Innovationssprünge behandelt. Vgl. Aghion/Howitt (1992a,b). 
proportional zur Innovationsrate. Durch die Endogenisierung des Humankapitals im Rahmen eines Overlapping-Generation-Modells und die damit verbundene Aufteilung des Produktionsfaktors Arbeit in unterschiedliche Qualifizierungsstufen nähert sich das Modell von Grossman/Helpman an das von Aghion/Howitt an. Die Autoren kommen zu einem ăhnlichen Ergebnis wie Lucas (1988). Die Effizienz des Bildungssektors ist eine entscheidende Determinante für die Innovationsfähigkeit der Volkswirtschaft und damit ihrer langfristigen Wachstumsrate. Die Ursache für diesen Zusammenhang liegt an dieser Stelle bei den Folgen von Ausbildungsmaßnahmen auf die relativen Preise, die für die unterschiedlich qualifizierten Arten von Arbeit bezahlt werden. Wird die Investition in Aus- und Weiterbildung produktiver, wird die Entlohnung der qualifizierten Arbeit im Vergleich zur unqualifizierten steigen, mehr Wirtschaftssubjekte werden ihr Humankapital verbessern wollen. Durch dieses Verhalten steigt aber die für die Forschung verfügbare Menge an Humankapital. Die Innovationsrate wird erhöht. Die Innovationsrate der Ökonomie war zuvor in allen Fällen abhängig vom Ressourcenbestand, d.h. der verfügbaren Menge an Arbeit. Die Einbeziehung eines weiteren Produktionsfaktors zeigt, daß Größe nicht zwangsläufig mehr Wachstum bedeutet. Die Relation zwischen qualifizierter und unqualifizierter Arbeit entscheidet maßgeblich über den Sektor, auf den sich eine Volkswirtschaft spezialisieren wird. Zuviel unqualifizierte Arbeit wird die Ökonomie auf die Herstellung von Endprodukten festlegen, die Forschungsanstrengungen sinken, die Innovationsrate und damit die Wachstumsrate des Outputs gehen zurück. ${ }^{143}$ Dies ist qualitativ kein anderes Ergebnis wie im zweiten Modell von Lucas (1988), in dem die unterschiedlichen Humankapitalmengen die Spezialisierung festlegten.

Weitere Modifikationen betreffen den Schritt von einer geschlossenen zu einer offenen Volkswirtschaft. Sowohl Fragen komparativer Vorteile als auch Aspekte der Produktimitation werden behandelt. Eng damit verbunden sind Hysterese-Effekte des Außenhandels. Der Gedanke der unvollständigen Ausschließbarkeit anderer Wirtschaftssubjekte von der Nutzung neu entdeckten Wissens kann auf internationale Wirtschaftsbeziehungen ausgedehnt werden. Stehen Volkswirtschaften miteinander in Kontakt, können sie jeweils von den Forschungsergebnissen der anderen profitieren. Dieser Gedanke stellt ja eine Grundlage der Konvergenzüberlegungen im traditionellen neoklassischen Wachstumsmodell dar. Mögliche Aufholprozesse setzen das Überschwappen neuen Wissen auf andere Volkswirtschaften voraus. Grossman/Helpman zeigen z.B., daß im Falle räumlich begrenzter externer Effekte der Wissensgenerierung das Land mit den anfänglich komparativen Vorteilen ein anderes Land aus der Forschung verdrängen kann. Einmal erreichte Vorsprünge bei der Produktivität bieten im technologisch führenden Land höhere Investitionsanreize. Es käme zu einem lock-in-Effekt beim Spezialisierungsmuster, d.h. die Volkswirtschaften würden ohne exogene Maßnahmen

143 Vgl. Grossman/Helpman (1991, S. 112ff.). 
dieses nicht ändern. Nur durch große Anstrengungen (big jump) wäre es einem Verfolgerstaat dann möglich aufzuholen. 144

\subsubsection{Fazit}

Die Modelle zur Erfassung der Wachstumswirkungen von Produktinnovationen erweitern die bisher diskutierten Ansätze der Neuen Wachstumstheorie um einige Aspekte. Vor allem die Einführung von Erwartungen sowie die Unsicherheit über die Ergebnisse des Forschungsprozesses stellen einen Fortschritt dar. Der Automatismus: Mehr Ressourcen in die Forschung führen zu technischem Fortschritt wurde zumindest relativiert. Darüber hinaus sind neue Produkte die Folge intentionaler Handlungen der Unternehmen. Das Streben nach Gewinnen und neuen Absatzmärkten ist die primäre Ursache für Investitionen in FuE-Aktivitäten. Stellt dies einen Schritt zu mehr Realitătsnähe dar, ist die Annahme, daß die Vermehrung des allgemeinen Know-hows nur ein Nebenprodukt darstellt, wie sie von Grossman/Helpman z.B. getroffen wurde, weiterhin problematisch. Ein weiterer Vorteil ist die explizite Berücksichtigung von Monopolen, die der realen Situation bei entstehenden Innovationen mehr entspricht als vollkommene Konkurrenz.

Darüber hinaus ist vor allem im Hinblick auf die Arbeiten von Aghion/Howitt und Grossman/Helpman die Konsistenz und Geschlossenheit des Ansatzes anzumerken, der in der Lage ist, viele beobachtbare Phänomene zumindest der Tendenz nach abzubilden. Dies bedeutet nicht, daß nicht auch die Arbeiten über Produktinnovationen kritisch gesehen werden müssen. Die vorzubringenden Kritikpunkte betreffen nicht nur die hier vorgestellten Modelle, sondern können gegenüber der Neuen Wachstumstheorie insgesamt vorgebracht werden.

\subsection{Kritische Anmerkungen zur Neuen Wachstumstheorie}

Die Modelle der Neuen Wachstumstheorie sind selbstverständlich nicht ohne Kritik geblieben. Sowohl auf modelltheoretischer als auch empirischer Ebene können Einwände gegen die Erklärungsansätze dieses neuen Zweigs der Wachstumstheorie vorgebracht werden. Im Mittelpunkt steht häufig die Frage, inwieweit überhaupt von einer Neuen Wachstumstheorie gesprochen werden kann. Auch die vorliegende Arbeit hat ja das Ziel, eventuelle Parallelen zur kaldoria-

144 Vgl. Grossman/Helpman (1991, Kap. 7 und 8). Weitere Beispiele für Lock-in-Effekte finden sich bei Krugman (1991) sowie Arthur (1994). Ein wichtiger Grund für dieses Phanomen sind steigende Skalenerträge. Die diversen Modelle zu diesem Themenkomplex zeigen u.a. auf, daß Marktergebnisse nicht unbedingt optimal sein mulssen. So kann sich z.B. eine eindeutig schlechtere Technologie am Markt durchsetzen, weil sie bei Markteinfuhrung den Anwendern im Vergleich zu einer anderen besseren Technologie höhere Nutzen bietet. Das wohl bekannteste und hăufig sehr heftig diskutierte Beispiel ist das der Videotechnik, wo sich das technisch schlechtere System VHS gegen Betamax durchsetzte. 
nischen Vorstellung über Wachstumsprozesse zu zeigen. Ein interessanter Aspekt ist die Tatsache, daß die Neue Wachstumstheorie sehr kritisch von den Vertretern der traditionellen neoklassischen Wachstumstheorie gesehen wird, während Ökonomen auf der anderen Seite des Spektrums der Wirtschaftstheorie erste Schritte zur Annäherung zwischen dem Mainstream und dessen Kritikern sehen. ${ }^{145}$

Im nächsten Abschnitt wird auf modelltheoretische Kritikpunkte eingegangen. Daran anschließend wird die Frage nach der empirischen Überprüfung genauer beleuchtet.

\subsubsection{Theoretische Kritikpunkte}

Die modelltheoretischen Kritikpunkte an der Neuen Wachstumstheorie werden hier in zwei Bereiche aufgeteilt. Einerseits stehen die unterstellten funktionalen Zusammenhänge im Vordergrund, andererseits wird auf wichtige nicht enthaltene Aspekte eingegangen.

\subsubsection{Kritik an den zugrundegelegten Funktionen}

Innerhalb der Neuen Wachstumstheorie wird vielfach zur Bestimmung der sozial optimalen Situation die intertemporale Nutzenfunktion eines repräsentativen Wirtschaftssubjekts verwendet. ${ }^{146}$ Die Problematik dieser Annahme vor dem Hintergrund des Arrowschen Unmöglichkeitstheorems wurde in Kapitel 2 angesprochen. Diese Annahme wirft aber auch Schwierigkeiten auf, wenn externe Effekte der Investitionen unterstellt werden. Wird die Annahme des repräsentativen Individuums mit der Existenz identischer Wirtschaftssubjekte gleichgesetzt, lassen sich Externalitäten nur intertemporal verstehen, wie es im Modell von Arrow (1962) oder Grossman/Helpman (1991) bzw. Aghion/Howitt (1992) der Fall ist. Selbst die Existenz eines generellen Wissensstandes, der durch das nicht-aneignenbare Know-how der Unternehmen gespeist wird, hilft nicht weiter. Im Falle eines einzigen Unternehmens ist dieser identisch mit dem unternehmensspezifischen Wissensstock.

145 Insbesondere Solow gehört zu den Kritikern der Neuen Wachstumstheorie. Vgl. Solow (1991, 1992a, b, 1994a, b). Ein Beispiel für eine offene Einstellung gegenüber den aktuellen Entwicklungen in der Wachstumstheorie ist Skott/Auerbach (1995).

146 Die Anwendung der Ramseyschen Optimierungsmethode anstelle einer konstanten Sparquote stoBt zum Beispiel bei Solow auf harsche Kritik:

"Maybe I reveal myself merely as old-fashioned, but I see no redeeming social value in using this construction (the intertemporally-optimizing representative agent, der Verf.), which Ramsey intended as a representation of the decision-making of an idealized policy-maker, as if it were a descriptive model of an industrial capitalist economy. It adds little or nothing to the story anyway, while encumbering it with unnecessary implausibilities and complexities." (Solow 1994a, S. 49.) Viele Studierende der Wachstumstheorie, die sich mit den Methoden der dynamischen Optimierung auseinandersetzen müssen, werden mit Sicherheit den letzten Halbsatz dieses Zitats unterschreiben. 
An der üblicherweise unterstellten Nutzenfunktion setzt ein weiterer Kritikpunkt an. Das einzige Argument innerhalb dieser Funktion ist der Konsum, den ein Wirtschaftssubjekt oder ein Haushalt pro Zeiteinheit tätigen kann. Mit Sicherheit greift diese Annahme zu kurz. Weitere Variablen müßten in die Präferenzstruktur einfließen. Eine Möglichkeit wäre die Beachtung des Nutzens, den Freizeit bringt. ${ }^{147}$ Eine derartige Veränderung würde die Ergebnisse für die Wachstumsraten verändern, da zusätzlich zur Entscheidung über Akkumulation und Konsum eine weitere Wahlmöglichkeit für die Zeitallokation hinzukommt. Wird z.B. in Lucas' erstem Modell die Nutzenfunktion um eine Freizeitkomponente erweitert, generiert es dieselben Ergebnisse wie das traditionelle neoklassische Wachstumsmodell, d.h. es ergibt sich keinerlei endogenes Wachstum. ${ }^{148}$ Darüber hinaus wird von einer über die Zeit hinweg stabilen Präferenzfunktion ausgegangen. Der Wachstumsproze $B$ hat keinerlei Konsequenzen auf die Bewertung des Konsums in der Nutzenfunktion. ${ }^{149}$

Nicht nur die Argumente, die in die Nutzenfunktion Eingang finden, können in Frage gestellt werden, sondern auch die generelle Konzentration auf die Dominanz der Haushalte. Unabhängig davon, ob ein Overlapping-Generation-Modell oder Individuen mit unendlich langer Lebensdauer unterstellt werden, die Unternehmer erfüllen weiterhin ihre vikarische Aufgabe, indem sie den Präferenzen der Wirtschaftssubjekte folgen. Eine eigenständige Investitionsfunktion fehlt. Die Allokation der Ressourcen bleibt den Konsumenten überlassen, die entsprechend ihrer Zeitpräferenz und ihrer intertemporalen Substitutionselastizität über die Struktur der Produktion entscheiden. Auch in den Modellen mit Produktinnovationen bleibt dieses weiterhin der Fall, da die Nutzenfunktion der Haushalte das Verhalten der Unternehmen vorherbestimmt. Ruft man sich die Überlegungen von Harrod zur Instabilität langfristiger Wachstumsprozesse aufgrund unerfullter Erwartungen der Unternehmer in Erinnerung, stellt diese Überlegung mit Sicherheit einen der wichtigsten Kritikpunkte dar. Die Annahme der problemlosen Verwendung der nicht für Konsum benötigten Ressourcen für investive Zwecke in den Modellen der Neuen Wachstumstheorie, sei es für Sachkapital, sei für Humankapital, läßt das Saysche Theorem auch hier wie im traditionellen Modell seine Gültigkeit haben.

Die Kritik betriff jedoch nicht nur die Nutzen- und die nicht vorhandene Investitionsfunktion, sondern die Annahme steigender Skalenerträge der Produktionsfunktion insgesamt bzw. der konstanten Skalenerträge bzgl. der akkumulierbaren Produktionsfaktoren in der Herstellung des Endprodukts und in der Akkumulationsfunktion des vermehrbaren Produktionsfaktors. Steigende Skalenerträge bei der Produktion des gesamtwirtschaftlichen Outputs sind keine hinreichende Bedingung für endogenes Wachstum, da weiterhin die Steady StateWachstumsrate nur von der Bevölkerungswachstumsrate und der Rate des exogenen technischen Fortschritts bestimmt wird. Zusătzlich hängt die gleichgewichtige Wachstumsrate von

147 Denkbar ware auch die Berucksichtigung von negativen Konsequenzen einer steigenden Guterversorgung, wie z.B. Umweltbelastungen.

$148 \mathrm{Vgl}$. Solow (1992a, S. 19ff.).

149 Vgl. Skott/Auerbach (1995, S. 390ff.). 
den Produktionselastizitäten, also technologischen Größen ab. Die Wachstumsrate der ProKopf-Größen ist zwar positiv, jedoch spielen Präferenzen keine Rolle. ${ }^{150}$

Wie zuvor gezeigt, sind die Produktionselastizitäten der vermehrbaren Produktionsfaktoren für positives Pro-Kopf-Wachstum entscheidend. Tatsächlich ist dies die einzige Annahme, die konstante, von Null verschiedene Pro-Kopf-Wachstumsraten zuläßt. Liegen steigende Skalenerträge vor, nimmt der Anreiz zur Investition ständig zu. Steady State im Sinne konstanter Wachstumsraten ist jedoch nicht mehr möglich und das Modell würde innerhalb endlicher Zeit einen unendlich hohen Output erreichen. ${ }^{151}$ Abnehmende Skalenerträge wiederum münden im selben Ergebnis wie das einfache Solow-Modell. Dies legt den Schluß nahe, daß das Ziel in diesem Falle den Weg bestimmt, d.h. die Annahmen über die Produktionstechnologie werden nicht entsprechend real beobachtbarer Phänomene getroffen, sondern unterliegen dem Diktat des Steady State-Gleichgewichts.

Die Notwendigkeit dieser Annahme wirft sofort eine weitere Frage auf. Wenn die Konstanz der Skalenerträge bzgl. der vermehrbaren Produktionsfaktoren so entscheidend ist und gleichzeitig externe Effekte der Investition diese Konstanz gewährleisten sollen, muß die Externalität die Differenz zwischen der kapitaleigenen Produktionselastizität und eins genau auffullen. Besonders deutlich wird dies an der Produktionsfunktion (3.18) dieses Kapitels:

$$
\mathbf{Y}=\mathbf{K}^{\alpha} \mathbf{L}^{1-\alpha} \mathbf{K}^{\theta}
$$

Damit konstante Skalenerträge bzgl. des Kapitals vorliegen, muß gelten:

$$
1-\alpha+\theta=1 \quad \text { bzw. } \alpha=\theta
$$

Der Zufall müßte beide Größen in Übereinstimmung bringen, damit Steady State-Wachstum entstehen kann. ${ }^{152}$ Wie sieht dann aber der Prozeß aus, der dieses gewăhrleistet? Diese Frage wird in den Modellen der Neuen Wachstumstheorie nicht geklärt.

Selbst wenn die Erfüllung obiger Bedingung angenommen wird, steht die Erklärung positiver Pro-Kopf-Wachstumsraten auf sehr wackeligen Beinen. Die entscheidende Determinante sind die Externalitäten der Akkumulation der verschiedenen Formen von Kapital. Nur wenn diese vorliegen, ist die Ökonomie dauerhaft zum Wachstum der Pro-Kopf-Größen fähig. Eine solche Erklärung von Wachstumsprozessen kann nicht befriedigen. Die Entwicklung des Wohlstands einer Nation scheint somit auf den ersten Blick ausschließlich das Ergebnis von Zufälligkeiten. Dem kann aber nicht ohne weiteres zugestimmt werden, da sich die Frage nach

150 Vgl. Solow (1991, S. 7). Solow selbst hat diese Möglichkeit 1970 erwăhnt, aber als unwichtig abgelehnt. 151 Im Anhang zu diesem Kapitel wird dieses Problem formal gezeigt. Vgl. Solow (1992a, S. 9 und 1994a, S. 49f.)

152 Die Willkürlichkeit der Erfullung dieser Bedingung wird in einer Aussage von Homburg (1995, S. 341) gut dargestellt: "...wenn Gott würelt, tritt $\alpha=\theta$ mit der Wahrscheinlichkeit Null auf." Im Original steht $\beta$ fur $\theta$. 
dem Ursache-Wirkungs-Zusammenhang stellt. Die Relevanz der Existenz von externen Effekten innerhalb der Modelle der Neuen Wachstumstheorie bedeutet nicht, daß diese die Ursache der für Wachstum notwendigen Investitionen sind, sondern sie sind Begleiterscheinungen der Akkumulation, die den Unterschied zwischen der sozialen Ertragsrate und der von den Unternehmen erkannten Ertragsrate der Investition widerspiegeln. Dieser Unterschied resultiert aus einem Informationsproblem, da über das Preissystem nicht alle positiven Wohlfahrtseffekte einer Handlung erfaßt werden. Hierin liegt mit das Verdienst der Neuen Wachstumstheorie. Sie macht auf das Auseinanderfallen beider Ertragsraten aufmerksam. Das Vorliegen positiver externer Effekte von Investitionsentscheidungen kann auch als anderer Ausdruck für die Tatsache gesehen werden, daß die tatsächliche Grenzproduktivität des Kapitals, unabhängig von der Art des Kapitals, höher ist als die von den Unternehmen ins Kalkül gezogene. Der gesamtwirtschaftliche Anreiz zur Investition bleibt dadurch erhalten, die Akkumulation von Sach- und Humankapital sowie des generell verfügbaren Wissens hält dauerhaft an. Der "Kapital"-Einsatz pro Arbeitseinheit nimmt somit ständig zu. Dies war im neoklassischen Grundmodell auch schon der Fall, denn dort sorgte der exogene technische Fortschritt für die Erhaltung eines Investitionsanreizes. Die Neue Wachstumstheorie verdeutlicht nun die Notwendigkeit des Investitionsprozesses für eine steigende Pro-KopfVersorgung. Die Konzentration auf externe Effekte scheint somit problematisch, die Betonung der herausragenden Bedeutung der Kapitalakkumulation ist ein Vorteil gegenüber dem traditionellen Modell, das zumindest im Steady State den Investitionen jeden Einfluß absprach.

Wenn Produktivitätsentwicklungen innerhalb einer Volkswirtschaft das Resultat einer Kapitalintensivierung sind und diese auch noch zur Erhöhung des technologischen Wissens führt, hat die in der Neoklassik verwurzelte Neue Wachstumstheorie einen ersten Schritt zur Überwindung eines Kritikpunkts vollzogen, der der neoklassischen Wachstumstheorie von postkeynesianischer Seite, insbesondere von Kaldor, vorgehalten wurde. Das Konzept der traditionellen Produktionsfunktion und des exogenen technischen Fortschritts trennen zwischen einer Substitutions- und einer Fortschrittskomponente bei Produktivitätssteigerungen. Die Unterscheidung von Bewegungen auf der Produktivitätskurve und Bewegungen der Kurve selbst sind jedoch nicht zu trennen. ${ }^{153}$ Die Aufgabe der Annahme exogenen technischen Fortschritts und der Versuch, mittels externer Effekte positive Wachstumsraten der Pro-KopfGrößen zu generieren, läßt die Neue Wachstumstheorie näher an die Überlegungen Kaldors rücken. Seine Kritik sowie das daraus entwickelte Konzept der technical progress function wird im vierten Kapitel diskutiert. Im fünften Kapitel werden beide Ansätze gegenübergestellt werden. 154

153 Dieses Problem außert sich auch bei der simultanen Bestimmung der Art und der Rate des technischen Fortschritts und der Substitutionselastizitat. Vgl. hierzu Gahlen (1972, insbes. S. 69ff. und S. 159ff.).

154 Vergleichbare Kritik wurde von Scott $(1991,1992)$ insbesondere am Growth-Accounting-Ansatz vorgebracht. Das unerklärte Residuum im Rahmen dieser Studien ist fur ihn die Folge von MeBfehlern. Der Investitionsbegriff im Rahmen dieser Studien ist zu eng. Versteht man unter Investitionen alle 
Direkt verbunden mit diesen Überlegungen ist die prinzipielle Kritik an neoklassischen Wachstumsmodellen, die ebenfalls von postkeynesianischer Seite vorgebracht wurde. Trotz heftiger Debatte über das neoklassische Konzept eines aggregierten Kapitalstocks im Rahmen von Wachstums- und Verteilungsmodellen, wird dieses Konzept weiterhin von den Vertretern der Neuen Wachstumstheorie verwendet. Auch die Disaggregierung des Kapitalstocks in einzelne Kapitalgüter im Modell von Romer $(1990 b, c)$ überwindet dieses Problem nicht. Letztendlich ergibt sich aufgrund der Modellannahmen wieder eine aggregierte Kapitalgröße. Durch die explizite Einführung des Humankapitalstocks als Produktionsfaktor bzw. die Zusammenfassung aller Kapitalarten zu einem Konzept wie im AK-Modell wird das Problem der Aggregation auf weiteren Ebenen eingeführ. Sieht man von Ausnahmen ab, scheint die CambridgeKontroverse fast spurlos an der Neuen Wachstumstheorie vorbeigegangen zu sein. Man kann nun entweder die Modelle der Neuen Wachstumstheorie aufgrund dieses Mangels pauschal ablehnen und eine Auseinandersetzung mit ihnen für nicht sinnvoll betrachten oder man betrachtet sie als eine Weiterentwicklung der traditionellen Modelle, die am Anfang steht und weiter spezifiziert werden muß.155

\subsubsection{Weitere Mängel}

Neben den Spezifikationen der funktionalen Zusammenhänge lassen sich weitere Kritikpunkte vorbringen, die in den Modellen der Neuen Wachstumstheorie nicht enthaltene Aspekte betreffen. Die Ausführungen zur Catching up-Hypothese haben die Bedeutung der sog. sozial capability für den Aufholprozeß von Volkswirtschaften hervorgehoben. Hierunter wurden auch institutionelle Rahmenbedingungen verstanden, die bei der Diffusion von technologischem Wissen hilfreich sein können. Die Arbeiten von North (1990) oder Olson (1965) haben die Relevanz von Institutionen verdeutlicht. Institutionen können durch die Senkung von Transaktionskosten produktivitätssteigernde Wirkungen zeitigen, wovon positive Effekte auf das

Ausgaben, die die zukünftigen Konsummöglichkeiten erhohen und die bestehenden Strukturen verändern, darf es keine Residualgroße mehr geben. Scott betont vor allem die Rolle sog. materieller Investitionen, die neben den Anlageinvestitionen auch Forschungs- und Entwicklungsausgaben umfassen. Aus- und Weiterbildungsmaßnahmen spielen in seinem Konzept eine untergeordnete Rolle. Aufgrund der Veränderungen der Produktions- und Konsumstrukturen, die durch die Investitionstătigkeit entstehen, entsteht neues Wissen. Der Ansatz von Scott folgt somit demselben Prinzip wie Teile der Neuen Wachstumstheorie. Wăhrend diese zwischen verschiedenen Kapitalformen unterscheidet, faBt Scott verschiedene Arten der Investition zu einem Konzept zusammen. Für eine Volkswirtschaft ist es aber nicht nur wichtig, daB investiert wird, sondern auch in welche Kapitalform investiert wird. Das Aufkommen der Informationsgesellschaft verlangt bspw. andere Investitionen als die Zeit nach dem Zweiten Weltkrieg.

155 Lucas (1988; S. 36) erwăhnt zumindest die Cambridge-Kontroverse und bestătigt, dab sie zugunsten des englischen Cambridge ausgegangen sei. Überblicke über die Inhalte und Ergebnisse dieser Debatte finden sich z.B. in Hagemann (1977, 1990b), Harcourt (1969, 1970, 1972, 1976), Kurz (1990a), Kurz/Salvadori (1995a). Die Vernachlässigung der Ergebnisse der Cambridge-Kontroverse wird von postkeynesianischer Seite immer wieder betont. Vgl. z.B. McCombie/Thirlwall (1994, S. 93) oder Pasinetti (1994, S. 356f.). 
Wachstum ausgehen können. Eine entgegengesetzte Wirkung kann von Institutionen aufgrund von Beharrungstendenzen und Inflexibilitäten ausgehen, die Innovationsprozesse hemmen können. Entsprechende Überlegungen bleiben aber in den Modellen der Neuen Wachstumstheorie außen vor. Die Einbeziehung institutioneller Sachverhalte wirf im Rahmen eines formalen Modells gewisse Schwierigkeiten auf. Vorstellbar wäre jedoch eine länderabhängige Funktion für die Erklärung der Diffusionsgeschwindigkeit von Technologien. Zur Verteidigung der Neuen Wachstumstheorie muß vorgebracht werden, daß zumindest implizit Institutionen Eingang finden, wie das Beispiel von Lucas zeigt, der die Notwendigkeit adäquater institutioneller Rahmenbedingungen für die Ausnutzung potentieller Externalitäten des Humankapitals diskutiert. 156

Des weiteren sei auf einen Kritikpunkt hingewiesen, der jedoch nicht nur die Neue Wachstumstheorie triff, sondern auch schon für das Grundmodell der neoklassischen Wachstumstheorie gilt. Die Nachfrageseite der Ökonomie wird vollständig ausgeblendet. Der Absatz der produzierten Güter wird ebenso wie die Beschäftigung des ständig wachsenden Kapitalstocks bzw. des zunehmenden Humankapitals vorausgesetzt. Nachfrageausfalle, die Schwankungen in der Auslastung der Produktionskapazitäten verursachen, werden nicht diskutiert, sondern wegdefiniert. Für die Betrachtung langfristiger Prozesse wird die Räumung der Faktormärkte als gegeben angenommen. Die Trennung zwischen Konjunktur und Wachstum, wie sie von Tinbergen (1942) gefordert wurde, wird hier weiterhin aufrechterhalten. Die Überlegungen Harrods zu den Zusammenhängen zwischen kurz- und langfristiger Entwicklung bleiben unberücksichtigt. ${ }^{157}$

Wachstumsprozesse, die vom technischen Fortschritt verursacht und begleitet werden, finden in der Realität jedoch nicht ohne Friktionen statt. Jede technologische Neuentwicklung wird, wenn sie nicht alle Bereiche einer Volkswirtschaft gleichmäßig triff, Anpassungsprozesse notwendig machen. Selbst wenn in allen Sektoren die Produktivitätssteigerungen gleich hoch sind, stellt sich immer noch das Problem einer technologischen Arbeitslosigkeit für den Fall nicht ausreichend hoher Nachfragezuwächse, die notwendig sind, um die gestiegenen Produktionsmöglichkeiten auszulasten. ${ }^{158}$ Tatsächlich wird der technologische Wandel die Sektoren einer Volkswirtschaft unterschiedlich stark berühren, so daß es zu Verschiebungen der strukturellen Zusammensetzung der Volkswirtschaft kommen wird. Dieser Prozeß kann z.B. zum Absterben nicht mehr wettbewerbsfähiger Sektoren führen, während neue Sektoren entstehen. Aus dem Strukturwandel werden sich somit auch Rückwirkungen auf die Arbeits-

156 Vgl. zur mangelnden Berücksichtigung institutioneller Aspekte z.B. Pasinetti (1994, S. 356), Solow (1994b, S. 378f.) und Skott/Auerbach (1995, 395ff.). Ein erster Schritt in diese Richtung sind die Modelle von Grossman/Helpman, die zwischen zwei Ländern unterscheiden. Hierbei werden unterschiedliche Außenhandelsregime unterstellt. Vgl. Grossman/Helpman (1991, Kap. 8 und 9).

157 Selbst Solow (1992b, S. 1 sowie 1994b, S. 379) weist auf diesen Mangel hin, der die Aussagekraft der Modelle auch seiner Meinung nach sehr stark einschränkt.

$158 \mathrm{Vgl}$. zu Fragen der technologischen Arbeitslosigkeit z.B. Hagemann (1985, 1994, 1995) und Hagemann/Kalmbach (1983). 
kräftenachfrage ergeben. In den Modellen mit Produktinnovationen stehen die negativen Folgen von neuentwickelten Produkten auf die Gewinnsituation der Monopolisten im Zentrum des Interesses, die Arbeitskräfte der Modellwirtschaft können ohne Schwierigkeiten an einem neuen Arbeitsplatz eingesetzt werden. Die Arbeiten zur Mismatch-Arbeitslosigkeit haben auf die Probleme, die sich durch spezifische Qualifikationsmuster der Arbeitskräfte bei der Suche nach einem neuen Beschäftigungsverhältnis ergeben können, hingewiesen. Wachstumsmodelle, die die Wichtigkeit des Humankapitals und des Wissens innerhalb einer Volkswirtschaft betonen, müssen diese Erkenntnisse berücksichtigen. Es muß deshalb nicht nur die Höhe des Humankapitals, sondern auch dessen Zusammensetzung in die Analyse einfließen. Hoch aggregierte Modelle können die Konsequenzen unterschiedlicher Qualifikationsstrukturen und die damit verbundene Mismatch-Problematik jedoch nicht erfassen. 159

Erste Schritte zur Integration des Arbeitsmarktes wurden von Aghion/Howitt (1991, 1994) unternommen, die ihr oben skizziertes Modell zum endogenen Wachstum mit einem Modell zur Sucharbeitslosigkeit verknüpfen. Der modellierte Wachstumsprozeß hat dabei zwei Wirkungen auf den Arbeitsmarkt. Zum einen erfordern, wie oben angesprochen, technologische Neuerungen eine Reallokation von Arbeitskräften, zum anderen erhöht die technologische Entwicklung die Ertragsrate der Investitionen in neue Produkte, wodurch zusätzliche Beschäftigungsmöglichkeiten geschaffen werden. 160

Eine weitere offene Frage ist die der richtigen Erwartungsstruktur innerhalb der Modelle der Neuen Wachstumstheorie. Die Wirtschaftssubjekte weisen entweder vollkommene Voraussicht auf (z.B. in den Arbeiten mit aggregierten Produktionsfunktionen) oder besitzen zumindest rationale Erwartungen bzgl. der Technologieentwicklung (z.B. Modelle mit Produktinnovationen) Die Unternehmen machen keine systematischen Fehler in allen Bereichen in denen sie tätig sind. Das Ergebnis der FuE-Aktivitäten tritt zwar nur mit einer bestimmten Wahrscheinlichkeit ein und unterliegt damit einem gewissen Risiko. Die Wahrscheinlichkeitsverteilung ist den Unternehmern aber bekannt. Kommt es dann zu einem erfolgreichen Ausgang der Forschungsbemühungen, wissen sie sofort, wie sie sich zu verhalten haben. Etwaige Trial-and-Error-Prozesse sind dann ausgeschlossen. Die Konsequenzen von falschen Erwartungen der Unternehmen werden nicht modelliert. Die falsche Allokation von Ressourcen ist prinzipiell ausgeschlossen. Das Vorliegen vollkommener Voraussicht bzw. rationaler Erwartungen tragen aber zum Gleichgewichtscharakter der Modelle bei. Ungleichgewichtige Entwicklungen, die das Erreichen einer Steady State Situation verhindern, sind damit ausgeschlossen. Inwieweit die Annahmen der Neuen Wachstumstheorie Forschungsprozesse und ihre Ergebnisse richtig abbilden, kann nicht adhoc entschieden werden. Unstrittig

159 Diese Art der Kritik wird verstăndlicherweise z.B. von Pasinetti (1994, S. 356ff.) vorgebracht, der in seinen eigenen Arbeiten Aspekte des strukturellen Wandels analysiert hat. Vgl. Pasinetti (1981, 1993a). Vgl. zum Verhalitnis der Neuen Wachstumstheorie zur strukturellen Komponente wirtschaftlichen Wachstums auch Syrquin (1994).

$160 \mathrm{Vgl}$. zu diesen ersten Anfängen Aghion/Howitt (1991, 1994) sowie Hahn (1994) und Ramser (1995). 
ist die Tatsache, daß es bei Forschungs- und Entwicklungsaktivitäten immer wieder zu Fehlinvestitionen kommt und das angestrebte Ziel nicht erreicht wird.

Als Gegenargument könnte vorgebracht werden, daß es keine Fehlinvestitionen innerhalb der Generierung neuen Wissens geben kann. Jede Forschungsanstrengung vermehrt das Knowhow einer Volkswirtschaft, und wenn es auch nur das Wissen erhöht, wie man etwas nicht machen sollte. Das Niveau des technologischen Wissens (A) muß dann direkt von den Investitionen abhängig gemacht werden, da jeder Konsumverzicht, der nicht für die Akkumulation von Sachkapital verwendet wird, zu einem höheren Know-how-Bestand führt. Ein Blick auf die oben geschilderten Modelle zeigt, daß dem so ist. Ressourcen, die nicht in die Güterproduktion fließen, kommen für investive Zwecke zum Einsatz und mehren dadurch das wie auch immer gemessene Wissen oder Humankapital der Volkswirtschaft. Nur in den Modellen mit Produktinnovationen wird diese Kette durch die Annahme, daß nur erfolgreiche Produkte das Know-how erhöhen, unterbrochen. Die Neue Wachstumstheorie muß sich somit der empirischen Überprüfung ihrer Annahmen stellen, da nur auf diesem Wege entschieden werden kann, ob diese auch sinnvoll sind. ${ }^{161}$

Die Konzentration auf gleichgewichtige Prozesse kann an sich schon kritisch betrachtet werden. Während im traditionellen neoklassischen Modell über die Variabilität des Kapitalkoeffizienten der Anpassungsprozeß zum Gleichgewichtspfad erläutert wurde, wird in vielen neueren Modellen die Existenz eines Steady State-Pfades belegt, der Weg dorthin jedoch vernachlässigt. Des weiteren wurde nicht diskutiert, welche Prozesse ablaufen, wenn das Gleichgewicht verlassen wird. Die Verwendung eines gleichgewichtigen Wachstumspfades als Referenzpfad für eine kapitalistisch organisierte und sich selbst überlassene Volkswirtschaft kann ihren Sinn haben, der Weg zu diesem Gleichgewicht scheint aber mindestens von gleichem Interesse. Die reale Entwicklung einer Volkswirtschaft unterliegt einer Vielzahl von endogenen und exogenen Einflüssen, die auch von der Neuen Wachstumstheorie nicht erfaßt werden. Die anfängliche Vernachlässigung des Anpassungsprozesses wurde für einige Ansätze von Barro/Sala-i-Martin (1995) aufgehoben, die die Stabilität der jeweiligen Gleichgewichte belegen. Sie können somit zeigen, wohin sich die jeweiligen Volkswirtschaften ohne exogene Einwirkungen (z.B. Schocks) entwickeln. 162

Schließlich und endlich muß die Frage gestellt werden, ob die Wachstumsraten, die in den Modellen der Neuen Wachstumstheorie ermittelt werden, nun endogen sind oder nicht. Bestimmen also die Entscheidungen über Sparen und Investieren bzw. Forschung und Ausbildung die langfristige Wachstumsrate dauerhaft? Wenn man die Sparquote und die Zeitpräferenzrate als Ausdruck der Präferenzen wählt, muß die Antwort ja sein. In allen Modellen spielten diese Größen eine Rolle. Kommt es zu Veränderungen beim Sparen, wird sich auch die

161 Vgl. zur Frage der Erwartungsbildung auch Verspagen (1992, S. 659) und Skott/Auerbach (1995, S. 387ff.). Die Vertreter der Neuen Wachstumstheorie sind sich der entsprechenden Einwendungen bewuBt. Vgl. z.B. Aghion (1994, S. 35ff.).

162 Zur Kritik am Festhalten am Steady State-Konzept vgl. z.B. Tichy (1991, S. 104f.). 
gleichgewichtige Wachstumsrate anpassen. Je nach Höhe dieser Parameter wird die Volkswirtschaft schneller oder langsamer wachsen. Auch im neoklassischen Grundmodell hatten beide Größen Einfluß auf die Entwicklung der Ökonomie. Dieser war jedoch auf die Lage des Wachstumspfades beschränkt, die Wachstumsrate der Pro-Kopf-Größen im Fall ohne technischen Fortschritt mußte langfristig gegen Null gehen. Deutlich wird auch, daß die vorhandene Menge des oder der akkumulierbaren Produktionsfaktors/en für die Wachstumsrate häufig entscheidend ist. Die Anfangsausstattung mit Ressourcen bestimmt somit die Wachstumsmöglichkeiten der Ökonomie. Die Wirtschaftssubjekte können durch Präferenzänderungen diesen Nachteil ausgleichen. 163

Der Grund für dieses Ergebnis liegt in den Annahmen über die Produktionstechnologie und über die externen Effekte der Investitionen. Sind diese Externalitäten nun exogen oder endogen, d.h. liegen sie im Einflußbereich der Wirtschaftssubjekte oder können diese nichts daran ändern? Mit Sicherheit wird ein Großteil der externen Effekte in der Natur der Technologie liegen und sich somit dem Einfluß einer Präferenzänderung entziehen und somit exogen sein. Natürlich könnte in einem Analogieschluß zu den in der Umweltökonomie diskutierten negativen externen Effekten eingewandt werden, daß die Wirtschaftssubjekte mit ihrer Entscheidung über die eingesetzte Technologie auch festlegen, welche Externalitäten möglich sind. Letztendlich wird aber auch bei der unter diesen Gesichtspunkten besten Produktionsweisen oder Bildungseinrichtungen ein letztes exogenes Element verbleiben. Sieht man von einem solchen Grad der Perfektion ab, können die Modelle der Neuen Wachstumstheorie durchaus als endogene Wachstumsmodelle bezeichnet werden, da es ihnen gelingt, ohne exogenen technischen Fortschritt auch in einem Steady State-Gleichgewicht positives Pro-KopfWachstum zu erzeugen.

\subsubsection{Empirische Überprüfung}

Eines der Hauptziele der Vertreter der Neuen Wachstumstheorie war neben der endogenen Erklärung der Steady State-Wachstumsrate die bessere Abstimmung der Modellaussagen mit den empirisch beobachtbaren Phänomenen. Ein solcher Anspruch läßt nun eine Vielzahl von Studien zur empirischen Überprüfung der aufgestellten Modelle erwarten. Tatsächlich aber bereiten die Ansätze der Neuen Wachstumstheorie bei der Umsetzung in testbare Hypothesen Schwierigkeiten. Die für endogenes Wachstum verantwortlichen Variablen wie Humankapital, technologischer Stand des Wissens und Externalitäten können nicht ohne weiteres statistisch erfaßt werden, d.h. es müssen Indikatoren gefunden werden, die zumindest indirekt Rück-

163 Auch im zweiten Modell von Lucas (1988), in dem die anfanglichen komparativen Vorteile das Spezialisierungsmuster bestimmen, kann eine Veränderung der Substitutionselastizităt andere Ergebnisse erzeugen. 
schlüsse auf die in der Wirtschaftstheorie ermittelten Wachstumsdeterminanten und ihre Effekte auf den Wachstumsprozeß einer Ökonomie zulassen.

Eine erste Überprüfung der Aussagen der Neuen Wachstumstheorie stellt die Überprüfung von Konvergenz bzw. Divergenzphänomenen dar. Aus der Existenz von Konvergenz zwischen Volkswirtschaften ist selbstverständlich keine pauschale Ablehnung endogener Wachstumsmodelle möglich, da auch diese Konvergenzprozesse zulassen. Es müßte dann geprüft werden, welche Determinanten zur Konvergenz gefürt haben. Ergeben dagegen die empirischen Tests keinen Hinweis auf die Gültigkeit der Konvergenzhypothese (absolute oder bedingte), kann vermutet werden, daß die Struktur des neoklassischen Grundmodells nicht geeignet ist, die realen Phänomene zu erklären. In einem zweiten Schritt ist dann zu fragen, welche Größen die Höhe der Wachstumsrate beeinflussen.

\subsubsection{Konvergenz- und Divergenz-Tests}

In Abschnitt zur Catching up-Hypothese wurden verschiedene Tests zur Frage vorgestellt, ob die Konvergenzhypothese, die von der neoklassischen Wachstumstheorie nahegelegt wird, in den realen Phänomenen Bestätigung findet oder nicht. Innerhalb relativ homogener Gruppen von Ländern ergaben sich Konvergenzphänomene und die Anwendung des Konzepts der bedingten Konvergenz ließ auch den Schluß zu, daß weltweit Volkswirtschaften sich an ihren Steady State annähern. Akzeptiert man diese Resultate, die einen ( $\beta$ )-Wert von ca. $2 \%$ bei allen Berechnungen ergaben, schließt sich sofort die Frage an, welche neoklassische Produktionsfunktion hier zugrunde liegen würde. Barro/Sala-i-Martin selbst zeigen, daß dies eine Produktionselastizität des Kapitals von 0,75 implizieren würde. Dies ist ein deutlich höherer Wert, als er normalerweise in Growth-Accounting-Studien unterstellt wird. Wenn nämlich das neoklassische Wachstumsmodell Gültigkeit haben soll, dann muß auch die neoklassische Verteilungstheorie gelten. Bei Entlohnung der Produktionsfaktoren entsprechend der Grenzproduktivitätstheorie müßten die Produktionselastizitäten von Kapital und Arbeit der Profitbzw. der Lohnquote entsprechen. Hier läßt sich im allgemeinen feststellen, daß auch in Zeiten steigender Profite und sinkender Reallöhne, ein Anteil der Gewinne am Volkseinkommen bei ca. einem Drittel liegt. Die über die Konvergenztests errechneten Produktionselastizitäten weisen aber mehr als das Doppelte aus. ${ }^{164}$ Auf die möglichen Implikationen dieses Ergebnisses wird etwas weiter unten eingegangen werden.

Eine weitere Lanze für das neoklassische Wachstumsmodell und somit indirekt ein Stab über die Neue Wachstumstheorie wird von Mankiw/Romer/Weil (1992) gebrochen. Die Autoren führen in einem ersten Schritt einen Test des "normalen" Solow-Modells durch, d.h.

164 Für die Autoren scheint dies kein Grund zur Sorge zu sein. Weitere Implikationen dieses Ergebnisses werden nicht diskutiert. Vgl. z.B. Barro/Sala-i-Martin (1995, S. 413). 
mit Hilfe einer Cobb-Douglas-Produktionsfunktion, die Harrod-neutralen technischen Fortschritt enthält, und unter Verwendung des Steady State-Werts der Kapitalintensität wird eine Schätzgleichung für das Steady State-Pro-Kopfeinkommen ermittelt:165

$$
\ln y_{t}=\ln A_{0}+t \gamma_{H}+\frac{\alpha}{1-\alpha} \ln (s)-\frac{\alpha}{1-\alpha} \ln \left(n+\gamma_{H}+\delta\right)
$$

Ziel ist der Beleg der positiven Abhängigkeit des Pro-Kopf-Einkommens von der Sparquote (s) sowie der negativen Abhängigkeit von der Summe $\left(n+\gamma_{H}+\delta\right)$. Unter der Annahme der Konstanz der Abschreibungsrate und der Rate des technischen Fortschritts sowie unter der Annahme, daß der natürliche Logarithmus des anfänglichen technologischen Stands $A_{0}$ in eine für alle in der Stichprobe enthaltenen Länder Konstante $(\Lambda)$ und einen länderspezifischen "Schock" (u) aufgeteilt werden kann, führen die Autoren die Modellgleichung in eine zusätzliche Schätzgleichung über: 166

$$
\ln y=\Lambda+\frac{\alpha}{1-\alpha} \ln (s)-\frac{\alpha}{1-\alpha} \ln \left(n+\gamma_{H}+\delta\right)+u
$$

Es wurden für drei verschiedene Ländergruppen Stichproben auf Basis der Daten von Summers/Heston (1988) für den Zeitraum 1960-1985 durchgeführt. In allen Gruppen erklärten die Sparquote und das Bevölkerungswachstum einen erheblichen Teil der Streuung der ProKopf-Einkommen. Der Einfluß dieser Variablen war jedoch zu groß, da sich eine implizite Schätzung der Produktionselastizität des Kapitals von ca. 0,60 für zwei Stichproben ergab. Wie in den Schätzungen von Barro/Sala-i-Martin könnte also ein Solow-Modell die empirisch beobachtbaren Phänomene abbilden, die Konsequenzen für die Produktionselastizitäten widersprechen aber den sonstigen Annahmen über die Produktionselastizität des Kapitals. ${ }^{167}$

Um dieses Ergebnis zu korrigieren, führen die Autoren als weiteren Produktionsfaktor das Humankapital ein. Die Produktionsfunktion wird dann zu:

$$
Y_{t}=K_{t}^{\alpha} H_{t}^{\beta}\left(A_{t} L_{t}\right)^{1-\alpha-\beta}
$$

Für beide Kapitalarten liegen somit abnehmende Grenzproduktivitäten vor. Externalitäten wie bei Romer (1986) oder Lucas (1988) sind ausgeschlossen. Die Akkumulation von Human- und

165 Vgl. Mankiw/Romer/Weil (1992, S. 410).

$166 \mathrm{Vgl}$. Mankiw/Romer/Weil (1992, S. 411). Es fallt auf, daß der Term $\left(t_{\mathrm{H}}\right)$ nicht mehr enthalten ist. Aus Einfachheitsgründen wird von den Autoren $\mathrm{t}=0$ gewahlt. Es handelt sich also um keine Steady StateGleichung.

167 Vgl. Mankiw/Romer/Weil (1992, S. 423ff.). Die Lăndergruppen waren erstens OECD Lănder mit einer Bevölkerung über einer Million, zweitens nicht-erdölproduzierende Lănder und drittens Lănder, die verlaßliche Daten und eine Bevolkerung über einer Million aufwiesen. 
Sachkapital ist identisch aufgebaut. Die Produktionsfunktion weist konstante Skalenerträge auf und ist somit typisch neoklassisch im Sinne der Solowschen Tradition. Derselben Vorgehensweise wie oben folgend ergibt sich als Grundlage für die Schätzgleichung:

$$
\ln y_{t}=\ln A_{0}+t \gamma_{H}+\frac{\alpha+\beta}{1-\alpha-\beta} \ln \left(s_{K}\right)-\frac{\alpha}{1-\alpha-\beta} \ln \left(n+\gamma_{H}+\delta\right)+\frac{\beta}{1-\alpha-\beta} \ln \left(s_{H}\right)
$$

Die Sparquoten $\left(s_{K}\right)$ und $\left(s_{H}\right)$ geben an, welcher Teil des Outputs für Investitionen in Sachkapital und welcher für Humankapitalinvestitionen verwendet wird. Als Indikator für den Humankapitalterm wird der Anteil der Bevölkerung im arbeitsfähigen Alter gewählt, der sich in weiterbildenden Schulen befindet. Die Einführung dieses Terms führt in allen Stichproben zu einer Reduktion der Produktionselastizităt des Kapitals. Die zuvor sehr hohen Werte teilen sich jetzt auf beide Kapitalarten auf. Nur innerhalb der OECD-Länder ergibt sich kein signifikantes Ergebnis.

Die Anwendung dieser beiden Modellstrukturen auf die Konvergenzhypothese liefert wieder eine Bestätigung der Aussagen von Barro/Sala-i-Martin. Es folgt ebenfalls ein Maß für die Konvergenz von $2 \%$ pro Jahr. Jedoch weist trotz der Erweiterung um Humankapital das Sachkapital eine sehr hohe Produktionselastizität auf von 0,4 bis 0,47 . Dennoch sprechen sich die Autoren für das Solow-Modell und gegen die Ansätze der Neuen Wachstumstheorie aus. ${ }^{168}$ Die Bestätigung der erweiterten traditionellen Modellstruktur muß jedoch nicht zwangsläufig zur Ablehnung einer anderen führen, die nicht direkt getestet wurde.

Eine weitere Modifikation des erweiterten Solow-Modells führte Gundlach (1995) durch. Anstelle der Sparquote für das Humankapital wurde von ihm eine Bestandsgröße eingeführt, die durch die Zahl der durchschnittlichen Schuljahre gemessen wurde. Vergleicht man seine Schätzungen für beide Versionen, ergeben sich im Falle der Bestandsgröße deutlich höhere Produktionselastizitäten des Sach- und des Humankapitals. In den führenden Volkswirtschaften deuten die Resultate auf das Vorliegen steigender Skalenerträge beider Kapitalarten insgesamt hin. Eine weitere Veränderung der Modellstruktur, bei der nur noch Humankapital und Sachkapital in die Produktion einfließen und die Humankapitalbildung als Produktionsfaktoren Humankapital und Arbeit aufweist, die zusammen konstante Skalenerträge besitzen, führt bei der Schätzung zu den gewünschten Produktionselastizitäten des Sachkapitals von ca. einem Drittel und einer sehr hohen Produktionselastizität des Humankapitals. 169

Die exemplarisch referierten Schätzungen scheinen ein Beleg für die Fähigkeit des SolowModells zu sein, durch einfache Modifikationen empirische Sachverhalte abbilden zu können. Tatsächlich jedoch erinnert manches an den durchgeführten Erweiterungen an einen Versuch, durch die Hinzunahme mehrerer Variablen in die Schätzgleichung, den traditionell neoklas- 
sischen Wert für die Produktionselastizität des Sachkapitals zu erzeugen. Die Ergebnisse der Autoren können auch dahingehend interpretiert werden, was z.B. bei Gundlach auch geschieht, daß das Humankapital eine sehr große Rolle für den Wachstumsprozeß der Volkswirtschaft spielt und somit auch die Modelle der Neuen Wachstumstheorie ihre Berechtigung haben.

\subsubsection{Determinanten des Pro-Kopf-Wachstums}

Eine weitere Form der empirischen Überprüfung von Wachstumsmodellen stellt die Durchführung von einfachen und multiplen Regressionen dar. Die abhängige Variable Wachstumsrate des Sozialprodukts wird einer Vielzahl von anderen als unabhängig angesehenen Größen gegenübergestellt. Insbesondere der Einfluß der Investitionsquote, des Ausbildungsstandes und wirtschaftspolitischer Maßnahmen wird dabei untersucht. Aber auch politische, soziale und kulturelle Aspekte werden zu erfassen versucht. 170

Ohne auf die große Menge an Ergebnissen eingehen zu wollen, werden hier nur zwei der wichtigsten angesprochen. Die Studien belegen vielfach einen positiven Einfluß der Investitionsquote auf die Wachstumsrate des Pro-Kopf-Einkommens. Ein solches Ergebnis kann als Beleg für die Aussagen der Neuen Wachstumstheorie interpretiert werden. Im AK-Modell z.B. führte eine höhere Sparquote und damit auch eine höhere Investitionsquote zu einer höheren gleichgewichtigen Wachstumsrate. ${ }^{171}$ Positive Wirkungen auf die Wachstumsmöglichkeiten besitzen auch die Bildung bzw. das Humankapital. Interessant ist hier, daß dieses Resultat unabhängig von der Wahl des Indikators ist. Auch dies könnte man als Bestätigung der Neuen Wachstumstheorie verstehen. Tatsächlich stellt sich die Erfassung des Humankapitals in empirischen Studien als sehr problematisch heraus. ${ }^{172}$

Darüber hinaus bieten die hohen Werte der Produktionselastizität des Sachkapitals in den Untersuchungen, die im vorigen Abschnitt angesprochen worden sind, eine mögliche für die Neue Wachstumstheorie positive Interpretation an. DeLong/Summers (1991, 1993) interpretieren ihre Resultate als Beweis für das Vorliegen von externen Effekten beim Investitionsverhalten. Selbst eine Produktionselastizität des Kapitals von 0,6 könnte in ihrem Fall auch nur die Hälfte der Wirkung der Investitionen auf die Wachstumsrate des Pro-Kopf-Einkommens erklären. Die soziale Ertragsrate des Sachkapitals, die durch ihre Regressionsrechnungen erfaßt wird, ist größer als die private Ertragsrate, die sich in den Profiten der Unternehmen wider-

170 Beispiele finden sich in Kormendi/Meguire (1985), Barro (1991), Barro/Sala-i-Martin (1995), Barro/Lee (1994), die vor allem die Ausbildung in den Mittelpunkt stellten, in Levine/Renelt (1992), die eine Vielzahl von verschiedenen Variablen miteinander korrelierten, in DeLong (1993), DeLong/Summers (1991, 1993), Summers (1990), bei denen die Investition im Vordergrund stand sowie Fisher (1993), der die makroökonomische Stabilităt besonders betonte, sowie in Sala-i-Martin (1994).

171 Vgl. z.B. Delong (1993, S. 19).

172 Vgl. z.B. Barro/Lee (1993) oder Schultz (1993). 
spiegelt. ${ }^{173}$ Dieses Ergebnis ist, wie gezeigt, typisch für das Verständnis der Neuen Wachstumstheorie von den Konsequenzen des Investitionsverhaltens. Angemerkt werden muß, daß hierbei nur Sachkapital und Arbeit in die Produktionsfunktion eingeflossen sind und die Autoren zugeben müssen, die vermuteten externen Effekte nicht direkt belegen zu können. ${ }^{174}$

Von Jones (1995) wurde anhand von Zeitreihen ein Test des AK-Modells sowie der Implikationen der Modelle mit Produktinnovationen durchgefürt. Für den Fall des AKModells wurden die gesamtwirtschaftiche Investitionsquote und die Wachstumsrate des ProKopf-Bruttoinlandsprodukts für den Zeitraum 1950-1988 für 15 OECD-Länder im Rahmen von Zeitreihentests gegenübergestellt. Die Investitionsquoten sind im Betrachtungszeitraum deutlich angestiegen, während die Wachstumsraten eher rückläufig waren. Das AK-Modell hätte dagegen einen Anstieg des Pro-Kopf-Wachstums nahegelegt. Bei den Modellen mit Produktinnovationen hatte die verfügbare Menge an Arbeitskräften einen positiven Einfluß auf die Wachstumsrate des Know-hows. Aus diesem Grund vergleicht Jones (1995) die Entwicklung der totalen Faktorproduktivität mit derjenigen der Zahl der Arbeitskräfte im Forschungssektor. Einem Anstieg der Zahl der Wissenschaftler in Westdeutschland, Frankreich, Japan und den USA standen in allen vier Ländern in der Zeit von 1960-1988 keine signifikanten Zunahmen der totalen Faktorproduktivität gegenüber. Auch die Modelle mit Produktinnovationen finden entsprechend diesem Verfahren keine Bestätigung in der Realität. Eine direkte Ablehnung der Neuen Wachstumstheorie kann aber aus dem Vergleich zweier Zeitreihen nicht folgen, da insbesondere im Fall der totalen Faktorproduktivität nicht nur die Zahl der Arbeitskräfte eine Rolle spielt, sondern diese von vielen Faktoren beeinflußt werden kann. Des weiteren können auch Meßfehler die Daten für die totale Faktorproduktivität verzerren. 175

Inwieweit nun aus dem Vorliegen ökonometrisch ermittelter Beziehungen zwischen der Wachstumsrate des Pro-Kopf-Einkommens und anderen Variablen auf die Gültigkeit des einen oder anderen Wachstumsmodells geschlossen werden kann, bleibt fraglich. Sicher ist die Notwendigkeit, das einfache Solow-Modell modifizieren zu müssen, damit empirisch relevante Beobachtungen erfaßt werden können. Der direkte empirische Beleg der Neuen Wachstumstheorie ist bisher nicht gelungen. Dies kann nur durch den Test eines oder mehrerer Modelle geschehen. Hier treten jedoch Probleme aufgrund der in den Modellen der Neuen Wachstumstheorie verwendeten Variablen auf. Wie soll die Messung des Humankapitals befriedigend erfolgen? Sind Patente der richtige Indikator für den Stand des technischen Wissens?

173 Vgl. z.B. DeLong/Summers (1991, S. 480ff.).

174 Vgl. z.B. DeLong/Summers (1991, S. 487). Die Ergebnisse zur Investitionsquote sind jedoch nicht sehr stabil, da bei Einfuhrung einer Dummy-Variablen für den jeweiligen Kontinent eines Landes der Einfluß nicht statistisch signifikant ist. (Vgl. auch Solow 1992b, S. 80ff.). Dariber hinaus beeinflussen auch Ausreißer bei den Daten die Resultate. Vgl. Auerbach/Hassett/Oliner (1994) und Delong/Summers (1994). 175 Vgl. Crafts (1996, S. 40f.). 
Zusätzlich stellt sich die Frage, ob die Untersuchung langfristiger Wachstumsphänomene nicht die Aufgabe der normalerweise durchgeführten Querschnittsanalysen über Länder hinweg erforderlich macht. Die Neue Wachstumstheorie betont immer wieder länderspezifische Unterschiede in wichtigen Variablen. Querschnittsuntersuchungen "rechnen" diese jedoch heraus. Vielfach sind diese Berechnungen sehr stark von der Gruppe der Länder abhängig, für die sie durchgeführt worden sind. Darüber hinaus sagen Regressionsrechnungen nichts über UrsacheWirkungs-Zusammenhänge aus. Vielmehr sind nur Tendenzaussagen möglich. Aus diesen Gründen scheint zumindest ergänzend zu den bisher durchgeführten Studien eine Längsschnittbetrachtung einzelner Länder sinnvoll. Hierbei können z.B. die Einflüsse des Investitionsverhaltens auf die Wachstumsrate eines einzelnen Landes deutlich gemacht werden. In einem zweiten Schritt könnten dann verschiedene Volkswirtschaften miteinander verglichen werden, um Rückschlüsse auf grundlegende Zusammenhänge ziehen zu können. Darüber hinaus sollten auch Fallstudien über einzelne Industrien durchgeführt werden, um z.B. weitere Hinweise auf die Existenz von Externalitäten oder monopolistischen Strukturen bei der Entstehung von technischem Fortschritt zu erhalten. 176

Festzuhalten bleibt, daß die bisherigen Untersuchungen zu den Determinanten des Wachstums vielfach mehr direkte oder indirekte Tests fur das traditionelle neoklassische Wachstumsmodell als die empirische Überprüfung der Aussagen der Neuen Wachstumstheorie darstellen. Zieht man die kurze Zeit in Betracht, seit der über die Neue Wachstumstheorie gearbeitet wird, kann dies jedoch nicht verwundern. Einerseits bieten sich bei den modelltheoretischen Strukturen noch Verbesserungsmöglichkeiten an, andererseits müssen auch erst adäquate statistische Indikatoren für die Modellvariablen entwickelt werden. Vom empirischen Standpunkt aus kann die Neue Wachstumstheorie deswegen genausowenig wie das traditionelle neoklassische Wachstumsmodell abgelehnt werden.

\subsection{Fazit}

Die Modelle der Neuen Wachstumstheorie, die aus der Kritik am Solow-Modell entstanden sind, zeigen verschiedene Wege auf, die es ermöglichen, die Abhängigkeit der langfristigen Wachstumsrate von exogenen Größen zu überwinden. Die Diskussion von Marktunvollkommenheiten aufgrund externer Effekte bei der Investition in die verschiedenen Formen des Kapitals und aufgrund monopolistischer Strukturen als Folge erfolgreicher Forschungs- und Entwicklungsaktivitäten hat genauso wie die explizite Einführung des Produktionsfaktors Humankapital das grundlegende neoklassische Wachstumsmodell weiterentwickelt.

Weiterhin jedoch weisen die Modelle Elemente auf, die kritisch zu betrachten sind. $\mathrm{Zu}$ nennen sind insbesondere die Verwendung einer intertemporalen Nutzenfunktion sowie die

176 Vgl. zu dieser Überlegung Pack (1994, insbes. S. 67ff.), Solow (1992a, b) und Maddison (1993). 
Annahme eines repräsentativen Individuums bzw. Haushalts oder Unternehmens. Auch das Festhalten am Steady State-Konzept schränkt die Modelle in ihren Aussagen stark ein. Problematisch ist die starke Abhängigkeit der Endogenität des Wachstums von Externalitäten zu sehen. Alle hier vorgestellten Ansätze benötigen externe Effekte bei der Investition bzw. die Annahme konstanter Skalenerträge bzgl. der akkumulierbaren Produktionsfaktoren. Wie gezeigt, stellt diese Annahme eine Gratwanderung dar. Steigende Skalenerträge des Kapitals verhindern ohne weitere Annahmen die Existenz konstanter Wachstumsraten, d.h. eines Steady States. Des weiteren ergibt sich das Problem eines unendlich hohen Outputs innerhalb einer endlichen Zeitspanne. Liegen dagegen sinkende Skalenerträge vor, sind wiederum exogene Faktoren notwendig, um positives Pro-Kopf-Wachstum auf Dauer zu erreichen.

Die Untersuchung hat auch gezeigt, daß endogenes Wachstum in allen Modellen mit dem Begriff Kapitalintensivierung umschrieben werden kann. In allen Ansätzen geht der Anreiz zur Akkumulation im Steady State nicht verloren. Die (soziale) Ertragsrate einer Investition in Sachkapital, Humankapital oder neue Produkte sinkt nicht unter die soziale Diskontrate, so $\mathrm{da} ß$ es immer lohnend ist, weiter auf Konsum zu verzichten, um in Zukunft mehr konsumieren zu können. Die Neue Wachstumstheorie reduziert somit die Erklärung des Wachstums auf die Investitionstätigkeit. Um so mehr muß das schon bei der Kritik angeführte Fehlen einer eigenständigen Investitionsfunktion bemängelt werden. Wenn Investitionen die Ursache des Wachstums sind, dann müssen die Beweggründe für die Investitionstätigkeit genauer analysiert und nicht nur als Ausdruck der Präferenzen der Konsumenten gesehen werden. Die Betonung der Bedeutung der Investitionen für langfristige Wachstumsprozesse läßt die Neue Wachstumstheorie in die Nähe der Überlegungen von Kaldor zur Frage des induzierten technischen Fortschritts rücken.

Das Vorliegen externer Effekte bzw. die Relevanz der Akkumulationsentscheidung für positives Pro-Kopf-Wachstum im Steady State bietet im Gegensatz zum neoklassischen Grundmodell die Möglichkeit mittels wirtschaftspolitischer Maßnahmen dauerhaft in den Wachstumsprozeß eingreifen zu können. Welche Implikationen sich innerhalb der Neuen Wachstumstheorie für eine Wachstumspolitik ergeben, soll weiter unten angesprochen werden, nachdem eine Gegenüberstellung mit den Ausführungen von Kaldor zur technical progress function und dem von ihm sehr stark betonten Verdoorn-Zusammenhang erfolgt ist.

Die Neue Wachstumstheorie stellt somit einen Schritt zur Integration neuer Faktoren, in die traditionelle Produktionsfunktion der Neoklassik dar. Die Modelle ermöglichen die Berücksichtigung typischer Elemente von Wachstumsprozessen, wie sie in den von Romer aufgestellten stilisierten Fakten zum Ausdruck kommen. Dies bedeutet nicht, daß sie alle Determinanten wirtschaftlichen Wachstums abbilden können. Eine solche Forderung wäre wohl von keiner Theorie zu erfullen. Vielmehr hat die Neue Wachstumstheorie allgemein akzeptierte Aspekte, (z.B. die Relevanz von Investitionen und Humankapital für die Wachstumsmöglichkeiten) mit neuen Methoden (intertemporale Optimierung) in weitgehend traditio- 
nelle Konzepte (Produktionsfunktion) integriert. Vergleicht man die Neue Wachstumstheorie mit einem Automobil, so stellt sie einen general überholten (Neo)Klassiker dar, der mit verfeinerten technischen Details manche Situation auf der holprigen Straße des Wachstums besser bewältigen kann, als es sein Vorgänger konnte.

\section{Anhang zu Kapitel 3:}

Bei der Diskussion der steigenden Skalenerträge als Ursache endogenen Wachstums wurde das Problem eines explodierenden Systems angesprochen. Innerhalb eines endlichen Zeitraums kann der Kapitalstock und mit ihm der Output einen unendlich hohen Wert erreichen. Der Beweis für diese Aussage wird im folgenden dargestellt. 177

Wenn man von einer Produktionsfunktion mit zwei Produktionsfaktoren ausgeht, z.B. Wissen (K) und Arbeit (L) und nimmt man Konstanz von (L), kann die Produktionsfunktion im Falle einer Cobb-Douglas-Produktionsfunktion vereinfacht als

(A.3.1) $\quad Y_{t}=F\left(K_{t}\right)=K_{t}^{\alpha}$

schreiben. Sieht man der Einfachheit von Abschreibungen ab und unterstellt eine konstante Sparquote (s), folgt für die Veränderung des Kapitalstocks die Gleichung:

$$
\dot{\mathrm{K}}_{\mathrm{t}}=\frac{\mathrm{dK}_{\mathrm{t}}}{\mathrm{dt}}=\mathrm{sF}\left(\mathrm{K}_{\mathrm{t}}\right)=\mathrm{sK}_{\mathrm{t}}^{\alpha}
$$

Dies ist eine Differentialgleichung, die in eine lineare Differentialgleichung überfürt werden kann. Mit $\mathrm{z}=\mathrm{K}^{1-\alpha} \operatorname{kann}(\mathrm{A} .3 .2)$ in

$$
\mathrm{dz}-(1-\alpha) \mathrm{sdt}=0
$$

überführt werden. ${ }^{178}$ Durch Umformung (A.3.3) ergibt sich eine nicht-homogene Differentialgleichung für (z):

$$
\text { (A.3.4) } \frac{\mathrm{dz}}{\mathrm{dt}}=(1-\alpha) \mathrm{s}
$$

177 Die Überlegungen basieren auf Solow (1994a, S. 50). In seinen etwas kurzen Ausführungen zu diesem Problem macht er jedoch nach Auffassung des Verfassers einen kleinen Fehler bei der Ermittlung der Lösung der Differentialgleichung für den Kapitalstock. Dieser andert aber nichts an den prinzipiellen Schlußfolgerungen. Auch von Meade (1962b) wird dieses Phănomen diskutiert.

178 Vgl. Chiang (1984, S. 491). 
Die Lösung einer solchen Gleichung ist durch

$$
\text { (A.3.5) } \quad z_{t}=c+(1-\alpha) s t
$$

bestimmt, wobei (c) eine noch zu bestimmende Konstante ist, für die $\left(z_{0}\right)$ als Anfangsgröße für (z) gesetzt werden kann. 179 Kehrt man nun zurück zur ursprünglichen Gleichung, die als Variable das Wissen (K) enthält, folgt

(A.3.6) $\quad \mathrm{K}_{\mathrm{t}}^{1-\alpha}=\mathrm{K}_{0}^{1-\alpha}+(1-\alpha)$ st.

Dieses Ergebnis kann nun verwendet werden, um zu zeigen, daß der Kapitalstock innerhalb eines bestimmbaren, endlichen Zeitraums einen unendlich hohen Wert annehmen kann. Es gilt:

(A.3.7) $\quad \mathrm{K}_{\mathrm{t}}^{1-\alpha}=\frac{\mathrm{K}_{\mathrm{t}}}{\mathrm{K}_{\mathrm{t}}^{\alpha}}=\frac{\mathrm{K}_{\mathrm{t}}}{\mathrm{F}\left(\mathrm{K}_{\mathrm{t}}\right)}$.

Der Beweis erfolgt auf einem indirekten Weg. Mit Hilfe des Integrals

$$
\int_{K_{0}}^{\infty} x^{-\alpha} d x
$$

kann gezeigt werden, zu welchem Zeitpunkt der Kapitalstock einen unendlich hohen Wert erreicht. Allgemein gilt:

$$
\int_{\mathbf{K}_{0}}^{\mathrm{K}_{\mathrm{t}}} \mathrm{x}^{-\alpha} \mathrm{dx}=\left|\frac{1}{1-\alpha} \mathrm{K}_{\mathrm{t}}^{1-\alpha}\right|_{\mathrm{K}_{0}}^{\mathrm{K}_{\mathrm{t}}}=\frac{1}{1-\alpha} \mathrm{K}_{\mathrm{t}}^{1-\alpha}-\frac{1}{1-\alpha} \mathbf{K}_{0}^{1-\alpha} .
$$

Geht nun $K_{t}$ gegen unendlich, wird (A.3.9) aufgrund dessen, daß $\alpha>1$ ist, gegen den Wert

$$
\text { (A.3.10) }-\frac{\mathbf{K}_{0}^{1-\alpha}}{1-\alpha}
$$

konvergieren, der erste Term der Differenz in (A.3.9) gegen Null strebt. An dieser Stelle kann man nun wieder (A.3.6) verwenden. Für die Differenz der Werte des Kapitalstocks zu zwei Zeitpunkten kann man schreiben:

179 Vgl. Chiang (1984, S. 473f.). 
(A.3.11) $\quad \mathrm{K}_{\mathrm{t} 1}^{1-\alpha}-\mathrm{K}_{\mathrm{t} 0}^{1-\alpha}=\mathrm{K}_{0}^{1-\alpha}+(1-\alpha) \mathrm{st}_{1}-\mathrm{K}_{0}^{1-\alpha}+(1-\alpha) \mathrm{st}_{0}=(1-\alpha) \mathrm{s}\left[\mathrm{t}_{1}-\mathrm{t}_{0}\right]$

Setzt man diesen Wert gleich mit (A.3.10), folgt für den Zeitpunkt des unendlichen Kapitalstocks

(A.3.12) $\quad(1-\alpha) s\left[t_{1}-t_{0}\right]=-\frac{K_{0}^{1-\alpha}}{1-\alpha} \quad$ bzw. $t_{1}=-\frac{K_{0}^{1-\alpha}}{s}+t_{0}$

Da aber auch (A.3.7) gilt, kann (A.3.12) auch folgendermaßen geschrieben werden:

(A.3.13) $\quad t_{1}=-\frac{K_{0}}{s F\left(K_{0}\right)}+t_{0}$

Auf den ersten Blick scheint dieses Ergebnis von der Höhe der Skalenerträge unabhängig. Es muß jedoch beachtet werden, daß nur im Fall $\alpha>1$ (A.3.8) gegen einen endlichen Wert konvergiert und so die Berechnung des endlichen Zeitpunkts $t_{1}$ möglich ist. 


\subsection{Vorbemerkungen}

Durch das Aufkommen der in Kapitel 3 besprochenen Neuen Wachstumstheorie seit Mitte der achtziger Jahre hat sich das Interesse der Ökonomen wieder vermehrt auf das Phänomen steigender Skalenerträge gerichtet. ${ }^{1}$ Die augenblicklich vorherrschende Behandlung dieses Themas im Rahmen der Neuen Wachstumstheorie vernachlässigt jedoch weitgehend Beiträge, die von postkeynesianischer Seite aus zu dieser Fragestellung gemacht wurden. Insbesondere die Arbeiten von Nicholas Kaldor liefern einige interessante Einblicke, wie sie durch die ausschließlich angebotsseitige Untersuchung durch die aktuellen neoklassischen Wachstumsmodelle nicht möglich sind.

Kaldors Beiträge zu wachstumstheoretischen Fragestellungen begründen sich in seiner kritischen Einstellung gegenüber dem neoklassischen Paradigma. Die Modelle der Allgemeinen Gleichgewichtstheorie in der Tradition von Walras und später von Arrow/Debreu müssen an der Erklärung realer Phänomene scheitern, da sie Annahmen treffen, die empirisch widerlegt werden können, diese Annahmen jedoch für die logisch konsistente Ableitung der Modellergebnisse von entscheidender Bedeutung sind. Eines der wichtigsten Beispiele in diesem Zusammenhang ist die Einführung konstanter Skalenerträge innerhalb der gesamtwirtschaftlichen Produktion. ${ }^{2}$ Unterliegt der Einsatz der Produktionsfaktoren dem Gesetz abnehmender Grenzerträge, folgt für die Produktionsfunktion insgesamt ein Skalenniveau von Eins. Diese Annahme ermöglicht eine konsistente Verbindung zwischen Wachstums- und Verteilungstheorie. Werden die Produktionsfaktoren entsprechend ihrer Grenzproduktivität entlohnt, wird das erzeugte Sozialprodukt vollständig ausgeschöpft. Folgt die Produktion jedoch steigenden Skalenerträgen, deren Ursachen bei den zu entlohnenden Produktionsfaktoren liegen, kommt es zu einer Überausschöpfung des Sozialprodukts. Die kumulierten Ansprüche der Produktionsfaktoren an das Sozialprodukt sind insgesamt zu hoch. Das neoklassische System zeigt Brüche. ${ }^{3}$

Darüber hinaus werden Axiome aufgestellt, die nach Kaldors Meinung falsch sind, wie z.B. die Eigenschaft der Preise, ein Datum für alle Wirtschaftssubjekte und der einzige Informationsträger im wirtschaftichen System zu sein. Tatsächlich jedoch orientieren sich die Akteure auch an Mengengrößen. Schließlich werden in der neoklassischen Theorie Ziel-

1 Das von James Buchanan und Yong Yoon herausgegebene Buch, das wichtige Originalbeitrage zu diesem Thema enthalt, tragt nicht nur wegen sprachlicher Eleganz allein den Titel The Return to Increasing Returns. Vgl. Buchanan/Yoon (1994).

2 Vgl. z.B. Kaldor (1985, S. 11).

3 Zur neoklassischen Verteilungstheorie siehe z.B. Külp (1994) oder Rose (1987). Eine kritische Betrachtung, die uber die Untersuchung der Produktionsbedingungen hinausgeht, bietet Preiser (1970a,b). 
funktionen, wie die Gewinn- und Nutzenmaximierung, unterstellt, die sich einer empirischen Überprüfung entziehen. ${ }^{4}$

Diese systematisierende Vorgehensweise, die versucht, allgemein gültige Gesetze abzuleiten, kann Kaldors Auffassung nach zur Erklärung realer Phänome nur wenig beitragen. Die ökonomische Theorie sollte in ihrer Eigenschaft als Sozialwissenschaft deswegen nicht dem Prinzip einer deduktiven, sondern vielmehr einer induktiven Forschung folgen. Die Entwicklung theoretischer Modelle soll der Erklärung der Fakten dienen, die sich als empirische Regelmäßigkeiten feststellen lassen. Eine solche Vorgehensweise impliziert nicht, daß diese Fakten als allezeit und allgemein gültige, unveränderbare Wahrheiten gesehen werden. Ähnlich der Forderung nach der ständigen Überprüfung von Hypothesen an der Wirklichkeit müssen auch die stilisierten Fakten immer wieder kontrolliert werden. ${ }^{5}$ Kaldors Arbeiten zur Wachstumstheorie sind deutlich durch diese Vorstellung geprägt. Sowohl seine theoretischen Beiträge zur technical progress function als auch seine Überlegungen zur angewandten Wachstumstheorie beginnen nicht mit dem Bau eines Modells, welches dann einem empirischen Test unterworfen wird, sondern referieren reale Phänomene, die durch wirtschaftstheoretische Thesen erklärt werden sollen. Das wohl berühmteste Beispiel sind Kaldors stylized facts, die er in seine Arbeiten der späten 1950er und der frühen 1960er Jahre als typisch für den Wachstumsprozeß einer kapitalistischen Volkswirtschaft ansah:

1.) Konstantes, positives Wachstum der Produktion und der Arbeitsproduktivität

2.) Konstantes Wachstum der Kapitalintensität

3.) eine konstante Profitrate

4.) Konstanter Kapitalkoeffizient

5.) eine enge Korrelation zwischen der Investitionsquote und der Profitquote

6.) größere Differenzen zwischen den Wachstumsraten der Arbeitsproduktivität in verschiedenen Volkswirtschaften, die mit der Investitionsquote und Profitquote zusammenhängen. 6

Die Fakten 1-4 entsprechen dabei exakt den Kennzeichen des Harrod-neutralen technischen Fortschritts. Aufbauend auf diesen Fakten entwirft Kaldor mehrere Wachstumsmodelle, die eine technical progress function als einen der wichtigsten Bausteine enthalten. Die Überlegungen zu dieser Funktion sind für die Gegenüberstellung mit den Ansätzen der Neuen Wachstumstheorie von besonderem Interesse. Im folgenden Abschnitt werden die zentralen Aussagen zu diesem Konzept diskutiert. Hierbei steht weniger die komplette Modellstruktur im Vordergrund, sondern vielmehr die Begründung der technical progress function sowie die

4 Vgl. Kaldor (1985, S. 11f. und S. 13f.).

5 Vgl. Kaldor $(1985$, S. 8f.).

6 Vgl. z.B. Kaldor (1961, S. 230f.) 
Beziehung zur Investitionstätigkeit und ihre Rolle für die gleichgewichtige langfristige Lösung innerhalb des Wachstumsmodells von Kaldor.

In dem sich daran anschließenden Abschnitt wird auf den Beitrag Kaldors zur angewandten Wachstumstheorie eingegangen. Hierbei wurde von Kaldor insbesondere der Verdoom-Zusammenhang als wichtiger Grund für die Erklärung divergierender Wachstumsprozesse angesprochen, weshalb eine umfassende Diskussion dieses Zusammenhangs erfolgt. Aufbauend auf dieser Darstellung werden mögliche Anwendungen dieser Bausteine diskutiert, die von Kaldor selbst gemacht wurden bzw. in einem erweiterten Modell der kumulativen Verursachung erfolgten. Eventuelle Lehren für wachstumspolitische Fragestellungen werden in einem gesonderten Kapitel der Arbeit diskutiert.

\subsection{Das Konzept der technical progress function}

Das Konzept der technical progress function wurde von Kaldor in mehreren Aufsätzen aufgestellt und diente der endogenen Erklärung der Veränderung der Arbeitsproduktivität. ${ }^{7}$ Schon der erste Absatz seines ersten Beitrags zu diesem Thema liest sich wie eine Einleitung zu einem Aufsatz der Neuen Wachstumstheorie:

"But more recently, there has been an increasing awareness of the fact that neither the proportion of income saved nor the rate of growth of productivity per man (...) are independent variables with respect to the rate of increase in production; and that the actual rate of progress of a capitalist economy is the outcome of the mutual interaction of forces which can adequately be represented only in the form of simple functional relationships rather than by constants." 8

Ein Wachstumsmodell soll somit eine endogene Erklärung des Wachstumsprozesses bieten und sich nicht auf exogene Größen zur Bestimmung der Arbeitsproduktivität verlassen.

Eine Möglichkeit zur Erfullung dieses Anspruchs wird von Kaldor in der technical progress function gesehen, die in ihrer ersten Version eine Beziehung zwischen der Wachstumsrate der Arbeitsproduktivität und derjenigen des Kapitalstocks pro Kopf darstellt: ${ }^{9}$

$$
\mathrm{p}_{\mathrm{t}}=\mathrm{F}\left(\mathrm{k}_{\mathrm{t}}-\mathrm{l}_{\mathrm{t}}\right) \quad \text { mit } \quad \mathrm{F}(0)>0 \text { und } \mathrm{F}^{\prime}(\mathrm{k}-1)>0 \text { und } \mathrm{F}^{\prime \prime}(\mathrm{k}-1)<0
$$

7 Vgl. Kaldor (1957, 1961) sowie Kaldor/Mirrlees (1962). Einen umfassenden Überblick über Kaldors Wachstumsmodelle bietet 2.B. Skott (1989).

8 Kaldor (1957, S. 591).

9 Auf eine Sache sei das Auge des Lesers an dieser Stelle gelenkt: Da in diesem Kapital in den betrachteten formalen Zusammenhăngen fast ausschließlich mit Wachstumsraten gearbeitet wird, werden anstelle des im Kapitel 3 verwendeten Dachs über den Variablen als Kennzeichen einer Wachstumsrate Kleinbuchstaben verwendet. Dies geschieht vor allem aus Gründen der Übersichtlichkeit der Formeln. Dariber hinaus entspricht es der Vorgehensweise in der zugrundegelegten Literatur. 
Eine mögliche Spezifikation ist die einer linearen technical progress function:

$$
\mathrm{p}_{\mathrm{t}}=\mathrm{a}+\mathrm{b}(\mathrm{k}-\mathrm{l})
$$

Abbildung (4.1) zeigt die graphische Darstellung von Gleichung (4.1) und (4.1')

\section{Abb. 4.1: Die technical progress function}

(a) allgemeine technical progress function

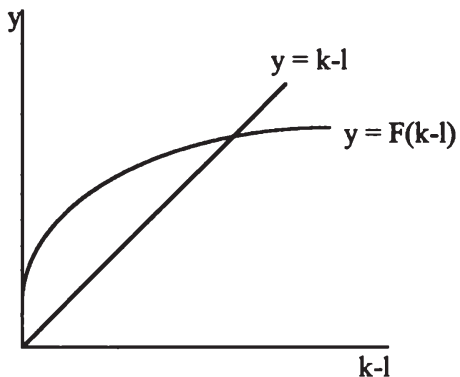

(b) lineare technical progress function

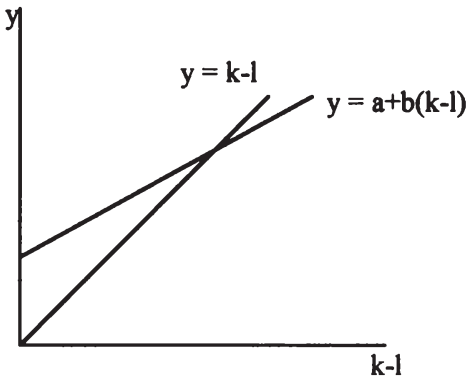

Quelle: Die Darstellung geht auf Kaldor (1957) zurück.

Die Begründung für diesen Zusammenhang sieht Kaldor in der Unmöglichkeit der Aufteilung der Produktivitätsfortschritte in eine Substitutionskomponente und eine Fortschrittskomponente. Kapitalintensivierung und die Erhöhung des technischen Wissens lassen sich nicht trennen, da zum einen jeder technische Fortschritt nach Auffassung Kaldors den Einsatz von mehr und effizienteren Maschinen notwendig macht und zum anderen die Erhöhung des Kapitaleinsatzes pro Arbeitskraft die Fähigkeit verlangt, mit der neuen Situation umgehen zu können. ${ }^{10}$ Der Kapitalstock ist somit nicht nur Träger des technischen Wissens, sondern Investitionen bieten auch Lernmöglichkeiten. Kapitalakkumulation und technischer Fortschritt sind unmittelbar miteinander verknüpft. Übertragen auf eine neoklassische Produktionsfunktion bzw. die Produktivitätsfunktion bedeutet dies, daß zwischen Bewegungen auf der Kurve (Kapitalintensivierung) und Bewegungen der Kurve selbst (exogener technischer Fortschritt) nicht unterschieden werden kann. ${ }^{11}$

Das Konzept der Produktionsfunktion ist zudem angreifbar, weil es ein Instrument einer statischen Theorie ist. Jeder Punkt auf der Produktionsfunktion steht für ein langfristiges stationäres Gleichgewicht, in dem sich die Produktionsverhältnisse genau an die im Punkt vorliegende Kapitalintensität angepaßt haben. Das System muß also ausreichend lange mit dieser

10 Vgl. zu dieser "Unmoglichkeit" Gahlen (1972).

11 Vgl. Kaldor (1957, S. 595f. und 1978, S. X2 
Kapitalintensität produzieren. Erst dann wird sich die Volkswirtschaft auf der Produktionsfunktion befinden. Dies erfordert aber zusätzlich die Konstanz des technischen Wissens. Verändert sich dieses, wird sich auch die Produktionsfunktion ständig verschieben, d.h. die Volkswirtschaft wird zu keiner Zeit einen Punkt auf der Produktionsfunktion realisieren können. Wenn dem so ist, können aber mit dieser keine Rückschlüsse auf das Preissystem und die Verteilung des Outputs gezogen werden. ${ }^{12}$

Kaldor sieht darüber hinaus ein Problem zwischen der Annahme konstanter Skalenerträge der traditionellen Produktionsfaktoren Arbeit und Kapital und der Exogenität des technischen Fortschritts. Technischer Fortschritt im Sinne des neoklassischen Modells ist ein kostenloser Produktionsfaktor, der kein eigenes Grenzprodukt erhält. Hierbei ergibt sich nun ein Unterschied zwischen dem privaten und dem sozialen Grenzprodukt der anderen Produktionsfaktoren. Die Annahme konstanter Skalenerträge bzgl. Arbeit und Kapital kann deshalb nicht aufrechterhalten werden. Technischer Fortschritt führt zum Vorliegen steigender Skalenerträge der Produktionsfunktion. Somit ist auch aus diesem Grunde nach Auffassung Kaldors jegliche Wachstumstheorie auf Basis einer neoklassischen Produktionsfunktion abzulehnen. ${ }^{13}$

Der Verlauf und die Lage der Kurve werden nach Kaldor durch die Eigenschaften des technischen Fortschritts bestimmt. Die technical progress function weist einen positiven Ordinatenabschnitt (a) auf, der exogene, nicht durch steigende Kapitalintensivierung entstandene Produktivitätsfortschritte erfassen soll. Hierunter sollen neben exogen entstehendem technologischen Wissen auch die Verbesserungen der Organisation innerhalb einer Produktionsstätte fallen, die ohne Investitionen entstehen können. Dieses Phänomen wurde unter dem Begriff des Horndahl-Effekts bei der Diskussion des Modells von Arrow schon angefuhrt. Kaldor schreibt somit einen Teil der im vorigen Kapitel oft so wichtigen Lerneffekte einer exogenen Komponente zu. Insgesamt soll dieser Achsenabschnitt den sog. technical dymamism der jeweiligen Volkswirtschaft beschreiben. Dieser Begriff ist sehr vage, da er nicht nur die Entstehung neuen Know-hows, sondern auch die Bereitschaft zum Wandel bestehender Strukturen sowie die Experimentierfreudigkeit einer Gesellschaft erfassen soll. Die Lage der Kurve ist von den Möglichkeiten einer Volkswirtschaft abhängig, neue Ideen und Technologien zu entwickeln und sie in den Produktionsprozeß einzufüren. Wenn dies auf konstantem Niveau gelingt, wird sich der Ordinatenabschnitt nicht ändern. Die Volkswirtschaft wird dauerhaft mit neuen Ideen versorgt. Technologische Sprünge wiederum vergrößern die Menge an technologischen Ideen. Die Chance, diese mittels Investitionen auszunutzen, wird der Volkswirtschaft höhere Produktivitätsfortschritte bei gleichen Wachstumsraten des Kapitalstocks pro Kopf ermöglichen. ${ }^{14}$

An dieser Stelle läßt sich erste Kritik an dem Konzept der technical progress function anbringen. Sie wurde konzipiert, um das Problem der Unmöglichkeit der Trennung zwischen

12 Vgl. Kaldor (1961, S. 260f. und 1986a, S. 193ff.).

13 Vgl. Kaldor (1961, S. 261f.).

14 Vgl. Thirlwall (1987, S. 175f.) und Hahn/Matthews (1964, S. 847). 
Verschiebung der Produktionsfunktion und Bewegung auf dieser zu vermeiden. Wenn nun aber der technical dynamism einer Volkswirtschaft über die Lage der technical progress function entscheidet und gleichzeitig die Rate der Kapitalakkumulation sowohl die Position auf dieser bestimmt als auch Rückwirkungen auf den technical dynamism hat, entsteht dieselbe Problematik nur auf einer anderen Ebene. ${ }^{15}$

Gleichzeitig werden abnehmende Grenzerträge der Kapitalintensivierung unterstellt, d.h. die Steigung der Kurve ist um so kleiner, je höher die Wachstumsrate der Kapitalausstattung pro Arbeitnehmer ist. Der Verlauf der Kurve gibt Auskunft über die Ausnutzung der vorhandenen Ideen. Wie im Falle des Kapitaleinsatzes in der Neoklassik, wo die Kapitalgüter zuerst auf die effizienteste Verwendung alloziiert werden, geht Kaldor davon aus, daß immer die ertragreichsten Ideen als erstes Anwendung finden. Dabei ist es uninteressant, ob es sich um neue oder alte Ideen handelt. Die abnehmende Steigung kann somit als abnehmende Fähigkeit zum Einsatz produktivitätssteigernder Technologien interpretiert werden. ${ }^{16}$

Innerhalb Kaldors Modell spielt somit die Investitionstätigkeit die maßgebliche Rolle für mögliche Produktivitätsfortschritte. Das Vorliegen technologischer Neuerungen reicht nicht aus, um Produktivitätssteigerungen zu ermöglichen. Vielmehr muß die Technologie auch umgesetzt werden, d.h. entsprechend wie im neoklassischen Vintage-Modell in die Produktionstechnologie via Investitionen einfließen. Um diesen Aspekt besser zu verdeutlichen, wird in einer Modifikation der ursprünglichen technical progress function nur noch das Wachstum der Arbeitsproduktivität der Arbeitnehmer an neu errichteten Maschinen und nicht mehr das Wachstum der Arbeitsproduktivităt insgesamt bestimmt. Die entscheidende Determinante für die Entwicklung der Produktivität der Arbeitskräfte an neu installierten Anlagen ist die Wachstumsrate der Bruttoinvestitionen pro Arbeitnehmer, da nicht nur zusätzliche Maschinen die Arbeitsproduktivität erhöhen, sondern auch neue, die alte abgeschriebene ersetzen. Diese Annahme stellt somit eine gewisse Parallelität zu Arrows Überlegungen dar. Eine hohe Wachstumsrate der Investitionen pro Kopf, d.h. erhöhen die Unternehmen in einem Zeitraum die Kapitalausstattung eines Arbeitsplatzes mehr, als sie es in der Vorperiode getan haben, wird die Arbeitsproduktivität der Arbeitskräfte an den neuen Maschinen steigern. Die technical progress function soll weiterhin einen positiven Ordinatenabschnitt aufweisen. ${ }^{17}$

Diese Form der technical progress function ist somit noch mehr als die vorige ein Vintage-Ansatz. Auch wenn vollständig auf das neoklassische Instrumentarium verzichtet wird, spiegelt sich doch dieselbe Grundidee wider. Im Gegensatz zur neoklassischen Überlegung ist der technische Fortschritt aber nicht ausschließlich exogener Natur. Vielmehr kann auch die Anwendung neuer Maschinen zum Lernen an diesen Maschinen führen, d.h. wie im Modell von Arrow entstehen Produktivitätsfortschritte nicht nur durch den Transport neuer Technologien

15 Vgl. Walter $(1969$, S. 216).

16 Vgl. Kaldor (1961, S. 265) und Walter (1969, S. 211f.).

17 Vgl. Kaldor/Mirrlees (1962, S. 176f.). 
in den Produktionsprozeß, sondern durch die Arbeit an den neuen Anlagen selbst. Kaldor/Mirrlees (1962) selbst sehen die Ursachen für den Einfluß der Wachstumsrate der Investitionen pro Kopf auf die Wachstumsrate der Arbeitsproduktivität an den neuesten Maschinen wohl mehr in der Ausnutzung vorhandener Technologien als in induzierten Lerneffekten. Diese werden nur in einer Fußnote erwähnt. Sie sollen primär die sinkende technische Effizienz von Maschinen aufgrund steigender Reparaturkosten ausgleichen. Somit scheinen die Lerneffekte an dieser Stelle mehr ein Mittel zum Zweck die konstante Produktivität der Maschinen eines Jahrgangs aufrecht zu erhalten denn eine zwangsläufige Folge der Investitionstätigkeit. Induzierter technischer Fortschritt aufgrund von learning by doing kann zwar mit der technical progress function in Verbindung gebracht werden, in den ursprünglichen Aufsätzen findet sich jedoch recht wenig dazu. Dies bedeutet nicht, daß Kaldor Produktivitätssteigerungen aufgrund von Lerneffekten vernachlässigte. Insbesondere in seinen Vorstellungen über die realen Wachstumsprozesse von Ökonomien wird dies im Zusammenhang mit der Besprechung des Verdoorn-Zusammenhangs deutlich. 18

Die Produktivitätssteigerungen als Ausdruck des technischen Fortschritts setzen sich aus autonomen und induzierten sowie korporierten und inkorporierten Elementen zusammen. Somit stellt Kaldors technical progress function eine Kombination von Modulen dar, die in der traditionellen neoklassischen Wachstumstheorie entscheidend waren, und Ideen, wie sie von Arrow (1962) mit seinem Konzept des learning by doing vorgebracht wurden. Der Vorteil des kaldorianischen Konzepts liegt in der Tatsache, daß keinerlei Produktionsfunktion notwendig ist. Diese Aussage muß jedoch relativiert werden, wenn man eine lineare technical progress function unterstellt, wie sie in Kaldors frühen Modellen (z.B. von 1957) enthalten ist. Die Überfuhhrung einer solchen Beziehung in eine Cobb-Douglas-Produktionsfunktion ist prinzipiell möglich. Bei der technical progress function handelt es sich in diesem Falle um eine Differentialgleichung, deren Stammfunktion eine Cobb-Douglas-Produktionsfunktion ist. Die Bestimmung der exakten Stammfunktion verlangt nun die Festlegung des Kapitalstocks und des Outputs zum Zeitpunkt (0). Es sind somit viele verschiedene Stammfunktionen denkbar. Für eine neoklassische Produktionsfunktion darf die Erstausstattung keine Rolle spielen. Darüber hinaus hat Black (1962) gezeigt, daß bei einer konvex verlaufenden technical progress function die Grenzproduktivität des Kapitals von der Rate der Kapitalakkumulation abhängig ist, während dies bei einer Cobb-Douglas-Produktionsfunktion nicht der Fall ist. Eine CobbDouglas-Produktionsfunktion kann somit nicht mit einer allgemeinen technical progress function in Verbindung gebracht werden. 19

Die Abhängigkeit sowohl der realisierten Produktivitätseffekte als auch der Entstehung neuer Ideen von der Kapitalbildung wirft die Frage nach der zugrundegelegten Investitions-

18 Vgl. auch Kaldor/Mirrlees (1962, S. 176 Fn 1), Kaldor (1959, S. 221) sowie Skott (1989, S. 31).

19 Eine lineare Form der technical progress function wurde von Kaldor nur aus Vereinfachungsgründen gewăhlt. Vgl. Kaldor (1961, Fn 2, S. 272), Black (1962) sowie Oppenländer (1963, S. 224ff.). Für die Anwendungen von Differentialgleichungen vgl. Chiang (1984). 
funktion bei Kaldor auf. Beide Sachverhalte stehen in enger Beziehung. Während die technical progress function die Möglichkeiten aufzeigt, durch Investitionen Produktivitätssteigerungen zu erzielen, gibt die Investitionsfunktion an, wieviel investiert wird, d.h., wie die vorhandenen Möglichkeiten ausgenutzt werden. Für Kaldor waren mehrere Determinanten für die Investitionsentscheidung der Unternehmen wichtig. Ziel der Unternehmen ist die Aufrechterhaltung eines konstanten Verhältnisses zwischen investiertem Kapital und Güterverkäufen, wobei dieses Verhältnis annahmegemäß mit steigenden Profiterwartungen zunimmt. Darüber hinaus sollen die Investitionen dafür sorgen, daß der gewünschte Kapitalstock dem tatsächlichen entspricht. Die Erwartungen über die Wachstumsrate der Verkäufe einer Periode entsprechen der tatsächlichen Wachstumsrate in der Vorperiode. Während er in seinem Modell von 1957 noch die Veränderung der Profitraten zwischen zwei Perioden als eine Größe in die Investitionsfunktion aufnimmt, betont Kaldor später (1962) die Bedeutung der Erwartungen über zukünftige Profite stärker. Im Modell von Kaldor/Mirrlees fehlt eine eigenständige Investitionsfunktion jedoch vollständig 20 Die kaldorianischen Vorstellungen zur Investitionsfunktion folgen somit dem Akzelerationsprinzip, wie es bei Harrod angesprochen wurde. Die Investitionen werden in jeder Periode von der Entwicklung des Outputwachstums beeinflußt. An dieser Stelle findet sich nun die Verbindung zwischen Investitionen und technical progress function. Das Wachstum des Outputs wird maßgeblich über die technical progress function durch die Entwicklung der Bevölkerung und den technischen Fortschritt beeinflußt.

Kaldor unterstellt den Unternehmen den Wunsch nach einem konstanten Kapital-OutputVerhältnis, d.h. stellen die Unternehmen fest, daß sie weniger akkumuliert haben, als es die eingetretenen Nachfragezuwächse verlangen, werden sie ihre Investitionstätigkeit ausdehnen, bis sie die gewünschte Situation erreicht haben. Geht man von einer konstanten Bevölkerung aus und liegt nun bspw. die Wachstumsrate des Outputs über der Wachstumsrate des Kapitalstocks, werden die Unternehmen ihre Kapitalnachfrage ausdehnen, um das von ihnen gewünschte Kapital-Output-Verhältnis zu erreichen. Diese Investitionsausdehnung führt wiederum aufgrund der technical progress function zu höherem Produktivitätswachstum und auch weiterem Produktionswachstum. Aufgrund der unterstellten abnehmenden "Grenzerträge" der Kapitalintensivierung bzw. der Investitionserhöhung pro Arbeitsplatz werden die Zuwächse bei der Wachstumsrate des Outputs geringer ausfallen als bei der Wachstumsrate des Kapitals. Das System konvergiert zu einem Gleichgewichtspunkt, in dem unabhängig von der gewählten technical progress function die Forderung nach der Konstanz des Kapitalkoeffizienten erfüllt ist und die Erwartungen der Unternehmen befriedigt sind. Eine der stilisierten Fakten ist erfüllt. ${ }^{21}$

20 Vgl. Kaldor (1957, S. 600f. und 1961, S. 27lf. insbes. S. 272 Fn 1). Vgl. auch Skott (1989, S. Kap. 4).

21 Vgl. z.B. Kaldor (1961, S. 273f.) und Oppenländer (1963, S. 205f.). Zu den Stabilitătsbedingungen siehe Kubota (1968) und Kaldor (1970b) sowie auch Skott (1989). Die Wirkungen des technischen Fortschritts im langfristigen Gleichgewicht entsprechen denen des Harrod-neutralen technischen Fortschritts. Vgl. Targetti (1992, S. 125). 
Dieses Ergebnis erinnert selbstverständlich an das des neoklassischen Modells. Einerseits ist auch dort die langfristige Situation durch die Konstanz des Kapitalkoeffizienten gekennzeichnet, andererseits lăuft der Anpassungsprozeß im neoklassischen Fall über die abnehmenden Grenzerträge des Kapitals bei steigender Kapitalintensivierung, während im vorliegenden Falle die abnehmenden Produktivitätseffekte der Kapitalintensivierung für eine Anpassung sorgen. Diese Ähnlichkeit wird noch deutlicher, wenn man z.B. die Lösung für die Wachstumsrate des Outputs pro Kopf (y) im Steady State im ersten Modell unter Annahme einer konstanten Bevölkerung betrachtet: 22

$$
y=\frac{a}{1-b}
$$

Auch im Modell von Kaldor/Mirrlees (1962) ergibt sich diese Lösung. Im Steady StateGleichgewicht wachsen die Arbeitsproduktivität an den neuesten Maschinen (Wachstumsrate: $\pi$ ) und die Investitionen pro Arbeitsplatz (Wachstumsrate: 1 ) mit derselben Rate. Es gilt: ${ }^{23}$

$$
\pi=\frac{s}{h}-1-\delta \quad \text { mit } \quad \pi=a+b(\mathfrak{l}) \quad \text { und } \pi=\mathfrak{l} \text {, }
$$

daraus folgt für die gleichgewichtige Wachstumsrate

$$
\pi=\imath=\frac{a}{1-b}=\frac{s}{h}-1-\delta
$$

Die langfristige Wachstumsrate wird in beiden Fällen durch die Parameter der technical progress function bestimmt. Die Volkswirtschaft kann mit keiner größeren Rate wachsen. Ein solches Ergebnis darf jedoch nicht verwundern. Die technical progress function ist eine exogen vorgegebene Beziehung, die im formalen Modell von Kaldor als nicht veränderbar angenommen wird. Wenn das Modell eine unverrückbare Beziehung enthält, und alle anderen Variablen im System - abgesehen vom gewünschten Kapitalkoeffizienten - flexibel sind, darf es

22 Vgl. Kaldor (1961, S. 274). Kaldor diskutiert auch den Fall einer wachsenden Bevolkerung. Die Pro-KopfWachstumsrate wird dabei gesenkt. Wenn die Effekte des technischen Fortschritts nicht ausreichen, die dämpfenden Effekte des Bevölkerungswachstums auszugleichen, ergibt sich die Möglichkeit des Nullwachstums. Daruber hinaus geht Kaldor auch auf verschiedene Stadien der Entwicklung ein, die sich bei der Leistungsfahigkeit und der Investitionsfunktion der Volkswirtschaft unterscheiden. Ähnlich dem Modell von Azariadis/Drazen (1990) muß erst ein Schwellenwert des Kapitalstocks erreicht werden, bevor die Volkswirtschaft uber dem Subsistenzniveau produzieren kann. Vgl. hierzu Kaldor (1957, S. 614ff.).

$23 \mathrm{Vgl}$. Kaldor/Mirrlees (1962, S. 178ff.). Hier steht (s) für die Sparquote, (h) für die gewünschte Amortisationsdauer einer Investition und ( $\delta$ ) fur die Abschreibungsrate. 
nicht verwundern, wenn die exogenen Größen die Wachstumsmöglichkeiten der Ökonomie bestimmen. 24

Wo liegen nun die Unterschiede zur neoklassischen Vorgehensweise? Der Vorteil der technical progress function liegt in der Tatsache, daß sie zumindest in ihrer Lage von den historischen Entwicklungen einer Gesellschaft bestimmt wird, während die Neoklassik in ihrer traditionellen Form als geschichtslos bezeichnet werden kann. Obwohl der Zusammenhang zwischen dem Investitionsprozeß und dem autonomen Teil der technical progress function nicht spezifiziert wird, taucht er zumindest in den verbalen Ausführungen Kaldors auf. In diesem Sinne stellt die technical progress function einen Weg zu mehr Endogenität des Wachstumsprozesses dar. Die kaldorianischen Überlegungen weisen des weiteren den Vorteil auf, daß die Investoren nicht als die Erfüllungsgehilfen der Haushalte gesehen werden, sondern eine von der Höhe der Ersparnisse unabhängige, eigenständige Entscheidung über die getätigten Investitionen fallen können. Prinzipiell bestimmt letztere über die Realisierung potentieller Wachstumschancen bzw. die Ausnutzung der Produktivitätseffekte. ${ }^{25}$

Darüber hinaus kann nicht bestritten werden, daß das Konzept ein direkter Vorläufer von Arrows Konzept des learning by doing ist. Sowohl in Kaldors als auch in Arrows Modell kann die Installation neuer Maschinen zu induzierten Lerneffekten führen, die zur Effizienzsteigerung späterer Investitionen führen. Während in Arrows Konzept das neu erlernte Wissen direkt in die Investitionsgüter der nächsten Generation fließt, wird der Übertragungsmechanismus bei Kaldors Konzept nicht so deutlich. Die Aufrechterhaltung eines gleichmäßigen Investitionsverhaltens ermöglicht hier die Übernahme der vorhandenen Technologien und sorgt für eine Aufrechterhaltung des technical dynamism, der die Lage der technical progress function bestimmt. Die Investitionstätigkeit eines Unternehmens hat deshalb externe Effekte auf nachfolgende Investitionen. Aus diesem Grunde kann Kaldors und Arrows Konzept in dieselbe Richtung interpretiert werden. Beidemal haben Investitionen externe Effekte in der Zukunft. Die Vergangenheit bestimmt damit die Gegenwart.

Im weiteren wird ein zweites zentrales Element in Kaldors Vorstellungen über Wachstumsprozesse in Marktwirtschaften untersucht werden, das noch mehr an empirisch feststellbaren Zusammenhängen orientiert ist, als es das Konzept der technical progress function war. Es wird sich zeigen, daß zwischen den grundsätzlichen Überlegungen zur technical progress function und dem sog. Verdoorn-Zusammenhang Beziehungen bestehen. Darüber hinaus werden die folgenden Aussagen mit Sicherheit den Eindruck eines sehr neoklassischen Nicholas Kaldors, dessen frühe Wachstumsmodelle nicht unbedingt postkeynesianische Resultate generieren, relativieren. Insbesondere der Glaube an die Instabilität marktwirtschaftlicher Systeme wird in seinen späteren Arbeiten besser erkennbar.

24 Vgl. Rothschild (1959, S. 168), der auch sehr stark die Annahme der Vollbeschăftigung bei Kaldor kritisiert.

25 Langfristig determinieren aber dennoch die exogenen Variablen den Wachstumsprozeß. 


\subsection{Angewandte Wachstumstheorie}

\subsubsection{Grundlagen}

Seit Mitte der sechziger Jahre konzentrierte sich Kaldor zunehmend auf eine mehr angewandte Wachstumstheorie, die Erklärungen für unterschiedliche Wachstumsraten von Volkswirtschaften geben konnte. Während in seinen rein wachstumstheoretischen Beiträgen immer das Konzept eines Steady States behandelt wird, ist seine Diskussion empirisch feststellbarer Zusammenhänge im Wachstumsprozeß durch die Betonung ungleichgewichtiger Entwicklungen, im Sinne kumulativer Prozesse, gekennzeichnet. Kaldors Arbeiten zur angewandten Wachstumstheorie basieren dabei im wesentlichen auf drei Elementen:26

1.) dem Prinzip der kumulativen Verursachung, wie es von Gunnar Myrdal in die ökonomische Diskussion eingebracht wurde.

2.) den Vorstellungen von Allyn Young zur Existenz und Ursache steigender Skalenerträge

3.) dem Verdoorn-Zusammenhang, der eine Beziehung zwischen Produktions- und Produktivitätswachstum unterstellt.

In den folgenden Abschnitten werden in einem ersten Schritt die wichtigsten Implikationen des Prinzips der kumulativen Verursachung in der Tradition von Gunnar Myrdal diskutiert. Daran anschließend werden die Überlegungen von Allyn Young (1928) zur Frage steigender Skalenerträge vorgestellt werden: Sie bilden eines der wichtigsten Verbindungselemente zwischen den Arbeiten Kaldors und der Neuen Wachstumstheorie. In einem weiteren Teilkapitel wird der sog. Verdoorn-Zusammenhang einer genaueren Untersuchung unterzogen werden.

\subsubsection{Das Prinzip der kumulativen Verursachung}

Der Begriff der kumulativen Verursachung geht auf Myrdal zurück, der in seinen Arbeiten zu den ökonomischen Problemen unterentwickelter Regionen bzw. Länder sehr deutlich herausarbeitet, wie systeminhärente Prozesse Ungleichheiten verstärken können. ${ }^{27}$ Hat ein soziales System eine gleichgewichtige Situation verlassen, werden Kräfte wirksam, die eine Rückkehr

26 Vgl. zu dieser Dreiteilung auch Hodgson (1989, S. 80f.).

27 Vgl. vor allem Myrdal (1959). Myrdal selbst weist auf die Existenz dieses Prinzips im Neuen Testament hin. Im Matthausevangelium (25, Vers 29, einem Teil der Bergpredigt) findet sich im Gleichnis Die anvertrauten Zentner, der Ausspruch: Denn wer da hat, dem wird gegeben werden, und er wird die Fülle haben; wer aber nicht hat, dem wird auch, was er hat, genommen werden. Einen Überblick gibt Ricoy (1987) und Thirlwall (1994). 
zum Gleichgewicht verhindern. Die Interdependenz der Systemvariablen kann bewirken, daß einmalige Veränderungen in ihren Folgen verstärkt werden, d.h. gleichgerichtete und nicht entgegengesetzte Reaktionen erzeugen. Es kommt zu einem circulus vitiosus, der einer automatischen Stabilisierung entgegensteht. Myrdal macht dies anhand der Situation der schwarzen Bevölkerung in den USA verständlich. Die zentralen Variablen sind der niedrige Lebensstandard der Farbigen und die Vorurteile der weißen Bevölkerung. Die Vorurteile bedingen einerseits die Diskriminierung der schwarzen Minderheit und sorgen dafür, daß diese ihren Lebensstandard nicht verbessern kann. Andererseits sehen sich die Weißen in ihren Vorurteilen bestätigt, da die soziale Situation der Farbigen genau ihren Vorstellungen entspricht. Dieses System kann stabil sein. Kommt es jedoch zu einer Veränderung einer der beiden Variablen, wird die andere sich auch verändern. Hierbei ist sowohl eine Entwicklung in die positive als auch in die negative Richtung möglich. Überwinden die Weißen ihre Vorurteile und diskriminieren sie die Farbigen nicht mehr, werden diese Chancen und Möglichkeiten haben, ihre Situation zu verbessern. Unterliegen gesellschaftliche Systeme dem Phänomen der zirkulären Verursachung bedeutet dies nicht, daß sie nicht auch in einer Ruhelage, d.h. einem Gleichgewicht verharren können. Kommt es zu einer Krafteinwirkung auf das System, die es aus dieser Ruhesituation bringt, dann bewirkt die veränderte Größe gleichgerichtete Änderungen weiterer Variablen, welche wiederum progressiv auf die Veränderung der anfänglich veränderten Größe wirken. Die einzelnen Teile des Systems sind durch positive Feedback-Effekte miteinander verknüpft. ${ }^{28}$

Übertragen auf ökonomische Fragestellungen impliziert dies eine Ablehnung der neoklassischen Vorstellung, daß ökonomische Systeme endogene Mechanismen aufweisen, die für die erneute Realisierung von Gleichgewichten nach exogenen Schocks verantwortlich sind. ${ }^{29}$ Die anfängliche gleichgewichtige Situation kann nicht mehr erreicht werden. Darüber hinaus können sich durch einen solchen circulus vitiosus wirtschaftiche Unterschiede zwischen Regionen oder Ländern vergrößern, d.h. Divergenz und nicht Konvergenz der Einkommensentwicklungen müßten weltweit zu erwarten sein. Während die neoklassische Theorie z.B. große Kapitalströme in unterentwickelte Regionen oder Länder aufgrund der dortigen hohen Grenzproduktivität des Kapitals und somit hohe Wachstumsraten des Sozialprodukts erwarten ließe, würde unter Gültigkeit des Prinzips der kumulativen Verursachung gerade die entgegengesetzte Richtung eingeschlagen werden. Expandiert ein Wirtschaftsraum, wird er aufgrund der steigenden Güternachfrage auch attraktiv für Investitionen sein. Mittels Multiplikatorprozeß führt dies wiederum zu steigenden Einkommen und steigender Nachfrage. Die ärmeren

28 Vgl. Myrdal (1959, S. 9ff.). Auch das Modell von Harrod ist in diesem Sinne ein Modell der kumulativen Verursachung.

29 Die automatische Ablehnung der neoklassischen Vorstellungen zur Stabilităt von Gleichgewichten ergibt sich aber nur, wenn man von Überlegungen, wie sie z.B. von Harrod (1973) oder von Leijonhufvud (1973) zur Existenz von "Stabilităts-Korridoren" gemacht wurden, absieht. Liegen nur geringe Abweichungen vom Gleichgewicht vor, treten nach dieser Idee nicht zwangslăufig Prozesse ein, die weiter vom Gleichgewicht wegfuhren. Vgl. auch Kapitel 2 zum Harrod-Modell. 
Regionen werden vor dem Problem der Kapitalflucht stehen. Die Kapitalanleger werden ihre Mittel in den Ländern investieren, die eine sichere Verzinsung bieten. Der auftretende Investitionseinbruch wird den kontraktiven Prozeß verstärken. Vergleichbares gilt für die Wanderungen von Arbeitskräften sowie den freien Güterhandel. Prosperierende Regionen attrahieren aufgrund des höheren Lohnniveaus mobile Arbeitskräfte, was zu sinkenden Lohnkosten in der Zielregion und einem Mangel an Arbeitskräften in der Abwanderungsregion führen kann. Außenhandel wiederum vergrößert die potentiellen Märkte, wodurch die Anbieter in den entwickelten Regionen Wettbewerbsvorteile realisieren können. ${ }^{30}$

Die Existenz einer divergierenden Entwicklung hängt mit von der Annahme über die zugrundegelegte Investitionsfunktion ab. Orientieren sich die Unternehmen an der Grenzproduktivität des Kapitals, werden sie bereit sein, unter sonst gleichen Umständen ihre Mittel in unterentwickelten Regionen zu investieren. Folgt das Investitionsverhalten jedoch keynesianischen Vorstellungen, d.h. sind die Grenzleistungsfähigkeit und das Akzelerationsprinzip die entscheidenden Konzepte, werden sich anfängliche Unterschiede in den Einkommen und der Güternachfrage zwischen Ländern oder Regionen vergrößern. Somit spielen die Erwartungen der Wirtschaftssubjekte eine wichtige Rolle. Nur falls die Investoren in den erfolgreichen Regionen ständig steigende Nachfrage erwarten, wird der Investitionsprozeß stabil sein. Ändern sich dagegen die Erwartungen, kann es zur Umkehr der Entwicklung kommen. Das Beispiel der Erwartungen zeigt, daß der Prozeß der kumulativen Entwicklung durch einen exogen Schock gestoppt oder umgekehrt werden, kann.

\subsubsection{Steigende Skalenerträge in der Tradition von Allyn Young ${ }^{31}$}

Ein zentrales Element der Arbeiten von Nicholas Kaldor zur Wachstumstheorie ist die auf Allyn Young zurückgehende Diskussion steigender Skalenerträge. ${ }^{32}$ Young untersuchte in seiner Presidential Address der ökonomischen Sektion der British Association for the Advancement of Science im Jahre 1928 die Zusammenhänge zwischen steigenden Skalenerträgen und ökonomischem Fortschritt. Ausgangspunkt seiner Überlegungen sind einerseits das Prinzip der Arbeitsteilung, wie es seit Adam Smith immer wieder diskutiert wurde und andererseits die Unterscheidung in externe, durch Reorganisation der Industrie entstandene

30 Vgl. hierzu Myrdal (1959, S. 26f.). Die Situation in der ehemaligen DDR in den Monaten nach dem Fall der Mauer stellt ein gutes Beispiel für diesen Aspekt der kumulativen Verursachung dar. Es wurde befürchtet, daß viele leistungsfahige Arbeitskräfte nach Westdeutschland übersiedeln würden, um dort eine Anstellung zu suchen. Um dies zu verhindern, wurde u.a. von den Tarifparteien eine schnelle Anpassung der Ostløhne an das westliche Niveau vereinbart. Die rasch steigenden Löhne haben die Wettbewerbsfahigkeit ostdeutscher Unternehmen zusatzlich verschlechtert.

31 Die nachfolgenden Überlegungen basieren auf dem berühmten Aufsatz von Allyn Young Increasing Returns and Economic Progress aus dem Jahr 1928. Vgl. zur Arbeit und Person von Young auch Blitch (1983b, 1990).

32 Vgl. z.B. Kaldor (1966, 1970a, 1972 oder 1981a). 
und interne, aus der Realisierung von Größenvorteilen resultierende Skalenerträge, wie sie von Alfred Marshall vorgeschlagen wurde.

Das Prinzip der Arbeitsteilung bildet in Smiths Wealth of Nations aufgrund seiner Bedeutung für die Entwicklungsmöglichkeiten von Volkswirtschaften einen der zentralen Untersuchungsgegenstände. Für Smith sind zwei maßgebliche Sachverhalte für die gesamtwirtschaftliche Produktion von Bedeutung:

"The annual labour of every nation is the fund which originally supplies it with all the necessaries and conveniences of life which it annually consumes, and which consist always, either in the immediate produce of that labour, or in what is purchased with that produce from other nations.

According therefore, as this produce, or what is purchased with it, bears a greater or smaller proportion to the number of those who are to consume it, the nation will be better or worse supplied with all necessaries and conveniences for which it has occasion.

But this proportion must in every nation be regulated by two different circumstances; first, by the skill, dexterity, and judgement with which its labour is generally applied; and, secondly, employed in useful labour, and that of those who are not so employed. Whatever the soil, climate, or extent of territory of any particular nation, the abundance or scantiness of its annual supply must, in that particular situation, depend upon those two circumstances. $" 33$

Die Arbeitsproduktivität und die Erwerbsquote (Anteil der Erwerbstätigen an der Bevölkerung) beeinflussen die Leistungsfähigkeit einer Volkswirtschaft. Zusätzlich wird die Effizienz durch den Kapitaleinsatz pro beschäftigter Arbeitskraft, d.h. die Kapitalintensität, beeinflußt. Kapitalintensive Produktionsweisen fördern die Produktivität der Arbeit, deren Entwicklung nicht getrennt von der Kapitalbildung analysiert werden kann. Arbeitsteilung wird durch die Kapitalakkumulation, verstanden als die Lagerbildung für Lebensmittel, Rohstoffe und Werkzeuge, erst ermöglicht. Beide Phänomene bedingen sich somit gegenseitig und sind im Zusammenhang zu untersuchen. Arbeit und Kapital sind komplementäre Produktionsfaktoren in Smiths Vorstellung. ${ }^{34}$

Die Produktivität der Arbeit wird im Smithschen System maßgeblich von der Arbeitsteilung bestimmt. Durch die zunehmende Spezialisierung der Arbeitskräfte erhöht sich deren Geschicklichkeit, da sie dieselben Tätigkeiten mit immer mehr Routine ausführen. Des weiteren reduziert sich der Zeitbedarf pro Arbeitsgang, da z.B. Rüstzeiten entfallen. Drittens gibt die Arbeitsteilung die Möglichkeit zur Erfindung neuer Maschinen. Die wiederholte Durchführung von ein und derselben Tätigkeit erleichtert es den Arbeitskräften, Mittel und Wege zu finden,

33 Smith (Introduction, [1993, S. 8[).

34 Vgl. Smith (Introduction, [1993 S. 98] und II. [1993, S. 159f.]). Im weiteren werden nur die für die Darstellung der Youngschen Überlegungen notwendigen Ausfuhrungen von Smith betrachtet. Für eine umfassendere Darstellung siehe z.B. Gourvitch (1940, S. 33ff.), Lowe (1954b, 1984 insbes. S. 188ff.), Groenewegen (1987), Gehrke (1990), Hagemann (1990a), Rostow (1990a) oder Kurz/Salvadori (1995a). 
die die entsprechende Arbeit schneller bzw. mit weniger Arbeitsaufwand erledigen. Zunehmende Arbeitsteilung hat somit den gestiegenen Einsatz von Maschinen zur Folge, da sie Rahmenbedingungen schaff, die zu neuen Erfindungen führen können. Smith läßt in seinen weiteren Ausführungen neben diesem endogenen technischen Fortschritt, der durch die Arbeitsteilung induziert ist, auch Raum für exogenen technischen Fortschritt, wie wissenschaftiche Entdeckungen der sog. makers of machines, die man nach heutigem Sprachgebrauch als Ingenieure bezeichnen würde. ${ }^{35}$

Die Bestimmungsgründe für das Phänomen der Arbeitsteilung liegen für Smith in der Neigung der Menschen zum Tausch. Sie ist zwangsläufiges Ergebnis des Tausches, nicht Folge von erzielten Erkenntnissen. Sie liegt somit in der menschlichen Natur und unterscheidet den Mensch vom Tier. ${ }^{36}$ Aufgrund der Abhängigkeit der Arbeitsteilung von Tauschvorgängen und damit von Märkten, folgert Smith, daß auch "...the extent of this division (of labour der Verf.) must always be limited by the extent of that power, or, in other words, by the extent of the market." 37 Erst große Absatzmöglichkeiten machen arbeitsteilige Produktionsweisen sinnvoll, da bei kleinen Märkten Spezialisierung aufgrund der zu geringen Nachfrage nach Gütern bzw. nach der entsprechenden Arbeitsleistung unrentabel wäre. Das Theorem der Begrenztheit der Arbeitsteilung durch die Größe des Marktes ist für Allyn Young "...one of the most illuminating and fruitful generalisations which can be found anywhere in the whole literature of economics." 38

Trotz dieser Begeisterung für die Smithsche Argumentationsweise hat dieser den zentralen Punkt der Arbeitsteilung, zumindest was moderne Industrienationen betriff, nach Youngs Meinung übersehen. Die ständig zunehmende Unterteilung anfänglich komplexer Produktionsprozesse in mehrere einfache Tätigkeiten führe nicht nur zu einer höheren Produktivität der Arbeitskräfte, weil diese Arbeiten besser ausführen können bzw. weil sie neue Maschinen erfinden. Entscheidend sei vielmehr die Tatsache, daß einfache Arbeitsschritte den Einsatz von Maschinen überhaupt erst ermöglichen. Die Verwendung kapitalintensiver Produktionsweisen wird deshalb durch das Ausmaß der Arbeitsteilung und somit indirekt durch die Größe des Marktes bestimmt. Nur falls ausreichend große Stückzahlen von einem Endprodukt abgesetzt werden können, werden Unternehmen von arbeitsintensiven auf kapitalintensive Herstellungsmethoden wechseln. Die Kapitalintensität ist somit eine Folge der Marktentwicklung und nicht irgendeines Lohn-Zins- bzw. Grenzproduktivitäten-Verhältnisses. ${ }^{39}$ Der

35 Erinnert sei an das Smithsche Beispiel eines Jungen, der die Selbstregulierung von Ventilen bei Dampfmaschinen erfand, um mehr Zeit zum Spielen mit anderen Jungen zu haben, die mit derselben Aufgabe in der Fabrik betraut waren. Vgl. Smith (I.i.7 [1993, S. 15ff.D.

36 Vgl. Smith (I.ii [1993, S. 21ff.]). Dieser Unterschied ist jedoch auch nur ein relativer. Menschenaffen z.B. zeigen ein Gruppenverhalten, das sehr wohl durch Arbeitsteilung gekennzeichnet ist. Vgl. auch Arrow $(1979$, S. 74).

37 Smith (I.iii.1 [1993, S. 2]).

38 Young (1928, S. 529).

39 Vgl. Kaldor $(1985$, S. 67). 
Einsatz von Maschinen macht nun einerseits die Realisierung von steigenden Skalenerträgen im Sinne der economies of machines 40 überhaupt erst möglich. Andererseits ermöglicht die intensivere Verwendung von Maschinen weitere Arbeitsteilung bei deren Produktion. ${ }^{41}$ Diese Überlegungen werden in folgendem Zitat am deutlichsten:

"It would be wasteful to make a hammer to drive a single nail; it would be better to use whatever awkward implement lies conveniently at hand. It would be wasteful to furnish a factory with an elaborate equipment of specially constructed jigs, gauges, lathes, drills, presses and conveyors to build a hundred automobiles; it would be better to rely mostly upon tools and machines of standard types, so as to make a relatively large use of directly-applied and a relatively smaller use of indirectly-applied labour. Mr. Ford's methods would be absurdly uneconomical if his output were very small, and would be unprofitable even if his output were what many other manufactures of automobiles would call large.

Then of course, there are economies of what might be called a secondary order. How far it pays to go in equipping factories with special appliances for making hammers or for constructing specialised machinery for use in making different parts of automobiles depends again upon how many nails are to be driven and how many automobiles can be sold." 42

Der Prozeß der ständig steigenden Arbeitsteilung erfolgt aber nicht nur innerhalb eines Unternehmens, sondern fürt zu einer gleichzeitigen Reorganisation der inter- und intraindustriellen Struktur. Wird innerhalb einer Industrie die Einführung kapitalintensiver Produktionsprozesse rentabel, entstehen für vorgelagerte Industrien ebenfalls größere Märkte. Interne Skalenerträge, die durch die Realisierung der Größenvorteile auf Unternehmensebene entstanden sind, ermöglichen in einem zweiten Schritt externe Skalenerträge durch die Umstrukturierung auf Branchen- bzw. gesamtwirtschaftlicher Ebene. Zunehmende Arbeitsteilung ist somit nicht nur ein Phänomen innerhalb einer Branche oder einer Industrie, sondern sie findet auch zwischen Industrien statt. Sie schaff die Voraussetzung für die Entstehung neuer Industrien. ${ }^{43}$

Diese Überlegung geht auf die von Marshall ausführlicher diskutierte Unterscheidung in interne und externe Skalenerträge zurück. Hierbei entstehen interne Skalenerträge aufgrund von Größenvorteilen, die sich durch die Ausdehnung der produzierten Stückzahlen innerhalb eines Unternehmens ergeben. Marshall führt hier vor allem die produktivitätssteigernden Effekte der firmeninternen Arbeitsteilung an, wie das gestiegene Wissen der Arbeitnehmer und den Einsatz von Maschinen sowie günstigere Konditionen beim Bezug von Waren und Vorlei-

40 Economies of machines wurden von Marshall neben den economies of skill und den economies of materials als die Hauptvorteile der Massenproduktion gesehen (vgl. Marshall 1920, S. 278ff.). Dies stellt eine Weiterentwicklung der Effekte der Arbeitsteilung dar, wie sie bei Adam Smith angesprochen worden sind.

41 Vgl. Young (1928, S. 529f.).

42 Young $(1928$, S. 530).

43 Vgl. Young (1928, S. 528). 
stungen ${ }^{44}$ Externe Skalenerträge, die nicht von der Größe eines einzelnen Unternehmens abhängen, sondern von der gesamten Branche, resultieren vor allem aus dem Zuwachs des Wissens über produktionstechnische Zusammenhänge. Steigende Arbeitsteilung erhöht das individuelle Know-how innerhalb der Unternehmen und aufgrund von verbesserten Kommunikationsmöglichkeiten, z.B. durch Bücher, Fachzeitschriften, aber auch durch die räumliche Nähe zu anderen Unternehmen wird sich dieses neue Wissen verbreiten. Es wird somit kostenlos in die Produktion anderer Firmen einfließen. ${ }^{45}$ Ergänzt wird diese Entwicklung durch das Aufkommen von vorgelagerten Industrien und die Verbesserung von Infrastruktureinrichtungen, die durch eine räumliche Ballung von Industrien erst rentabel werden.

Das Marshallsche Konzept der internen und externen Skalenerträge betont deren dynamische Komponente. Führen intern Lemprozesse und Investitionen in Sachkapital zu überproportionalen Outputzuwächsen, hat extern die zunehmende Arbeitsteilung zwischen Firmen und Industrien eine Steigerung der Effizienz ohne externen technischen Fortschritt zur Folge. Sie resultieren aus der ständigen Veränderung des Produktionsprozesses und sind sowohl Ergebnis als auch Ursache des ökonomischen Wandels. Marshall selbst betonte insbesondere die langfristige Bedeutung dieser Effekte. ${ }^{46}$ Zugleich sind die externen Skalenerträge

44 Vgl. hierzu insbesondere Marshall (1920, Buch IV, Kapitel 9, 11 und 13).

45 Marshall diskutiert diese Aspekte z.B. im zehnten und elften Kapitel des vierten Buches der Principles sehr ausfürlich. Konzentrieren sich Unternehmen, die der gleichen Branche zuzurechnen sind, an einem Ort, entsteht zum einen die Möglichkeit zum Erfahrungsaustausch und zum anderen ein lokaler Arbeitsmarkt mit qualifizierten Arbeitskräften, die Wissen von einem Unternehmen zum anderen mitnehmen können. Vgl. z.B. Marshall (1920, S. 271).

46 Die von Marshall getroffene Unterscheidung in externe und interne Skalenerträge und deren Anwendung in einem statischen Preismodell hat zu einer größeren Debatte bzgl. der Konsequenzen für die Wettbewerbssituation auf den entsprechenden Märkten und dem Verlauf der Angebotskurven der Unternehmen gefuhrt. (Marshall selbst leitete aus seinen Überlegungen zur Produktionstechnik fallende Angebotskurven ab.) Liegen ausschließlich interne steigende Skalenertrage vor, kann von der Unvereinbarkeit dieser mit vollkommenem Wettbewerb ausgegangen werden. Sraffa (1926) betont eine weitere Konsequenz steigender Skalenertrăge, die sich auch in den Allyn Youngs Überlegungen wiederfindet. Die Ermittlung einer gleichgewichtigen Marktlosung für ein einzelnes Gut erfordert, daB desse Produktion und die Nachfrage nach diesem unabhängig voneinander sind. Zusătzlich müssen die Angebots- und die Nachfragekurven auf jedem einzelnen Markt wiederum unabhăngig vom Geschehen auf allen anderen Märkten sein. Nur unter diesen Bedingungen hat die Partialanalyse ihre Berechtigung. Liegen nun steigende Skalenerträge vor, entscheidet ihr Charakter ubber die Vereinbarkeit mit der partialanalytischen Untersuchungsmethode. Externe Skalenerträge, wie sie oben angesprochen wurden, sind aufgrund dieser Überlegung nicht mit dem Wettbewerbsgleichgewicht kompatibel, da hier die Kostensituationen der verschiedenen Unternehmer voneinander abhăngig sind. Für interne Skalenertrăge gilt, daB auch sie eine Abkehr von den Wettbewerbsbedingungen implizieren. In diesem Falle sind die Nachfrage und das Angebot auf einem spezifischen Markt miteinander verbunden. Steigende Skalenerträge dieser Art resultieren letztendlich im natürlichen Monopol. Für Sraffa haben Skalenerträge, die zwar für ein Unternehmen extern, für die gesamte Branche jedoch intern sind, kaum empirische Relevanz, so daß sich die Analyse mehr der Monopoltheorie zuwenden sollte. Chipman (1970) zeigt jedoch, daß im Fall externer steigender Skalenerträge ein Wettbewerbsgleichgewicht moglich ist. Eine zentrale Annahme dabei ist, daß die Unternehmen jedes für sich genommen davon ausgehen, daß sie mit konstanten Skalenerträgen produzieren. Vgl. zur Diskussion "Steigende Skalenertrăge und Wettbewerb" z.B. Knight (1921, 1924 und 1925), Sraffa (1926), Chamberlin (1933), Robinson (1933), Stigler (1941 und 1951), Chipman (1965, 1970), Erber (1980). An dieser Stelle sollen jedoch die Implikationen der vorgestellten Konzeption für die wachstumstheoretischen Überlegungen von Young und Kaidor im Mittelpunkt stehen. 
Externalitäten technologischer Art. Sie erzeugen nicht über das Marktsystem erfaßte Kostenvorteile für andere Unternehmen. Natürlich beinhaltet das Marshallsche Konzept auch pekuniäre Externalitäten. Wenn ein Unternehmen aufgrund zunehmender Arbeitsteilung ein Gut günstiger herstellen kann, wird der Preis für dieses Gut auf Konkurrenzmärkten sinken, und die Kostensituation der anderen Unternehmen verbessert sich. Dadurch, daß Marshall aber auch die Wissensentstehung an den Prozeß der Arbeitsteilung knüpft, und dieses Wissen kostenlos Verbreitung findet, können andere Unternehmen davon profitieren ohne, daß sich Preise verändern. Diese Vorgehensweise findet sich später in den Modellen der Endogenen Wachstumstheorie wieder. Erinnert sei z.B. an Romer (1986) oder Lucas (1988).

Young kombiniert in seiner Argumentation die Smithschen und Marshallschen Überlegungen zu den Konsequenzen der Arbeitsteilung und erweitert sie um eine weitere Annahme, die den Zusammenhang zwischen Marktgröße und Maß der Arbeitsteilung betrifft. Im Modell von Smith bestimmt die Größe des potentiellen Marktes das Maß der möglichen Arbeitsteilung. Gleichzeitig wird dadurch eine Entscheidung über die Produktivität der jeweiligen Industrie getroffen und implizit festgelegt, wie hoch die Einkommen in dieser Branche sein werden. Die durch die Produktionstätigkeit entstandene Kaufkraft determiniert nun neben anderen Variablen die Güternachfrage der Haushalte, d.h. die Größe des Marktes und die Chance für weitere Arbeitsteilung. M.a.W.: die Arbeitsteilung wird indirekt durch die Arbeitsteilung bestimmt Diese Argumentationsweise folgt dem Sayschen Theorem, d.h. der Determinierung des volkswirtschaftlichen Outputs durch die Angebotsseite. ${ }^{47}$

Hieraus entwickelt Young die Vorstellung, daß wirtschaftliches Wachstum einem kumulativen Prozeß folgt. ${ }^{48}$ Verändert sich an einem Punkt der Industrie die Produktionsorgansisation, werden an anderen Stellen im System Reaktionen auftreten, die wiederum Rückwirkungen zur Folge haben werden. Erfolgt die Produktion der Güter unter steigenden Skalenerträgen und werden Kostensenkungen in die Preise weitergegeben, d.h. unterliegt der Markt für dieses Gut dem Wettbewerb, kann die Ausdehnung der Produktion in diesem Bereich zur Ausdehnung der Produktion in anderen Sektoren führen. Zu diesem Zweck greift Young auf die Marshallianischen reziproken Nachfragekurven zurück und geht von einer elastischen Reaktion der Nachfrage aller Güter aus, indem eine Erhöhung des Angebots eines Produkts A eine Ausdehnung der produzierten Menge der Güter bewirkt, die für A eingetauscht werden kann. In diesem Falle bedeutet mehr Angebot eines Gutes automatisch mehr

47 Vgl. Young (1928, S. 532ff.) sowie Kaldor (1972, S. 383).

48 Auch in Smiths System folgt der Wachstumsprozeß einem zirkulăren Prozeß. Das Zusammenspiel von Arbeitsangebot, Arbeitsteilung und Akkumulation ermoglicht dauerhaftes Wachstum. Führt eine steigende Beschäftigung zu einer Ausdehnung der Märkte, wird die Arbeitsteilung zunehmen und weitere Produktivitătssteigenungen werden möglich. Wenn in Verbindung damit die Gewinnerwartungen der Unternehmer steigen, wird mehr investiert. Dies hat einen Anstieg der Arbeitskraftenachfrage zur Folge. Bei temporär begrenztem Arbeitsangebot werden die Reallohne über dem Existenzminimum liegen. Ist das Arbeitsangebot endogen (vgl. Smith I.viii), d.h. fuhrt der hohe Reallohn zu steigenden Geburtenzahlen, ist zumindest langfristig mehr Beschăftigung realisierbar, wodurch wieder die Nachfrage nach Gütern steigt. Angebots- und nachfrageseitige Prozesse bedingen sich somit gegenseitig. Vgl. Lowe (1984, S. 193ff.). 
Nachfrage nach anderen Gütern. Das Wachstum eines Sektors induziert einerseits das Wachstum anderer Sektoren, andererseits kann es dieses aber auch begrenzen. Für die Expansionsmöglichkeiten einer Volkswirtschaft sind somit die Elastizitäten der Güternachfrage von Bedeutung. Nur wenn entsprechende Verbindungen zwischen den einzelnen Sektoren bestehen, ist Fortschritt ohne exogene Faktoren möglich. ${ }^{49}$

Ein Weg, der den von Young diskutierten kumulativen Prozeß ermöglicht, ist mit dem Begriff der induzierten Investitionen verknüpft. Steigt die Produktion eines Gutes an, bedeutet dies nicht automatisch eine Veränderung der Produktionsstruktur. Damit die angesprochenen externen Skalenerträge auftreten können, muß es zur Investitionsausdehnung kommen, d.h. die Unternehmen müssen an ihrer Kapazitätsgrenze produzieren und dauerhafte Nachfragesteigerungen erwarten. Das Akzelerationsprinzip muß zum Tragen kommen. Kurzfristige Veränderungen der Absatzmöglichkeiten werden keinen anhaltenden Effekt auf die Produktionsstruktur haben. Die elastische Nachfragereaktion, wie sie von Young angeführt worden ist, stellt somit eine notwendige, aber keine hinreichende Bedingung für einen kumulativen Wachstumsprozeß dar. ${ }^{50}$

Aus den Überlegungen von Young lassen sich abschließend mehrere wichtige Aussagen für die weitere Diskussion festhalten:51

1.) Steigende Skalenerträge sind kein rein firmenspezifisches Phänomen, sondern liegen auch auf inter- und intraindustrieller Ebene vor.

2.) Steigende Skalenerträge sind das Resultat zunehmender Arbeitsteilung, die mit kapitalintensiven Produktionsweisen verbunden sind.

3.) Das Maß der Arbeitsteilung hängt von der Größe des Marktes ab. Gleichzeitig bestimmt die Arbeitsteilung durch ihre Wirkungen auf die Produktivität die Kaufkraft und bestimmt somit die Größe des Marktes.

4.) Aufgrund steigender Skalenerträge kann es zu kumulativen Prozessen kommen.

5.) Die Tendenz zu kumulativen Prozessen erfordert, daß bei der Diskussion und Analyse von ökonomischen Entwicklungsprozessen sowohl angebots- als auch nachfrageseitige Aspekte zu berücksichtigen sind.

49 Vgl. Young (1928, S. 537f.); Kaldor (1972, S. 384ff.); Blitch (1983a, S. 369f.).

$50 \mathrm{Vgl}$. hierzu Kaldor $(1972,386 \mathrm{ff}$.) Auf diesen Zusammenhang wird weiter unten noch einmal eingegangen.

51 Die drei ersten Aussagen finden sich direkt bei Young am Ende seines Aufsatzes. Vgl. Young (1928, S. 539). 
Kaldors Konzept der technical progress function bzw. Arrows Konzept des learning by doing sind durch eine große Nähe zu den Vorstellungen von Young gekennzeichnet. In beiden Ansätzen spielen die Kapitalintensivierung und damit verbundene steigende Skalenerträge eine wichtige Rolle.

\subsubsection{Der Verdoorn-Zusammenhang}

Ein weiterer zentraler Baustein der Beiträge Kaldors zur Wachstumstheorie und -politik stellt der sog. Verdoorn-Zusammenhang dar, der eine enge empirische Beziehung zwischen der Wachstumsrate der Arbeitsproduktivität und derjenigen der Produktion im industriellen Sektor unterstellt. Aufgrund der vehementen Betonung der Relevanz dieser Beziehung durch Kaldor, wird in diesem Zusammenhang auch vom zweiten Kaldorschen Gesetz gesprochen. ${ }^{52}$ Verdoorn selbst war zur Zeit der Erstellung seines viel zitierten Beitrags Fattori che regolano lo sviluppo della produttività del lavoro, ${ }^{53}$ der im Jahre 1949 in italienischer Sprache erschienen ist, Mitarbeiter der Economic Commission for Europe (ECE) der Vereinten Nationen. Als Mitglied der Abteilung Planning and Research war er mit Fragen der langfristigen Vorausschätzung der ökonomischen Entwicklung Europas betraut, wobei insbesondere das Wachstum der Arbeitsproduktivität auf Erwerbstätigenbasis von Interesse war.

Verdoorn versuchte eine Möglichkeit zu finden, die langfristige Entwicklung der Arbeitsproduktivität abzuschätzen. Nur falls diese bekannt sei, könnten Rückschlüsse auf die Beziehung zwischen Produktion und Beschäftigung gezogen werden. Die Überprüfung der Zeitreihen für die Arbeitsproduktivität und die industrielle Produktion in verschiedenen Ländern veranlaßte Verdoorn zur Untersuchung der Beziehung zwischen den Wachstumsraten dieser beiden Variablen. Er führt einerseits Vergleiche zwischen den industriellen Sektoren der USA, der Schweiz, dem Vereinigten Königreich und Deutschlands und andererseits eine Querschnittsanalyse auf Länderebene durch. Der Vergleich zeigt eine relative Konstanz des Verhältnisses beider Wachstumsraten, das in der Literatur als Verdoorn-Koeffizient oder Verdoorn-Elastizität bezeichnet wird. Je nach Land und Zeitraum lag es zwischen 0,41 und 0,57 (vgl. Tab. 4.1). Die Regressionsrechnung, welche die Wachstumsrate der Produktion als unabhängige und diejenige der Produktivität als abhängige Variable enthält, ergab für die Zeit zwischen dem Ersten und Zweiten Weltkrieg auf Basis einer Stichprobe von 14 Ländern einen Regressionskoeffizienten von 0,573 (vgl. Tab. 4.2). Dies impliziert eine Steigerung der Ausbringung pro Kopf von 0,57\%, wenn die Produktion um ein Prozent erhöht wird. ${ }^{54}$ Leider gibt

52 Vgl. z.B. Thirlwall (1983).

53 Ins Deutsche übersetzt: Bestimmungsgründe des Wachstums der Arbeitsproduktivităt.

54 Vgl. Verdoorn (1949, S. 45f bzw. 1993, S. 59). Leider gibt Verdoorn keine Auskunft über das verwendete Verfahren bzw. Bestimmtheitsmaße. Bei Durchführung einer Kleinst-Quadrat-Schătzung ergibt sich die in Tabelle (4.2) angefürte Regressionsgleichung. 
Verdoorn das von ihm verwendete Schätzverfahren nicht bekannt. Führt man mit seinen Originaldaten eine Kleinst-Quadrat-Schätzung durch, ergeben sich von Verdoorns Gleichung abweichende Resultate. Der Regressionskoeffizient für das Produktionswachstum $(b=0,61)$ entspricht in etwa dem angegebenen Wert, der Absolutterm der Regressionsgleichung weicht jedoch deutlich ab. Wie weiter unten gezeigt werden wird, kann die Abschätzung des Verdoorn-Zusammenhangs durch eine Regressionsrechnung zu Problemen bei der Interpretation der gewonnenen Resultate für den Verdoorn-Koeffizienten führen.

Tabelle 4.1: Jährlicher Anstieg der Produktion und der Arbeitsproduktivität in der Industrie (Ländervergleich)

\begin{tabular}{|l|c|c|c|c|}
\hline \multicolumn{1}{|c|}{ Land } & Zeitraum & $\mathbf{y}$ & $\mathbf{p}$ & $\mathbf{V}=\frac{\mathbf{p}}{\mathbf{y}}$ \\
\hline Schweiz & $1913-1930$ & 2,40 & 1,03 & 0,43 \\
\hline Vereinigtes Königreich & $1841-1907$ & 2,40 & 0,98 & 0,41 \\
\hline dito & $1907-1930$ & 1,28 & 0,605 & 0,47 \\
\hline USA & $1869-1899$ & 5,61 & 2,31 & 0,42 \\
\hline dito & $1899-1939$ & 3,35 & 1,91 & 0,57 \\
\hline Deutschland & $1882-1907$ & 4,38 & 2,14 & 0,49 \\
\hline
\end{tabular}

Quelle: Verdoorn (1949, S. 48 bzw. 1993, S. 60).

Unabhängig von der Wahl der zugrundegelegten Produktionsstruktur läßt sich die Elastizität des Produktivitätswachstums bzgl. des Produktionswachstums (V) durch 55

$$
\mathrm{V}=\frac{\dot{\mathrm{P}}}{\mathrm{P}}: \frac{\dot{\mathrm{Y}}}{\mathrm{Y}}=1-\frac{1}{\mathrm{y}}
$$

bestimmen.

Für eine Cobb-Douglas-Produktionsfunktion mit den Produktionsfaktoren Arbeit $(\mathrm{L})$ und Kapital (K)

$$
\mathrm{Y}=\mathrm{L}^{\beta} \mathrm{K}^{\alpha}
$$

55 Hierbei bezeichnet $(\mathrm{P})$ die Arbeitsproduktivităt, $(\mathrm{Y})$ die Produktion und $(\mathrm{L})$ die eingesetzte Arbeitsmenge. Kleinbuchstaben stehen fur die Wachstumsraten der jeweiligen Großen. Punkte über den Variablen stehen für Verănderungen der Variablen. 
ergibt sich durch Logarithmieren und Differentiation nach der Zeit für die Wachstumsrate des Sozialprodukts

$$
y=\beta 1+\alpha k \text { bzw. } \frac{y}{1}=\beta+\alpha \frac{k}{1}
$$

Tab. 4.2: Jährlicher Anstieg der Produktion und der Arbeitsproduktivität in der Industrie (Regressionsrechnung)

\begin{tabular}{|l|c|c|c|c|}
\hline \multicolumn{1}{|c|}{ Land } & Zeitraum & $\mathbf{y}$ & $\mathbf{p}$ & $\mathbf{V}=\frac{\mathbf{p}}{\mathbf{y}}$ \\
\hline Schweiz & $1924-1938$ & 5,0 & 5,3 & 1,06 \\
\hline Japan & $1926-1938$ & 6,7 & 3,4 & 0,51 \\
\hline Finnland & $1924-1938$ & 5,1 & 3,2 & 0,63 \\
\hline Ungarn & $1927-1938$ & 3,4 & 2,8 & 0,82 \\
\hline Holland & $1924-1938$ & 2,3 & 2,6 & 1,13 \\
\hline Norwegen & $1924-1938$ & 2,6 & 2,5 & 0,96 \\
\hline Dänemark & $1924-1938$ & 3,5 & 1,9 & 0,54 \\
\hline Polen & $1927-1938$ & 1,6 & 1,9 & 1,18 \\
\hline Vereinigtes Königreich & $1924-1938$ & 1,4 & 1,5 & 1,07 \\
\hline USA & $1924-1939$ & 0,6 & 1,0 & 1,67 \\
\hline Kanada & $1924-1938$ & 1,6 & 1,0 & 0,63 \\
\hline Tschechoslowakei & $1927-1938$ & 0,4 & 0,7 & $0,57^{*}$ \\
\hline Estland & $1927-1938$ & 0,8 & 0,4 & 0,5 \\
\hline Italien & $1924-1938$ & 0,8 & 0,2 & 0,25 \\
\hline Regressionsgleichung: $\mathrm{p}=0,00239+0,573 \mathrm{y}($ Verdoorn $)$ & & \\
\hline Regressionsgleichung: $\mathrm{p}=0,48+0,61 \mathrm{y} \mathrm{R}^{2}=0,71 \mathrm{t}-\mathrm{Wert}=3,9(\mathrm{eig} . \mathrm{Berech})$. \\
\hline
\end{tabular}

Quelle: Verdoorn (1949, S. 48 bzw. 1993, S. 60).

*Verdoorn gibt hierfür keinen Wert an.

Eingesetzt in die Gleichung für den Verdoorn-Koeffizienten (V) folgt:

$$
\mathrm{V}=1-\frac{1}{\beta+\alpha \frac{\mathrm{k}}{1}} \text { bzw. } \mathrm{V}=1-\frac{1}{\beta+\alpha \zeta} \text { mit } \zeta=\frac{\mathrm{k}}{1}
$$

Die Konstanz von (V) ist gewährleistet, wenn einerseits konstante Produktionselastizitäten und andererseits der Quotient $(\zeta)$ konstant bleibt. Dies impliziert jedoch nicht, daß die Kapital- 
intensität konstant bleibt. Nur für den Wert $\zeta=1$ variiert diese nicht. Die Höhe des VerdoornKoeffizienten wiederum ist abhängig vom Niveau der Produktionselastizitäten $(\alpha)$ und $(\beta)$ sowie dem Verhältnis der Wachstumsraten des Kapital- und des Arbeitseinsatzes ( $\zeta$ ). Falls $\zeta=1$, ist für den Wert von (V) ausschließlich das Niveau der Skalenerträge von Bedeutung. Steigende Skalenerträge, d.h. $\alpha+\beta>1$, bedeuten, daß $\mathrm{V}<1$ ist. Bei sinkenden Skalenerträgen, d.h. $\alpha+\beta<1$, gilt $V<0$. Für konstante Skalenerträge ist der Verdoorn-Koeffizient bei den getroffenen Annahmen nicht definiert.

Eindeutige Aussagen bzgl. Konstanz und Höhe von (V) sind somit nur möglich, falls weitere Informationen über die Entwicklung des Kapital- und des Arbeitseinsatzes sowie über die Konstanz der Produktionsfunktion vorliegen. Festgehalten werden kann an dieser Stelle, daß aufgrund des Vorliegens eines signifikant von Null verschiedenen Verdoorn-Koeffizienten nicht ohne weiteres auf die Existenz steigender Skalenerträge geschlossen werden kann. Verdoorn selbst gibt ein Beispiel dafür, welche Variablen auf (V) Einfluß nehmen können, indem er auf das zuvor vorgestellte Modell von Tinbergen (1942) zurückgreift. Hierbei unterstellt er folgende Funktionen. Die Produktion bestimmt sich wie oben nach

$$
\mathrm{Y}=\mathrm{L}^{\beta} \mathrm{K}^{\alpha}
$$

Für die Arbeitsnachfrage gilt unter Annahme der Grenzproduktivitätsentlohnung:56

$$
w=\beta \frac{Y}{L}
$$

Die Löhne sind eine lineare Funktion der Durchschnittsproduktivität und steigen somit mit derselben Wachstumsrate wie die Arbeitsproduktivität. Das Arbeitsangebot ist gegeben durch:

$$
w=\beta\left(\frac{L}{B}\right)^{\gamma} e^{\lambda t}
$$

Dies bedeutet ein steigendes Arbeitsangebot bei steigenden Löhnen. In Verbindung mit der Annahme über die Arbeitsnachfrage und der impliziten Vollbeschäftigungsannahme hat dies zur Folge, daß jeder Anstieg der Arbeitsproduktivität zu Lohnsteigerungen führt, was wiederum mehr Arbeitsanbieter anlockt. 57

Das Kapitalangebot (=Sparen) bzw. die Kapitalnachfrage (=Investition) bestimmt sich nach

$$
\dot{\mathrm{K}}=\chi \mathrm{Y}
$$

56 Hierbei steht (w) für den Reallohn.

57 Vgl. hierzu Rowthorn (1979). 
Die Bevölkerung (B) wächst exponentiell mit der Rate (n) und ergibt sich durch

$$
B=\mathbf{e}^{\text {nt }}
$$

Durch Lösung dieses Gleichungssystems ergibt sich für den Verdoorn-Koeffizienten folgender Term: 58

$$
\mathrm{V}=\frac{\gamma\left(1-\frac{1-\beta}{\alpha} \frac{\mathrm{n}}{\chi}\right)+\frac{1-\beta}{\alpha} \frac{\lambda}{\chi}}{\gamma\left(1+\frac{\beta}{\alpha} \frac{\mathrm{n}}{\chi}\right)+1-\frac{\beta}{\alpha} \frac{\mathrm{n}}{\chi}}
$$

Bei unterstellter Konstanz der Produktionselastizitäten ( $\alpha$ und $\beta$ ), der Lohnflexibilität $(\gamma)^{59}$ und der Sparquote $(\chi)$ entscheiden das Bevölkerungswachstum (n) und die Wachstumsrate der Löhne $(\lambda)$ über die Stabilität und die Höhe des Verdoorn-Koeffizienten. Verdoorn selbst war der Überzeugung, daß sehr große Veränderungen der entsprechenden Variablen vorliegen müßten, damit $(V)$ deutlich von dem ursprünglich gefundenen Wert abweichen würde.

Ursprünglich als Hilfsmittel für die Durchführung von Schätzungen gedacht, hat Verdoorns Gesetz vor allem nach seiner Wiederentdeckung durch Kaldor zu wirtschaftstheoretischen Debatten und der Erstellung einer großen Anzahl von ökonometrischen Studien gefürt, die alle der Beantwortung der Frage gewidmet waren, wie die im Zeitablauf in unterschiedlicher Stärke zu beobachtende Beziehung zwischen Produktions- und Produktivitätswachstum richtig zu interpretieren sei. ${ }^{60}$ Verdoorn selbst hat sich zu seinem Gesetz noch einmal 1980 mit einem Beitrag im Economic Journal geäußert, in dem er deutlich macht, daß seine ursprünglichen Aussagen bzgl. der Konstanz von (V) auf eine Steady State-Situation bezogen gewesen seien. Ausgehend von einer Cobb-Douglas-Funktion mit Hicks-neutralem technischen Fortschritt

$$
Y_{t}=e^{v t} L_{t}^{\beta} K_{t}^{\alpha}
$$

und unter Zuhilfenahme der typisch neoklassischen Gleichungen für die Arbeitsmenge $\left(\mathrm{L}_{t}\right)$ und die Kapitalbildung $\left(\dot{\mathrm{K}}_{\mathrm{t}}\right)$

58 Zur Ableitung dieser Lơsung bestimmt Verdoorn zuerst Gleichungen für die Wachstumsraten des Kapitalund des Arbeitseinsatzes, welche wiederum zu jedem Zeitpunkt von den Absolutwerten der jeweiligen Variable abhängig sind, d.h. es liegen Differentialgleichungen vor. Verdoorn muß somit Annahmen über den Ausgangswert von (K), (B) und $(L)$ treffen, was automatisch $(Y)$ und $(w)$ festlegt. Verdoorn wahlt für die Berechnung von $(V)$ das Jahr $t=0$. Alle frei festzusetzenden Variablen sollen den Wert Eins annehmen. Vgl. Verdoom (1949, S. 52 bzw. 1993, S. 66).

59 Siehe hierzu auch die Ausfuhrungen in Kapitel 2 zum Modell von Tinbergen.

$60 \mathrm{Vgl}$. zu dieser Debatte weiter unten. 
(4.12) $\quad \mathrm{L}_{\mathrm{t}}=\mathrm{L}_{0} \mathrm{e}^{\mathrm{nt}}$

(4.11) $\quad \dot{\mathrm{K}}_{\mathrm{t}}=\chi \mathrm{Y}_{\mathrm{t}}$

zeigt er die Konstanz der Verdoorn-Elastizität für den Steady State-Fall. Für die Wachstumsrate des Sozialproduktes ergibt sich zu jedem Zeitpunkt die Gleichung:61

$$
y_{t}=\frac{n \beta+v}{1-\alpha}\left(1+\alpha \frac{\chi(1-\alpha)-v_{0}(n \beta+v)}{\chi(1-\alpha)\left[e^{(n \beta+v) t}-1\right]+v_{0}(n \beta+v)}\right)
$$

Für $t \rightarrow \infty$ folgt als Grenzwert für $\left(y_{t}\right)$ :

(4.16) $\lim _{t \rightarrow \infty} y_{t}=\frac{n \beta+v}{1-\alpha}$.

Daraus folgt für die Wachstumsrate der Arbeitsproduktivität $\left(\mathrm{p}_{\mathrm{t}}\right)$ im Steady State:

$$
\lim _{t \rightarrow \infty} p_{t}=\frac{n \beta+v}{1-\alpha}-n \quad \text { bzw. } \quad \lim _{t \rightarrow \infty} p_{t}=\frac{n(\alpha+\beta-1)+v}{1-\alpha}
$$

bzw. fur die Verdoom-Elastizität $\left(\mathrm{V}_{t}\right)$

$$
\lim _{t \rightarrow \infty} V_{t}=\frac{n(\alpha+\beta-1)+v}{n \beta+v}
$$

Folgt man dieser Interpretation des Verdoorn-Gesetzes, sind Prognosen auf Basis dieses Zusammenhanges nicht sinnvoll, wenn die Rahmenbedingungen des Basiszeitraumes der Schätzung großen Veränderungen unterliegen. Die Stabilität des Zusammenhangs zwischen dem Wachstum der Arbeitsproduktivität und der Produktion kann deshalb nicht ohne weiteres erwartet werden.

61 Zur Ableitung dieser Gleichung siehe Verdoorn (1959) bzw. (1980). 


\subsubsection{Die Renaissance des Verdoorn-Zusammenhangs: Kaldors Inaugural Lecture}

\subsubsection{Der Verdoorn-Zusammenhang im Verarbeitenden Gewerbe}

Im Rahmen seiner Antrittsvorlesung an der Universität von Cambridge im Jahre 1966 griff Kaldor zur Erklärung der im internationalen Vergleich geringen Wachstumsraten der britischen Volkswirtschaft in der Zeit nach dem Zweiten Weltkrieg den Verdoorn-Zusammenhang wieder auf. ${ }^{62}$ Kaldor sah die Wachstumsmöglichkeiten einer Volkswirtschaft als maßgeblich durch die Entwicklung des zweiten Sektors bestimmt an. Hohe Wachstumsraten des industriellen Bereichs ermöglichen auch hohes gesamtwirtschaftiches Wachstum. Als Beleg für diese Aussage berechnet er eine Regressionsgleichung, bei der als exogene Variable die Wachstumsrate der Produktion des Mamufacturing Sectors ( $\approx$ Verarbeitendes Gewerbe) dient und die Wachstumsrate des Bruttoinlandsprodukts für den Zeitraum zwischen 1953/54 und 1963/64 in zwölf OECD-Ländern erklären sollte. ${ }^{63}$ Hierbei ergab sich eine sehr enge statistisch signifikante Beziehung zwischen den beiden Wachstumsraten, die auch als erstes Wachstumsgesetz von Kaldor bezeichnet wird. Die Elastizität des BIP-Wachstums bezogen auf das Wachstum des Manufacturing Sectors betrug 0,614, d.h. ein Prozent mehr Wachstum im sekundären Sektor induziert 0,614\% Wachstum des Bruttoinlandsprodukts. In Verbindung mit dem Absolutwert der Regressionsgleichung leitet Kaldor, ohne es in einem ersten Schritt genauer zu belegen, aus diesem Ergebnis einen Zusammenhang zwischen der Wachstumsrate des Bruttoinlandsprodukts und der Differenz zwischen der Wachstumsrate des Mamufacturing Sectors und derjenigen der restlichen Volkswirtschaft ab. Je größer diese Differenz sei, um so höher sei die gesamtwirtschaftiche Wachstumsrate. ${ }^{64}$ Darüber hinaus würden Unterschiede zwischen den Wachstumsraten nicht durch Veränderungen der Beschäftigtenzahlen, sondern durch Differenzen im Produktivitätswachstum erklärt, weshalb technische Zusammenhänge für den Einfluß des Manufacturing Sectors verantwortlich seien. Die Ursachen für einen positiven Einfluß des Wachstums des sekundären Sektors auf die gesamtwirtschaftliche Produktivität sind nach Kaldors Ansicht nicht im überdurchschnittlich hohen Produktivitätsniveau oder in der höheren Rate des exogenen technischen Fortschritts in diesem Sektor zu suchen, sondern vielmehr im Vorliegen steigender Skalenerträge. ${ }^{65}$ Dies aber sei das charakteristische Element des

62 Wahrend einer Vorlesungsreihe, die Kaldor 1967 an der Cornell University uber strategische Faktoren innerhalb der wirtschaftlichen Entwicklung hielt, griff er diese Vorlesung in fast identischer Form wieder auf, so daß hier primăr auf seine Antrittsvorlesung Bezug genommen wird.

63 Kaldors Stichprobe enthielt Belgien, die Bundesrepublik Deutschland, Dänemark, Frankreich, Italien, Japan, Kanada, die Niederlande, Norwegen, Österreich, das Vereinigte Königreich und die USA.

64 In dieser Beziehung spiegeln sich die Produktivitătseffekte des strukturellen Wandels wider, die von Kaldor im Laufe seiner Vorlesung weiter diskutiert werden.

65 Seine Ablehnung gegenüber der ersten moglichen Ursache griundet er auf eine Untersuchung von Beckerman aus dem Jahre 1965, die nur einen sehr geringen Einfluß der sektoral unterschiedlichen Produktivitătsniveaus auf die Entwicklung der gesamtwirtschaftichen Produktivităt ergab. Gegen das zweite Argument wendet er ein, daß technischer Fortschritt keine typische Eigenschaft des industriellen 
verarbeitenden Bereichs. Es können dabei statische und dynamische Skalenerträge unterschieden werden. Während erstere durch Größenvorteile der Massenproduktion entstehen und prinzipiell umkehrbar sind, resultieren die letzteren aus induziertem technischen Fortschritt, Produktionsexternalitäten und/oder learning by doing und sind irreversibel. Steigende Skalenerträge sind für Kaldor ganz im Sinne von Smith und Young das Ergebnis zunehmender Arbeitsteilung, da sie das Ausbildungsniveau und die Fähigkeiten der Arbeitnehmer erhöht und gleichzeitig erfahrungsinduzierte Innovationen ermöglicht. Arbeitsteilung wirkt deshalb auf beide Produktionsfaktoren (Arbeit und Kapital). Darüber hinaus sind die von Young (1928) diskutierten Folgen einer sich intensivierenden Spezialisierung auf die Struktur der Volkswirtschaft, d.h. dem Entstehen neuer Industrien im Verarbeitenden Sektor von Bedeutung. Der Verdoom-Zusammenhang ist somit für Kaldor nicht ausschließlich die Folge der Massenproduktion im industriellen Sektor, sondern wird auch aufgrund des durch die Spezialisierung induzierten technischen Fortschritts hervorgerufen. ${ }^{66}$ Obwohl Kaldor technischen Fortschritt nicht als ein Spezifikum des sekundären Sektors ansah, hat dieser seiner Ansicht nach im Gegensatz zu den anderen Bereichen der Volkswirtschaft ein größeres Potential für induzierten technischen Fortschritt.

\section{Tab. 4.3: Kaldors Wachstumsgesetze}

\begin{tabular}{|c|c|c|c|c|}
\hline & Beziehung & Regressionsgleichung & $\mathbf{R}^{2}$ & t-Wert \\
\hline Gesetz 1 & $y_{M}$ und $y$ & $y=1,153+0,614 y_{M}$ & 0,959 & 15,35 \\
\hline Gesetz 2a & $\mathrm{y}_{M}$ und $\mathrm{p}_{\mathrm{M}}$ & $p=1,035+0,484 y_{M}$ & 0,826 & 6,91 \\
\hline Gesetz 2b & $\mathrm{y}_{M}$ und $\mathrm{l}_{\mathrm{M}}$ & $1=-1,028+0,516 y_{M}$ & 0,844 & 7,37 \\
\hline Gesetz 3a* & $\mathrm{l}_{\mathrm{M}}$ und $\mathrm{y}$ & $y=2,665+1,0661_{M}$ & 0,828 & 9,69 \\
\hline Gesetz 3b* & $\mathrm{l}_{\mathrm{NM}}+\mathrm{l}_{\mathrm{M}}$ un & $y_{M}=2,899+0,821 l_{M}-1$ & 0,842 & $4,86 / 3,22$ \\
\hline
\end{tabular}

Quellen: Kaldor (1966, S. 285 u. 289), Kaldor (1968, S. 338).

Bei einer Regression mit dem Produktionswachstum als Regressor und dem Produktivitätswachstum als Regressand scheint ein enger statistisch signifikanter Zusammenhang kaum verwunderlich. Die unabhängige Variable ist gleichzeitig ein Teil der abhängigen Größe. Für den Fall eines Beschäftigungswachstums von Null sind beide Wachstumsraten identisch. Der Zusammenhang wäre perfekt. ${ }^{67}$ Aus diesem Grund gibt Kaldor alternativ zur traditionellen Darstellung des Verdoom-Zusammenhangs, welche die Beziehung zwischen Produktivitätsund Produktionswachstum zum Inhalt hat, eine Regression zwischen Beschäftigungs- und Produktionswachstum an. Hier liegt ein Koeffizient vor, der sich von Eins signifikant unter-

Sektors sei. Vielmehr würde die Landwirtschaft sehr hohe Produktivitătsfortschritte realisieren. Kaldor bezieht sich des weiteren auf eine Untersuchung von Salter (1960). Vgl. Kaldor (1966, S. 286f.).

66 Vgl. Kaldor (1966, S. 287f.).

67 Vgl. Kaldor (1975, S. 892 und 1985, S. 63ff.). 
scheidet. Dies bedeutet, daß 1\% Produktionswachstum nur ca. 0,5\% Wachstum bei der Beschäftigung zur Folge haben. Die Erhöhung der Produktion erfordert nur ein unterproportionales Wachstum des Inputfaktors Arbeit und kann als Beleg für das Vorliegen steigender Skalenerträge interpretiert werden. 68

Die zentrale Rolle dynamischer Skalenerträge ist mit ein Grund für Kaldor, die Umkehrung des Verdoom-Zusammenhangs, d.h. das Produktionswachstum des Verarbeitenden Gewerbes als Folge autonomen Produktivitätswachstum zu sehen, abzulehnen. Bei Gültigkeit einer solchen Kausalität wäre kein Platz für induzierten technischen Fortschritt. Sämtliche Produktivitätssteigerungen wären exogen, d.h. Ergebnis von Forschungs- und Entwicklungsanstrengungen, die außerhalb des ökonomischen Systems stattfinden. Sowohl Kaldors eigene Überlegungen zur technical progress function als auch die damit verwandten Ergebnisse von Arrows Modell (1962) zu den Folgen des learning by doing würden durch den Glauben an einen inversen Verdoorn-Zusammenhang übergangen. Kaldor selbst gibt ein anschauliches Beispiel, das eine solche Vorgehensweise in Frage stellt. Wenn Produktivitätssteigerungen vollständig autonom sind, ist z.B. fraglich, warum es zwischen den einzelnen Töchtern von amerikanischen Automobilunternehmen deutliche Unterschiede bei der Wachstumsrate der Arbeitsproduktivität gibt. Zumindest innerhalb ein und desselben Konzerns müßte das vorhandene technische Know-how ein öffentliches Gut sein. ${ }^{69}$ Dieses Beispiel läßt eine voreilige Ablehnung der getroffenen Annahme eines exogenen Produktivitätsfortschritts natürlich nicht zu. Es weist jedoch auf eine der zentralen Schwierigkeiten innerhalb der neoklassischen Wachstumstheorie der sechziger Jahre hin, die sich im Modell ergebenden Konsequenzen autonomen technischen Fortschritts für die Entwicklung von Volkswirtschaften mit den realen Gegebenheiten in Einklang zu bringen.

Der Verarbeitende Sektor ist somit der Wachstumsmotor schlechthin. Wenn es gelingt, das Wachstum im industriellen Bereich zu beschleunigen, wird auch das gesamtwirtschaftliche Wachstum zunehmen. Die Ausbeutung der steigenden Skalenerträge in diesem Teil der Volkswirtschaft wird einen größeren Beitrag zum Wachstum leisten als Produktionszunahmen im primären und tertiären Sektor. Inwieweit diese Effekte realisiert werden können, wird durch die Faktoren bestimmt, die das Wachstum des Verarbeitenden Sektors beeinflussen. Sowohl angebots- als auch nachfrageseitige Größen sind hierbei von Belang. Die Entwicklung der Nachfrage nach den Produkten des sekundären Sektors wird in großem Umfang von drei Komponenten beeinflußt: Konsum, Investitionen und (Netto-)Export. Je nach Entwicklungsstand einer Volkswirtschaft liegt eine hohe oder niedrige Einkommenselastizität der Nachfrage

68 Vgl. Kaldor (1966, S. 11f.) und Hahn (1989, S. 49). Diese Interpretation Kaldors wurde später durch eine modifizierte Darstellung des Verdoorn-Zusammenhangs kritisiert. Vgl. den năchsten Abschnitt.

69 Kaldor bezieht sich hier auf die Produktivitătsfortschritte in der Automobilindustrie in Westdeutschland und Großbritannien im Zeitraum 1954-1960. Wahhrend in der Bundesrepublik die Produktivităt um 7 Prozent p.a. in dieser Zeit stieg, konnten die britischen Automobilbauer nur ein Wachstum von jăhrlich 2,7 Prozent erreichen. Vgl. Kaldor (1966, S. 290f.). 
nach Gütern des Verarbeitenden Sektors vor. ${ }^{70}$ In einer Phase starker Reaktionen der Nachfrage in diesem Bereich auf Realeinkommensänderungen hat eine Zunahme der Produktion und somit des Realeinkommens im Verarbeitenden Sektor eine stark steigende Konsumgüternachfrage zur Folge, die wiederum zur Ausdehnung der Güterproduktion führen wird und die Realisierung steigender Skalenerträge mit den entsprechenden Produktivitätsfortschritten zur Folge hat. Auch bei der Investitionsgüternachfrage sind vergleichbare Prozesse denkbar. Die Expansion des Investitionsgütersektors wird die Nachfrage nach dessen Produkten aufgrund der notwendigen Investitionen erhöhen, wodurch ein steigendes Angebot in diesem Sektor profitabel wird. Eine weitere Möglichkeit zur Steigerung der Produktion des sekundären Sektors wird im Export gesehen, da hierdurch der relevante Markt vergrößert und somit, wie schon von Smith diskutiert, die Möglichkeit zu weiterer Arbeitsteilung bestehen bleibt. $^{71}$

Die Argumentationsweise Kaldors folgt an dieser Stelle einerseits keynesianischen Linien. Die Erhöhung der Güternachfrage schafft durch mehr Produktion steigende Einkommen, was wiederum mehr Nachfrage zur Folge hat. Multiplikator- und Akzeleratoreffekte gehen Hand in Hand. Andererseits jedoch führt die Ausdehnung der Güterproduktion zu induzierten Produktivitätssteigerungen, die in der kurzfristig orientierten, d.h. an der Behebung konjunktureller Einbrüche ausgerichteten keynesianischen Betrachtungsweise nicht beachtet wurden. Diese Produktivitătseffekte führen in der kaldorianischen Sicht jedoch nicht zur Freisetzung, sondern schaffen zum einen die Möglichkeit für hohe Wachstumsraten der Volkswirtschaft aufgrund der bedeutenden Rolle des Verarbeitenden Sektors insgesamt, zum anderen können sie durch angebotsseitige Entwicklungen, z.B. dem Mangel an Arbeitskräften, gedämpft werden. ${ }^{72}$ Speziell für das Großbritannien der 1950er und 1960er Jahre sah Kaldor das Problem in einer Angebotsbeschränkung auf dem Arbeitsmarkt. Der steigende Bedarf an Arbeitskräften in der Industrie wurde lange Zeit durch den landwirtschaftlichen Sektor gedeckt, der durch eine Art versteckte Arbeitslosigkeit gekennzeichnet war. Technischer Fortschritt auf dem Gebiet der landwirtschaftlichen Produktion hat es ermöglicht, Arbeitskräfte freizusetzen, ohne Rückgänge bei der Produktion hinnehmen zu müssen. Man kann hier wirklich von Freisetzung sprechen, da dieser Begriff nicht im Sinne einer eleganten Bezeichnung für den Weg in die Arbeitslosigkeit verwendet wird, sondern die Produktivitätsfortschritte im primären Sektor die Entwicklung im industriellen Sektor ermöglichen. Kommt dieser Strom an Arbeitskräften zum Erliegen,

70 Erinnert sei hier nur an die Drei-Sektoren-Hypothese von Fourastié oder das Engelsche Gesetz.

71 Vgl. Kaldor (1966, S. 295ff.).

72 Des weiteren sind Engpässe bei Ressourcen und Vorleistungen sowie Zahlungsbilanzschwierigkeiten als Wachstumsschranken denkbar. Die Notwendigkeit einer ausgeglichenen Zahlungsbilanz kann zum Phănomen der Listschen Arbeitslosigkeit führen. Treten eine hohe Importelastizităt der Nachfrage und eine geringe Wettbewerbsfahigkeit eines Landes gleichzeitig auf, bedingen die Leistungsbilanzdefizite, die in einer Expansionsphase zwangslăufig auftreten, restriktive Maßnahmen zur Sicherung der Stabilităt der Wahrung. Vgl. hierzu Kaldor (1980) und Hagemann (1984). Insbesondere McCombie/Thirlwall (1994) haben sich den Konsequenzen der dritten Beschrănkung gewidmet. 
werden die Wachstumspotentiale des Verarbeitenden Sektors zurückgehen. Die Wachstumsraten werden in diesem Bereich sinken und bei Gültigkeit des Verdoorn-Zusammenhangs konsequenterweise auch das Produktivitätswachstum verlangsamen. Das Problem der britischen Volkswirtschaft war nach Kaldors Ansicht das Erreichen einer Situation der Reife, in der die sektorale Verteilung der Arbeitskräfte den Wechsel von unproduktiven im primären Sektor angesiedelten hin zu produktiven Arbeitsplätzen im sekundären Sektor nicht mehr erlaubt. Dies bedeutet nicht, daß ein kleiner Anteil des landwirtschaftlichen Sektors an der Gesamtzahl der Erwerbstätigen automatisch ein Indikator für solch einen Zustand ist, sondern $\mathrm{da} ß$ es ein unbegrenztes Arbeitsangebot im Sinne von Arthur Lewis $(1954,1955)$ und den damit verbundenen Wachstumsmöglichkeiten nicht mehr gibt. ${ }^{73}$ Ein Anzeichen für das Vorhandensein eines Arbeitskräfteüberschusses im kaldorianischen Sinn wäre der schnelle Anstieg der Arbeitsnachfrage in hochproduktiven Teilen der Volkswirtschaft bei gleichzeitig steigender Rate des Arbeitsplatzwechsels in diesen Bereich. Dies gelte auch für den Fall sinkender Lohndifferentiale zwischen den Sektoren. Eine Angleichung der sektoralen Lohnniveaus kann durch die steigende Arbeitsproduktivität im primären Sektor erklärt werden. Die sektorale Verteilung der Erwerbstätigen wird deshalb durch die Arbeitsnachfrage des Verarbeitenden Gewerbes determiniert. ${ }^{74}$

\subsubsection{Die Rolle des Arbeitsangebots}

Die sektorale Wanderung der Arbeitskräfte kann darüber hinaus auch bei Nichtexistenz von steigenden Skalenerträgen im Verarbeitenden Sektor das gesamtwirtschaftliche Wachstum erhöhen. Es muß gewährleistet sein, daß der Produktionsbeitrag, der von ihnen im industriellen Sektor erbracht wird, über ihrem vorigen liegt. M.a.W.: sie müssen durch ihren sektoralen Übertritt einer effizienteren Verwendung zugeführt werden. Für den Fall sinkender Skalenerträge in den Abwanderungsbereichen bzw. dem Vorliegen von versteckter Arbeitslosigkeit ist diese Forderung erfüllt. Des weiteren hat der Verarbeitende Sektor gegenüber anderen Sektoren den Vorteil, daß er notwendige Ressourcen selbst erzeugen kann und keinen direkten natürlichen Beschränkungen unterliegt wie z.B. die Landwirtschaft. Selbstverständlich sind damit eventuelle Knappheiten bei Inputfaktoren nicht ausgeschlossen. Der industrielle Produktionsprozeß weist jedoch zumindest mittel- bis langfristig einen höheren Grad an Substitutionalität beim Faktoreinsatz als andere Sektoren auf. ${ }^{75}$ Eine positive Korrelation der gesamtwirt-

73 Lewis entwickelte seine Theorie ursprünglich für Entwicklungslănder. Eine gewisse Relevanz für den Wachstumsprozeß von Industrienationen läbt sich aber nicht leugnen. Die Zahl und die Qualităt der Arbeitskrăfte kann als limitierender Faktor zumindest auf sektorale Ebene auftreten.

74 Ein Beispiel fur eine solche Fehlinterpretation ist der kritische Beitrag von Wolfe (1968). Vgl. zur Definition der Situation der Reife Kaldor (1966, S. 302f.) sowie Kaldor (1968, S. 385f.).

75 Vgl. Kaldor (1968, S. 386 Fn 1) und Thirlwall (1983, S. 354). 
schaftichen Wachstumsrate mit der Wachstumsrate der Beschäftigung im verarbeitenden Bereich bei einem gleichzeitig negativen Zusammenhang mit der Wachstumsrate der Beschäftigung in den anderen Sektoren wäre ein erster Hinweis auf diese Überlegung. Tatsächlich ergab sich für die von Kaldor verwendeten Daten eine entsprechende Regressionsgerade, die in einer Studie von Cripps/Tarling bestätigt wurden. ${ }^{76}$

Die Relevanz des Arbeitsangebots für die Wachstumsmöglichkeiten kann durch eine alternative, in Verdoorns Original-Modell implizit enthaltene Darstellung des VerdoornZusammenhangs gezeigt werden, die speziell die Variablen des Arbeitsmarktes berücksichtigt. Ausgangspunkt sind hierbei die Funktionen furr die Arbeitsnachfrage (4.9) und das Arbeitsangebot (4.10). Ohne spezielle Annahmen über die zugrundegelegte Produktionsfunktion zu treffen, kann man einen Zusammenhang zwischen der Entwicklung der Güterproduktion und derjenigen der Arbeitsproduktivität ableiten. Wie oben angesprochen, implizieren die unterstellten Beziehungen zwischen der Arbeitsproduktivität und dem Lohnsatz sowie der Abhängigkeit des Arbeitsangebots vom Lohnsatz, daß steigende Effizienz in der Produktion zu steigenden Löhnen und konsequenterweise zu einem größeren Arbeitsangebot führt. Aufgrund der angenommenen Gleichheit von Arbeitsangebot und Arbeitsnachfrage werden die zusätzlichen Arbeitskräfte auch beschäftigt. Durch das Gleichgewicht auf dem Arbeitsmarkt ergibt sich: 77

$$
\beta \frac{Y}{L}=\beta L^{\gamma} e^{(\lambda-\gamma n) t}
$$

bzw.

$$
\ln \frac{Y}{L}=\gamma \ln L+(\lambda-\gamma n) t
$$

Durch Differentiation nach ( $t$ ) folgt:

$$
\mathrm{p}=\gamma l+\lambda-\gamma \mathrm{n}
$$

Durch Umformung nach (l) ergibt sich:

$$
1=-\frac{\lambda-\gamma \mathrm{n}}{\gamma}+\frac{1}{\gamma} \mathrm{p}
$$

76 Vgl. Kaldor (1968, S. 387f.) und Cripps/Tarling, (1973, Kap 3., S. 2 lff.). Kaldors Daten ergaben die Regressionsgleichung $y=2,899+0,8211_{M}-1,1831_{N M}$. Diese Beziehung wird auch als drittes Wachstumsgesetz von Kaldor bezeichnet. Vgl. Thirlwall (1983, S. 354), Targetti (1992, S. 170ff.) und auch Tab. (4.3). Die Rolle der Arbeitskraftewandenung wurde in formalisierter Form auch in den Beiträgen von Thirlwall (1986, 1992), Targetti (1985), Canning (1988), Dutt (1992) diskutiert.

$77 \mathrm{Vgl}$. zu dieser Interpretation Rowthorn (1979), der leider nur das Endergebnis präsentiert. 
Das Produktionswachstum (y) setzt sich definitionsgemäß aus dem Wachstum der Arbeitsproduktivität $(p)$ und demjenigen der Beschäftigung (l) zusammen. Daraus folgt für den Verdoorn-Zusammenhang:

$$
\mathrm{p}=\frac{\lambda-\gamma \mathrm{n}}{1+\gamma}+\frac{\gamma}{1+\gamma} \mathrm{y}
$$

bzw. für die von Kaldor bevorzugte Schreibweise:

$$
1=-\frac{\lambda-\gamma \mathrm{n}}{1+\gamma}+\frac{1}{1+\gamma} \mathrm{y}
$$

Durch diese Vorgehensweise wird der Verdoorn-Zusammenhang durch die Bedingungen auf dem Arbeitsmarkt bestimmt. Insbesondere der Verdoorn-Koeffizient hängt ausschließlich von der Lohnflexibilität des Arbeitsangebotes ab. Die Interpretation des statistisch signifikanten Regressionskoeffizienten läßt in diesem Falle keine direkten Rückschlüsse auf die Produktionsstruktur zu, sondern nur auf Angebotsparameter des Arbeitsmarktes. Ein Wert von 0,5 würde eine Lohnflexibilität von $\gamma=1$ implizieren. Dies entspricht genau dem von Tinbergen angenommenen Wert. In diesem Falle würde jede Lohnsteigerung dieselbe Reaktion bei der Erwerbsquote zur Folge haben. Je höher der Verdoorn-Koeffizient ist, um so niedriger muß die Lohnflexibilität des Arbeitsangebots sein. Die Darstellung des Verdoorn-Zusammenhangs wirf die Frage nach den theoretischen Erklärungen auf, die eine Bestimmung des VerdoornKoeffizienten durch die Lohnflexibilität erlauben. Je niedriger die Lohnflexibilität des Arbeitsangebots ist, desto stärker wird die Erwerbsquote bei gegebenen Lohnsteigerungen reagieren. Dies führt in einem nächsten Schritt zur Steigerung der produzierten Gütermenge, die in ihrer Höhe von den produktionstechnischen Relationen abhängt. Die Schätzungen legen nun einen Anstieg der Arbeitsproduktivität nahe. Dies impliziert jedoch eine Produktionstechnik, die durch eine steigende Durchschnittsproduktivität der Arbeit und damit steigende Skalenerträge gekennzeichnet ist. Welche Eigenschaften diese Produktionsfunktion jedoch genau aufweist, läßt sich aus dieser Interpretation nicht erkennen. Deutlich wird dagegen, daß die Realisierung der steigenden Skalenerträge eine ständige Zunahme des Arbeitskräfteangebots für den industriellen Sektor benötigt. Eine solche Interpretation ist schwerlich mit den Vorstellungen Kaldors zu vereinbaren, der der keynesianischer Tradition folgend die Abhängigkeit des Arbeitsmarktes von der Güternachfrage annahm. Dies führt direkt zu einer weiteren Frage, die im folgenden Abschnitt besprochen wird. 


\subsubsection{Die Wahl der richtigen exogenen Variable: Beschäftigung oder Output?}

Der Hinweis auf den Mangel an surplus labour, der eine Ursache des unbefriedigenden Wachstums der britischen Volkswirtschaft darstellen kann, wurde teilweise als Beleg dafür interpretiert, daß Kaldor die Höhe der Beschäftigung als unabhängige, exogene Größe betrachten würde, die immer und überall die Grenze des Wachstums bilden würde. ${ }^{78}$ Aus diesem Grund wurde das Verdoom-Gesetz durch die Gleichung

$$
\mathrm{p}=\mathrm{a}+\mathrm{bl}
$$

spezifiziert. Diese Darstellung kann durch die Verwendung der Identität $p \equiv q-1$ und Umformung aus der ursprünglichen Definition des Verdoorn-Zusammenhangs ermittelt werden. Damit steigende Skalenerträge vorliegen, muß $b>0$ sein, da zunehmender Arbeitseinsatz Produktivitätswachstum mit sich bringen muß. Wäre $b<0$ würde dies bedeuten, daß bei zunehmendem Arbeitseinsatz die Arbeitsproduktivität abnehmen, die Produktionstechnik somit sinkenden Skalenerträgen unterliegen muß. Unter Verwendung dieser Gleichung und Kaldors Daten kommt es zu einem instabilen Zusammenhang zwischen Produktivitäts- und Beschäftigungswachstum. Sobald die japanischen Daten aus der Stichprobe herausgenommen werden, ist der Zusammenhang beider Größen nicht mehr signifikant. Eine Aussage über die Existenz steigender Skalenerträge ist dann nicht mehr möglich. ${ }^{79}$

Spezifiziert man dagegen den Verdoorn-Zusammenhang in der Form, die Kaldor bevorzugte, verringert der Ausschluß Japans aus der Stichprobe die Güte der Korrelation, jedoch ist der Koeffizient des Produktionswachstums signifikant von Eins verschieden, d.h. die Regressionsergebnisse deuten auf das Vorliegen steigender Skalenerträge hin. In Tabelle 4.4 sind zur Veranschaulichung Kaldors und Rowthorns Spezifikationen jeweils mit und ohne die Werte furr die japanische Volkswirtschaft aufgeführt.

78 Beispiele für eine solche Interpretation finden sich in Cripps/Tarling (1973), Chatterji/Wickens (1981, 1982), und Rowthorn (1975a).

79 Vgl. Rowthorn (1975a, S. 15f.). Aufgrund dieses Ergebnisses wurde überlegt, ob der VerdoornZusammenhang nicht nur das Ergebnis des Catching up-Prozesses ist. Nimmt man an, dab das Produktivitătswachstum einer Volkswirtschaft unabhăngig vom Produktionswachstum ist, kann es dennoch bei einer internationalen Querschnittsanalyse zu einem positiven Zusammenhang zwischen beiden Wachstumsraten kommen, wenn in den einzelnen Volkswirtschaften jeweils hohes (geringes) exogenes Produktivitătswachstum auch hohes (geringes) Produktionswachstum zur Folge hat. Die Ursachen hierfuir können in der Social Capability der einzelnen Lănder liegen (vgl. hierzu auch Abschnitt 3.2). Der Verdoorn-Zusammenhang wäre dann das Ergebnis des statistischen Verfahrens und nicht empirischer Ausdruck der zugrundeliegenden Produktionstechnologie. Gegen diese Interpretation sprechen jedoch die Resultate von Längsschnittuntersuchungen, die für einzelne Lănder induzierte Produktivitătseffekte ergaben. Vgl. hierzu z.B. Gomulka (1971), Cornwall $(1968,1976,1977)$. 
Tab. 4.4: Diverse Spezifikationen des Verdoorn-Zusammenhangs

\begin{tabular}{|c|c|c|c|}
\hline Beziehung & Regressionsgleichung & $\mathbf{R}^{\mathbf{2}}$ & t-Wert \\
\hline $\mathrm{p}_{M}$ und $\mathrm{l}_{M}$ incl. J & $\mathrm{p}_{M}=2,632+0,626 \mathrm{l}_{M}$ & 0,447 & 2,85 \\
\hline $\mathrm{p}_{M}$ und $\mathrm{l}_{M}$ excl. J & $\mathrm{p}_{M}=3,237+0,183 \mathrm{l}_{M}$ & 0,050 & 0,69 \\
\hline $\mathrm{l}_{M}$ und $\mathrm{y}_{M}$ incl. J & $\mathrm{l}_{M}=-1,028+0,516 \mathrm{q}_{M}$ & 0,844 & 7,37 \\
\hline $\mathrm{l}_{M}$ und $\mathrm{y}_{M}$ excl. J & $\mathrm{l}_{M}=-1,331+0,574 \mathrm{q}_{M}$ & 0,685 & 4,42 \\
\hline
\end{tabular}

Quelle: $\quad$ Rowthorn (1975a, S. 14), Kaldor (1975, S. 892). (M) als Index steht für Manufacturing Sector.

Dieses auf den ersten Blick paradox erscheinende Ergebnis hat seine Ursache in der Ungenauigkeit der empirischen Schätzung. Durch einfache Umformung kann die Spezifikation des Verdoorn-Zusammenhangs, wie sie z.B. von Rowthorn (1975a) gewählt wurde, in eine Umkehrregression der kaldorianischen Interpretation $(l=c+d q)$ überführt werden. Mit $\mathrm{p} \equiv \mathrm{q}-1$ folgt aus

$$
\mathrm{p}=\mathrm{a}+\mathrm{bl}
$$

direkt

$$
q=a+(1+b) l
$$

Für die Regressionskoeffizienten beider Gleichungen gilt:

$$
(1+b) d=R^{2}
$$

Je höher somit der Regressionskoeffizient der einen Gleichung ausfältt, um so kleiner muß derjenige der anderen sein. Gibt die Kaldor-Gleichung einen Hinweis auf steigende Skalenerträge, kann dies dennoch von den Ergebnissen der Umkehrregression in Zweifel gezogen werden. Nur im Falle einer perfekten Korrelation $\left(R^{2}=1\right)$ spielt die Spezifikation keine Rolle. Je kleiner der Verdoorn-Koeffizient ausfällt, um so höher ist die Wahrscheinlichkeit, daß die Spezifikation des Verdoorn-Zusammenhangs durch die Wahl des Beschäftigungswachstums als unabhängige Variable zu einem kontroversen Ergebnis führt. ${ }^{80}$

Die Interpretation des Verdoorn-Zusammenhangs als eine Beziehung zwischen Produktivitäts- und Beschäftigungswachstum, in der die Beschäftigungsmenge die unabhängige Variable darstellt, widerspricht Kaldors grundsätzlich keynesianischer Vorstellung der Endo-

80 Vgl. hierzu Kaldor (1975, S. 892f.), McCombie/Thirlwall (1994, S. 182ff.), McCombie (1983, S. 416) und Rowthorn (1975a, S. 16ff.). 
genität des Beschäftigungsniveaus. Die in einer Volkswirtschaft eingesetzte Arbeitsmenge wird bestimmt durch die produzierte Gütermenge, d.h. die Unternehmen legen mit ihrer Produktionsentscheidung fest, wie viele Arbeitskräfte benötigt werden. Das Maß der Produktion wiederum ist eine Konsequenz der Absatzchancen, d.h. der Güternachfrage. Die Kausalität des Verdoorn-Zusammenhangs muß somit vom Produktionswachstum zum Beschäftigungswachstum erfolgen, "since I (Kaldor, d.Verf.) regarded output as being in general the exogenous variable (determined by demand)..."81 Volkswirtschaften sind in ihren Wachstumsmöglichkeiten dieser Überlegung nach nicht durch die Menge an Produktionsfaktoren, sondern durch die Nachfrage nach Gütern beschränkt. Kaldor gab später seine 1966 vehement vertretene These der Arbeitsangebotsbeschränkung für die britische Volkswirtschaft auf. Das Problem der geringen Wachstumsraten würde vielmehr in der geringen internationalen Wettbewerbsfähigkeit der britischen Industrie dieser Zeit zu finden sein. 82

Diese Betonung der Exogenität der Güternachfrage kann kritisiert werden, da sie die Unabhängigkeit der Nachfrage von anderen Variablen des Systems beinhaltet. ${ }^{83}$ Wäre dies der Fall, muß die Frage nach der Existenz eines Prozesses der kumulativen Verursachung gestellt werden. Ohne Rückwirkungen der Beschäftigungs- und Produktivitätsentwicklung auf die Güternachfrage ist ein zirkulärer Prozeß schwer vorstellbar. Kaldor hätte sich also selbst widersprochen und durch eine Annahme über die Richtung der Kausalität der eigenen Vorstellung über die Zusammenhänge der ökonomischen Entwicklung den Boden entzogen. Kaldor war sich dieser Kritik jedoch bewußt und hat sie selbst vorweggenommen:

"In saying that growth is explained by the increase in demand which is 'exogenous' to the growing sectors I am conscious of the fact that this statement itself is a simplification but one which does not invalidate the statistical inferences derived from it. The growth of industrial output for any region is governed in part by the growth in productivity which itself influences demand through the change in competiveness which is induced by it. It is this reverse link which accounts for the cumulative and circular nature of growth processes. There is a two-way relationship from growth to productivity growth and from productivity growth to demand growth; but the second relationship is, in may view, far less regular and systematic than the first." 84

Der Wahl der Wachstumsrate der Beschäftigungsmenge als exogene Variable kann zusätzlich entgegengehalten werden, daß sich auch hier die Frage der Interdependenz der Variablen stellt. Führen Steigerungen der Beschäftigung zu Produktivitätswachstum, muß nach den Rückwirkungen auf die Produktionsmenge und damit die Beschäftigungsmenge gefragt werden. Niveau und Wachstum der eingesetzten Arbeitsmenge werden von der Produktions- und

81 Kaldor (1975, S. 892).

82 Vgl. Kaldor (1975, S. 895).

83 Vgl. z.B. Rowthorn (1975b, S. 898ff.).

84 Kaldor (1975, S. 895 Fn 1). 
Produktivitätsentwicklung bestimmt und sind wie die beiden anderen Größen eine endogene Variable. In Abschnitt (4.5) werden diese Zusammenhänge noch einmal aufgegriffen.

Von diesem Teil der Verdoorn-Debatte bleibt festzuhaiten, daß Kaldor den Wachstumsprozeß kapitalistischer Volkswirtschaften maßgeblich als durch das Wachstum der "exogenen" Güternachfrage bestimmt sieht. Es sind in seiner Argumentationsweise aber auch Situationen denkbar, in denen die verfügbare Arbeitsmenge die Wachstumsmöglichkeiten zumindest zeitweise beschränken kann.

\subsubsection{Fazit}

Kaldors Erklärung für die im internationalen Vergleich niedrigen Wachstumsraten des britischen Sozialprodukts konzentriert sich in großem Umfang auf die Existenz des VerdoornZusammenhangs im industriellen Sektor. Die obigen Ausführungen haben gezeigt, daß die empirisch festgestellte Beziehung zwischen der Wachstumsrate der Arbeitsproduktivität und dem Outputwachstum auf unterschiedliche Art und Weise abgeleitet werden kann. Die Bestimmungsgründe der Elastizität der Produktivitätswachstumsrate bzgl. derjenigen der Produktion sind je nach Vorgehensweise entweder produktionstechnischer Art oder durch den Arbeitsmarkt bestimmt. Auf den ersten Blick entsteht der Eindruck der Willkür bei der theoretischen Erklärung des empirisch festgestellten Zusammenhangs. Zusammengenommen zeigen beide Interpretationen zwei wesentliche Aspekte, die zur Existenz outputinduzierter Produktivitätseffekte beitragen können: einerseits steigende Skalenerträge, andererseits ein ausreichend flexibles Arbeitsangebot, damit die potentiellen Skalenerträge genutzt werden können.

Gelingt es einer Volkswirtschaft nicht, diese Potentiale zu realisieren, werden die Folge niedrige Wachstumsraten sein. Eine Möglichkeit, einer solchen Wachstumsfalle zu entkommen, sieht Kaldor in der effizienteren Verwendung des Produktionsfaktors Arbeit, was er leider nicht genauer spezifiziert. Mit Sicherheit dachte er dabei an eine bessere Ausbildung der Arbeitskräfte sowie an eine Steigerung der Effizienz der Allokation von Arbeitskräften, "...to limit the absorption of labour into those sectors in which...the marginal social product is likely to be appreciably below the marginal private product." 85 Eine weitere Chance sah er in der technologischen Entwicklung auf dem Gebiet der Mikroelektronik und Automatisierung, d.h. das Problem der technologischen Arbeitslosigkeit hervorgerufen durch den verstärkten Einsatz neuer Technologien schien ihm zu diesem Zeitpunkt nicht relevant. Eine dritte Möglichkeit bot eine stärkere Integration in den Welthandel, um durch die damit verbundene steigende internationale Arbeitsteilung, sich auf die Bereiche konzentrieren zu können, die durch hohe

85 Kaldor (1966, S. 303). 
Skalenerträge gekennzeichnet waren. Insbesondere die letzte Strategie kann für ein Land auch Nachteile bieten (vgl. Abschnitt 4.5).

\subsection{Zur Erklärung des Verdoorn-Zusammenhangs}

Kaldors Ausfuhrungen zum geringen Wachstum der britischen Volkswirtschaft und insbesondere seine Rückbesinnung auf den Verdoorn-Zusammenhang haben eine umfangreiche Diskussion seiner Ideen zur Folge gehabt. Vor allem die starke Betonung eines inflexiblen Arbeitsangebots als mögliches Hemmnis für dauerhaftes Wachstum und die Frage nach der richtigen Spezifikation und der Stabilität des Verdoorn-Zusammenhangs standen im Zentrum der Auseinandersetzungen und weiterer empirischer Untersuchungen. Da der VerdoornZusammenhang eines der zentralen Elemente in der kaldorianischen Erklärung länderspezifischer Wachstumsraten und, wie später noch gezeigt wird, auch von Divergenzprozessen ist, wird im folgenden die Diskussion über diesen Zusammenhang zusammengefaßt. Schon der Abschnitt über die eigentliche Vorgehensweise von Verdoorn hat gezeigt, daß der VerdoornKoeffizient auf sehr unterschiedlichen Determinanten basieren kann. Da die Begründung des Verdoorn-Zusammenhangs durch eine neoklassische Cobb-Douglas-Produktionsfunktion nicht den Vorstellungen Kaldors entspricht, der mit seiner technical progress function gerade dieses Konzept überwinden wollte, wurde versucht, eine andere theoretische Erklärung zu finden. Darüber hinaus wurden Schätzungen auf Längs- und Querschnittsbasis sowie auf internationaler, nationaler und sektoraler Ebene durchgeführt. In den folgenden Abschnitten werden neben den Ergebnissen der theoretischen Überlegungen auch immer Ergebnisse empirischer Studien eingebracht.

\subsubsection{Theoretische Begründungen des Verdoorn-Zusammenhangs auf Basis der Cobb-Douglas-Produktionsfunktion}

\subsubsection{Begriffliche Klärung: Verdoorn-Elastizität versus Verdoorn-Koeffizient}

Wie oben gezeigt, hat Verdoorn aufgrund empirischer Beobachtungen die Konstanz der Elastizität des Produktivitätswachstum bzgl. des Produktionswachstums mit Hilfe einer CobbDouglas-Produktionsfunktion zu erklären versucht. Kaldor wiederum griff auf diese Überlegungen zurück, um Aussagen über den Wachstumsprozeß entwickelter Industrienationen treffen zu können. Von ihm wurde eine lineare Einfachregression verwendet, die das Produktions- und das Produktivitätswachstum in Beziehung zueinander setzt. Der Regressionskoeffizient der Produktionswachstumsrate wurde im weiteren Verlauf der Diskussion über den Verdoorn-Zusammenhang als Verdoorn-Koeffizient bezeichnet. Bevor die Frage nach der 
Stabilität und Gültigkeit dieser Relation zu klären versucht wird, soll der Unterschied zwischen dem ursprünglich von Verdoorn untersuchten Koeffizienten (V) und dem im weiteren Verlauf der Debatte so bezeichneten Verdoorn-Koeffizienten herausgearbeitet werden.

Verdoom definierte (V) durch folgende Quotienten:

$$
\mathrm{V}=\frac{\dot{\mathrm{P}}}{\mathrm{P}}: \frac{\dot{\mathrm{Y}}}{\mathrm{Y}}
$$

Er stellte somit das gesamte Produktivitätswachstum in Verhältnis zum Produktionswachstum. Die Erhöhung der Produktivität muß nicht vollständig durch die Steigerung der Produktion erklärt sein. (V) gibt vielmehr nur ein Verhältnis zwischen den beiden Wachstumsraten wieder. Welches die Ursachen des Produktivitätsanstiegs sind, wird durch die Berechnung dieser Elastizität nicht erklärt. Es müssen alle denkbaren Einflußgrößen berücksichtigt werden, d.h. sowohl exogen als auch endogen bedingte Effizienzsteigerungen sind als Determinanten denkbar. Welche Faktoren letztendlich für die Existenz und Stabilität von (V) verantwortlich sind, kann nur durch eine theoretische und empirische Analyse geklärt werden. Verdoorn zeigte mit Hilfe einer statischen Cobb-Douglas-Funktion, daß (V) durch:

$$
V=1-\frac{1}{\beta+\alpha \frac{k}{1}}
$$

bestimmt ist. Für den Fall einer Cobb-Douglas-Produktionsfunktion mit technischem Fortschritt $\mathrm{Y}=\mathrm{e}^{\mathrm{vt}} \mathrm{L}^{\beta} \mathrm{K}^{\alpha}$ folgt:86

$$
V=1-\frac{1}{\beta+\alpha \frac{k}{1}+\frac{v}{1}}
$$

Je nachdem, welche Art der Produktionsfunktion unterstellt wird, verändert sich die Zahl der Determinanten der Verdoorn-Elastizität. Führt man dagegen eine Kleinst-Quadrat-Schätzung durch, um den Verdoorn-Zusammenhang mittels einer Einfachregression zu bestimmen, muß beachtet werden, daß sich die Regressionsgleichung aus zwei Teilen zusammensetzt. Allgemein gilt für die Schätzung des Verdoorn-Zusammenhangs:

$$
p=a+b y
$$

86 Vgl. Boulier (1984, S. 261). 
Der Regressionskoeffizient (b) kann nicht mit der Verdoorn-Elastizität (V) gleichgesetzt werden. Denn es gilt:

$$
V=\frac{p}{y}
$$

und somit:

$$
V=\frac{p}{y}=\frac{a+b y}{y}=b+\frac{a}{y}
$$

Der Regressionskoeffizient (b) stellt somit nur einen verzerrten Schätzer der eigentlichen Verdoorn-Elastizität dar, die sowohl die Effekte der autonomen (a/y) als auch der produktionsinduzierten Produktivitätssteigerungen (b) erfaßt.

Diese Überlegung sei anhand eines Beispiels kurz dargestellt. Mit Hilfe der Wachstumsratengleichung für die Cobb-Douglas-Produktionsfunktion und unter Verwendung der Identität $\mathrm{y}=\mathrm{p}+1$ stellt sich der Zusammenhang zwischen Produktionswachstum und Produktivitätswachstum folgendermaßen dar: ${ }^{87}$

$$
p=\frac{\alpha}{\beta} k+\frac{\beta-1}{\beta} y
$$

Die Höhe des Verdoorn-Koeffizienten hängt bei dieser Vorgehensweise ausschließlich von der Produktionselastizität der Arbeit ab. Der autonome Teil der Regressionsgleichung würde die Beiträge des Kapitals zum Produktivitätswachstum erfassen, was aber die Annahme der Konstanz der Kapitalwachstumsrate impliziert. Würde man aus dieser Spezifikation Rückschlüsse auf die Parameter der Produktionsfunktion ziehen, würden sich u.U. sehr hohe Skalenniveaus ergeben. Geht man z.B. von einem Wert von 0,5 für den Regressionskoeffizienten aus, folgt für die Produktionselastizität der Arbeit $\beta=2$, d.h. die Produktionsfunktion müßte auf alle Fälle, d.h. falls ein positiver Beitrag des Kapitals zur Produktion $(\alpha>0)$ vorliegt, steigende Skalenerträge aufweisen. Die Veränderung des Arbeitseinsatzes um $1 \%$ würde den Output um 2,0\% steigern. Die traditionelle Annahme abnehmender Grenzproduktivitäten der Produktionsfaktoren wäre dann nicht mehr haltbar. Ein Grund für diesen hohen Wert für $(\beta)$ kann in der unterstellten Produktionsfunktion liegen, da technischer Fortschritt explizit nicht berücksichtigt wurde, d.h. die Schätzwerte für die Produktionselastizitäten beinhalten u.U. auch die Wirkungen des technischen Fortschritts. ${ }^{88}$ Erweitert man die Produktionsfunktion um 
Hicks-neutralen technischen Fortschritt mit der Rate (v), beinhaltet der Absolutterm noch weitere Determinanten:

$$
\mathrm{p}=\frac{\alpha}{\beta} \mathrm{k}+\frac{\nu}{\beta}+\frac{\beta-1}{\beta} \mathrm{y}, \text { d.h. } a=\frac{\alpha}{\beta} \mathrm{k}+\frac{v}{\beta}
$$

Würde man beide Gleichungen entsprechend der Vorgehensweise von Verdoorn umformen, um zur Verdoorn-Elastizität zu kommen, würden sich selbstverständlich die oben angeführten Definitionen von (V) ergeben. Der Verdoorn-Koeffizient ist nur für den Fall einer homogenen Regression ein unverzerrter Schätzer für die Verdoorn-Elastizität. Sobald der Test für das Absolutglied der Regressionsgleichung die Ablehnung der Nullhypothese anzeigt, stellt der Regressionskoeffizient des Regressors keine exakte Schätzung für (V) dar. Diese Überlegungen machen deutlich, daß ein empirischer Test des Verdoorn-Zusammenhangs nicht auf eine Einfachregression beschränkt werden kann, sondern er müßte zusätzliche exogene Variablen, wie z.B. die Wachstumsrate des Kapitalstocks, beinhalten. 89

Der Verdoorn-Zusammenhang kann auch als durch Parameter des Arbeitsmarkts bestimmt angesehen werden kann. Es ergab sich hier die Gleichung:

$$
\mathrm{p}=\frac{\lambda-\gamma \mathrm{n}}{1+\gamma}+\frac{\gamma}{1+\gamma} \mathrm{y}
$$

Wird diese Struktur zugrundegelegt, sind keinerlei Rückschlüsse auf das Vorliegen von Skalenerträgen möglich, sondern nur auf die Lohnflexibilität des Arbeitsangebots. Die Verdoorn-Elastizität (V) würde in diesem Falle durch

$$
\mathrm{V}=\frac{\lambda-\gamma(\mathrm{n}+\beta 1+\alpha \mathrm{k})}{(1+\gamma)(\beta 1+\alpha \mathrm{k})}
$$

bestimmt. Neben den Parametern des Arbeitsmarktes beeinflussen die Produktionselastizitäten und die Wachstumsraten der Beschäftigung und des Kapitalstocks die Höhe von (V).

In Abschnitt 4.3.1.3 wurde Verdoorns eigene Vorgehensweise zur Ermittlung von (V) vorgestellt, die eine Kombination der Spezifikation des Verdoorn-Zusammenhangs auf Basis einer statischen Cobb-Douglas-Produktionsfunktion und der Funktionen des Arbeitsmarktes ist. Die Verwendung eines simultanen Gleichungssystems ergibt somit das Identifikationsproblem bei der Höhe der Skalenerträge. Inwieweit steigende Skalenerträge vorliegen, hängt maßgeblich von der Wahl der Parameter der Arbeitsnachfrage- und der Arbeitsangebotsfunktion ab. Eindeutige Rückschlüsse sind nicht möglich. 90

89 Auf die Konsequenzen dieser Verănderung wird weiter unten eingegangen werden.

90 Vgl. McCombie (1980, S. 5) und Rowthorn (1979, S. 131f.). 


\subsubsection{Der Einfluß der Kapitalbildung auf den Verdoorn-Zusammenhang}

Bei den Spezifikationen des Verdoorn-Zusammenhangs, die in den vorigen Abschnitten diskutiert wurden, zeigte sich, daß das Absolutglied der Einfachregression mehrere Variablen enthält, darunter auch die Wachstumsrate des Kapitalstocks. Welchen Beitrag die Kapitalbildung auf die Entwicklung der Produktivität hat, wird hierdurch nicht deutlich. Sowohl Arrows Behandlung des learning by doing als auch Kaldors technical progress function legen eine wichtige Rolle des Investitionsverhaltens für die Entwicklung der Arbeitsproduktivität nahe. Kaldor führte z.B. mit Hilfe der von ihm ermittelten Schätzung für den VerdoornZusammenhang einen Vergleich zwischen dem tatsächlichen Produktivitätswachstum der Länder in seiner Stichprobe und dem potentiellen, sich aus der Regressionsgleichung ergebenden Produktivitätsanstieg durch. Die Volkswirtschaften, die höheres Produktivitätswachstum erzielen konnten als erwartet wurde, waren durch eine hohe Investitionstätigkeit gekennzeichnet. Als Indikator verwendete Kaldor das Verhältnis zwischen dem Anteil der Realinvestitionen an der industriellen Produktion und der Wachstumsrate der Industrieproduktion (ICOR=incremental capital output ratio). Je höher diese Maßzahl war, desto besser schnitten die Volkswirtschaften bei Kaldors Test ab. ${ }^{91}$ Eine genauere Analyse müßte bei empirischen Untersuchungen eine weitere exogene Variable einführen, um die Effekte der Investitionen zu berücksichtigen. Im folgenden soll zum einen auf die notwendigen Veränderungen bei der Spezifikation des Verdoorn-Zusammenhangs, zum anderen auf durchgefürte Untersuchungen zu diesem Sachverhalt eingegangen werden.

Die traditionelle Schreibweise lautet dann furr den Fall ohne technischen Fortschritt:

$$
p=\frac{\beta-1}{\beta} y+\frac{\alpha}{\beta} k
$$

Für den Fall mit technischem Fortschritt:

$$
p=\frac{v}{\beta}+\frac{\beta-1}{\beta} y+\frac{\alpha}{\beta} k
$$

Wird das Beschäftigungswachstum als Regressand gewählt, stellt sich der Zusammenhang wie folgt dar:

91 Für Kaldor waren diese Ergebnisse gleichzeitig ein Beleg für die außerordentliche Relevanz von steigenden Skalenerträgen. Unterschiede im Investitionsverhalten könnten nur Lücken bei der Erklărung von divergierendem Produktivitătswachstum schließen. Leider bleibt er den Beweis für diese Behauptung schuldig. Die Berechnung einer Regression zwischen dem ICOR und der Wachstumsrate der Produktivităt hătte u.U. Aufschluß über die Richtigkeit dieser Aussage geben können. Vgl. Kaldor (1966, S. 292f.) und Kaldor (1967, S. 20). 


$$
1=\frac{1}{\beta} y-\frac{\alpha}{\beta} k
$$

bzw.

$$
1=-\frac{v}{\beta}+\frac{1}{\beta} y-\frac{\alpha}{\beta} k
$$

Aus dieser Spezifikation lassen sich zwei Schlüsse für die Schätzung ziehen. Liegt kein technischer Fortschritt vor, muß das Absolutglied der Regressionsgleichung gegen Null gehen, bzw. ist es signifikant von Null verschieden, kann auf autonomen technischen Fortschritt geschlossen werden. Prinzipiell ist fraglich, welche Veränderung die Einbeziehung des Kapitals als erklärende Variable für die Güte und Höhe der Regressionskoeffizienten hat. Falls die Wachstumsrate des Kapitals konstant ist, wird der Absolutterm der Regressionsgleichung die Effekte der Kapitalbildung erfassen. Die Schätzwerte für den Verdoom-Koeffizienten werden mit und ohne Berücksichtigung von (k) identisch sein. Ist dagegen der Kapitalkoeffizient (v) konstant, verändert sich der Informationsgehalt des Regressionskoeffizienten. Die Gleichungen (4.37-4.40) können durch gleichzeitige Addition und Subtraktion von $\frac{\alpha}{\beta} y$ umgeschrieben werden $\mathrm{zu}: 92$

$$
\mathrm{p}=\frac{\alpha+\beta-1}{\beta} \mathrm{y}+\frac{\alpha}{\beta}(\mathrm{k}-\mathrm{y}) \text { und }\left(4.38^{\prime}\right) \mathrm{p}=\frac{\nu}{\beta}+\frac{\alpha+\beta-1}{\beta} \mathrm{y}+\frac{\alpha}{\beta}(\mathrm{k}-\mathrm{y})
$$

bzw.

$$
1=\frac{1+\alpha}{\beta} y-\frac{\alpha}{\beta}(k-y) \text { und }
$$$$
\left(4.40^{\prime}\right) 1=-\frac{v}{\beta}+\frac{1+\alpha}{\beta} y-\frac{\alpha}{\beta}(k-y)
$$

Unterstellt man diese Schreibweisen, so ist der Verdoorn-Koeffizient bei Konstanz von (v), d.h. die Produktion wächst mit der gleichen Rate wie der Kapitaleinsatz, in der Einfachregression ein unverzenter Schätzer von $\frac{\alpha+\beta-1}{\beta}$ bzw. der Regressionskoeffizient des Produktionswachstums in der alternativen Schreibweise ein unverzerrter Schätzer von $\frac{1+\alpha}{\beta}$. Das gleiche gilt für den Fall der Orthogonalität von ( $k$ ) und $(y)$. In beiden Fällen können keine Rückschlüsse aus dem ermittelten Koeffizienten der Einfachregression auf die Höhe der Skalenerträge gezogen werden. Liegt dagegen Kollinearität zwischen (y) und (k) vor, die z.B.

92 Vgl. für den Fall der traditionellen Schreibweise des Verdoorn-Zusammenhanges Bairam (1987, S. 30f.). 
aufgrund des Akzeleratorprinzips erwartet werden kann ${ }^{93}$, oder Kollinearität zwischen (k-y) und $(y)$ vor, werden sich Veränderungen des Verdoorn-Koeffizienten ergeben.

Ein weiteres Problem ist die Wahl des richtigen Indikators für den Kapitalstock. Eine mögliche Variable, um die Effekte der Kapitalbildung zu erfassen, ist die Bruttoinvestitionsquote (I/Y) ${ }^{94}$ Sie bildet aber nur unter der Annahme der Konstanz des Kapitalkoeffizienten $(v=K / Y)$ eine verläßliche Schätzung für die Wachstumsrate des Kapitalstocks, denn es gilt:95

$$
\frac{I}{Y}=\frac{d K}{K} \frac{K}{Y}=k v
$$

Die Bruttoinvestitionsquote ergibt sich aus dem Produkt von Kapitalkoeffizient und der Wachstumsrate des Kapitals. Nur für $v=1$, ist (I/Y) ein exakter Indikator für das prozentuale Wachstum des Kapitalstocks. Variiert (v), kann aus der Investitionsquote nicht mehr direkt auf (k) geschlossen werden. Im kaldorianischen Gedankengebäude stellt die Annahme der Konstanz des Kapitalkoeffizienten kein großes Hindernis dar, denn eine von Kaldors berühmten stilisierten Fakten ist die Stabilität von (v). Bei internationalen Vergleichen muß zusätzlich zur Konstanz von (v) auch die Identität der nationalen Kapitalkoeffizienten angenommen werden, da ansonsten unterschiedliche Bruttoinvestitionsquoten auf differierenden Kapitalkoeffizienten basieren und nicht auf unterschiedlichen Wachstumsraten des Kapitals.

Wie oben erwähnt wurde, war Kaldor schon in seiner Antrittsvorlesung auf die Investitionsproblematik eingegangen. Aufgrund des Mangels an Daten referierte er erst 1967 über Berechnungen, die explizit die Investitionsquote als erklärende Variable berücksichtigten. Für dieselbe Gruppe von Ländern, die er 1966 verwendete, berechnete er für die gesamte Industrie für den Zeitraum 1953/54 bis 1963/64 sowohl die Einfachregressionen als auch die Mehrfachregressionen des Verdoorn-Zusammenhangs. Es zeigte sich, daß die Güte des Zusammenhangs von der Berücksichtigung Kanadas in der Stichprobe abhängt (vgl. Tab. 4.5). Leider gibt Kaldor keine Berechnung seiner eigenen Schreibweise des Verdoorn-Zusammenhangs für den erweiterten Fall an, sondern wählt das Produktionswachstum als endogene Variable und das Beschäftigungswachstum und die Investitionsquote als unabhängige Variable. Dies ist eine sehr neoklassische Vorgehensweise, denn sie impliziert die Unabhängigkeit des Kapital- und Arbeitseinsatzes von der Nachfrage nach Gütern und widerspricht somit den sonstigen Ausführungen von Kaldor zu dieser Fragestellung. 96

93 Vgl. Kaldor (1968, S. 389).

94 Vgl. z.B. Kaldor (1967), Cripps/Tarling (1973). Für Längsschnittanalysen siehe z.B. Schnur (1990).

95 Vgl. Bairam $(1987$, S. 31).

96 Vgl. Kaldor (1967, S. 81ff.). 
Tab. 4.5: Der einfache und der erweiterte Verdoorn-Zusammenhang im Industriesektor für 12 OECD-Länder (1953/54-1963/64)

\begin{tabular}{|c|c|c|c|}
\hline Nr. & Stichprobe & Regressionsgleichung & $\mathbf{R}^{2}$ \\
\hline 1 & 12 Länder & $\mathrm{p}=0,888+\underset{(0,060)}{0,446 y}$ & 0,847 \\
\hline 2 & 12 Länder & $\begin{array}{r}1=-0,888+0,554 y \\
(0,060)\end{array}$ & 0,893 \\
\hline 3 & 12 Länder & $\mathrm{p}=0, \underset{(0,079)}{0,527} \underset{(0,029)}{0,356 y}$ & 0,880 \\
\hline 4 & 12 Länder & $y=0,0,835+\underset{(0,168)}{1,3671}+\underset{(0,037)}{0,097} \frac{\mathrm{I}}{\mathrm{Y}}$ & 0,940 \\
\hline 5 & 11 Länder* & $\mathrm{p}=\underset{(0,049)}{0,709+\underset{(0,017)}{0,268 y}}$ & 0,960 \\
\hline 6 & 11 Länder* & $\mathrm{y}=0,937+\underset{(0,085)}{1,3201}+\underset{(0,018)}{0,105} \frac{\mathrm{I}}{\mathrm{Y}}$ & 0,986 \\
\hline
\end{tabular}

Quelle: Kaldor (1967, S. 81-83). * ohne Kanada

Interpretiert man die Regressionsgleichungen (4) und (6) als richtige Darstellung einer CobbDouglas-Produktionsfunktion, würde diese ein Skalenniveau zwischen 1,425 und 1,464 aufweisen. ${ }^{97}$ Die These steigender Skalenerträge im industriellen Sektor wäre bestätigt. Die Annahme der Grenzproduktivitätsentlohnung müßte aufgegeben werden. Der Produktionsfaktor Arbeit würde steigende Grenzproduktivitäten aufweisen, da seine Produktionselastizität deutlich über Eins liegt. Die Rate des technischen Fortschritts würde 0,709 bzw. 0,937 betragen.

Um aus den Regressionsgleichungen, die das Produktivitätswachstum als unabhängige Variable beinhalten, auf das Niveau der Skalenerträge schließen zu können, sind Annahmen über das Verhältnis der Produktionselastizitäten zueinander notwendig. Die Summe der Regressionskoeffizienten des Produktions- und des Kapitalwachstums ergibt: 98

$$
\frac{\alpha+\beta-1}{\beta}
$$

Für die ermittelten Werte aus Kaldors Beispiel folgt für Gleichung (5) für diese Summe 0,341. Der Regressionskoeffizient des Kapitalwachstums steht für $(\alpha / \beta)$ und gibt deshalb Informa-

97 Die Regressionsgleichungen wăren mit der Darstellung der Produktionsfunktion in Wachstumsratenform identisch: $y=v+\beta 1+\alpha k$.

98 Vgl. auch die Ausführungen zur Unverzerrtheit der Schătzer bei Vernachlässigung des Kapitalbeitrags weiter oben. Die Summe der Regressionskoeffizienten der Mehrfachregression entspricht dem Regressionskoeffizienten der Einfachregression bei Annahme der Konstanz des Kapitalkoeffizienten. 
tionen über das Verhältnis dieser beiden Parameter. Im vorliegenden Fall gilt deshalb $\alpha=0,073 \beta$. Eingesetzt in Gleichung (4.42) folgt für $(\beta)$ ein Wert von 1,366 und für $(\alpha)$ ein Wert von 0,0997 , d.h. das Skalenniveau würde 1,4657 betragen. Dieser nicht überraschende Wert entspricht ziemlich genau dem Ergebnis der direkten Schätzung der Cobb-DouglasProduktionsfunktion. Andere Autoren (z.B. McCombie 1983, oder McCombie/Thirlwall 1994) akzeptieren zwar die Annahme über die Konstanz des Kapitalkoeffizienten, verwenden aber nicht die Information über die Höhe des zweiten Regressionskoeffizienten für das Verhältnis $(\alpha / \beta)$. Vielmehr erfolgt hierbei eine indirekte Schätzung der Skalenerträge die, indem auf Gleichung (4.37) und den sich daraus ergebenden Verzerrungen des Verdoorn-Koeffizienten zurückgegriffen wird, Informationen über den Kapitalkoeffizienten notwendig macht. Die Ergebnisse für die Höhe der Skalenerträge werden in diesem Fall vom unterstellten Verhältnis von $(\alpha / \beta)$ beeinflußt. ${ }^{99}$

Weitere Möglichkeiten zur Integration der Kapitalbildung in den Verdoorn-Zusammenhang ergeben sich durch die Verwendung der Veränderungsraten des Bruttoanlagevermögens (mit und ohne Wohnungsbau) und der Kapitalintensität sowie der logarithmischen Abweichung der Investitionen von der Trendentwicklung. 100 Auch diese Spezifikationen sehen sich dem Problem der Multikollinearität zwischen Produktions- und Kapitalvariable gegenüber. Ein weiteres Problem ergibt sich bei der Spezifikation des Verdoorn-Zusammenhangs, wenn als erklärende Variable die Veränderung der Kapitalintensität gewählt wird. Eine solche Spezifikation würde die Bedeutung der Substitution zwischen Produktionsfaktoren explizit berücksichtigen. Steigerungen der Kapitalintensität erhöhen die Pro-Kopf-Produktion, d.h. sie bewirken Produktivitätswachstum. Im traditionellen neoklassischen Modell unterliegt dieser Vorgang jedoch dem Gesetz des abnehmenden Grenzertrags. Eine stetige, gleichmäßige absolute Erhöhung der Kapitalintensität würde abnehmende Wachstumsraten des Pro-KopfOutputs zur Folge haben. Konstante Wachstumsraten bei der Kapitalintensität führen in einer Cobb-Douglas-Welt zur Konstanz der Wachstumsrate des Pro-Kopf-Produkts. Dies kann anhand einer Pro-Kopf-Produktionsfunktion gezeigt werden. Es gilt bei $\alpha+\beta=1$ und ohne technischen Fortschritt:

$$
\frac{\mathrm{Y}}{\mathrm{L}}=\mathrm{P}=\frac{\mathrm{L}^{\beta} \mathrm{K}^{\alpha}}{\mathrm{L}}=\left(\frac{\mathrm{K}}{\mathrm{L}}\right)^{\alpha}
$$

bzw. in Wachstumsraten:

$$
\mathrm{p}=\alpha(\mathrm{k}-1)
$$

99 Vgl. McCombie (1983, S. 418) und McCombie/Thirlwall (1994, S. 176ff.).

100 Vgl. z.B. Pusse (1975, 1990), Schnur (1990), McCombie/DeRidder (1983), Michl (1985) sowie McCombie/Thirlwall (1994). 
Ist die Produktionsfunktion durch konstante Skalenerträge gekennzeichnet, besteht eine lineare Beziehung zwischen der prozentualen Veränderung der Kapitalintensität und der Wachstumsrate der Arbeitsproduktivität. Die Einbeziehung von Hicks-neutralem technischen Fortschritt fügt der Wachstumsratengleichung nur einen Absolutterm hinzu.

Will man an einer allgemeinen Cobb-Douglas-Produktionsfunktion als theoretischer Grundlage festhalten, müssen die Regressionskoeffizienten reinterpretiert werden, da sich ihre Bestimmungsgrößen verändern. Es kann gezeigt werden, daß durch diese Vorgehensweise die Wachstumsrate der Beschäftigung auf der Seite der unabhängigen Variablen der Regressionsgleichung hinzukommt und daraus vergleichbare Probleme wie bei der Vernachlässigung der Kapitalwachstumsrate als exogene Variable resultieren. Die Veränderungsrate der Kapitalintensität entspricht der Differenz zwischen der Wachstumsrate des Kapitalstocks und derjenigen der Beschäftigung. Die Gleichungen für den Verdoorn-Zusammenhang müssen deshalb entsprechend erweitert werden. Für die traditionelle Schreibweise folgt:

$$
p=\frac{v}{\beta}+\frac{\alpha}{\beta} 1+\frac{\beta-1}{\beta} y+\frac{\alpha}{\beta}(k-1)
$$

Der Absolutterm der Regressionsgleichung würde aus diesem Grund (l) enthalten. Nur wenn (l) konstant ist, müßte sie nicht als weitere exogene Variable berücksichtigt werden. In diesem Fall würde sich die Wachstumsrate der Kapitalintensität wie die Kapitalstockwachstumsrate verhalten. Eine Unterscheidung zwischen den beiden Raten wäre nicht notwendig, die durchgeführte Erweiterung somit überflüssig. Führt man dagegen einen weiteren Umformungsschritt durch, verändert sich die Struktur der Regressionskoeffizienten und es sind keine Annahmen über die Beschäftigungsentwicklung mehr notwendig. Es gilt dann:101

$$
p=\frac{v}{\alpha+\beta}+\frac{\alpha+\beta-1}{\alpha+\beta} y+\frac{\alpha}{\alpha+\beta}(k-1)
$$

Der Verdoorn-Koeffizient bietet sich nun als Indikator für die Höhe der Skalenerträge direkt an. Noch deutlicher wird dies, wenn man die entsprechenden Manipulationen für die kaldorianische Schreibweise durchführt. Analog steht nun:

$$
1=-\frac{v}{\beta}+\frac{1}{\alpha+\beta} y-\frac{\alpha}{\beta}(k-1)
$$

101 Durch die Addition von $\frac{\alpha}{\beta} y-\frac{\alpha}{\beta} y$ in Gleichung (4.45) folgt $p=\frac{v}{\beta}-\frac{\alpha}{\beta}(y-1)+\frac{\alpha+\beta-1}{\beta} y+\frac{\alpha}{\beta}(k-1)$. Mit $\mathrm{p}=\mathrm{y}-1$ steht im nălchsten Schritt: $\left(1+\frac{\alpha}{\beta}\right) \mathrm{p}=\frac{\nu}{\beta}+\frac{\alpha+\beta-1}{\beta} \mathrm{y}+\frac{\alpha}{\beta}(\mathrm{k}-1)$. Durch Division mit $\frac{\alpha+\beta}{\beta}$ ergibt sich Gleichung (4.46). 
Über den Kehrwert des Regressionskoeffizienten kann nun das Skalenniveau ermittelt werden. Ist die Schätzung des Koeffizienten signifikant kleiner als Eins, liegen steigende Skalenerträge vor. Problematisch bei dieser Spezifikation ist jedoch die Tatsache, daß die Wachstumsrate der Beschäftigung indirekt als erklärende Variable für sich selbst verwendet wurde. Liegt kein Wachstum des Kapitalstocks vor, verkürzt sich (k-1) zu (-1). Die Schätzung der Regressionsgleichung kann dann nicht korrekt sein, da durch $(-1)$ die komplette Streuung der abhängigen Variable erklärt, aber noch ein weiterer Regressor (y) vorhanden wäre. Die Regressionsgleichung wäre fehlspezifiziert. 102

Die Ausführungen zur Berücksichtigung des Beitrags des Produktionsfaktors Kapital zum Produktivitätswachstum bzw. zur Beschäftigungsentwicklung haben gezeigt, daß je nach Wahl der Variable, die für die Kapitalentwicklung stehen soll, die Regressionskoeffizienten unterschiedlich interpretiert werden können. Darüber hinaus hat sich an dieser Stelle gezeigt, daß hinter der empirisch ermittelten Schätzgleichung unterschiedliche Annahmen über die Veränderung der Variablen und ihre Beziehungen zueinander stecken können. Die Frage, inwieweit sich Kaldors These steigender Skalenerträge im industriellen Sektor aufrechterhalten läßt, kann durch die Einbeziehung des Kapitals als weitere exogene Variable nicht abschließend beantwortet werden. Während nämlich die Spezifikation durch Gleichung (4.46) eindeutige Rückschlüsse auf das Skalenniveau aufgrund des Regressionskoeffizienten zuläßt, müssen bei Anwendung von Gleichung (4.45) für diesen Zweck Annahmen über das Verhältnis der Produktionselastizitäten zueinander getroffen werden.

Empirische Untersuchungen, die eine entsprechende Erweiterung des VerdoornZusammenhangs um eine Kapitalvariable durchfüren, weisen als ein Ergebnis ein negatives Vorzeichen beim Absolutterm der Regressionsgleichung auf. ${ }^{103}$ Ist dieser signifikant kleiner als Null, würde dies bei Verwendung einer Cobb-Douglas-Produktionsfunktion entweder eine negative Rate des exogenen technischen Fortschritts $(v)$ oder eine negative Produktionselastizität der Arbeit $(\beta)$ bedeuten. Beide Interpretationen widersprechen jedoch sonstigen Ergebnissen empirischer Analysen bzw. den Annahmen über die Eigenschaften der Produktionsfunktion. Somit stellen sich auch in diesem Zusammenhang die Fragen, inwieweit der Verdoorn-Zusammenhang durch eine Cobb-Douglas-Produktionsfunktion richtig wiedergegeben wird und welche Meßfehler bei den Variablen zu ökonomisch zweifelhaften Ergebnissen führen. statistischen Testgroßen. 


\subsubsection{Statischer versus dynamischer Verdoorn-Zusammenhang}

Neben dem im vorigen Abschnitt angesprochenen Identifikationsproblem, das sich aus Verdoorns Gleichungssystem ergibt, tritt bei der Untersuchung des Verdoorn-Zusammenhangs eine weitere Schwierigkeit auf, die eine Cobb-Douglas-Produktionsfunktion als Grundlage dieser Beziehung in Frage stellt. Für die Schätzung des Verdoorn-Koeffizienten müßte es unerheblich sein, ob man die Beziehung zwischen der Arbeitsproduktivitätsentwicklung und dem Produktionswachstum mit Niveaugrößen oder mit Wachstumsraten ermittelt. Geht man wie oben von einer Standard-Cobb-Douglas-Produktionsfunktion

$$
\mathrm{Y}=\mathrm{e}^{\mathrm{vt}} \mathrm{L}^{\beta} \mathrm{K}^{\alpha}
$$

aus, ergibt sich für die Niveaugrößen:

$$
\ln P=\frac{v t+\alpha \ln K}{\beta}+\frac{\beta-1}{\beta} \ln Y
$$

und für die Wachstumsraten durch Differenzierung nach der Zeit:

$$
p=\frac{\alpha}{\beta} k+\frac{\beta-1}{\beta} y .
$$

bzw. für die von Kaldor bevorzugte Schreibweise:

$$
\ln L=\frac{v t+\alpha \ln K}{\beta}+\frac{1}{\beta} \ln Y
$$

und

$$
1=\frac{v+\alpha}{\beta} k+\frac{1}{\beta} y
$$

Wenn die Beziehung zwischen den statischen und dynamischen Gleichungen eindeutig ist, müssen sich auf den ersten Blick identische Werte für die Regressionskoeffizienten ergeben. Diese Aussage ist aber nur eingeschränkt gültig. Aufgrund des Zeitterms im Absolutglied der Regressionsgleichung bereitet die Schätzung des statischen Verdoorn-Zusammenhangs Probleme, wenn mehrere Jahre in die Berechnung einfließen bzw. Wachstumsraten für längere 
Zeiträume (z.B. zehn Jahre) zugrundegelegt werden. Der Wert des Absolutglieds würde sich in Abhängigkeit von der Zeit verändern. Werden dagegen Regressionsrechnungen auf Länderbasis durchgefürt, die auf ein bestimmtes Jahr bezogen sind, ist keine Korrektur notwendig.

Von McCombie (1982) wurden für die von Kaldor (1966) verwendete Ländergruppe Schätzungen für eine statische und eine dynamische Version des Verdoorn-Zusammenhangs für die Zeit von 1950 bis 1973 durchgeführt. Hierbei wurden einerseits Regressionsgleichungen für einzelne Jahre und andererseits für den kompletten Zeitraum ermittelt. Um im letzteren Fall das Problem des Zeitterms im Absolutglied der Regression zu berücksichtigen, wurden Dummies in die Regression mit aufgenommen. Als Ausgangspunkt seiner Schätzungen wählt McCombie in Anlehnung an Black (1962) eine Produktionsfunktion, die nur die Beschäftigungsmenge als Produktionsfaktor enthält: 104

$$
Y=\left(\frac{L}{A e^{v t}}\right)^{-b}
$$

Durch Umformung folgt für die Höhe der Beschäftigung:

$$
L=A e^{v t} Y^{b}
$$

Aus dieser Gleichung läßt sich durch Logarithmieren die statische Version und durch anschlieBende Differenzierung nach der Zeit die dynamische Form der von Kaldor bevorzugten Schreibweise des Verdoorn-Zusammenhangs einfach gewinnen:

$$
\begin{aligned}
& \ln L=\ln A+v t+b \ln Y \\
& 1=v+b y
\end{aligned}
$$

Die Ergebnisse der Schätzungen für den statischen Verdoorn-Zusammenhang legen konstante bzw. nur sehr geringe steigende Skalenerträge nahe (vgl. Tab. 4.6) Die Regressionskoeffizienten waren dabei für einzelne Jahre nicht signifikant von Eins verschieden, jedoch für den Gesamtzeitraum. Berechnungen für den gleichen Zeitraum unter Verwendung von Wachstumsraten ergaben dagegen Hinweise auf deutlich über Eins liegende Skalenniveaus. McCombie bezieht sich in diesem Zusammenhang, ohne es besonders deutlich zu machen, auf die Studie von Cripps/Tarling (1973). Die Entscheidung, den Verdoorn-Zusammenhang mittels Niveaugrößen oder mittels Wachstumsraten zu schätzen, beeinflußt somit den Interpretationsspielraum der sich ergebenden Regressionskoeffizienten.

104 Vgl. McCombie (1982, S. 285ff.). 
Tab. 4.6: Schätzungen für den statischen Verdoorn-Zusammenhang

\begin{tabular}{|c|c|c|c|c|c|}
\hline $\begin{array}{c}\mathbf{N} \\
\text { r. }\end{array}$ & Jahr & Regressionskoefinient & $\overline{\mathbf{R}}^{2}$ & t-Wert & SEE \\
\hline 1 & 1951 & 0,806 & 0,876 & $1,92^{* 0}$ & 0,10111 \\
\hline 2 & 1956 & 0,861 & 0,875 & 1,35 & 0,1029 \\
\hline 3 & 1960 & 1,027 & 0,934 & 0,26 & 0,1033 \\
\hline 4 & 1965 & 0,886 & 0,904 & $1,24^{\circ}$ & 0,0914 \\
\hline 5 & 1970 & 0,925 & 0,946 & 1,07 & 0,0698 \\
\hline 6 & Alle Jahre (1-5) & 0,898 & 0,913 & $2,85^{*}$ & 0,0357 \\
\hline
\end{tabular}

Quelle: McCombie (1982, S. 287).

* Der Koeffizient ist bei einem Konfidenzlevel von 0,95 signifikant von Eins verschieden.

ODiese t-Werte weichen von denen im Beitrag von McCombie ab. Die dort angegebenen Werte sind nicht aus den anderen Angaben ermittelbar.

Deutlicher werden die Unterschiede zwischen der statischen und der dynamischen Gleichung, wenn explizit die Kapitalmenge als erklärende Variable in die Regressionsgleichung aufgenommen wird. Die entsprechenden Gleichungen ändern sich in:

$$
\ln \mathrm{L}=-\frac{\ln \mathrm{A}+\mathrm{vt}}{\beta}+\frac{1}{\beta} \ln \mathrm{Y}-\frac{\alpha}{\beta} \ln \mathrm{K}
$$

bzw.

$$
1=-\frac{v}{\beta}+\frac{1}{\beta} y-\frac{\alpha}{\beta} k
$$

Eine Untersuchung von McCombie/DeRidder für die US-amerikanischen Bundesstaaten (ohne Alaska und Hawaii) ergaben bei dieser Spezifikation des Verdoorn-Zusammenhangs markante Differenzen zwischen den beiden Versionen. Für die Schätzung des dynamischen Zusammenhangs wurde als abhängige Variable die Wachstumsrate der Beschäftigung (prozentuale Veränderung der Beschäftigtenzahl) und als unabhängige Variablen die Wachstumsraten der realen Bruttowertschöpfung und des Kapitalstocks, der durch eine Kombination aus der Perpetual-Inventory-Methode und den kumulierten Bruttoinvestitionen ermittelt wurde, gewählt. Der Berechnungszeitraum erstreckte sich von 1963-1973. Um regionale Unterschiede zu berücksichtigen, wurden Dummy-Variablen für die einzelnen Regionen (Nord-Ost, NordZentral, Süden und Äußerer Westen) eingeführt. Die statische Version wurde auf Basis der gepoolten Daten der Jahre 1963 und 1973 geschätzt. Das oben angesprochene Problem der zeitlichen Abhängigkeit des Absolutglieds der Regressionsgleichung wurde wiederum mit Hilfe 
von Dummy-Variablen beachtet. 105 Während sich im dynamischen Fall ein Skalenniveau in Höhe von 1,45 ergab, das bei einem Konfidenzniveau von 0,99 signifikant größer als Eins ist, wies die statische Regressionsgleichung nur einen Wert von 1,02 auf, der denselben Test nicht bestand. 106 Parallel hierzu führten die Autoren auch Berechnungen für den Fall durch, daß die Produktionswachstumsrate die endogene und die Beschäftigungswachstumsrate die exogene Variable ist. Sowohl für die statische als auch die dynamische Schreibweise wurden die zuvor genannten Ergebnisse bestätigt. Nur das Skalenniveau betrug im letzteren Fall nicht 1,45, sondern 1,33 (vgl. Tab. 4.7). ${ }^{107}$

Tab. 4.7: Schätzungen des dynamischen und statischen Verdoorn-Zusammenhangs (US-amerikanische Bundesstaaten 1963-1973 bzw. 1963 und 1973)

\begin{tabular}{|c|c|c|c|c|}
\hline Version & Regressionsgleichung & SKE & $\overline{\mathbf{R}}^{2}$ & SEE \\
\hline Allgemein & $\begin{array}{l}\text { Dynamisch: } \\
1=a_{0}+a_{1} d_{1}+a_{2} d_{2}+a_{3} d_{3}+b_{1} y+b_{2} k \\
y=a_{0}+a_{1} d_{1}+a_{2} d_{2}+a_{3} d_{3}+b_{1} l+b_{2} k \\
\text { Statisch: } \\
\ln L=a+t+d_{1}+d_{2}+d_{3}+b_{1} \ln Y+b_{2} \ln K \\
\ln Y=a+t+d_{1}+d_{2}+d_{3}+b_{1} \ln L+b_{2} \ln K\end{array}$ & & & \\
\hline \multirow{2}{*}{$\begin{array}{l}\text { Dynamisch } \\
(n=49)\end{array}$} & 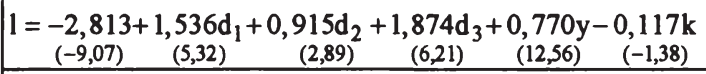 & 1,45 & 0,887 & 0,649 \\
\hline & 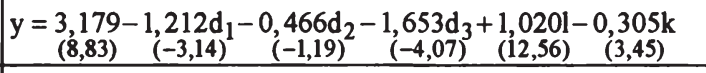 & 1,33 & 0,876 & 0,746 \\
\hline \multirow{2}{*}{$\begin{array}{l}\text { Statisch } \\
(n=98)\end{array}$} & $\ln \mathrm{L}=\mathrm{a}+\mathrm{t}+\mathrm{d}_{1}+\mathrm{d}_{2}+\mathrm{d}_{3}+\underset{(16,78)}{1,087 \ln \mathrm{Y}}-\underset{(-1,65)}{0,109 \ln \mathrm{K}}$ & $1,02^{*}$ & 0,991 & 0,136 \\
\hline & $\ln Y=a+t+d_{1}+d_{2}+d_{3}+\underset{(16,78)}{0,695 \ln L}+\underset{(7,72)}{0,319 \ln K}$ & $1,01^{*}$ & 0,995 & 0,108 \\
\hline
\end{tabular}

Quelle: McCombie/DeRidder (1984, S. 274 und 276), (SKE=Skalenelastizităt).

* Statistisch signifikant bei Konfidenzniveau 0,99; Werte in Klammer sind t-Werte.

Diese gravierenden Unterschiede lassen sich auf verschiedene Ursachen zurückführen. Eine Möglichkeit besteht in Meßfehlern bei den verwendeten Zahlenreihen. Bei Kleinst-QuadratSchätzern führen Fehler bei der Ermittlung der unabhängigen Variablen zu einer nach unten verzerrten Schätzung der Regressionskoeffizienten. 108 So sind einerseits aufgrund unterausgelasteter Kapazitäten in den Anfangs- und Endjahren des betrachteten Zeitraums größere

105 Die Autoren geben leider nur einen Teil ihrer Vorgehensweise bzw. durchgefuhrten Berechnungen bekannt. Vgl. McCombie/DeRidder (1984, S. 273ff.).

106 Das Skalenniveau ergibt sich mit $b_{1}=\frac{1}{\beta}$ und $b_{2}=-\frac{\alpha}{\beta}$ aus $\frac{1-b_{2}}{b_{1}}=\alpha+\beta$.

107 Vgl. McCombie/DeRidder (1984, S. 274 und 278).

108 Vgl. zu diesen okonometrischen Schwierigkeiten z.B. Kennedy (1992, S. 91ff.). 
Fehler bei der dynamischen Abschätzung möglich. Andererseits beeinflussen dauerhafte Fehler bei der Ermittlung der Variablen die Ergebnisse für die statische Version mehr. Welche der beiden Regressionsgleichungen einem größeren Fehlerrisiko unterliegt, läßt sich nicht ohne weiteres bestimmen. 109

Des weiteren können verzerrte Schätzungen durch die Nichtberücksichtigung relevanter Variablen entstehen. Dieser Einwand ist vor allem bei der Einfachregression zu machen, die im Falle des Verdoorn-Zusammenhangs den Beitrag des Kapitals zum Produktivitätswachstum vollständig außen vor läßt. Jedoch auch bei einer Mehrfachregression besteht die Möglichkeit, Determinanten zu vergessen. Sind die vernachlässigten Variablen orthogonal zu den berücksichtigten, entstehen bei der Abschätzung der Regressionskoeffizienten der letzteren keine Verzerrungen. Darüber hinaus trifft der Vorwurf einer falschen Wahl der unabhängigen Variablen beide Arten des Verdoorn-Zusammenhangs. Die Differenzen zwischen den sich ergebenden Skalenniveaus lassen sich somit nicht unbedingt auf das "Vergessen" wichtiger erklärender Größen zurückführen. 110

Verzerrte Schätzungen können auch durch die falsche Spezifikation des Zusammenhanges zwischen unabhängigen und abhängigen Variablen entstehen. Lineare Mehrfachregressionen gehen von einem eindeutigen Ursache-Wirkungs-Zusammenhang aus. Vor allem für die Entwicklung der Beschäftigung und der Produktion sowie der Produktivität wird man von einer gegenseitigen Beeinflussung ausgehen können. Wie beim vorigen Argument können die Folgen eines solchen Fehlers für die Ermittlung der dynamischen und statischen Regressionen nicht eindeutig bestimmt werden. ${ }^{111}$

Neben Meß- und Verfahrensproblemen bietet sich eine andere Erklärung für das Phänomen des Auseinanderfallens von statischer und dynamischer Lösung an. Identische Ergebnisse müssen sich nur ergeben, falls die Beziehung für die Wachstumsraten und für die Niveaugrößen eindeutig ineinander überführt werden können. Parallel zur Diskussion über den Zusammenhang zwischen der technical progress function von Kaldor und einer Cobb-Douglas Produktionsfunktion kann die Frage aufgeworfen werden, ob der Verdoorn-Zusammenhang überhaupt durch eine Cobb-Douglas-Produktionsfunktion erklärt wird. Durch Logarithmieren und Differenzierung nach der Zeit ergibt sich die lineare Gleichung für den VerdoornZusammenhang. Bei der Integration dieser Gleichung ergeben sich aber Identifikationsprobleme. Die Ermittlung der Stammfunktion ist abhängig von der gewählten Integrationskonstanten. Dies kann zum einen als Beleg für die Relevanz der Ausgangsbedingungen, d.h. der Anfangsausstattungen der Volkswirtschaft mit Produktionsfaktoren interpretiert werden, oder

109 Vgl. McCombie (1982, S. 288).

110 Vgl. auch hier Kennedy (1992, S. 91ff.) sowie McCombie (1982, S. 288f).

111 Vgl. auch hier Kennedy (1992, S. 91 ff.) sowie McCombie (1982, S. 288f.). 
zum anderen als Indiz dafür, daß auch andere funktionale Zusammenhänge die lineare Beziehung zwischen Produktivitäts- bzw. Beschäftigungs- und Produktionswachstum begründen. ${ }^{112}$

Denkbar ist z.B. eine Lernkurve, wie sie von Arrow (1962) und Verdoom (1956) diskutiert wurde. ${ }^{113}$ Die Höhe der Arbeitsproduktivität (P) ist positiv bzw. der Arbeitseinsatz (L) negativ abhängig von der Höhe des kumulierten Outputs. Eine mögliche Funktion ist:114

$$
P_{t}=a\left(\int_{0}^{t} Y_{t} d t\right)^{b} \text { mit } b<1
$$

Für langfristige Betrachtungen kann eine konstante Wachstumsrate des Sozialprodukts $(\mu)$ unterstellt werden, so daß die Höhe des Sozialprodukts zu einem bestimmten Zeitpunkt $\left(Y_{t}\right)$ gegeben ist durch:

$$
Y_{t}=Y_{0} e^{\mu t}
$$

Setzt man (4.60) in (4.59) ein, folgt:

$$
P_{t}=a\left(\int_{0}^{t} Y_{0} e^{\mu t} d t\right)^{b}
$$

Nach Auflösung des Integrals ergibt sich für das Produktivitätsniveau $\left(\mathbf{P}_{\mathbf{t}}\right):^{115}$

$$
P_{t}=a\left(\frac{Y_{t}-Y_{0}}{\mu}\right)^{b}
$$

Durch Logarithmieren folgt:

$$
\ln P_{t}=\ln a+b \ln \left(Y_{t}-Y_{0}\right)-b \ln \mu
$$

Bei ausreichend großem $(\mathrm{t})$ kann $\left(\mathrm{Y}_{0}\right)$ vernachlässigt werden und es kann dadurch eine lineare Gleichung für den statischen Verdoom-Zusammenhang bestimmt werden:

112 Vgl. zur Beziehung zwischen technical progress function und Cobb-Douglas-Produktionsfunktion Black (1962) sowie zur Anwendung dieser Überlegungen auf den Verdoorn-Zusammenhang McCombie (1982, S. 289ff.), McCombie/DeRidder (1984, S. 277) und McCombie/Thirlwall (1994, S. 209ff.).

113 Vgl. Arrow $(1962$, S. 156) und Verdoorn $(1956$, S. 434).

114 Vgl. Verdoorn (1956, S. 434).

115 Es gilt: $\int_{0}^{t} Y_{0} e^{\mu t} d t=\left|\frac{1}{\mu} Y_{0} e^{\mu t}\right|_{0}^{t}=\frac{1}{\mu} Y_{0} e^{\mu t}-\frac{1}{\mu} Y_{0} e^{0}=\frac{Y_{t}-Y_{0}}{\mu}$ 


$$
\ln P_{t}=\ln a-b \ln \mu+b \ln Y_{t}
$$

sowie für den dynamischen Fall:116

$$
\mathrm{p}=\text { by }
$$

Der Vollständigkeit halber seien an dieser Stelle auch noch die Gleichungen für die kaldorianische Schreibweise aufgeführt:

$$
\ln \mathrm{L}_{\mathrm{t}}=-\ln \mathrm{a}+\mathrm{b} \ln \mu+(1-\mathrm{b}) \ln \mathrm{Y}_{\mathrm{t}}
$$

und:

$$
1=(1-b) y
$$

Bei dieser Vorgehensweise stellt sich der dynamische Verdoorn-Zusammenhang als eine homogene Funktion dar. Der Koeffizient (b) entspricht genau der Verdoorn-Elastizität, wie sie von Verdoorn (1949) definiert wurde. Da Gleichung (4.65) aufgrund sehr einschränkender Steady State-Annahmen ermittelt wurde, kann nicht automatisch geschlossen werden, daß eine Lernkurve die Basis des Verdoorn-Zusammenhangs bildet. Verdoorn selbst sah sowohl in seinem Aufsatz aus dem Jahre 1949 als auch später (z.B. 1956) Lerneffekte als eine Möglichkeit an, eine Beziehung zwischen dem Produktivitäts- und dem Produktionswachstum zu erklären. Eine Cobb-Douglas-Produktionsfunktion mit steigenden Skalenerträgen als Begründung für das nach ihm benannte Gesetz lehnte er ebenfalls nicht vollständig ab. ${ }^{117}$

Diese Vorgehensweise weist aber dieselben Probleme auf wie bei der Verwendung einer Cobb-Douglas-Produktionsfunktion. Auch hier werden z.B. Variablen bei der Schätzung der statischen Gleichungen außen vor gelassen, d.h. es können dieselben ökonometrischen Einwände gemacht werden wie zuvor. Darüber hinaus muß bei Regressionen geprüft werden, ob das Absolutglied der Regressionsgleichung von Null verschieden ist, da die Lernkurve eine Beziehung zwischen den Wachstumsraten nahelegt, die kein autonomes Produktivitätswachstum beinhaltet. Viele empirische Untersuchungen weisen aber auf einen nicht-homogenen Verdoorn-Zusammenhang hin. Viele Regressionsgleichungen weisen einen Absolutterm ungleich Null auf, wobei einschränkend angemerkt werden muß, daß häufig keine Angaben zu den statistischen Testgrößen gemacht werden. Positive Ordinatenabschnitte ergaben z.B. die Untersuchungen von Boyer/Petit (1991b) und Michl (1985). In allen Fällen waren sowohl das

116 Der Term, der die Wachstumsrate des Outputs enthalt, wird bei der Differenzierung nach der Zeit Null, da die Annahme der Konstanz dieser Größe getroffen wurde.

$117 \mathrm{Vgl}$. Verdoorn (1980). 
Absolutglied als auch der Verdoorn-Koeffizient statistisch signifikant von Null verschieden. ${ }^{118}$ Auch in der Untersuchung von Erber (1994), in der der Verdoorn-Zusammenhang für alle OECD-Länder auf gesamtwirtschaftlicher Ebene für den Zeitraum 1960-1993 geschätzt wurde, ergaben sich mehrfach von Null verschiedene Absolutglieder des Verdoorn-Zusammenhangs. Aufgrund dieses Ergebnisses kann nicht generell ein homogener Zusammenhang zwischen Produktions- und Produktivitätswachstum erwartet werden. Auch diese Fundierung kann damit nicht vollständig überzeugen. 119

\subsubsection{Eine kaldorianische Begründung des Verdoorn-Zusammenhangs}

Die bisherigen Ausführungen über den Verdoom-Zusammenhang basierten weitgehend auf der Annahme, daß sich die Beziehung zwischen Produktions- und Produktivitätswachstum aus einer Cobb-Douglas-Funktion ableiten läßt. Obwohl Verdoorn selbst diese Vorgehensweise in seinen Beiträgen zu diesem Themenkomplex gewählt hat, ist fraglich, inwieweit sie mit den Vorstellungen Kaldors über die Funktionsweisen einer kapitalistisch organisierten Volkswirtschaft vereinbar sind. Wie oben ausgeführt, entwickelte Kaldor als Alternative zum neoklassischen Konzept einer gesamtwirtschaftichen Produktionsfunktion seine technical progress function. Darüber hinaus sah er in Phänomenen wie Lernen und Ausbildung sowie externen Effekten, wie sie von Young diskutiert worden sind, die Ursachen für den VerdoornZusammenhang. Aus diesem Grund bietet es sich an, den Verdoorn-Zusammenhang als alternative Schreibweise einer solchen Fortschrittsfunktion zu sehen.

\subsubsection{Der Verdoorn-Zusammenhang und die technical progress function}

Geht man von einer linearen technical progress function aus, bei der die Wachstumsrate der Arbeitsproduktivität linear von der Veränderungsrate der Kapitalausstattung pro Arbeitseinheit abhängt: 120

$$
\mathrm{p}=\theta+\pi \kappa
$$

$118 \mathrm{Vgl}$. Abschnitt (4.4.8).

119 Vgl. Erber (1994, S. 28ff.).

120 Hier steht $(\theta)$ für die Rate des unverkörperten technischen Fortschritts, (k) für die Wachstumsrate der Kapitalausstattung pro Arbeitnehmer bzw. pro Arbeitseinheit, d.h. der Verănderung der Kapitalintensitatt. Es gilt: $k=\mathrm{k}-\mathrm{l}$. Vgl. für die Ableitung des Verdoorn-Zusammenhangs aus der technical progress function Dixon/Thirlwall (1975, S. 208ff.) und McCombie/Thirlwall (1994). 
und macht man $(\kappa)$ und $(\theta)$ abhängig von der Wachstumsrate des Outputs, steht für die Rate des unverkörperten technischen Fortschritts:

$$
\theta=\varepsilon_{1}+\tau_{1} \mathbf{y}
$$

und für die Wachstumsrate des Kapitaleinsatzes pro Arbeitseinheit:

$$
\kappa=\varepsilon_{2}+\tau_{2} y .
$$

Setzt man beide Gleichungen in (4.68) ein, ergibt sich der Verdoorn-Zusammenhang als

$$
p=\left(\varepsilon_{1}+\pi \varepsilon_{2}\right)+\left(\tau_{1}+\pi \tau_{2}\right) y
$$

Der Verdoorn-Koeffizient wird somit durch die Rate des induzierten unverkörperten technischen Fortschritts, die Rate der induzierten Kapitalbildung und das Ausmaß des unverkörperten technischen Fortschritts bei der Kapitalbildung bestimmt. Die Schätzung des VerdoornZusammenhangs und einer der beiden Bestimmungsgleichungen für die Wachstumsrate der Kapitalintensität bzw. die Rate des unverkörperten technischen Fortschritts ließe Rückschlüsse auf die Parameter der technical progress function zu. Tatsächlich ist die Ermittlung einer Regressionsschätzung für den Zusammenhang zwischen der Wachstumsrate der Kapitalintensität und derjenigen des Outputs naheliegender, da ansonsten ein Indikator für den unverkörperten technischen Fortschritt gefunden werden müßte.

Die Interpretation des Verdoorn-Zusammenhangs als eine mögliche Schreibweise einer linearen technical progress function trifft die Vorstellungen Kaldors mit Sicherheit besser als die einer Cobb-Douglas-Funktion, jedoch besteht bei jeder linearen technical progress function die Möglichkeit sie durch Integration in eine Cobb-Douglas Produktionsfunktion zu überführen. Der Verdoorn-Zusammenhang kann somit ohne weiteres rein formal sowohl als Ausdruck einer Produktionsfunktion mit neoklassischen Eigenschaften als auch einer kaldorianischen technical progress function gesehen werden.

\subsubsection{Die technical progress function versus die Cobb-Douglas-Produktionsfunktion}

Wie nahe beide Möglichkeiten einander sind, zeigt ein Vergleich zwischen dem erweiterten Verdoorn-Zusammenhang, bei dem sowohl die Kapital- als auch die Produktionswachstumsrate berücksichtigt wurden, und der ursprünglichen technical progress function, die den Einfluß der Kapitalintensivierung auf das Produktivitätswachstum zum Inhalt hat. ${ }^{121}$ Aus-

121 Also einer technical progress function, wie sie ursprünglich von Kaldor (1957) vorgeschlagen wurde. 
gehend von der traditionellen Schreibweise des Verdoorn-Zusammenhangs, die anstelle der Kapitalwachstumsrate diejenige der Kapitalintensität beinhaltet:

$$
\mathrm{p}=\frac{v}{\alpha+\beta}+\frac{\alpha+\beta-1}{\alpha+\beta} \mathrm{y}+\frac{\alpha}{\alpha+\beta}(\mathrm{k}-1)
$$

läßt sich zeigen, daß bei konstanten Skalenerträgen $(\alpha+\beta=1)$ sich die Gleichung einer traditionellen technical progress function ergibt, nämlich:

$$
p=v+\alpha(k-1)
$$

Liegen dagegen steigende Skalenerträge vor $(\alpha+\beta>1)$, könnte man von einer erweiterten technical progress function sprechen, die zu den Effekten der Kapitalintensivierung auch noch produktionsinduzierten technischen Fortschritt beinhaltet. Der Funktionsterm wäre allgemein durch

$$
\mathrm{p}=\mathrm{a}+\mathrm{by}+\mathrm{c}(\mathrm{k}-1)
$$

gegeben. ${ }^{122}$ Dieser Vergleich läßt noch eine weitergehende Aussage zu. Eine lineare technical progress function kann, wie von Black (1962) gezeigt, in eine Cobb-Douglas-Produktionsfunktion überfuhrt werden. Andererseits kann aber letztere nur dann in eine technical progress function in der Form von Gleichung (4.73) überführt werden, wenn sie konstante Skalenerträge aufweist oder das Beschäftigungswachstum Null ist. Ausgehend von der Wachstumsratengleichung der Cobb-Douglas-Funktion

$$
y=v+\alpha k+\beta 1
$$

entsteht durch die Subtraktion der Wachstumsrate der Beschäftigung (l) auf beiden Seiten der Gleichung:

$$
p=y-1=v+(\beta-1) 1+\alpha k \text {. }
$$

Für den Fall konstanter Skalenerträge gilt $\beta-1=-\alpha$, so daß

$$
p=y-1=v-(1-\beta) 1+\alpha k=v+\alpha(k-1)
$$

122 Vgl. hierzu Michl (1985, S. 476f,) der - sehr bildlich - von einer Heirat zwischen der technical progress function und dem Verdoorn-Zusammenhang spricht. 
Liegen dagegen andere Skalenerträge vor, z.B. steigende, können die Wachstumsraten der beiden Produktionsfaktoren zwar zusammengefaßt werden, jedoch verbleibt immer ein negativer oder ein positiver Beitrag des Beschäftigungswachstums zur Produktivitätsentwicklung. Gleichung (4.77) kann durch Umformung in

$$
p=v+(\beta-1) l+\alpha l+\alpha k-\alpha l=v+(\alpha+\beta-1) l+\alpha(k-1)
$$

überführt werden. Bei steigenden Skalenerträgen ist $\alpha+\beta>1$, so daß das Beschäftigungswachstum die Wachstumsrate der Arbeitsproduktivität beeinflußt. Im Falle sinkender Skalenerträge ist $\alpha+\beta<1$. Die Beschäftigungszunahme erbringt negative Produktivitätseffekte. Diese Wirkungen sind zu erwarten, da sie genau die Eigenschaften der jeweiligen Skalenerträge widerspiegeln. Konstante Skalenerträge bedeuten bei der Durchführung einer proportionalen Faktorvariation, d.h. k=l, daß die Arbeitsproduktivität nur im Maße des exogenen technischen Fortschritt steigt. Liegen dagegen sinkende (steigende) Skalenerträge vor, führt die proportionale Faktorvariation zu einer abnehmenden (zunehmenden) Arbeitsproduktivität.

Die Höhe des Skalenniveaus spielt keine Rolle, wenn die Beschäftigung mit der Rate Null wächst. In diesem Fall verkürzt sich die Gleichung (4.78) zu

$$
p=v+\alpha k
$$

Das Produktivitätswachstum wird ausschließlich von der Wachstumsrate des Kapitals und dem ungebundenen exogenen technischen Fortschritt bestimmt.

Ist die technical progress function dagegen nicht linear, kann sie nicht generell in eine Cobb-Douglas-Produktionsfunktion überführt werden. Entsprechende Überlegungen bzgl. der Rolle der Skalenerträge sind deshalb nicht von Interesse. Darüber hinaus kann ein linearer Verdoorn-Zusammenhang aus einer solchen technical progress function nur abgeleitet werden, wenn die Bestimmungsfunktionen für die Wachstumsrate der Kapitalintensität und für die Rate des unverkörperten technischen Fortschritts entsprechende Bedingungen erfüllen. Die ursprünglich von Kaldor vorgeschlagene technical progress function weist abnehmende Steigungen bei zunehmender Wachstumsrate der Kapitalintensität auf. Eine mögliche Form einer solchen Funktionsgleichung ist:

$$
\mathrm{p}=\theta+\pi \mathrm{\kappa}^{\vartheta} \text { mit } 0<\vartheta<1
$$

Um einen linearen Verdoorn-Zusammenhang zu erhalten, muß die Gleichung für die Wachstumsrate der Kapitalintensität durch 


$$
\kappa=\left(\varepsilon_{2}+\tau_{2} y\right)^{\vartheta^{-1}}
$$

gegeben sein. Einsetzen von (4.81) in (4.80) ergibt wieder:

$$
p=\left(\varepsilon_{1}+\pi \varepsilon_{2}\right)+\left(\tau_{1}+\pi \tau_{2}\right) y
$$

Prinzipiell ist es möglich, aus einer nicht-linearen technical progress function einen VerdoornZusammenhang abzuleiten. Es sind aber sehr spezifische Annahmen für die Bestimmungsgleichung der Wachstumsrate der Kapitalintensität notwendig. Wenn nun der VerdoornZusammenhang als Ausdruck einer solchen technical progress function verstanden werden kann und gleichzeitig aber auch für eine Cobb-Douglas-Produktionsfunktion stehen kann, ist eine Verbindung zwischen beiden Konzepten vorhanden. Die Diskussion über die wahren Determinanten der positiven Relation zwischen Produktions- und Produktivitätswachstum wurde durch die technical progress function um einen weiteren Aspekt bereichert. Eindeutige Rückschlüsse auf die Ursachen des Verdoorn-Zusammenhangs sind weiterhin nicht möglich.

\subsubsection{Der Verdoorn-Zusammenhang und Zeitreihenuntersuchungen}

Zur ökonometrischen Ermittlung des Verdoorn-Zusammenhangs stehen zwei Möglichkeiten zur Verfügung. Zum einen kann mit Querschnittsanalysen auf Länder- oder Industriebasis die Beziehung zwischen den Wachstumsraten der Arbeitsproduktivität und des Outputs bzw. der Beschäftigung und des Outputs bestimmt werden. Zum anderen ist es möglich, mit Hilfe von Zeitreihenuntersuchungen für ein Land oder für eine Industrie spezifisch die jeweiligen Regressionsgleichungen zu berechnen. Der Großteil der empirischen Untersuchungen zum Verdoorn-Zusammenhang konzentriert sich auf die erste Vorgehensweise. Für einen bestimmten Zeitraum werden z.B. für jedes in der Stichprobe enthaltene Land die durchschnittlichen Wachstumsraten der jeweiligen Variablen ermittelt und die Regressionsrechnung durchgeführt. Wählt man bei dieser Methode für den Anfang und das Ende des Untersuchungszeitraums jeweils Jahre, in denen gleiche konjunkturelle Situationen vorliegen, also z.B. Hochpunkte des Konjunkturzyklusses, dann werden durch die Durchschnittsbildung Effekte, die durch die unterschiedliche Auslastung der Kapazitäten auftreten, herausgerechnet.

Zeitreihenanalysen dagegen müssen dieses Problem berücksichtigen. Unabhängig von der Wahl des betrachteten Zeitraums kann das Ergebnis für den Verdoorn-Zusammenhang durch kurzfristige Phänomene verzerrt werden. Das Hineinwachsen in die Vollauslastung der vorhandenen Kapazitäten kann ein Wachstum der Arbeitsproduktivität erzeugen, das kurzfristiger Natur ist, jedoch keine Rückschlüsse auf Skaleneffekte erlaubt. Vor allem wenn die Arbeitsproduktivität durch den Output pro Erwerbstätigen oder pro Beschäftigten gemessen 
wird, kommt es zu diesen Wirkungen. Reagieren Unternehmen bei sinkendem Output nicht sofort mit der Freisetzung von Arbeitskräften bzw. stellen sie bei steigender Produktion nicht sofort mehr Arbeitnehmer ein, d.h. kann von der Gültigkeit des Okunschen Gesetzes ausgegangen werden, entwickeln sich Arbeitsproduktivität und Produktion in dieselbe Richtung. Die Entwicklung der Produktivität hat in diesem Falle nichts mit den technischen Gegebenheiten, d.h. mit der Existenz von steigenden Skalenerträgen und/oder technischem Fortschritt, zu tun, sondern ist vielmehr eine statistische Schimäre, die durch das Verhalten der Unternehmen erzeugt wird. 123

Konsequenterweise müssen die Variablen für die Beschäftigung und, wenn es berücksichtigt wird, für das Kapital zur Vermeidung dieser Effekte korrigiert bzw. Indikatoren gewählt werden, die gegen konjunkturelle Einflüsse dieser Art immun sind. Aus diesem Grunde empfiehlt es sich, die Arbeitsproduktivität als Stunden- und nicht als Personenproduktivität zu messen. Hierüber werden auch die Effekte von Arbeitszeitverkürzungen auf die Produktivität berücksichtigt. Sinkt die Arbeitszeit pro Kopf ab, ist der betroffene Arbeitnehmer c.p. weniger produktiv, wenn dieser pro Zeiteinheit die gleiche Outputmenge erzeugt. Die Stundenproduktivität bleibt somit konstant, die Erwerbstätigenproduktivität entwickelt sich negativ. Soll dennoch das personenbezogene Konzept der Produktivitätsmessung Verwendung finden, kann die Arbeitszeitentwicklung als zusätzliche Variable in eine erweiterte Verdoorn-Gleichung übernommen werden. 124

Popularität hat der auf Zeitreihen basierende Verdoorn-Zusammenhang im Rahmen der Diskussion über die massiven Beschäftigungsprobleme der neunziger Jahre erhalten. Die Wachstumsrate der Beschäftigung ergibt sich, unabhängig davon, ob sie in Stunden oder Erwerbstätigenzahl gemessen wird, aus der Differenz zwischen der Wachstumsrate der Arbeitsproduktivität und des Outputs:

$$
1=y-p
$$

Für den Fall, daß y=p verändert sich die Beschäftigungsmenge innerhalb der Volkswirtschaft nicht. Der einfache Verdoorn-Zusammenhang bietet die Möglichkeit, eine Aussage über die Wachstumsrate des Outputs zu treffen, die notwendig ist, um zumindest ein konstantes Beschäftigungsvolumen zu erreichen. Gemeinhin wird diese Wachstumsrate als Beschäftigungsschwelle bezeichnet. ${ }^{125}$ Sie bestimmt sich durch das Einsetzen von $\mathrm{y}=\mathrm{p}$ in die Gleichung des Verdoorn-Zusammenhangs:

123Vgl. zum Zusammenhang zwischen dem Verdoorn-Zusammenhang und Okuns Gesetz Erber (1994).

124 Vgl. hierzu z.B. Chatterji/Wickens (1981, 1982 und 1983), Kaldor (1981b), Pusse (1990), Schnur (1990) und Hof (1994).

125 Vgl. z.B. Buttler/Hof (1977), Klauder (1990), Hof (1991,1994), Erber (1994). 


$$
y=a+b y \Leftrightarrow y^{B S}=\frac{a}{1-b}
$$

Je höher der autonome Produktivitätsfortschritt und der Verdoorn-Koeffizient sind, desto höher wird die Beschäftigungsschwelle sein. Die Beschäftigung kann nur steigen, wenn die Volkswirtschaft ein Wachstum realisiert, das über dem kritischen Wert ( $\left.y^{\mathrm{BS}}\right)$ liegt. Geringere Wachstumsraten führen aufgrund der stärker steigenden Produktivität zur Freisetzung von Arbeitskräften.

\section{Abb. 4.2: Die Bestimmung der Beschäftigungsschwelle}

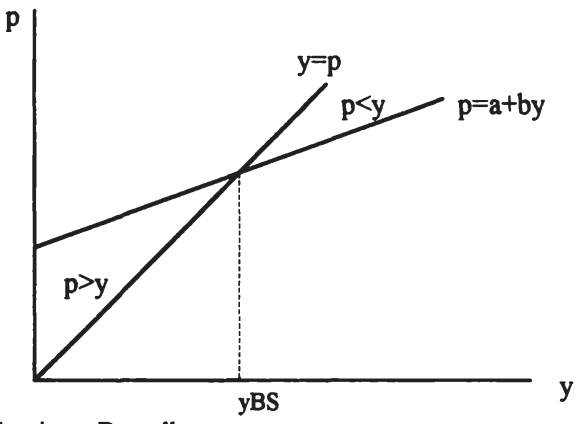

Quelle: eigene Darstellung.

Das rechnerische Ergebnis für den Wert der Beschäftigungsschwelle zeigt den Einfluß des Absolutwertes der Regressionsgleichung und des Verdoorn-Koeffizienten. Je höher diese sind, desto höher ist die Wachstumsrate, die für die Konstanz der Beschäftigung erreicht werden muß. Empirische Untersuchungen für die Bundesrepublik Deutschland zeigen einen Rückgang der Beschäftigungsschwelle in der Zeit zwischen 1960 bis 1992.126 Verantwortlich waren hierfür der zurückgehende Absolutterm der Regressionsgleichung und je nach Teilperiode auch ein sinkender Verdoorn-Koeffizient. Abnehmende Werte für (a) werden gemeinhin als Rückgang des exogenen technischen Fortschritts interpretiert. ${ }^{127}$ Die Überlegungen zur theoretischen Begründung des Verdoorn-Zusammenhangs durch eine Cobb-Douglas-Produktionsfunktion haben gezeigt, daß dieser Teil des einfachen Verdoorn-Zusammenhangs sowohl von der Rate des technischen Fortschritts als auch von der Wachstumsrate des Kapitalstocks beeinflußt wird. Aus der Veränderung des sich aus der Regressionsrechnung ergebenden Absinkens des nicht durch das Produktionswachstum induzierten Produktivitätswachstums lassen sich keine 
eindeutigen Rückschlüsse auf die Rate des exogenen technischen Fortschritts in den jeweiligen Zeitabschnitten ziehen.

Tab. 4.8: Die Entwicklung der Beschäftigungsschwelle in der Bundesrepublik Deutschland

\begin{tabular}{|c|c|c|c|}
\hline Zeitraum & Regressionsgleichung & $\mathbf{y}^{\mathbf{B S}}$ & $\mathbf{R}^{\mathbf{2}}$ \\
\hline $1960-1973$ & $\mathrm{p}=1,86+0,50 \mathrm{y}$ & 3,7 & 0,78 \\
\hline $1973-1980$ & $\mathrm{p}=0,89+0,60 \mathrm{y}$ & 2,2 & 0,75 \\
\hline $1980-1989$ & $\mathrm{p}=0,27+0,62 \mathrm{y}$ & 0,7 & 0,71 \\
\hline $1980-1992$ & $\mathrm{p}=0,40+0,41 \mathrm{y}$ & 0,7 & 0,70 \\
\hline $1973-1992$ & $\mathrm{p}=0,69+0,51 \mathrm{y}$ & 1,4 & 0,70 \\
\hline $1960-1992$ & $\mathrm{p}=0,78+0,62 \mathrm{y}$ & 2,1 & 0,79 \\
\hline
\end{tabular}

Quelle: Hof (1994, S. 135). Die Produktivităt wurde auf Erwerbstătigenbasis gemessen. Als Outputmaß wurde die Bruttowertschöpfung gewählt. Weitere Informationen über die Güte des statistischen Zusammenhangs werden leider nicht gemacht.

Erfolgt die Erweiterung durch eine Kapitalstockvariable ergeben sich vielfach negative Werte für den Absolutterm. ${ }^{128}$ Dies würde, wie oben gezeigt, aber bei Annahme einer Cobb-DouglasProduktionsfunktion entweder eine negative Produktionselastizität der Arbeit oder eine negative Rate des technischen Fortschritts bedeuten. Dieses Problem bleibt häufig unbeachtet, d.h. es wird zwar einerseits mit einer Cobb-Douglas-Produktionsfunktion als Basis für den Verdoom-Zusammenhang gearbeitet, die Konsequenzen aus den Schätzungen für die Parameter der Produktionsfunktion bleiben jedoch außen vor.

Die Verwendung des einfachen bzw. erweiterten Verdoorn-Zusammenhangs im Rahmen der Diskussion um die Beschäftigungsschwelle dient hauptsächlich als Hilfsmittel zur Ermittlung wichtiger Einflußgrößen auf die Entwicklung der Arbeitsproduktivität. Die theoretische Begründung des Zusammenhanges wird zwar jeweils kurz angesprochen, von vorrangigem Interesse scheint jedoch die Güte der ermittelten Regression zu sein. Die Wirtschaftstheorie liefert hierbei Hinweise auf mögliche Variablen, die bei der empirischen Analyse der Produktivitätsentwicklung zu berücksichtigen sind, wie z.B. die zunehmende Substitution von Arbeit durch Kapital, die Investitionsquote, Produktionswachstum, autonomer technischer Fortschritt etc. Wie diese Faktoren letztendlich produktionstechnisch miteinander verbunden sind, scheint zweitrangig und wird nicht immer erläutert. Es kann jede lineare Multiple-Regressionsgleichung in diesem Zusammenhang als Ausdruck einer Cobb-Douglas-Produktionsfunktion 
verstanden werden. 129 Inwieweit sich hieraus zwingend nachvollziehbare ökonomische Zusammenhänge ergeben, bleibt offen. $\mathrm{Da}$ die Untersuchungen zur Beschäftigungsschwelle primär der Ermittlung der Höhe und der Bestimmung ihrer Determinanten sowie der Prognose der Entwicklung der Arbeitsproduktivität dienen, stehen solche Aspekte nicht zwangsläufig im Vordergrund des Interesses.

Die Renaissance des Verdoorn-Zusammenhangs als Prognoseinstrument stellt in gewissem Sinne eine Rückkehr zum Ausgangspunkt der Debatte um diese Beziehung zwischen dem Wachstum der Produktion und der Produktivität dar. Hatte Verdoorn (1949) noch die Lösung der Frage nach der Begrenzung der Produktionsmöglichkeiten durch das Arbeitsangebot im Sinn, hat sich in den neunziger Jahren gerade die umgekehrte Fragestellung ergeben. Wieviel Wachstum ist notwendig, damit die Beschäftigung zumindest konstant bleiben kann? Die Diskussion um die Beschäftigungsschwelle kann aus diesem Grunde als die Konsequenz der Debatten über die Freisetzungs- und Einsparungsberechnungen in den sechziger und siebziger Jahren verstanden werden. Mit Hilfe von Prognosen für das Produktivitäts- und das Produktionsniveau wurde ermittelt, wie groß das hypothetische Freisetzungs- und Einsparungspotential an Arbeitsplätzen durch die gestiegene Effizienz der Volkswirtschaft ist. Die Ergebnisse solcher Berechnungen lassen jedoch keine Aussage über die tatsächliche Entwicklung auf dem Arbeitsmarkt zu, sondern stellen nur denkbare Szenarien dar. Einer der Hauptkritikpunkte an diesen Kalkulationen richtet sich gegen die Annahme der Unabhängigkeit des Produktivitätswachstums vom Produktionswachstum. Liegt ein Zusammenhang dieser beiden Größen vor, muß dies bei der Prognose berücksichtigt werden. Falls der Verdoorn-Zusammenhang Gültigkeit hat, muß das Produktivitätswachstum um so höher angenommen werden, je höher das Produktionswachstum geschätzt wird. Wird Nullwachstum bei der Produktion unterstellt, kann auch nur der Produktivitätsfortschritt in Höhe des produktionsunabhängigen, autonomen Wachstums der Arbeitsproduktivität, d.h. des Werts des Achsenabschnitts der Gleichung für den Verdoorn-Zusammenhang, angesetzt werden. 130 Die Bestimmung der Beschäftigungsschwelle berücksichtigt nun die Interdependenzen zwischen der Entwicklung der Arbeitsproduktivität und des Produktionswachstums. Prognosen, die auf der Basis dieses Konzepts erstellt werden, ermöglichen bessere Vorhersagen als die traditionellen Freisetzungs- und Einsparungsberechnungen über die künftige Beschäftigungsentwicklung. Dies gilt jedoch nur bei Abwesenheit von Strukturbrüchen, d.h. löst sich der Verdoorn-Zusammenhang auf bzw.

129 Genauer gesagt kann die Regressionsgleichung als Ausdruck einer Pro-Kopf-(oder Pro-Stunde)CobbDouglas-Produktionsfunktion interpretiert werden, wobei die Regressionskoeffizienten nicht zwangsläufig den Produktionselastizităten entsprechen müssen.

130 Hohen Bekanntheitsgrad erzielten die Berechnungen des ifo-Instituts Ende der sechziger Jahre, die zum einen Freisetzungs- und Einsparungspotentiale rulckwirkend für die Zeit von 1950 bis 1968 ermittelten, zum anderen Prognosen fur die den Zeitraum 1968-1980 versuchten [vgl. Uhlmann/Huber (1971) sowie grundlegend Kuhlo (1965)]. Die Veroffentlichung der Ergebnisse fuhrte zu kontroversen Diskussionen und Fehlinterpretationen der hypothetischen Werte [vgl. hierzu z.B. Kluge (1968), Kühlewind/Schnur (1970), Klauder (1971) und Uhlmann (1978)]. Zur Notwendigkeit der Berücksichtigung des VerdoornZusammenhangs bei diesen Berechnungen siehe z.B. Kalmbach (1983) und Uhlmann (1987). 
verändern sich die Regressionskoeffizienten, werden auf Vergangenheitswerten basierende Prognosen nicht erfolgreich sein.

\subsubsection{Weitere Ergebnisse für den Verdoorn-Zusammenhang}

Im Laufe der Diskussion wurden verschiedene Beispiele für die empirische Ermittlung des Verdoorn-Zusammenhangs vorgestellt. An dieser Stelle werden die Ergebnisse zwei weiterer Berechnungen vorgestellt werden, die beide eine Auflösung des Verdoorn-Zusammenhangs in der Zeit nach 1973 attestieren.

Boyer/Petit (1991b) ermittelten eine Regression zwischen den Wachstumsraten der Arbeitsproduktivität und des Produktionswachstums im Verarbeitenden Gewerbe von zwölf OECD-Ländern. ${ }^{131}$ Die Untersuchung erstreckte sich auf den Zeitraum 1950 bis 1988 . Für das gesamte Intervall konnte die Gültigkeit des Verdoorn-Zusammenhangs nachgewiesen werden. Die Autoren verwendeten dabei die Daten für acht Konjunkturzyklen in den betrachteten Ländern. Darüber hinaus wurden drei Intervalle gebildet: 1950-1973, 1973-1988 und 19791988. Der Verdoorn-Zusammenhang scheint sich in diesem Zeitraum aufzulösen. Sowohl der Verdoorn-Koeffizient als auch das Bestimmtheitsmaß waren rückläufig (vgl. Tabelle 4.9).

Tab. 4.9: Der Verdoorn-Zusammenhang im Verarbeitenden Gewerbe in zwölf OECD-Ländern 1950-1988

\begin{tabular}{|c|c|c|c|c|c|}
\hline Zeitraum & Absolutterm & $\begin{array}{c}\text { Verdoorn- } \\
\text { Koeffizient }\end{array}$ & $\mathbf{R}^{\mathbf{2}}$ & Beobachtungen & SER \\
\hline $1950-1988$ & $\begin{array}{c}2,4 \\
(9,7)\end{array}$ & $\begin{array}{c}0,5 \\
(11,8)\end{array}$ & 0,59 & 96 & 1,5 \\
\hline $1950-1973$ & $\begin{array}{c}2,1 \\
(5,1)\end{array}$ & $\begin{array}{c}0,54 \\
(9,6)\end{array}$ & 0,61 & 60 & 1,5 \\
\hline $1973-1988$ & 2,8 & $\begin{array}{c}0,35 \\
(2,8)\end{array}$ & 0,16 & 36 & 1,5 \\
\hline $1979-1988$ & $\begin{array}{c}2,7 \\
(5,9)\end{array}$ & $\begin{array}{c}0,29 \\
(1,9)\end{array}$ & 0,10 & 24 & 1,5 \\
\hline
\end{tabular}

Quelle: Boyer/Petit (1991b, S. 51). Die Werte in der Klammer sind t-Werte.

Von Michl (1985) wurden ebenfalls für das Verarbeitende Gewerbe der gleichen zwölf Länder Berechnungen durchgeführt. Hier wurden der Zeitraum 1950-1980 in sechs Konjunkturzyklen

131 Die zwölf Lănder waren Belgien, Dănemark, Frankreich, Großbritannien, Italien, Japan, Kanada, Niederlande, Norwegen, Schweden, USA und Westdeutschland. 
unterteilt. Der Verdoorn-Zusammenhang ist in allen Phasen nachweisbar. Der VerdoornKoeffizient ist immer statistisch signifikant von Null verschieden.

Tab. 4.10: Der Verdoorn-Zusammenhang im Verarbeitenden Gewerbe in zwölf OECD-Ländern 1950-1980

\begin{tabular}{|c|c|c|c|c|c|}
\hline Zeitraum & Absolutterm & $\begin{array}{c}\text { Verdoorn- } \\
\text { Koeffizient }\end{array}$ & $\mathbf{R}^{\mathbf{2}}$ & Beobachtungen & SER \\
\hline $1951-1956 / 57$ & $\begin{array}{c}0,9734 \\
(2,501)\end{array}$ & $\begin{array}{c}0,490 \\
(8,880)\end{array}$ & 0,897 & 11 & 0,699 \\
\hline $1955-1961 / 62$ & $\begin{array}{c}1,688 \\
(4,621)\end{array}$ & $\begin{array}{c}0,513 \\
(9,799)\end{array}$ & 0,923 & 10 & 0,637 \\
\hline $1959-1965 / 66$ & $\begin{array}{c}1,228 \\
(1,095)\end{array}$ & $\begin{array}{c}0,626 \\
(3,674)\end{array}$ & 0,574 & 12 & 1,058 \\
\hline $1965-1970$ & $\begin{array}{c}2,171 \\
(2,499)\end{array}$ & $\begin{array}{c}0,680 \\
(4,993)\end{array}$ & 0,713 & 12 & 1,207 \\
\hline $1970-1973 / 74$ & $\begin{array}{c}3,252 \\
(2,787)\end{array}$ & $\begin{array}{c}0,578 \\
(2,616)\end{array}$ & 0,406 & 12 & 1,375 \\
\hline $1973-1979 / 80$ & $\begin{array}{c}2,754 \\
(4,782)\end{array}$ & $\begin{array}{c}0,579 \\
(2,465)\end{array}$ & 0,336 & 14 & 1,557 \\
\hline $1951-1980$ & $\begin{array}{c}2,568 \\
(8,012)\end{array}$ & $\begin{array}{c}0,479 \\
(8,796)\end{array}$ & 0,528 & 71 & 1,448 \\
\hline
\end{tabular}

Quelle: Michl (1985, S. 483). Werte in der Klammer sind t-Werte.

Die Ergebnisse dieser Studie legen den Schluß nahe, daß sich der Verdoorn-Zusammenhang im betrachteten Zeitraum nicht aufgelöst hat. Stellt man beide Studien gegenüber, scheint sich insbesondere in der Zeit nach 1980 der Verdoorn-Zusammenhang abzuschwächen. Eindeutige Aussagen über die Entwicklung der Beziehung zwischen Produktivitäts- und Produktionswachstum sind jedoch nicht möglich. Es kann nur festgestellt werden, daß sich bei Querschnittsanalysen im allgemeinen eine Zunahme des Absolutterms und ein mehr oder minder starker Rückgang des Verdoorn-Koeffizienten zeigt. Aus diesem Ergebnis kann auf eine gestiegene Bedeutung der autonomen Fortschrittskomponente gegenüber der induzierten Komponente geschlossen werden. Gegen eine solche Interpretation sprechen aber die Resultate der Längsschnittanalyse für die Bundesrepublik Deutschland von Hof (1994). Man kann somit von einem Verdoorn-Puzzle sprechen. 132

132 In Untersuchungen von Vacagio (1975), der eine Querschnittsanalyse für den Zeitraum 1950-1969 für eine Stichprobe aus 18 Industrielăndern durchführte, und von Rayment (1981), der sich bei Lăngs- und Querschnittsanalysen für neun Industrieländer für den Zeitraum 1950-1970 auf den industriellen Sektor konzentrierte, ergab sich eine Bestătigung des Verdoorn-Zusammenhangs. Gegen Ende der Betrachtungszeitrăume schwächte sich der Zusammenhang jedoch etwas ab. 
Insgesamt läßt sich festhalten, daß die Frage nach der Auflösung des VerdoornZusammenhangs nicht ohne weiteres geklärt werden kann. Nur für die Zeit nach 1980 kann von einer Abschwächung bei internationalen Vergleichen ausgegangen werden. Die Resultate solcher Berechnungen sind jedoch auch das Ergebnis der Entwicklung einzelner Volkswirtschaften. So kann sich bei Querschnittsanalysen eine Auflösung des Zusammenhangs anbieten, während innerhalb eines Landes genau die umgekehrte Entwicklung stattfindet. Welche Vorgehensweise gewählt wird, hängt vom Untersuchungszweck ab. Die Suche nach generell gültigen Zusammenhängen legt die Querschnittsanalyse nahe, die Untersuchung einer einzelnen Volkswirtschaft dagegen die Längsschnittuntersuchung.

\subsubsection{Verdoorn-Zusammenhang - was bleibt?}

Die Ausführungen zum Verdoorn-Zusammenhang haben gezeigt, daß zwar im allgemeinen eine Beziehung zwischen dem Produktions- und dem Produktivitätswachstum bestehen kann, diese aber nicht in dem Sinne stabil ist, daß sie immer in gleicher Höhe vorliegt. Rückschlüsse auf die zugrundeliegende Produktionsverhältnisse sind nicht möglich. Nimmt man z.B. an, daß eine Cobb-Douglas-Produktionsfunktion die richtige Spezifikation des Verdoorn-Zusammenhangs darstellt, kann nicht eindeutig auf das Niveau der Skalenerträge dieser Produktionsfunktion geschlossen werden. Je nachdem, welche Gleichung zum Test des Verdoorn-Zusammenhangs verwendet wird, gibt der Regressionskoeffizient fur das Outputwachstum nur bedingt Auskunft über die Art der Skalenerträge. Auch die Erweiterung um eine Komponente des Kapitalstockwachstums kann hier nicht weiterhelfen. Darüber hinaus entstehen zusätzliche Probleme bei der Wahl des richtigen Indikators für diese Kapitalkomponente. Die Untersuchung der in Frage kommenden Variablen ergab, daß in Abhängigkeit von den gewählten Variablen einschränkende Annahmen getroffen werden müssen. Prinzipiell sprechen gegen diese Art der Fundierung des Verdoorn-Zusammenhangs der Hin- und Beweis von Verdoorn (1980) selbst, daß die Stabilität der Beziehung zwischen Produktions- und Produktivitätswachstum für den Fall einer Cobb-Douglas-Produktionsfunktion nur im Steady State möglich ist. In allen anderen Situationen kann kein stabiler Zusammenhang erwartet werden. Der zweite prinzipielle Einwand gegen eine Cobb-Douglas-Produktionsfunktion ergibt sich aus dem Unterschied zwischen der statischen und der dynamischen Version des Verdoorn-Zusammenhangs. Die Ergebnisse fielen für beide Fälle sehr unterschiedlich aus. Ein Hauptgrund für dieses Resultat kann in der Fehlspezifikation des Verdoorn-Zusammenhangs durch eine Cobb-Douglas-Produktionsfunktion liegen. Vergleichbar mit der Überlegung, inwieweit aus einer technical progress function auf eine Cobb-Douglas-Produktionsfunktion geschlossen werden kann, ist auch hier notwendig, Annahmen über die Integrationskonstante zu treffen. Die Anfangsausstattungen der Ökonomie mit Produktionsfaktoren spielen deshalb eine entscheidende Rolle. 
Aufgrund dieser Überlegungen wurde die mögliche Begründung des VerdoornZusammenhangs durch andere Produktionsstrukturen wie z.B. einer Lernkurve oder einer technical progress function diskutiert. Relativierend muß angemerkt werden, daß eine Lernkurve die Probleme zwischen statischer und dynamischer Schätzung nicht lösen kann und zusätzlich Steady State-Annahmen zur Ableitung einer dynamischen Verdoorn-Gleichung benötigt. Die Verwendung einer technical progress function wird mit Sicherheit den Vorstellungen von Kaldor über die Ursachen des Verdoorn-Zusammenhangs gerecht. Um einen Verdoorn-Zusammenhang eindeutig aus der technical progress function ableiten zu können, muß diese jedoch linear sein. Ansonsten sind sehr einschränkende Annahmen über die technical progress function selbst und die Funktion der Wachstumsrate der Kapitalintensität notwendig, damit beide Konzepte zusammenpassen.

Eine Anwendung des Verdoorn-Zusammenhangs in Längsschnittanalysen kann zwar Auskunft über die längerfristige Beziehung zwischen Produktions- und Produktivitätswachstum geben, jedoch sieht sich diese Form der Überprüfung dem Problem konjunktureller Überlagerungen gegenüber. Zyklische Schwankungen der Arbeitsproduktivität, die das Ergebnis der Reaktionen der Unternehmen auf die kurzfristige Nachfrageentwicklung sind, können die Resultate für den Verdoorn-Zusammenhang verzerren. Ein Ausweg aus diesem Dilemma besteht in der Betrachtung nur kompletter Konjunkturzyklen, so daß die kurzfristigen Schwankungen sich gegenseitig aufheben. Dies erfordert die Annahme gleichen Verhaltens der Unternehmen in Auf- und in Abschwungsphasen bzgl. der Beschäftigung. Die Forderung nach Längsschnittanalysen, die bei der Kritik zur Neuen Wachstumstheorie am Ende von Kapitel drei angesprochen wurde, steht vor demselben Problem. Schätzungen über einen längeren Zeitraum sind nicht nur Ausdruck des langfristigen Trends der Entwicklung, sondern können auch das Ergebnis konjunktureller Schwankungen sein. Sowohl die Berechnungen zum Verdoom-Zusammenhang als auch andere langfristige empirische Untersuchungen müssen dies berücksichtigen. Darüber hinaus stellt sich bei solchen Schätzungen, die vielfach zu Prognosezwecken verwendet werden, das Problem von Strukturbrüchen.

Der von Kaldor so stark betonte Verdoorn-Zusammenhang ist somit weder ein eindeutiger Indikator für das Vorliegen steigender Skalenerträge innerhalb einer Cobb-DouglasProduktionsfunktion noch läßt er Rückschlüsse darauf zu, ob überhaupt etwas für das Vorliegen einer Produktionsstruktur im Sinne einer Cobb-Douglas-Funktion spricht. Auch eindeutige Rückschlüsse auf die Existenz einer kaldorianischen technical progress function bzw. einer Lernkurve sind nicht möglich. Darüber hinaus ergeben sich bei der empirischen Überprüfung sehr verschiedene Resultate, so daß auch nicht eindeutig auf die Existenz des Zusammenhangs geschlossen werden kann. Dies impliziert jedoch nicht die Ablehnung des VerdoornZusammenhangs per se. Wenn innerhalb einer Volkswirtschaft bzw. eines Sektors eine solche Beziehung zwischen Produktions- und Produktivitätswachstum nachgewiesen wird, kann die Frage gestellt werden, welche möglichen Konsequenzen sich für die Volkswirtschaft daraus 
ergeben. Ein solches Beispiel wurde verbal von Kaldor selbst in einem Aufsatz von (1970a) gegeben und später von Dixon/Thirlwall (1975) und McCombie/Thirlwall (1994) sowie von Boyer/Petit (1989, 1991a, b) wieder aufgegriffen. Die sich anschließenden Ausführungen werden weitere Hinweise auf mögliche Gründe für die uneinheitlichen Ergebnisse für den Verdoorn-Zusammenhang geben.

\subsection{Kumulative Verursachung und Wachstum}

\subsubsection{Grundüberlegungen}

Bei der Besprechung der Ideen von Kaldor zur Erklärung der im internationalen Vergleich niedrigen Wachstumsraten Großbritanniens nach dem Zweiten Weltkrieg wurden mehrere Elemente des kumulativen Prozesses deutlich. Eine zentrale Rolle spielte der VerdoornZusammenhang. Wenn dieser innerhalb einer Volkswirtschaft vorliegt, und dies unabhängig von der Produktionsstruktur darauf hindeutet, daß steigende Skalenerträge existieren, wird diese Volkswirtschaft durch die Konzentration auf diesen Sektor dauerhaftes Produktivitätswachstum erreichen. Kann dagegen eine Volkswirtschaft einen solchen Sektor nicht aufweisen bzw. unterliegen die Produktionstätigkeiten konstanten bzw. abnehmenden Skalenerträgen, ist ihr dieser Weg zu mehr Wohlstand verschlossen.

Ein zweites Element stellte ein ausreichend großes Arbeitsangebot dar, das die Arbeitskräftenachfrage des industriellen Sektors bedienen kann. Verringert sich der Strom der Arbeitskräfte aus unproduktiven Verwendungen, d.h. aus dem primären Sektor, wird die Realisierung der Produktivitätsfortschritte nicht mehr gewährleistet sein. Obwohl Kaldor die Bedeutung des Arbeitsangebots relativierte, kann nicht bestritten werden, daß solche Beschränkungen auftreten können. Die Anwerbung von ausländischen Arbeitnehmern Anfang der sechziger Jahre durch Westdeutschland ist ein historisches Beispiel für diese Überlegungen. Die Schließung der Zonengrenze durch die DDR verringerte den Zustrom an Arbeitskräften, der bis zu diesem Zeitpunkt die westdeutschen Unternehmen vor allzu stark steigenden Arbeitskosten verschont hatte. In den neunziger Jahren unseres Jahrhunderts ist jedoch die Annahme einer Beschränkung der Produktionsmöglichkeiten durch ein zu geringes Arbeitskräfteangebot allenfalls auf sektoraler Ebene aufgrund von Mismatch-Phänomenen vorstellbar.

Ein dritter Faktor, der in Kaldors Antrittsvorlesung nicht explizit angesprochen wird, jedoch für den Prozeß der kumulativen Verursachung relevant ist, ist die Rückwirkung der Produktivitätseffekte auf die Nachfrageseite. Young (1928) sah eine Möglichkeit in Marshalls reziproken Nachfragekurven, d.h. die Erhöhung des Angebots eines Gutes erhöht auch die Nachfrage nach anderen Gütern. Diese Überlegung sichert jedoch nicht automatisch einen kumulativen Prozeß. Die Ausdehnung der Nachfrage nach einem Gut, bedeutet nämlich u.U. 
auch die Reduzierung der Nachfrage nach einem anderen, so daß nur in Abhängigkeit der jeweiligen Nachfrageelastizitäten auf einen Anstieg der Gesamtnachfrage geschlossen werden kann. Für einen kumulativen Prozeß ist aber eine Steigerung der Gesamtnachfrage notwendig. ${ }^{133}$

Eine Möglichkeit besteht in induzierten Investitionen. Hierbei sind zwei Formen denkbar. Zum einen kann eine Veränderung der Nachfragebedingungen zu Reaktionen bei den Lagerbeständen führen. Während in einer Welt Walrasscher Prägung keinerlei Unterschiede zwischen Produktion und Nachfrage auftreten können, ist realiter ein solcher Ausgleich nie zu erwarten. Vielmehr sind Pufferbestände notwendig, um die entsprechenden Ungleichgewichte auf den Gütermärkten auszugleichen. Möglicher Erfüllungsgehilfe kann hier der Handel sein. Steigt z.B. die Produktion von Gütern an, und der Handel hat die Erwartung stabiler Preise, so wird dieser den entsprechenden Produktionszuwachs aufkaufen. Diese Investition in Lagerbestände wiederum schaff Einkommen, das weitere Nachfrage induziert. Auf diese Art und Weise können nachfrageinduzierte Produktivitätseffekte wiederum zu steigender Nachfrage führen. Zum anderen können Investitionen in Form von Erweiterungsinvestitionen in den Sektoren auftreten, in denen nach der Auffassung von Young aufgrund steigender Arbeitsteilung die Güternachfrage zunimmt. Stellen die Unternehmen fest, daß ihre Güterproduktion nicht ausreicht, die Nachfrage zu decken, und erwarten sie gleichzeitig ein Anhalten der Nachfrage, werden sie entsprechend dem Akzelerationsprinzip ihre Produktionskapazitäten ausdehnen und somit weitere Nachfrage schaffen. Die Schaffung einer Verbindung zwischen Produktivitäts- und Produktionswachstum ist deshalb von den Erwartungen der Akteure abhängig. Die Händler müssen stabile Preise erwarten, die Produzenten wiederum müssen sich an die Nachfrageentwicklungen anpassen und ihre Erwartungen verändern, d.h. dauerhaft mehr Nachfrage erwarten, wenn die Nachfrage sich erhöht hat. ${ }^{134}$

Das Verhalten der Unternehmen beinhaltet zusätzlich eine Lösung des zuvor angesprochenen Problems denkbarer Angebotsbeschränkungen aufgrund eines Arbeitskräftemangels. Erweiterungsinvestitionen zur Bedienung der erwarteten Nachfragesteigerungen implizieren auch eine Steigerung der Arbeitsproduktivität, wenn man das Konzept der technical progress function berücksichtigt. Durch diesen Prozeß sind Freisetzungen von Arbeitskräften möglich, die für andere Produktionszwecke eingesetzt werden können. Interessant ist, daß von Kaldor die Auflösung versteckter Arbeitslosigkeit im primären Sektor als Lieferant von Arbeitskrätten gesehen wird, er die Wirkungen des technischen Fortschritts innerhalb des industriellen Sektors jedoch vernachlässigt.

133 Vgl. Young (1928, S. 537f.) und Kaldor (1972, S. 386ff.). Der Zusammenhang zwischen der Steigerung der Arbeitsproduktivităt und Zunahmen der Güternachfrage wurde von Pasinetti (1981) als eine notwendige Bedingung für die Aufrechterhaltung der Vollbeschaftigung bei technischem Fortschritt angesehen. Neben seinen Freisetzungswirkungen kann technischer Fortschritt in Form von Produktinnovationen zu einer Erhöhung der Güternachfrage führen.

134 Vgl. Kaldor (1972, S. 387f.) und speziell zu den Erwartungen im Handel Kaldor (1939) sowie Hahn (1989, S. 50ff.). 
Die Rolle des Handels und der produzierenden Unternehmen zeigt die Relevanz der Nachfrageentwicklung auf induzierte Produktivitätseffekte für das Vorliegen eines kumulativen Prozesses, der auch als selbsttragendes Wachstum bezeichnet werden kann. Die Aufgabe der Schaffung von ausreichend Nachfrage durch Unternehmer, wenn man Produzenten und Händler zu einer Gruppe zusammenfaßt, ist hier genauso entscheidend wie das Verhalten der Unternehmer im Modell von Harrod. Die Erwartungsstruktur dieser Wirtschaftssubjekte determiniert den Weg der Volkswirtschaft. Beeinflußten bei Harrod die Erwartungen der Unternehmer die Stabilität des Systems, legen sie im vorliegenden Fall fest, ob es zu einem kumulativen Prozeß kommen kann oder nicht.

Nur am Rande taucht eine weitere Bedingung auf, die für kumulative Prozesse relevant ist. Die Finanzierung der relevanten Investitionen macht ein akkommodierendes Geldangebot notwendig. Das Bankensystem muß die Unternehmen mit ausreichenden Krediten versorgen, da ansonsten eine Kreditrationierung die Verbindung zwischen Produktivitäts- und Nachfrageentwicklung durchtrennen würde. Inwieweit die Ausdehnung und Reduzierung von Krediten den Investitionsprozeß anregen oder dämpfen kann, wurde schon von Wicksell (1898) in seinem Werk Geldzins und Güterpreise veranschaulicht, weshalb diese Bedingung von Kaldor wohl nur beiläufig erwähnt wurde. ${ }^{135}$

Wendet man die Ideen des kumulativen Prozesses auf offene Volkswirtschaften an, kann die Einführung von internationalem Güterhandel bzw. die sich daraus ergebende Spezialisierung zu divergierenden Entwicklungen in den Ländern führen. Stellt man sich zwei Volkswirtschaften vor, die beide im landwirtschaftlichen und im industriellen Sektor tätig sind, können die Folgen von Handelsbeziehungen unterschiedlich ausgerichtete Wachstumsprozesse sein. Weist ein Land komparative Kostenvorteile im industriellen Sektor auf, wird es die entsprechenden Güter kostengünstiger anbieten können als das andere Land. Die Nachfrage nach und die Produktion der Industriegüter wird sich entsprechend der klassischen Handelstheorie auf das Land konzentrieren, das billiger produzieren kann. Wenn nun die Herstellung im industriellen Sektor steigenden Skalenerträgen unterliegt, wird die Industrienation ihre Kostenvorteile weiter ausbauen können, während das Agrarland in den Bereich der sinkenden Skalenerträge gedrängt wird. Die Wachstumsraten der beiden Länder werden sich dauerhaft unterscheiden. Es kommt zur Divergenz der Entwicklung. Man könnte nun argumentieren, daß durch den Außenhandel der Bezug der Industriegüter für die Agrarnation günstiger wird und somit Ressourcen für die Produktion der landwirtschaftichen Güter freigesetzt werden, wodurch sich der Wohlstand des Landes erhöhen würde. Dem ist zwar zuzustimmen, die relative Position des Agrarlandes wird sich jedoch verschlechtern. Darüber hinaus ist eine vollständige Verdrängung der Agrarnation aus dem industriellen Bereich vorstellbar. ${ }^{136}$ 
Die Produktivitätsfortschritte, die sich aus der Realisierung von Skalenerträgen ergeben, reichen für sich genommen noch nicht aus, um dauerhafte Unterschiede bei den Wachstumsraten zu erzeugen. Die tatsächlichen Kosten der Produktion von Gütern und damit ihre internationale Wettbewerbsfähigkeit werden maßgeblich davon bestimmt, wie sich die Lohnstruktur eines Landes entwickelt. Führt nun das Wachstum der Produktivität zu überproportionalen Lohnsteigerungen, werden sich die Lohnstückkosten, die efficiency wages im keynesianischen Sinne erhöhen. Nur wenn es gelingt, diese konstant zu halten bzw. zu senken, wird der Vorteil der steigenden Skalenerträge sich vollständig in der relativen Position des Landes niederschlagen. Diese Lohnkomponente bei der Wirkung ist ein Grund für die Konzentration von Kaldor auf regionale Entwicklungen, um die Wirkungen des Verdoorn-Zusammenhangs zu zeigen. In den Regionen eines Landes werden sich die Löhne in etwa konform entwickeln, was im internationalen Vergleich nicht der Fall ist. Innerhalb eines Landes kann auch die räumliche Mobilität der Arbeit den Lohndruck reduzieren, um steigenden Lohnstückkosten entgegenzuwirken. Volkswirtschaften wiederum haben die Möglichkeit der Protektion bzw. der Abwertung. ${ }^{137}$ Ein Blick auf die Situation der neuen Bundesländer macht diese Überlegungen recht anschaulich. Durch die Investitionen entstehen zwar zumindest lokal große Produktivitätsfortschritte, die jedoch durch die steigenden Löhne aufgefressen werden. Die Preiswettbewerbsfahigkeit der ostdeutschen Industrie ist aus diesem Grunde niedrig. Die Abwertungsstrategie, wie sie z.B. von anderen Ländern des ehemaligen Comecon-Blocks angewandt worden ist, blieb den neuen Bundesländern verschlossen.

Die skizzierte Entwicklung bei der Spezialisierung ist abhängig von der Existenz des Verdoorn-Zusammenhangs. Löst sich dieser auf, werden sich auch mögliche Produktivitätsvorsprünge nicht mehr einstellen. Recht deutlich wird dies in einer formalen Behandlung des Modells von Kaldor durch Dixon/Thirlwall (1975), die im nächsten Abschnitt zur Sprache kommt.

\subsubsection{Formalisierung}

Die oben skizzierten Überlegungen treten in einer formalen Struktur mehr hervor, in der die zentralen Elemente abgebildet werden. Dixon/Thirlwall haben in einem Aufsatz von 1975 den Fall regionaler Entwicklungen zu erfassen versucht. Durch die Konzentration auf Regionen werden Probleme wie Zahlungsbilanzungleichgewichte, die für ganze Volkswirtschaften bestehen, ausgeblendet. Zusätzlich kann die Nachfrage bei einer regionalen Betrachtung mehr als auf Länderebene als autonom angenommen werden. Aufgrund der Überlegungen zu Hicks' Supermultiplikator wird davon ausgegangen, daß nur Exportnachfrage vorliegt, die das

137 Vgl. Kaldor (1970a, S. 343f.). 
Wachstum der Investitionen und des Konsums bestimmt. ${ }^{138}$ Folgt man diesem Gedanken, kann das Wachstum des Outputs zu jedem Zeitpunkt als durch (4.85) bestimmt angesehen werden.

$$
y_{t}=\gamma x_{t}
$$

Hierbei steht $\left(\mathrm{y}_{\mathrm{t}}\right)$ für die Wachstumsrate des Outputs, $\left(\mathrm{x}_{t}\right)$ für die Wachstumsrate der Exporte und $(\gamma)$ für eine (konstante) Elastizität des Outputwachstums bzgl. der Wachstumsrate der Exporte. Die ausschließliche Abhängigkeit des Outputwachstums von der Entwicklung der Exporte macht es notwendig, die Exportnachfrage zu erklären. Hierfür wird angenommen, daß sich diese multiplikativ nach folgender Formel ergibt:

$$
X_{t}=\Pi_{\mathrm{dt}}^{\eta} \Pi_{\mathrm{ft}}^{\delta} z_{t}^{\varepsilon}
$$

wobei $\left(X_{t}\right)$ die Exportmenge in $(t),(\eta)$ die Preiselastizität der Exportnachfrage, $\left(\Pi_{d t}\right)$ den Inlandspreis in $(t),(\delta)$ die Kreuzpreiselastizität der Exportnachfrage, $\left(\Pi_{\mathrm{ft}}\right)$ den Weltmarktpreis in $(t),(\varepsilon)$ die Einkommenselastizität der Exportnachfrage und $\left(Z_{t}\right)$ das Einkommensniveau weltweit in ( $t$ ) angibt. In Wachstumsraten ausgedrückt folgt dann:

$$
\mathrm{x}_{\mathrm{t}}=\eta \pi_{\mathrm{dt}}+\delta \pi_{\mathrm{ft}}+\varepsilon \mathrm{z}_{\mathrm{t}}
$$

Für eine einzelne Region wird dabei die Entwicklung aller Größen bis auf die von $\left(\Pi_{\mathrm{dt}}\right)$ als gegeben angenommen. Der Inlandspreis unterliegt einer Aufschlagskalkulation. Das Preisniveau der im Inland produzierten Güter verändert sich folglich mit den Lohnstückkosten. Hiermit wird die oben angesprochene Rolle der Löhne für den Entwicklungsprozeß in das Modell integriert. Die Preisgleichung bestimmt sich nach

$$
\Pi_{\mathrm{dt}}=\frac{\mathrm{W}_{\mathrm{t}}}{\mathrm{P}_{\mathrm{t}}} \mathrm{T}_{\mathrm{t}}
$$

wobei $\left(W_{t}\right)$ für das Niveau der Geldlöhne in $(t),\left(P_{t}\right)$ für die Arbeitsproduktivität im Exportsektor in $(t)$ und $\left(T_{t}\right)$ für den Aufschlag auf die Stückkosten in $(t)$ steht. Auch hier kann eine Wachstumsratengleichung abgeleitet werden:

$$
\pi_{d t}=w_{t}-p_{t}+\tau_{t}
$$

138 Vgl. Kaldor (1970, S. 342), Dixon/Thirlwall (1975, S. 203) sowie Hicks (1950). 
Vervollständigt wird dieses Modell durch die Gleichung für den Verdoorn-Zusammenhang:

$$
\mathrm{p}_{\mathrm{t}}=\mathrm{p}_{\mathrm{a}}+\lambda \mathrm{y}_{\mathrm{t}}
$$

Dabei steht $\left(\mathrm{p}_{\mathrm{a}}\right)$ für das autonome Produktivitätswachstum und $(\lambda)$ für den Verdoorn-Koeffizienten. Kombiniert man alle Wachstumsratengleichungen, ergibt sich als Lösung für die Wachstumsrate des Outputs folgende Gleichung:139

$$
y_{t}=\gamma \frac{\eta\left(w_{t}-p_{t}+\tau_{t}\right)+\delta \pi_{f t}+\varepsilon\left(z_{t}\right)}{1+\gamma \eta \lambda}
$$

Die Wirkung des Verdoorn-Koeffizienten ist trotz des positiven Vorzeichens im Nenner dieser Gleichung positiv, da die Preiselastizität der Exportnachfrage ( $\eta$ ) kleiner Null ist. Gleichung (4.91) relativiert die Relevanz des Verdoorn-Zusammenhangs für die Erklärung regionaler Wachstumsdifferenzen. Unterschiede bei den Wachstumsraten zwischen Regionen sind nur möglich, falls sich der Verdoorn-Koeffizient regional unterscheidet oder anfängliche Unterschiede bei anderen Variablen bestehen, die durch die induzierten Produktivitätseffekte aufgrund von Nachfragesteigerungen verstärkt werden. Trotzdem zeigt das Vorliegen des Verdoorn-Zusammenhangs die von Kaldor diskutierten Wirkungen. Steigende Skalenerträge führen zu kumulativ zirkulären Prozessen, die das Auseinanderklaffen von Wachstumsraten bestimmen. Die Startvoraussetzungen einer Region oder, wenn für einen Moment von Zahlungsbilanzfragen abgesehen wird, eines Landes bestimmen in diesem Modell den Entwicklungsprozeß der jeweiligen Volkswirtschaft. 140

An dieser Stelle werden die oben ausgeführten Überlegungen zum Verdoorn-Zusammenhang wieder interessant. Der Verdoorn-Koeffizient konnte als Ergebnis sehr unterschiedlicher Annahmen über die Produktionsstruktur interpretiert werden. Abgesehen von den Einwänden, die in Abschnitt (4.4) zu den jeweiligen Spezifikationen vorgebracht wurden, würden in die Bestimmungsgleichung der Wachstumsrate (4.91) mehrere zusätzliche Parameter einfließen. Bei Verwendung einer Cobb-Douglas-Produktionsfunktion war der Verdoorn-Koeffizient durch die Produktionselastizität der Arbeit bestimmt, während der autonome Produktivitätsfortschritt durch das Kapitalwachstum und den exogenen technischen Fortschritt festgelegt waren. Die Substitution des Verdoorn-Koeffizienten und des autonomen Produktivitätsfort-

139 Vgl. Dixon/Thirlwall (1975, S. 205).

140 Dixon/Thirlwall (1975, S. 207f.) zeigen anhand einer Modifikation der Gleichung für die Exportnachfrage, in die ein Zeitlag von einer Periode eingefüht wird, welche Bedingungen für die Divergenz bei den Wachstumsraten erfullt sein müssen. Unter Annahme einer konstanten Exportquote und einer Preiselastizităt der Exportnachfrage kleiner Zwei sowie einem Verdoorn-Koeffizienten von ca. 0,5 deutet das Modell auf konstante Abstande zwischen den Wachstumsraten verschiedener Volkswirtschaften hin. Die Einbeziehung einer beschränkenden Wirkung der Zahlungsbilanz andert an den prinzipiellen Ergebnissen zur Rolle des Verdoorn-Zusammenhangs nichts entscheidendes. Vgl. McCombie/Thirlwall (1994, insbes. Kap. 7) sowie die dort angegebene Literatur. 
schritts impliziert, daß mehr Kapitalwachstum und/oder technischer Fortschritt sowie eine hohe Produktionselastizität der Arbeit zu höheren Produktivitätsfortschritten führen. Alle drei Größen sind in diesem Modell jedoch exogener Natur. Die Berücksichtigung des Kapitalstockwachstums könnte im gewissen Sinne als endogener Faktor verstanden werden. Je mehr die Wirtschaftssubjekte bereit sind zu investieren, um so mehr Wachstum wird die Volkswirtschaft erzielen. Das Modell läßt somit Einflüsse der Investitionsquote auf die Wachstumsrate auch für den Fall einer Cobb-Douglas-Produktionsfunktion mit konstanten Skalenerträgen zu. Problematisch ist jedoch, daß die Rate der Kapitalbildung nicht erklärt wird.

Sieht man wiederum die technical progress function als Basis des Verdoorn-Zusammenhangs an, kommen endogene Elemente des technischen Fortschritts in Gleichung (4.91) zum Tragen. ${ }^{141}$ Der Verdoorn-Koeffizient wird unter den oben gemachten Annahmen durch die Rate des nicht-gebundenen induzierten technischen Fortschritts, der Rate der wachstumsinduzierten Kapitalakkumulation und der Rate des kapitalgebundenen technischen Fortschritts bestimmt. In die autonome Komponente des Produktivitätswachstums fließen die autonome Rate des nicht-gebundenen technischen Fortschritts, die autonome Rate der Kapitalbildung und die Rate des kapitalgebundenen technischen Fortschritts ein. Im Unterschied zur Verwendung der Cobb-Douglas-Produktionsfunktion bestimmen hier zusätzlich induzierte Größen das Produktionswachstum. Erklärungsbedürftig bleiben aber auch bei dieser Vorgehensweise die exogenen Komponenten des technischen Fortschritts bzw. der Kapitalbildung. Festgehalten werden kann, daß sowohl die Verknüpfung des Verdoorn-Zusammenhangs mit einer CobbDouglas-Produktionsfunktion als auch mit einer technical progress function die Rolle des technischen Wissens und der Kapitalbildung für das Wachstum einer Region oder eines Landes verdeutlichen.

Das Ergebnis der Überlegungen von Kaldor entspricht dem des zweiten Modells von Lucas (1988), in dem die Anfangsausstattungen mit Humankapital festlegten, auf welche Güterproduktion sich eine Volkswirtschaft konzentriert. Ein anfänglicher Vorteil bei den Produktionsfaktoren determinierte die weitere Entwicklung. Auch im formalisierten KaldorModell werden Unterschiede bei der Startsituation verstärkt. Hat z.B. ein Land einen Vorsprung bei der Herstellung von Gütern mit hoher Preiselastizität, wird sich seine Wachstumsrate erhöhen, da produktivitätsinduzierte Preissenkungen Nachfragesteigerungen zur Folge haben werden. Der Verdoorn-Zusammenhang wird diese noch verstärken. Für andere Volkswirtschaften wird es immer schwieriger diesen Vorteil auszugleichen. Während im Modell von Lucas die Annahme über die Entwicklung des produktspezifischen Humankapitals für den Ausbau des Vorsprungs sorgte, erfült in Kaldors Überlegungen der VerdoornZusammenhang diese Aufgabe. Sowohl die Humankapitalbildung im Lucas-Modell als auch die nicht-spezifizierten steigenden Skalenerträge im Kaldor-Verdoorn-Fall sorgen für induzierte Produktivitätseffekte, die weitere Kostenvorteile bewirken.

141 Vgl. Dixon/Thirlwall (1975, S. 20f.). 
Trotz unterschiedlicher Spezifikation dieser Verstärkerfunktionen ist beiden Modellen die Erhöhung der Produktivität der Produktionsfaktoren gemein. Die Ausführungen von Lucas setzen dabei an den Produktionsfaktoren direkt an. Mehr Produktion eines Gutes führt über Lerneffekte zu einem höheren Bestand an Humankapital, das wiederum die Effizienz des Einsatzes der anderen Produktionsfaktoren verbessert. Weshalb die für die Lerneffekte notwendige Güterproduktion stattfindet, wird hierbei nicht spezifiziert. Im kaldorianischen Fall wird ebenfalls implizit eine Funktion unterstellt, die bei steigender Produktion zu einer höheren Produktivität des Faktoreinsatzes führt. Im Gegensatz zum Modell von Lucas wird hier die Art und Weise, wie es zu den Produktivitätssteigerungen kommt, nicht genau spezifiziert. Wie oben gezeigt, spielen auch in den Vorstellungen von Kaldor Lerneffekte eine zentrale Rolle. Der Schwerpunkt des Kaldor-Modells liegt bei der Frage, wie es dazu kommt, daß potentielle Skalenerträge ausgenutzt werden können. Die von Lucas als selbstverständlich angenommene Güterproduktion bzw. deren Steigerung im Laufe der Zeit wird von Kaldor als durch die Nachfrage bestimmt angesehen. M.a.W.: es muß ausreichend Güternachfrage vorliegen, damit Lerneffekte überhaupt möglich sind.

Beide Modelle generieren somit qualitativ dieselben Resultate. Der Weg dorthin ist bei beiden Autoren durch ihre prinzipielle Einstellung gegenüber den Determinanten des Wachstums bestimmt. So bietet jedes Modell allein nur einen begrenzten Ausschnitt der wachstumsrelevanten Variablen. Zusammengesehen zeigen sie, wie wichtig nachfrage- und angebotsseitige Rahmenbedingungen für endogene Wachstumsprozesse sind.

\subsubsection{Weitere Überlegungen zu den Bedingungen der kumulativen Verursachung}

\subsubsection{Grundsätzliches}

Die Ausfuhrungen Myrdals über die Situation der farbigen Bevölkerung in den USA, die der Veranschaulichung des Prinzips der kumulativen, genauer gesagt der zirkulären kumulativen, Verursachung dienten, machten deutlich, daß bei kumulativen Prozessen auf einen Impuls immer eine Reaktion einer anderen Variablen erfolgen muß, die den ursprünglichen Anstoß verstärkt. Übertragen auf ökonomische Modelle bedeutet dies, daß der Prozeß der kumulativen Verursachung sowohl die Abhängigkeit der Produktivitätsentwicklung von der Nachfrageentwicklung als auch eine umgekehrte Ursache-Wirkungs-Kette beinhalten muß. Innerhalb der Überlegungen von Kaldor erfüllte der Verdoorn-Zusammenhang die erste Aufgabe, während die Reaktionen der Händler und der Unternehmen bzw. die Reaktionen der Auslandsnachfrage aufgrund komparativer Kostenvorteile die zweite Beziehung darstellte. Fällt eine der beiden Wirkungsketten aus, ist das Prinzip der kumulativen Verursachung hinfällig. Gemeinhin wird in diesem Zusammenhang auch von Produktivitäts- und Nachfrageregimen gesprochen, die die 
wechselseitigen Beziehungen erfassen. Das Modell der kumulativen Verursachung könnte auf zwei Gleichungen reduziert werden, die diese Bedingungen erfassen. 142

$$
\begin{aligned}
& \mathrm{p}=\mathrm{f}(\mathrm{y}) \quad \text { mit } \mathrm{f}^{\prime}>0 \quad \text { Spezialfall: } \quad \mathrm{p}=\mathrm{a}+\text { by } \\
& \text { (4.93) } \quad y=g(p) \\
& \text { mit } g^{\prime}>0 \quad \text { Spezialfall: } \quad y=c+d p
\end{aligned}
$$

Die Gleichung (4.92) steht für das Produktivitäts-, Gleichung (4.93) für das Nachfrageregime. Abbildung (4.3) zeigt die graphische Umsetzung dieser Gleichungen.

Abb. 4.3: Produktivitäts- und Nachfragegleichung im Modell der kumulativen Verursachung

a) Stabiles Gleichgewicht

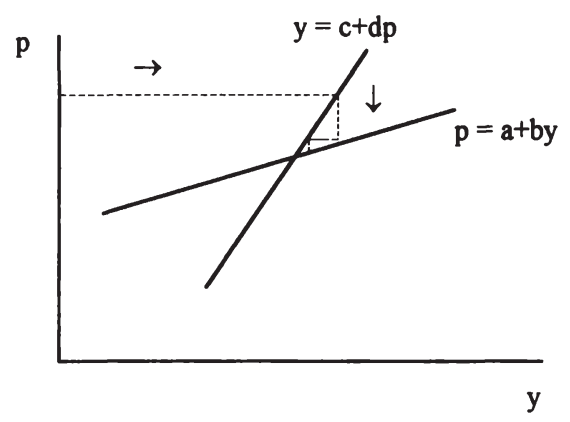

b) Instabiles Gleichgewicht

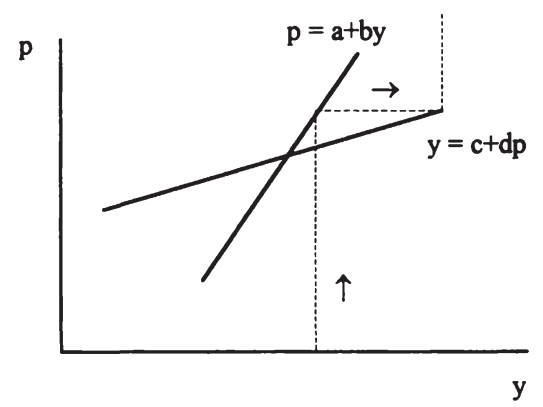

Quelle: Boyer/Petit (1989, S. 26).

142 Vgl. z.B. Boyer/Petit (1991, S. 490). 
Das Produktivitätsregime erfaßt die Wirkungen des Produktions- bzw. Nachfragewachstums auf das Produktivitätswachstum, während das Nachfrageregime die Effekte steigender Produktivität auf die Nachfrageentwicklung widerspiegelt. Der Verlauf beider Funktion determiniert den Wachstumsprozeß der Volkswirtschaft. Es sind sowohl Situationen mit konstanten als auch mit variablen Wachstumsraten des Outputs und der Produktivität möglich. In Schaubild (4.3a) wird sich z.B. unabhängig vom Startpunkt eine stabile Wachstumsrate einstellen, die sich im Schnittpunkt von (4.92) und (4.93) ergibt. Die Konstellation in Schaubild (4.3b) ist ein System mit ständig steigenden Wachstumsraten, wenn die Startsituation rechts vom Schnittpunkt der Geraden liegt, und fallenden Wachstumsraten, wenn die Startsituation links vom Schnittpunkt liegt. Diese Grafik verdeutlicht, daß das Vorliegen des VerdoornZusammenhangs nicht ausreicht für die Erklärung eines Wachstumsprozesses mit zunehmenden oder fallenden Wachstumsraten, sondern durchaus auch mit konstanten Wachstumsraten des Outputs einhergehen kann. Die Ausführungen Kaldors bedürfen deshalb einer genaueren Spezifikation, unter welchen Bedingungen es zu einem kumulativen Prozeß kommen kann, der steigende Wachstumsraten bedingt.

Die graphische Darstellung läßt noch einen weiteren Schluß zu. Instabile Situationen, d.h. dauerhaft zunehmende bzw. dauerhaft sinkende Wachstumsraten werden vorliegen, wenn die Steigung der Nachfragekurve größer als die des Verdoorn-Zusammenhangs ist, wenn man die Steigungen beider Kurven von der Abszisse aus betrachtet. Exakt ausgedrückt bedeutet dies, daß die Steigung der Umkehrfunktion der Nachfragekurve größer als diejenige des VerdoornZusammenhangs sein muß. Für stabile Gleichgewichtssituationen muß somit gelten: $g^{\prime}<\mathbf{f}^{\prime}$.

\subsubsection{Nachfrageinduziertes Produktivitätswachstum}

Der Verdoorn-Zusammenhang stellt bei dieser Überlegung das Kondensat aller relevanten Beziehungen dar, die eine positive Relation zwischen dem Wachstum der aggregierten Nachfrage und der Produktivitätsentwicklung bewirken können. Hierbei kann an verschiedene Aspekte gedacht werden, die zusammengenommen die empirisch ermittelte lineare Regressionsgleichung ergeben. Die von Kaldor angesprochene Tatsache, daß bei Existenz eines statistisch signifikant von Null verschiedenen Verdoorn-Koeffizienten jedes Prozent Produktionswachstum zu einem Anstieg der Wachstumsrate der Produktivität führt, muß dann nicht mehr automatisch ein Indiz für das Vorliegen einer Produktionsfunktion mit steigenden Skalenerträgen sein. Tatsächlich ist das nachfrageinduzierte Produktivitätswachstum u.U. auf andere Variablen zurückzuführen. 143

143 Vgl. für die möglichen Bestimmungsgründe Boyer/Petit (1989, S. 28ff. und 1991a, S. 493ff.) und Targetti (1992, S. 185ff.). 
Selbstverständlich werden auch statische steigende Skalenerträge aufgrund von Größenvorteilen eine Rolle spielen. Aber schon die Hinweise von Young (1928), Kaldor (1966) oder Arrow (1962) auf dynamische Skalenerträge, die durch Lerneffekte oder steigende Arbeitsteilung entstehen, erweitern das Bild. Auch das Konzept der technical progress function sowie Vintage-Überlegungen insgesamt können hier angeführt werden. Da Bruttoinvestitionen einen Teil der gesamtwirtschaftlichen Güternachfrage darstellen, werden ständig neue Technologien in den Produktionsprozeß eingebracht. Die Produktivität der Arbeitskräfte wird entsprechend steigen. Nachfrageinduziertes Produktivitätswachstum wird somit von der Diffusion des technischen Fortschritts in der Volkswirtschaft abhängen, d.h. Investitions- und Abschreibungsquote entscheiden mit über die Gültigkeit des Verdoorn-Zusammenhangs. Die Ausführungen in Abschnitt (4.4.1.2) haben die möglichen Probleme der Berücksichtigung einer Kapitalvariablen am Beispiel der Cobb-Douglas-Produktionsfunktion als Basis des Verdoorn-Zusammenhangs gezeigt. Darüber hinaus kann auch nachfrageinduzierter technischer Fortschritt, wie er z.B. von Schmookler (1966) diskutiert wurde, den Verdoorn-Zusammenhang bewirken. Nachfrageausdehnungen drängen die Unternehmen zur Forschung und Entwicklung, um die Bedürfnisse der Kunden befriedigen zu können. Tatsächlich wird es auch in Zeiten sinkender Nachfrage zu technischem Fortschritt kommen, wenn die Unternehmen ihre Kunden durch neue Produkte zu halten versuchen. Produktivitätssteigerungen treten dann bei negativen Wachstumsraten der Nachfrage auf.

Das Zusammenspiel dieser Punkte läßt den technischen Fortschritt, verstanden als Produktivitätswachstum, zu einer zumindest teilweise endogenen Variablen werden. Die Erhöhung der Nachfrage regt die Entstehung neuen technologischen Wissens an, indem einerseits Innovationen induziert werden und andererseits Lerneffekte und Externalitäten im Sinne von Young auftreten können. Der Verdoorn-Zusammenhang kann somit als ein Ausdruck der Endogenität des technischen Fortschritts interpretiert werden. Die Aufzählung möglicher versteckter Determinanten der positiven Relation zwischen Produktions- und Produktivitätswachstum veranschaulicht des weiteren mögliche Ursachen für das Aufbrechen des VerdoornZusammenhangs über die Zeit. Verdoorn sah die Existenz einer Steady State-Situation als notwendige Voraussetzung für die Stabilität des nach ihm benannten Zusammenhangs an, wenn ihm eine Cobb-Douglas-Produktionsfunktion zugrundegelegt wird. Diese Überlegung kann auf die hier vorgestellte Interpretation übertragen werden. Sämtliche Komponenten des Produktivitätsregimes müssen zueinander in stabilen Beziehungen stehen. Verändert sich z.B. die Rate des gebundenen technischen Fortschritts oder reagieren Unternehmen auf Nachfragesteigerungen in ihren Forschungs- und Entwicklungsentscheidungen anders, d.h. ist die Rate des nachfrageinduzierten technischen Fortschritts nicht stabil, kann auch kein stabiler Verdoorn-Zusammenhang erwartet werden, da dieser die reduzierte Form der skizzierten Einflußgrößen auf die Produktivitätsentwicklung darstellt. 
Die Ermittlung des Verdoorn-Zusammenhangs steht aus diesem Grund bei internationalen Vergleichen vor demselben Problem wie die Versuche, die Konvergenz-Hypothese nachzuweisen. Innerhalb der Konvergenz-Diskussion wurde deutlich, daß sich nur für Volkswirtschaften mit identischen Parameterwerten identische Steady State-Situationen ergeben. Absolute Konvergenz ist in der Realität nicht zu erwarten. Entsprechendes gilt für die Bestimmung des Verdoorn-Zusammenhangs als Ausdruck eines international gültigen Produktivitätsregimes. Wird eine heterogene Stichprobe von Ländern gewählt, kann dies zu nicht-signifikanten Ergebnissen für den Verdoorn-Koeffizienten führen. Es ist deshalb in einer Gruppe von Volkswirtschaften, die sich sehr ähnlich sind und enge Beziehungen zueinander unterhalten, mit größerer Wahrscheinlichkeit eine signifikante Korrelation zwischen Produktions- und Produktivitätswachstum möglich als in einer Gruppe, die diese Voraussetzung nicht erfüllt. Schätzungen, die den Verdoorn-Zusammenhang für verschiedene Zeiträume ermitteln und eine Auflösung attestieren, könnten als Beleg für den Wechsel der Produktivitätsbedingungen interpretiert werden. Dieser Schlußfolgerung kann jedoch nicht uneingeschränkt zugestimmt werden, da zum einen die ökonometrischen Probleme, d.h. die Datenlage für die Abschwächung der Relation verantwortlich sein können, zum anderen die Stichprobe aufgrund der Entwicklung einzelner Länder nicht mehr homogen ist. Japan z.B. muß zum heutigen Zeitpunkt der Gruppe der industrialisierten Länder hinzugerechnet werden, während dies in der Zeit vor dem Zweiten Weltkrieg nicht angebracht gewesen wäre.

\subsubsection{Produktivitätsinduzierte Nachfrageveränderungen}

Das zweite Element des Prozesses der kumulativen Verursachung stellt die Reaktion der Nachfrage auf das Produktivitätswachstum dar. Auch hier sind mehrere Wirkungsketten denkbar und zu berücksichtigen. Die Erhöhung der Produktivität beeinflußt die Entwicklung von Preisen, Löhnen, Profiten sowie verschiedener Nachfragekomponenten. Dabei können diese Komponenten nicht als voneinander unabhängig gesehen werden. Das Wachstum der Produktivität bedingt z.B. ein Ansteigen der Reallöhne, so daß aufgrund des gestiegenen Realeinkommens der Haushalte eine höhere Konsumnachfrage zu erwarten ist. Gleichzeitig kann die gestiegene Effizienz des Arbeitseinsatzes zu einer Reduktion der Arbeitsnachfrage führen, wenn der Output nicht entsprechend wächst. Die Wirkungen auf das Realeinkommen der Haushalte sind somit nicht ohne weiteres bestimmbar. 144

144 Bei Reallohnsteigerungen ist zwar eine Zunahme der Konsumnachfrage zu erwarten, jedoch werden nicht alle Konsumgütermärkte im gleichen Maße davon berührt werden. Wenn von der Gültigkeit des Engelschen Gesetzes ausgegangen wird, wird sich mit steigendem Realeinkommen der Haushalte auch deren Nachfragestruktur verändern. Technischer Fortschritt und die damit verbundenen Produktivitătsfortschritte geben somit aufgrund ihrer Wirkungen auf die Einkommenssituation einen Anstoß zum strukturellen Wandel. Neben diesem nachfrageseitigen Impuls hat der technische Fortschritt auch angebotsseitige Konsequenzen, da er sich nicht nur in Form von Prozeßinnovationen, sondern auch 
Auch das Investitionsverhalten der Unternehmen ist entscheidend für die Umsetzung von Produktivitätswachstum in eine steigende Güternachfrage. Kaldor betonte die Notwendigkeit der induzierten Investitionen, die für ihn maßgeblich von den Erwartungen der Unternehmen und des Handels über die Veränderung der Profite geprägt waren. Die möglichen Profite wiederum hängen von der Entwicklung der Reallöhne (negativ) und den möglichen absetzbaren Gütermengen (positiv) ab. Eindeutige Rückschlüsse sind auch hier nicht möglich. ${ }^{145}$

Neben der Investitions- und der Konsumnachfrage stellt die Exportnachfrage, genauer der Exportüberschuß den dritten Baustein der Güternachfrage dar. Dies wurde sowohl bei Kaldor (1972) als auch bei der vorgestellten Formalisierung berücksichtigt. Die Exportnachfrage wurde sogar als die allein entscheidende Nachfragekomponente angesehen. Die Produktivitätsentwicklung bedingt eine gestiegene Preiswettbewerbsfähigkeit der inländischen Unternehmen. Steigende Effizienz in der Produktion bedeutet sinkende Kosten, die c.p. unter Annahme einer Aufschlagskalkulation zu sinkenden Preisen führen. Tatsächlich müssen jedoch die Lohnstückkosten beachtet werden, d.h. auch an dieser Stelle kann die Lohnentwicklung die Nachfrage beeinflussen. Während bei der Konsumnachfrage steigende Löhne einen positiven Effekt haben, liegen hier eindeutig negative Wirkungen vor. ${ }^{146}$ Neben den Lohnkosten sind jedoch auch nicht-preisliche Komponenten entscheidend für die Wettbewerbsfähigkeit von Unternehmen. Die Qualität von Gütern sowie Serviceleistungen können preisliche Nachteile überwinden helfen. Dasselbe gilt für die Fähigkeit, neue Güter und Problemlösungen anbieten zu können.

Die Reaktionen der Nachfrage auf Produktivitätssteigerungen sind somit nicht eindeutiger Natur, sondern maßgeblich von der Entwicklung von Löhnen und Profiten bestimmt. Der Lohnfindungsprozeß sowie Fragen der Preisbildung gewinnen dabei an Bedeutung, d.h. institutionelle und soziale Verhältnisse beeinflussen die Umsetzung der erzielten Produktivitätssteigerungen in zusätzliche Nachfrage mit. Mehr noch als das Produktivitätsregime ist die Nachfrageseite des Modells der kumulativen Verursachung für Veränderungen der zugrundeliegenden Strukturen anfällig. Entsprechend der Vorgehensweise beim Verdoorn-Zusammenhang könnte eine Regressionsgleichung als Ausdruck der oben skizzierten Beziehungen

als Produktinnovationen manifestiert. Das Angebot an neuen Gütern wird ebenfalls Folgen für die Zusammensetzung der Konsumgüternachfrage haben. Die Frage, welche Sektoren nun Zuwächse bzw. Rückgänge aufweisen werden, kann nur in Abhăngigkeit von den Einkommenselastizităten der Nachfrage beantwortet werden. Pasinetti $(1981,1993)$ hat in seinen Modellen die Bedingungen aufgezeigt, die erfullt sein müssen, damit eine Volkswirtschaft im Prozeß des strukturellen Wandels die Vollbeschäftigung der Arbeitskraffte erreichen kann. Produktivitătsfortschritte, Nachfrageentwicklung und die Reallokation der Arbeitskraffte müssen hierfür sektoral aufeinander abgestimmt sein. Auch diese Überlegungen legen nahe, $\mathrm{daB}$ eindeutige Rückschlüsse von Produktivitătssteigenungen auf eine steigende Güternachfrage nicht mogglich sind.

145 Vgl. Boyer/Petit (1989, S. 28ff. und 1991a, S. 490ff.) und Targetti (1992, S. 185ff.)

146 Die im internationalen Vergleich zu hohen Lohnkosten sind das zentrale Argument der Arbeitgeber, wenn es um die internationale Wettbewerbsfahigkeit von Unternehmen geht. 
geschätzt werden, wobei die Wachstumsrate der Produktivität als unabhängige, diejenige der Nachfrage als abhängige Variable gesehen werden kann.

Der Verlauf und die Lage einer solchen Regressionsgeraden, wenn sie in linearer Form ermittelt werden soll, spiegelt die unterschiedliche Gewichtung der einzelnen Übertragungsmechanismen wider. ${ }^{147}$ Eine positive Beziehung zwischen Produktivitäts- und Produktionswachstum ergibt sich, wenn z.B. die Löhne mit der Produktivität wachsen und die Unternehmen ihre Investitionen aufgrund des Akzeleratorprinzips sehr stark ausdehnen, und die internationale Preiswettbewerbsfahigkeit kein Hindernis darstellt. Diese Situation wird auch als reines keynesianisches Nachfrageregime bezeichnet. Dieselben Wirkungen auf die Regressionsgerade würden sich bei reinen klassischen Verhältnissen ergeben. Hierbei folgen die Löhne der Produktivität nicht, und sinkende Lohnstückkosten haben mehr Exporte zur Folge.

Mischformen würden zu einer negativen Beziehung führen. Schlägt sich z.B. das gestiegene Produktivitätsniveau in den Löhnen nieder und besteht gleichzeitig ein hohes Maß an internationaler Konkurrenz bei den Preisen, kann u.U. das Mehr beim Konsum die nachfragesenkenden Wirkungen der gestiegenen Löhne auf den Export und die Profite und somit die Investitionen nicht ausgleichen. Die gleichen Konsequenzen ergeben sich, falls die gestiegene Effizienz nicht an die Arbeitnehmer weitergegeben wird und gleichzeitig der Export keine Rolle spielt. ${ }^{148}$

\subsubsection{Empirische Ergebnisse für das erweiterte Modell der kumulativen Verursachung}

Das Modell der kumulativen Verursachung versucht eine Vielzahl von Komponenten zu erfassen. Die Reduktion auf zwei Gleichungen, die stellvertretend für die Nachfrage- und die Produktivitätsverhältnisse sind, greift dabei zu kurz. Tatsächlich ergibt eine Schätzung zweier Regressionsgleichungen, bei denen für die eine Gleichung das Produktivitätswachstum als unabhängige und das Outputwachstum als abhängige Variable und für die andere Gleichung die Beziehung umgekehrt angenommen wird, keinen Sinn. Es würde nur die Umkehrregression geschätzt. Es entsteht somit dasselbe Problem, das bei der Frage nach der richtigen exogenen Variablen des Verdoorn-Zusammenhangs auftauchte. Je höher der Regressionskoeffizient der einen Gleichung ausfält, um so kleiner muß er bei der anderen sein. Der steigende Einfluß des Produktionswachstums im Verdoorn-Zusammenhang reduziert in diesem Fall die Wirkung des

147 Vgl. hierzu z.B. (Boyer/Petit 1991a, S. 498ff.).

148 Die USA werden hier als Beispiel angefuhrt. Vgl. Boyer/Petit (1991a, S. 490). Eine thnnliche Argumentationsweise findet sich z.B. bei Krugman (1994, S. 268ff.), der der Debatte uber internationale Wettbewerbsfahigkeit recht kritisch gegenübersteht. Für ihn sind Produktivitätssteigerungen innerhalb der USA, die geringer ausfallen als in anderen Volkswirtschaften, kein Problem, da der Export nur eine untergeordnete Rolle fur die Nachfrage in den Vereinigten Staaten spielt. 
Produktivitätswachstums auf die Wachstumsrate des Outputs. Ein solches Ergebnis kann als Dominanz des Produktivitätsregimes gedeutet werden. Dies schreibt der Regressionsanalyse zu viel Erklärungsgehalt zu. Insbesondere wenn man eine weitere Implikation der Schätzung dieser Art betrachtet. In beiden Regressionsgleichungen werden die Regressionskoeffizienten der unabhängigen Variablen, d.h. des Produktionswachstums beim Verdoom-Zusammenhang und des Produktivitätswachstums in der Nachfragegleichung, dasselbe Vorzeichen haben. Dies bedeutet, daß das Vorliegen des Verdoorn-Zusammenhangs automatisch ein keynesianisches oder klassisches Nachfrageregime zur Folge hat. In einem Modell, das sehr viele Komponenten incl. den institutionellen Gegebenheiten als entscheidend für den Transmissionsmechanismus von Produktivitätssteigerungen in Nachfrageveränderungen ansieht, kann dies nicht befriedigen. Die beiden Teile des Modells sind zwar interdependent, dennoch muß die Vorgehensweise bei der Schätzung Spielraum für unterschiedliche Kombinationen der einzelnen Regimeformen lassen. Die adäquate Überprüfung des Modells erfordert deshalb ein Mehrgleichungssystem, in dem sowohl der Verdoorn-Zusammenhang als auch die Nachfragegleichung des Modells indirekt geschätzt werden. 149

Boyer/Petit (1991a) stellen in einem Überblick über verschiedene Schätzungen von Produktivitäts- und Nachfrageregimen mit Hilfe von Mehrgleichungsmodellen mehrere interessante Ergebnisse zusammen:

1.) Innerhalb einer Stichprobe von sechs europäischen Ländern, die leider nicht genannt werden, wurde eine Elastizität der Produktivität bzgl. des Produktionswachstums von 0,6 festgestellt, wobei steigenden Skalenerträgen fast hundert Prozent dieses Zusammenhangs zugeschrieben wurden. Die Steigung des Nachfrageregimes ist positiv. Die positiven Effekte der internationalen Wettbewerbsfähigkeit reichen nicht aus, um die negativen Wirkungen gestiegener Produktivität auf die Arbeitskräftenachfrage auszugleichen. Die Ergebnisse sind bei einem Konfidenzniveau von zehn Prozent statistisch signifikant, d.h. es liegen steigende Skalenerträge vor. ${ }^{150}$

2.) Für die USA ergab sich in der Zeit zwischen 1899 und 1976 eine Elastizität der Produktivität bzgl. des Produktionswachstums von 0,5. Maßgebliche Komponenten sind dabei die Investitionen und die Innovationen. Für das Nachfrageregime wurde ein steigender Verlauf ermittelt, wobei Reallohnsteigerungen und Exportzuwächse sehr

149 Ein erster Schritt stellt z.B. das Modell von Parikh (1978) dar, der auf der Produktivitătsseite die Folgen der Investitionen, des exogenen technischen Fortschritts und der verbesserten Arbeitsorganisation mit Hilfe eines Mehrgleichungsmodells, in das angebots- und nachfrageseitige Großen in die Regressionsgleichung für das Produktions- und in diejenige für das Produktivitătswachstum einfließen, untersuchte. In seinem Modell wurde die These des nachfragebegrenzten Wachstums gestutzt. Vgl. Parikh (1978, insbes. S. 86 und 89f.).

150 Vgl. Boyer/Petit (1991a, S. 501f.). 
stark auf eine erhöhte Produktivität reagieren. Die Nachfrage steigt insgesamt um 0,43 Prozentpunkte, wenn die Produktivität um ein Prozent wächst. Der statistische Zusammenhang ist auf hohem Niveau gesichert. ${ }^{151}$

3.) Bei einem Vergleich von drei Schätzungen in den Zeiträumen 1960 bis 1973, 1973 1979 und 1979-1987 für zehn europäische Länder plus USA und Japan ergab sich für den ersten Zeitraum die Bestätigung der Vorstellungen über das Modell der kumulativen Verursachung. Insbesondere die These steigender Skalenerträge konnte bestätigt werden. Im zweiten Zeitraum kam es zu einer Auflösung dieses Zusammenhangs. Die Bedeutung steigender Skalenerträge ging zurück, während die Bedeutung von Forschungs- und Entwicklungsausgaben stieg. Auf der Nachfrageseite ergab sich die Tendenz zu einem negativen Zusammenhang zwischen Produktivitäts- und Produktionswachstum. Für die achtziger Jahre konnte keine eindeutige Aussage getroffen werden. 152

In einer vergleichbar aufgebauten Untersuchung von Pini (1995), die den Zeitraum 1960-1990 umfaßte und neun ausgewählte OECD-Länder in der Stichprobe enthielt, ergab sich für alle Teilzeiträume und alle Ländergruppen immer ein positiver Verlauf des indirekt geschätzten Verdoorn-Zusammenhangs und eine positive Beziehung zwischen Produktivitäts- und Produktionswachstum auf der Nachfrageseite des Modells. ${ }^{153}$ Es konnten jedoch Unterschiede zwischen den Ländergruppen festgestellt werden. Innerhalb der in der Stichprobe enthaltenen Länder der Europäischen Union (EU) wies die Verdoorn-Gerade für den Gesamtzeitraum einen höheren Absolutterm und eine niedrigere Steigung $(0,66)$ auf als in den Nicht-EULändern $(0,73)$. Ein Prozent Produktionswachstum bewirkte in den Nicht-EU-Ländern somit mehr zusätzliches Wachstum bei der Produktivität als in den restlichen Volkswirtschaften der Stichprobe. Der hohe autonome Anteil am Produktivitätswachstum ließ aber dennoch im Bereich der tatsächlich realisierten Wachstumsraten des Outputs die Verdoorn-Gerade in der EU-Gruppe ständig über derjenigen der Nicht-EU-Länder liegen. Gleichzeitig bewirkte ein Prozentpunkt Produktivitätswachstum in den Nicht EU-Ländern mehr Wachstum der Nachfrage als in der EU-Gruppe. Dies trägt mit zur Erklärung des geringen Beschäftigungswachstums in den EU-Volkswirtschaften im Vergleich zu Volkswirtschaften wie der USA bei. 154

151 Vgl. Boyer/Petit (1991a, S. 504ff.)

152 Vgl. Boyer/Petit (1991a, S. 508ff. und (1991b, S. 59).

153 Die Lănder waren Belgien, Frankreich, GroBbritannien, Italien, Japan, Kanada, Niederlande, USA und Westdeutschland. Vgl. Pini (1995, S. 185).

154 Die genauen Werte der Koeffizienten werden vom Autor nur für die Steigungen der Kurven angegeben. Alle anderen Angaben können nur einer Grafik entnommen werden, in der die unterschiedlichen Lagen der Verdoorn-Geraden abzulesen sind. Vgl. Pini (1995, S. 200 und 203ff.). 
Bei der Aufteilung in zwei Zeitintervalle (1960-1976 und 1976-1990) zeigte sich, daß sich im zweiten Zeitraum die Nachfragegerade zur Ordinate hin verschoben hatte. Das Produktivitätswachstum regte deshalb weniger Produktionswachstum an als in der Zeit zwischen 19601976. Bei der Verdoorn-Geraden ergaben sich jedoch nur geringe Veränderungen bei der Lage und der Steigung. ${ }^{155}$ Für alle durchgeführten Berechnungen lag die Steigung des VerdoornZusammenhangs unter derjenigen der Nachfragekurve, d.h. entsprechend den Überlegungen von Abschnitt (4.5.3.1) liegt in allen Fällen eine Tendenz zu konstanten Wachstumsraten vor. Die Hypothese eines Zusammenbruchs des Verdoorn-Zusammenhangs wurde durch diese Studie im Vergleich zu den zuvor von Boyer/Petit angefuhrten Untersuchungen somit nicht belegt. Eine Ursache kann in der Zahl und Art der verwendeten Variablen liegen. Aber auch die Ländergruppen unterscheiden sich. Darüber hinaus ist es auch möglich, daß der längere Zeitraum, insbesondere die Einbeziehung der kompletten achtziger Jahre, für dieses Ergebnis verantwortlich ist.

\subsubsection{Fazit}

Kaldors Überlegungen zum Verdoorn-Zusammenhang im Rahmen seiner Antrittsvorlesung in Cambridge haben die Relevanz dieser Beziehung innerhalb eines Modells der kumulativen Verursachung erkennen lassen. Deutlicher wurde die Rolle der positiven Beziehung zwischen Produktions- und Produktivitätswachstum für den Wachstumsprozeß von Volkswirtschaften durch die Integration einer expliziten Nachfragefunktion, die in Kaldors Modell zur Erklärung divergierender Wachstumsprozesse durch die Exportfunktion eingeführt worden ist. Die alleinige Existenz des Verdoorn-Zusammenhangs ist nicht ausreichend, um kumulative Prozesse erklären zu können. Die Reaktionen der Nachfragekomponenten müssen die Impulse einer gestiegenen Produktivität entsprechend verstärken. Es ist genauso gut vorstellbar, daß die Nachfrageseite den Impuls des Produktivitätswachstums abbremsen und den kumulativen Prozeß zum Erliegen bringen kann.

Die Erweiterung dieses einfachen Modells durch zusätzliche Variablen überwindet die Reduktion der Ursachen des Verdoorn-Zusammenhangs auf eine einfache Cobb-DouglasProduktionsfunktion oder eine lineare technical progress function. Der Verdoorn-Zusammenhang kann vielmehr als Ausdruck komplexer Strukturen verstanden werden, die die Auswirkungen von Nachfragesteigerungen auf die Produktivitätsentwicklung abbilden. Eine monokausale Erklärung für die Existenz bzw. die Auflösung der Beziehung zwischen Produktionsund Produktivitätswachstum kann deshalb nicht gegeben werden. Dasselbe gilt für die Nachfrageseite. Die alleinige Konzentration auf die Exportfunktion ist nicht ausreichend. Das erweiterte Modell der kumulativen Verursachung bezieht die Wirkungen auf die Konsum- und 
Investitionsnachfrage in die Erklärung ein, indem auch die Effekte der Produktivitätssteigerungen auf die Lohn- und Profitentwicklung Beachtung finden.

Die Modifikation des ursprünglichen Kaldor-Modells stellt einen Schritt zu mehr Erklärungsgehalt dar. Die bisher empirisch durchgeführten Untersuchungen zeigen, daß zumindest in den sechziger Jahren die These Kaldors über die Rolle des Verdoorn-Zusammenhangs Gültigkeit besessen hat. Die durchgeführten Erweiterungen der Überlegungen Kaldors versetzen das Modell in die Lage, auch andere Situationen als die typisch kaldorianischen abbilden zu können.

\subsection{Zusammenfassung}

Dieses Kapitel hat gezeigt, daß der Beitrag Kaldors zur Erklärung wirtschaftlichen Wachstums nicht auf ein Teilgebiet der Wachstumstheorie beschränkt ist. Während seine frühen Arbeiten auf diesem Gebiet - Kaldor war bei der Veröffentlichung seiner Papiere zur technical progress function knapp fünfig Jahre alt - noch sehr stark durch Steady State-Überlegungen geprägt waren, enthalten seine Beiträge seit Mitte der sechziger Jahre den Glauben an die Instabilität des ökonomischen Systems. Beide Teile seiner wachstumstheoretischen Arbeiten haben zum besseren Verständnis von Wachstumsprozessen beigetragen.

Das Konzept der technical progress function stellt neben dem Konzept des learning by doing von Arrow einen ersten Schritt zur endogenen Erklärung des technischen Fortschritts dar. Die Investitionshöhe bzw. die Entwicklung der Investitionen pro Arbeitsplatz legen fest, welche Zuwächse bei der Arbeitsproduktivität entstehen können. Die Einbeziehung von investitionsinduzierten Lerneffekten stellt dabei einen Zusammenhang zwischen der Kapitalakkumulation und der Entstehung des technischen Fortschritts her. Die Diskussion der technical progress function hat darüber hinaus die Rolle der Geschichte einer Volkswirtschaft verdeutlicht. Die Gleichmäßigkeit der Investitionstätigkeit bestimmt die Übernahme neuer Technologien in den Produktionsprozeß sowie die Möglichkeit, neue Ideen im Sinne von neuem technologischem Wissen zu schaffen. Kaldors Behandlung der technical progress function vertieft jedoch nicht die genaue Entstehung des technologischen Wissens. Die technical progress function ist in seinen Modellen vielmehr eine feste Funktion, die sich zwar durch eine Veränderung des technical dynamism in ihrer Lage und durch Veränderung der Bereitschaft und Fähigkeit neue Technologien zu übernehmen in ihrer Steigung verändern kann, die innerhalb der Analyse von Kaldor aber als vorgegeben angenommen wird. Aus diesem Grund konnte auch die Abhängigkeit der Steady State-Wachstumsrate von den Parametern der technical progress function nicht überraschen. Trotz dieser Einwände stellt die technical progress function einen Fortschritt gegenüber der neoklassischen Theorie des exogenen technischen Fortschritts dar. Investitionen führen zu intertemporalen externen Effekten, da sie die 
Effizienz zukünftiger Kapitalgüter positiv beeinflussen. Darüber hinaus wird die Bedeutung der Kapitalintensivierung für die Produktivitätsentwicklung verdeutlicht.

Die Rolle externer Effekte im Sinne von Young (1928) und der daraus resultierenden steigenden Skalenerträge, die in Kaldors Vorstellungen zu den treibenden Kräften des Wachstumsprozesses gehören, wurde in der Diskussion über den Verdoorn-Zusammenhang erkennbar. Die sowohl bei Längs- als auch Querschnittsanalysen statistisch ermittelbare Beziehung zwischen dem Produktions- und dem Produktivitätswachstum wurde von Kaldor als ein Beleg für die Existenz steigender Skalenerträge angesehen. Die Ausführungen zu den möglichen theoretischen Begründungen des Verdoorn-Zusammenhangs ergaben, daß nicht eindeutig auf ein Skalenniveau größer Eins geschlossen werden kann, wenn eine Cobb-Douglas-Produktionsfunktion unterstellt wird. Selbst wenn der geschätzte Regressionskoeffizient auf steigende Skalenerträge hinweist, bleibt das Problem der signifikanten Unterschiede zwischen dem statischen und dem dynamischen Verdoorn-Zusammenhang, das sich für den Fall einer CobbDouglas-Produktionsfunktion ergibt. Aus diesem Grund wurden andere theoretische Begründungen für den Verdoorn-Zusammenhang wie die technical progress function bzw. eine Lernkurve diskutiert. Prinzipiell ist es zwar möglich, aus diesen Funktionen eine lineare Beziehung zwischen den Wachstumsraten von Produktion und Produktivität abzuleiten, jedoch sind dafür ebenfalls sehr einschränkende Annahmen notwendig. Die tatsächliche theoretische Struktur des Verdoorn-Zusammenhangs ist zu komplex, als daß sie durch eine einfache Funktion bestimmt werden kann.

Trotz dieser Einwände kann der Verdoorn-Zusammenhang zur Erklärung der von Kaldor betonten Prozesse der kumulativen Verursachung herangezogen werden. Anfängliche Unterschiede bei Wachstumsraten können bei Existenz dieser Beziehung bestehen bleiben bzw. sich verstärken. Sowohl Young als auch Kaldor haben erkannt, daß ein kumulativer Prozeß nur möglich ist, falls die Nachfrage auf die Produktivitätssteigerungen reagiert, d.h. Produktivitätserhöhungen müssen weitere Nachfragesteigerungen nach sich ziehen. Nicht zwangsläufig ergibt sich daraus ein Prozeß ständig steigender oder sinkender Wachstumsraten. Vielmehr haben die Ausfuihrungen gezeigt, daß sich auch eine stabile Wachstumsrate einstellen kann. Vergleicht man nun verschiedene Länder miteinander, besteht die Möglichkeit, daß sich eine Volkswirtschaft zu einem Gleichgewicht mit einer geringeren Wachstumsrate entwickelt als ein anderes Land. Die Divergenz von Wachstumsraten ist somit nur eines von vielen Ergebnissen innerhalb des Modells der kumulativen Verursachung.

Das Zusammenspiel von Verdoorn-Zusammenhang und Nachfrageregime bestimmt über die Wachstumsraten. Für beide Teile des Modells sind unterschiedliche Beziehungen denkbar, die für die Feedback- und Spillover-Effekte zwischen Produktions- und Produktivitätswachstum verantwortlich sein können. Die Erweiterung des ursprünglichen Eingleichungsmodells von Kaldor durch die genauere Untersuchung der Produktivitäts- und Nachfrageregime stellt einen großen Schritt zu mehr Erklärungsgehalt dar. Es werden zusätzliche 
Determinanten beachtet, die im einfachen Verdoorn-Zusammenhang außen vor bleiben. Dies stellt auch einen großen Vorteil gegenüber den Überlegungen der Neuen Wachstumstheorie dar.

Kaldors Vorstellungen über die Bestimmungsgründe tatsächlich beobachtbarer Wachstumsprozesse haben somit einen wichtigen Beitrag zur Generierung von Modellen mit mehr Realitätsnähe geleistet, indem sie den Blick über den Tellerrand der neoklassischen Sichtweise hinaus angeregt haben. 
Stephan Seiter - 978-3-631-75406-1

Downloaded from PubFactory at 01/11/2019 05:22:48AM

via free access 
5 Neue Wachstumstheorie und Kaldors Beitrag zur Erklärung wirtschaftlichen Wachstums im Vergleich

\subsection{Vorbemerkungen}

Die vorigen Kapitel haben sowohl die grundlegenden Vorstellungen der Neuen Wachstumstheorie als auch die Kernelemente des Verständnisses von Kaldor über Wachstumsprozesse gezeigt. Am Ende der jeweiligen Kapitel wurden die wichtigsten Aussagen und Ergebnisse zusammengefaßt. In diesem Abschnitt der Arbeit werden beide Herangehensweisen verglichen, um einerseits Gemeinsamkeiten und andererseits Unterschiede zu verdeutlichen. Daran anschließend wird auf mögliche wachstumspolitische Konsequenzen beider Ansätze eingegangen. Den Abschluß bildet eine kurze Zusammenfassung der wichtigsten Aussagen dieses Kapitels.

\subsection{Unterschiede und Gemeinsamkeiten}

\subsubsection{Unterschiede}

Bei einer Gegenüberstellung der Ideen und Vorgehensweisen der Neuen Wachstumstheorie mit den Gedanken und der Methodik von Kaldor ist es einfacher, mit den Unterschieden zu beginnen. Die kaldorianische Kritik am neoklassischen Paradigma, in dem die Neue Wachstumstheorie verwurzelt ist, hat die gegensătzlichen Positionen beider Vorstellungen über den richtigen Weg zu Erklärung von Wachstumsprozessen schon in den sechziger Jahren deutlich gemacht. Tatsăchlich wird sich auch mehr Gegensătzliches finden als Vereinendes, dennoch zeigt sich eine gewisse Annăherung in einigen Punkten.

\subsubsection{Die Vorstellungen über die Produktionsstruktur}

Am Anfang von Kapitel 4 wurde die Kritik von Kaldor an der neoklassischen Produktionsfunktion erläutert. Die Unmöglichkeit der Trennung von Substitutions- und Fortschrittskomponente ist für ihn ein Grund, die neoklassische Produktionsfunktion abzulehnen. Technischer Fortschritt mache immer Investitionen zur Erhöhung der Kapitalausstattung pro Arbeitsplatz notwendig, da sie sowohl Träger als auch Ursache neuen technologischen Wissens darstellen. Bewegungen auf der Produktionsfunktion, d.h. Kapitalintensivierung, und Verschiebungen der Kurve, d.h. das Vorliegen exogenen technischen Fortschritts, können nicht getrennt werden. Die Verănderung des technischen Wissens hat des weiteren die Implikation, 
daß eine Volkswirtschaft in der Überzeugung Kaldors nie in der Lage sein wird, einen Punkt auf der Produktionsfunktion zu erreichen. Die Ökonomie hat nicht ausreichend lange genug Zeit, mit konstanter Kapitalintensität zu produzieren. Des weiteren führe technischer Fortschritt zu steigenden Skalenerträgen in der Produktion, und die Annahme einer Produktionsfunktion mit einem Skalenniveau von Eins könne nicht aufrechterhalten werden. Ein Wachstumsmodell müsse deshalb eine Beziehung zwischen der relativen Zunahme der Kapitalausstattung pro Kopf bzw. der relativen Veränderung der Investitionen pro Arbeitsplatz und der Wachstumsrate der Arbeitsproduktivität insgesamt bzw. der Arbeitsproduktivität an den neuesten Maschinen beinhalten.

Die Ablehnung einer gesamtwirtschaftichen Produktionsfunktion verstärkt sich in den späteren Beiträgen von Kaldor. Innerhalb der Ausführungen zur kumulativen Verursachung kommen Überlegungen zur Produktionstechnologie nur im Hinblick auf das Vorliegen steigender Skalenerträge zur Sprache. Modellstrukturen, wie sie in den rein wachstumstheoretischen Arbeiten Kaldors verwendet werden, stehen dabei nicht zur Debatte. Vielmehr wird anhand empirisch nachweisbarer Beziehungen versucht, Rückschlüsse auf mögliche Ursachen eines endogenen Wachstumsprozesses zu ziehen.

Die Neue Wachstumstheorie hält am Konzept einer gesamtwirtschaftichen Produktionsfunktion fest. Sowohl innerhalb der Modelle von Romer als auch von Lucas sowie in den Ansätzen mit Produktinnovationen bildet sie das Kernelement, um endogenes Wachstum nachweisen zu können. Die prinzipiellen Einwände Kaldors lassen sich deshalb weiterhin vorbringen. Den Ansätzen der Neuen Wachstumstheorie ist es aber zumindest an einer Stelle gelungen, die Kritik Kaldors abzuschwächen. Durch den Wegfall des exogenen technischen Fortschritts und wegen der Existenz von Externalitäten können die Annahmen der Grenzproduktivitätsentlohnung und steigende Skalenerträge in der gesamtwirtschaftlichen Produktion zusammen analysiert werden. Solange das Skalenniveau aller zu entlohnenden Produktionsfaktoren gleich eins ist, besteht das Problem der Überausschöpfung nicht. Die Ersetzung der exogenen Fortschrittskomponente durch Externalitäten ändert jedoch nichts an der Tatsache, daß weiterhin ein kostenloser Produktionsfaktor enthalten ist, der wie steigende Skalenerträge der entlohnten Inputs wirkt. Das soziale und private Grenzprodukt von Sachkapital und Arbeit fallen weiter auseinander.

Die Anwendung einer gesamtwirtschaftlichen Produktionsfunktion bleibt mit Sicherheit einer der zentralen Unterschiede zwischen den Arbeiten in der Nachfolge von Kaldor und den Beiträgen zur Neuen Wachstumstheorie. Hiermit eng verbunden ist ein weiterer Punkt, der unterschiedlich behandelt wird. Das traditionelle neoklassische Wachstumsmodell ist ein einsektorales Modell, in dem unter Verwendung der Produktionsfaktoren Arbeit und Kapital sowie unter Einsatz des technologischen Wissens ein Gut hergestellt wird, das sowohl konsumiert als auch investiert werden kann. Innerhalb der Neuen Wachstumstheorie findet sich derselbe Mechanismus wieder. In den Unternehmen wird unter Verwendung verschiedener 
Produktionsfaktoren ein Gut hergestellt, das wiederum für Konsum- und Investitionszwecke verwendet werden kann. Im Modell von Romer (1986a) z.B. konnte dieses Endprodukt in das Kapitalgut Wissen umgewandelt werden. Das erste Modell von Lucas (1988) bildet eine Volkswirtschaft ab, die zwar zwei Arten von Kapitalgütern enthält, jedoch können beide ineinander überführt werden. Humankapital und Sachkapital werden zur Herstellung des Endprodukts verwendet, das nun in Sachkapital umgewandelt werden kann. Das Humankapital wiederum entsteht durch den Einsatz von Humankapital und Zeit, die nicht für die Konsumgutproduktion verwendet wird. Die Verwendung von Zeit für Bildung stellt Konsumverzicht dar und unterscheidet sich nicht von der Sachkapitalinvestition. Das Endprodukt kann in beiden Fällen in Kapital umgewandelt werden. Die Modelle zur Produktinnovation stellen einen ersten Schritt in Richtung eines mehrsektoralen Modells dar, da sie zum einen verschiedene Güter auf Konsumebene unterscheiden und zum anderen zusätzliche Sektoren einführen, die unterschiedliche Arten von Arbeit erfordern. Die Umwandlung von Konsumgütern in Kapitalgüter kann hier nicht ohne weiteres stattfinden wie in den anderen Modellen. Am Beispiel von Romers Modell (1990) wurde aber gezeigt, daß durch die getroffenen Annahmen die heterogenen Kapitalgüter wieder in ein einziges Kapitalgut überführt werden können. Der große Vorteil war jedoch die Berücksichtigung monopolistischer Marktstrukturen.

Auch die ersten Modelle von Kaldor sind Ein-Gut-Modelle. Es wird weder zwischen unterschiedlichen Sektoren hinsichtlich der Produktionstechnik, der Marktform oder den Qualifikationserfordernissen der Arbeitskräfte differenziert. Erst in seiner Antrittsvorlesung in Cambridge führt Kaldor eine Betrachtung ein, die zwischen dem primären, dem sekundären und dem tertiären Sektor unterscheidet. Diese Bereiche unterliegen unterschiedlichen Produktionstechnologien. Den steigenden Skalenerträgen der industriellen Produktion stehen die abnehmenden im primären und (so die Annahme) im tertiären Sektor gegenüber. ${ }^{1}$ Die Konsequenzen dieser Aufteilung werden von Kaldor jedoch nicht anhand eines formalen Modells untersucht, sondern in verbaler Form. Der Übergang von Arbeitskräften vom landwirtschaftlichen in den warenproduzierenden Teil der Volkswirtschaft ermöglicht hohe endogene Wachstumsraten. Bei der Formalisierung seiner Vorstellungen zur kumulativen Verursachung, wie z.B. durch Dixon/Thirlwall (1975) oder Boyer/Petit (1991a,b), wenden sich die Autoren vorrangig dem industriellen Bereich zu, während die anderen Sektoren nur implizit enthalten sind. Die kaldorianische Vorgehensweise ist somit prinzipiell an der strukturellen Komponente des Wachstumsprozesses interessiert. Die sektorale Zusammensetzung einer Ökonomie kann sich über die Zeit hinweg verändern. Das neoklassische Wachstum dagegen ist strukturkonstant.

1 Inwieweit im tertiăren Sektor abnehmende oder steigende Skalenerträge vorliegen, bleibt offen. Die Entwicklung im Bereich der Computertechnologien kann durchaus auch steigende Skalenerträge innerhalb dieses Bereichs zur Folge haben. Da der Produktionsfaktor Wissen bei der Herstellung der Produkte dieses Sektors relevant ist, sind Wissensexternalitatten wahrscheinlich. Auch GroBBenvorteile sind denkbar, wenn man an die Herstellung von Software denkt. 


\subsubsection{Individuum versus Gesellschaft}

In den Modellen der Neuen Wachstumstheorie wird mit Hilfe einer intertemporalen Nutzenfunktion eines repräsentativen Individuums oder Haushalts eine Form der mikroökonomischen Fundierung der Entscheidungen über Sparen und damit über die Investitionshöhe zu erreichen versucht. Die Schwierigkeiten, die eine solche Vorgehensweise bei Vorliegen externer Effekte aufwerfen kann, wurden im Rahmen von Kapitel 3 angesprochen. Prinzipiell ist es problematisch, makroökonomische Wachstumsprozesse vollständig auf repräsentatives individuelles Verhalten zurückzuführen. Gesamtwirtschaftiche Phänomene sind das Resultat des Zusammenspiels unterschiedlicher Akteure, die für sich allein oder gemeinsam als Gruppe eigene Interessen verfolgen, die nicht unbedingt in einer einzigen Nutzenfunktion abgebildet werden können. Die Reduzierung der Komplexität der mannigfaltigen Interaktionen zwischen den Wirtschaftssubjekten auf das Konzept des repräsentativen Individuums greift zu kurz.

Die von Solow an dieser Vorgehensweise vorgebrachte Kritik, daß die Anwendung der Vorgehensweise Ramseys nur Schwierigkeiten bringt und keine zusätzlichen bahnbrechenden Erkenntnisse, kann mit großer Wahrscheinlichkeit von kaldorianischer Seite aus zugestimmt werden. Anstelle des Versuchs einer Quasi-Mikrofundierung ist die Konzentration auf die Erklärung stilisierter Fakten sinnvoller. ${ }^{2}$ Für das Sparverhalten bedeutet dies z.B., daß für die Erklärung von Wachstumsprozessen die Annahme konstanter Sparquoten durchaus sinnvoll sein kann, wenn dies in der Realität beobachtet wird. Sowohl die rein theoretischen Beiträge als auch die Arbeiten zur angewandten Wachstumstheorie von Kaldor versuchen diesem Anspruch zu folgen. So sind in seinen Modellen ausschließlich Funktionen auf gesamtwirtschafticher Ebene zu finden. Die Sparfunktion ist nicht aus der Nutzenmaximierung abgeleitet, sondern das Resultat der Einkommensverteilung und der für Lohn- und Profiteinkommensbezieher jeweils als konstant angenommenen Sparquoten. Die Investitionsfunktion stellt eine eigenständige Funktion dar, die von den Erwartungen der Unternehmen und den technologischen Möglichkeiten der Volkswirtschaft bestimmt wird. Insgesamt ergibt sich das Bild einer homogenen Investorengruppe. Die Annahmen über die Funktion selbst sind aber aus makroökonomischen Beobachtungen abgeleitet.

Auch innerhalb des Modells der kumulativen Verursachung wird das gesamtwirtschaftliche Geschehen nicht auf die Entscheidungen des Haushalts verkürzt. Mehr noch als in den rein theoretischen Arbeiten von Kaldor kommt in seinen Überlegungen zum VerdoornZusammenhang die Konzentration auf makroőkonomische Relationen zum Ausdruck. Zentrales Element seiner Erklärung kumulativer Wachstumsprozesse ist die Existenz der Beziehung zwischen den Wachstumsraten der Arbeitsproduktivität und des Outputs im Verarbeitenden Gewerbe. Die Entwicklung der Nachfragegrößen Konsum, Investition und Export legt fest, welches Wachstum bei der Produktion möglich ist und welche Produktivitätssteigerung

2 Vgl. Solow (1994a, S. 49). 
erwartet werden kann. Auch die Weiterentwicklungen seiner Ideen im Rahmen des am Ende des letzten Kapitels vorgestellten Modells der kumulativen Verursachung weichen von dieser Vorgehensweise nicht ab. Es werden ebenfalls Annahmen über makroökonomische Funktionen getroffen, die dann einem ökonometrischen Test unterzogen werden.

Die Fixierung auf rein gesamtwirtschaftliche Beziehungen kann jedoch genauso wenig überzeugen wie das Konzept des repräsentativen Wirtschaftssubjekts. Tatsächlich muß eine Integration erfolgen. Kaldor selbst hat am Ende seines wachstumstheoretischen Schaffens auf die Notwendigkeit eines solchen Schritts hingewiesen:

"A macro-economic model needs to be supplemented by a micro-economic analysis on the level of the single decision making unit, the firm or business, and also of the relations between groups of competing firms [without such a supplementation it is not obvious how the forces isolated in the macro-model actually operate; and it is impossible to say how far (how effectively, or how quickly or slowly) they operate]."3

Insbesondere das Unternehmensverhalten schien für Kaldor erklärungsbedürftig zu sein, da die Unternehmer durch ihre Akkumulationsentscheidung Multiplikator-, Kapazitäts- und Produktivitätseffekte erzeugen. Diese Forderung Kaldors steht in Einklang mit dem am Ende des Kapitels zur Neuen Wachstumstheorie gesagten. Die Erklärung von Wachstumsprozessen muß auch Methoden wie z.B. Fallstudien anwenden, um mehr über die Motivationsstrukturen der Unternehmer und deren Erfolgsfaktoren zu erfahren.

Ein wesentlicher Unterschied zwischen den Arbeiten Kaldors und der Neuen Wachstumstheorie stellt somit die Konzentration auf das repräsentative Individuum bzw. auf makroökonomische Verhaltensfunktionen dar. Dies ist jedoch kein spezifischer Unterschied, der den hier durchgeführten Vergleich betriff, sondern ein prinzipieller. Postkeynesianische Arbeiten versuchen gemeinhin, reale ökonomische Probleme mit Hilfe von angepaßten Modellstrukturen zu erklären, die stilisierte Fakten berücksichtigen. Die neoklassische Tradition wiederum hat das Ziel, unter Verwendung eines vorgegebenen Sets von Verhaltensannahmen reale Phänomene zu analysieren.

\subsubsection{Konstante versus variable Wachstumsraten}

Ein weiterer wesentlicher Unterschied zwischen den in Kapitel 3 und 4 diskutierten Vorstellungen über Wachstumsprozesse ist die Frage nach der Stabilität der Wachstumsrate des Outputs. Die Neue Wachstumstheorie ist maßgeblich durch das Konzept des Steady StateWachstums gekennzeichnet. Die Konstanz der langfristigen Wachstumsrate wird wie die Vollbeschäftigung der Produktionsfaktoren als Bedingung eingeführt, d.h. in einem ersten Schritt

3 Kaldor (1986b, S. 20). 
werden nur gleichgewichtige Situationen betrachtet. Diese Vorgehensweise erklärt sich aus der Zielsetzung der Neuen Wachstumstheorie, im Steady State eine positive Wachstumsrate der Pro-Kopf-Größen ohne exogenen technischen Fortschritt zu erreichen. Das neoklassische Grundmodell wies in der Anpassungsphase an einen Gleichgewichtspfad z.B. nach einer Sparquotenerhöhung durchaus von Null verschiedene Wachstumsraten des Pro-Kopf-Einkommens auf. Nur im langfristigen Gleichgewicht entstand aufgrund der abnehmenden Grenzproduktivität des Kapitals das Ergebnis konstanter Pro-Kopf-Größen. Will nun die Neue Wachstumstheorie diesen Punkt verbessern, muß sie in einem ersten Schritt Steady State-Situationen untersuchen. Problematisch ist das prinzipielle Festhalten an diesem Konzept. Der Beweis der Endogenität der Wachstumsrate sollte nur eine Zwischenetappe sein, um sich dann auf ungleichgewichtige Situationen im Sinne von variablen Wachstumsraten zu konzentrieren.

Die frühen Arbeiten Kaldors zur Wachstumstheorie weisen hier ebenfalls eine Parallelität zur Neoklassik auf, denn auch die Lösungen seiner Modelle sind Steady State-Situationen, die durch konstante Wachstumsraten gekennzeichnet sind. In Abschnitt (4.2) wurde auf die Ähnlichkeit zwischen der technical progress function und der neoklassischen Produktionsfunktion hingewiesen. Diese Modellergebnisse sollten erzeugt werden, da die Konstanz der Wachstumsrate des Outputs und der Arbeitsproduktivität von Kaldor als eine der stilisierten Fakten angenommen wurde. Das Prinzip der kumulativen Verursachung, das auch variable Wachstumsraten des Outputs und der Arbeitsproduktivität zuläßt, wird von Kaldor erst später berücksichtigt. Steady State-Situationen stellen nur eine Möglichkeit innerhalb des Modells der kumulativen Verursachung dar. Die induzierten Wirkungen von Produktivitätssteigerungen auf die Nachfrage und die sich daran anschließenden Feedback-Effekte auf die Produktivität bestimmen, ob ein System zu einer gleichgewichtigen oder einer ungleichgewichtigen Situation kommt.

Die Modelle der Neuen Wachstumstheorie sind prinzipiell in der Lage kumulatives Wachstum zu erzeugen. Es muß dafür z.B. in einem AK-Modell nur die Annahme steigender Grenzproduktivitäten des Kapitals getroffen werden. Jede zusätzlich investierte Kapitaleinheit wird den Output überproportional erhöhen und die Wachstumsraten werden ständig zunehmen. Die Frage, ob ständig steigende oder fallende Wachstumsraten in einem Modell vorliegen, kann nur in Abhängigkeit von den getroffenen Annahmen beantwortet werden. Die Ansätze der Neuen Wachstumstheorie unterscheiden sich deshalb von der kaldorianischen Vorgehensweise nicht dadurch, daß instabile Situationen in den aktuellen Modellen der neoklassischen Wachstumstheorie unmöglich sind, sondern durch die Zielsetzung, diese gar nicht erst zu betrachten. Die Ausführungen im dritten Kapitel haben in diesem Zusammenhang die Notwendigkeit konstanter Skalenerträge bzgl. der akkumulierbaren Produktionsfaktoren gezeigt, die einzig in der Lage waren, endogenes Steady State-Wachstum zu erzeugen. Die Unterschiede zwischen der kaldorianischen Tradition und der Neuen Wachstumstheorie sind deshalb sehr stark durch die grundsätzliche Einstellung gegenüber dem marktwirtschaftlichen System geprägt. Post- 
keynesianische Autoren werden aufgrund der von ihnen angenommenen inhärenten Instabilität der Marktwirtschaft kumulative Prozesse analysieren, die zu instabilen Situationen führen, während Vertreter der neoklassischen Theorie die Tendenz des sich selbst überlassenen Systems zu einer Steady State-Lösung betonen werden. Prinzipiell sind in beiden Ansätzen diese Ergebnisse möglich.

\subsubsection{Determinanten des Wachstumsprozesses}

Einer der Hauptunterschiede zwischen der Neuen Wachstumstheorie und den Ansätzen Kaldors bzw. der Weiterentwicklungen liegt in den Faktoren, die für den Wachstumsprozeß als entscheidend bzw. beschränkend angesehen werden. In einem Satz könnte verkürzt festgestellt werden: In der Neuen Wachstumstheorie bestimmen angebotsseitige Bedingungen den Wachstumsprozeß, in der kaldorianischen Welt spielen zusätzlich auch nachfrageseitige Zusammenhänge eine Rolle.

Die Vertreter der Neuen Wachstumstheorie stellen wie ihre Vorgänger der Solowschen Tradition die Quantität und Qualität der Produktionsfaktoren in den Mittelpunkt ihrer Untersuchung. Die Wirtschaftssubjekte, genauer gesagt das repräsentative Individuum oder der repräsentative Haushalt, sehen sich einem Set von Produktionsmöglichkeiten gegenüber, aus dem sie entsprechend ihrer Präferenz- bzw. Nutzenfunktion und ihren Kenntnissen über die Akkumulationsfunktionen der im Modell enthaltenen Kapitalarten (Sach-, und/oder Humankapital sowie technologisches Know-how) eine Möglichkeit auswählen, die den Nutzen über den Betrachtungszeitraum hinweg maximiert. Die sich aus diesem Kalkül ergebende Steady StateWachstumsrate des Pro-Kopf-Einkommens wird dabei allgemein durch die Präferenzen der Wirtschaftssubjekte, insbesondere durch die Zeitpräferenzrate, die Effizienzparameter bei der Vermehrung der enthaltenen Kapitalart, die Wachstumsrate des vermehrbaren Produktionsfaktors sowie durch die Anfangsausstattungen nicht wachsender Inputs bestimmt. Die Annahmen über die Produktionstechnik gewährleisten, daß die Steady State-Wachstumsrate positiv ist. Veränderungen in den Präferenzen und/oder Anfangsausstattungen haben entweder direkt oder indirekt über die beschleunigte Akkumulation der verschiedenen Kapitalarten dauerhafte Wirkungen auf die Höhe der Wachstumsrate. Die Rolle der Startbedingungen für die Wachstumsmöglichkeiten führt ein Element der Pfadabhängigkeit ein, das in den traditionellen Modellen nicht vorhanden war. Während im neoklassischen Grundmodell die abnehmende Grenzproduktivität des Kapitals die Existenz positiven Pro-Kopf-Wachstums ohne exogenen technischen Fortschritt verhinderte und die Steady State-Wachstumsrate ausschließlich von der Rate des technischen Fortschritts bestimmt wurde, bedingt in den Modellen der Neuen Wachstumstheorie das endogene Wachstum der verschiedenen Kapitalstöcke positives ProKopf-Wachstum. 
Die Entwicklung der Nachfragebedingungen spielt für die Überlegungen in der Neuen Wachstumstheorie keine Rolle. Alle erzeugten Güter, Kapital- und Konsumgüter, werden vollständig nachgefragt. Überkapazitäten sind nicht möglich, da einerseits für den Arbeitsmarkt die Vollbeschäftigungsannahme getroffen wird und andererseits der komplette ökonomische Prozeß das Resultat von Wirtschaftssubjekten mit rationalen Erwartungen oder vollkommener Voraussicht ist. Die Haushalte werden in einer solchen Welt ihre Erfüllungsgehilfen, die Unternehmen, keine Güter produzieren lassen, die nicht nachgefragt werden.

Im Gegensatz hierzu entscheidet die Entwicklung der Nachfrage in den kaldorianischen Modellen mit über die Wachstumschancen einer Volkswirtschaft. Jedoch ist auch hier zwischen dem frühen und dem späten Kaldor zu unterscheiden. Die rein wachstumstheoretischen Arbeiten enthalten im Unterschied zur neoklassischen Theorie keine Produktionsfunktion im traditionellen Sinne, sondern die technical progress function. Tatsächlich stellt auch diese eine Art Möglichkeitenkurve dar, die festlegt, welches Produktivitätswachstum bei welcher Investitionstätigkeit erreicht werden kann. Die Güternachfrage fließt nur indirekt über die Erwartungen der Investoren in das Modell ein. Die Investitionsfunktion ist abhängig von den erwarteten Nachfrageveränderungen. Langfristig resultiert auch hier ein Wachstumsgleichgewicht, in dem das Pro-Kopf-Wachstum von den Parametern der technical progress function bestimmt wird. Die langfristige Wachstumsrate ist somit durch exogene Parameter bestimmt, d.h. auch diese Volkswirtschaft kann nicht über die von der technical progress function vorgegebenen Grenzen springen. In diesem Sinne ist sie ressourcenbeschränkt und damit neoklassisch.

Der Vorwurf der Beschränkung der Wachstumsmöglichkeiten aufgrund eines Arbeitskräftemangels und damit einer Angebotsbeschränkung wurde gegen die Ausführungen in Kaldors Antrittsvorlesung in Cambridge vorgebracht (vgl. Abschnitt 4.3). Die herausragende Rolle der Wanderung von Arbeitskräften aus dem primären in den sekundären Sektor einer Volkswirtschaft, die Kaldor damals sehr stark betonte, ließ den Eindruck entstehen, daß er die Wachstumsmöglichkeiten als durch das Arbeitsangebot begrenzt ansehen würde. Diese Interpretation wurde von Kaldor später vehement zurückgewiesen. Das Wachstum ist für ihn primär nachfragedeterminiert. Nur wenn die Nachfrage und damit der Output der Volkswirtschaft wächst, können die potentiellen steigenden Skalenerträge realisiert werden. Die Notwendigkeit einer entsprechenden Nachfragereaktion auf die so entstandenen Produktivitätssteigerungen wird jedoch erst in der expliziten Behandlung des Produktivitäts- und des Nachfrageregimes im Rahmen des Modells der kumulativen Verursachung deutlich. Hier ist der Wachstumsprozeß von beiden Seiten abhängig. Je nach Lage und Verlauf der Verdoornund der Nachfragegeraden wird sich die Wachstumsrate entwickeln. Der langfristige Wachstumsprozeß ist damit nicht nur ausschließlich von der Angebotsseite der Volkswirtschaft bestimmt. Das Modell der kumulativen Verursachung ist trotz der Einbeziehung von Nachfragezusammenhängen kein Konjunkturmodell. Vielmehr wird der langfristige Wachstumsprozeß als eine Abfolge von kurzfristigen Ereignissen gesehen. Unterausgelastete Kapazitäten können 
zwar den Wachstumsprozeß aufgrund von Pfadabhängigkeiten beeinflussen, sie sind aber nicht das zentrale Element der Untersuchung. Die in wachstumstheoretischen Untersuchungen im Vordergrund stehende Definition von Wachstum als Steigerung des Produktionspotentials ist auch hier gegeben. Das Zusammenspiel von Nachfrage- und Produktivitätsregime bestimmt die langfristig mögliche Wachstumsrate des Pro-Kopf-Einkommens. Dies wird am besten deutlich in einem stabilen System der kumulativen Verursachung, in dem sich eine konstante Wachstumsrate einstellt. 4

Eng mit der Unterscheidung in nachfrage- und angebotsseitige Determinanten des Wachstumsprozesses einer Volkswirtschaft hängt ein Aspekt zusammen, der in der Neuen Wachstumstheorie zumindest in der formalen Struktur der Modelle außen vor bleibt. Institutionelle und gesellschaftiche Rahmenbedingungen werden nicht betrachtet, während im Modell der kumulativen Verursachung über die Nachfragegleichung Zusammenhänge wie z.B. der Lohnbildungsprozeß oder die Preisbildung berücksichtigt werden. Die Ansätze der neoklassischen Wachstumstheorie vernachlässigen aber nicht nur diesen Aspekt, sondern gehen von stabilen Nutzen- und Produktionsfunktionen aus. Der Wandel essentieller Parameter oder die Reaktion der Präferenzen auf den Wachstumsprozeß stehen nicht zur Debatte. Ganz anders in den Modellen der kaldorianischen Tradition. Die Veränderung der Rahmenbedingungen wird zumindest in der empirischen Überprüfung beachtet. Strukturkonstanz ist damit ausgeschlossen. Der Wachstumsprozeß einer Volkswirtschaft wird zum Ergebnis verschiedener Phasen unterschiedlicher Produktivitäts- und Nachfrageregime.

Zur Verteidigung der Vorgehensweise der Neuen Wachstumstheorie gegenüber derjenigen der Vertreter des Modells der kumulativen Verursachung könnte vorgebracht werden, daß mit Hilfe einer an der langen Frist orientierten Theorie, die dauerhaft gültigen Beziehungen abgebildet werden sollen. Die neoklassische Analyse berücksichtigt deshalb nur die Aspekte, die langfristig Bestand haben. Die Veränderungen aller anderen Größen sind nicht Gegenstand einer wachstumstheoretischen Untersuchung. Dem können zwei Argumente entgegengehalten werden. Wenn langfristige Trends untersucht werden sollen, so kann mit Sicherheit davon ausgegangen werden, daß sowohl die Produktionstechnik als auch die Präferenzen im Betrachtungszeitraum nicht konstant sein werden. Solows' Kritik am Harrod-Modell richtete sich ja gerade gegen die Annahme der Inflexibilität des Kapitalkoeffizienten und der Kapitalintensität im Rahmen eines Wachstumsmodells. Langfristig sind die Einsatzverhältnisse variabel. Diese Kritik kann zurückgegeben werden. In der langen Frist können sich nicht nur die Faktoreinsatzverhältnisse bei Gültigkeit einer einzigen Produktionsfunktion verändern, sondern es kommt durch technischen Fortschritt zu einer ständigen Verschiebung der Produktionsfunktion. Zusätzlich ist es aber auch möglich, daß die Produktionsfunktion ein neues Aussehen erhält. Ein Wachstumsmodell müßte deshalb zumindest eine endogen bestimmte Produktions-

4 Vgl. z.B. Skott (1990, S. 273). 
technologie aufweisen. Dieselben Einwände können auch gegen die Konstanz der Präferenzen der Wirtschaftssubjekte vorgebracht werden. ${ }^{5}$

Ein zweites Argument gegen die Vernachlässigung von strukturellen Veränderungen kann mit Lock-in-Effekten und Pfadabhängigkeiten begründet werden. Autoren wie Arthur (1994), David (1985) oder Krugman (1991) haben gezeigt, daß bei Vorliegen von steigenden Skalenerträgen in der Produktion bzw. bei der Nutzung von Gütern multiple Gleichgewichte möglich sind. Welches von diesen realisiert wird, ist abhängig von "historischen" Entscheidungen. Auf dem Markt für PC-Prozessoren legte sich z.B. IBM zu Beginn des PC-Zeitalters auf die Technologie von Intel und nicht auf die von Motorola fest. Technisch gesehen war der MotorolaChip zwar wesentlich leistungsfähiger, jedoch sorgte die Entscheidung von IBM für eine schnelle Diffusion der Intel-Technologie. Aufgrund steigender Skalenerträge in der Herstellung und Netzwerkexternalitäten für die Nutzer gewann die schwächere Technik den Markt für sich. ${ }^{6}$ Mittel- und langfristige Ergebnisse von Marktprozessen können die Folge einer singulären Entscheidung sein. Übertragen auf Wachstumsprozesse impliziert diese Überlegung die Abhängigkeit der langfristigen Wachstumsrate von kurzfristigen Veränderungen, d.h. die Variationen in den Rahmenbedingungen können das System auf einen neuen Wachstumspfad bringen. Die "Geschichte" einer Volkswirtschaft wird hierbei zu einem entscheidenden Faktor.

Die Relevanz der Vergangenheit für die Zukunft wurde von Kaldor immer wieder betont. Sowohl das Konzept der technical progress function (vgl. Abschnitt 4.2) als auch die Ausführungen Kaldors zum Verdoorn-Zusammenhang (vgl. Abschnitt 4.3 und 4.5) bilden dieses Prinzip ab, das ein Kernelement der Theorie der kumulativen Verursachung darstellt. Hier liegt ein weiterer Unterschied zwischen der kaldorianischen und der neoklassischen Analyse von Wachstumsprozessen. Dieser Unterschied ergibt sich hauptsächlich durch die Festlegung der Modelle der Neuen Wachstumstheorie auf die Analyse von Steady State-Situationen. Tatsächlich sind historische Elemente durchaus enthalten. Die Annahme intertemporaler externer Effekte von Investitionsentscheidungen, die von Arrow (1962) eingeführt wurde und in der Neuen Wachstumstheorie sehr häufig zum Tragen kommt, verbindet den heutigen Erfolg von Investitionen mit den Entscheidungen, die in Vorperioden getroffen wurden. Auch die Abhängigkeit der Steady State-Wachstumsrate von den Präferenzen der Wirtschaftssubjekte kann als Beleg für die Integration der Bedeutung "historischer" Entscheidungen verstanden werden. Eine Veränderung der Präferenzstruktur hat dauerhafte Konsequenzen auf die Steigung des Wachstumspfades. Würde zusätzlich die für die Erreichung eines Steady State-Wachstumspfades notwendige Bedingung der konstanten Skalenerträge für die akkumulierbaren Produk-

5 Hier stellt sich natürlich sofort die Frage nach der Endogenităt und Exogenităt der Determinanten, die eine Veränderung der Produktionstechnologie bedingen. Vgl. auch Skott/Auerbach (1995, S. 390).

6 Netzwerkexternalităten sind bestimmt durch die Zahl der Anwender einer Technologie. Sind z.B. zwei Computerstandards verfugbar, wird die Entscheidung, welches System sich ein Neueinsteiger kauf, nicht nur von den direkten Vorteilen des Standards bestimmt, sondern auch von den indirekten. Nutzt schen eine große Zahl von Anwendern ein System, wird ein Neueinsteiger dieses auswăhlen, wenn der Nutzen aus der Kompatibilităt mit anderen Computern die rein technischen Vorteile des zweiten Systems überwiegt. 
tionsfaktoren aufgegeben, könnten auch in den Modellen der Neuen Wachstumstheorie kumulative Prozesse abgebildet werden und die Aussage: "History matters" würde noch mehr Gültigkeit erhalten.

\subsubsection{Gemeinsamkeiten}

In den vorigen Abschnitten wurden mehrere Unterschiede zwischen der Neuen Wachstumstheorie und den kaldorianischen Vorstellungen über Wachstumsprozesse diskutiert, die teilweise jedoch eher durch die Zielsetzungen und Annahmen der Autoren als durch die Leistungsfähigkeit der jeweiligen Modelle entstanden sind. Im folgenden wird auf zwei Aspekte eingegangen, die man durchaus als verbindende Elemente, wenn nicht sogar als Gemeinsamkeiten zwischen beiden Gedankengebäuden ansehen kann.

\subsubsection{Die Rolle der Externalitäten}

Die mit Sicherheit auffälligste Gemeinsamkeit zwischen der Neuen Wachstumstheorie und Kaldors Ausführungen ist die Einbeziehung externer Effekte von Investitionsentscheidungen. In Kapitel 3 wurde bei der Diskussion der Vorgehensweise innerhalb der Neuen Wachstumstheorie die entscheidende Rolle von Externalitäten für den Wachstumsprozeß verdeutlicht. Eine Investitionsentscheidung hat nicht nur Auswirkungen auf das Unternehmen, in dem die Investition erfolgt, sondern auch auf andere Investoren. Diese Überlegung kann auf alle Arten des Kapitals übertragen werden, d.h. die wachstumsfördernden Wirkungen können mit dem Sach-, dem Humankapital oder dem gesamtwirtschaftichen Bestand an technologischem Wissen verknüpft werden.

Die Ursache der Externalitäten liegt im öffentlichen Gutscharakter des Wissens. Im Modell von Arrow führen investitionsinduzierte Lemprozesse zur Erhöhung des technologischen Know-hows, das in den Kapitalgütern der Folgegenerationen enthalten ist. Die zum Zeitpunkt $(\mathrm{t}=0)$ getroffene Investitionsentscheidung hat damit einen externen Effekt auf alle Investitionen zu späteren Zeitpunkten. Die Überlegung, daß durch Investitionen der Bestand des Wissens steigt, von dem andere Wirtschaftssubjekte nicht ausgeschlossen werden können, findet sich in allen Modellen der Neuen Wachstumstheorie wieder. Sie diente der Erklärung für die Konstanz des Grenzprodukts der vermehrbaren Faktoren. Die Modelle, die mehrere Kapitalgüter einführen, benötigen diese Annahme nicht zwingend, jedoch müssen insgesamt konstante Skalenerträge für diese Kapitalarten angenommen werden und die Produktion der entsprechenden Kapitalgüter muß ebenfalls konstanten Skalenerträgen unterliegen. Externe 
Effekte sind ein Mittel zur Generierung endogenen Wachstums. Ihre Existenz kann mit Sicherheit nicht geleugnet werden, ihr tatsächliches Ausmaß ist jedoch schwierig zu belegen. ${ }^{7}$

In den kaldorianischen Vorstellungen findet sich ebenfalls das Konzept externer Effekte wieder. Produktivitätsfortschritte sind im Konzept der technical progress function das Ergebnis des exogenen technischen Fortschritts, unter den auch organisatorische Verbesserungen aufgrund von Lerneffekten fallen, und der Investitionstätigkeit. Die Lage und der Verlauf der technical progress function sind abhängig von den bisher getätigten Investitionen, die den technical dynamism einer Volkswirtschaft mitbestimmen. Aufgrund dieser Beziehung haben Investitionen in vergangenen Perioden eine Wirkung auf die Eigenschaften heutiger. Je mehr der technical dynamism der Volkswirtschaft als Folge vorangegangener Kapitalbildung ausgeprägt ist, um so höhere Produktivitätsfortschritte können bei einer gegebenen Wachstumsrate der Investitionen erzielt werden. Die Akkumulation von Kapital führt hier ebenfalls zur Vergrößerung des Bestands eines öffentlichen Gutes, dem technical dymamism. Die Grundüberlegung Arrows und vieler Vertreter der Neuen Wachstumstheorie findet sich somit in Grundzügen in den wachstumstheoretischen Modellen von Kaldor. ${ }^{8}$

Die Relevanz der externer Effekte kommt in der Diskussion zum Verdoorn-Zusammenhang noch deutlicher ans Licht. Kaldor war in seinen späten Arbeiten davon überzeugt, daß der Wachstumsprozeß marktwirtschaftlich organisierter Volkswirtschaften dem Prozeß einer kumulativen Verursachung folgt. Ein zentrales Element dabei ist der Verdoorn-Zusammenhang als Ausdruck für steigende Skalenerträge im industriellen Sektor. Diese setzen sich nach Kaldors Auffassung aus statischen Skalenerträgen, d.h. aus reinen Größenvorteilen und aus dynamischen Skalenerträgen zusammen. Letztere werden durch die steigende Arbeitsteilung erzeugt, die auf Unternehmens- und industrieller Ebene stattfindet. Die zunehmende Aufteilung der Arbeitsprozesse führt zu Lerneffekten (Arrow), zur Erfindung neuer Maschinen und Anlagen (Smith), macht den Einsatz von Maschinen überhaupt erst lohnend und führt zum Entstehen neuer Industrien (Young). Die mit der Arbeitsteilung einhergehende Kapitalintensivierung bedingt nun entsprechend der technical progress function Produktivitätswachstum aufgrund der Integration des vorhandenen technologischen Wissens in die Produktion. Gleichzeitig ist mit der zunehmenden Kapitalintensivierung ein Anstieg des technologischen Wissens verbunden (Marshall), welches öffentlichen Gutscharakter hat. Die zunehmende Arbeitsteilung führt in Verbindung mit dem gestiegenen Kapitaleinsatz zu endogenem technischen Fortschritt. Der Verdoorn-Zusammenhang, in der Form, wie er von Kaldor verstanden wurde, stellt somit einen Vorläufer der Neuen Wachstumstheorie dar. Diese Verwandtschaft erklärt sich nicht nur aus der Tatsache, daß sowohl für den Verdoorn-Zusammenhangs als auch in der Neuen Wachstumstheorie das Phänomen steigender Skalenerträge eine Rolle spielt, sondern auch die

7 An dieser Stelle sei noch einmal ein Hinweis gemacht. Die in der Neuen Wachstumstheorie auftauchenden Externalităten sind nicht mit dem Konzept des learning by doings gleichzusetzen. Learning by doing ist ein Weg zu neuem Wissen, welches dann allgemein genutzt werden kann.

8 Vgl. Abschnitt (4.2). 
Begründung Kaldors für die Existenz des Verdoorn-Zusammenhangs ist mit der Erklärung endogenen Wachstums in den aktuellen Modellen der neoklassischen Wachstumstheorie identisch.

\subsubsection{Die Rolle der Investitionen}

Mit den im vorangegangenen Abschnitt getätigten Überlegungen ist ein weiterer Aspekt verbunden, der die Neue Wachstumstheorie und kaldorianische Vorstellungen miteinander verbindet. Im traditionellen neoklassischen Wachstumsmodell hatte die Investitionsquote keinerlei Einfluß auf die langfristige Wachstumsrate, da der Kapitaleinsatz dem Gesetz vom abnehmenden Grenzertrag unterlag. Der Weg zur Überwindung dieser Schwäche war der exogene technische Fortschritt, der dafür sorgte, daß die Unternehmen mehr investierten als für die Aufrechterhaltung einer konstanten Kapitalintensität bei einer wachsenden Bevölkerung notwendig war. Es ist die Funktion des exogenen technischen Fortschritts, mittels seiner Produktivitätseffekte den Investitionsanreiz für die Unternehmen zu erhalten.

In den Modellen der Neuen Wachstumstheorie erfullen diese Aufgabe die externen Effekte der Investition in die verschiedenen Kapitalarten und/oder die Annahme der konstanten Skalenerträge der vermehrbaren Produktionsfaktoren. Die Konstanz der (Netto)Ertragsrate des Kapitaleinsatzes sorgt dafür, daß sich diese nicht an die soziale Diskontrate annähert. Die zukünttigen Erträge aus der Ausdehnung des Kapitalstocks pro Arbeitseinheit sind deshalb immer größer als der Nutzenentgang aus dem für die Investition notwendigen Konsumverzicht. Der Wachstumsprozeß ist folglich durch eine ständig steigende Kapitalintensivierung gekennzeichnet. Sehr deutlich wird dies anhand von Modellen, die von einer konstanten Bevölkerung ausgehen, wie z.B. Romer (1990b, c), oder im Standard AK-Modell. ${ }^{9}$ Das endogene Wachstum des Pro-Kopf-Einkommens und der Arbeitsproduktivităt ist die Folge der Investitionstätigkeit. Dieser Zusammenhang ist unabhängig von der Art des Kapitals, in das investiert wird. Aufgrund dieser Überlegung können die verschiedenen Kapitalformen zumindest bildlich gesprochen entsprechend eines Aspekts aggregiert werden: unabhängig davon, ob es sich um Sachkapital, Humankapital oder technologisches Wissen handelt, für endogenes Wachstum muß sich der Bestand des im jeweiligen Modell enthaltenen Kapitals pro Arbeitseinheit erhöhen.

Diese Eigenschaft der Ansätze der Neuen Wachstumstheorie treibt einen Keil zwischen die Wirkungen des Kapitals im Modell und den Inhalten, die gemeinhin mit einem bestimmten Kapitalbegriff verbunden werden. Für die Generierung endogenen Wachstums ist es uninteressant, welche Kapitalsorte man verwendet. Sie muß nur vermehrbar sein und muß gesamtwirtschaftlich konstante Skalenerträge aufweisen bzw. es muß bei ihrer Akkumulation ein

9 Vgl. Abschnitt (3.3.2.2). 
externer Effekt auftreten, der zu Differenzen zwischen der privaten und sozialen Ertragsrate führt. Obwohl begrifflich getrennt, werden alle Kapitalarten vergleichbar. Der einzige Unterschied besteht in der Tatsache, daß das Kapitalgut Know-how nicht vollständig von demjenigen angeeignet werden kann, der den Bestand des Wissens vermehrt hat.

Der Fortschritt gegenüber dem neoklassischen Modell ist, daß sich die Investitionen in den Modellen der Neuen Wachstumstheorie den Anreiz zur ständigen Kapitalintensivierung über die externen Effekte selbst schaffen. Man könnte daher von einem Münchhausen-Effekt sprechen. Jede Investition bewirkt eine Erhöhung des frei verfügbaren Gutes Wissen, das die Ertragsrate des Kapitals konstant hält. Die Investoren ziehen sich damit selbst aus dem "Sumpf" der abnehmenden Grenzproduktivität der Kapitalbildung. Aufgrund der herausragenden Bedeutung der Investitionstätigkeit erweist sich das Fehlen einer eigenständigen Investitionsfunktion in den Modellen der Neuen Wachstumstheorie als ein großer Mangel.

Die zentrale Bedeutung des Investitionsprozesses für die Wachstumsmöglichkeiten einer Volkswirtschaft wurde von Kaldor immer wieder betont. Seine Kritik am Konzept der neoklassischen Wachstumstheorie alter Prägung baut im wesentlichen auf der Ablehnung des Konzepts der Produktionsfunktion auf. Die Unmöglichkeit der Trennung zwischen Kapitalintensivierung und technischem Fortschritt führt die Annahme exogener Produktivitätssteigerungen ad absurdum. Produktivitätswachstum macht die Erhöhung der Kapitalausstattung pro Kopf notwendig, wenn man vom Ordinatenabschnitt der technical progress function einmal absieht. Kaldors Verständnis des Verdoorn-Zusammenhangs beinhaltet ebenfalls diese Überlegungen. Die Erhöhung der Arbeitsproduktivität ist untrennbar mit der Vergrößerung der Kapitalausstattung pro Kopf verbunden. Wie oben angeführt, bedingt eine steigende Arbeitsteilung die Ausdehnung des Kapitalstocks, die damit verbundenen Investitionen bringen das neueste technologische Wissen in den Produktionsprozeß ein und induzieren gleichzeitig die Entstehung neuen Wissens.

In beiden Sichtweisen ist die Produktivitätsentwicklung eine Funktion der Investitionstätigkeit, genauer gesagt der Kapitalintensivierung. Diese Ähnlichkeit wird noch deutlicher, wenn man für den Fall eines AK-Modells die Beziehung zwischen der Wachstumsrate der Kapitalintensivierung und dem Output pro Kopf betrachtet, d.h. eine technical progress function erster Generation ableitet. Für das AK-Modell galt folgende Produktionsfunktion

$$
\mathrm{Y}=\mathrm{AK}
$$

und die Wachstumsrate der Kapitalintensität ergab sich durch Gleichung (3.22)

$$
\frac{\dot{\mathrm{k}}}{\mathrm{k}}=\mathrm{sA}-(\mathrm{n}+\delta)
$$


Die Wachstumsrate der Kapitalintensität ist in diesem Modell immer gleich der Wachstumsrate der Arbeitsproduktivität:

$$
\frac{\dot{\mathrm{y}}}{\mathrm{y}}=\frac{\dot{\mathrm{k}}}{\mathrm{k}}
$$

Überträgt man (3.22) und (5.1) in ein gemeinsames Schaubild ergibt sich Abbildung (5.1).

\section{Abb. 5.1: Graphische Darstellung der Wachstumsrate der Kapitalintensität im AK- Modell}

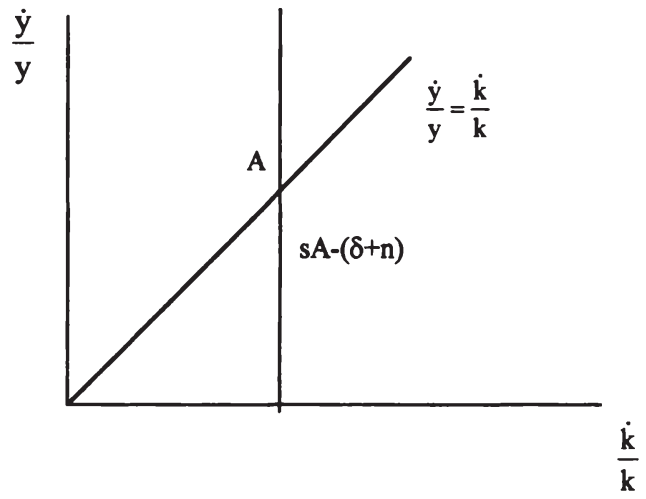

Quelle: eigene Darstellung

Die erste Winkelhalbierende könnte in diesem Fall als eine Art technical progress function interpretiert werden, die keinen Ordinatenabschnitt aufweist. Welche Wachstumsrate der Kapitalintensität und des Pro-Kopf-Outputs letztendlich gewählt wird, entscheiden die Sparer mit ihrer Sparquote (Punkt A). ${ }^{10}$ Sie können aus einem Angebot an verschiedenen Wachstumspfaden auswählen, das durch die Punkte auf der ersten Winkelhalbierenden gegeben ist. Bei mehr als einem vermehrbaren Produktionsfaktor, z.B. Sachkapital und Humankapital, ist das Pro-Kopf-Wachstum das Ergebnis der Erhöhung der Einsatzmengen beider Produktionsfaktoren. Die Wachstumsraten des Humankapitals und des Sachkapitals definieren zusammen die Wachstumsrate des Outputs. Bei gegebenem Arbeitseinsatz bestimmen die eingesetzten Human- und Sachkapitalmengen die Produktionsmenge. Ein zusätzlicher Anstieg der verfügbaren Arbeitsmenge hat vergleichbare Wirkungen mit der Erhöhung des technologischen

$10 \mathrm{Um}$ einen positiven Ordinatenabschnitt zu erhalten, hătte exogener technischer Fortschritt angenommen werden mussen, der die Arbeitsproduktivităt auch ohne Kapitalintensivierung steigen ließe. 
Wissens im Grundmodell. Die Produktionsfunktion wird bei jedem Kapitaleinsatz einen höheren Wert aufweisen. 11

An dieser Stelle wird die Rolle der Größe einer Volkswirtschaft noch einmal deutlich. In den Modellen der Neuen Wachstumstheorie wird vielfach entweder von einer konstanten Ausstattung mit Arbeitskräften bzw. mit Humankapital ausgegangen (z.B. Romer 1986, 1990b, c Grossman/Helpman 1991 oder Aghion/Howitt 1992a) oder es gibt nur einen Produktionsfaktor Kapital wie im AK-Modell. Die Lösungen für die gleichgewichtigen Wachstumsraten in der ersten Gruppe haben gezeigt, daß diese um so höher sind, je größer die Anfangsausstattung mit Arbeit oder Humankapital ist. Im AK-Modell bestimmte das Bevölkerungswachstum die Höhe der langfristigen Wachstumsrate mit. Wenn die Anfangsausstattung jedoch stetig wächst oder Arbeit als weiterer Produktionsfaktor in die Produktionsfunktion im AKModell eingefürt wird, ist Steady State-Wachstum nicht mehr möglich, da zu dem Wachstumsbeitrag des Kapitals auch noch das Wachstum eines weiteren Produktionsfaktors hinzukommt. Die Produktionsfunktion hätte in Verbindung mit der Annahme der konstanten Skalenerträge des (Human)Kapitals bzw. Wissens insgesamt steigende Skalenerträge bzgl. aller vermehrbaren Inputs. Die Wachstumsrate wäre nicht mehr stabil. Die Konstanz der Arbeitsmenge bzw. die Nichtberücksichtigung der Arbeit als Produktionsfaktor ist eine notwendige Annahme für die Stabilität des Modells.

Beim Konzept der technical progress function stellte diese die Möglichkeiten zu Produktivitätssteigerungen in Abhängigkeit von der Wachstumsrate der Kapitalintensität dar. Im Falle der Neuen Wachstumstheorie rückt an die Stelle der traditionellen Pro-Kopf-Produktionsfunktion ebenfalls eine Beziehung zwischen der Wachstumsrate der Kapitalintensität und der Wachstumsrate des Pro-Kopf-Outputs. Die Wirtschaftssubjekte bestimmen entsprechend ihrer Präferenzen, welchen Pfad der Kapitalbildung sie bevorzugen und bestimmen damit auch die Wachstumsrate der Pro-Kopf-Größen. Im traditionellen neoklassischen Modell bestimmte die Rate des exogenen technischen Fortschritts die Wachstumsrate der Kapitalintensität und des Pro-Kopf-Einkommens auf dem Steady State-Pfad. In Kaldors Modellen legten ebenfalls vorgegebene Größen, die Parameter der technical progress function, die mögliche Wachstumsrate der Arbeitsproduktivität und der Kapitalintensität fest. Die gleichgewichtige Lösung ist in der Neuen Wachstumstheorie das Ergebnis der Präferenzen der Wirtschaftssubjekte, während sie bei Kaldors Wachstumsmodellen unabhängig von der unterstellten Investitionsfunktion determiniert ist. Nur wenn man die Verschiebung der technical progress function aufgrund des vergangenen Investitionsverhaltens zuläßt, wäre auch hier eine endogene Festlegung des Gleichgewichts erreicht. In den Ansätzen, die auf dem Prinzip der kumulativen Verursachung basieren, ist jedoch eine endogene Bestimmung der Wachstumsrate gewähr-

11 Im neoklassischen Grundmodell definierten der Arbeits- und der Kapitaleinsatz eine Ebene, uber die sich die Produktionsfunktion wölbte. Im Falle der Neuen Wachstumstheorie bestimmen die Einsatzmengen der Kapitalarten diese Flăche. Mehr Arbeit verschiebt die "Decke", d.h. die Produktionsmoglichkeiten nach oben. 
leistet. Die wechselseitigen Reaktionen der Nachfrage- und Produktivitätsveränderungen legen die Entwicklung der Wachstumsrate des Pro-Kopf-Einkommens bzw. der Arbeitsproduktivität fest.

In beiden Ansätzen stellt die Akkumulation die Ursache des Produktivitätswachstums dar. Durch die Konzentration auf externe Effekte ist es der Neuen Wachstumstheorie gelungen, die im ursprünglichen neoklassischen Wachstumsmodell notwendige exogene Verschiebung der Produktionsfunktion zu überwinden. Technischer Fortschritt verstanden als Steigerung der Arbeitsproduktivität ist nicht länger getrennt von der Investitionsentscheidung und die Präferenzen beeinflussen die langfristige Wachstumsrate. Im kaldorianischen Modell ermöglicht erst die Hinzunahme der Nachfrageseite die Endogenität der Wachstumsrate.

\subsubsection{Fazit}

Die vergleichenden Ausführungen zur Neuen Wachstumstheorie und kaldorianischen Vorstellung über Wachstumsprozesse haben gezeigt, daß sich insbesondere bei den methodischen Vorgehensweisen und der prinzipiellen Sichtweise marktwirtschaftlicher Systeme weiterhin große Unterschiede abzeichnen. Auch die Frage nach den wichtigsten Determinanten von Wachstumsprozessen wird auf verschiedene Art und Weise beantwortet. Trotz dieser Differenzen drückt sich in der Einbeziehung von externen Effekten in die Modelle der Neuen Wachstumstheorie und die daraus resultierende Relevanz der Spar- und Akkumulationsentscheidung furr die langfristige Rate des Wachstums der Pro-Kopf-Größen eine Annäherung an die Überlegungen von Kaldor und seiner Nachfolger aus.

Darüber hinaus können die Analysen und Ergebnisse der Neuen Wachstumstheorie auch für das Modell der kumulativen Verursachung hilfreiche Anregungen geben. Obwohl die Hypothesen der Neuen Wachstumstheorie empirisch schwierig zu überprüfen sind, verdeutlichen sie mögliche Determinanten des Produktivitätswachstums innerhalb einer Volkswirtschaft. Das Set an Beziehungen, das für die Schätzung des Produktivitätsregimes verwendet wird, könnte erweitert werden, indem z.B. neben Variablen für die Forschungs- und Entwicklungsanstrengungen auch Humankapitalindikatoren eingeführt werden.

Die unterschiedlichen Vorgehensweisen in beiden Ansätzen bieten zusätzlich die Möglichkeit, Wachstumsprozesse aus unterschiedlichen Perspektiven zu betrachten. Insbesondere für wachstumspolitische Maßnahmen ist dies von Bedeutung. Die ausschließliche Konzentration auf angebots- oder nachfrageseitige Strategien zur Förderung von Wachstum greift zu kurz. Die Reaktionen der Wirtschaftspolitik auf die Folgen der Ölpreisverteuerung sind nur ein Beispiel für die Konsequenzen einer einseitigen Sichtweise des Wirtschaftsprozesses. Im nächsten Abschnitt wird deshalb auf mögliche Schlußfolgerungen eingegangen, die sich unter Einbeziehung beider Sichtweisen ergeben. 


\subsection{Lehren für die Wachstumspolitik?}

Die Auseinandersetzung mit Modellen der Wachstumstheorie sollte auch eine Diskussion möglicher Konsequenzen der Ergebnisse der theoretischen Analyse für wirtschaftspolitische Fragestellungen beinhalten. Selbstverständlich muß dabei eine Übertragung der Ergebnisse der theoretischen Untersuchungen überdacht werden, da in Modellen mit einem so hohen Aggregationsgrad zu viele Aspekte außen vor bleiben müssen. Es sind aber zumindest Ansatzpunkte für wachstumspolitische Maßnahmen benennbar.

\subsubsection{Die Neue Wachstumstheorie}

Auf Seiten der neoklassischen Theorie werden wirtschaftspolitische Eingriffe in das Marktgeschehen traditionell skeptisch betrachtet. Auch das traditionelle Wachstumsmodell macht keine Ausnahme. Die Unabhängigkeit der langfristigen Wachstumsdynamik von Spar- und Investitionsentscheidungen und die Annahme exogenen technischen Fortschritts scheinen eine Beeinflussung des Wachstumsprozesses zu verbieten. Für reale Entwicklungen spielt aber nicht nur die Steady State-Situation eine Rolle, sondern auch die Aussage des Modells, daß durch einen Anstieg der Spar- bzw. Investitionsquote das Niveau des Wachstumspfades erhöht werden kann. Maßnahmen zur Investitionsförderung würden zumindest in Anpassungsphasen Wirkungen zeigen. Auch die Rate des technischen Fortschritts kann durch Forschungspolitik erhöht werden, die zur Entwicklung effizienterer Produktionsverfahren führt. Hierunter können auch bildungspolitische Maßnahmen subsumiert werden. Der Wachstumspolitik kommt auch im neoklassischen Grundmodell eine gewisse Aufgabe und Gestaltungsmöglichkeit zu. ${ }^{12}$

Im Gegensatz hierzu bergen die Modelle der Neuen Wachstumstheorie sehr viele Hinweise auf wachstumspolitischen Spielraum. ${ }^{13}$ Die Existenz von externen Effekten legt den Einsatz von Instrumenten zu ihrer Internalisierung nahe. Im Falle positiver Externalitäten würde dies eine Subvention der entsprechenden Investitionstätigkeit bedeuten. ${ }^{14}$ Sämtliche betrachteten Modelle, bis auf diejenigen zur Verbesserung der Produktqualität, zeigten, daß die sozial optimale Wachstumsrate von derjenigen eines Systems ohne allwissenden Planer nach oben abweicht. Unter Wettbewerbsbedingungen würde zu wenig investiert. Dieses Ergebnis tauchte bekanntlich schon im Modell von Arrow (1962) auf und ist kein spezifisches Ergebnis der Neuen Wachstumstheorie. Durch einen staatlichen Eingriff könnte nun die Wachstumsrate

12 Vgl. z.B. auch Solow (1992b, S. 6)

$13 \mathrm{Zu}$ den wirtschaftspolitischen Implikationen der Neuen Wachstumstheorie vgl. z.B. auch Stern (1992), Shaw (1992), Stolpe (1993), Paqué (1993, 1995), Straßberger (1995).

14 Internalisierungsstrategien auf Basis des Coase-Theorems unterliegen schon in der statischen Betrachtung einer Vielzahl von Einschrănkungen, so daß ihre Übertragung auf die Probleme der Neuen Wachstumstheorie an dieser Stelle nicht diskutiert wird. Vgl. zum Coase-Theorem Boadway/Wildasin (1984, S. 128ff.), Coase (1960), Tirole (1992, S. 49f.). 
der Volkswirtschaft gesteigert werden. Tatsächlich steht eine solche Strategie vor dem Problem, daß das Ausmaß der externen Effekte bekannt sein muß, damit die Internalisierung zum Optimum führt.

Neben dem Ausmaß der externen Effekte muß auch deren Art geklärt werden. Für die "richtige" Wirtschaftspolitik ist es wichtig, ob technologische oder pekuniäre Externalitäten vorliegen. Während technologische externe Effekte nicht über das Preissystem erfaßt werden, verändern pekuniäre externe Effekte die Preisstruktur. Unter Gesichtspunkten der statischen Pareto-Effizienz machen die zuletzt genannten keine Maßnahmen zur Internalisierung notwendig. Die Überlegungen zu den Konsequenzen der steigenden Arbeitsteilung und der damit verbundenen Kapitalintensivierung haben gezeigt, daß unter dynamischen Aspekten auch diese Art der externen Effekte die Dynamik einer Volkswirtschaft bestimmen. So ermöglichen beispielsweise sinkende Preise für Kapitalgüter höhere Investitionen und damit ein gestiegenes Produktivitätsniveau sowie induzierte Lerneffekte, die wiederum technologische Externalitäten darstellen. Beispiele für pekuniäre Externalitäten waren die sinkenden Kosten für Kapitalgüter im Modell von Romer (1986b, 1987) zur Spezialisierung sowie der Konsumentenrenten- und der Profitzerstörungseffekt im Modell von Grossman/Helpman (1991). Beide Fälle zeigten, daß die Vernachlässigung dieser Wirkungen zu einer zu geringen Akkumulationstätigkeit führen. Wird durch die Investitionstätigkeit der allgemein verfügbare Stand des technologischen Wissens vermehrt, liegen dagegen technologische Externalitäten vor. Die Differenzierung zwischen pekuniären und technologischen externen Effekten ist für die Art der Internalisierung relevant, nicht jedoch für die Klärung der Frage nach ihren Folgen für den Wachstumsprozeß. 15

Eine Wachstumspolitik, die auf die Internalisierung externer Effekte setzt, wird durch einen weiteren Sachverhalt erschwert. Nur solange eine einzige Externalität im Modell enthalten ist, kann eine Aussage über die Konsequenzen für die gleichgewichtige Wachstumsrate gemacht werden. Liegen dagegen wie im Fall der Modelle mit Qualitätsverbesserungen mehrere Wirkungen des individuellen Verhaltens auf andere Wirtschaftssubjekte vor, kann das Ergebnis des freien Spiels der Marktkräfte eine zu niedrige oder eine zu hohe Wachstumsrate im Vergleich zum sozialen Optimum sein. Eine erfolgreiche Internalisierungsstrategie steht damit vor einem großen Informationsproblem. Selbstverständlich wird dieser Aspekt von den Kritikern wirtschaftspolitischer Maßnahmen besonders hervorgehoben. Weshalb sollte staatlichen Stellen gelingen, wozu die direkt involvierten Unternehmen nicht in der Lage sind? Nämlich die volle Tragweite ihres individuellen Handelns zu erkennen, um so aufgrund der positiven externen Effekte mehr zu investieren. ${ }^{16}$

Die konkrete Wachstumspolitik muß aber an diesem Informationsproblem nicht scheitern. Die Internalisierung von Externalitäten wird ein höheres Aktivitätsniveau bei der Tätigkeit

15 Vgl. auch Paqué (1995).

16 Vgl. z.B. Paqué (1992, S. 276f., 1995, S. 250f.), Stolpe (1993, S. 369). 
bewirken, die sie erzeugen. Davon zu unterscheiden ist aber die Ausnutzung der vorhandenen Externalitäten. Der Wissenstransfer zwischen den Unternehmen oder zwischen Universitäten und den Unternehmen kann z.B. gefördert werden, indem Kommunikationsmöglichkeiten geschaffen werden. Technologieparks wie die japanischen Technopolies sind ein Beispiel für solche Maßnahmen. Es müssen staatlicherseits ausreichend Infrastrukturinvestitionen getätigt werden, um die Diffusion neuen Wissens zu ermöglichen.

Wichtige Komponenten für den Wachstumsprozeß waren aber nicht nur die externen Effekte der Kapitalbildung, sondern auch die Bedingungen, unter denen diese stattfindet. Im ersten Modell von Lucas (1988) und in Romers Modell (1990b) bestimmte z.B. die Effizienz der Aus- und Weiterbildungsmaßnahmen bzw. des Forschungssektors die langfristige Wachstumsrate. Wirtschaftspolitische Instrumente, die in diesem Bereich ansetzen, können dauerhafte Wirkungen zeigen. Eine Senkung der Ausgaben für Bildungszwecke wäre in diesem Zusammenhang kontraproduktiv. Die daraus ableitbare Förderung der Effizienz des Forschungs- und Bildungssektors einer Volkswirtschaft entspricht dem Ergebnis der traditionellen Wachstumstheorie, in der die Erhöhung der Rate des technischen Fortschritts zum gleichen Ergebnis führte.

Eng mit dieser Überlegung ist ein weiterer Aspekt verbunden, der vor allem in den Ansätzen in der Tradition Schumpeters deutlich wird. Die Bereitschaft, das Risiko einer Investition in Forschungs- und Entwicklungsaktivitäten einzugehen, wird maßgeblich von dem Ertrag bestimmt, der aus dieser Handlung gezogen werden kann. Ein wichtiger Faktor ist in diesem Zusammenhang der Grad der Monopolmacht und die Zeitspanne, in der ein Unternehmen als Monopolist Extraprofite erwirtschaften kann. Höhere Gewinnerwartungen werden höhere Investitionen zur Folge haben, d.h. je besser ein Monopol geschützt ist, um so mehr sind die Investoren willens, ein solches zu erreichen. Ein zu umfangreicher Schutz des Monopolisten vor unliebsamen Konkurrenten ist aber nicht nur aufgrund der Ergebnisse der statischen Mikroökonomie bedenklich, sondern verhindert u.U. die positiven Wirkungen des Monopols in der dynamischen Betrachtung. Wenn es furr andere Unternehmen aussichtslos erscheint, ein bestehendes Monopol aufzubrechen, werden möglicherweise die Anreize zur Forschung und Entwicklung zum Erliegen kommen. Staatliche Aktivităten zur Förderung der Entstehung von neuen Produkten müssen deshalb das Problem eines optimalen Patentschutzes lösen.

Die Ausgestaltung konkreter wirtschaftspolitischer Maßnahmen auf Basis der Ergebnisse der Neuen Wachstumstheorie erweist sich aufgrund der angesprochenen Probleme als recht schwierig, dennoch sind einige generelle Schlußfolgerungen möglich. Der Vergleich der verschiedenen Modelle der Neuen Wachstumstheorie hat die überragende Bedeutung der Investitionen für den Wachstumsprozeß gezeigt. Eine Wachstumspolitik im Sinne dieses Resultats wird an der Förderung der Akkumulation der verschiedenen Kapitalformen ansetzen müssen. $\mathrm{Da}$ die Präferenzen der Wirtschaftssubjekte über den Wachstumspfad mitentscheiden, scheint 
eine Sparförderpolitik, die die Zeitpräferenz der Wirtschaftssubjekte senkt, eine optimale wachstumspolitische Maßnahme. Tatsächlich ist dies nur in einem Modell sinnvoll, in dem das Sparen automatisch zum Investieren wird. ${ }^{17}$ Der Glaube an den durchschlagenden Erfolg einer solchen Wirtschaftspolitik hieße, all das, was an Bedenken nicht nur von (post)keynesianischer Seite an diesem Zusammenhang zum Ausdruck gebracht wurde, über Bord zu werfen. Eine stabile Investitionsfunktion, wie sie von der neoklassischen Theorie unterstellt wird, ist unrealistisch. Dennoch muß der Hinweis der Neuen Wachstumstheorie, daß wirtschaftliches Wachstum langfristig nicht ohne den Verzicht auf den Konsum von Ressourcen möglich ist, bei wachstumspolitischen Maßnahmen beachtet werden.

Ein zweiter wichtiger Aspekt ist die Entwicklung neuer Produkte, die das Potential fuir Lerneffekte schaffen. Learning by doing ist auf Dauer nur möglich, wenn sich ständig neue Situationen einstellen, die zum Lernen anregen. Eine Volkswirtschaft, der dies nicht gelingt, wird mittel- bis langfristig von dieser Seite aus sinkende Wachstumsraten der Produktivität hinnehmen müssen. Neben diesen induzierten Effizienzsteigerungen führen die mit der Suche nach neuen Gütern verbundenen Forschungs- und Enwicklungsinvestitionen zu neuem technologischen Wissen, welches wiederum Spillover-Effekte auf andere Industrien haben kann. Aus der Neuen Wachstumstheorie läßt sich damit die Forderung nach einer gezielten Förderung von neuen Produkten ableiten. Selbstverständlich besteht auch hier ein Informationsproblem. Niemand ist in der Lage, heute schon die erfolgreichen Güter der Zukunft zu kennen. Der Suchprozeß muß den Unternehmen überlassen bleiben, da sie über mehr Marktkenntnisse verfügen als staatliche Instanzen und sie ein Eigeninteresse am Erfolg ihrer Investitionen haben. Staatlicherseits ist deshalb keine Politik des "Picking the winners" anzustreben, sondern vielmehr die Setzung von Rahmenbedingungen, die es den Unternehmen ermöglichen, die "Winners" zu entwickeln. Eine solche Wachstumspolitik beinhaltet gleichzeitig eine Absage an strukturkonservierende Maßnahmen.

Ein drittes Element einer auf der Neuen Wachstumstheorie aufbauenden Wachstumspolitik ist die Bildung des Humankapitals. Die Diskussion der Struktur der endogenen Wachstumsmodelle hat gezeigt, daß jeder vermehrbare Produktionsfaktor prinzipiell die Rolle des Wachstumsmotors übernehmen kann, wenn er nur konstante Skalenerträge aufweist. Im Unterschied zur Investition in Sachkapital weist die Humankapitalakkumulation mit größerer Wahrscheinlichkeit externe Effekte auf. Die Erhöhung des individuellen Humankapitals im ersten

17 Vgl. zur Rolle der Zeitpraferenz und den Folgen ihrer Veränderung für den Wachstumsprozeß z.B. Neumann $(1990,1994)$, der einen Grund für sinkende Wachstumsraten in den gewandelten Grundeinstellungen gegenüber Konsum und Sparen sieht. Insbesondere die Vererbung von Vermögen kann die Zeitpraferenz der Erben erhöhen. Neumann nennt dies das Buddenbrook-Symdrom. Im Roman von Thomas Mann baute der Großvater das Vermogen aus, der Sohn erhielt es und die Enkelgeneration sorgte durch ihr Konsumverhalten fur den Niedergang. Vgl. Neumann (1990, S. 51). Dieser Zusammenhang erinnert an das Schicksal mancher Unternehmensfamilie. Nicht umsonst ubergaben erfolgreiche Firmengründer die Leitung des Unternehmens an Dritte oder Stiftungen und beschrănkten die Familie im Zugriff auf das Unternehmensvermögen. 
Lucas-Modell (1988) führte zu positiven Wirkungen auf die anderen Produktionsfaktoren. Die Qualifikation der Arbeitnehmer stellt darüber hinaus eine wichtige Vorbedingung für die zuvor angesprochene Entwicklung neuer Produkte dar. Die Forderung nach einer Verbesserung der Qualifikation der Arbeitskräfte bedeutet jedoch nicht, möglichst viele Menschen auf die Universitäten zu schicken. Die Zahl der Ingenieure ist nicht zwangsläufig positiv mit der Wachstumsrate korreliert. Dieser Aspekt kommt in den Modellen der Neuen Wachstumstheorie aufgrund der Annahme substitutionaler Produktionsfaktoren zu kurz. In der Realität jedoch sind unterschiedlich qualifizierte Arbeitskräfte notwendig, die einerseits flexibel auf neue Arbeitsinhalte reagieren und andererseits eigenständig und kreativ agieren können. Insbesondere die sich aktuell vollziehende Entwicklung zur Informationsgesellschaft macht dies notwendig. So hat die EU-Kommission in ihrem Weißbuch, das 1993 der Öffentlichkeit vorgestellt wurde, die steigende Bedeutung des sog. immateriellen Kapitals betont. Unter diesem Begriff wird insbesondere der Inputfaktor Wissen subsumiert. ${ }^{18}$ Die Mikroelektronik hat in den neunziger Jahren die Möglichkeit geschaffen, standardisierte industrielle Produktionsverfahren an fast jedem Standort der Erde plazieren zu können. Über Investitionen in diesem Bereich werden deshalb maßgeblich die Lohnstückkosten entscheiden. Beschäftigungspotentiale für im internationalen Vergleich teuere Arbeit werden sich nur noch in den Sektoren ergeben, in denen die Qualifikation und Kreativität der Arbeitskräfte eine vorrangige Rolle spielen. Die technologische Entwicklung erfordert deshalb Investitionen in das Humankapital. Staatlicher Wirtschaftspolitik kommt in diesem Zusammenhang die Aufgabe zu, das Bildungswesen an die Erfordernisse anzupassen und für den Einzelnen, Anreize zur Aus- und Weiterbildung zu schaffen. 19

Alle angesprochenen Maßnahmen unterliegen einer gemeinsamen Restriktion, die den Erfolg der Wachstumspolitik beeinträchtigen kann. Staatliche Wachstumspolitik verlangt den Einsatz finanzieller Mittel, die von den privaten Wirtschaftssubjekten aufgebracht werden müssen. Die Erhebung einer Steuer wird die private Ertragsrate der Investitionen senken, an der sich die Unternehmen orientieren. Konsequenterweise wird sich ein Rückgang der Kapitalakkumulation einstellen. Die wachstumsfördernde Maßnahme hat wachstumssenkende Begleiterscheinungen. Dieses Problem tauchte schon bei der kurzen Behandlung öffentlicher Güter in endogenen Wachstumsmodellen auf. Der Staat steht damit vor einem Trade-off zwischen den positiven und negativen Folgen der Wachstumspolitik. Das oben angesprochene Informationsproblem erhält dadurch eine zusätzliche Komponente. ${ }^{20}$

18 EU-Kommission (1994, S. 80ff.). Vgl. zur Rolle dieser Komponente auch Romer (1993).

19 Die Folgen einer zunehmenden Trennung zwischen den Unternehmenszielen und den Interessen eines ganzen Landes, im Sinne von was gut für Daimler-Benz ist, ist nicht mehr zwingenderweise auch gut für Baden-Württemberg, werden z.B. von Reich (1991) sehr drastisch dargestellt.

20 Die Wirkungen einer Einkommensteuer auf die langfristige Wachstumsrate einer Volkswirtschaft hăngt in den Modellen der Neuen Wachstumstheorie im wesentlichen von den getroffenen Annahmen ab, d.h. die Ergebnisse sind recht instabil. Vgl. z.B. Barro (1990), Barro/Sala-i-Martin (1990, 1992, 1995), Jones/Manuelli (1990), King/Rebelo (1990), Rebelo (1991), Saint-Paul (1992) und Lessat (1994). Auch die Laffer-Kurve erlebt eine kleine Renaissance durch die Neue Wachstumstheorie. Vgl. hierzu Ireland (1994). 
Insgesamt läßt sich festhalten, daß sich aus den Modellen der Neuen Wachstumstheorie in erster Linie eine Wachstumspolitik zur Verbesserung der Standortqualität ableiten läßt. ${ }^{21}$ Wirtschaftspolitische Maßnahmen, die die Qualität und die Quantität der Produktionsfaktoren Sachkapital, technologisches Wissen, Humankapital und somit auch der Arbeit erhöhen, stellen die vorrangige Aufgabe einer erfolgreichen Strategie zur Erreichung hoher Wachstumsraten dar. Dies umfaßt die Förderung aller Arten der Infrastruktur, vor allem der Kommunikationstechnologie, die Unterstützung von Forschungs- und Entwicklungsaktivitäten, die Förderung des Bildungswesens incl. unternehmensinterner Aus- und Weiterbildung und die Verbesserung des Dialogs zwischen Wirtschaft und Wissenschaft. Ein Blick auf diesen Maßnahmenkatalog zeigt, daß die Regierungen vieler Länder diesen Empfehlungen mehr oder weniger schon längere Zeit gefolgt sind. Die Neue Wachstumstheorie liefert in gewissem Sinne die wirtschaftstheoretische Fundierung für bisher getätigte Wirtschaftspolitik. Des weiteren weisen viele Industrieländer, vor allem die USA, bei den angesprochenen Faktoren Defizite auf. Das öffentliche Bildungssystem in den Vereinigten Staaten z.B. kann die Qualifikation aller Bevölkerungsschichten nicht mehr gewährleisten. Die Beschäftigung mit der Neuen Wachstumstheorie dient somit auch der Gewinnung von Einsichten über aktuelle Probleme der Wirtschaftspolitik. 22

\subsubsection{Die kaldorianische Sichtweise}

In den wachstumstheoretischen Vorstellungen von Kaldor stand die technical progress function im Mittelpunkt des Interesses, die in Lage und Verlauf von dem technical dynamism einer Volkswirtschaft bestimmt war. Wachstumspolitik bedeutet in diesem Zusammenhang die Stärkung dieser Komponente. Die Bereitschaft und Fähigkeit zur Entwicklung und Übernahme neuer Technologien in den Produktionsprozeß muß gefördert werden. Vergleicht man dies mit den Überlegungen der Neuen Wachstumstheorie ergibt sich eine große Ähnlichkeit. Die zuvor aufgefürten wachstumspolitischen Maßnahmen dienen insgesamt der Verbesserung der Innovationsfähigkeit einer Volkswirtschaft. Mehr Humankapital, mehr Forschungs- und Entwicklungsaktivitäten und adäquat ausgebaute Infrastrukturnetzwerke erhöhen die Wahrscheinlichkeit, technologische Fortschritte in Form von Prozeß- und Produktinnovationen zu realisieren.

Die Aufrechterhaltung eines steten Ideenflusses, der ein mögliches Set an Know-how bietet, stellt aber nur eine Seite des Wachstumsprozesses dar. Kaldor betonte nicht nur die Fähigkeit zur Generierung neuen Wissens, sondern auch die Bereitschaft dieses Potential aus-

21 Vgl. z.B. auch Shaw (1992) und Stern (1992).

22 Die vorliegenden Defizite stellen mit groBer Sicherheit einen Grund für die Beschăftigung mit diesen Fragen im Rahmen der Neuen Wachstumstheorie dar. 
zunutzen. Es bedarf der Investitionen der Unternehmen, um den Vorrat an Wissen umzusetzen. Die Bestimmungsgründe der Investitionsfunktion gewinnen dadurch an Bedeutung. Im rein wachstumstheoretischen Modell von Kaldor war die Form der Investitionsfunktion und damit ihrer Determinanten in der letztendlich realisierten Steady State-Situation aufgrund der Dominanz der technical progress function nicht entscheidend. In seinen Vorstellungen zu den kumulativen Eigenschaften von Wachstumsprozessen ändert sich dies. Investitionen erfullen zwei Funktionen. Sie sind wie im Fall der technical progress function Träger der neuesten Technologie und gleichzeitig bewirken sie als Teil der gesamtwirtschaftlichen Nachfrage induzierte Produktivitätseffekte. Eine hohe Investitionsquote kann aus diesem Grund als eines der wichtigen Ziele wirtschaftspolitischen Handelns aufgefaßt werden. Die langfristige Betrachtung ergibt dieselben Forderungen wie die kurzfristig orientierte keynesianische Sichtweise. Die Aufrechterhaltung der Investitionen hat auf konjunktureller Ebene die Aufgabe, via Multiplikatoreffekt die Vollauslastung der Kapazitäten zu erreichen, auf Wachstumsebene dienen sie der Generierung und Realisierung von Produktivitätsfortschritten.

Das Prinzip der kumulativen Verursachung macht hier noch mehr als die Modelle der Neuen Wachstumstheorie die Beziehung zwischen kurzfristigen und langfristigen Entwicklungen deutlich. Wie schon im Harrod-Modell können einzelne Entscheidungen eine Entwicklung auslösen, die zu sinkenden oder steigenden Wachstumsraten führen wird. Bleiben z.B. die Investitionen der Unternehmen auf niedrigem Niveau, verschenkt die Volkswirtschaft nach kaldorianischer Auffassung Wachstumspotentiale. Die Pfadabhängigkeit des Wachstumsprozesses stellt die Verbindung zwischen konjunktureller und langfristiger Entwicklung her. Durch diese Abhängigkeit gewinnt die Konjunkturpolitik eine langfristige Dimension, die aus wachstumstheoretischer Sichtweise zumeist vernachlässigt wird, die aber im Harrodschen Wachstumsmodell schon zu erkennen war: die Erreichung langfristig gleichgewichtiger Wachstumsprozesse setzt dort konjunkturelle Stabilităt voraus.

Überträgt man diese Überlegungen auf reale Fragestellungen, muß sich die Wirtschaftspolitik zu jedem Zeitpunkt fragen, inwieweit sie durch die Erreichung kurzfristiger Ziele, die Chance auf langfristige Wachstumspotentiale verschenkt. Eine restriktive Geldpolitik zur Stabilisierung des Preisniveaus wird deshalb z.B. nicht nur vor dem Hintergrund ihrer Effekte auf die aktuelle Konjunktursituation zu beurteilen sein, sondern muß auch die langfristigen Konsequenzen berücksichtigen. Entsprechendes gilt für eine Fiskalpolitik, die durch hohe Defizite zur Finanzierung konsumtiver Zwecke die Anlage investiver Mittel in Staatsanleihen rentabler macht als die Investition in Forschungs- und Entwicklungsausgaben. Wirtschaftspolitik muß aus Sicht des Modells der kumulativen Verursachung nicht nur an ihren Beschäftigungs- und Preisniveaueffekten, sondern zusätzlich an ihren Folgen für die lange Frist beurteilt werden.

Die Einbeziehung der Produktivitäts- und Nachfrageverhältnisse in die Untersuchung von Wachstumsprozessen eröffnet mehrere Ansatzpunkte für eine auf Wachstum angelegte Wirt- 
schaftspolitik. Am Anfang dieses Abschnitts wurde die Sinnhaftigkeit der von der Neuen Wachstumstheorie nahegelegten Maßnahmen im Rahmen der kaldorianischen Vorstellungen schon angesprochen, so daß eine Diskussion dieser Aktivitäten nicht notwendig ist. Da der Verdoorn-Zusammenhang als Ausdruck steigender Skalenerträge auf industrieller Ebene verstanden wird, bietet sich eine Konzentration auf die entsprechenden Wirtschaftszweige an. Eine vergleichbare Forderung wurde von Kaldor schon 1966 aufgestellt, der eine Lösung zur Überwindung der niedrigen Wachstumsraten der britischen Ökonomie in der Förderung der Nachfrage nach den Produkten des sekundären Sektors empfahl. Eine Wirtschaftspolitik, die es sich zum Ziel gesetzt hat, die Wirtschaftszweige mit steigenden Skalenerträgen zu fördern, steht vor demselben Dilemma, das im Falle der Internalisierung externer Effekte auftrat. Eine angepaßte Förderpolitik muß zuerst das sich stellende Informationsproblem lösen. Zwar wird der Nachweis steigender Skalenerträge leichter fallen als derjenige von externen Effekten, da aber die Ursachen der steigenden Skalenerträge nicht ohne weiteres feststellbar sind, muß offen bleiben, ob sie vielleicht nur temporärer Natur sind und Fördermaßnahmen vielleicht zu spät kommen.

Eine Ursache steigender Skalenerträge wurde in den theoretischen Analysen immer wieder betont. Steigende Arbeitsteilung bewirkt überproportionale Produktionszuwächse bei zunehmendem Einsatz von Inputfaktoren. Smith und Young haben die Verbindung zwischen der Größe des Marktes und dem Grad der Arbeitsteilung veranschaulicht. Eine Strategie zur Realisierung der mit der Arbeitsteilung verbundenen Produktivitätseffekte besteht in der Vergrößerung der relevanten Märkte von Unternehmen. Eine solche Forderung stellt selbstverständlich eine Verbindung zu außenwirtschaftlichen Überlegungen dar. Die Erschließung internationaler Märkte kann von privater Seite nicht vollständig alleine geleistet werden. Verfolgt die Regierung eines Landes eine Politik der Importrestriktion, sind Verhandlungen auf Regierungsebene notwendig, um ausländischen Unternehmen den Zutritt auf die abgeschotteten Märkte zu ermöglichen. Die Schaffung des gemeinsamen Binnenmarktes in der Europäischen Union stellt eine solche Maßnahme dar.

Neben den angebotsseitigen Variablen im System der kumulativen Verursachung kommen auch den Nachfragereaktionen auf Produktivitätssteigerungen Bedeutung zu. In diesem Zusammenhang wurden in Kapitel 4 mehrere Aspekte angesprochen. Die Entwicklung der Konsum-, Investitions- und Exportnachfrage wird sowohl positiv als auch negativ von einem zunehmenden Produktivitätsniveau berührt. Die Interdependenz zwischen Produktivitäts-, Lohn- und Preisentwicklungen bestimmen maßgeblich die Rückwirkungen von Produktivitätssteigerungen auf die Entwicklung der Nachfrage. Der Wachstumsprozeß wird folglich von lohn-, preis- und verteilungspolitischen Aspekten abhängen. ${ }^{23}$ Vergleichbar mit dem zuvor

23 Dies darf in einem auf den Arbeiten von Kaldor aufbauenden Modell nicht verwundern. Der Zusammenhang zwischen Wachstum und Verteilung war der Ausgangspunkt der Arbeiten von Kaldor zur Verteilungs- und Wachstumstheorie. 
angesprochenen wachstumspolitischen Charakter der Konjunkturpolitik erhalten wirtschaftspolitische Entscheidungen zur Lohn- und Preispolitik sowie zur Verteilungspolitik eine langfristige Relevanz. Dieser Bereich fält sowohl in das Aufgabengebiet der Tarifparteien als auch des Staates. Für Lohnverhandlungen läßt sich aus dem Modell der kumulativen Verursachung die Schlußfolgerung ziehen, daß bei der Festlegung der Löhne nicht nur Kostengesichtspunkte, sondern auch Rückwirkungen auf die gesamtwirtschaftliche Nachfrage beachtet werden müssen. Dies bezieht sich nicht nur auf die Entwicklung im Inland, sondern schließt auch außenwirtschaftliche Zusammenhänge ein. Ähnliches gilt für die staatliche Verteilungspolitik. Einerseits dürfen die Profite der Unternehmen nicht zu stark belastet werden, andererseits müssen beispielsweise die Konsequenzen von Sozialausgabenkürzungen für die Konsumgüternachfrage ins Kalkül aufgenommen werden. Hier wird gleichzeitig die institutionelle gesellschaftiche Komponente von Wachstumsprozessen erkennbar. Bestehende institutionelle Regelungen müssen immer wieder auf ihre Sinnhaftigkeit überprüft werden. ${ }^{24}$

Die Gewinnung konkreter wirtschaftspolitischer Handlungsempfehlungen aus Kaldors Arbeiten bzw. dem Modell der kumulativen Verursachung steht vor demselben Problem, das zuvor bei der Neuen Wachstumstheorie auftrat. Die Ergebnisse des Modells können auf relevante Beziehungen und Fallstricke innerhalb der Wachstumspolitik aufmerksam machen. Ohne die Kenntnis der tatsächlich vorliegenden Strukturen kann sie nur in allgemeinen Hinweisen und Konzepten verhaftet bleiben. Der Vorteil gegenüber den Modellen der Neuen Wachstumstheorie ist die Berücksichtigung des Einflusses der Nachfragebedingungen auf die Wachstumschancen einer Volkswirtschaft. Im Unterschied zur neoklassischen Tradition bleibt eine auf den Ergebnissen der kaldorianischen Tradition aufbauende Wachstumspolitik nicht in angebotsseitigen Maßnahmen stecken.

\subsubsection{Fazit}

Die wirtschaftspolitischen Implikationen der Neuen Wachstumstheorie und der Vorstellungen Kaldors bzw. des Modells der kumulativen Verursachung weisen eine große Nähe auf. In beiden Ansätzen können die Förderung von Forschungs- und Entwicklungsaktivitäten, der Humankapitalbildung und die Verbesserung der Rahmenbedingungen für die Entstehung von Produkt- und Prozeßinnovation das Produktivitäts- und somit das Wachstumspotential einer Volkswirtschaft steigern. Die Ableitung konkreter wirtschaftspolitischer Maßnahmen muß in beiden Fällen am hohen Aggregationsgrad der Modelle scheitern. Dennoch wurden Anknüpfungspunkte für wachstumspolitische Handlungsmöglichkeiten deutlich.

Eine Strategie zur Förderung der Wachstumskräfte darf unter Berücksichtigung der Ergebnisse beider Ansätze nicht auf die Produktionsfaktoren begrenzt bleiben. Die Qualität

24 Erinnert sei in diesem Zusammenhang an die Arbeiten von Olson (1965 und 1982). 
und Quantität des Sach- und Humankapitals sowie das technologische Wissen sind nicht zu vernachlässigende Determinanten eines erfolgreichen Wachstumsprozesses. Die Investitionen in diesem Bereich schaffen das Potential für mögliche Produktivitätsfortschritte. Die Realisierung dieses Potentials kann nur erfolgen, wenn die Nachfrage nach Gütern komplementär zum Angebot ist und darüber hinaus selbst positive Wirkungen auf die Effizienz des Faktoreinsatzes ausübt. Eine erfolgreiche Wachstumspolitik erfordert des weiteren eine Berücksichtigung konjunktureller und gesellschaftlicher Zusammenhänge.

Beide Ansätze beinhalten eine weitere Konsequenz für wachstumspolitisches Handeln. Der Wachstumsprozeß ist in den vorgestellten Sichtweisen beeinflußbar, d.h. Wachstum ist kein von außen vorgegebenes Phänomen, sondern das Resultat der Entscheidungen der Wirtschaftssubjekte über Sparen, Konsum und Investieren. Diese Implikation ist Chance und Gefahr zugleich. Der Einfluß unternehmerischer und staatlicher Handlungen kann einerseits wachstumsfördernde und andererseits wachstumshemmende Folgen haben. Welche Seite letztendlich die Oberhand gewinnt, wird zu einem großen Teil von der richtigen Diagnose der Ursachen für Wachstumsschwächen durch die Träger der Wirtschaftspolitik abhängen.

\subsection{Zusammenfassung}

In diesem Kapitel wurden die neue Wachstumstheorie und die kaldorianische Sichtweise gegenübergestellt. In den jeweiligen Abschnitten wurden die wichtigsten Ergebnisse schon zusammengefaßt, so daß an dieser Stelle lediglich die zentralen Punkte noch einmal angeführt werden. Sowohl der Vergleich der theoretischen Eigenschaften als auch die Diskussion möglicher wirtschaftspolitischer Implikationen haben Unterschiede und Gemeinsamkeiten ergeben. Die prinzipiellen Positionen in beiden Ansätzen bezüglich der methodischen Instrumente (z.B. Produktionsfunktion, Nutzenfunktion) und der Frage nach der Stabilität marktwirtschaftlich organisierter Volkswirtschaften liegen weiterhin auseinander. Beide Konzepte befassen sich jedoch mit denselben Fragestellungen und führen das Wachstum der Produktivität auf den Investitionsprozeß und die damit verbundenen Externalitäten zurück. Neben der ehemals vorrangigen Rolle des Sachkapitals werden in diesem Zusammenhang zunehmend immaterielle Produktionsfaktoren berücksichtigt. Dies geschieht teilweise direkt wie in den Modellen der Neuen Wachstumstheorie oder indirekt wie bei der Diskussion des Verdoorn-Zuammenhangs.

Die Konzentration auf vergleichbare Fragestellungen und Wachstumsdeterminanten ergibt auch eine Konvergenz bei möglichen wachstumspolitischen Schlußfolgerungen, die aus den Modellen gezogen werden können. Die Fähigkeit zur Entwicklung und Übernahme des technischen Fortschritts sowie die Diffusion technologischer Neuerungen sind in beiden Ansätzen eine Möglichkeit zur Schaffung von mehr Wachstum. Die Überlegungen zum Modell der kumulativen Verursachung haben diese angebotsseitige Bestimmung des Wachstumsprozesses 
durch Nachfragezusammenhänge ergänzt, welche die relevanten Rückwirkungen der Nachfrage auf realisierte Produktivitätsfortschritte abbilden.

Für eine erfolgversprechende Wachstumspolitik folgt hieraus die Notwendigkeit, sich nicht nur auf die Produktionsseite zu konzentrieren, sondern auch die langfristigen Konsequenzen der Beschäftigungs-, Lohn-, Preis- und Verteilungspolitik im Hinblick auf ihre Folgen für die gesamtwirtschaftliche Nachfrage in einem integrierten Konzept zu berücksichtigen. Wachstumspolitik muß somit verschiedene Bereiche der Wirtschaftspolitik integrieren, um dauerhaftes Wachstum erreichen zu können. 
Die im Rahmen der vorliegenden Arbeit durchgeführten Untersuchungen dienten der Klärung der Frage nach dem Beitrag von Nicholas Kaldor zur Neuen Wachstumstheorie, die seit Mitte der achtziger Jahre für eine Wiederbelebung des Interesses an der Erklärung von Wachstumsprozessen gesorgt hat. Auf den ersten Blick scheint die Antwort auf die gestellte Frage eine klare Verneinung eines Zusammenhangs zwischen Kaldors wachstumstheoretischen Arbeiten und den aktuellen Entwicklungen auf diesem Gebiet zu sein. Kaldor entwickelte seine Modelle als Alternative $\mathrm{zu}$ den neoklassischen Beiträgen von Solow und anderen, wobei insbesondere das Konzept der Produktionsfunktion im Mittelpunkt seiner Kritik stand, die durch die technical progress function ersetzt wurde. Die kategorische Ablehnung des Instrumentariums der jeweils anderen Sichtweise verstellt jedoch den Blick für eventuelle Berührungspunkte und Anregungen, die sich aus den Ergebnissen des anderen "Lagers" ergeben.

Die zu Anfang dieser Arbeit diskutierten traditionellen Wachstumsmodelle postkeynesianischer und neoklassischer Provenienz zeigten die prinzipiellen Differenzen zwischen beiden Paradigmen. Während Ökonomen in der Tradition von Keynes die inhärente Instabilität des marktwirtschaftlich organisierten Systems betonten, gingen und gehen neoklassische Ökonomen von einer Tendenz des Marktes zu stabilen Gleichgewichten aus. Auf Wachstumsprozesse übertragen bedeutet dies, daß in der postkeynesianischen Sichtweise die Existenz einer langfristigen Entwicklung mit ausgelasteten Produktionskapazitäten und konstanten Wachstumsraten, d.h. eines Steady State-Wachstumspfades, für möglich, die tatsächliche Realisierung aber für sehr unwahrscheinlich gehalten wird. Im Gegensatz hierzu wird im neoklassischen System ein solcher Pfad von einer Volkswirtschaft, die keinen exogenen Einflüssen unterliegt, immer angestrebt.

Die traditionelle Wachstumstheorie hatte nicht nur die Frage nach der prinzipiellen Stabilität von Wachstumsprozessen zum Inhalt, sondern untersuchte auch die Wirkungen des technischen Fortschritts, der sich in einer Erhöhung der Faktorproduktivitäten ausdrückt. Insbesondere im neoklassischen Grundmodell kam der als exogen angenommenen technologischen Entwicklung eine zentrale Funktion zu, da sich ohne diese Komponente das Wachstum der Pro-Kopf-Versorgung nicht erklären ließ. Das System erreicht zwar eine Steady StateSituation, aber ohne technischen Fortschritt ist diese durch das Nullwachstum aller Pro-KopfGrößen gekennzeichnet. Erst bei Vorliegen technischen Fortschritts ist positives Wachstum des Pro-Kopf-Einkommens möglich. Aufgrund der Ergebnisse des neoklassischen Wachstumsmodells in Kombination mit der Annahme, daß technischer Fortschritt international ein öffentliches Gut darstellt, wurde bei Vergleichen eine hohe Wachstumsrate bei kapitalarmen Volkswirtschaften und eine Konvergenz der Wachstumsraten erwartet. Eine weitergehende Interpretation ging von einer Konvergenz der Pro-Kopf-Einkommen aus. 
Dieses sowohl aus theoretischen als auch empirischen Gründen unbefriedigende Ergebnis der exogenen Bestimmung der langfristigen Wachstumsrate einer Volkswirtschaft und der damit verbundenen Irrelevanz von Spar- und Investitionsentscheidungen für die Steigung des Steady State-Wachstumspfades wurde mit Hilfe von investitionsinduziertem technischen Fortschritt zu überwinden versucht. Insbesondere die in Kapitel 3 diskutierte Arbeit von Arrow (1962) zum Konzept des learning by doing stellt einen zentralen Beitrag in diesem Zusammenhang dar. Trotz der Abhängigkeit der gleichgewichtigen Pro-Kopf-Wachstumsrate vom Bevölkerungswachstum bilden Arrows Überlegungen einen wichtigen Schritt in Richtung endogener Bestimmung des Wachstumsprozesses. Die Annahme externer Effekte individueller Investitionsentscheidungen auf die Investitionen in der Zukunft ermöglicht es für den Produktionsfaktor Kapital in einem ersten Schritt auf gesamtwirtschafticher Ebene konstante Skalenerträge anzunehmen.

Aufbauend auf der Arbeit von Arrow wurden von den Vertretern der Neuen Wachstumstheorie Modelle entwickelt, die den Einfluß der Sachkapitalbildung auf das technologische Wissen, die Rolle des Humankapitals und die Bedeutung von Forschungs- und Entwicklungsausgaben zum Inhalt hatten. In allen Fällen liegen externe Effekte der getätigten Investitionen vor, die mit der Vermehrung eines zumindest teilweise nicht dem Ausschlußprinzip unterliegenden Bestands an Wissen einhergehen. Durch die Annahme konstanter Skalenerträge für die vermehrbaren Produktionsfaktoren wird endogenes Wachstum möglich, da die Ertragsrate der Investitionen sich nicht an die soziale Diskontrate bzw. Zeitpräferenz annähert. Auch ohne exogenen technischen Fortschritt werden die Unternehmen ihre Investitionen aufrechterhalten und so eine steigende Kapitalintensivierung bewirken. Das Wachstum in den Modellen der Neuen Wachstumstheorie resultiert aus der Erhöhung des Kapitaleinsatzes pro Arbeitsplatz, wobei unter dem Begriff Kapital auch Humankapital oder der Bestand des technischen Wissens verstanden werden kann. Das Verhältnis zwischen den durch Konsumverzicht vermehrbaren Produktionsfaktoren und dem Arbeitseinsatz wird ständig steigen, d.h. Produktivitätsfortschritte sind das Ergebnis der zunehmenden Kapitalintensivierung.

Die Verbindung zwischen Investitionen und technischem Fortschritt wurde von Kaldor in seinen Beiträgen zur Wachstumstheorie durch das Konzept der technical progress function hervorgehoben. Produktivitätssteigerungen sind hier weitgehend die Folge der Investitionstätigkeit, die darüber entscheidet, welche Technologien in den Produktionsprozeß einfließen. Die technical progress function ist Ausdruck des technical dynamism einer Volkswirtschaft, der die Fähigkeit und Bereitschaft einer Gesellschaft beinhaltet, neue Technologien zu entwikkeln und sie anzuwenden. Die historische Abfolge der Investitionen ist dabei nicht nur Ergebnis des technical dynamism, sondern bestimmt diesen auch. Aufgrund dieser Beziehung liegen auch hier externe Effekte der Investitionsentscheidung vor. Trotz der Kritik an der Vorgehensweise der Neoklassik ist in Kaldors Wachstumsmodellen die gleichgewichtige Wachstumsrate von exogenen Parametern abhängig. 
Von kaldorianischer Seite aus wird eine weitere Erklärung für endogene Wachstumsprozesse angeboten, die auf der empirisch feststellbaren linearen Beziehung zwischen der Wachstumsrate der Produktion und derjenigen der Arbeitsproduktivität, dem sog. VerdoornZusammenhang aufbaut. Hierbei werden produktionsinduzierte Produktivitätssteigerungen als Ausdruck steigender Skalenerträge in der Güterproduktion angesehen. Die Gründe für diese sind zum einen in den Vorteilen der Massenproduktion, zum anderen in Lerneffekten, Wissensexternalitäten und der zunehmenden Kapitalintensität zu suchen. Letztere ist, wie Smith und Young gezeigt haben, eine Folge der zunehmenden Arbeitsteilung, die einerseits die Größe der Märkte bestimmt und andererseits selbst eine Folge der Ausdehnung der Märkte ist.

Der Verdoorn-Zusammenhang läßt jedoch keine direkten Rückschlüsse auf die ihm zugrundeliegende Produktionsstruktur zu. Neben der Fundierung durch eine Cobb-DouglasProduktionsfunktion kann sowohl aus einer Lernkurve als auch aus einer technical progress function eine lineare Beziehung zwischen Produktions- und Produktivitätswachstum abgeleitet werden. In allen drei Fällen sind dazu aber mehrere Annahmen notwendig, die den Schluß nahelegen, daß der Verdoorn-Zusammenhang nicht als Indikator für eine spezifische Produktionsfunktion interpretiert werden kann, sondern vielmehr das Ergebnis des Zusammenspiels mehrerer Faktoren darstellt, die neben den Effekten der Arbeitsteilung und der damit verbundenen kapitalintensiveren Produktionsweise auch Komponenten des nachfrageinduzierten technischen Fortschritts sowie der Effizienz von Forschungs- und Entwicklungsaktivitäten enthält.

Der Einfluß der Arbeitsteilung und der Größe des Marktes auf die Produktivitätsverhältnisse einer Volkswirtschaft macht zusätzlich den Einfluß der Nachfrage auf die Wachstumsmöglichkeiten einer Volkswirtschaft deutlich. Das von Kaldor betonte kumulative Element von Wachstumsprozessen kann durch den Verdoorn-Zusammenhang jedoch nicht vollständig abgebildet werden. Myrdals Analyse zirkulärer kumulativer Prozesse hat die Notwendigkeit der Existenz von Feedback-Effekten verdeutlicht. Für den Entwicklungsprozeß von Volkswirtschaften impliziert dies eine Rückwirkung des nachfrageinduzierten Produktivitätsanstiegs auf das Wachstum der Güternachfrage. Hierbei sind sowohl positive als auch negative Reaktionen beim Konsum, den Investitionen und der Exportnachfrage möglich. Die Entwicklung von Löhnen, Profiten, Beschäftigung und Preisen determiniert, welche Konsequenzen letztendlich zu erwarten sind. Der Wachstumsprozeß wird damit auch durch institutionelle Verhältnisse beeinflußt.

Der Vergleich beider Sichtweisen zeigt vor allem Unterschiede bei Aspekten, die schon immer konträr diskutiert wurden. Zu nennen sind insbesondere die vikarische Funktion der Investoren in der Neoklassik, das Konzept des repräsentativen Individuums und die Anwendung einer gesamtwirtschaftlichen Produktionsfunktion sowie die Beurteilung des Steady State-Konzepts. Auch die Frage nach möglichen Wachstumsbeschränkungen wird unterschiedlich beurteilt. Die Gemeinsamkeiten und damit der Beitrag Kaldors zur Neuen Wachs- 
tumstheorie finden sich in der herausragenden Rolle, die externen Effekten und der Investitionstätigkeit für die Existenz endogenen Wachstums zugewiesen wird. Produktivitătssteigerungen erfordern in beiden Fällen die Erhöhung des Kapitaleinsatzes pro Arbeitskraft.

Aufgrund dieser Parallelen können beide Modellstrukturen vergleichbare Ergebnisse generieren. Externe Effekte und steigende Skalenerträge ermöglichen trotz der prinzipiellen Unterschiede zwischen der Neuen Wachstumstheorie und der kaldorianischen Sichtweise in beiden Fällen kumulative oder stabile Wachstumsprozesse. Während die in der Tradition der Neoklassik verwurzelte Neue Wachstumstheorie Steady State-Situationen als Untersuchungsgegenstand wählt, lassen Modelle auf Basis der Überlegungen Kaldors aufgrund ihrer postkeynesianischen Ausrichtung beide Möglichkeiten offen. Beiden Ansätzen ist dabei die Abhängigkeit der langfristigen Wachstumsrate von der "Geschichte" der Volkswirtschaft gemein. Neben den Anfangsausstattungen mit Ressourcen bewirken in der Neuen Wachstumstheorie heutige Investitionsentscheidungen externe Effekte auf zukünftige, wodurch Produktivitätsfortschritte in der Zukunft festgelegt werden. Der Einfluß einmaliger Entscheidungen auf die Wachstumsmöglichkeiten einer Volkswirtschaft tritt in den Kaldor-Verdoorn-Modellen noch deutlicher zu Tage. Die Spezialisierung auf einen bestimmten industriellen Sektor determiniert z.B., ob die Ökonomie einen Pfad wachsender oder sinkender Wachstumsraten einschlägt.

Die Struktur der Modelle impliziert einen wachstumspolitischen Spielraum, der sich aus dem Vorliegen externer Effekte, der Relevanz der Investitionsentscheidungen für die langfristige Wachstumsrate und der Rolle der Nachfrage für die Verstärkung der induzierten Produktivitätseffekte ergibt. In beiden Fällen sind Maßnahmen zur Förderung der Entstehung und der Diffusion neuen Wissens zu ergreifen, die nicht nur auf finanzielle Fördermaßnahmen begrenzt sind, sondern auch Rahmenbedingungen zur Ausnutzung der Externalităten schaffen. Hierbei ist in erster Linie an die Forschungsforderung und die Verbesserung der Qualität des Bildungswesens zu denken. Aus kaldorianischer Sichtweise ergeben sich zusätzlich Konsequenzen für die Lohn-, Preis-, und Verteilungspolitik, da diese mit über die Rückwirkungen von Produktivitätssteigerungen auf die Nachfrageentwicklung entscheiden. Aufgrund des hohen Aggregationsgrads der Modelle gestaltet sich aber die Ableitung konkreter wachstumspolitischer Maßnahmen recht schwierig. Die theoretische Analyse kann nur auf mögliche Aktivitätsfelder hinweisen und mögliche Zielkonflikte darlegen.

$\mathrm{Faßt}$ man die hier noch einmal skizzierten Untersuchungsergebnisse zusammen, bleibt festzuhalten, daß die wachstumstheoretischen Arbeiten von Kaldor einen Beitrag zur Neuen Wachstumstheorie darstellen. Neben der von ihm propagierten Ausrichtung ökonomischer Modelle an den stilisierten Fakten der Realität finden sich vor allem seine Ansichten über die Untrennbarkeit von technischem Fortschritt und Investitionen sowie über die Relevanz von externen Effekten in den Ergebnissen der aktuellen neoklassischen Wachstumsmodelle wieder. Eng damit verbunden ist Kaldors Vorstellung, daß Wachstumsprozesse nicht nur ein Ergebnis 
gegenwärtigen Handelns sind, sondern auch die Vergangenheit einen maßgeblichen Einfluß auf die zukünftigen Wachstumschancen hat. Die frühe Vorwegnahme der Kernaussagen der Neuen Wachstumstheorie durch Kaldor stellt somit ein weiteres Beispiel für die Aussage von Paul Samuelson dar, der in einem anderen Zusammenhang über Kaldor sagte: ${ }^{1}$

"But once Kaldor was confronted with the problem, he did see how it had to go!"

1 Samuelson (1988, S. 321). Samuelson sprach uber die Generation von Okonomen, die in der Zeit zwischen 1930 bis 1980 die Wirtschaftswissenschaften seiner Auffassung nach maßgeblich geprägt haben. Er berichtet dabei uber Kaldors eigenstăndige Entdeckungen von Ergebnissen, die sich in Arbeiten von Pareto und von Neumann finden, ohne daß er die Originale kannte. 
Stephan Seiter - 978-3-631-75406-1

Downloaded from PubFactory at 01/11/2019 05:22:48AM

via free access 


\section{Literaturverzeichnis}

Abramovitz, M. (1956), Resource and Output Trends in the United States since 1870, in: American Economic Review, Bd. 46, Nr. 2, S. 5-23.

Abramovitz, M. (1979), Rapid Growth Potential and Realization, in: Malinvaud, E. (1979), Economic Growth and Resources, Bd. 1, Proceedings of the Fifth World Congress of the International Economic Association held in Tokyo (Japan), London, S. 1-30, hier Nachdruck in: Abramovitz, M. (1989), Thinking about Growth, Cambridge, S.187-219.

Abramovitz, M. (1986), Catching up, Forging Ahead, and Falling Behind, in: Journal of Economic History, Bd. 46, Nr. 2, S. 385-406, hier Nachdruck in: Abramovitz, M. (1989), Thinking about Growth, Cambridge, S. 220-242.

Abramovitz, M. (1989), Thinking about Growth, Cambridge.

Abramovitz, M. (1990), The Catch-Up Factor in Postwar Economic Growth, in: Economic Inquiry, Bd. 28, S. 1-18.

Abramovitz, M. (1994), Catch-up and Convergence in the Postwar Boom and After, in: Baumol, W.J., Nelson, R.R., Wolff, E.N. (Hrsg.) (1994), Convergence of Productivity: Cross-National Studies and Historical Evidence, Oxford u.a., S. 86-125.

Aghion, Ph. (1994), Endogenous Growth: a Schumpeterian Approach, Papier vorgestellt auf dem Workshop "Endogenous Growth and Development" of The International School of Economic Research, Universität Siena - C.N.R., at the Certosa di Pontignano, Siena, 3.-9. Juli 1994, Vorlesungsband, S. 5-45.

Aghion, Ph., Howitt, P. (1991), Unemployment - A Symptom of Stagnation or Side-Effect of Growth?, in: European Economic Review, Bd. 35, S. 535-541.

Aghion, Ph., Howitt, P. (1992a), A Model of Economic Growth through Creative Destruction, in: Econometrica, Bd. 60, Nr. 2, S. 323-351.

Aghion, Ph., Howitt, P. (1992b), The Schumpeterian Approach to Technical Change and Growth, in: Siebert, H. (Hrsg.) (1992), Economic Growth in the World Economy, Tübingen, S. 55-76.

Aghion, Ph., Howitt, P. (1994), Growth and Unemployment, in: Review of Economic Studies, Bd. 61, S. 477-494.

Amsden, A. (1989), Asia's Next Giant: South Korea and Late Industrialization, New York und Oxford.

Arbeitsgemeinschaft Deutscher Wirtschaftswissenschaftlicher Forschungsinstitute (Hrsg.) (1994), Wachstumsperspektiven in den neunziger Jahren, Tagungsband der Arbeitsgemeinschaft Deutscher Wirtschaftswissenschaftlicher Forschungsinstitute e.V. (gleichzeitig Beiheft der Konjunkturpolitik, Bd. 42), Berlin.

Arestis, Ph., Kitromilides, Y. (Hrsg.) (1990), Theory and Policy in Political Economy: Essays in Pricing, Distribution, and Growth, Aldershot. 
Arestis, Ph., Marshall, M.G. (Hrsg.) (1995), The Political Economy of Full Employment, Aldershot.

Arestis, Ph., Sawyer, M. (Hrsg.) (1994), The Elgar Companion to Radical Political Economy, Aldershot.

Arrow, K.J. (1962), The Economic Implications of Learning by Doing, in: Review of Economic Studies, Bd. 29, S. 155-173.

Arrow, K.J. (1963), Social Choice and Individual Values, New York.

Arrow, K.J. (1979), The Division of Labour in the Economy, the Polity, and Society, in: O'Driscoll, G.P.jr. (Hrsg.) (1979), Adam Smith and Modern Political Economy, Iowa, S. 153-164, hier Nachdruck in: Buchanan, J.M., Yoon, Y.J. (Hrsg.) (1994), The Return to Increasing Returns, Ann Arbor, S. 69-81.

Arrow, K.J., Chenery, H.B., Minhas, B.S., Solow, R.M. (1961), Capital-Labor Substitution and Economic Efficiency, in: Review of Economics and Statistics, Bd. 43, S. 225-250.

Arrow, K.J., Karlin, S., Suppes, P. (Hrsg.) (1959), Mathematical Methods in the Social Sciences, Stanford.

Arthur, B.W. (1994), Increasing Returns and Path Dependence in the Economy, Ann Arbor.

Auerbach, A.J., Hassett, K.A., Oliner, S.D. (1994), Reassessing the Social Returns to Equipment Investment, in: Quarterly Journal of Economics, Bd. 109, Nr. 3, S. 789-802.

Azariadis, C., Drazen, A. (1990), Treshold Externalities in Economic Development, in: Quarterly Journal of Economics, Bd. 105, S. 501-526.

Bairam, E. (1987), The Verdoorn Law, Returns to Scale and Industrial Growth: A Review of the Literature, in: Australian Economic Papers, Bd. 26, S. 20-42.

Baranzini, M., Scazzeri, R. (Hrsg.) (1990), The Economic Theory of Structure and Change, Cambridge.

Barnett, W.A. et al. (Hrsg.) (1991), Equilibrium Theory and Applications, Cambridge.

Barro, R.J. (1990), Government Spending in a Simple Model of Endogenous Growth, in: Journal of Political Economy, Bd. 98, S. 107-125.

Barro, R.J. (1991), Economic Growth in a Cross Section of Countries, in: Quarterly Journal of Economics, Bd. 106, Nr. 2, S. 407-443.

Barro, R.J., Lee, J.-W. (1993), International Comparisons of Educational Attainment, in: Journal of Monetary Economics, Bd. 33, Nr. 3, S. 363-394.

Barro, R.J., Lee, J.-W. (1994), Sources of Economic Growth, in: Carnegie-Rochester Conference Series on Public Policy, S. 1-46.

Barro, R.J., Sala-i-Martin, X. (1990), Public Finance in Models of Economic Growth, NBER Working Paper Nr. 3362, Boston. 
Barro, R.J., Sala-i-Martin, X. (1991), Convergence across States and Regions, in: Brookings Papers on Economic Activity, Nr. 1, S. 107-182.

Barro, R.J., Sala-i-Martin, X. (1992a), Convergence, in: Journal of Political Economy, Bd. 100, Nr. 2, S. 223-251.

Barro, R.J., Sala-i-Martin, X. (1992b), Public Finance in Models of Economic Growth, in: Review of Economic Studies, Bd. 59, Nr. 4, S. 645-661.

Barro, R.J., Sala-i-Martin, X. (1995), Economic Growth, New York u.a.

Baumol, W.J. (1986), Productivity Growth, Convergence, and Welfare: What the Long-Run Data Show, in: American Economic Review, Bd. 76, Nr. 5, S. 1072-1085.

Baumol, W.J., Blackman, S.A.B., Wolff, E.N. (1989), Productivity and American Leadership: The Long View, Cambridge (Mass.) und London.

Baumol, W.J., Nelson, R.R., Wolff, E.N. (Hrsg.) (1994), Convergence of Productivity: CrossNational Studies and Historical Evidence, Oxford u.a.

Baumol, W.J., Wolff, E.N. (1988), Productivity Growth, Convergence and Welfare: Reply, in: American Economic Review, Bd. 78, Nr. 5, S. 1155-1159.

Becker, G.S. (1964), Human Capital. A Theoretical and Empirical Analysis with Special Reference to Education, New York.

Becker, G.S., Murphy, K.M., Tamura, R. (1990), Human Capital, Fertility, and Economic Growth, in: Journal of Political Economy, Bd. 98, Nr. 5, S. S12-S37.

Beckerman, Wilfried (1965), The British Economy in 1975, Cambridge.

Black, J. (1962), The Technical Progress Function, in: Economica, Bd. 29, S. 166-170.

Blanchard, O.J., Fischer, St. (1989), Lectures on Macroeconomics, Cambridge (Mass.) und London.

Blitch, Ch.P. (1983a), Allyn Young on Increasing Returns, in: Journal of Post Keynesian Economics, Bd. 5, Nr. 3, S. 359-372.

Blitch, Ch.P. (1983b), Allyn A. Young: a Curious Case of Professional Neglect, in: History of Political Economy, Bd. 15, Nr. 1, S. 1-24.

Blitch, Ch.P. (1990), Kaldor's Lecture Notes from Allyn Young's London School of Economics Class, 1928-29, in: History of Political Economy, Bd. 22, Nr. 3, S. 443-463.

Boadway, R.W., Wildasin, D.E. (1984), Public Sector Economics, 2. Auflage, Boston und Toronto.

Boltho, A., Holtham, G. (1992), The Assessment: New Approaches to Economic Growth, in: Oxford Review of Economic Policy, Bd. 8, Nr. 4, S. 1-14.

Bombach, G. (1965), Wirtschaftswachstum, in: Hanawörterbuch der Sozialwissenschaften, Stuttgart, S. 763-801. 
Bombach, G. (1985), Post-War Growth Revisited, Amsterdam.

Boulier, B.L. (1984), What lies behind Verdoorn's Law?, in: Oxford Economic Papers, Bd. 26, S. 259-267.

Boyer, R., Petit, P. (1989), The Cumulative Growth Model Revisited, in: Political Economy: Studies in the Surplus Approach, Bd. 4, Nr. 1, S. 23-43.

Boyer, R., Petit, P. (1991a), Kaldor's Growth Theories: Past, Present and Prospects for the Future, in: Nell, E.J., Semmler, W. (Hrsg.) (1991), Nicholas Kaldor and Mainstream Economics, London, S. 485-517.

Boyer, R., Petit, P. (1991b), Technical Change, Cumulative Causation and Growth: Accounting for the Contemporary Productivity Puzzle with Some Post Keynesian Theories, in: OECD (Hrsg.) (1991), Technology and Productivity: the Challenge for Economic Policy, the Technology Economy Programme, Paris, S. 47-67.

Buchanan, J.M., Yoon, Y.J. (Hrsg.) (1994), The Return to Increasing Returns, Ann Arbor.

Butler, G., Hof, B. (1977), Bevölkerung und Arbeitsmarkt bis zum Jahr 2000, Köln.

Buttler, F., Kühl, J., Rahmann, B. (Hrsg.) (1985), Staat und Beschäftigung, Angebots- und Nachfragepolitik in Theorie und Praxis, Beiträge zur Arbeitsmarkt- und Berufsforschung, Bd. 88, Nürnberg.

Canning, D.J. (1988), Increasing Returns in Industry and the Role of Agriculture in Growth, in: Oxford Economic Papers, Bd. 40, Nr. 3, S. 463-476.

Cass, D. (1965), Optimum Growth in an Aggregative Model of Capital Accumulation, in: Review of Economic Studies, Bd. 32, S. 233-240.

Cass, D., Shell, K. (1976a), Introduction to Hamiltonian Dynamics in Economics, in: Journal of Economic Theory, Bd. 12, Nr. 1, S. 1-10.

Cass, D., Shell, K. (1976b), The Structure and Stability of Competitive Dynamical Systems, in: Journal of Economic Theory, Bd. 12, Nr. 1, S. 31-70.

Chamberlin, E. (1933), The Theory of Monopolistic Competition, Cambridge (Mass.).

Chatterii, M. (1992), Convergence Clubs and Endogenous Growth, in: Oxford Review of Economic Policy, Bd. 8, Nr. 4, S. 57-69.

Chatterii, M., Wickens, M. (1981), Verdoorn's Law - The Externalities Hypothesis and Economic Growth in the U.K., in: Currie, D., Nobay, R., Peell, D. (Hrsg.) (1981), Macroeconomic Analysis: Essays in Macroeconomics and Econometrics, London, S. 405429.

Chatterji, M., Wickens, M. (1982), Productivity, Factor Transfer and Economic Growth in the U.K., in: Economica, Bd. 49, S. 21-38.

Chatterii, M., Wickens, M. (1983), Verdoorn's Law and Kaldor's Law: a Revisionist Interpretation?, in: Journal of Post Keynesian Economics, Bd. 5, Nr. 3, S. 396-413. 
Chiang, A.C. (1984), Fundamental Methods of Mathematical Economics, 3. Auflage, New York u.a.

Chipman, J.S. (1965), A Survey of the Theory of International Trade: Part 2, The NeoClassical Theory, in: Econometrica, Bd. 33, S. 685-760.

Chipman, J.S. (1970), External Economies of Scale and Competitive Equilibrium, in: Quarterly Journal of Economics, Bd. 84, S. 347-385, hier Nachdruck in: Buchanan, J.M., Yoon, Y.J. (Hrsg.) (1994), The Return to Increasing Returns, Ann Arbor, S. 121-166.

Chowdhury, A., Islam, I. (1993), The Newly Industrialising Economies of East Asia, London und New York.

Coale, A.J. (Hrsg.) (1976), Economic Factors in Population Growth, Proceedings of a Conference held by the International Economic Association at Valescure, France, London.

Coase, R.H. (1960), The Problem of Social Cost, in: Journal of Law and Economics, Bd. 3, S. $1-44$.

Cornwall, J. (1968), Postwar Growth in Western Europe. A Re-evaluation, in: Review of Economics and Statistics, Bd. 50, Nr. 3, S. 361-367.

Cornwall, J. (1976), Diffusion, Convergence and Kaldor's Law, in: Economic Journal, Bd. 86, S. 307-314.

Cornwall, J. (1977), Modern Capitalism: Its Growth and Transformation, London.

Crafts, N.F.R. (1996), "Post-Neoclassical Endogenous Growth Theory": What are its Policy Implications?, in: Oxford Review of Economic Policy, Bd. 12, Nr. 2, S. 30-47.

Cripps, T.F., Tarling, R.J. (1973), Growth in Advanced Capitalist Economics 1950-1970, Cambridge.

Currie, D., Nobay, R., Peell, D. (Hrsg.) (1981), Macroeconomic Analysis: Essays in Macroeconomics and Econometrics, London.

David, P. (1985), Clio and the Economics of QWERTY, in: American Economic Review, Bd. 75, Nr. 2, S. 332-337.

DeLong, J.B. (1988), Productivity Growth, Convergence, and Welfare: Comment, in: American Economic Review, Bd. 78, Nr. 5, S. 1138-1154.

DeLong, J.B. (1993), Growth in the World Economy, ca. 1870-1990, in: Siebert, H. (Hrsg.) (1993), Economic Growth in the World Economy, Tübingen, S. 3-26.

DeLong, J.B., Summers, L.H. (1991), Equipment Investment and Economic Growth, in: Quarterly Journal of Economics, Bd. 106, Nr. 2, S. 445-502.

DeLong, J.B., Summers, L.H. (1993), How Strongly Do Developing Economies Benefit from Equipment Investment?, in: Journal of Monetary Economics, Bd. 32, Nr. 3, S. 395-416.

Delong, J.B., Summers, L.H. (1994), Equipment Investment and Economic Gowth: Reply, in: Quarterly Journal of Economics, Bd. 109, Nr. 3, S. 803-807. 
Denison, E.F. (1962), The Sources of Economic Growth in the United States and Alternatives Before Us, New York.

Denison, E.F. (1967), Why Growth Rates Differ: Post-War Experience in Nine Western Countries, Washington, D.C.

Denison, E.F. (1972), Classification of Sources of Growth, in: Review of Income and Wealth, Bd. 18, S. 1-25.

Denison, E.F. (1974), Accounting for United States Economic Growth 1929-1969, Washington, D.C.

Denison, E.F. (1979), Accounting for Slower Economic Growth, Washington, D.C.

Denison, E.F. (1984), Accounting for Slower Economic Growth: An Update, in: Kendrick. J.W. (Hrsg.) (1984), International Comparisons of Productivity and Causes of the Slowdown, Cambridge (Mass.), S. 1-45.

Denison, E.F. (1985), Trends in American Economic Growth, 1929-1982, Washington, D.C.

Diamond, P. (Hrsg.) (1990), Growth/Productivity/Employment: Essays to Celebrate Bob Solow's Birthday, Cambridge (Mass.).

Dixit, A.K. (1990), Optimization in Economic Theory, 2. Auflage, Oxford.

Dixit, A.K., Stiglitz, J.E. (1977), Monopolistic Competition and Optimum Product Diversity, in: American Economic Review, Bd. 67, S. 297-308.

Dixon, R., Thirlwall, A.P. (1975), A Model of Regional Growth-Rate Differences on Kaldorian Lines, in: Oxford Economic Papers, Bd. 27, Nr. 2, S. 201-214.

Domar, E.D. (1946), Capital Expansion, Rate of Growth and Employment, in: Econometrica, Bd. 14, S. 137-147.

Domar, E.D. (1947), Expansion and Employment, in: American Economic Review, Bd. 37, Nr. 1, S. 34-55.

Dosi, G., Fabiani, S. (1994), Convergence and Divergence in the Long-term Growth of Open Economies, in: Silverberg, G., Soete, L. (Hrsg.) (1994), The Economics of Growth and Technical Change: Technologies, Nations, Agents, Aldershot, S. 119-146.

Dosi, G., Freeman, C., Fabiani, S., Aversi, R. (1994), The Diversity of Development Patterns: Catching Up, Forging Ahead and Falling Behind, in: Pasinetti, L.L., Solow, R.M. (Hrsg.) (1994), Economic Growth and the Structure of Long-Term Development: Proceedings of the IEA Conference held in Varenna (Italy), New York, S. 132-167.

Douglas, P.H. (1934), The Theory of Wages, New York.

Dowrick, S., Gemmell, N. (1991), Industrialization, Catching Up and Economic Growth: A Comparative Study Across the World's Capitalist Economies, in: Economic Journal, Bd. 101, S. 263-275. 
Dowrick, S., Nguyen, D.T. (1989), OECD Comparative Economic Growth 1950-1985: CatchUp and Convergence, in: American Economic Review, Bd. 79, Nr. 5, S. 1010-1030.

Dutt, A. K. (1992), A Kaldorian Model of Growth and Development Revisited: A Comment on Thirlwall, in: Oxford Economic Papers, Bd. 44, Nr. 1, S. 156-168.

Eatwell, J., Milgate, M., Newman, P. (Hrsg.) (1987), The New Palgrave: a Dictionary of Economics, London.

Eatwell, J., Milgate, M., Newman, P. (Hrsg.) (1990), The New Palgrave: Capital Theory, London.

Eltis, W. (1987), Harrod-Domar Growth Model, in: Eatwell, J., Milgate, M., Newman, P. (Hrsg.) (1987), The New Palgrave: a Dictionary of Economics, London, S. 602-604.

Erber, G. (1980), Skalenerträge, Wettbewerb versus Monopol und die neoklassische Theorie der Unternehmung: Prolegomena einer Kritik der ökonomischen Theorie, Berlin.

Erber, G. (1994), Verdoorn's or Okun's Law? Employment and Growth Experiences in OECD Countries 1960-1993, DIW-Diskussionspapiere Nr. 98, Berlin.

Ethier, W.J. (1982), National and International Returns to Scale in the Modern Theory of International Trade, in: American Economic Review, Bd. 72, S. 389-405.

EU-Kommission (1994), Wachstum, Wettbewerbsfăhigkeit, Beschäftigung: Herausforderungen der Gegenwart und Wege ins 21. Jahrhundert, Weißbuch, Luxemburg.

Fischer, St. (Hrsg.) (1987), National Bureau of Economic Research Annual 1987, Cambridge (Mass.).

Fischer, St. (1993), The Role of Macroeconomic Factors in Growth, in: Journal of Monetary Economics, Bd. 32, Nr. 3, S. 485-512.

Flemmig, J., Götz, G. (1993), Externaltitäten, Nichtkonvexitäten und endogener technischer Fortschritt: ein Einblick in die Funktionsweise der "neuen" Wachstumstheorie, in: Jahrbuch für Sozialwissenschaft, Bd. 44, S. 203-215.

Frey, B.S. (1969), Eine einfache Einfuhrung zu Pontryagins Maximum-Prinzip im Wirtschaftswachstum, in: Weltwirtschaftliches Archiv, Bd. 103, S. 213-228.

Frey, B.S. (1970), Optimales Wachstum; Übersicht und Kritik, in: Jahrbücher für Nationalökonomie und Statistik, Bd. 184, S. 9-30.

Gahlen, B. (1972), Der Informationsgehalt der neoklassischen Wachstumstheorie für die Wirtschaftspolitik, Tübingen.

Gahlen, B., Hesse, H., Ramser, H.J. (Hrsg.) (1991), Wachstumstheorie und Wachstumspolitik: ein neuer Anlauf, Schriftenreihe des Wirtschaftswissenschaftlichen Seminars Ottobeuren, Bd. 20, Tübingen.

Gehrke, Chr. (1990), Wachstumstheoretische Vorstellungen bei Adam Smith, in: Kurz, H.D. (Hrsg.) (1990b), Adam Smith (1723-1790): ein Werk und seine Wirkungsgeschichte, Marburg, S. 129-151. 
Gehrke, Chr., Rühl, Chr. (1988), Verlangsamung des Produktivitätswachstums? Erklärungsansätze und empirische Befunde für die Bundesrepublik Deutschland, Bremen.

Giersch, H., Wolter, F. (1983), Towards an Explanation of the Productivity Slowdown: an Acceleration-Deceleration Hypothesis, in: Economic Journal, Bd. 93, S. 35-55.

Gomulka, S. (1971), Inventive Activity, Diffusion, and the Stages of Economic Growth, Aarhus.

Gourvitch, A. (1940), Survey of Economic Theory on Technological Change and Employment, Nachdruck 1966, New York.

Groenewegen, P. (1987), Division of Labour, in: Eatwell, J., Milgate, M., Newman, P. (Hrsg.) (1987), The New Palgrave: a Dictionary of Economics, London, S. 901-907.

Grossman, G.M., Helpman, E. (1991), Innovation and Growth in the Global Economy, Cambridge (Mass.).

Gundlach, E. (1995), The Role of Human Capital in Economic Growth: New Results and Alternative Interpretations, in: Weltwirtschaftliches Archiv, Bd. 131, Nr. 2, S. 383-402.

Hacche, G. (1979), The Theory of Economic Growth: an Introduction, New York.

Hagemann, H. (1977), Rate of Return und Profitrate: eine kapitaltheoretische Kontroverse zwischen Neoklassikern und Postkeynesianern im Rahmen der Cambridge-Debatte, Schriften zur wirtschaftswissenschaftlichen Forschung, Bd. 125, Meisenheim am Glan.

Hagemann, H. (1984), Lohnsenkungen als Mittel der Krisenbekämpfung? Überlegungen zum Beitrag der 'Kieler Schule' in der beschäftigungspolitischen Diskussion am Ende der Weimarer Republik, in: Hagemann, H., Kurz, H.D. (Hrsg.) (1984), Beschäftigung, Verteilung und Konjunktur: zur Politischen Okonomik der modernen Gesellschaft. Festschrift für Adolph Lowe, Bremen, S. 97-129.

Hagemann, H. (1985), Freisetzungs- und Kompensationseffekte neuer Technologien: Zur Gefahr einer technologischen Arbeitslosigkeit, in: Buttler, F., Kühl, J., Rahmann, B. (Hrsg.) (1985), Staat und Beschäftigung, Angebots- und Nachfragepolitik in Theorie und Praxis, Beiträge zur Arbeitsmarkt- und Berufsforschung, Bd. 88, Nürnberg, S. 291-335.

Hagemann, H. (1990a), Akkumulation, neue Technologien und Beschäftigung - Zu den quantitativen und qualitativen Beschäftigungswirkungen des technischen Fortschritts bei Adam Smith, in: Kurz, H.D. (Hrsg.) (1990b), Adam Smith (1723-1790): ein Werk und seine Wirkungsgeschichte, Marburg, S. 153-174.

Hagemann, H. (1990b), Capital Goods, in: Eatwell, J., Milgate, M., Newman, P. (Hrsg.) (1990), The New Palgrave: Capital Theory, London, S. 123-127.

Hagemann, H. (1990c), The Structural Theory of Economic Growth, in: Baranzini, M., Scazzeri, R. (Hrsg.) (1990), The Economic Theory of Structure and Change, Cambridge, S. 144-171. 
Hagemann, H. (1991), A Kaldorian Saving Function in a Two-Sectoral Linear Growth Model, in: Nell, E.J., Semmler, W. (Hrsg.) (1991), Nicholas Kaldor and Mainstream Economics, London, S. 449-468.

Hagemann, H. (1992), Traverse Analysis in a Post-Classical Model, in: Halevi, J., Laibman, D., Nell, E.J. (Hrsg.) (1992), Beyond the Steady State. A Revival of Growth Theory, London, S. 235-263.

Hagemann, H. (1994), Technological Unemployment, in: Arestis, Ph., Sawyer, M. (Hrsg.) (1994), The Elgar Companion to Radical Political Economy, Aldershot, S. 410-415.

Hagemann, H. (1995), Technological Unemployment, in: Arestis, Ph., Marshall, M.G. (Hrsg.) (1995), The Political Economy of Full Employment, Aldershot, S. 36-53.

Hagemann, H. (1996), Von der Konjunktur- zur Wachstumstheorie: Kontinuität und Evolution im Forschungsprogramm Adolf Löwes, in: Rieter, H. (Hrsg.) (1996), Studien zur Entwicklung der ökonomischen Theorie, Schriften des Vereins für Socialpolitik, N.F., Bd. 115/XV, S. 123-156.

Hagemann, H., Kalmbach, P. (Hrsg.) (1983), Technischer Fortschritt und Arbeitslosigkeit, Fankfurt a.M. und New York.

Hagemann, H., Kurz, H.D. (Hrsg.) (1984), Beschäftigung, Verteilung und Konjunktur: zur Politischen Okonomik der modernen Gesellschaft. Festschrift für Adolph Lowe, Bremen.

Hahn, F.H. (1989), Kaldor on Growth, in: Cambridge Journal of Economics, Bd. 13, S. 4757.

Hahn, F.H., Matthews, R.C.O. (1964), The Theory of Growth. A Survey, in: Economic Journal, Bd. 74, S. 779-902.

Hahn, F.R. (1994), Wachstum und Arbeitslosigkeit. Neue Erkenntnisse aus der Wachstumstheorie, in: ifo-Studien, Bd. 40, S. 305-319.

Halevi, J., Laibman, D., Nell, E.J. (Hrsg.) (1992), Beyond the Steady State. A Revival of Growth Theory, London.

Harcourt, G.C. (1969), Some Cambridge Controversies in the Theory of Capital, in: Journal of Economic Literature, Bd. 7, S. 369-405.

Harcourt, G.C. (1970), A Reply, in: Journal of Economic Literature, Bd. 8, S. 44-45.

Harcourt, G.C. (1972), Some Cambridge Controversies in the Theory of Capital, Cambridge.

Harcourt, G.C. (1976), The Cambridge Contorversies: Old Ways and New Horizons? - Or Dead End?, Oxford Economic Papers, Bd. 28, S. 25-65.

Harrod, R.F. (1939), An Essay in Dynamic Theory, in: Economic Journal, Bd. 49, S. 14-33.

Harrod, R.F. (1948), Towards a Dynamic Economics, London und New York.

Harrod, R.F. (1960), Second Essay in Dynamic Theory, in: Economic Journal, Bd. 70, S. $277-$ 293. 
Harrod, R.F. (1973), Economic Dynamics, London.

Helpman, E. (1992), Endogenous Macroeconomic Growth Theory, in: European Economic Review, Bd. 36, S. 237-267.

Hey, J.D. (Hrsg.) (1992), The Future of Economics, Oxford.

Hicks, J.R. (1932), The Theory of Wages, London.

Hicks, J.R. (1949), Mr. Harrod's Dynamic Theory, in: Economica, Bd. 16, S. 106-121.

Hicks, J.R. (1950), The Trade Cycle, Oxford.

Hicks, J.R. (1973), Capital and Time, Oxford.

Hodgson, G. (1989), Institutional Rigidities and Economic Growth, in: Cambridge Journal of Economics, Bd. 13, S. 79-101.

Hof, B. (1991), Die Beschäftigungsschwelle, in: Wirtschaftswissenschaftliches Studium (WiSt), Nr. 6, S. 281-286.

Hof, B. (1994), Beschäftigungsschwelle und Wachstum - was besagt die Empirie?, in: ifoStudien, Bd. 40, S. 127-144.

Homburg, St. (1995), Humankapital und endogenes Wachstum, in: Zeitschrift für Wirtschaftsund Sozialwissenschaften, Bd. 115, S. 339-366.

Inada, K-I. (1963), On a Two-Sector Model of Economic Growth: Comments and a Generalization, in: Review of Economic Studies, Bd. 30, S. 119-127.

Ireland, P.N. (1994), Supply-side Economics and Endogenous Growth, in: Journal of Moentary Economics, Bd. 33, S. 559-571.

Ito, T. (1992), The Japanese Economy, Cambridge (Mass.).

Johansen, L. (1959), Substitution versus Fixed Production Coefficients in the Theory of Economic Growth. A Synthesis, in: Econometrica, Bd. 27, S. 157-176.

Jones, C.I. (1995), Time Series Tests of Endogenous Growth Models, in: Quartely Journal of Economics, Bd. 110, Nr. 2, S. 495-525.

Jones, L.E., Manuelli, R.E. (1990), A Convex Modell of Equilibrium Growth: Theory and Policy Implications, in: Journal of Political Economy, Bd. 98, Nr. 5, S. 1008-1038.

Jorgenson, D. W., Griliches, Z. (1967), The Explanation of Productivity Change, in: Review of Economic Studies, Bd. 34, S. 249-280.

Jorgenson, D.W. (Hrsg.) (1995), Productivity, Vol. I+II, Cambridge (Mass.).

Judd, K.L. (1985), On the Performance of Patents, in: Econometrica, Bd. 55, Nr. 3, S. 567585 . 
Kaldor, N. (1939), Speculation and Economic Stability, in: Review of Economic Studies, Bd. 7, S. 1-27.

Kaldor, N. (1955/56), Alternative Theories of Distribution, in: Review of Economic Studies, Bd. 23, Nr. 2, S. 83-100, hier Nachdruck in: Targetti, F., Thirlwall, A.P. (Hrsg.) (1989), The Essential Kaldor, London, S. 201-228.

Kaldor, N. (1957), A Model of Economic Growth, in: Economic Journal, Bd. 67, S. 591-624.

Kaldor, N. (1959) Economic Growth and the Problem of Inflation, in: Economica, Bd. 26, S. 212-226 und S. 287-298.

Kaldor, N. (1961), Capital Accumulation and Economic Growth, in: Lutz, F.A., Hague, D.C. (Hrsg.) (1961), The Theory of Capital, London und New York, hier Nachdruck in: Targetti, E., Thirlwall, A.P. (Hrsg.) (1989), The Essential Kaldor, London, S. 229-281.

Kaldor, N. (1966), Causes of the Slow Rate of Economic Growth in the United Kingdom, Inaugural Lecture at the University of Cambridge, Cambridge, hier Nachdruck in: Targetti, E., Thirlwall, A.P. (Hrsg.) (1989), The Essential Kaldor, London, S. 282-310.

Kaldor, N. (1967), Strategic Factors in Economic Development, Ithaca.

Kaldor, N. (1968), Productivity and Growth in Manufacturing Industry: A Reply, in: Economica, Bd. 35, S. 385-391.

Kaldor, N. (1970a), The Case for Regional Policies, in: Scottish Journal of Political Economy, Bd. 17, Nr. 3, S. 337-348.

Kaldor, N. (1970b), Some Fallacies in the Interpretation of Kaldor, in: Review of Economic Studies, Bd. 37, S. 1-7.

Kaldor, N. (1972), The Irrelevance of Equilibrium Economics, in: Economic Journal, Bd. 82, S. 1237-1255, hier Nachdruck in: Targetti, E., Thirlwall, A.P. (Hrsg.) (1989), The Essential Kaldor, London, S. 373-398.

Kaldor, N. (1975), Economic Growth and the Verdoorn Law. A Comment on Mr. Rowthorn's Article, in: Economic Journal, Bd. 85, S. 891-896.

Kaldor, N. (1978), Introduction, in: Kaldor, N., Further Essays on Economic Theory, London, S. VII-XXIX.

Kaldor, N. (1980), The Foundations of Free Trade Theory and their Implications for the Current World Recession, in: Malinvaud, E., Fitoussi, J.P. (Hrsg.) (1980), Unemployment in Western Countries, London, hier Nachdruck in: Kaldor, N. (1989), Further Essays on Economic Theory and Policy, hrsg. von Targetti, F. und Thirlwall, A.P., London, S. 224234.

Kaldor, N. (1981a), The Role of Increasing Returns, Technical Progress and Cumulative Causation in the Theory of International Trade and Economic Growth, in: Économie Appliqué, Nr. 34, S. 543-617. 
Kaldor, N. (1981b), Discussion [von Chatterii and Wickens], in: Currie, D., Nobay, R., Peell, D. (Hrsg.) (1981), Macroeconomic Analysis: Essays in Macroeconomics and Econometrics, London, S. 430-433.

Kaldor, N. (1985), Economics without Equilibrium, Armonk (N.Y.).

Kaldor, N. (1986a), Limits on Growth, in: Oxford Economic Papers, Bd. 38, S. 187-198.

Kaldor, N. (1986b), Recollections of an Economist, in: Banca Nazionale del Lavoro Quarterly Review, Bd. 39, S. 3-26.

Kaldor, N., Mirrlees, J.A. (1962), A New Model of Economic Growth, in: Review of Economic Studies, Bd. 29, S. 174-192.

Kalmbach, P. (1972), Wachstum und Verteilung in neoklassischer und postkeynesianischer Sicht, Berlin.

Kalmbach, P. (1983), Die Berechnung der Freisetzung und Einsparung von Arbeitskräften: Einige Überlegungen anläßlich der ex-post-Betrachtung einer Prognose, in: Hagemann, $\mathrm{H}$., Kalmbach, P. (Hrsg.) (1983), Technischer Fortschritt und Arbeitslosigkeit, Frankfurt a.M. und New York, S. 296-311.

Kendrick. J.W. (Hrsg.) (1984), International Comparisons of Productivity and Causes of the Slowdown, Cambridge (Mass.).

Kennedy, P. (1992), A Guide to Econometrics, 3. Auflage, Oxford und Cambridge (Mass.).

Keynes, J.M. (1936), The General Theory of Employment, Interest, and Money, London, Nachdruck San Diego u.a. 1964.

Kim, J.W. (1989), Evolution of Industrial Policy in Korea, in: Klenner, W. (Hrsg.) (1989), Trends of Economic Development in East Asia, Berlin und Heidelberg, S. 461-473.

King, R.G., Rebelo, S. (1990), Public Policy and Economic Growth: Developing Neoclassical Implications, in: Journal of Political Economy, Bd. 98, S. S126-S150.

Klauder, W. (1971), Zur Aussagekraft und Problematik von Freisetzungsberechnungen, in: Rationalisierungskuratorium der Deutschen Wirtschaft e.V. (Hrsg.) (1971), Produktivität und Rationalisierung. Chancen, Wege, Forderungen, Frankfurt a.M., S. 86-91.

Klauder, W. (1990), Zur Entwicklung von Produktivität und Beschäftigungsschwelle, in: Mitteilungen aus der Arbeitsmarkt- und Berufsforschung, Bd. 23, Nr. 1, S. 86-99.

Klenner, W. (Hrsg.) (1990), Trends of Economic Development in East Asia, Berlin und Heidelberg

Kluge, M. (1968), Technischer Fortschritt und Beschäftigung, Berichte des Deutschen Instituts für Wirtschaftspolitik, Bd. 2, Nr. 5.

Knight, F.H. (1921), Risk, Uncertainty and Profit, Chicago.

Knight, F.H. (1924), Some Fallacies in the Interpretation of Social Cost, in: Quarterly Journal of Economics, Bd. 38, S. 582-606. 
Knight, F.H. (1925), Decreasing Cost and Comparative Cost: A Rejoinder, in: Quarterly Journal of Economics, Bd. 39, S. 332-333.

König, H. (Hrsg.) (1968), Wachstum und Entwicklung der Wirtschaft, Köln und Berlin.

Komiya, R., Okuno, M., Suzumura, K. (Hrsg.) (1988), Industrial Policy of Japan, Tokio u.a.

Koopmans, T.C. (1970), On the Concept of Optimal Growth, in: Scientific Papers of T.C. Koopmans, Berlin u.a., S. 485-547.

Kormendi, R.C., Meguire, P.G. (1985), Macroeconomic Determinants of Growth: CrossCountry Evidence, in: Journal of Monetary Economics, Bd. 16, Nr. 2, S. 141-163.

Kosai, Y. (1988), The Reconstruction Period, in: Komiya, R., Okuno, M., Suzumura, K. (Hrsg.) (1988), Industrial Policy of Japan, Tokio u.a., S. 25-48.

Krengel, R. (1958), Anlagevermögen, Produktion und Beschäftigung der Industrie im Gebiet der Bundesrepublik von 1924 bis 1956, Berlin.

Krengel, R. (1963), Some Reasons for the Rapid Economic Growth of the German Federal Republic, in: Banca Nazionale del Lavoro Quarterly Review, Bd. 64, S. 121-144.

Krugman, P.R. (1987), The Narrow Moving Band, the Dutch Disease, and the Competitive Consequences of Mrs. Thatcher: Notes on Trade in Presence of Dynamic Scale Economies, in: Journal of Development Economics, Bd. 27, S. 41-55, hier Nachdruck in:: Krugman, P.R. (Hrsg.) (1990), Rethinking International Trade, Cambridge (Mass.), S. 106-120.

Krugman, P.R. (Hrsg.) (1990), Rethinking International Trade, Cambridge (Mass.).

Krugman, P.R. (1991), History versus Expectations, in: Quarterly Journal of Economics, Bd. 106, Nr. 2, S. 651-667.

Krugman, P.R. (1994), Peddling Prosperity: Economic Sense and Nonsense in the Age of Diminished Expectations, New York und London.

Kubota, K. (1968), A Re-examination of the Existence and Stability Propositions in Kaldor's Growth Models, in: Review of Economic Studies, Bd. 35, S. 353-360.

Kühlewind, G., Schnur, P. (1970), Darstellung der Gesamtproblematik von Freisetzungsberechmungen anhand vorliegender Modelle einschließlich einer Darstellung von Forschungslücken. Expertise im Auftrag des Arbeitskreises Automation, Erlangen.

Kuhlo, K.-Chr. (1965), Begriffe und Berechnungen von Freisetzungen, in: ifo-Studien, Bd. 11, S. 45-103.

Külp, B. (1994), Verteilungstheorie, 3., neubearbeitete und erweiterte Auflage, Stuttgart und New York.

Kurz, H.D. (1984), Akkumulation, Einkommensverteilung und effektive Nachfrage, in: Hagemann, H., Kurz, H.D. (Hrsg.) (1984), Beschäftigung, Verteilung und Konjunktur: zur Politischen Ökonomik der modernen Gesellschaft. Festschrift für Adolph Lowe, Bremen, S. 161-185. 
Kurz, H.D. (1990a), Debates in Capital Theory, in: Eatwell, J., Milgate, M., Newman, P. (Hrsg.) (1990), The New Palgrave: Capital Theory, London, S. 79-93.

Kurz, H.D. (Hrsg.) (1990b), Adam Smith (1723-1790): ein Werk und seine Wirkungsgeschichte, Marburg.

Kurz, H.D., Salvadori, N. (1995a), The Theory of Production: a Long-Period Analysis, Cambridge.

Kurz, H.D., Salvadori, N. (1995b), Theories of 'Endogenous' Growth in Historical Perspective, Papier präsentiert auf dem 11. Weltkongreß der International Economic Association, Tunis.

Leijonhufvud, A. (1973), Effective Demand Failures, in: Swedish Journal of Economics, Bd. 75, S. 27-58.

Lessat, V. (1994), Endogenes Wirtschaftswachstum. Theoretische Modelle und wirtschaftspolitische Implikationen, Frankfurt a.M. u.a.

Levhari, D. (1966a), Further Implications of Learning by Doing, in: Review of Economic Studies, Bd. 33, S. 31-38.

Levhari, D. (1966b), Extensions of Arrow's "Learning by Doing", in: Review of Economic Studies, Bd. 33, S. 117-131.

Levine, R., Renelt, D. (1992), A Sensitivity Analysis of Cross-Country Growth Regressions, in: American Economic Review, Bd. 82, Nr. 4, S. 942-963.

Lewis, W.A. (1954), Economic Development with Unlimited Supplies of Labour, in: The Manchester School, S. 139-191.

Lewis, W.A. (1955), The Theory of Economic Growth, London.

Lindbeck, A. (1983), The Recent Slowdown of Productivity Growth, in: Economic Journal, Bd. 93, S. 13-34.

Lowe, A. (1954a), The Classical Theory of Economic Growth, in: Lowe, A (1954b)., Essays in Political Economics: Public Control in a Democratic Society, Brighton, S. 107-131.

Lowe, A. (1954b), Essays in Political Economics: Public Control in a Democratic Society, Brighton.

Lowe, A. (1976), The Path of Economic Growth, Cambridge.

Lowe, A. (1984), Politische Okonomik - On Economic Knowledge, Königstein/Ts. (dt. Übersetzung von On Economic Knowledge, 2. Auflage, New York, 1965).

Lucas, R.E. jr. (1988), On the Mechanics of Economic Development, in: Journal of Monetary Economics, Bd. 22, Nr. 1, S. 3-42.

Lucas, R.E. jr. (1990), Why doesn't Capital Flow from Rich to Poor Countries?, in: American Economic Review, Bd. 80, Nr. 2, Papers and Proceedings, S. 92-96. 
Lucas, R.E. jr. (1993), Making a Miracle, in: Econometrica, Bd. 61, Nr. 2, S. 251-272.

Lutz, F.A., Hague, D.C. (Hrsg.) (1961), The Theory of Capital, London und New York.

Maddison, A. (1982), Phases of Capitalist Development, New York.

Maddison, A. (1989), The World Economy in the 20th Century, Paris.

Maddison, A. (1993), Comment on J. Bradford DeLong, 'Growth in the World Economy, ca. 1870-1990', in: Siebert, H. (Hrsg.) (1993), Economic Growth in the World Economy, Tübingen, S. 27-30.

Maddison, A. (1994), Explaining the Economic Performance of Nations, 1820-1898, in: Baumol, W.J., Nelson, R.R., Wolff, E.N. (Hrsg.) (1994), Convergence of Productivity: Cross-National Studies and Historical Evidence, Oxford u.a., S. 20-61.

Malinvaud, E. (1979), Economic Growth and Resources, Bd. 1, Proceedings of the Fifth World Kongress of the International Economic Association held in Tokyo (Japan), London.

Malinvaud, E., Fitoussi, J.P. (Hrsg.) (1980), Unemployment in Western Countries, London.

Mankiw, N.G., Romer, D., Weil, D.N. (1992), A Contribution to the Empirics of Economic Growth, in: Quarterly Journal of Economics, Bd. 107, S. 407-437.

Marschak, J., Radner, R. (1972), Economic Theory of Teams, New Haven, Monograph Cowles Foundation for Economic Research in Economics, Bd. 22.

Marshall, A. (1920), Principles of Economics, 8. Auflage, London.

Matthews, R.C.O. (1964), "The New View of Investment": Comment, in: Quarterly Journal of Economics, Bd. 78, S. 164-176.

McClelland, D.C. (1961), The Achieving Society, Princeton u.a.

McCombie, J.S.L. (1980), A Comment on Some Recent Interpretations of Verdoorn's Law, Hull Economic Research Papers Nr. 69, University of Hull.

McCombie, J.S.L. (1982), Economic Growth, Kaldor's Laws and the Static-Dynamic Verdoorn Law Paradox, in: Applied Economics, Bd. 14, S. 279-294.

McCombie, J.S.L. (1983), Kaldor's Laws in Retrospect, in: Journal of Post Keynesian Economics, Bd. 5, Nr. 3, S. 414-429.

McCombie, J.S.L., DeRidder, J.R. (1984), "The Verdoorn Law Controversy": Some New Empirical Evidence Using U.S. State Data, in: Oxford Economic Papers, Bd. 36, S. 268284.

McCombie, J.S.L., Thirlwall, A.P. (1994), Economic Growth and the Balance-of-Payments Constraint, London.

Meade, J.E. (1952), External Economies and Diseconomies in a Competitive Situation, in: Economic Journal, Bd. 62, S. 54-67. 
Meade, J.E. (1962a), A Neoclassical Theory of Economic Growth, 2. Auflage, London.

Meade, J.E. (1962b), The Effects of Savings on Consumption in a State of Steady Growth, in: The Review of Economic Studies, Bd. 29, S. 227-234.

Michel, Ph. (1982), On the Transversality Condition in Infinite Horizon Optimal Problems, in: Econometrica, Bd. 50, Nr. 4, S. 975-986.

Michl, Th.R. (1985), International Comparisons of Productivity Growth: Verdoorn's Law Revisited, in: Journal of Post Keynesian Economics, Bd. 7, Nr. 4, S. 474-492.

Mincer, J. (1958), Investment in Human Capital and Personal Income, in: Journal of Political Economy, Bd. 66, S. 281-302.

Morishima, M. (1982), Why has Japan "succeeded"?: Western Technology and the Japanese Ethos, Cambridge.

Müller, K.W., Ströbele, W. (1985), Wachstumstheorie, München und Wien.

Myrdal, G. (1959), Ökonomische Theorie und unterentwickelte Regionen, Stuttgart. (Dt. Übersetzung von Economic Theory and Underdeveloped Regions, London 1957).

Nell, E.J., Semmler, W. (Hrsg.) (1991), Nicholas Kaldor and Mainstream Economics, London.

Nelson, R. (1995), Recent Evolutionary Theorizing About Economic Change, in: Journal of Economic Literature, Bd. 33, Nr. 1, S. 48-90.

Neumann, M. (1990), Zukunftsperspektiven im Wandel: lange Wellen in Wirtschaft und Politik, Studien in den Grenzbereichen der Wirtschafts- und Sozialwissenschaften, Bd. 66, Tübingen.

Neumann, M. (1994), Wirtschaftswachstum durch Innovationen und Investitionen: Zusammenhänge und wirtschaftspolitische Perspektiven, in: Arbeitsgemeinschaft Deutscher Wirtschaftswissenschaftlicher Forschungsinstitute (Hrsg.) (1994), Wachstumsperspektiven in den neunziger Jahren, Tagungsband der Arbeitsgemeinschaft Deutscher Wirtschaftswissenschaftlicher Forschungsinstitute e.V. (gleichzeitig Beiheft der Konjunkturpolitik, Bd. 42), Berlin, S. 11-21.

Niehans, J. (1963), Economic Growth with Two Endogenous Factors, in: Quarterly Journal of Economics, Bd. 77, S. 349-371.

North, D.C. (1990), Institutions, Institutional Change and Economic Performance, Cambridge u.a.

OECD (Hrsg.) (1991), Technology and Productivity: the Challenge for Economic Policy, the Technology Economy Programme, Paris.

Olson, M.(1965), The Logic of Collective Action, Cambridge (Mass.).

Olson, M. (1982), The Rise and Decline of Nations, New Haven.

Oppenländer, K. (1963), Die moderne Wachstumstheorie, Berlin und München. 
Pack, H. (1994), Endogenous Growth Theory: Intellectual Appeal and Empirical Shortcomings, in: The Journal of Economic Perspectives, Bd. 8, Nr. 1, S. 55-72.

Paqué, K.H. (1993), A Recipe for Prosperity? Policy Implications of the New Growth Theories, in: Siebert, H. (Hrsg.) (1993), Economic Growth in the World Economy, Tübingen, S. 273-287.

Paqué, K.H. (1995), Technologie, Wissen und Wirtschaftspolitik - Zur Rolle des Staates in Theorien des endogenen Wachstums, in: Die Weltwirtschaft, Nr. 3, S. 237-253.

Parikh, A. (1978), Differences in Growth Rates and Kaldor's Laws, in: Economica, Bd. 45, S. 83-91.

Pasinetti, L.L. (1962), Rate of Profit and Income Distribution in Relation to the Rate of Economic Growth, in: Review of Economic Studies, Bd. 29, S. 267-279.

Pasinetti, L.L. (1981), Structural Change and Economic Growth - A Theoretical Essay on the Dynamics of the Wealth of Nations, Cambridge.

Pasinetti, L.L. (1993a), Structural Economic Dynamics - A Theory of the Economic Consequences of Human Learning, Cambridge.

Pasinetti, L.L. (Hrsg.) (1993b), Italian Economic Papers, Vol. II, Oxford.

Pasinetti, L.L. (1994), The Structure of Long-Term Development: Concluding Comments, in: Pasinetti, L.L., Solow, R.M. (Hrsg.) (1994), Economic Growth and the Structure of LongTerm Development: Proceedings of the IEA Conference held in Varenna (Italy), New York, S. 353-362.

Pasinetti, L.L., Solow, R.M. (Hrsg.) (1994), Economic Growth and the Structure of LongTerm Development: Proceedings of the IEA Conference held in Varenna (Italy), New York.

Phelps, E.S. (1961), The Golden Rule of Accumulation: A Fable for Growthmen, in: American Economic Review, Bd. 51, S. 638-643.

Phelps, E.S. (1962), The New View of Investment: A Neoclassical Analysis, in: Quarterly Journal of Economics, Bd. 76, S. 548-567.

Phelps, E.S. (1966), Golden Rules of Economic Growth, New York.

Pini, P. (1995), Economic Growth, Technological Change and Employment: Empirical Evidence for a Cumulative Growth Model with External Causation for Nine OECD Countries: 1960-1990, in: Structural Change and Economic Dynamics, Bd. 6, S. 185-213.

Pitchford, J.D. (1974), Population in Economic Growth, Contributions to Economic Analysis, Bd. 85, Amsterdam u.a.

Pontryagin, L.S. (1962), Ordinary Differential Equation, Reading (Mass.).

Pontryagin, L.S., Boltyanskii, V.G., Gamkrelidze, R.V., Mischchenko, E.R. (1962), The Mathematical Theory of Optimal Processes, New York. 
Preiser, E. (1970a), Erkenntniswert und Grenzen der Grenzproduktivitätstheorie, in: Preiser, E. (1970c), Bildung und Verteilung des Volkseinkommens, 4. Auflage, Göttingen, S. 265289.

Preiser, E. (1970b), Distribution, in: Preiser, E. (1970c), Bildung und Verteilung des Volkseinkommens, 4. Auflage, Göttingen, S. 290-318.

Preiser, E. (1970c), Bildung und Verteilung des Volkseinkommens, 4. Auflage, Göttingen.

Pusse, L. (1975), Zur Analyse und Prognose der Arbeitsproduktivität auf produktionstheoretischer Basis, in: Mitteilungen aus der Arbeitsmarkt- und Berufsforschung, Bd. 8, Nr. 3, S. 231-242.

Pusse, L. (1990), Überlegungen zur formalen und empirischen Bestimmung der Beschäftigungsschwelle, in: Mitteilungen aus der Arbeitsmarkt- und Berufsforschung, Bd. 23, S. 100-105.

Ramser, H.J. (1993), Grundlagen der Neuen Wachstumstheorie, in: Wirtschaftswissenschaftliches Studium (WiSt), Nr.3, S. 117-123.

Ramser, H.J. (1995), Arbeitslosigkeit und Wirtschaftswachstum, Diskussionsbeiträge der Fakultät für Wirtschaftswissenschaften und Statistik der Universität Konstanz, Serie I Nr. 278, Oktober.

Ramsey, F.P. (1928), A Mathematical Theory of Savings, in: Economic Journal, Bd. 38, S. 543-559.

Rationalisierungskuratorium der Deutschen Wirtschaft e.V. (Hrsg.) (1971), Produktivität und Rationalisierung. Chancen, Wege, Forderungen, Frankfurt a.M.

Rayment, P.B.W. (1981), Structural Change in Manufacturing Industry and the Stability of the Verdoorn Law, in: Economica Internazionale, Bd. 34, S. 104-122.

Rebelo, S. (1991), Long-Run Policy Analysis and Long-Run Growth, in: Journal of Political Economy, Bd. 99, Nr. 3, S. 500-521.

Reich, R.B. (1991), The Work of Nations: A Blueprint for the Future, New York.

Ricoy, Ch.J. (1987), Cumulative Causation, in: Eatwell, J., Milgate, M., Newman, P. (Hrsg.) (1987), The New Palgrave: a Dictionary of Economics, London.

Rieter, H. (Hrsg.) (1996), Studien zur Entwicklung der okonomischen Theorie, Schriften des Vereins für Socialpolitik, N.F., Bd. 115/XV.

Robinson, J. (1933), The Economics of Imperfect Competition, London.

Robinson, J. (1956), The Accumulation of Capital, London.

Romer, D. (1996), Advanced Macroeconomics, New York u.a.

Romer, P.M. (1986a), Increasing Returns and Long-Run Growth, in: Journal of Political Economy, Bd. 94, Nr. 5, S. 1002-1037. 
Romer, P.M. (1986b), Increasing Returns, Specialization, and External Economies: Growth as Described by Allyn Young, Rochester Center for Economic Research Working Paper Nr. 64, University of Rochester, New York.

Romer, P.M. (1987a), Growth Based on Increasing Returns Due to Specialization, in: American Economic Review, Bd. 77, Nr. 2, S. 56-62.

Romer, P.M. (1987b), Crazy Explanations for the Productivity Slowdown, in: Fischer, St. (Hrsg.) (1987), National Bureau of Economic Research Anmual 1987, Cambridge (Mass.), S. 163-202.

Romer, P.M. (1990a), Are Nonconvexities Important for Understanding Growth?, in: American Economic Review, Bd. 80, Nr. 2, Papers and Proceedings, S. 97-103.

Romer, P.M. (1990b), Endogenous Technical Change, in: Journal of Political Economy, Bd. 98, Nr. 5, S. S71-S102.

Romer, P.M. (1990c), Capital, Labor, and Productivity, in: Brookings Papers on Economic Activity: Microeconomics, S. 337-367.

Romer, P.M. (1991), Increasing Returns and New Developments in Growth Theory, in: Barnett, W.A. et al. (Hrsg.) (1991), Equilibrium Theory and Applications, Cambridge, S. 83-110.

Romer, P.M. (1993), Idea Gaps and Object Gaps, in: Journal of Monetary Economics, Bd. 32, S. 543-573.

Romer, P.M. (1994), The Origins of Endogenous Growth, in: Journal of Economic Perspectives, Bd. 8, Nr. 1, S. 3-22.

Rose, K. (1987), Grundlagen der Wachstumstheorie: eine Einfiihrung, 5. Aufl., Göttingen.

Rosen, S. (1987), Human Capital, in: Eatwell, J., Milgate, M., P. Newman, P. (Hrsg.) (1987), The New Palgrave: a Dictionary of Economics, London, S. 681-690.

Rostow, W.W. (1990), Theorists of Economic Growth from David Hume to the Present: with a Perspective on the Next Century, New York und Oxford.

Rostow, W.W. (1990b), The Stages of Economic Growth. A Non-communist Manifesto, 3. Auflage, Cambridge.

Rothschild, K.W. (1959), The Limitations of Economic Growth Models: Critical Remarks on Some Aspects of Mr. Kaldor's Model, in: Kyklos, Bd. 12, Nr. 4, S. 567-586.

Rowthorn, R.E. (1975a), What Remains of Kaldor's Law?, in: Economic Journal, Bd. 85, S. 10-19.

Rowthorn, R.E. (1975b), A Reply to Lord Kaldor's Comment, in: Economic Journal, Bd. 85, S. 897-901.

Rowthorn, R.E. (1979), A Note on Verdoorn's Law, in: Economic Journal, Bd. 89, S. 131133. 
Saint-Paul, G. (1992), Fiscal Policy in an Endogenous Growth Model, in: Quarterly Journal of Economics, Bd. 107, S. 1243-1259.

Sala-i-Martin, X. (1994), Cross-Sectional Regressions and the Empirics of Economic Growth, in: European Economic Review, Bd. 38, S. 739-747.

Sala-i-Martin, X. (1996), The Classical Approach to Convergence Analysis, in: Economic Journal, Bd. 106, S. 1019-1036.

Salter, W.E.G. (1960), Productivity and Technological Change, Cambridge.

Samuelson, P.A. (1988), The Passing of the Guard in Economics, in: Eastern Economic Journal, Bd. 14, Nr. 4, S. 319-329.

Sato, R. (1968), Fiskalpolitik in einem neoklassischen Wachstumsmodell: eine Untersuchung der Zeitdauer des Anpassungsprozesses, in: König, H. (Hrsg.) (1968), Wachstum und Entwicklung der Wirtschaft, Köln und Berlin, S. 175-184.

Schmookler, J. (1966), Invention and Economic Growth, Cambridge (Mass.).

Schneider, J., Ziesemer, Th. (1995), What's New and What's Old in New Growth Theory: Endogenous Technology, Microfoundation and Growth Rate Predictions: a Critical Overview, in: Zeitschrift für Wirtschafts- und Sozialwissenschaften, Bd. 115, S. 429-472.

Schnur, P. (1990), Investitionstätigkeit und Produktivitätsentwicklung. Empirische Analyse auf der Basis eines erweiterten Verdoorn-Ansatzes, in: Mitteilungen aus der Arbeitsmarkt- und Berufsforschung, Bd. 23, Nr. 1, S. 106-110.

Schultz, T.P. (1993), The Role of Education and Human Capitel in Economic Development: An Empirical Assessment, in: Siebert, H. (Hrsg.) (1993), Economic Growth in the World Economy, Tübingen, S. 145-164.

Schultz, T.W. (1961), Investment in Human Capital, in: American Economic Review, Bd. 51, Nr. 1, S. 1-17.

Schumann, J. (1969), Zur Theorie optimalen wirtschaftlichen Wachstums, in: Zeitschrift für die gesamte Staatswissenschaft, Bd. 125, S. 1-16.

Schumpeter, J.A. (1934), Theorie der wirtschaftlichen Entwicklung: eine Untersuchung über Unternehmergewinn, Kapital, Kredit, Zins und den Konjunkturzyklus, 4. Auflage, 7. Nachdruck, Berlin.

Scitovsky, T. (1954), Two Concepts of External Economies, in: Journal of Political Economy, Bd. 64, S. $143-151$.

Scott, M.F. (1991), A New View of Economic Growth, Oxford.

Scott, M.F. (1992), A New Theory of Endogenous Economic Growth, in: Oxford Review of Economic Policy, Bd. 8, Nr. 4, S. 29-42.

Sen, A. (Hrsg.) (1970), Growth Economics, Hartmondsworth. 
Shaw, G.K. (1992), Policy Implications of Endogenous Growth Theory, in: Economic Journal, Bd. 102, S. 611-621.

Shell, K. (1967a), Optimal Programs of Capital Accumulation for an Economy in Which There Is Exogenous Technological Change, in: Shell, K. (Hrsg.) (1967c), Essays on the Theory of Optimal Economic Growth, Cambridge (Mass.), S. 1-30.

Shell, K. (1967b), A Model of Inventive Activity and Capital Accumulation, in: Shell, K. (Hrsg.) (1967c), Essays on the Theory of Optimal Economic Growth, Cambridge (Mass.), S. 67-85.

Shell, K. (Hrsg.) (1967c), Essays on the Theory of Optimal Economic Growth, Cambridge (Mass.).

Sheshinski, E. (1967), Optimal Accumulation with Learning by Doing, in: Shell, K. (Hrsg.) (1967c), Essays on the Theory of Optimal Economic Growth, Cambridge (Mass.), S. 31-52.

Siebert, H. (Hrsg.) (1993), Economic Growth in the World Economy, Tübingen.

Silverberg, G., Soete, L. (Hrsg.) (1994), The Economics of Growth and Technical Change: Technologies, Nations, Agents, Aldershot.

Skott, P. (1989), Kaldor's Growth and Distribution Theory, Frankfurt a.M. u.a.

Skott, P. (1990), Vicious Circles and Cumulative Causation, in: Arestis, Ph., Kitromilides, Y. (Hrsg.) (1990), Theory and Policy in Political Economy: Essays in Pricing, Distribution, and Growth, Aldershot, S. 270-300.

Skott, P., Auerbach, P. (1995), Cumulative Causation and the "New" Theories of Economic Growth, in: Journal of Post Keynesian Economics, Bd. 17, Nr. 3, S. 381-402.

Smith, A. (1993), An Inquiry into the Nature and Causes of the Wealth of Nations, hrsg. mit einer Einleitung und einem Kommentar von K. Sutherland in der Reihe The World's Classics, Oxford.

Soete, L., Verspagen, B. (1994), Competing for Growth: The Dynamics of Technology Gaps, in: Pasinetti, L.L., Solow, R.M. (Hrsg.) (1994), Economic Growth and the Structure of Long-Term Development: Proceedings of the IEA Conference held in Varenna, (Italy) New York, S. 272-299.

Solow, R.M. (1956), A Contribution to the Theory of Economic Growth, in: Quarterly Journal of Economics, Bd. 70, Nr. 1, S. 65-94, hier Nachdruck in: Sen, A. (Hrsg.) (1970), Growth Economics, Hartmondsworth, S. 161-192.

Solow, R.M. (1957), Technical Change and the Aggregate Production Function, in: Review of Economics and Statistics, Bd. 39, S. 312-320.

Solow, R.M. (1959), Investment and Technical Progress, in: Arrow, K.J., Karlin, S., Suppes, P. (Hrsg.) (1959), Mathematical Methods in the Social Sciences, Stanford, S. 89-104.

Solow, R.M. (1971), Wachstumstheorie: Darstellung und Anwendung, Göttingen (Dt. Übersetzung von Growth Theory: An Exposition, Oxford, 1970). 
Solow, R.M. (1991), New Directions in Growth Theory, in: Gahlen, B., Hesse, H., Ramser, H.J. (Hrsg.) (1991), Wachstumstheorie und Wachstumspolitik: ein neuer Anlauf, Schriftenreihe des Wirtschaftswissenschaftlichen Seminars Ottobeuren, Bd. 20, Tübingen, S. 3-17.

Solow, R.M. (1992a), Siena Lectures on Endogenous Growth, Siena.

Solow, R.M. (1992b), Policies for Economic Growth, in: DeEconomist, Bd. 140, S. 1-15.

Solow, R.M. (1994a), Perspectives on Growth Theory, in: Journal of Economic Perspectives, Bd. 8, Nr. 1, S. 45-54.

Solow, R.M. (1994b), Concluding Comments, in: Pasinetti, L.L., Solow, R.M. (Hrsg.) (1994), Economic Growth and the Structure of Long-Term Development: Proceedings of the IEA Conference held in Varenna (Italy), New York, S. 376-379.

Solow, R.M., Samuelson, P. A. (1953), Balanced Growth under Constant Returns to Scale, in: Econometrica, Bd. 21, S. 412-424.

Solow, R.M., Tobin, J., Weizsäcker, C.Chr.v., Yaari, M. (1966), Neoclassical Growth with Fixed Factor Proportions, in: Review of Economic Studies, Bd. 33, S. 79-115.

Sraffa, P. (1926), The Laws of Returns under Competitive Conditions, in: Economic Journal, Bd. 36, S. 535-550.

Steinmann, P.G. (1974), Bevölkerungswachstum und Wirtschaftsentwicklung. Neoklassische Wachstumsmodelle mit endogenem Bevölkerungswachstum, Berlin.

Stern, N. (1992), The Determinants of Growth, in: Hey, J.D. (Hrsg.) (1992), The Future of Economics, Oxford, S. 122-133.

Stigler, G. (1941), Production and Distribution Theories, New York.

Stigler, G. (1951), The Division of Labor is Limited by the Extent of the Market, in: Journal of Political Economy, Bd. 59, S. 185-193.

Stokey, N. (1988), Learning by Doing and the Introduction of New Goods, in: Journal of Political Economy, Bd. 96, S. 701-717.

Stokey, N. (1991), Human Capital, Product Quality, and Growth, in: Quarterly Journal of Economics, Bd. 106, S. 587-616.

Stolpe, M. (1992), Ansätze der neuen Wachstumstheorie - ein Literaturüberblick, Kieler Arbeitspapier Nr. 508, Institut für Weltwirtschaft, Kiel.

Stolpe, M. (1993), Industriepolitik aus Sicht der neuen Wachstumstheorie, in: Die Weltwirtschaft, Nr. 3, S. 361-377.

Straßberger, F. (1995), Technischer Wandel und wirtschaftliches Wachstum: Einige jüngere Entwicklungen, empirische Ergebnisse und wirtschaftspolitische Konsequenzen, in: $D I W$ Vierteljahreshefte, Bd. 64, S. 200-220. 
Summers, L.H. (1990), What is the Social Return to Capital Investment?, in: Diamond, P. (Hrsg.) (1990), Growth/Productivity/Employment: Essays to Celebrate Bob Solow's Birthday, Cambridge (Mass.), S. 113-141.

Summers, R., Heston, A. (1988), A New Set of International Comparisons of Real Economic Product and Prices for One Hundred and Thirty Countries, 1950-1985, in: Review of Income and Wealth, Bd. 34, Nr.1, S. 1-25.

Swan, T.W. (1956), Economic Growth and Capital Accumulation, in: Economic Record, Bd. 32, S. 334-361.

Syrquin, M. (1994), Structural Transformation and the New Growth Theory, in: Pasinetti, L.L., Solow, R.M. (Hrsg.) (1994), Economic Growth and the Structure of Long-Term Development: Proceedings of the IEA Conference held in Varenna (Italy), New York, S. 3-21.

Targetti, F. (1985), Growth and the Terms of Trade: A Kaldorian Two Sector Model, in: Metroeconomica, Bd. 37, S. 79-96.

Targetti, F. (1992), Nicholas Kaldor: the Economics and Politics of Capitalism as a Dynamic System, Oxford.

Targetti, F., Thirlwall, A.P. (Hrsg.) (1989), The Essential Kaldor, London.

Thirlwall, A.P. (1980), Rowthorn's Interpretation of Verdoorn's Law, in: Economic Journal, Bd. 90 , S. 386-388.

Thirlwall, A.P. (1983), A Plain Man's Guide to Kaldor's Growth Laws, in: Journal of Post Keynesian Economics, Bd. 5, Nr. 3, S. 345-358.

Thirlwall, A.P. (1986), A General Model of Growth and Development on Kaldorian Lines, in: Oxford Economic Papers, Bd. 38, S. 199-219.

Thirlwall, A.P. (1987), Nicholas Kaldor, New York.

Thirlwall, A.P. (1992), A Kaldorian Model of Growth and Development Revisited: A Rejoinder to Dutt, in: Oxford Economic Papers, Bd. 44, S. 169-172.

Thirlwall, A.P. (1994), Cumulative Causation, in: Arestis, Ph., Sawyer, M. (Hrsg.) (1994), The Elgar Companion to Radical Political Economy, Aldershot, S. 62-65.

Tichy, G. (1991), Wachstumstheorie und moderne Makroökonomik: (K)ein neuer Anlauf, in: Gahlen, B., Hesse, H., Ramser, H.J. (Hrsg.) (1991), Wachstumstheorie und Wachstumspolitik: ein neuer Anlauf, Tübingen, S. 91-109.

Tinbergen, J. (1942), Zur Theorie der langfristigen Wirtschaftsentwicklung, in: Weltwirtschaftliches Archiv, Bd. 55, Nr. 3, S. 511-549.

Tirole, J. (1995), Industrieökonomik, München.

Tobin, J. (1955), A Dynamic Aggregative Model, in: Journal of Political Economy, Bd. 63, Nr. 2, S. 103-115. 
Tsuru, S. (1993), Japan's Capitalism: Creative Defeat and Beyond, Cambridge.

Tsuruta, T. (1988), The Rapid Growth Era, in: Komiya, R., Okuno, M., Suzumura, K. (Hrsg.) (1988), Industrial Policy of Japan, Tokio u.a., S. 49-87.

Tu, P.N.V. (1994), Dynamical Systems: an Introduction with Applications in Economics and Biology, 2. durchgesehene und erweiterte Auflage, Berlin u.a.

Uekusa, M. (1988), The Oil Crisis and After, in: Komiya, R., Okuno, M., Suzumura, K. (Hrsg.) (1988), Industrial Policy of Japan, Tokio u.a., S. 89-117.

Uhlmann, L. (1978), Freisetzung: ein Denkmodell wurde Wirklichkeit, in: ifo-Schnelldienst, Bd. 31, Nr. 36, S. 3-8.

Uhlmann, L. (1987), Freisetzungsberechnungen. Rückblickende Überlegungen und eine neue Prognose, in: ifo-Studien, Bd. 24, Nr. 33, S. 195-222.

Uhlmann, L., Huber, G. (1971), Technischer Wandel und struktureller Wandel in der wachsenden Wirtschaft. Zweiter Band des Forschungsprojekts des RationalisierungsKuratoriums der Deutschen Wirtschaft (RKW) e.V. "Wirtschaftliche und soziale Aspekte des technischen Wandels in der Bundesrepublik Deutschland", Frankfurt a.M.

Uzawa, H. (1965), Optimum Technical Change in an Aggregative Model of Economic Growth, in: International Economic Review, Bd. 6, Nr. 1, S. 18-31.

Vaciago, G. (1975), Increasing Returns and Growth in Advanced Economies: A Reevaluation, in: Oxford Economic Papers, Bd. 27, S. 232-239.

Varian, H.R. (1991), Grundzüge der Mikroökonomie, 2. Auflage, München und Wien (dt. Übersetzung von Intermediate Microeconomics, $2^{\text {nd }}$ ed., New York).

Verdoorn, P.J. (1949), Fattori che regolano lo sviluppo della produttività del lavoro, in: L'Industria, S. 45-53.

Verdoorn, P.J. (1956), Complementary and Long-Range Projections, in: Econometrica, Bd. 24, S. 429-450.

Verdoorn, P.J. (1959), Capital and Technical Development in Long-Term Projections Models, in: Cahiers Economiques de Bruxellles, Bd. 5, S. 59-69.

Verdoorn, P.J. (1980), Verdoorn's Law in Retrospect: A Comment, in: Economic Journal, Bd. 90 , S. 382-385.

Verdoorn, P.J. (1993), On the Factors Determining the Growth of Labour Productivity, in: Pasinetti, L.L. (Hrsg.) (1993b), Italian Economic Papers, Vol. II, Oxford, S. 59-68. [Englische Übersetzung von Verdoorn, P.J. (1949), Fattori che regolano lo sviluppo della produttività del lavoro, in: L'Industria, S. 45-53 durch A.P. Thirlwall.]

Vernon, R. (1966), International Investment and International Trade in the Product Cycle, in: Quarterly Journal of Economics, Bd. 80, S. 190-207.

Verspagen, B. (1992), Endogenous Innovation in Neo-Classical Models: A Survey, in: Journal of Macroeconomics, Bd. 14, Nr. 4, S. 631-662. 
Verspagen, B. (1994), Technology and Growth: the Complex Dynamics of Convergence and Divergence, in: Silverberg, G., Soete, L. (Hrsg.) (1994), The Economics of Growth and Technical Change: Technologies, Nations, Agents, Aldershot, S. 154-183.

Viner, J. (1932), Cost Curves and Supply Curves, in: Zeitschrift für Nationalökonomie, Bd. 3, S. 23-46.

Walter, H. (1969), Der technische Fortschritt in der neueren ökonomischen Theorie: Versuch einer Systematik, Berlin.

Walter, H. (1977), Technischer Fortschritt I: in der Volkswirtschaft, in: Handwörterbuch der Wirtschaftswissenschaften (HdWW), Stuttgart u.a., S. 569-583.

Walter, H. (1983), Wachstums- und Entwicklungstheorie, Stuttgart u.a.

Weber, M. (1904), Die protestantische Ethik und der "Geist" des Kapitalismus I, in: Archiv für Sozialwissenschaft und Sozialpolitik, Bd. 20, S. 1-54.

Weber, M. (1905), Die protestantische Ethik und der "Geist" des Kapitalismus II, in: Archiv fuir Sozialwissenschaft und Sozialpolitik, Bd. 21, S. 1-10.

Wegner, E. (1981), Die personelle Verteilung der Arbeitseinkommen: betriebliche Herrschaft und Lohnstruktur, Frankfurt a.M. und New York.

Weizsäcker, C.Chr. v. (1966), Zur ökonomischen Theorie des technischen Fortschritts, Göttingen.

Weltbank (1987), Korea: Managing the Industrial Transition, Vol. I+II, A Worldbank Case Study, Washington, D.C.

Wicksell, K. (1898), Geldzins und Güterpreise, Jena.

Wolfe, J.N. (1968), Productivity and Growth in Manufacturing Industry: Some Reflections on Professor Kaldor's Inaugural Lecture, in: Economica, Bd. 35, S. 117-126.

Young, A.A. (1928), Increasing Returns and Economic Growth, in: Economic Journal, Bd. 38, S. 527-542.

Young, A. (1991), Learning by Doing and the Dynamic Effects of International Trade, in: Quarterly Journal of Economics, Bd. 106, S. 369-406.

Ziesemer, Th. (1987), Economic Theory of Underdevelopment, Regensburg.

Ziesemer, Th. (1991), Human Capital, Market Structure and Taxation in a Growth Model with Endogenous Technical Progress, in: Journal of Macroeconomics, Bd. 13, Nr. 1, S. 47-68. 
Stephan Seiter - 978-3-631-75406-1

Downloaded from PubFactory at 01/11/2019 05:22:48AM

via free access 
Symbolverzeichnis (ausgewăhlte Symbole)

\section{a) Kapitel 2}

A Stand des technologischen Wissens

$\mathrm{E}_{\mathrm{Yi}} \quad$ Produktionselastizităt des Produktionsfaktors i

$F_{i} \quad$ Grenzproduktivităt des Produktionsfaktors i

G Wachstumsrate in den Zitaten von Kaldor

H Hamiltonfunktion

$\mathrm{H}_{\mathrm{t}} \quad$ Stand des durch Harrod-neutralen technischen Fortschritt erzeugten Wissens

I Investitionen

K Kapitalstock

L Arbeitsmenge

Leff effiziente Arbeitseinheiten

P Profitsumme

S Sparen

$S_{t} \quad$ Stand des durch Solow-neutralen technischen Fortschritt erzeugten Wissens

U Nutzenfunktion

W Lohnsumme

Y Output bzw. Sozialprodukt

br brutto

c Pro-Kopf-Konsum

g Wert einer Variablen auf dem optimalen Wachstumspfad ("Goldene Regeln")

k Kapitalintensităt

$k^{\text {eff }}$ Kapitalintensităt in effizienten Arbeitseinheiten gemessen

n Wachstumsrate der Bevolkerung

$\overline{\mathbf{r}} \quad$ durchschnittlicher Zinssatz

s Sparquote

Sp Sparquote aus Profiten

SW Sparquote aus Löhnen

t Zeitterm

u (a) Arbeitskoeffizienten

(b) Nutzen aus Konsum

v Kapitalkoeffizient

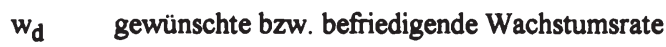

$w_{t} \quad$ tatsăchliche Wachstumsrate

y Pro-Kopf-Output

$\Omega \quad$ Produktionskapazităt 
$\alpha \quad$ Produktionselastizität des Kapitals

$\beta_{\mathrm{r}} \quad$ Akzelerator

$\gamma \quad$ Rate des exogenen technischen Fortschritts

$\gamma_{\mathrm{H}} \quad$ Rate des exogenen Harrod-neutralen technischen Fortschritts

$\gamma_{\mathrm{HI}}$ Rate des exogenen Hicks-neutralen technischen Fortschritts

$\gamma_{\mathrm{S}} \quad$ Rate des exogenen Solow-neutralen technischen Fortschritts

$\delta \quad$ Abschreibungsrate

$\xi \quad$ Kapitalgebundener technischer Fortschritt im Phelps-Modell

I Durchschnittsproduktivität der Investitionen

$\lambda$ Grenznutzenelastizität

$\rho \quad$ Zeitpräferenzrate

$\sigma \quad$ Intertemporale Substitutionselastizität

$\tau$ Parameter in Hamiltonfunktion

$\phi \quad$ Funktionsterm

\section{Tinbergen-Modell}

$\begin{array}{ll}\text { a } & \text { Arbeitsmenge } \\ \mathrm{b} & \text { Bevölkerungsmenge } \\ \mathrm{i}^{\mathrm{r}} & \text { Realzins } \\ \mathrm{I}^{\mathrm{r}} & \text { Reallohn } \\ 1^{\mathrm{o}} & \text { Konstante in Gleichung furr Normallohn } \\ 1^{\circ} & \text { Normallohn } \\ \mathrm{u} & \text { Outputmenge } \\ \varepsilon & \text { Stand des technischen Wissens } \\ \theta & \text { Steigerungsrate des Normallohns } \\ \lambda & \text { Produktionselastizität der Arbeit } \\ \chi & \text { Spar- bzw. Investitionsquote } \\ \varphi & \text { Substitutionsparameter }\end{array}$

\section{b) Kapitel 3 und Kapitel 5}

Catching up-Hypothese und empirische Überprüfung

$\mathrm{P}_{\mathrm{t}} \quad$ Produktivitätsniveau

T Zeitterm

pot Potentielle Wachstumsrate des Produktivitätsniveaus 


$\begin{array}{ll}\mathrm{p}_{\text {tat }} & \text { Tatsächliche Wachstumsrate des Produktivitätsniveaus } \\ \mathrm{s}_{\mathrm{i}} & \text { Spar- bzw. Investitionsquote bei Kapitalart } \mathrm{i} \\ \mathrm{y} & \text { Pro-Kopf-Einkommen } \\ \hat{\mathrm{y}} & \text { Wachstumsrate des Pro-Kopf-Einkommens } \\ \mathrm{t} & \text { Zeitterm } \\ \mathrm{u} & \text { Störterm in Regressionsgleichungen } \\ \Lambda & \text { Konstante in Regressionsrechnung } \\ \alpha & \text { Absoluter Regressionskoeffizient } \\ \beta & \text { Konvergenzparameter }\end{array}$

Arrow-Modell

G Summe der kumulierten Bruttoinvestitionen bzw. Seriennummer der Kapitalgüter

L Arbeitsmenge

N Stückzahl

Y Output bzw. Sozialprodukt

a Arbeitskoeffizient

b Parameter der Lernkurve

n Parameter der Lemkurve

$\lambda$ Multiplikator bei Faktorvariation

Romer-Modell $(1986,1987)$ und AK-Modell

A Stand des technologischen Wissens

C Konsum

E Menge der begrenzten Faktoren

K (a) Kapitalstock

(b) Gesamtwirtschaftliches Know-how

M Zahl der Zwischenprodukte

N (Nichtrivalisierende) Produktionsfaktoren

R (a) Rivalisierende Produktionsfaktoren

(b) Ressource für Produktion des Zwischenprodukts

Z Zwischenprodukt

c Pro-Kopf-Konsum

g Kostenfunktion

k Kapitalintensität

$\mathbf{k}_{\mathbf{i}} \quad$ Wissen des Unternehmens $\mathrm{i}$

s Sparquote 
n Wachstumsrate der Bevölkerung

v Kapitalkoeffizient

$x_{i} \quad$ Vektor aller als konstant angenommenen Produktionsfaktoren

$\delta \quad$ Abschreibungsrate

Lucas-Modelle $(1988,1993)$

H Bestand des durchschnittlichen Humankapitalbestands weltweit

$\mathrm{L}_{\mathrm{h}} \quad$ Menge der Arbeit mit Humankapitalbestand $\mathrm{h}$

L e Effektive Arbeitsmenge

h Individueller Humankapitalbestand

ha Durchschnittlicher individueller Humankapitalbestand

$h_{i} \quad$ Humankapital im Sektor i

q Preisverhältnis zwischen den Gütern im Gleichgewicht

$u_{i} \quad$ Nutzen aus dem Konsum von Gut $i$

$u_{h} \quad$ Anteil der Nichtfreizeit, die für Produktionszwecke verwendet wird.

v Wachstumsrate des Humankapitals

vopt Wachstumsrate des Humankapitals im sozialen Optimum

vlf Wachstumsrate des Humankapitals im Laisser-faire-Gleichgewicht

$x_{i} \quad$ Produktionsmenge des Gutes $i$

$\alpha_{i} \quad$ Parameter der Nutzenfunktion

B Produktionselastizität des Humankapitalbestands bei der Akkumulation des Humankapitals

$\gamma \quad$ Produktionselastizität des durchschnittlichen Humankapitalbestands

$\varepsilon \quad$ Effizienzparameter der Humankapitalbildung

$\rho \quad$ (a) Diskontrate bzw. Zeitpräferenzrate

(b) Substitutionsparameter der Nutzenfunktion

$\sigma \quad$ Intertemporale Substitutionselastizität

$\varphi \quad$ Substitutionselastizität

Romer-Modell $(1990 b, c)$
A Stand des technologischen Wissens
$\mathrm{H}_{\mathrm{i}} \quad$ Menge des im Sektor $\mathrm{i}$ eingesetzten Humankapitals
K Kapitalstock
L Arbeitsmenge
Y Sozialprodukt
$x_{i} \quad$ Menge des Kapitalgutes i 
$\alpha \quad$ Produktionselastizität der Arbeit in der Produktion des Endprodukts

$\beta \quad$ Produktionselastizität des Humankapitals in der Produktion des Endprodukts

$\varepsilon \quad$ Effizienzparameter bei der Wissensentstehung

$\eta \quad$ Menge des für die Herstellung einer Kapitalguteinheit notwendigen Konsumverzichts

Grossman/Helpman (1991) und Aghion/Howitt (1992)

A Stand des technologischen Wissens

D Konsumindex

U Nutzen

$L_{i} \quad$ Arbeitmenge im Sektor $i$

Lq Menge der qualifizierten Arbeit

Ls Menge der unspezialisierten Arbeit

Luq Menge der unqualifizierten Arbeit

a Arbeitskoeffizient

n Zahl der vorhandenen Produkte

$x_{i} \quad$ Menge des Konsumgutes i

$\alpha \quad$ Substitutionsparameter

$\rho \quad$ Zeitpräferenz bzw. Diskontrate

C) Kapitel 4

A Stand des technologischen Wissens

B Bevölkerung

I Investitionen

K Kapitalstock

L Arbeitsmenge

P Arbeitsproduktivität

T Aufschlagssatz bei Preiskalkulation

V Verdoorn-Elastizität

X Export

Y Output

W Niveau der Geldlöhne

Z Einkommensniveau weltweit

d Dummy-Variable

k Wachstumsrate des Kapitalstocks

l Wachstumsrate der Arbeitsmenge 
n Wachstumsrate der Bevölkerung

p Wachstumsrate der Arbeitsproduktivităt

t Zeitterm

v Kapitalkoeffizient

w Reallohn

x Wachstumsrate der Exporte

y Wachstumsrate des Ouputs

$\theta$ Autonome Komponente in der technical progress function

$\Pi_{d} \quad$ Inlandspreis

$\Pi_{\mathrm{f}} \quad$ Weltmarktpreis

$\alpha \quad$ Produktionselastizität des Kapitals

B Produktionselastizität der Arbeit

$\gamma \quad$ (a) Lohnflexibilität

(b) Elastizität des Outputwachstums bzgl. des Exportwachstums

$\delta \quad$ Kreuzpreiselastizität der Exportnachfrage

$\varepsilon \quad$ (a) Einkommenselastizität der Exportnachfrage

(b) Parameter

$\zeta \quad$ Quotient aus k und 1

$\eta \quad$ Preiselastizität der Exportnachfrage

P Parameter

K Wachstumsrate der Kapitalintensität

$\lambda \quad$ (a) Wachstumsrate der Löhne

(b) Verdoorn-Koeffizient

$\boldsymbol{\mu} \quad$ Wachstumsrate des Sozialprodukts

$v \quad$ Hicks-neutraler technischer Fortschritt

$\pi \quad$ Parameter in technical progress function

$\tau \quad$ Parameter in technical progress function

$\chi \quad$ Spar-bzw. Investitionsquote 


\section{HOHENHEIMER VOLKSWIRTSCHAFTLICHE SCHRIFTEN}

Band 1 Walter Deffaa: Anonymisierte Befragungen mit zufallsverschlüsselten Antworten. Die Randomized-Response-Technik (RRT). Methodische Grundlagen, Modelle und Anwendungen. 1982.

Band 2 Thomas Michael Baum: Staatsverschuldung und Stabilisierungspolitik in der Demokratie. Zur neoinstitutionalistischen Kritlik der keynesianischen Fiskalpolitik. 1982.

Band 3 Klaus Schröter. Die wettbewerbspolitische Behandlung der leitungsgebundenen Energiewirtschaft. Dargestellt am Beispiel der Fernwărmewirtschaft der Bundesrepublik Deutschland. 1986.

Band 4 Hugo Mann: Theorie und Politik der Steuerreform in der Demokratie. 1987.

Band 5 Max Christoph Wewel: Intervallarithmetische Dependenzanalyse in der Ökonometrie. Ein konjekturaler Ansatz. 1987.

Band 6 Heinrich Pascher: Die U.S.-amerikanische Deregulation Policy im Luftverkehrs- und Bankenbereich. 1987.

Band 7 Harald Lob: Die Entwicklung der franzősischen Wettbewerbspolitik bis zur Verordnung Nr. 86-1243 vom 01. Dezember 1986. Eine exemplarische Untersuchung der Erfassung der Behinderungsstrategie auf der Grundlage des Konzepts eines wirksamen Wettbewerbs. 1988.

Band 8 Ulrich Kirschner: Die Erfassung der Nachfragemacht von Handelsunternehmen. Eine Analyse der ókonomischen Beurteilungskriterien und der wettbewerbsrechtlichen Instrumente im Bereich der Verhaltenskontrolle.1988.

Band 9 Friedhelm Herb: Marktwirtschaftliche Innovationspolitik. 1988.

Band 10 Claus Schnabel: Zur ókonomischen Analyse der Gewerkschaften in der Bundesrepublik Deutschland. Theoretische und empirische Untersuchungen von Mitgliederentwicklung, Verhalten und Einfluß auf wirtschaftliche Großßen. 1989.

Band 11 Jan B. Rittaler: Industrial Concentration and the Chicago School of Antitrust Analysis. A Critical Evaluation on the Basis of Effective Competition. 1989.

Band 12 Thomas Mărtz: Interessengruppen und Gruppeninteressen in der Demokratie. Zur Theorie des Rent-Seeking. 1990.

Band 13 Andreas Maurer: Statistische Verfahren zur Ermittlung von oligopolistischen Strukturen. 1990.

Band 14 Peter Mendler. Zur ókonomischen und politisch-institutionellen Analyse öffentlicher Kredithilfen. 1992.

Band 15 Heinrich J. Engelke: Die Interpretation der Rundfunkfreiheit des Grundgesetzes: Eine Analyse aus Okonomischer Sicht. 1992.

Band 16 Thomas Fischer: Staat, Recht und Verfassung im Denken von Walter Eucken. Zu den staats- und rechtstheoretischen Grundlagen einer wirtschaftsordnungspolitischen Konzeption. 1993.

Band 17 Stefan Elßer: Innovationswettbewerb. Determinanten und Unternehmensverhalten. 1993.

Band 18 Reinhard Scharff: Regionalpolitik und regionale Entwicklungspotentiale. Eine kritische Analyse. 1993.

Band 19 Karin Beckmann: Probleme der Regionalpolitik im Zuge der Vollendung des Europăischen Binnenmarktes. Eine Okonomische Analyse. 1995. 
Band 20 Bernd Nolte: Engpaßfaktoren der Innovation und Innovationsinfrastruktur. Eine theoretische und empirische Analyse für lândliche Wirtschaftsrăume in Baden-Württemberg. 1996.

Band 21 Klaus-Rainer Brintzinger: Die Nationalökonomie an den Universităten Freiburg, Heidelberg und Tübingen 1918 - 1945. Eine institutionenhistorische, vergleichende Studie der wirtschaftswissenschaftlichen Fakultäten und Abteilungen südwestdeutscher Universitäten. 1996.

Band 22 Steffen Binder: Die Idee der Konsumentensouverănităt in der Wettbewerbstheorie. Teleokratische vs. nomokratische Auffassung. 1996.

Band 23 Alexander Burger: Deregulierungspotentiale in der Gesetzlichen Rentenversicherung. Reformnotwendigkeiten versus Reformmőglichkeiten. 1996.

Band 24 Burkhard Scherer: Regionale Entwicklungspolitik. Konzeption einer dezentralisierten und integrierten Regionalpolitik. 1997.

Band 25 Frauke Wolf: Lorenzkurvendisparităt. Neuere Entwicklungen, Erweiterungen und Anwendungen. 1997.

Band 26 Hans Pitlik: Politische Ökonomie des Föderalismus. Föderative Kompetenzverteilung im Lichte der konstitutionellen Ökonomik. 1997.

Band 27 Stephan Seiter: Der Beitrag Nicholas Kaldors zur Neuen Wachstumstheorie. Eine vergleichende Studie vor dem Hintergrund der Debatte über den Verdoorn-Zusammenhang. 1997. 
Wolfgang Hermann Müller

\section{Prinzipien wirtschaftlichen Handelns und ihre Anwendung}

\section{Umriß einer Wirtschaftsphilosophie}

Frankfurt/M., Berlin, Bern, New York, Paris, Wien, 1996. 145 S., 1 Abb. ISBN 3-631-30477-3 · br. DM 48. - *

Die enge Verbindung von Wirtschaft und Weltgeschehen verlangt nach einer exakten Untersuchung ihrer Notwendigkeit, Möglichkeiten und Grenzen. Die Ökonomie wird auf ihre erlernbaren theoretischen Prinzipien, die Möglichkeiten ihrer Anwendung und die Bedingungen ihrer praktischen Durchsetzbarkeit hin untersucht. Ergebnis ist eine gründliche Neubestimmung des Verhältnisses von Theorie, Anwendung und Praxis. Die historisch bekannten Wirtschaftsschulen und -richtungen werden auf ihren Geltungsanspruch hinterfragt. Politische und ideologische Rahmenbedingungen werden in ihrem Einfluß auf Privat- und Gemeinwirtschaft erläutert und Modelle zur Überprüfung von Wirtschaftspolitik und Wirtschaftsethik aufgezeigt.

Aus dem Inhalt: Wissenschaftsanspruch, Definition und Methoden der Ökonomie - Einfluß von politischen und ideologischen Rahmenbedingungen . Privat- und Gemeinwirtschaft - Wirtschaftsschulen und Wirtschaftsmodelle • Wirtschaftsrecht und Wirtschaftsethik · Ökonomische Begriffsbildung und Wissenschaft · Ökonomie und Philosophie

Frankfurt/M - Berlin - Bern - New York · Paris - Wien

Auslieferung: Verlag Peter Lang AG

Jupiterstr. 15, CH-3000 Bern 15

Telefax (004131) 9402131

*inklusive Mehrwertsteuer

Preisänderungen vorbehalten 
Stephan Seiter - 978-3-631-75406-1

Downloaded from PubFactory at 01/11/2019 05:22:48AM

via free access 